University of Louisville

ThinkIR: The University of Louisville's Institutional Repository

\title{
$5-2013$
}

\section{Theoretical investigations of photophysics and spectroscopic properties in B12 cofactors.}

\author{
Karina Kornobis \\ University of Louisville
}

Follow this and additional works at: https://ir.library.louisville.edu/etd

\section{Recommended Citation}

Kornobis, Karina, "Theoretical investigations of photophysics and spectroscopic properties in B12 cofactors." (2013). Electronic Theses and Dissertations. Paper 772.

https://doi.org/10.18297/etd/772

This Doctoral Dissertation is brought to you for free and open access by ThinkIR: The University of Louisville's Institutional Repository. It has been accepted for inclusion in Electronic Theses and Dissertations by an authorized administrator of ThinkIR: The University of Louisville's Institutional Repository. This title appears here courtesy of the author, who has retained all other copyrights. For more information, please contact thinkir@louisville.edu. 


\title{
THEORETICAL INVESTIGATIONS OF PHOTOPHYSICS AND SPECTROSCOPIC PROPERTIES IN B ${ }_{12}$ COFACTORS
}

\author{
By \\ Karina Kornobis \\ MS, Wroclaw University of Technology, 2007 \\ A Dissertation \\ Submitted to the Faculty of the \\ College of Arts and Sciences of the University of Louisville \\ in Partial Fulfillment of the Requirements \\ for the Degree of \\ Doctor of Philosophy \\ Department of Chemistry \\ University of Louisville \\ Louisville, Kentucky
}

May 2013 



\title{
THEORETICAL INVESTIGATIONS OF PHOTOPHYSICS AND SPECTROSCOPIC PROPERTIES IN B ${ }_{12}$ COFACTORS
}

\author{
By \\ Karina Kornobis \\ MS, Wroclaw University of Technology, 2007 \\ A Dissertation Approved On \\ April 12th, 2013 \\ by the following Dissertation Committee: \\ Dr. Pawel M. Kozlowski, Dissertation Director
}

Dr. Craig A. Grapperhaus

Dr. George R. Pack

Dr. Francis P. Zamborini

Dr. Jacek M. Zurada 


\section{DEDICATION}

To my loving Mom and Grandparents. 


\section{ACKNOWLEDGEMENTS}

This dissertation appeared as a consequence of combined effort of many people whose advice, faith, understanding, and support helped me to complete it and who I would like to acknowledge. I start with my advisor, Dr. Pawel Kozlowski. We both know that it was often a struggle but I thank you for directing the projects into a publishable research and for all these discussions which helped us both to achieve common goals. I express my gratitude to my dissertation committee members: Dr. Craig Grapperhaus, Dr. George Pack, Dr. Francis Zamborini, and Dr. Jacek Zurada for accepting this challenge exploring my research and opening to computational chemistry. Your questions and comments in response to my seminars were always invaluable helping me to improve every consecutive paper, presentation, and research strategy where I tried to implement your advices. Special thanks to Dr. Grapperhaus for giving me an additional weekend to finish this work in the light of my unexpected condition and to Dr. Zamborini for creating a joyful and pleasant atmosphere and proving that fun in work environment makes a visible difference. I truly appreciate every smile, joke, and caring question that I received a number of times from Dr. Richard Wittebort. Thank you for making these short stops when seeing me regardless of the constant rush, you were in. My five years of teaching experience were interesting not only because of the students, who I met on the way, but also because of all instructors I had a pleasure to interact with and hereby, I would like to acknowledge: Dr. Richard Baldwin, Dr. Donald DuPre, Dr. Richard Higashi, Dr. Aleeta Powe, Dr. Francis Zamborini, and Dr. Xiang Zhang.

I thank all my collaborators and coauthors for professionalism, courtesy in communication, and efficient work. A special gratitude goes to Kenneth Ruud for 
offering me an opportunity to visit the CTCC in Norway and to conduct a research project in a geographically exceptional and magical Tromsø. Your kindness, knowledge, professionalism, always honest assistance, and care have been truly inspiring, exposing the truth about the mentorship. I greatly appreciate having this opportunity to work with you. Thank you Harald for your patience, willingness to help, pleasant cooperation, and sharing your success when you defended your $\mathrm{PhD}$ degree. Bruno, Adam, Antoine, Dan, Michal, Krzysztof, Joanne, Emmanuelle, John, and Inge you made my stay in Troms $\emptyset$ unforgettable. Kasia, thank you for sharing the northern experience with me.

I would not be in Louisville if I was not fortunate to meet and work with Tadeusz Andruniów and I would not survive without Dr. John Nyland. Tadeusz, thank you for inspiring me and showing me where the light can be found in computational chemistry. I immensely value your guidance, disinterested help, support, and friendship. Our connection has been truly amazing as you are always there for me regardless of the way I am calling you. Dr. Nyland, you taught me how to walk again and how to always get up, and stand on my feet. You are my Angel. Thank you for sharing with me and involving me in your family life.

Renia, Janusz, Karol, Damian, and Adrian I am so grateful to be a part of your family. Thank you for all holidays and meetings that we shared, I received from you so much and so often. I also thank my aunt Krysia who first showed me the USA, drove me to Louisville despite the heavy snow and helped me many times.

My stay in Louisville was enriched by exceptional people, I met and who will always be a part of me. Monica, thank you for your honest friendship and much more than words can express. Jose Carlos, your love, understanding, and time we shared will stay in my heart and memory forever.

Thank you Janek for recognizing me speaking Polish and introducing me to the Polish crew in town as well as cultural entertainments. Maciek, Ewa, Sylwia, Malwina, and Magda, our meetings have been a real fun. Maciek, thank you for helping with a watered laptop emergency and great talks.

Gilberto, Celeste, Ramiro, and Valeria, you have brought so much joy ev- 
ery time we were meeting. I love spending time with you even if you only speak Spanish.

I would not stay positive without huge support from my friends from Poland. Mikołaj we went through so many things together. Thank you for your support, care, and friendship. Ola and Tomek, Magda and Łukasz, Beata and Piotr, although far, we are always close and connected. Thank you for making going outs, weddings, staying ins, baby sittings, and all meetings so true and happy. Ania we always remember and think about each other. Thank you for our travels through life and being there for me so many times. Kasia, our friendship started in high school and gives me strength every day since. I am so grateful for every positive word or thought, you have sent during my studies and over this whole time.

I thank my international friends for making the stay in Louisville so colorful. Irina, your natural attitude is so valuable. Thank you for talks in Russian, making me laugh spontaneously and for your care. Artur, thank you for eager rescuing me from many emergencies and your smiles. Neeraj and Deepika thanks for your support and understanding in many moments. Shu, Davinder, and Shahbaz thanks for playing badminton with me. Iga and Manoj, I am glad we met and shared the same lab.

My biggest gratitude belongs to my Mom, my Grandparents, my Sister, and my Father. You made me who I am and I would not be here without you. Mamusiu, your unconditional love goes beyond any measures. You are with me and for me always, everywhere and in every moment that I need it. There are no words which can express how thankful I am to have you as my Mom. Babciu, Dziadziusiu and Ciociu Stasiu your love builds me every day, your undisturbed faith in me has been uplifting. Gosiu, I know that I can always count on you. Thank you for believing in me and cheering me up. Tatusiu, thank you for your support.

Finally, I thank you my dear Radovan for being with me on this journey since the moment we met. I would not get to the finish line without you. Thank you for caring about me, supporting me and making me believe that I can always count on you. Your love is my blessing. 


\begin{abstract}
THEORETICAL INVESTIGATIONS OF PHOTOPHYSICS AND SPECTROSCOPIC PROPERTIES IN $\mathrm{B}_{12}$ COFACTORS
\end{abstract}

Karina Kornobis

April 12th, 2013

Enzymes incorporating $\mathrm{B}_{12}$ cofactors (cobalamins) are significant biological components as they are involved in the metabolism of every cell of the body. The absorbing riddle, yet unresolved, is the enormous acceleration of the reaction rate that is strictly related to the $\mathrm{Co}-\mathrm{C}$ bond cleavage initiating every catalytic cycle. Consequently, the realistic explanation of the processes controlled by $\mathrm{B}_{12}$ derivatives demands a profound analysis of their structure coupled with the examination of factors influencing subsequent steps of the reaction as well as properties of the reagents and intermediates. Herein, the photochemical study of cobalamins was presented as it provides a sensitive probe for the investigation of the reactivity of the cobalt-carbon bond in terms of any structural or environmental modification. Theoretical investigations, guided by Density Functional Theory (DFT), Time-Dependent DFT (TD-DFT) and correlated $a b$ initio methods were performed in order to explore the nature of electronically excited states of different vitamin $B_{12}$ derivatives and the mechanism of events following the photon excitation in vitamin $\mathrm{B}_{12}$. Initially, the most appropriate density functional to study the photochemistry of these complex molecules was determined. Its accuracy was evaluated with respect to both its agreement with available experimental data and higher level ab initio outcomes. 
Next, the absorption (Abs), circular dichroism (CD) and magnetic CD were calculated for the first time. Based on the examination of certain spectral regions the electronic properties of $\mathrm{B}_{12}$ derivatives were examined. The correlation between the nature of specific electronic transitions and the structure was extensively discussed. In addition, the environmental effect on the character of excited states was investigated as the calculations were performed both in gas phase as well as in water solution modeled via the polarizable continuum model (PCM) or conductor-like screening model (COSMO). In addition, the study of cob(I)alamin, lacking axial ligands, is of significant value especially considering the fact that the complete model of cob(I)alamin was applied as opposed to a majority of previous theoretical efforts. Finally, the lack of photodissociation in CNCbl as opposed to other $\mathrm{B}_{12}$ analogs was investigated in detail. The electronically excited states of $\mathrm{CNCbl}$ were examined as a function of two axial ligands calculated at TD-DFT level of theory. By analyzing the shapes of potential energy surfaces (PESs) and the nature of its lowest excited states in different environments, the explanation of subsequent steps following photon excitation of that system was provided. 


\section{TABLE OF CONTENTS}

ACKNOWLEDGEMENTS

ABSTRACT vii

LIST OF TABLES X xiii

LIST OF FIGURES X Xviii

CHAPTER $\quad$ Page

1 INTRODUCTION 1

$2 \quad$ ELECTRONICALLY EXCITED STATES OF VITAMIN B B $_{12}$

2.1 Introduction .................... 17

2.2 Computational Details . . . . . . . . . . . . . . 20

2.2.1 Structural Models . . . . . . . . . . . 20

2.2 .2 TD-DFT Calculations . . . . . . . . . . . 20

2.2.3 Correlated $a b$ initio calculations . . . . . . . . . . 21

2.2 .4 Basis set . . . . . . . . . . . . . 22

2.3 Results and Discussion . . . . . . . . . . . . . 23

2.3.1 Analysis of absorption data . . . . . . . . . 23

2.3.2 Lambda diagnostic for low-lying excited states . . . . . 29

2.3.3 Comparison with $a b$ initio calculations . . . . . . . 32

2.3.4 Assignment of Electronic Excitations . . . . . . . . . 37

2.4 Summary and Conclusions . . . . . . . . . . . . 45

3 ELECTRONIC STRUCTURE OF THE $S_{1}$ STATE IN METHYLCOBALAMIN

3.1 Introduction . . . . . . . . . . . . 47

3.2 Computational details . . . . . . . . . . . . 50 
3.2.1 Structural models . . . . . . . . . . . . . . . . 50

3.2.2 CASSCF/MC-XQDPT2 calculations . . . . . . . . 51

3.2 .3 EOM-CCSD calculations . . . . . . . . . . . 52

3.2.4 TD-DFT calculations with different functionals . . . . . 53

3.3 Results and discussion . . . . . . . . . . . . . . 55

3.3.1 Experimental information about the low-lying excited states of MeCbl . . . . . . . . . . . . . . 55

3.3.2 Electronic excitations resulting from the CASSCF/MCXQDPT2 calculations . . . . . . . . . . . . . 58

3.3.3 Electronic excitations resulting from the EOM-CCSD calculations . . . . . . . . . . . . . . . 6 65

3.3.4 Comparison of TD-DFT and $a b$ initio wave-function . . . 69

3.3.5 Adiabatic nature of the $S_{1}$ transition $\ldots \ldots \ldots 77$

3.4 Summary and Conclusions $\ldots \ldots \ldots \ldots$

\section{ELECTRONICALLY EXCITED STATES OF VITAMIN $B_{12}$ AND METHYL- COBALAMIN: THEORETICAL ANALYSIS OF ABSORPTION, CD AND MCD DATA}

4.1 Introduction $\ldots \ldots \ldots \ldots \ldots \ldots \ldots$

4.2 Computational Details . . . . . . . . . . . . 86

4.2 .1 Structural models . . . . . . . . . . . . . . . 86

4.2.2 Abs and CD calculations . . . . . . . . . 87

4.2 .3 MCD calculations . . . . . . . . . . . . . . 88

4.2 .4 Functionals and basis set . . . . . . . . . . . 88

4.3 Results and Discussion . . . . . . . . . . . . . . . 89

4.3.1 Absorption spectra of $\mathrm{CNCbl}$ and $\mathrm{MeCbl} \ldots \ldots$

4.3.2 CD spectra of CNCbl and MeCbl . . . . . . . . . . . 94

4.3.3 MCD spectra of CNCbl and MeCbl . . . . . . . . . 96

4.3.4 Comparison between BP86 and CAM-B3LYP results . . . 97

4.3.5 Solvent effects . . . . . . . . . . . . . . . . . . . . 98 


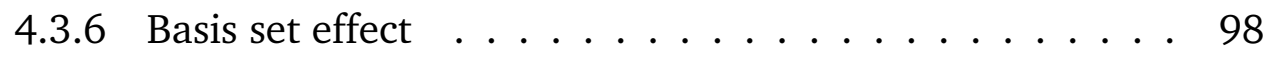

4.3.7 Assignment of electronic transitions . . . . . . . . . . 101

4.4 Summary and Conclusions . . . . . . . . . . . . 110

\section{COB(I)ALAMIN: INSIGHT INTO THE NATURE OF ELECTRONICALLY} EXCITED STATES 113

5.1 Introduction $\ldots \ldots \ldots \ldots \ldots \ldots \ldots$

5.2 Computational details $\ldots \ldots \ldots \ldots 116$

5.2.1 Structural models . . . . . . . . . . . . . . . . . . 116

5.2.2 Abs, CD and MCD spectra in response theory TD-DFT calculations . . . . . . . . . . . . . . 117

5.2.3 CASSCF/MC-XQDPT2 calculations . . . . . . . 120

5.3 Results and discussion . . . . . . . . . . . . . . . 124

5.3.1 Brief summary of earlier work . . . . . . . . . . . 124

5.3 .2 Abs spectra . . . . . . . . . . . . . . . 126

5.3.3 CD and MCD spectra . . . . . . . . . . . . . . . 129

5.3.4 Assignment of electronic transitions . . . . . . . . . . 131

5.3.5 Truncated versus full model . . . . . . . . . . . . 136

5.4 Conclusions . . . . . . . . . . . . . . . . . . . . . . 142

\section{ELECTRONIC AND STRUCTURAL PROPERTIES OF LOW-LYING EX-} CITED STATES OF VITAMIN B 12

6.1 Introduction . . . . . . . . . . . . . . . . . 144

6.2 Computational Details . . . . . . . . . . . . . . . 146

6.3 Results and Discussion . . . . . . . . . . . . . . . . . . 148

6.3.1 Structural Model of CNCbl . . . . . . . . . . . . . . 148

6.3.2 NBO Charges Analysis of CNCbl . . . . . . . . . . . 148

6.3.3 Potential Energy Curves along the Co-C Bond Stretch . . 154

6.3.4 Comparative Analysis of Axial Bonding in CNCbl and MeCbl . . . . . . . . . . . . . . . 157 
6.3.5 Excited States of CNCbl Computed as a Function of Axial Bonds . . . . . . . . . . . . . . . 160

6.3.6 Energy Minima on $\mathrm{S}_{1} / \mathrm{CNCbl}$ and $\mathrm{T}_{1} / \mathrm{CNCbl}$ Surfaces . . 165

6.3.7 Electronic Properties of $S_{1}$ and $T_{1}$ States in CNCbl . . . 166

6.4 Comparison with Transient Absorption Spectroscopy . . . . . 175

6.5 Comparison of $S_{1}$ States in CNCbl and MeCbl . . . . . . . 179

6.6 Summary and Conclusions . . . . . . . . . . . . . . 179

$\begin{array}{lll}7 & \text { CONCLUSIONS } & 181\end{array}$

$\begin{array}{ll}\text { REFERENCES } & 186\end{array}$

$\begin{array}{ll}\text { APPENDIX } & 204\end{array}$

$\begin{array}{ll}\text { CURRICULUM VITAE } & 222\end{array}$ 


\section{LIST OF TABLES}

TABLE

Page

2.1 Vertical excitation energies (eV) of Im- $\left[\mathrm{Co}^{\mathrm{III}}\right.$ (corrin) $]-\mathrm{CN}^{+}$calculated for the first four low-lying states with different methods and the 6-

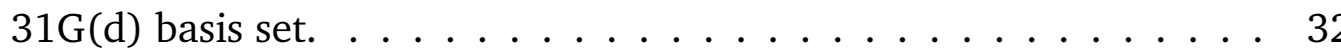

2.2 The first four low-lying excited states of $\mathrm{Im}-\left[\mathrm{Co}^{\mathrm{III}}\right.$ (corrin) $]-\mathrm{CN}^{+}$obtained from CASSCF(12,12)/MC-XQDPT2 calculations ${ }^{a}$. . . . . . 37

2.3 Composition of low-lying electronically excited states of Im-[Co ${ }^{\mathrm{III}}$ (corrin)]$\mathrm{CN}^{+}$based on TD-DFT calculations with the use of different density functionals and the $6-31 \mathrm{G}(\mathrm{d})$ basis set. . . . . . . . . . . . 38

2.4 TD-DFT/BP86/6-31G(d)-based electronic transitions ${ }^{a}$ for the singlet states of Im-[Co ${ }^{\mathrm{III}}($ corrin $\left.)\right]-\mathrm{CN}^{+}$. . . . . . . . . . . . . . 39

2.5 TD-DFT/BP86/6-31G(d)-based electronic transitions ${ }^{a}$ for the singlet states of $\mathrm{Im}-\left[\mathrm{Co}^{\mathrm{III}}\right.$ (corrin) $]-\mathrm{CN}^{+}$calculated with the use of the COSMO $\left(\mathrm{H}_{2} \mathrm{O}\right)$ solvation model.

3.1 Vertical excitation energies $(\mathrm{eV})$ of the lowest four excited states of Im- $\left[\mathrm{Co}^{\mathrm{III}}\right.$ (corrin) $]-\mathrm{CH}_{3}^{+}$calculated with different methods and the 631G(d) basis set. . . . . . . . . . . . . . . 56

3.2 The ground and four lowest excited states of $\mathrm{Im}-\left[\mathrm{Co}{ }^{\mathrm{III}}\right.$ (corrin) $]-\mathrm{CH}_{3}^{+}$ obtained from the CASSCF-based MC-XQDPT2 calculations. CSF stands for a configuration state function. . . . . . . . . . . . . 64

3.3 The lowest three excited states of Im- $\left[\mathrm{Co}^{\mathrm{III}}\right.$ (corrin) $]-\mathrm{CH}_{3}^{+}$obtained from EOM-CCSD calculations. . . . . . . . . . . . 65 
3.4 LC-BLYP/6-31G(d) electronic transitions of Im-[Co ${ }^{\mathrm{III}}$ (corrin) $]-\mathrm{CH}_{3}^{+}$ calculated as a function of the $\mu$ range-separation parameter together with their composition and character. . . . . . . . . . . 70

3.5 Orbital composition of the $\mathrm{S}_{1}$ excited state of Im-[Co ${ }^{\mathrm{III}}$ (corrin) $]-\mathrm{CH}_{3}^{+}$ resulting from the TD-DFT calculations with different density func-

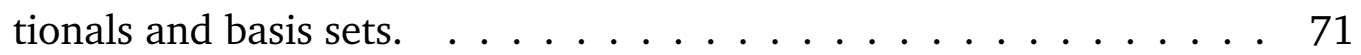

3.6 Electronic transitions of Im- $\left[\mathrm{Co}{ }^{\mathrm{III}}\right.$ (corrin) $]-\mathrm{CH}_{3}^{+}$calculated with B3LYP/6-

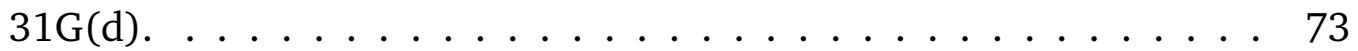

3.7 The first three excited states of $\mathrm{Im}-\left[\mathrm{Co}{ }^{\mathrm{III}}\right.$ (corrin) $]-\mathrm{CH}_{3}^{+}$calculated with BP86/6-311++G(d,p). . . . . . . . . . . . . 74

4.1 Electronic excitations of CNCbl based on BP86/aug-cc-pVDZ calculations in gas phase ${ }^{a} . \ldots \ldots \ldots$. . . . . . . . . . 95

4.2 Electronic excitations of MeCbl based on BP86/aug-cc-pVDZ calculations in gas phase ${ }^{a} . \ldots \ldots \ldots \ldots \ldots$

4.3 Electronic excitations of CNCbl based on BP86/aug-cc-pVDZ calculations in solution (water, PCM) ${ }^{a} . \quad \ldots \ldots \ldots \ldots$. . . . . . 106

5.1 Excitation energies ${ }^{a}$ together with oscillator and rotatory strengths ${ }^{b}$ (CD) of 38 states of cob(I)alamin (simplified model) calculated with

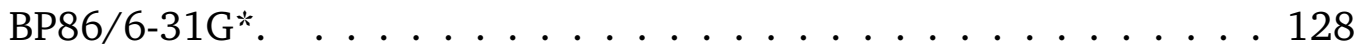

5.2 Excitation energies ${ }^{a}$ together with oscillator and rotatory strengths ${ }^{b}$ (CD) of 38 states of cob(I)alamin (simplified model) calculated with CAM-B3LYP/6-31G*.

5.3 First 10 excited states of cob(I)alamin calculated with MC-XQDPT2 presented in terms of $\%$ contributions. Note that only contributions larger than $5 \%$ were shown. . . . . . . . . . . . . . . . . 135

5.4 Excitation energies ${ }^{a}$ together with oscillator and rotatory strengths ${ }^{b}$ (CD) of 43 states of cob(I)alamin (full structure) calculated with BP86/6-31G*. . . . . . . . . . . . . . . . . 138 
5.5 Excitation energies ${ }^{a}$ together with oscillator and rotatory strengths ${ }^{b}$ (CD) of 30 states of cob(I)alamin (full structure) calculated with CAM-B3LYP/6-31G*.

6.1 Selected geometrical parameters ${ }^{a}$ of ground state and low-lying excited states corresponding to axial bonding in $\mathrm{Im}-\left[\mathrm{Co}{ }^{\mathrm{III}}\right.$ (corrin) $]-\mathrm{CN}^{+}$. 149

6.2 Selected geometrical parameters for $\mathrm{Im}-\left[\mathrm{Co}{ }^{\mathrm{III}}\right.$ (corrin) $]-\mathrm{CN}^{+} \ldots \ldots \ldots$

6.3 Selected geometrical parameters for $\mathrm{Im}-\left[\mathrm{Co}{ }^{\mathrm{III}}\right.$ (corrin) $]-\mathrm{CN}^{+}$(the values obtained from calculations with COSMO solvation model and water as solvent). . . . . . . . . . . . . . . . . . . . 151

6.4 $\mathrm{NBO}$ charges for $\mathrm{Im}-\left[\mathrm{Co}^{\mathrm{III}}\right.$ (corrin) $]-\mathrm{CN}^{+}$calculated in gas phase and in water solution based on COSMO $/ \mathrm{H}_{2} \mathrm{O}$ model. . . . . . . . . 152

6.5 Mulliken charges for $\mathrm{Im}-\left[\mathrm{Co}{ }^{\mathrm{III}}\right.$ (corrin) $]-\mathrm{CN}^{+}$calculated in gas phase and in water solution based on COSMO/ $\mathrm{H}_{2} \mathrm{O}$ model. . . . . . . 153

6.6 NBO and Mulliken charges for the $\mathrm{Im}-\left[\mathrm{Co}{ }^{\mathrm{III}}\right.$ (corrin) $]-\mathrm{CN}^{+}$model with side chains of the corrin ring calculated in gas phase and in water solution based on the COSMO/ $\mathrm{H}_{2} \mathrm{O}$ model (overall charge on the side chains is denoted as $\mathrm{R}) . \quad \ldots \ldots$. . . . . . . . . . 154

6.7 NBO analysis for cobalt axial bonds in gas phase and in water solution. . . . . . . . . . . . . . . . . . . . 159

6.8 Singlet electronic excitations calculated with BP86/6-31G(d). . . . 171

6.9 Triplet electronic excitations calculated with BP86/6-31G(d). . . . 173

A.1 Scaling parameters used to simulate absorption spectra of Im-[Co ${ }^{\mathrm{III}}$ (corrin)]$\mathrm{CN}^{+}$calculated in vacuo and water solution (PCM) with the 6-31G(d) basis set. . . . . . . . . . . . . . . . . . . 204

A.2 Scaling parameters used to simulate absorption spectra of Im-[Co ${ }^{\text {III }}$ (corrin)]$\mathrm{CN}^{+}$calculated in vacuo with the TZVP basis set. . . . . . . . . 204

A.3 Twenty low-lying excited states of $\mathrm{Im}-\left[\mathrm{Co}^{\mathrm{III}}\right.$ (corrin) $]-\mathrm{CN}^{+}$computed with the CASSCF method. . . . . . . . . . . . . . . . 205 
A.4 Twenty low-lying excited states of $\mathrm{Im}-\left[\mathrm{Co}{ }^{\mathrm{III}}\right.$ (corrin) $]-\mathrm{CH}_{3}^{+}$computed with the CASSCF method. . . . . . . . . . . . . . . . 206

A.5 Orbital energies and composition of the Im- $\left[\mathrm{Co}^{\mathrm{III}}\right.$ (corrin) $]-\mathrm{CH}_{3}^{+}$molecular orbitals calculated with BLYP/6-31G(d). . . . . . . . . . . . 207

A.6 Orbital energies and composition of the Im- $\left[\mathrm{Co}^{\mathrm{III}}\right.$ (corrin) $]-\mathrm{CH}_{3}^{+}$molecular orbitals calculated with MPW1PW91/6-31G(d) . . . . . . . . 213

A.7 Orbital energies and composition of the Im- $\left[\mathrm{Co}^{\mathrm{III}}\right.$ (corrin) $]-\mathrm{CH}_{3}^{+}$molecular orbitals calculated with MPWPW91/6-31G(d) . . . . . . . . . 213

A.8 Orbital energies and composition of the Im- $\left[\mathrm{Co}{ }^{\mathrm{III}}\right.$ (corrin) $]-\mathrm{CH}_{3}^{+}$molecular orbitals calculated with TPSSh/6-31G(d). . . . . . . . . . . . 214

A.9 Orbital energies and composition of the Im- $\left[\mathrm{Co}^{\mathrm{III}}\right.$ (corrin) $]-\mathrm{CH}_{3}^{+}$molecular orbitals calculated with TPSS/6-31G(d). . . . . . . . . . . . . 214

A.10 Orbital energies and composition of the Im- $\left[\mathrm{Co}{ }^{\mathrm{III}}\right.$ (corrin) $]-\mathrm{CH}_{3}^{+}$molecular orbitals calculated with BP86/6-31G(d)， . . . . . . . . . 215

A.11 Orbital energies and composition of the $\mathrm{Im}-\left[\mathrm{Co}^{\mathrm{III}}\right.$ (corrin) $]-\mathrm{CH}_{3}^{+}$molecular orbitals calculated with B3LYP/6-31G(d). . . . . . . . . . . 215

A.12 The first five excited states calculated with BP86/6-311G(d,p). . . . 216

A.13 The first five excited states calculated with BP86/6-311++G(d,p). . 216

A.14 Lambda parameter for DBI-[CoIII (corrin) $]-\mathrm{CN}^{+}$calculated with different methods. The values $\leq 0.30$ are indicated with red color, $\leq$ 0.40 with green. . . . . . . . . . . . . . . . . . . . 217

A.15 Lambda parameter for DBI-[Co ${ }^{\mathrm{III}}$ (corrin) $]-\mathrm{CH}_{3}^{+}$calculated with different methods. The values $\leq 0.30$ are indicated with red color, $\leq$ 0.40 with green. . . . . . . . . . . . . . . . . . . . 218

A.16 First 19 excited states of cob(I)alamin calculated with MC-XQDPT2 presented in terms of \% contributions of CAS states (see Table A.17 for the nature of individual states) . . . . . . . . . . . . . . . . 219

A.17 First 19 excitations calculated with CASSCF. . . . . . . . . . . . 220 
A.18 MO energy and fragments contributions for singlet states of Im-[Co ${ }^{\mathrm{III}}$ (corrin)]$\mathrm{CN}^{+}$in gas phase and COSMO/ $\mathrm{H}_{2} \mathrm{O}$. The results coming from calculations with solvent model are shown in parenthesis. . . . . . . . . 221 


\section{LIST OF FIGURES}

\section{FIGURE}

1.1 Molecular structure of vitamin $\mathrm{B}_{12}(\mathrm{CNCbl}=$ cyanocobalamin, $\mathrm{R}=$ $\mathrm{CN})$, methylcobalamin $(\mathrm{R}=\mathrm{Me})$ and adenosylcobalamin $(\mathrm{R}=\mathrm{Ado})$. The nucleotide loop is connected to $\mathrm{C}_{17}$ of the corrin ring. . . . . . . 2

1.2 The catalytic cycle of methionine synthase coupled with the reactivation of coenzyme proceeding through a $\mathrm{Co}^{+2} / \mathrm{Co}^{+1}$ reduction. . . . . 4

1.3 General catalytic mechanism for AdoCbl-dependent enzymes. . . . . 5

1.4 Simplified model of $\mathrm{B}_{12}$ cofactor. $\mathrm{R}=\mathrm{CN}$ or $\mathrm{Me}$, or Ado; Base $=$ Imidazole (Im) or 5,6-dimethylbenzimidazole (DBI) . . . . . . . . . 16

2.1 Two structural models of CNCbl used in excited states calculations. Left with imidazole (Im), right with dimethylbenzimidazole (DBI) as axial base, denoted as B-[Co ${ }^{\mathrm{III}}($ corrin $\left.)\right]-\mathrm{CN}^{+}(\mathrm{B}=\mathrm{Im}$ or DBI$) .. .21$

2.2 Simulated electronic absorption spectra of CNCbl computed with the use of different functionals and the 6-31G(d) basis set (left: vacuo, right: water solution, PCM). See Table A.1 (Appendix) for details regarding the scaling factors. Experimental spectra were reproduced from Ref. 67 with permission. . . . . . . . . . . . . . . . 24

2.3 Simulated electronic absorption spectra of CNCbl computed with the use of different functionals and the TZVP basis set (vacuo). See Table A.2 (Appendix) for details regarding the scaling factors. Experimental spectra were reproduced from Ref. 67 with permission. . . 24

2.4 Vertical excitation energies calculated with LC-BLYP/6-31G(d) as a function of the range separation parameter, $\mu . \ldots 26$ 
2.5 The simulated absorption spectra of Im-[Co ${ }^{\mathrm{III}}$ (corrin) $]-\mathrm{CN}^{+}$based on LC-BLYP/6-31G(d) calculations in vacuo computed as a function of $\mu$ value (indicated in the top right corner). Experimental spectra were reproduced from Ref. 67 with permission. . . . . . . . . . . . . 27

2.6 The simulated absorption spectra of $\mathrm{Im}-\left[\mathrm{Co}^{\mathrm{III}}\right.$ (corrin) $]-\mathrm{CN}^{+}$based on LC-BLYP/6-31G(d) calculations in water solution (PCM) computed as a function of $\mu$ value (indicated in the top right corner). Experimental spectra were reproduced from Ref. 67 with permission. . . . 28

2.7 Ten lowest electronic transitions of $\mathrm{Im}-\left[\mathrm{Co}^{\mathrm{III}}\right.$ (corrin) $]-\mathrm{CN}^{+}$calculated with different density functionals and basis sets together with the corresponding $\Lambda$ diagnostic values (presented as numbers). . . . . 30

2.8 Ten lowest electronic transitions of DBI-[Co ${ }^{\mathrm{III}}$ (corrin) $]-\mathrm{CN}^{+}$calculated with different density functionals and basis sets together with the corresponding $\Lambda$ diagnostic values (presented as numbers). . . . 31

2.9 Active space orbitals of $\mathrm{Im}-\left[\mathrm{Co}^{\mathrm{III}}\right.$ (corrin) $]-\mathrm{CN}^{+}$used in $\operatorname{CASSCF}(12,12)$

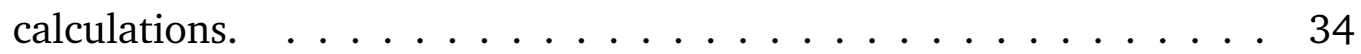

2.10 Energies of the lowest twenty excited states computed at the CASSCF level and the corresponding four low-lying states using MC-XQDPT2. The lines between CASSCF and MC-XQDPT2 indicate mixing of states upon inclusion of second order perturbation theory (see Table 2.2 for

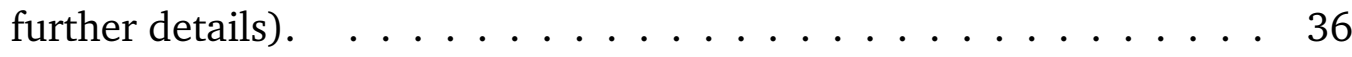

2.11 Isosurface plots of frontier MOs for the ground state of CNCbl model based on BP86/6-31G(d) calculations in vacuo and with the COSMO/ $\mathrm{H}_{2} \mathrm{O}$ model. . . . . . . . . . . . . . . . . . . . . . . . 41

3.1 Structural model of MeCbl, abbreviated as Im- $\left[\mathrm{Co}^{\mathrm{III}}\right.$ (corrin) $]-\mathrm{CH}_{3}^{+}$, used in the present study. . . . . . . . . . . . . . . . 51

3.2 Active orbitals of Im-[Co ${ }^{\mathrm{III}}$ (corrin) $]-\mathrm{CH}_{3}^{+}$used in the $\operatorname{CASSCF}(12,12)$ and subsequent MC-XQDPT2 $(12,12)$ calculations. . . . . . . 60 
3.3 The lowest twenty excited states computed at the $\operatorname{CASSCF}(12,12)$ level and the four lowest-energy excited states resulting from the corresponding MC-XQDPT2 calculations. The lines between the CASSCF and MC-XQDPT2 energy levels indicate mixing of states upon the incorporation of dynamical correlation effects via the second order perturbation theory (see Table 3.2 for further details). . . . . . . . . 63

3.4 Canonical RHF orbitals used in the CCSD and EOM-CCSD calcula-

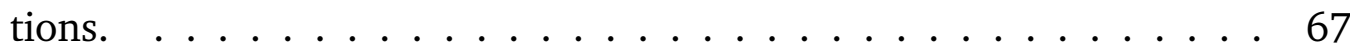

3.5 Molecular orbitals obtained with BP86/6-311++G(d,p). $\quad \ldots \ldots .75$

3.6 Natural transition orbitals (NTOs) describing the three lowest singlet excited states for the Im-[CoIII (corrin) $]-\mathrm{CH}_{3}^{+}$model complex. Results obtained from the TD-DFT/BP86/6-311++G(d,p) calculations. . . . 76

3.7 Electron density differences between the $\mathrm{S}_{0}$ and $\mathrm{S}_{1}, \mathrm{~S}_{0}$ and $\mathrm{S}_{2}$, and $\mathrm{S}_{0}$ and $\mathrm{S}_{3}$ states for the Im-[Co ${ }^{\mathrm{III}}$ (corrin) $]-\mathrm{CH}_{3}^{+}$model complex: (a) isosurface plot value of 0.003 and (b) cross-section contour plot along the axial bonding. Results obtained from the TD-DFT/BP86/6$311++\mathrm{G}(\mathrm{d}, \mathrm{p})$ calculations. . . . . . . . . . . . . . . .

3.8 Left panel - potential energy surfaces of the $S_{0}$ and $S_{1}$ states resulting from the BP86 and TD-DFT/BP86/6-31G(d) calculations plotted as a function of axial bond lengths (in $\AA$ ). Right panel - vertical projection of the potential energy surface of the $S_{1}$ state. . . . . . . . . . 80

3.9 A comparison of the BP86/6-31G(d)-optimized geometries of the ground (top) and first excited (middle) state of Im-[Co ${ }^{\mathrm{III}}$ (corrin)]$\mathrm{CH}_{3}^{+}$, along with the alignment of their structures (bottom). . . . . .

3.10 The isosurface plots of dominant MOs corresponding to the first electronic transition $\left(\mathrm{S}_{0} \rightarrow \mathrm{S}_{1}\right.$ ) of Im- $\left[\mathrm{Co}{ }^{\mathrm{III}}\right.$ (corrin) $]-\mathrm{CH}_{3}^{+}$at the TD-DFT/BP86/6$31 \mathrm{G}(\mathrm{d})$-optimized geometry of the $\mathrm{S}_{1}$ excited state. . . . . . . . .

4.1 Simplified structural models of the MeCbl (left) and CNCbl (right) used in calculations and denoted as DBI-[Co ${ }^{\mathrm{III}}$ (corrin) $]-\mathrm{Me}^{+}$and DBI$\left[\mathrm{Co}^{\mathrm{III}}\right.$ (corrin) $]-\mathrm{CN}^{+}$, respectively. . . . . . . . . . . . . . 
4.2 Abs (top), CD (middle) and MCD (bottom) computed spectra (solid line) and experimental (dotted line) of CNCbl. Simulated TD-DFT spectra correspond to gas phase calculations with BP86/aug-cc-pVDZ (left panel) and CAM-B3LYP/aug-cc-pVDZ (right panel). Experimental spectra were reproduced from Ref. 108 with permission. . . . . . 91

4.3 Abs (top), CD (middle) and MCD (bottom) computed spectra (solid line) and experimental (dotted line) of MeCbl. Simulated TD-DFT spectra correspond to gas phase calculations with BP86/aug-cc-pVDZ (left panel) and CAM-B3LYP/aug-cc-pVDZ (right panel). Experimental spectra were reproduced from Ref. 108 with permission. . . . . . 92

4.4 Energy (eV) diagram representing frontier MOs of DBI-[Co ${ }^{\mathrm{III}}$ (corrin)]$\mathrm{CN}^{+}$based on BP86/aug-cc-pVDZ calculations: left panel in gas phase and right panel in water solution, PCM. . . . . . . . . . . . 93

4.5 Absorption and CD spectra of CNCbl (panel A) and MeCbl (panel B) calculated with CAM-B3LYP*. Experimental spectra were reproduced from Ref. 108 with permission. . . . . . . . . . . . . . . . . . . 99

4.6 Comparison of low-energy regions of $\mathrm{MeCbl}$ absorption and $\mathrm{CD}$ spectra computed with BP86/aug-cc-pVDZ. Experimental data were taken from Ref. 108 with permission. . . . . . . . . . . . . . . . . 100

4.7 Energy (eV) diagram representing frontier MOs of DBI-[C ${ }^{\mathrm{III}}$ (corrin)]$\mathrm{Me}^{+}$gas phase BP86/aug-cc-pVDZ calculations. . . . . . . . 103

4.8 Comparison of low-energy regions of CNCbl absorption and CD spectra computed with BP86/aug-cc-pVDZ. Experimental data were taken from Ref. 108 with permission. . . . . . . . . . . . . . . . . 104

5.1 Molecular structure of super-reduced form of vitamin $B_{12}$ (cob(I)alamin or $\mathrm{B}_{12 \mathrm{~s}}$ ). Note that the lower axial position is unoccupied as opposed to another reduced derivative, i.e., cob(II)alamin $\left(\mathrm{B}_{12 \mathrm{r}}\right)$. The nucleotide loop is connected to $\mathrm{C}_{17}$ of the corrin ring. . . . . . . 114 
5.2 Structural models of cob(I)alamin employed in calculations: simplified (left) and full (right) without lower axial base (i.e., cob(I)inamide). 117

5.3 CASSCF Active Space Orbitals used in the calculations of the cob(I)alamin model system. The last inset shows the coordinate system used to label MOs in present study. Note that $\mathrm{C}_{2}$ symmetry axis coincides with $\mathrm{x}$ axis in this frame while $\mathrm{n}$ denotes lone pair on corrin nitrogens. See Section 5.2 .3 for details. . . . . . . . . . . . . . . . 122

5.4 Abs, CD and MCD spectra of cob(I)alamin (simplified model) calculated with BP86/6-31G* (left) and CAM-B3LYP/6-31G* (right). Experimental data (plotted in red) were taken from Ref. 110. . . . . . 123

5.5 Abs, CD and MCD spectra of cob(I)alamin (full structure) calculated with BP86/6-31G* (left) and CAM-B3LYP/6-31G* (right). Experimental data (plotted in red) were taken from Ref. $110 . \quad \ldots \ldots . .123$

5.6 Molecular structure of vitamin $\mathrm{B}_{12 s}$ (cob(I)alamin). Note that both axial positions are unoccupied. The nucleotide loop is connected to $\mathrm{C}_{17}$ of the corrin ring. $\mathrm{R}_{1}=\mathrm{CH}_{2} \mathrm{CONH}_{2}, \mathrm{R}_{2}=\mathrm{CH}_{2} \mathrm{CH}_{2} \mathrm{CONH}_{2}, \mathrm{R}_{3}=$ $\left(\mathrm{CH}_{2}\right)_{2} \mathrm{CONHCH}_{2} \mathrm{CH}\left(\mathrm{CH}_{3}\right) \mathrm{OPO}_{3}^{-} \ldots \ldots \ldots \ldots \ldots$

5.7 BP86/6-31G*-computed electronic spectra of cob(I)alamin (full structure) based on 11 isolated excitations localized mainly on corrin. . . 140

6.1 Structural model of vitamin $\mathrm{B}_{12}$ used in calculations and denoted as Im-[Co ${ }^{\mathrm{III}}$ (corrin) $]-\mathrm{CN}^{+} . \ldots \ldots \ldots$

6.2 Potential energy curves of low-lying singlet (red) and triplet (blue) states of CNCbl (vacuo and water) and MeCbl (vacuo) generated as vertical excitations along the optimized Co-C distance of $\mathrm{S}_{0}$ state. See Figure 6.3 for the corresponding $\Lambda$ diagnostic test analysis. . . 155

6.3 The $\Lambda$ diagnostic along the $\mathrm{Co}-\mathrm{C}_{\mathrm{CN}}$ bond stretch computed at $\mathrm{BP} 86 / 6$ $31 \mathrm{G}(\mathrm{d})$. The values are presented for the four lowest vertical excitations (upper panel) and for the $\mathrm{S}_{1}$ state in the optimized geometry (lower panel) calculated in gas phase and in water solution. . . . . 156 
6.4 (Left) Potential energy surfaces for the two lowest singlet states generated as vertical excitations and plotted as a function of axial bond lengths (expressed in $\AA$ ). (Right) Vertical projections of the $S_{1}$ and $S_{2}$ potential energy surfaces. . . . . . . . . . . . . . . . . . . . 161

6.5 (Left) Potential energy surfaces for two lowest triplet states plotted as a function of axial bond lengths (expressed in $\AA$ ). (Right) Vertical projections of the $T_{1}$ and $T_{2}$ potential energy surfaces. Both singlet $\left(\mathrm{S}_{0}\right)$ and first triplet $\left(\mathrm{T}_{1}\right)$ geometries were optimized. The higher triplet states were obtained as vertical excitations from $\mathrm{T}_{1}$. . . . 163

6.6 Potential energy curves of the lowest-excited singlet $\left(S_{1}\right.$, red $)$ and triplet $\left(\mathrm{T}_{1}\right.$, blue) states of the CNCbl model along the $\mathrm{Co}-\mathrm{C}_{\mathrm{CN}}$ bond stretch optimized at BP86/6-31G(d) without solvent (dashed lines) and with solvent (solid lines) using the $\mathrm{COSMO} / \mathrm{H}_{2} \mathrm{O}$ model. See Figure 6.3 for the corresponding $\Lambda$ diagnostic test analysis. . . . 167

6.7 Molecular orbital diagram for $\mathrm{Im}-\left[\mathrm{Co}{ }^{\mathrm{III}}\right.$ (corrin) $]-\mathrm{CN}^{+}$in the $\mathrm{S}_{0}, \mathrm{~S}_{1}(\mathrm{~A})$ and $S_{1}(C)$ optimized geometry. . . . . . . . . . . . . . . . . 169

6.8 Molecular orbital diagram for Im-[CoII (corrin) $]-\mathrm{CN}^{+}$in the $\mathrm{S}_{0}, \mathrm{~S}_{1}(\mathrm{~A})$ and $S_{1}(C)$ optimized geometry based on the BP86/6-31G(d) calculations with the use of COSMO/ $\mathrm{H}_{2} \mathrm{O}$ model. . . . . . . . . . . . . . . 170

6.9 Electron density difference between the $\mathrm{S}_{0}$ and $\mathrm{S}_{1}$ states for $\mathrm{CNCbl}$ and MeCbl: (a) isosurface plot value of 0.003 and (b) cross-section contour plot along the axial bonding (in two different section planes). The yellow and red colors denote electron space, blue color - hole space. . . . . . . . . . . . . . . . . . . . . 172

6.10 Electron density difference between the $S_{0}$ and $S_{1}$ states for CNCbl and MeCbl based on the BP86/6-31G(d) calculations with the use of COSMO solvatation model: (a) isosurface plot value of 0.003 and (b) cross-section contour plot along the axial bonding (in two different section planes). The yellow and red colors denote electron space, blue color - hole space. . . . . . . . . . . . . . . . . . . 174 
6.11 Electron density difference between the $\mathrm{S}_{0}$ and $\mathrm{T}_{1}$ states for $\mathrm{CNCbl}$ based on the BP86/6-31G(d) calculations with the use of COSMO solvatation model: (a) isosurface plot value of 0.003 and (b) crosssection contour plot along the axial bonding (in two different section planes). The yellow and red colors denote electron space, blue color - hole space. . . . . . . . . . . . . . . . . . . . 176

6.12 Potential energy curves of the lowest vertical excited singlet (red dot) and the optimized first excited state (red solid line) of the CNCbl model along the structural parameter q computed at BP86/6-31G(d) without solvent (left) and with solvent (right) using the COSMO/ $\mathrm{H}_{2} \mathrm{O}$ model.

A.1 Absorption and CD spectra of MeCbl calculated in solution (PCM) using: BP86/aug-cc-pVDZ (A) and CAM-B3LYP/aug-cc-pVDZ (B) levels of theory. Experimental spectra were reproduced from Ref. 108 with permission.

A.2 Absorption and CD spectra of CNCbl calculated in gas phase using: BP86/6-31G(d) (A) and CAM-B3LYP/6-31G(d) (B) levels of theory. Experimental spectra were reproduced from Ref. 108 with permission. . . . . . . . . . . . . . . . . . . . . . . . . . . . . . . . 209

A.3 Absorption and $\mathrm{CD}$ spectra of MeCbl calculated in gas phase using: BP86/6-31G(d) (A) and CAM-B3LYP/6-31G(d) (B) levels of theory. Experimental spectra were reproduced from Ref. 108 with permission. . . . . . . . . . . . . . . . . . . . . 210

A.4 Absorption and CD spectra of CNCbl calculated in solution (PCM) using: BP86/6-31G(d) (A) and CAM-B3LYP/6-31G(d) (B) levels of theory. Experimental spectra were reproduced from Ref. 108 with permission. . . . . . . . . . . . . . . . . . 211 
A.5 Absorption and CD spectra of MeCbl calculated in solution (PCM) using: BP86/6-31G(d) (A) and CAM-B3LYP/6-31G(d) (B) levels of theory. Experimental spectra were reproduced from Ref. 108 with permission. . . . . . . . . . . . . . . . . . . 212 


\section{CHAPTER 1}

\section{INTRODUCTION}

Since the discovery of a positive impact of liver intake in diet on patients suffering pernicious anemia, ${ }^{1,2}$ vitamin $B_{12}$ has become a vividly studied therapeutic agent also required for its treatment. It was isolated in late $1940 \mathrm{~s}^{3}$ and then crystallized in the laboratory of D. C. Hodgkin, ${ }^{4}$ contributing to her Nobel Prize award in 1964. Subsequent years brought the achievements in total synthesis of the cofactor which was eventually reported by Woodward and Eschenmoser in $1972 .{ }^{5}$ The following efforts of scientists were directed towards gaining insight into chemical, biochemical, and enzymatic aspects of $\mathrm{B}_{12}$ nature and catalysis. It was also discovered that although vitamin $\mathrm{B}_{12}$ is an essential component in biological reactions, the higher organisms do not posses mechanisms to synthesize it within their bodies. In contrast, the variety of microorganisms developed effective biosynthesis pathways that can be implemented both in aerobic and anaerobic conditions.

The cyanocobalamin ( $\mathrm{CNCbl}$ or vitamin $\mathrm{B}_{12}$ ) itself does not have a known physiological role. It is a water-soluble molecule present in the most common nutritional supplements that are commercially available. ${ }^{6,7}$ When ingested, CNCbl is converted to one of its biologically active forms, namely, methylcobalamin ( $\mathrm{MeCbl}$ ) or adenosylcobalamin (AdoCbl or coenzyme $\mathrm{B}_{12}$ ), which act in cytosol and mitochondria, respectively. ${ }^{8}$ All these forms are indispensable human nutrients that belong to cobalamins, a class of octahedral Co(III) complexes based on the corrin macrocycle (Figure 1.1). The centered metal position in these molecules is occupied by a Co atom which is equatorially coordinated by four corrin nitrogens. The corrin system resembles the porphyrin, apart from the fact that one of the bridging methine groups is missing and that the corrin macrocycle is partially saturated. 


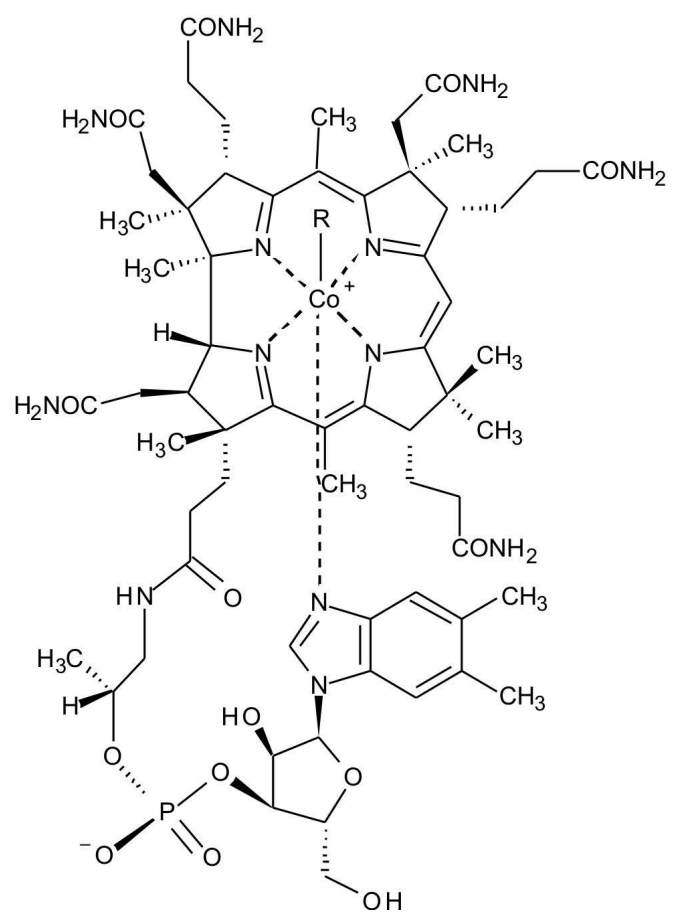

$\mathrm{R}=\mathrm{CN}, \mathrm{Me}$, Ado

CN:<smiles>[14CH2]N</smiles>

Me:<smiles>CC</smiles>

Ado: 5'-deoxy-5'-adenosyl

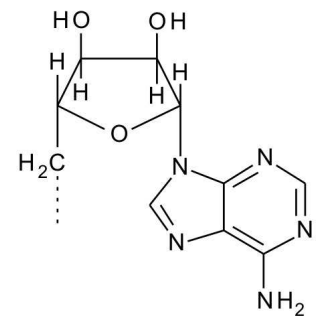

Figure 1.1. Molecular structure of vitamin $\mathrm{B}_{12}(\mathrm{CNCbl}=$ cyanocobalamin, $\mathrm{R}=\mathrm{CN})$, methylcobalamin $(\mathrm{R}=\mathrm{Me})$ and adenosylcobalamin $(\mathrm{R}=\mathrm{Ado})$. The nucleotide loop is connected to $\mathrm{C}_{17}$ of the corrin ring.

Whereas the lower axial ligand ( $\alpha$-phase), 5,6-dimethylbenzimidazole (DBI), connects to one of the seven amide chains of corrin, the upper one ( $\beta$-phase) binds only to the cobalt atom and is occupied either by a $\mathrm{CN}$ group in the parent vitamin $\mathrm{B}_{12}$, a methyl group in $\mathrm{MeCbl}$ or by a 5'-deoxyadenosyl group in AdoCbl, respectively (Figure 1.1). The nature of the upper axial ligands sets ground for another popular division of $\mathrm{B}_{12}$ derivatives into alkyl and non-alkyl cobalamins. The former compounds include organocobalamins such as the aforementioned MeCbl or AdoCbl with a methyl or 5'-deoxy-5'-adenosyl group, respectively, in which the upper axial ligand is C-bonded to the Co atom. On the other hand, $\mathrm{CNCbl}$ with the $\mathrm{CN}^{-}$group is classified as non-alkylcobalamin. The other known examples that belong to this group are: aquocobalamin $\left(\mathrm{H}_{2} \mathrm{OCbl}\right)$, hydroxycobalamin (HOCbl), and azidcobal$\operatorname{amin}\left(\mathrm{N}_{3} \mathrm{Cbl}\right)$. 
Enzymes incorporating $B_{12}$ cofactors are significant biological components as they are involved in the metabolism of every cell of the body. They catalyze a variety of chemical processes that can be divided into two categories. ${ }^{9-28}$ The methyltransfer reactions, employing $\mathrm{MeCbl}$, proceed by the heterolytic cleavage of the Co-C bond, leaving behind the methyl cation and $\operatorname{cob}(\mathrm{I})$ alamin. ${ }^{19}$ In contrast, the radical-induced rearrangements or ribonucleotide reduction, utilizing AdoCbl, follows with a Co-C bond homolysis and production of an adenosyl radical and cob(II)alamin. ${ }^{29,30}$ Although the family of cobalamin-dependent enzymes consists of a broad range of $\mathrm{B}_{12}$ analogs, they all are classified as either isomerases, methyltransferases, or reductive dehalogenases. ${ }^{20}$ The isomerases control fermentation processes in bacteria, are involved in the DNA replication and repair (ribonucleotide reductase), and are responsible for numerous radical reactions including carbon skeleton rearrangements, heteroatom eliminations, and intramolecular amino group migrations. ${ }^{20}$ Methyltransferases participate in amino acids and one-carbon metabolism or $\mathrm{CO}_{2}$ fixation, and are especially important for anaerobic organisms in their energy generation processes. ${ }^{19,20,26,27}$ The reductive dehalogenases play an essential role in the dehalospiration, a way to derive an energy by various microorganisms. ${ }^{20}$ During the dehalogenation reaction the halogen substituents are removed and this process is conjugated with an electron transfer chain. Such an ability to break down the halogenated pollutants has also the ecological merit as the bacteria can be efficiently utilized in the environmental detoxification through bioremediation. The significance of $\mathrm{B}_{12}$-dependent enzymes in vital processes is undeniable and therefore cobalamins have been vividly studied and discussed in the literature. ${ }^{14,17,20,21,23,25,27,31-33}$ However, only two MeCbl- and AdoCbl- based enzymes have been found in mammals. ${ }^{34,35}$ Methionine synthase (MetH) is a methyltransferase that regenerates methionine (Met) from homocysteine (Hcy) by methyl group transfer. ${ }^{23,25,27,32,33}$ This process is coupled with a demethylation of methyltetrahydrofolate $\left(\mathrm{CH}_{3}-\mathrm{H}_{4}\right.$-folate) to tetrahydrofolate $\left(\mathrm{H}_{4}\right.$-folate). Whereas Met is an essential aminoacid, $\mathrm{H}_{4}$-folate participates in the biosynthesis of amino acids, purine and pyrimidine and therefore is crucial for the synthesis of nucleic acids. 


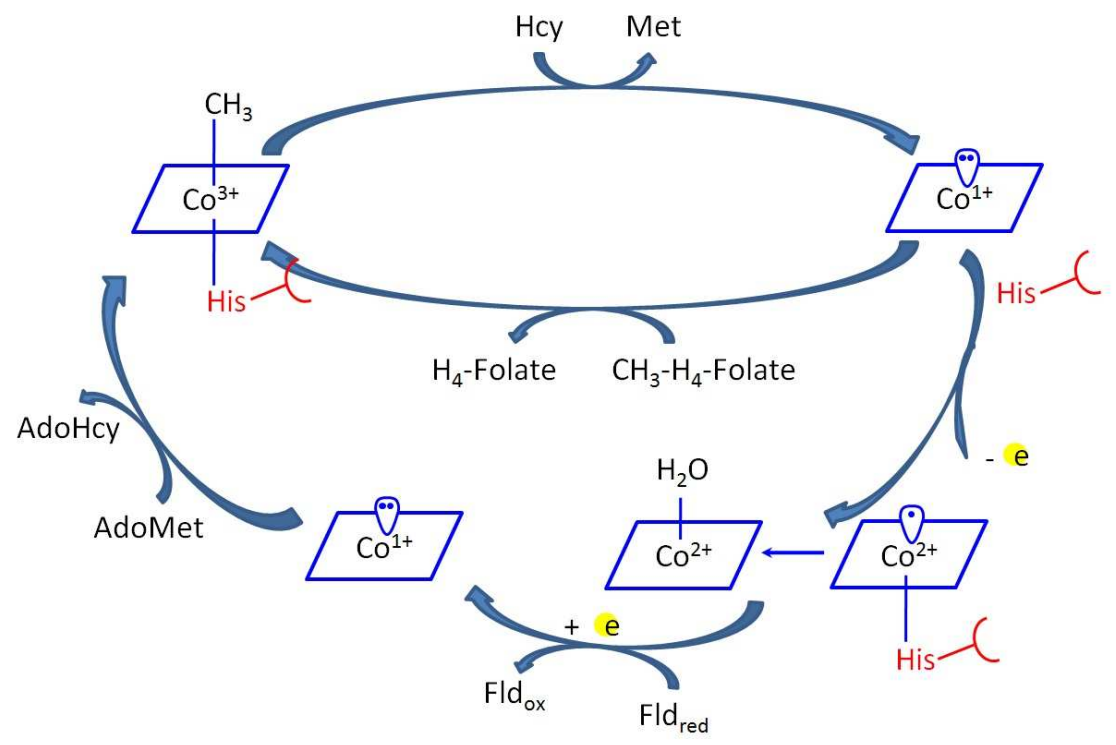

Figure 1.2. The catalytic cycle of methionine synthase coupled with the reactivation of coenzyme proceeding through a $\mathrm{Co}^{+2} / \mathrm{Co}^{+1}$ reduction.

The reaction mechanism of MetH (Figure 1.2) is a common mechanism shared by methyltransferases and is initiated by the heterolytic cleavage of the Co-C bond. In the primary turnover of the MetH-guided enzymatic catalysis the Co atom cycles between +3 and +1 oxidation states. However, in microaerophilic conditions the $\mathrm{Co}^{+1}$ form (cob(I)alamin) is oxidized to the $\mathrm{Co}^{+2}$ ( $\mathrm{Cob}(\mathrm{II})$ alamin) derivative, which is an inactive form of the enzyme. This process happens approximately every 2000 turnovers and prohibits further proper catalytic functioning of the enzyme. In order to secure its activity, the cob(II)alamin is reduced back to cob(I)alamin by accepting an electron from the biological electron donor, flavodoxin. This process is then followed by a methyl group abstraction by adenosyl-methionine (AdoMet) and regeneration of MeCbl. The second cobalamin found in mammals is methylmalonyl-CoAmutase (MMCM) which incorporates AdoCbl as a cofactor. ${ }^{36-38}$ It belongs to a broad class of AdoCbl-dependent enzymes which catalyze reactions proceeding through a 1,2-type hydrogen exchange with a functional group on adjacent carbon atoms (Figure 1.3). In contrast to the MeCbl-dependent catalysis, the AdoCbl-dependent rearrangements are initiated by a generation of a radical pair: deoxyadenosyl (Ado ${ }^{\circ}$ ) 


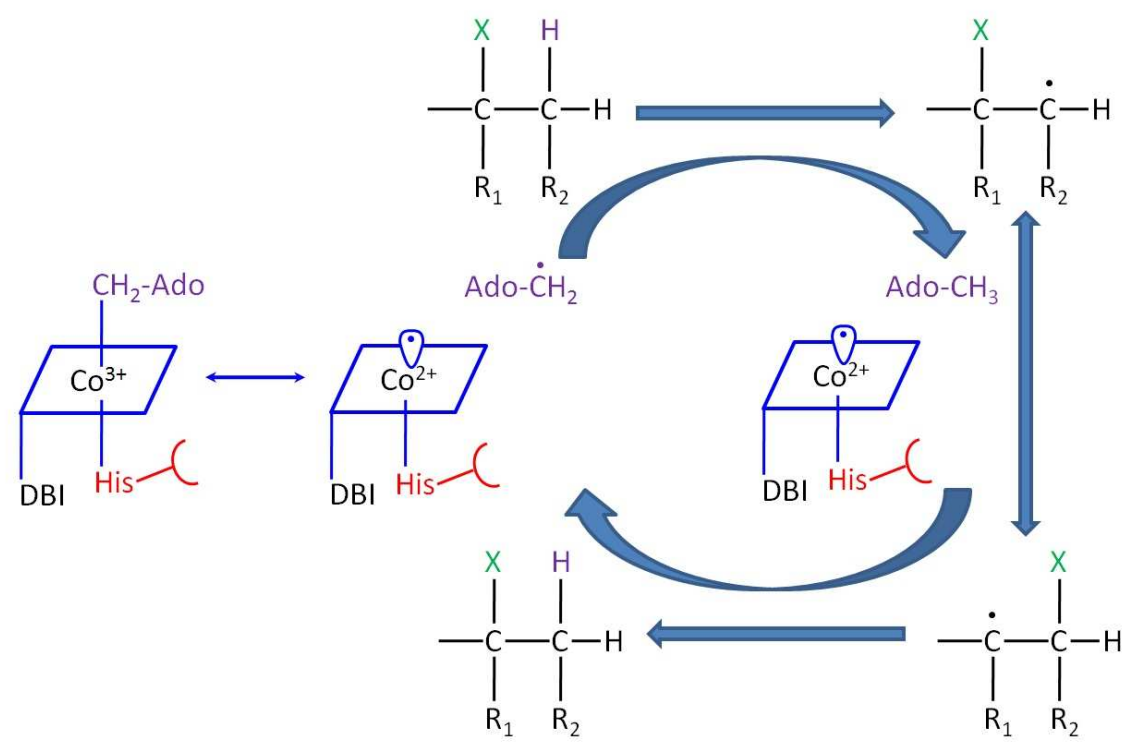

Figure 1.3. General catalytic mechanism for AdoCbl-dependent enzymes.

and cob(II)alamin through a heterolytic cleavage of the $\mathrm{Co}-\mathrm{C}$ bond. The subsequent steps include: the hydrogen abstraction by Ado ${ }^{\bullet}$ from the substrate, the rearrangement in a radical intermediate and its transformation to a product alike radical, followed by a reabstraction of the hydrogen atom from Ado moiety and the formation of the product. The proper functioning of MMCM is required for the catabolism of odd-chain fatty acids, cholesterol and branched chain amino acids during which methylmalonyl-CoA is converted to succinyl-CoA. Any disruption in the activity of MMCM can lead to an accumulation of methylmalonyl-CoA which consequently results in acidosis. In general, apart from the historically discovered pernicious anemia, the vitamin $\mathrm{B}_{12}$ deficiency can lead to serious hematologic, neurologic, and even psychiatric disorders. Considering the indisputable role of vitamin $\mathrm{B}_{12}$ in chemistry, biology, and enzymology as well as its potential in the environment preservation or medical treatment, including recently designed bioconjugates delivering anti-tumors or diagnostic labels, ${ }^{39-41}$ cobalamins are still a desirable subject of study. In particular, it is intriguing to ask how nature succeeds in the enormous acceleration of the reactions as compared to the same processes conducted in the absence of the catalysts. ${ }^{23,27,32,42}$ In addition, the specific role of the alkyl 
group, Me or Ado, in different reaction mechanisms remains uncertain. The foundations for apprehension of this problem lay in the insightful analysis of cobalt carbon (Co-C) bond cleavage that initiates all known $\mathrm{B}_{12}$-catalyzed reactions. The complete understanding of its mechanism can be achieved with the comprehensive analyses of structure and properties of the cofactors, proper determining the intermediates created during catalysis, as well as conditions at which they are formed.

To this date, various experimental techniques have been employed to study isolated and enzyme-bound $\mathrm{B}_{12}$ cofactors. However, the complexity of these compounds makes them challenging systems to study. For many years X-ray measurements did not deliver sufficient information about the structures of cobalamins due to the low resolutions of reported data. ${ }^{24,43,44}$ The majority of studies relied on searching analogies with predictions for simple models. ${ }^{45-47}$ Only recently, in consequence of technological developments, the more accurate structural data became available. These breakthroughs concern not only cobalamins, but also $\mathrm{B}_{12}$-dependent enzymes as well as protein binding domains and proteins involved in the transport. ${ }^{24,25,48-50}$ The additional amount of structural information for organocobalamins comes from NMR spectroscopy. This technique provides data complementary to these obtained with X-ray crystallography as the spectra are recorded for samples in solutions. Such studies have been employed to identify numerous cobalamins supporting thermodynamic and kinetic studies of these compounds. ${ }^{23}$ Whereas X-ray and NMR methods are important tools for chemical analysis of $B_{12}$ cofactors, they are insufficient to resolve mechanistic issues of the $B_{12}$ chemistry, which relies on $\mathrm{Co}-\mathrm{C}$ bond cleavage. Under nonenzymatic conditions the Co-C bond cleavage can be induced thermally ${ }^{51-55}$ or photochemically. ${ }^{56-65} \mathrm{Nu}-$ merous experiments have been performed to probe properties of the $\mathrm{Co}-\mathrm{C}$ bond in vitamin $B_{12}$ and related cobalamins. Among them spectroscopy is a particularly useful tool as it offers the insightful approach towards understanding the electronic structures of these molecules. The interactions of molecules with electromagnetic fields result in the changes of their electronic and vibrational states. These properties can be probed by analyzing the positions and intensities of bands in recorded 
spectra which are characteristic for investigated systems. The remarkable feature of cobalamins is their variation of colors that can be attributed to a manifold of lowlying excited states whose energies are sensitive to the nature of axial ligand as well as oxidation state of metal ion. ${ }^{66}$ This is a reason why photoabsorption (Abs) spectroscopy has been a widely used method among various experimental techniques applied to probe excited states of cobalamins. The resulting Abs spectra can be characterized by several prominent bands that fall into the following four categories: (i) the $\alpha$ and $\beta$ bands, which are a series of low to medium intensity transitions in the 420-600 $\mathrm{nm}$ region, (ii) the D and E bands, which are bands of low intensity in the 390-420 $\mathrm{nm}$ region, (iii) the $\gamma$ band, which is the most intense photoabsorption peak in the 350-370 nm region, usually accompanied by a shoulder on the high-energy side, and (iv) the $\delta$ band, which is characterized by a low to medium intensity in the $300-330 \mathrm{~nm}$ region. ${ }^{67,68}$ The bands observed in the Abs spectra of cobalamins were named after their analogs in porphyrin spectra, since these $\alpha, \beta, \gamma$ and $\delta$ bands are shared by both classes of metal complexes.

Electronic spectra of different $B_{12}$ analogs have been actively studied for past decades. The origin of individual bands is of particular importance as it provides insights into their complex electronic structures. Traditionally, the main photoabsorption features characterizing cobalamins have been interpreted as electronic transitions associated with the corrin ring, ${ }^{69-71}$ which is a recurring structural motif in all $\mathrm{B}_{12}$ complexes. The evidence supporting this interpretation has been provided by studies on cobalt-free corrin derivative, ${ }^{72}$ where it has been shown that its Abs spectrum displays maxima in the visible region that coincide with those observed for aquacobamides. Similarly, the early theoretical studies of $\mathrm{B}_{12}$ derivatives based on semiempirical calculations including extended Hückel or Pariser-Parr-Pople methods, arrived in conclusions supporting $\pi \rightarrow \pi^{*}$ assignments of main electronic transitions of these compounds. ${ }^{73-79}$ The following years have revolved around timedependent density functional theory (TD-DFT) ${ }^{80-84}$ which became a significant and powerful tool to explore electronic structure of cobalamins. ${ }^{85-106}$ Abs spectra of various $\mathrm{B}_{12}$ analogues including: dicyanocobinamide (DCC) ${ }^{107}$ and CNCbl, ${ }^{108} \mathrm{MeCbl},{ }^{105}$ 
AdoCbl, ${ }^{106} \mathrm{H}_{2} \mathrm{OCbl}$, cob(I)alamin, ${ }^{109,110}$ as well as metal free corrins ${ }^{111}$ have been examined. These efforts brought the attention to the differences in shapes of the spectra and the continuous changes in the bands together with the change of axial ligand allowing for insight into to the nature of electronic transitions but also raising new questions and uncovering the issues related to limitations of methods.

The clear interpretation of experimental spectra requires support from theoretical findings. However, computational methods are based on approximations which have to be validated before final assignments are done. Depending on the aim of the studies there are different approaches to justify validity of computed results. In the case of electronic excitations of cobalamins the first option is to compare simulated spectra with the existing experimental ones. If the overall shapes correspond one to another, it seems legitimate to extend the analyses towards subsequent explanation of the nature of electronic transitions. Such studies advance the description of particular bands pointing out the number of excitations contributing to a particular region. By matching the calculated excitation energies and intensities with the experimental peaks, the proposals about their assignments can be provided. In addition, a good agreement with experiment of the major bands visible in spectra allows to assume that the neighboring parts can be characterized with a similar accuracy. Whereas these predictions are crucial for explaining spectral differences between $\mathrm{B}_{12}$ analogs, the understanding of their photochemistry requires larger confidence in methods applied to identify the excited states. Consequently, when the target of investigation is the nature of selected excitations, which is not available experimentally, simpler calculations have to be benchmarked against higher level theories. Such an approach allows to validate methods used to study electronic properties of cobalamins which can be further applied to explore photochemical processes guided by $\mathrm{B}_{12}$ cofactors. Chapters 2 and 3 in this dissertation discuss the choice of an appropriate and practical methodology to investigate excited states of vitamin $B_{12}$ and methylcobalamin. The results coming from various TD-DFT calculations have been compared with higher level $a b$ initio calculations. Since the size and complexity of $\mathrm{B}_{12}$ cofactors significantly limits the use of ad- 
vanced wave function based methods due to their cost, only low-lying excitations of $\mathrm{MeCbl}$ and $\mathrm{CNCbl}$ were accessed at this level addressing the existing controversies with respect to the nature of these transitions. At the same time, the consequent elucidation of a density functional producing data matching these findings for the lowest excited states, prepared solid foundations for analysis of energetically higher spectral bands with TD-DFT method.

Although, as already aforementioned, the Abs spectra of various $B_{12}$ analogues have been extensively discussed in scientific reports, revealing more subtle differences between them, ${ }^{108,110,112,113}$ the origin of individual bands still remains elusive. The supporting information comes from circular dichroism (CD) and magnetic CD (MCD) spectroscopy. The systematic analysis of these spectra along with the Abs data for non-alkyl as well as alkyl cobalamins, has become the foundation for the classification of the corresponding electronic spectra as "normal" and "anomalous". The former one was associated with CNCbl and was identified by the presence of the prominent $\alpha, \beta, \mathrm{D} / \mathrm{E}$ and $\delta$ bands, and a large-intensity, sharp $\gamma$ band in the high energy region. The latter spectral type has been attributed to MeCbl, and its primary manifestation is a decrease in intensity of the $\alpha$ band compared to the neighboring $\beta$ band coupled with an intensity reduction of the $\gamma$ band and its distribution among several similarly intense transitions in the UV region. In spite of the gradual changes in the Abs spectra of $\mathrm{B}_{12}$ derivatives induced by the substitution of the upper axial position by different ligands, the lower-energy $\alpha$ band has traditionally been characterized as a $\pi \rightarrow \pi^{*}$ excitation, whereas the neighboring $\beta$ region of the Abs spectrum has usually been interpreted as originating from a vibrational progression within a single electronic $\mathrm{S}_{0} \rightarrow \mathrm{S}_{1}$ transition. In other words, the $\alpha / \beta$ band has typically been interpreted as a superposition of the $0-0$ and $0-1$ components of the lowest-energy electronic $\pi \rightarrow \pi^{*}$ transition associated with the vibrational mode that primarily involves the $\mathrm{C}=\mathrm{C}$ stretching of the corrin ring.

This assignment is, however, difficult to justify experimentally as a band shape analysis may provide misleading information regarding to the number of excitations contributing to a particular part of the Abs spectrum. Some improvement 
extracting the individual transitions for certain spectral regions (as in the case of $\alpha / \beta$ band) can be achieved with the aim of CD and MCD spectroscopy. The opposite sign of these excited states facilitates their distinction. In addition, the appearance of two such consecutive features, one of which possesses a positive whereas the second bears a negative intensity value in $\mathrm{CD}$ and MCD spectra, presents an indirect evidence to exclude the possibility of vibrational progression including these two transitions. Despite the advantages of $\mathrm{CD}$ and MCD spectra, the information delivered by their analysis might still be insufficient if several excitations are energetically close. Some of them might be missed when hidden under particular band shape. Therefore, the only way to distinguish between them and to provide an insight at the molecular level is to employ theoretical tools.

Herein, it is important to draw attention to the results originating from TD-DFT ${ }^{80-84}$ calculations in the context of the interpretation of the energetically lowest $\mathrm{S}_{0} \rightarrow \mathrm{S}_{1}$ band in cobalamins described as $\pi \rightarrow \pi^{*}$ excitations within the corrin conjugation. ${ }^{107,109,111}$ The early TD-DFT analyses based on the hybrid B3LYP functional $^{114,115}$ (which has been recommended as one of the most appropriate functionals to study bioinorganic molecules ${ }^{116}$ ) carried out for models of vitamin $\mathrm{B}_{12}$ have demonstrated that the lowest-energy photoabsorption band is associated with a single electronic transition. The subsequent spectroscopic and computational studies reported by Brunold and co-workers ${ }^{108}$ have resulted in the assignment of major spectral features and the generation of the experimentally validated electronic-structure description based on the Abs, CD, and MCD spectra. To explain the difference between the $\alpha / \beta$ regions of the "typical" and "unique" Abs spectra of cobalamins (note that the alternative terms "normal" and "anomalous" were used for these systems in the older studies), a quantitative band-shape analysis for several molecular models was performed within the framework of time-dependent Heller theory and TD-DFT, where both non-alkyl and alkyl cobalamins were taken into consideration. Based on TD-DFT/B3LYP computations, it was concluded that the energetically lowest excited state can indeed be described as a $\pi \rightarrow \pi^{*}$ transition, in accordance with the original assignment found and several previous calculations. 
However, some inconsistencies were noticed, when the assumption of a single electronic transition was applied to the lowest-energy band. In the case of CNCbl, it was concluded that the $\alpha / \beta$ part of the Abs spectrum in the visible region should result from the corrin-based HOMO $\rightarrow$ LUMO transition assigned as a $\pi \rightarrow \pi^{*}$ excitation. For the $\alpha / \beta$ region of the MeCbl Abs spectrum, it was suggested that the considerably higher intensity of the $\beta$ band and the occurrence of a prominent shoulder in that band originated from an additional electronic excitation. The mechanism of vibrational progressions associated with a single electronic excitation was further questioned by different polarizations of electronic transitions and analyses of excitation profiles in the resonance Raman (RR) spectrum. Moreover, the oppositely signed features revealed in the $\mathrm{CD}$ and MCD spectra of MeCbl exposed the presence of two distinct electronic excitations in the $\alpha / \beta$ band region. To reconcile the differences between the original assignments and their own spectroscopic data, Brunold and co-workers concluded that vibrational progression associated with two different electronic transitions was required to explain the lowest-energy $\alpha / \beta$ band of MeCbl. Despite this observation, the controversies regarding to the energetically low part of cobalamin spectra have not been further explored leaving a room for speculations with respect to both a number of contributing excitations (and consequently vibrational progression) as well as their nature. In response, Chapters 4 and 5 address this problem from a novel molecular perspective. In the thereby presented studies the $\mathrm{CD}$ and MCD spectra of MeCbl, CNCbl and cob(I)alamin were simulated for the first time using TD-DFT calculations and supplementing data extracted from Abs spectra. In order to obtain the excitation energies and corresponding oscillator and rotational strengths required for the reproduction of Abs and CD spectra the poles and residues of linear response functions were calculated leading to a stick type of the spectra. To facilitate the comparison with experiment the Lorentzian broadening was then applied. In the case of MCD spectra, the Lorentzian line-shape function was obtained directly from calculations as a consequence of the utilization of the complex polarization propagator (CPP) approach with an empirical damping parameter. 
In addition, the poles and residues of quadratic as opposed to linear response functions were computed. Chapters 4 and 5 provide more detailed information about these calculations.

Whereas the electronic spectra of $\mathrm{B}_{12}$ cofactors carry information that allows to identify and differentiate the particular forms created during catalytic cycles, the understanding of their electronic structure is required to explore the mechanism of the Co-C bond cleavage. Enzyme catalyzed reactions proceed either through homolysis or heterolysis. In order to recreate this event in laboratory conditions, the Co-C dissociation is induced either thermally or photochemically leading to a pair of radical products (cob(II)alamin and an axial group radical) which are formally expected during the enzymatic homolysis. ${ }^{117}$ The difference between these two approaches is the amount of electronic states that can be probed. Through a thermal cleavage one can only access the lowest electronic state, while breaking Co-C bond with the photons involves the manifold of these states. The determination of particular states engaged in the photodissociation process of various $\mathrm{B}_{12}$ analogs as well as its overall mechanism have been insufficiently addressed at the molecular level. At the same time the photochemistry of $\mathrm{B}_{12}$ cofactors has over the years drawn a significant attention, especially due to the developments in the area of transient absorption spectroscopy (TAS). Its principles lie in recording spectra of molecules initially promoted to their excited states at different time delays. The advantages of such an approach include a possibility to reveal the states which are "dark" in linear Abs spectra as well as probing the dynamics of photoinduced processes. TAS measurements performed on cobalamins indicated their sensitivity to excitation wavelength depending on the upper axial ligand. In the case of $\mathrm{MeCbl}$, the excitation at $400 \mathrm{~nm}$ led to a prompt dissociation as well as formation of a long-lived $\mathrm{S}_{1}$ state. However, the excitation at $520 \mathrm{~nm}$ was followed only by formation of a long-lived $S_{1}$ state. ${ }^{56,58,65}$ In contrast, the photolysis of AdoCbl was found to be wavelength independent resulting in a creation of a geminate radical pair within $100-130$ ps at room temperature. ${ }^{57,59,62}$ The same studies for ethyland n-propylcobalamin indicated the formation of radical pair when samples were 
excited at both 400 and $520 \mathrm{~nm}$ but the rapid dissociation contribution was mainly observed following the higher energy excitation. At the same time, the short-lived $\mathrm{S}_{1}$ state, which could be a spectral counterpart to the one occurring after $\mathrm{MeCbl}$ excitation, was more prominent following the excitation at $520 \mathrm{~nm} \cdot{ }^{60,63}$ Examination of the nature of intermediate states populated following excitation revealed the subsequent differences among the $\mathrm{B}_{12}$-derivatives. Whereas the metastable $\mathrm{S}_{1}$ state in $\mathrm{CNCbl}$ was assigned as corresponding to ligand-to-metal charge transfer (LMCT) transition, ${ }^{64}$ the $S_{1}$ in MeCbl was described as metal-to-ligand charge transfer (MLCT). ${ }^{56,58,60}$ On the other hand, the $S_{1}$ for coenzyme $B_{12}$ presented different character (MLCT or LMCT) depending on the environment. ${ }^{65}$

In the light of the above findings, the photochemistry of cobalamins was further explored with the aim of theoretical studies. The photodissociation of $\mathrm{MeCbl}$ and ethylcobalamin (EtCbl) was simulated using TD-DFT calculations. ${ }^{118,119}$ The mechanism of photolytic cleavage of Co-C bond was explained as a consequence of an intersystem crossing between singlet and repulsive ${ }^{3}\left(\sigma_{\mathrm{Co}-\mathrm{C}} \rightarrow \sigma_{\mathrm{Co}-\mathrm{C}}^{*}\right)$ triplet state. Moreover, the immediate photolysis observed at $400 \mathrm{~nm}$ was shown to result from a low energetic barrier between high-lying singlet states and the dissociative one. At the same time, the absorption at $520 \mathrm{~nm}$ was suggested to proceed through an excitation to the lowest $S_{1}$ state from which either internal conversion to $\mathrm{S}_{0}$ state or photolysis through intersystem crossing was expected. Although the above statements were supported by reasonable arguments, the proposal became contradictory to the successive experimental measurements of the magnetic field effect (MFE). ${ }^{120}$ These results suggest that the photodissociation of $\mathrm{MeCbl}$ and AdoCbl must proceed through a singlet state. Clearly, more comprehensive theoretical investigations are required to provide a reliable explanation of light induced photolysis in $\mathrm{B}_{12}$ cofactors. In particular, the structural changes in the response to the interaction with electromagnetic fields have to be discussed. This means that in addition to previously applied approach, where electronic excitations were calculated mainly as vertical excitations from optimized structures of the ground state of particular $\mathrm{B}_{12}$ derivatives, the structures of their excited states should be also op- 
timized and inspected with respect to the photo-induced structural modifications. This perspective is presented in Chapter 6, where the photophysical properties of vitamin $\mathrm{B}_{12}$ are explored. In particular, the underlying reasons of the lack of observed Co-C photodissociation in $\mathrm{CNCbl}$, in contrast to the photolysis occurring in alkylcobalamins, are addressed with the aim of TD-DFT results. By examining the potential energy surfaces (PESs) corresponding to calculated electronic excited states of CNCbl, the nature of these states was determined and correlated with existing TAS findings. In addition, the comparisons of electronic properties and photophysics between $\mathrm{CNCbl}$ and $\mathrm{MeCbl}$, representing two distant responses to light excitation, are presented.

The main goals of hereby presented research are directed towards the understanding of electronic structure of $\mathrm{B}_{12}$ derivatives, the assignment of characteristic bands observed in Abs, $C D$ and MCD spectra, the identification of the nature of individual transitions and consequent elucidation of the events following photon excitation in vitamin $\mathrm{B}_{12}$. All investigations are carried out at molecular level by applying theoretical tools. In Chapter 2 and 3 the limitations of quantum chemical methods for studying electronic properties of cobalamins are discussed. A considerable effort was invested to determine both an adequate and practical theoretical framework. In particular, the choice of a proper density functional used in TD-DFT calculations is extensively discussed. The low-lying excited states of CNCbl (Chapter 2) and $\mathrm{MeCbl}$ (Chapter 3) are carefully examined preparing solid grounds for further insight into the photochemistry and photophysics of these cofactors which consequently can lead to an explanation of mechanisms of the reactions catalyzed by $\mathrm{B}_{12}$-dependent enzymes. Chapters 4 and 5 focus on a detailed analysis of Abs, $\mathrm{CD}$ and MCD spectra of MeCbl, CNCbl and cob(I)alamin. Whereas the former two compounds have been a subject of many scientific investigations, the latter one, lacking axial ligands, represents a very reactive species making it a very difficult system to study. Accessing its properties with spectroscopic techniques coupled with detailed analyzes at molecular level through theory is, thus, basic and required before the mechanistic studies of $B_{12}$ catalyzed reactions. Consequently, the advances in 
the assignments of individual spectral bands varying between different $\mathrm{B}_{12}$ analogs, presented in Chapters 4 and 5 are secured by utilizing a novel approach, namely a simulation of CD and MCD, in addition to already studied Abs spectra, employing TD-DFT calculations. The advantage of the CD and MCD spectra of cobalamins is that they provide complementary information to these extracted from Abs spectra. Specifically, this concerns ligand field $(\mathrm{d} \rightarrow \mathrm{d}$ ) transitions which can be enhanced in $\mathrm{CD}$ and MCD, being, at the same time, dimly pronounced in the Abs spectra. Therefore, the rationale to compute these spectra is to present new assignments of the electronic transitions of $\mathrm{B}_{12}$ derivatives focusing mainly on the controversial issues, to examine their properties as a function of axial ligand, as well as, to further validate the applied theoretical schemes in terms of their applicability to such complex systems.

An additional aspect of this research comprises the environmental dependencies. As already mentioned the TAS measurements have indicated different responses of various cobalamins to the light depending on whether samples were excited in a gas phase, solution or protein matrix. Taking this into account, the influence of the environment on the electronically excited states of MeCbl and CNCbl are explored in the studies described in Chapters 2, 4, and 6. In Chapter 6 this aspect is discussed thoroughly with respect to the nature of energetically low excitations of CNCbl. It is demonstrated that in the case of this system a different description of events following photon excitation is obtained when the calculations are performed with or without solvent effects.

An important issue demanding attention before the further step forward in theoretical studies of $\mathrm{B}_{12}$ cofactors will be made, is the determination of proper structural models used in calculations. Although the majority of previous computations has been performed successfully with simplified corrin structures, lacking side chains (Figure 1.4), the arising question is to what extent such simplifications are justified and what type of studies or properties do not suffer remarkable limitations caused by these manipulations. In response, the investigations carried out for cob(I)alamin system (Chapter 5) relied on the calculations employing both simpli- 


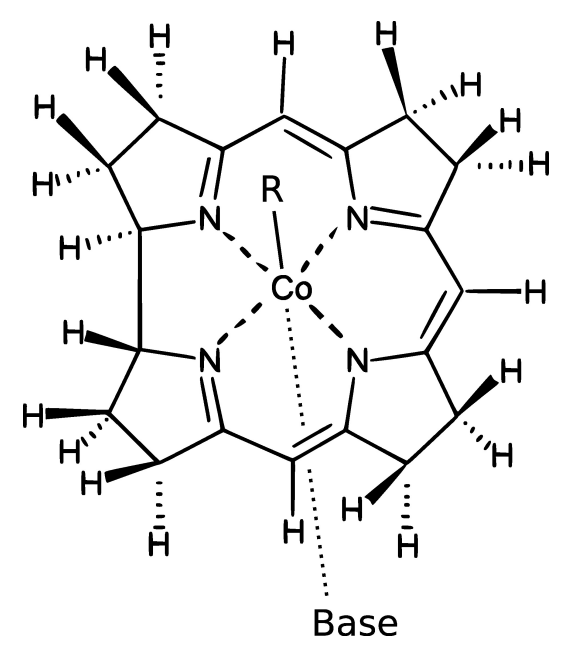

Figure 1.4. Simplified model of $\mathrm{B}_{12}$ cofactor. $\mathrm{R}=\mathrm{CN}$ or Me, or Ado; Base $=$ Imidazole (Im) or 5,6-dimethylbenzimidazole (DBI).

fied and full structure of this cofactor. Despite the additional issues resulting from limitations of applied methodologies when full structure was used, a new light has been shed on the description of electronic excitations of cob(I)alamin in particular and vitamin $\mathrm{B}_{12}$ cofactors in general. 


\section{CHAPTER 2}

\section{ELECTRONICALLY EXCITED STATES OF VITAMIN $B_{12}{ }^{1}$}

\subsection{Introduction}

Since its initial formulation, ${ }^{80}$ linear-response time-dependent density functional theory (TD-DFT) ${ }^{121,122}$ has become one of the most widely used computational tools to study electronically excited states of complex molecules. ${ }^{81,83,84}$ It is generally accepted (at least for medium to large systems up to 300 second-row atoms) that certain electronic excitations can be accurately described within the TD-DFT framework. For generalized gradient approximation (GGA) $)^{123,124}$ or hybrid exchange-correlation functionals, ${ }^{114}$ computed excitation energies are often accurate to within a few tenths of an electronvolt. This applies to electronic transitions involving minimal charge transfer that lie well below the ionization potentials. However, for excitations to Rydberg states or for charge-transfer (CT) states, a significant underestimation of computed energies has been noticed. ${ }^{125-132}$ While the former can be corrected using an asymptotic correction, the latter are more problematic mainly due to errors associated with self-interaction. ${ }^{133,134}$

CT-type excitation energies are often significantly underestimated by TD-DFT calculations employing conventional exchange-correlation functionals. In order to partially correct for this shortcoming, range-separated exchange-correlation functionals, such as Coulomb-attenuated B3LYP (CAM-B3LYP) ${ }^{135}$ or long-range corrected BLYP (LC-BLYP), ${ }^{136}$ have been designed for accurately describing CT excitations in TD-DFT calculations. Nevertheless, the view that CT excitations are

\footnotetext{
${ }^{1}$ The material presented in this Chapter was reprinted (adapted) with permission from K. Kornobis, N. Kumar, B. Wong, P. Lodowski, M. Jaworska, T. Andruniów, K. Ruud, and P. M. Kozlowski, J. Phys. Chem. A 115, 1280 (2011). Copyright 2011 American Chemical Society.
} 
significantly underestimated by TD-DFT calculations needs to be interpreted with caution since these errors typically refer to long-range excitations with a low degree of spatial overlap between the occupied and virtual orbitals involved in the excitation. In order to judge the reliability of excitation energies, a simple test (referred to as the $\Lambda$ diagnostic $)^{137}$ has been suggested. For a given excitation, the degree of overlap is calculated using this $\Lambda$ diagnostic, which involves the inner product of the moduli of individual occupied and virtual Kohn-Sham orbitals. Its numerical value can range from 0 to 1 , with values near zero signifying long-range excitation, while values near unity signifying a short-range, localized excitation. Based on its definition, excitation energies are significantly underestimated when $\Lambda$ is very small. It has further been proposed that an excitation with $\Lambda<0.4$ from a GGA or $\Lambda<0.3$ from a hybrid functional is likely to be significantly underestimated. ${ }^{138,139}$ However, a high $\Lambda$ value does not guarantee that the excitation is correctly described by TD-DFT.

The use of range separated exchange-correlation functionals, such as CAM-B3LYP, does not necessarily guarantee that results are improved over conventional functionals either. In fact, the predicted spectra may worsen, as has been recently found for two cobalamins: CNCbl and MeCbl (see Chapter 4 or Ref. 140), where absorption (Abs), circular dichroism (CD) and magnetic CD (MCD) spectra based on TD-DFT calculations were simulated for direct comparison with experiment. For these two bioinorganic complexes, the conventional BP86 functional performed better than CAM-B3LYP. It should also be noted that the $\Lambda$ diagnostic was computed for each excited state under consideration, and all computed $\Lambda$ values were greater than 0.4 , indicating that the TD-DFT calculations were not affected by the CT problem. The unexpected poor performance of the CAM-B3LYP functional may indicate that the inclusion of long-range Hartree-Fock exchange into TD-DFT overestimates the energy gap between the occupied and virtual orbitals, thus making the virtual-occupied energy difference a poor estimation for excitations energies. 
This type of sensitivity with respect to the treatment of exact exchange seems to play a more important role in systems with transition metals rather than in organic molecules. ${ }^{141,142}$

In the present chapter, we continue our focus on vitamin $B_{12}$ (Figure 1.1) as one of the most important vital bioinorganic complexes. Electronically excited states of vitamin $\mathrm{B}_{12}$ derivatives have been extensively investigated employing a variety of experimental techniques. ${ }^{64-66,68,108,113}$ However, their excited-state properties remain poorly understood from an electronic structure point of view. Since the TD-DFT framework is currently the only practical tool that can be used to routinely study excited states of these complex systems, the question naturally arises on how to select the appropriate functional for these studies. To some extent, this depends on the kind of spectroscopic properties one intends to investigate. If the target is the overall electronic spectrum and the objective is to assign certain electronic transitions, a number of excited states need to be computed to simulate the spectrum for direct comparison with experimental data. The performance of the functional is then judged based on how well the experimental spectrum is reproduced by the simulated one. However, when the target is an individual electronic state (or a manifold of low-lying excited states) the situation becomes somewhat more complicated. The performance based on a comparison with experiment is only possible if the identity of the particular electronic state is well established. ${ }^{143}$ When an assignment is uncertain, the performance of the functional is analyzed by comparisons with excited state calculations carried out by high-level $a b$ initio methods. Typically second-order approximate coupled cluster singles and doubles model (CC2) or complete active space self-consistent field followed by second-order perturbation correction (CASSCF/CASPT2) calculations are used to calibrate TD-DFT computed excited states. ${ }^{144-146}$ In the present chapter, we discuss both our TDDFT and $a b$ initio calculations and compare their accuracy in predicting vitamin $\mathrm{B}_{12}$ excitation properties. 


\subsection{Computational Details}

\subsubsection{Structural Models}

The full structure of vitamin $\mathrm{B}_{12}$ was extracted from available high-resolution X-ray crystallographic data. ${ }^{147}$ To decrease the computational cost associated with the complexity of the system (Figure 1.1), some simplifications to the initial structure were introduced. All side chains of the corrin ring as well as the nucleotide loop were replaced by hydrogen atoms. Previous studies have demonstrated that such simplified models of $\mathrm{B}_{12}$ cofactors were appropriate for studying their structural and electronic properties. ${ }^{104}$ The full structure of CNCbl also possesses dimethylbenzimidazole (DBI) as the lower axial base. In certain classes of $\mathrm{B}_{12}$-dependent enzymes ${ }^{16}$ the DBI base is replaced by histidine (His) from the protein side chain. Hence, two structural models with different lower axial ligands were utilized in the present calculations. The first structural model contains imidazole (Im), and the second is axially ligated to DBI (Figure 2.1). Both models were optimized at the BP86/6-31G(d) level of theory using the Gaussian 09 suite of programs. ${ }^{148}$ Taking into account that the main features observed in the electronic absorption spectra of cobalamins correspond to corrin $\pi \rightarrow \pi^{*}$ transitions, both models are appropriate to study electronically excited states of vitamin $B_{12}$.

\subsubsection{TD-DFT Calculations}

Several types of TD-DFT calculations have been carried out to explore the electronically excited states of CNCbl. To reproduce the electronic absorption spectrum of $\mathrm{CNCbl}$, the 35 lowest excited states were calculated. All vertical excitation energies were computed at the BP86/6-31G(d) optimized ground state geometries described previously. Three different functionals, including BP86, B3LYP, and LC-BLYP were utilized. The range-separated LC-BLYP functional, which recovers the exact $-1 / r$ exchange dependence at large interelectronic distances, was calculated as a function of the range-separation parameter $\mu$. Starting from $\mu=0.00$, which corresponds to a pure exchange-correlation density functional, the range-separation 

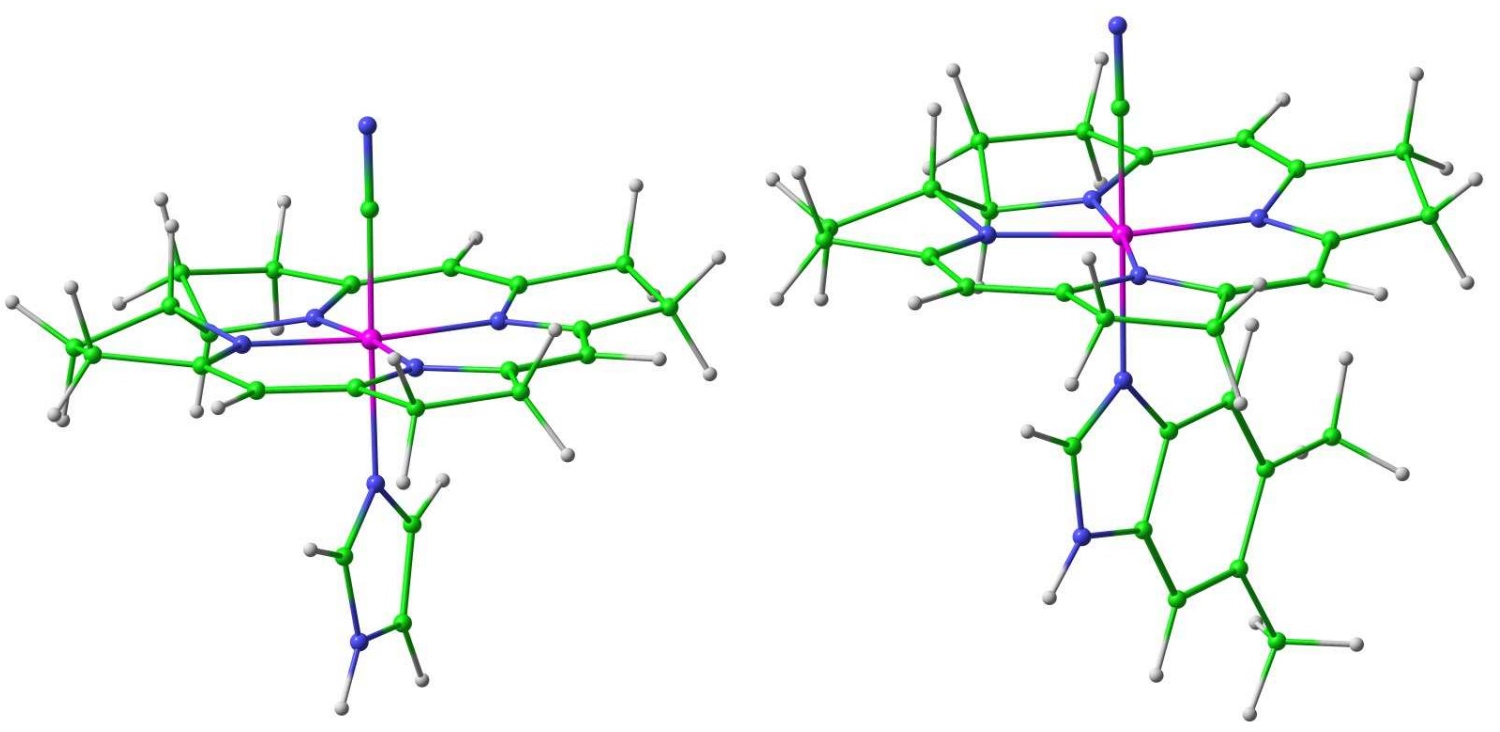

Figure 2.1. Two structural models of CNCbl used in excited states calculations. Left with imidazole (Im), right with dimethylbenzimidazole (DBI) as axial base, denoted as $\mathrm{B}-\left[\mathrm{Co}^{\mathrm{III}}\right.$ (corrin) $]-\mathrm{CN}^{+}(\mathrm{B}=\mathrm{Im}$ or $\mathrm{DBI})$.

value was systematically increased up to 0.90 in increments of 0.05 . The TD-DFTbased absorption spectra were analyzed in both gas phase and water solution environments, with the latter being modeled using a polarizable continuum model (PCM) and conductor-like screening model (COSMO). ${ }^{149}$ The discussion about the nature of the low-lying excited states, however, focuses mainly on gas phase calculations which can be directly compared with high-level, correlated ab initio results. In certain instances, the electronically excited states were computed by employing the CAM-B3LYP functional. The TD-DFT calculations have been performed using the GAMESS, ${ }^{150}$ Turbomole, ${ }^{151}$ and Dalton ${ }^{152}$ programs.

\subsubsection{Correlated $a b$ initio calculations}

Since one of the main objectives of the study presented in this chapter is an assessment of electronically excited states computed with different functionals, two types of $a b$ initio calculations have been performed for benchmarking purposes. The CC2 and CASSCF computations have been carried out in order to determine the first four low-lying excited states. The former were performed with Turbomole while 
the latter were computed with PC-GAMESS/Firely QC package. ${ }^{153}$ Due to a lack of dynamic correlation energy, the CASSCF calculations alone are not sufficiently accurate and need to be corrected by second order perturbation theory. A modified version of quasi-degenerate perturbation theory with multi-configurational selfconsistent field reference functions, referred to as MC-XQDPT2, ${ }^{154}$ as implemented in the PC GAMESS/Firefly, was applied. In order to carry out the MC-XQDPT2 analysis, state average (SA) CASSCF calculations were first calculated using 12 electrons and 12 orbitals in the active space. It should be noted that QDPT2 correction mixes and alters the order of low-lying excited states based on the initial CASSCF calculations. Thus, in order to obtain the four lowest excited states at the CASSCF/MCXQDPT2, it was required to initially compute 20 states at the SA-CASSCF level.

\subsubsection{Basis set}

In order to make a meaningful comparison between TD-DFT and $a b$ initio calculations, the same basis set was used in both calculations. Taking into account the complexity of vitamin $B_{12}$, a double-zeta 6-31g(d) basis set was selected to perform the $a b$ initio calculations. The TD-DFT results were re-evaluated with a larger TZVP triple zeta basis set. The structural model containing DBI as the lower axial ligand was also examined with a much larger aug-cc-pVDZ, basis set. It should be mentioned that the basis set quality significantly increases computational cost, and balancing accuracy against a reasonable computational time is crucial for the feasibility of the computations. Consequently, $a b$ initio calculations with the triplezeta quality basis set were not considered as a practical scheme, and these calculations were performed only with the 6-31G(d) basis set. We also note that since the CASSCF method largely considers nondynamical electronic correlation effects, and thus depends less on the nodal structure of the wave function, the 6-31G(d) basis will suffice for giving reasonable results for the MCSCF calculations. For the more dynamical correlation-oriented CC2 method, the 6-31G(d) basis may be less adequate, but we will see that there are other concerns regarding the reliability of the CC2 results. 


\subsection{Results and Discussion}

The performance of DFT in modeling electronic and structural properties associated with the ground state of $\mathrm{B}_{12}$ cofactors has been the subject of several studies. ${ }^{100,155}$ It was concluded that GGA-type functionals (such as BP86) are particularly suitable for describing their ground state properties. The recent review by Jensen and Ryde ${ }^{156}$ provides a more detailed discussion. Other properties such as absorption spectra, $\mathrm{CD}$, and MCD data were also analyzed based on realistic corrinbased models. The results based on the BP86 functional were in much better agreement with experimental data than the results based on CAM-B3LYP, demonstrating the improved performance of BP86 as compared to hybrid or Coulomb-attenuated functionals (see Chapter 4 or Ref. 140).

\subsubsection{Analysis of absorption data}

The manifold of low-lying excited states was computed to cover the spectral range up to $250 \mathrm{~nm}$ (ca. $5 \mathrm{eV}$ ). The calculated 35 vertical excitations and oscillator strengths were used to simulate the absorption spectra for direct comparison with experiment (Figures 2.2 and 2.3) in both gas-phase and PCM-based water solvent model. In order to compare with the experimental spectrum, ${ }^{67}$ each TD-DFT electronic transition was broadened with a Gaussian function having a width of $\Gamma=0.174 \mathrm{eV}$. Since the LC-BLYP and BP86-simulated spectra were almost identical, we performed additional computations using the LC-BLYP functional as a function of the range-separation parameter, $\mu$ (Figure 2.4). By varying the values of $\mu$, the influence of the relative contributions of DFT and HF to the exchange-correlation energy on the spectra were assessed. The spectra corresponding to $\mu$ values ranging from 0.00 to 0.85 were simulated and directly compared with experiment (Figures 2.5 and 2.6). The absorption profiles obtained in both environments (gas phase, and PCM-based water solution) showed the best agreement with experiment only for the first few low $\mu$ values, indicating that the use of GGA functionals is a better choice in predicting electronic properties of CNCbl. 

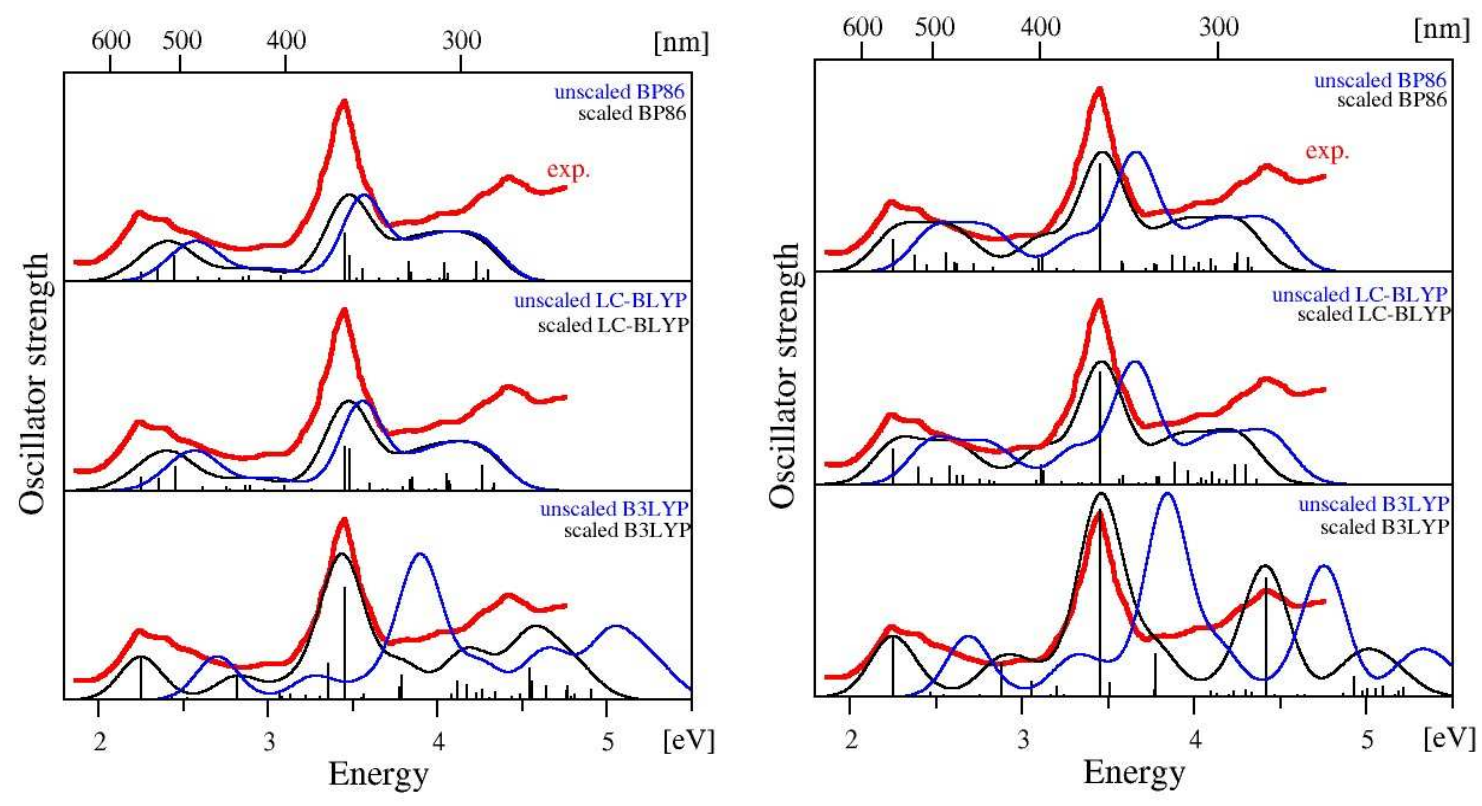

Figure 2.2. Simulated electronic absorption spectra of CNCbl computed with the use of different functionals and the 6-31G(d) basis set (left: vacuo, right: water solution, PCM). See Table A.1 (Appendix) for details regarding the scaling factors. Experimental spectra were reproduced from Ref. 67 with permission.

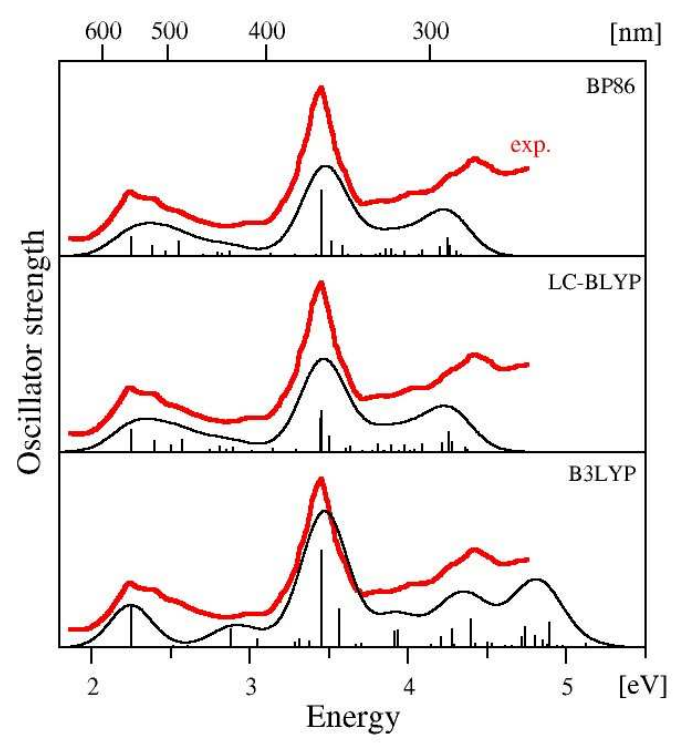

Figure 2.3. Simulated electronic absorption spectra of $\mathrm{CNCbl}$ computed with the use of different functionals and the TZVP basis set (vacuo). See Table A.2 (Appendix) for details regarding the scaling factors. Experimental spectra were reproduced from Ref. 67 with permission. 
Previous studies on cobalt corrinoids have demonstrated that electronic excitations based on TD-DFT calculations utilizing hybrid functionals were systematically overestimated. ${ }^{107}$ Since the overestimation was systematic, it could be compensated by scaling. Initially, a simple shift of the electronic transition energies was applied for models of vitamin $\mathrm{B}_{12}$; however, for the alkylcobalamins, such as methylcobalamin ( $\mathrm{MeCbl}$ ) or adenosylcobalamin (AdoCbl), a better agreement with experiment was obtained when two scaling parameters were applied. ${ }^{105,106}$ To achieve better correlation with experimental data, each electronic transition was scaled as $E_{\text {scaled }}^{i}=\chi E_{\mathrm{TD} \text {-DFT }}^{i}+E_{\text {shift }}$, where $\chi$ and $E_{\text {shift }}$ are a specific set of parameters for a given functional and corrinoid. The determination of the optimal pair of parameters was based on the assumption that electronic excitations associated with $\alpha$ and $\gamma$ bands should be reproduced accurately.

The comparison of the simulated absorption spectra (Figures 2.2 and 2.3) indicates overall good agreement with experiment regardless of the functional chosen in TD-DFT calculations. However, the detailed analysis of electronic transitions reveals some differences. Whereas the high energy $\gamma$ band is in accordance with experiment and gains the largest intensity in the B3LYP-simulated spectrum, the oscillator strengths of the same band predicted by the remaining two methods are significantly lowered. Moreover, in contrast to the B3LYP results, the BP86 and LC-BLYP gas phase data indicate the involvement of two (instead of one) intense electronic transitions. Based only on the reproduction of the experimental $\gamma$ band in gas phase, one can conclude the better suitability of the B3LYP functional in simulating absorption spectra of CNCbl. At first glance, this may imply that different functionals are good for different parts of the spectrum. However, it is important to notice that the change of the environment improves the intensity pattern in the $\gamma$ region for all three functionals and reveals the presence of a single electronic transition regardless of the method applied. In addition, the $\alpha / \beta$ band seems to be better reproduced by the BP86 and LC-BLYP functionals. The interpretation of the simulated CD and MCD spectra implied the existence of a manifold of electronic excitations in the $\alpha / \beta$ part of the CNCbl absorption spectrum. ${ }^{140}$ The data shown 


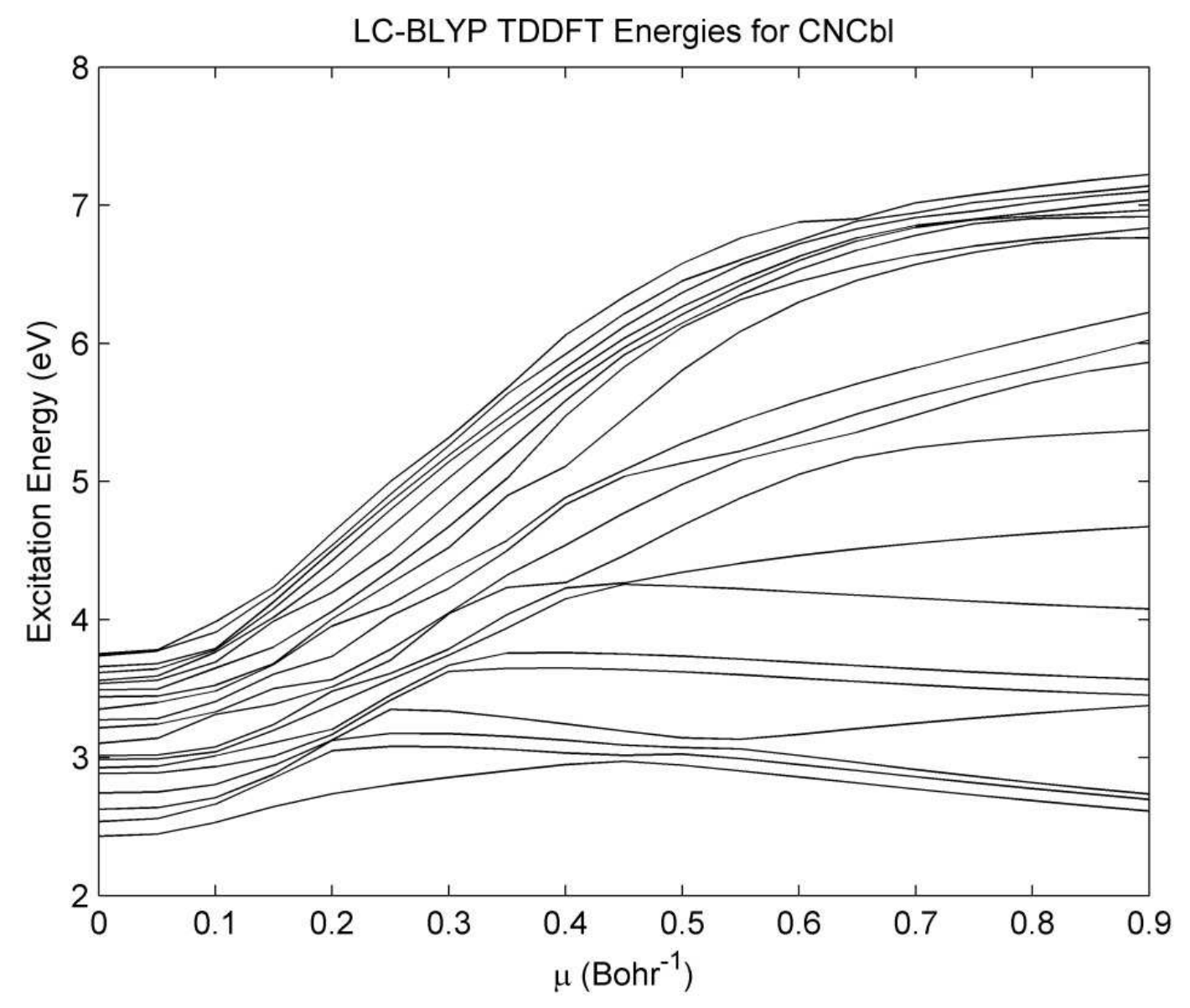

Figure 2.4. Vertical excitation energies calculated with LC-BLYP/6-31G(d) as a function of the range separation parameter, $\mu$. 


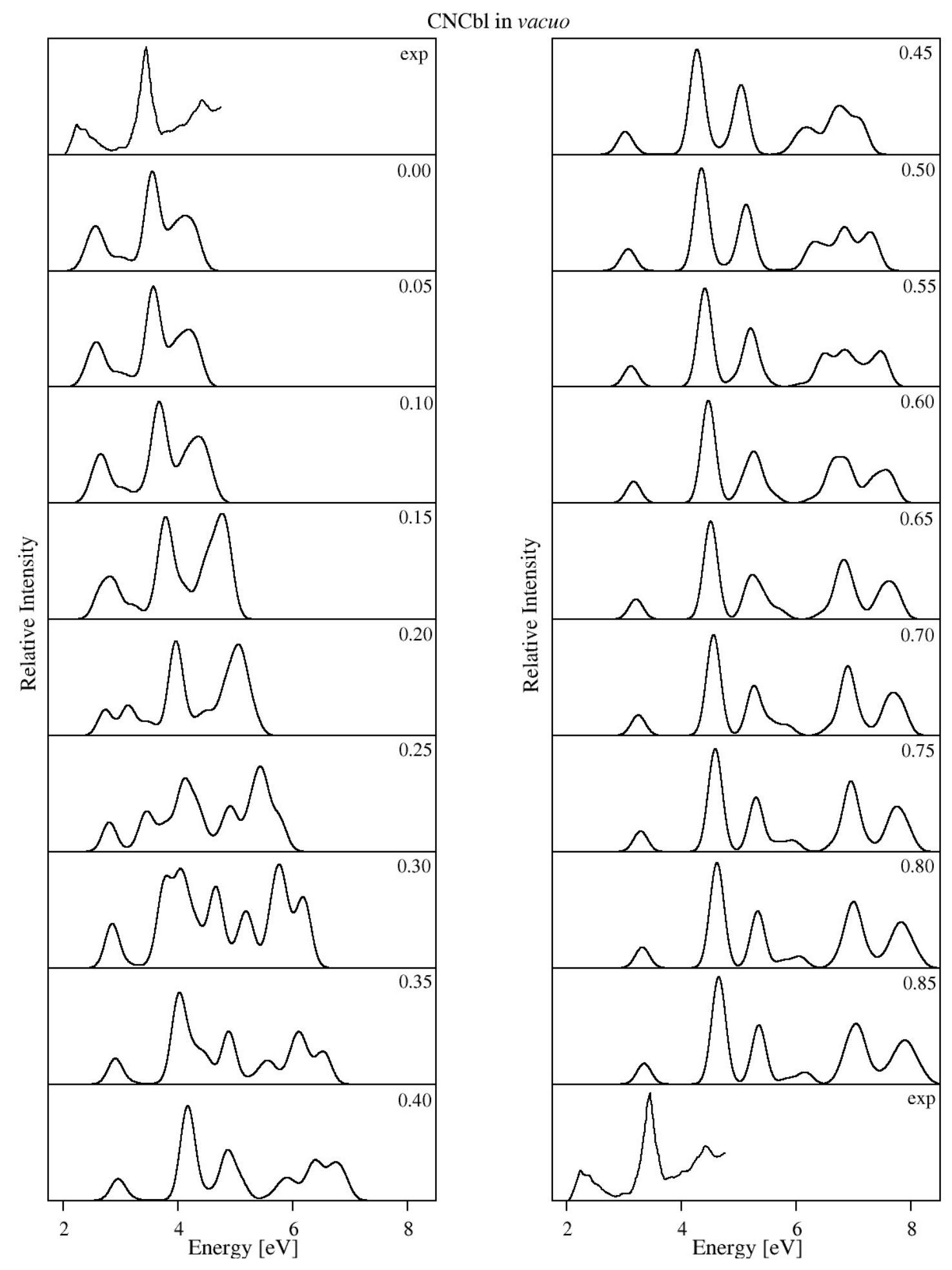

Figure 2.5. The simulated absorption spectra of Im-[Co ${ }^{\mathrm{III}}$ (corrin) $]-\mathrm{CN}^{+}$based on LC-BLYP/6-31G(d) calculations in vacuo computed as a function of $\mu$ value (indicated in the top right corner). Experimental spectra were reproduced from Ref. 67 with permission. 


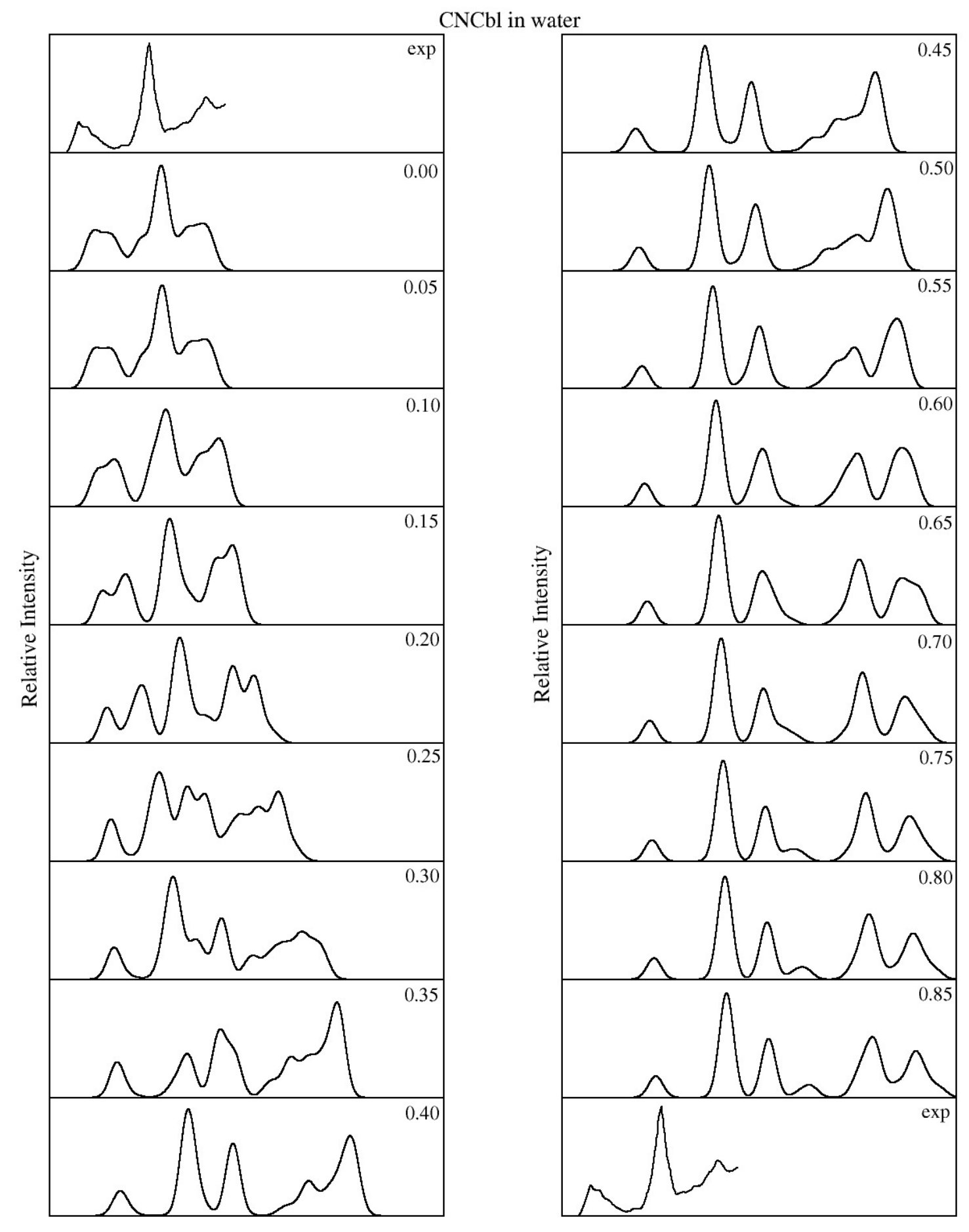

Figure 2.6. The simulated absorption spectra of Im-[Co ${ }^{\mathrm{III}}$ (corrin) $]-\mathrm{CN}^{+}$based on LC-BLYP/6-31G(d) calculations in water solution (PCM) computed as a function of $\mu$ value (indicated in the top right corner). Experimental spectra were reproduced from Ref. 67 with permission. 
in the present study indicates that there are multiple excitations present in the lowenergy part of the spectra only if BP86 or LC-BLYP is applied. Both gas phase as well as PCM data (Figure 2.2), obtained from BP86 and LC-BLYP calculations, confirm such assignment. In contrast, the $\alpha / \beta$ band in the B3LYP-computed profiles is composed of one intense excitation regardless of the environment (Figure 2.2). These results are more in line with earlier proposals, suggesting vibrational progression of a single electronic transition in the $\alpha / \beta$ region of CNCbl spectrum. ${ }^{66,68,108}$ As can be noticed, the choice of the exchange-correlation functional is crucial in terms of a reliable description of particular bands in $\mathrm{CNCbl}$ absorption spectra. However, the final statement about the correct applicability of the particular functional demands a detailed analysis of the nature of the excited states coupled with a reasonable evaluation of the theoretical methods in terms of their correspondence to the experimental results. Taking into account the photochemistry of $B_{12}$ cofactors, the most important salient features arise from the examination of the first few lowlying electronic excited states. Consequently, the comparison of TD-DFT results with correlated $a b$ initio data (presented further in this paper) allows for a reliable justification of the suitability of a particular exchange-correlation functional to study electronic properties of CNCbl.

\subsubsection{Lambda diagnostic for low-lying excited states}

Up to this point, the performance of the different functionals, including GGA and hybrid, has been discussed in the context of how well they reproduce absorption spectra. Although functionals analyzed in the present study tend to provide satis-

factory agreement with experiment, the CT-type excitations are often very poorly described by TD-DFT. In order to justify the reliability of vertical excitations of CNCbl predicted at the TD-DFT framework with different functionals, calculations involving the $\Lambda$ diagnostic were performed. Figure 2.7 displays the $\Lambda$ values corresponding to the manifold of low-lying excited states of the vitamin $\mathrm{B}_{12}$ model having imidazole as the axial base, i.e. Im-[Co ${ }^{\mathrm{III}}$ (corrin) $]-\mathrm{CN}^{+}$(Figure 2.1, upper panel). As can be already seen with the 6-31G(d) basis set, none of the computed 


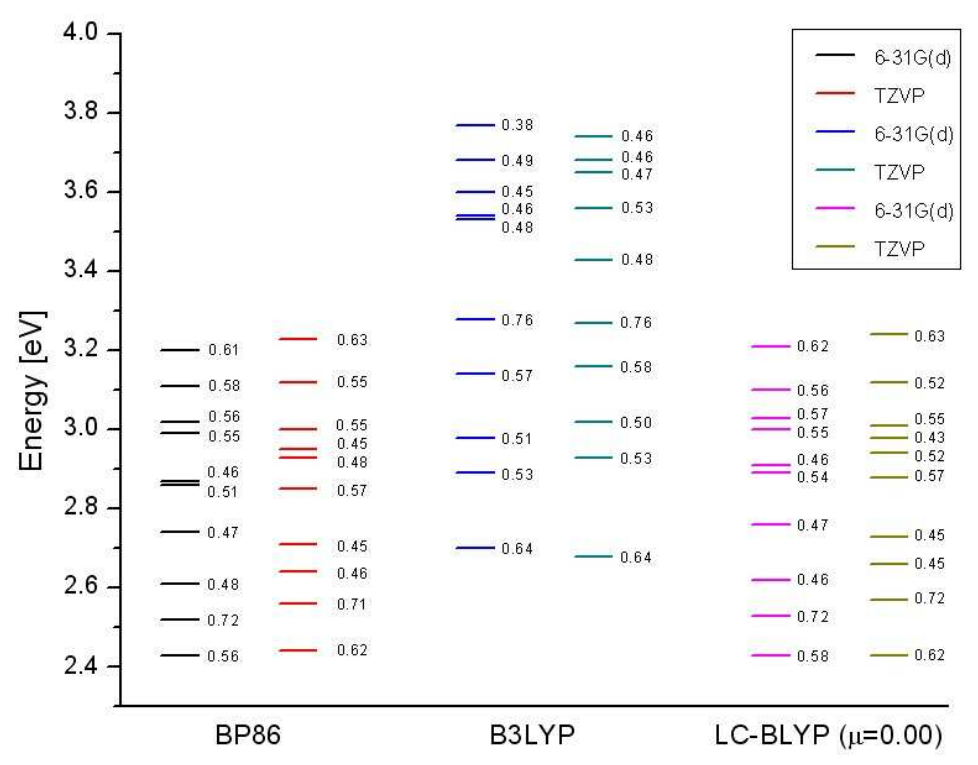

Figure 2.7. Ten lowest electronic transitions of Im-[CoII (corrin) $]-\mathrm{CN}^{+}$calculated with different density functionals and basis sets together with the corresponding $\Lambda$ diagnostic values (presented as numbers).

electronic transitions have noticeably low $\Lambda$ values, indicating that our calculations do not suffer from the CT problem. We have found that an increase in the basis set does not dramatically influence the $\Lambda$ values. On the other hand, when the same calculations were carried out for the model having DBI as the axial base, i.e. DBI-[Co ${ }^{\text {III }}$ (corrin) $]-\mathrm{CN}^{+}$(Figure 2.1, lower panel) the problem related to the CT issue was more noticeable (Figure 2.8). It appears that this model is more susceptible to CT problems due to the larger lower axial base having more extensive aromatic character and a longer Co-N (axial) distance (2.09 ̊̊ as compared to 2.05 $\AA$ for Im-[Co ${ }^{\mathrm{III}}$ (corrin) $]-\mathrm{CN}^{+}$model). According to the $\Lambda$ diagnostic, the predictions from the BP86/6-31G(d) calculations indicate that the $S_{4}$ and $S_{5}$ states have CT character since their $\Lambda$ values are lower than 0.4. The problem disappears with an increase in basis set size, although this is not due to a change in nature of the transition induced by the change of the basis set, but rather the improved ability of the more diffuse aug-cc-pVDZ basis set to model the rather short-ranged CT of this excitation. The results based on calculations with the TZVP basis set show improvement 


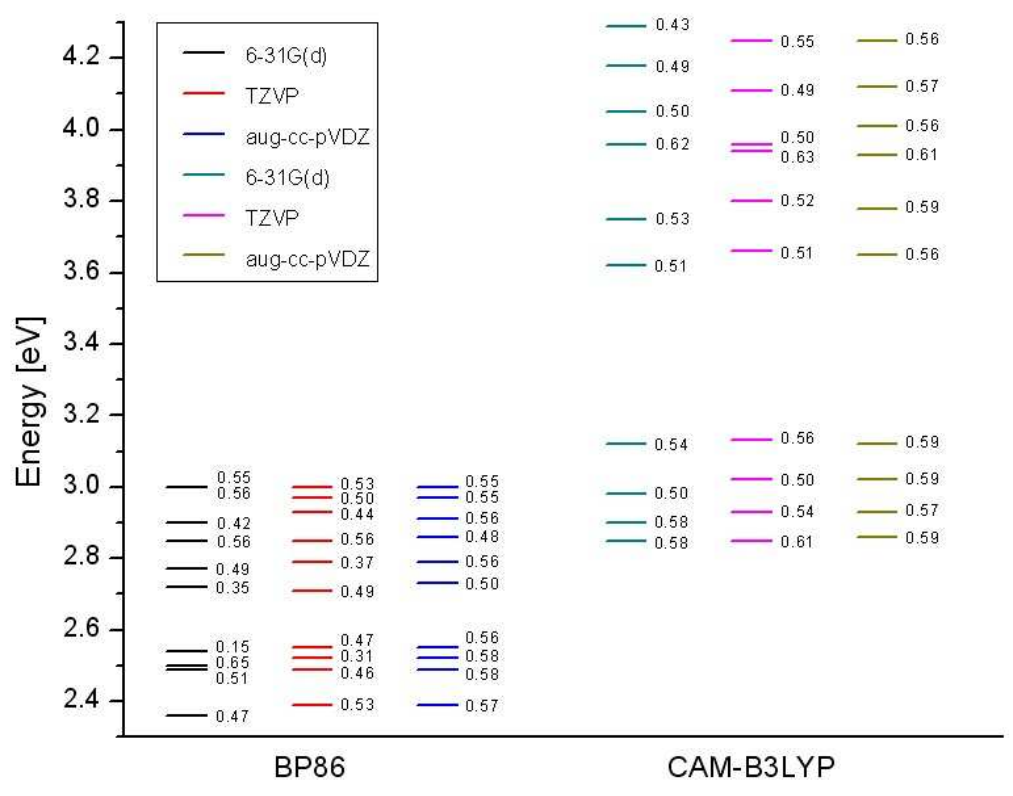

Figure 2.8. Ten lowest electronic transitions of DBI-[Co ${ }^{\mathrm{III}}$ (corrin) $]-\mathrm{CN}^{+}$calculated with different density functionals and basis sets together with the corresponding $\Lambda$ diagnostic values (presented as numbers).

for $S_{4}$ and $S_{5}$ states but simultaneously decrease the $\Lambda$ values for $S_{3}$ and $S_{6}$. At the same time, the calculations for low-lying excited states of DBI-[CoII (corrin)]- $\mathrm{CN}^{+}$, employing the aug-cc-pVDZ basis set, do not produce excitation energies suffering from the CT failure (Figure 2.8). However, it is important to note that the size of the basis set doubles (from $\sim 600$ to $\sim 1200$ ), making such calculations feasible but not practical.

The conclusion coming from our analysis indicates that the CNCbl excitation energies calculated using TD-DFT are not affected by CT failure. The lower $\Lambda$ values in the case of DBI-[Co ${ }^{\mathrm{III}}$ (corrin) $]-\mathrm{CN}^{+}$model are related to the quality of the basis set and can not be a reason for disregarding TD-DFT/BP86-based results. Moreover, the increased basis set does not change the character of the excitations, allowing for a reasonable comparison of the nature of low-lying transitions predicted by TD-DFT and $a b$ initio methods employing the 6-31G(d) basis set. 


\subsubsection{Comparison with $a b$ initio calculations}

In order to analyze the nature of the low-lying electronic transitions of CNCbl in detail, we turn our attention to ab initio methods. For medium-sized systems, the CC2 method is commonly used to compute excitation energies to serve as accurate benchmarks for TD-DFT calculations. We initially focused our attention to the four lowest states of $\mathrm{CNCbl}$ in order to estimate their energies at the CC2 level of theory. However, as shown in Table 2.1, the CC2 excitation energies are severely underestimated due to a significant multi-reference character indicated by the D1 diagnostic. ${ }^{157}$ The D1 diagnostic essentially measures the quality of the HF reference wave function, with values less than 0.05 indicating that a single determinant is appropriate. We found that all the 4 lowest electronic states of CNCbl were significantly above the 0.05 threshold, demonstrating a severe deficiency in the HF reference wave function for these metal-containing complexes.

TABLE 2.1. Vertical excitation energies (eV) of Im-[Co ${ }^{\text {III }}$ (corrin) $]-\mathrm{CN}^{+}$calculated for the first four low-lying states with different methods and the 6-31G(d) basis set.

\begin{tabular}{lcccc}
\hline \hline Method & $\mathrm{S}_{1}$ & $\mathrm{~S}_{2}$ & $\mathrm{~S}_{3}$ & $\mathrm{~S}_{4}$ \\
\hline TD-DFT & & & & \\
B3LYP & 2.75 & 2.83 & 2.93 & 3.10 \\
BP86 & 2.43 & 2.53 & 2.62 & 2.73 \\
LC-BLYP $(\mu=0.00)$ & 2.43 & 2.54 & 2.62 & 2.74 \\
LC-BLYP $(\mu=0.10)$ & 2.53 & 2.66 & 2.70 & 2.82 \\
LC-BLYP $(\mu=0.20)$ & 2.74 & 3.05 & 3.12 & 3.14 \\
LC-BLYP $(\mu=0.30)$ & 2.86 & 3.08 & 3.17 & 3.33 \\
Ab initio & & & & \\
CC2 & 1.80 & 2.27 & 2.57 & 2.63 \\
CASSCF(12,12) & 3.44 & 3.48 & 3.60 & 4.75 \\
CASSCF/MC-XQDPT2 & 2.08 & 2.32 & 2.68 & 2.98 \\
\hline \hline
\end{tabular}


Consequently, CASSCF calculations were applied as a more appropriate method for studying electronically excited states of CNCbl. The CASSCF approach is very challenging when applied to molecules containing transition metals, and the results strongly depend on the choice of active space. Due to the presence of cobalt metal in $\mathrm{CNCbl}$, the proper selection of active space requires the use of the metal's d orbitals. In addition, a complementary set of $3 \mathrm{~d}$ orbitals has been added to the active space in order to consider the double-shell effect. ${ }^{158}$ When the complex contains a tetrapyrrolic ligand (such as corrin) in addition to cobalt $\mathrm{d}$ orbitals, the proper active space also requires inclusion of the $\pi$ orbitals of corrin as well as $\sigma$ orbitals associated with axial bonding. Keeping in mind these constraints, the active space was initially selected using orbitals previously applied in CASSCF calculations for cob(I)alamin ${ }^{159}$ as well as corrole ${ }^{160}$ systems. Several possible compositions have been tested, leading to the conclusion that the most appropriate choice was 12 active electrons distributed in 12 active orbitals. More specifically, five $\mathrm{d}$ Co and one $\pi$ corrin occupied MOs were combined with the corresponding virtual orbitals, and their isosurface plots are presented on Figure 2.9.

The analysis of vertical excitations predicted at the $\operatorname{CASSCF}(12,12)$ level of theory shows an overestimation of the energies in comparison to those computed at the TD-DFT level with different functionals (Table 2.1). The inspection of the nature of the energetically lowest CASSCF $(12,12)$ excitations (Table A.3 in the Appendix) revealed that $\mathrm{d} \rightarrow \mathrm{d}$ transitions were dominant, contrary to all other results (Table 2.3). This finding also contradicts the expectation that electronic excitations possessing non-zero transition dipole moments mainly involve corrin $\pi \rightarrow \pi^{*}$ transitions. In order to obtain reliable results, second-order perturbation theory was applied using the MC-XQDPT2 based on the SA-CASSCF wave function. Inclusion of dynamical correlation energy changes the nature of the electronic transitions and more states having mixed $\mathrm{d} / \pi \rightarrow \pi^{*}$ character are produced (Table 2.2). The specific mixing is shown in Figure 2.10, where only the four lowest MC-XQDPT2 excited states were depicted as those are the only ones considered for comparison with the TDDFT results. None of the first few electronically excited states predicted 

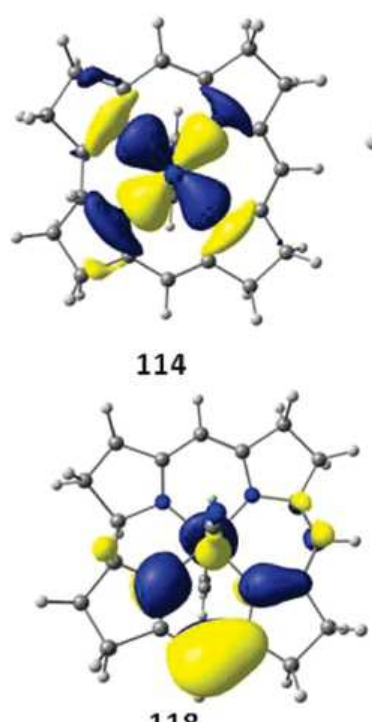

118

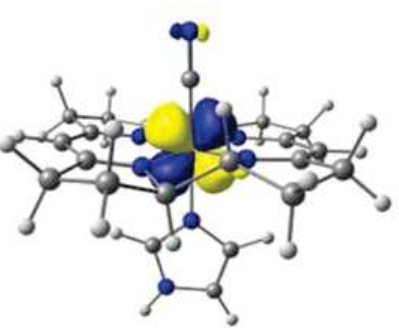

115
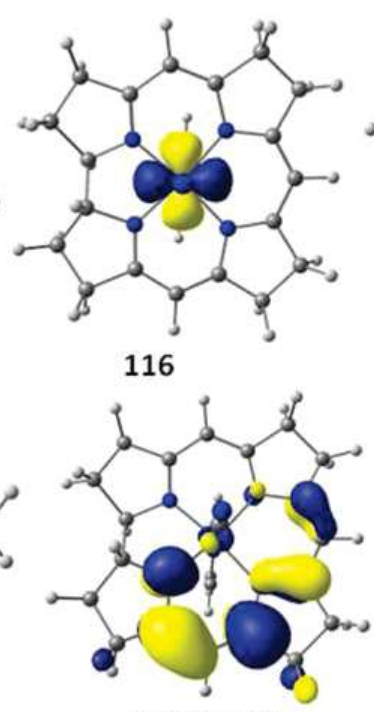

120-LUMO

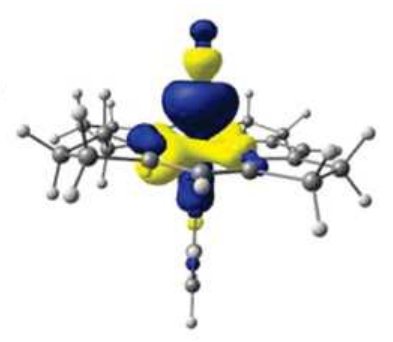

117

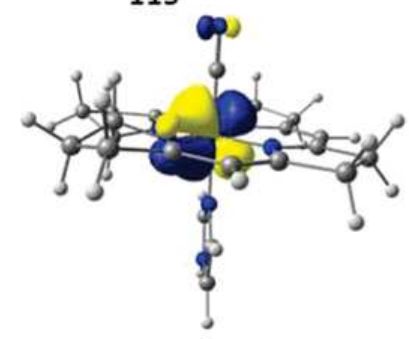

119-HOMO

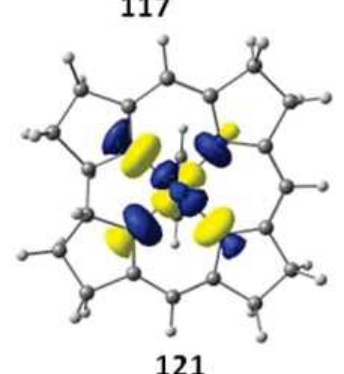

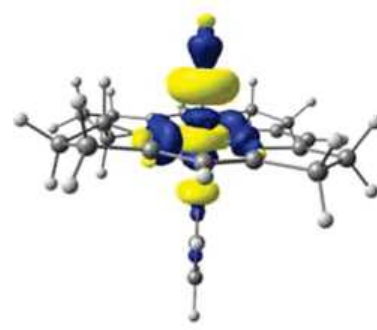

122

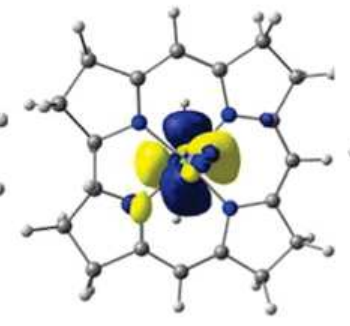

123

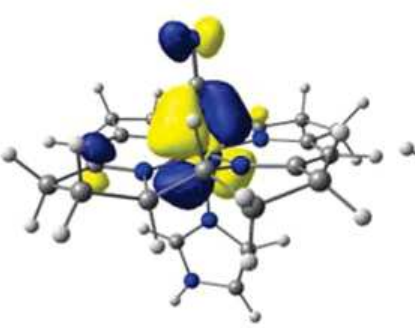

124

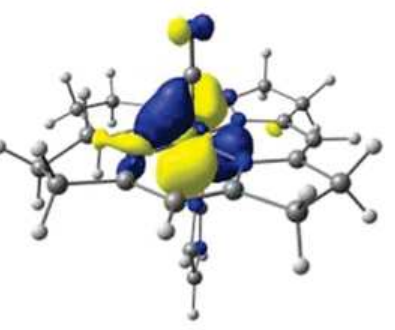

125

Figure 2.9. Active space orbitals of $\mathrm{Im}-\left[\mathrm{Co}^{\mathrm{III}}\right.$ (corrin) $]-\mathrm{CN}^{+}$used in $\mathrm{CASSCF}(12,12)$ calculations. 
at the MC-XQDPT2 level retain the same character as the CASSCF states. In addition, the involvement of dynamic correlation varies for different states and becomes more significant in states having $\mathrm{d} \rightarrow \pi^{*}$ and $\pi \rightarrow \pi^{*}$ transitions.

To further explore the nature of the low-lying excited states of CNCbl, we compared the results obtained from TD-DFT and MC-XQDPT2 calculations (Tables 2.2 and 2.3). The electronic absorption spectra of cobalamins (at least the most intense transitions) are dominated by corrin $\pi \rightarrow \pi^{*}$ excitations. However, as noted before, experiments focusing on the photochemistry of $\mathrm{B}_{12}$ cofactors suggest the possibility of CT character for the energetically lowest excitations. Indeed, the data presented in Table 2.2 confirm such predictions as none of the MC-XQDPT2calculated states have a pure nature. In fact, the $S_{1}, S_{3}$, and $S_{4}$ states are of mixed $\mathrm{d} \rightarrow \pi^{*}$ and $\pi \rightarrow \pi^{*}$ character, whereas $\mathrm{S}_{2}$ is primarily a $\mathrm{d} \rightarrow \pi^{*}$ transition. Based on this assignment and the TD-DFT results presented in Table 2.3, it can be concluded that hybrid functionals fail in predicting the correct nature of the electronic excited states of CNCbl. On the other hand, the excitations computed by BP86 show good agreement with MC-XQDPT2 calculations. The $S_{1}$ and $S_{3}$ states are composed of mixed $\mathrm{d} / \pi \rightarrow \pi^{*}$ and $\pi \rightarrow \pi^{*}$ transitions, and $\mathrm{S}_{2}$ is dominated by $\mathrm{d} / \pi \rightarrow \pi^{*}$. Similar results are obtained with the LC-BLYP functional when the range-separation parameter $\mu$ is zero (which essentially reduces the LC-BLYP formalism to a GGA-type functional). However, in order to evaluate the impact of increasing the amount of exact HF exchange on the character of low-lying electronic transitions, the performance of the LC-BLYP method was analyzed by varying the value of $\mu$ from 0.00 to 0.90 (see Ref. ${ }^{161}$ ). The description of the low-lying excited states of CNCbl worsens with a large value of $\mu$. Therefore, we conclude that the most reliable description of CNCbl excitations is predicted by BP86 calculations which imply the involvement of corrin $\pi \rightarrow \pi^{*}$, as well as charge transfer transitions involving cobalt $\mathrm{d}$ orbitals. 


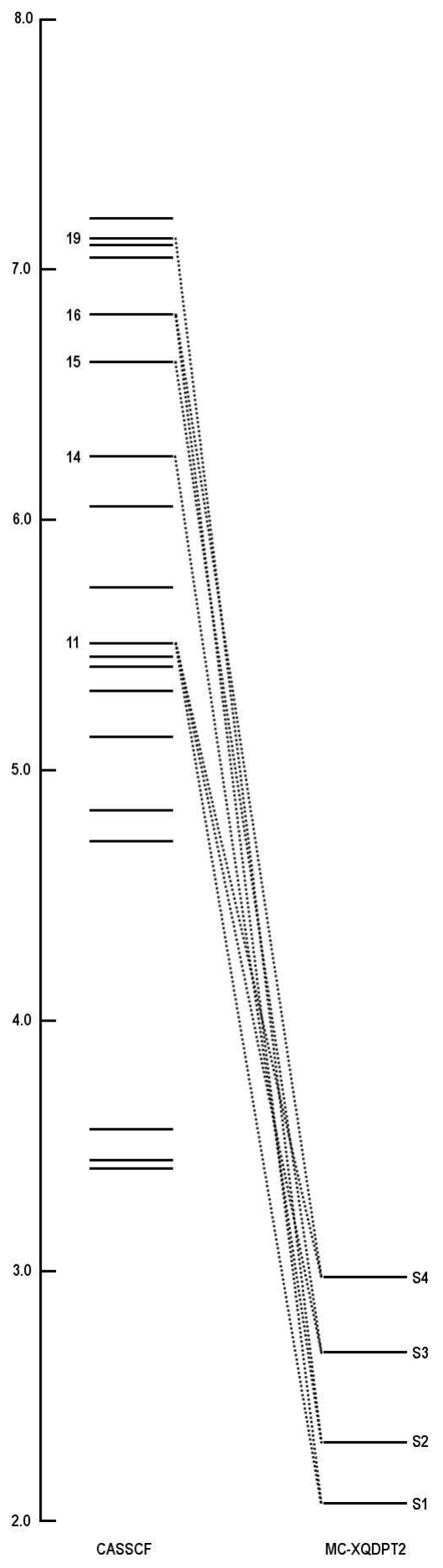

Figure 2.10. Energies of the lowest twenty excited states computed at the CASSCF level and the corresponding four low-lying states using MC-XQDPT2. The lines between CASSCF and MC-XQDPT2 indicate mixing of states upon inclusion of second order perturbation theory (see Table 2.2 for further details). 
TABLE 2.2. The first four low-lying excited states of Im-[CoIII (corrin) $]-\mathrm{CN}^{+}$obtained from CASSCF $(12,12) /$ MC-XQDPT2 calculations ${ }^{a}$.

\begin{tabular}{|c|c|c|c|c|c|c|}
\hline State & $\mathrm{E}(\mathrm{eV})$ & $\begin{array}{r}\text { Coeff. of CAS } \\
\text { state in MC/XQDPT2 }\end{array}$ & $\begin{array}{l}\text { Weight (\%) of CAS } \\
\text { state in MC-XQDPT2 }\end{array}$ & $\begin{array}{r}\text { CAS } \\
\text { state }\end{array}$ & $\begin{array}{r}\text { Coeff. } \\
\text { CSF (\%) }\end{array}$ & Character \\
\hline $\mathrm{S}_{0}$ & 0.00 & -0.924238 & 85 & 1 & & \\
\hline \multirow[t]{5}{*}{$\mathrm{S}_{1}$} & 2.08 & -0.601547 & 36 & 16 & $\begin{array}{l}24 \\
10\end{array}$ & $\begin{aligned} \mathrm{d}_{y z} & \rightarrow \pi^{*} \\
\mathrm{~d}_{x z} & \rightarrow \pi^{*}\end{aligned}$ \\
\hline & & & & & 4 & $\mathrm{~d}_{x z} / \pi \rightarrow \pi^{*} / \sigma^{*}\left(\mathrm{~d}_{z^{2}}\right)$ \\
\hline & & -0.482393 & 23 & 11 & 18 & $\pi \rightarrow \pi^{*}$ \\
\hline & & & & & 12 & $\sigma\left(\mathrm{d}_{z^{2}}\right) \rightarrow \pi^{*}$ \\
\hline & & & & & 11 & $\mathrm{~d}_{x y} \rightarrow \pi^{*}$ \\
\hline \multirow[t]{5}{*}{$S_{2}$} & 2.32 & -0.793131 & 63 & 14 & 31 & $\mathrm{~d}_{x^{2}-y^{2}} \rightarrow \pi^{*}$ \\
\hline & & & & & 8 & $\mathrm{~d}_{x z} \rightarrow \pi^{*}$ \\
\hline & & 0.530156 & 28 & 19 & $\begin{array}{r}8 \\
16\end{array}$ & $\left.\mathrm{~d}_{x^{2}-y^{2} /\left(z^{2}\right.} \rightarrow \pi^{*}\right)\left(\mathrm{d}_{z^{2}}\right)$ \\
\hline & & & & & 15 & $\mathrm{~d}_{x^{2}-y^{2}} / \pi \rightarrow \pi^{*} / \mathrm{d}_{x y}+\mathrm{n}$ \\
\hline & & & & & 7 & $\mathrm{~d}_{x y} / \mathrm{d}_{x^{2}-y^{2}} \rightarrow \pi^{*} / \sigma^{*}\left(\mathrm{~d}_{z^{2}}\right)$ \\
\hline \multirow[t]{6}{*}{$\mathrm{S}_{3}$} & 2.68 & -0.751397 & 56 & 15 & 21 & $\mathrm{~d}_{x z} \rightarrow \pi^{*}$ \\
\hline & & & & & 16 & $\mathrm{~d}_{y z} \rightarrow \pi^{*}$ \\
\hline & & & & & 6 & $\mathrm{~d}_{x^{2}-y^{2}} \rightarrow \pi^{*}$ \\
\hline & & -0.323601 & 10 & 11 & $\begin{array}{r}6 \\
18\end{array}$ & $\mathrm{~d}_{x y} \rightarrow \pi^{*}$ \\
\hline & & 0.020001 & & & 11 & $\sigma\left(\mathrm{d}_{z^{2}}\right) \rightarrow \pi^{*}$ \\
\hline & & & & & 11 & $\mathrm{~d}_{x y} \rightarrow \pi^{*}$ \\
\hline \multirow[t]{6}{*}{$\mathrm{S}_{4}$} & 2.98 & 0.454615 & 21 & 16 & 24 & $\mathrm{~d}_{y z} \rightarrow \pi^{*}$ \\
\hline & & & & & 10 & $\mathrm{~d}_{x z} \rightarrow \pi^{*}$ \\
\hline & & & & & 4 & $\mathrm{~d}_{x z} / \pi \rightarrow \pi^{*} / \sigma^{*}\left(\mathrm{~d}_{z^{2}}\right)$ \\
\hline & & -0.443262 & 20 & 11 & 18 & $\pi \rightarrow \pi^{*}$ \\
\hline & & & & & 11 & $\sigma\left(\mathrm{d}_{z^{2}}\right) \rightarrow \pi^{*}$ \\
\hline & & & & & 11 & $\mathrm{~d}_{x y} \rightarrow \pi^{*}$ \\
\hline
\end{tabular}

${ }^{a}$ CSF stands for Configuration State Function.

\subsubsection{Assignment of Electronic Excitations}

The previous studies employing the B3LYP functional lead to the assignment of the majority of bands observed in the absorption spectrum of CNCbl. ${ }^{107,108}$ As in other corrinoid species, the B3LYP calculations yield a much more blue shifted transition with respect to experimental values. Although the spectral shifts can be compensated by scaling, other problems with the B3LYP functional such as the underestimation of the Co-C bond dissociation energy, overestimation of Co- $\mathrm{N}_{\text {axial }}$ bond distance, as well as the description of the nature of low-lying excited states can not be disregarded. Hence, we focus our discussion on our TD-DFT/BP86 calculations in order to present a re-assignment of the electronic transitions of CNCbl.

The description of the singlet excited states of CNCbl is presented in Table 2.4 and 5. The longest wavelength part of the $\mathrm{CNCbl}$ absorption spectrum $(\alpha / \beta$ region) shows several bands at $550,517,485 \mathrm{sh}$ (sh = shoulder) $\mathrm{nm}$, which were originally 
TABLE 2.3. Composition of low-lying electronically excited states of Im$\left[\mathrm{Co}^{\mathrm{III}}\right.$ (corrin) $]-\mathrm{CN}^{+}$based on TD-DFT calculations with the use of different density functionals and the 6-31G(d) basis set.

\begin{tabular}{clcl}
\hline \hline State & Functional & Energy $(\mathrm{eV})$ & Character \\
\hline $\mathrm{S}_{1}$ & B3LYP & 2.75 & $79 \%\left(\pi \rightarrow \pi^{*}\right)$ \\
& BP86 & 2.43 & $60 \%\left(\pi \rightarrow \pi^{*}\right)+30 \%\left(\mathrm{~d}_{x z}+\pi_{\mathrm{CN}}+\pi \rightarrow \pi^{*}\right)$ \\
& LC-BLYP $(\mu=0)$ & 2.43 & $72 \%\left(\pi \rightarrow \pi^{*}\right)+26 \%\left(\mathrm{~d}_{x z}+\pi \rightarrow \pi^{*}\right)$ \\
$\mathrm{S}_{2}$ & B3LYP & 2.83 & $22 \%\left(\mathrm{~d}_{x z}+\pi_{\mathrm{CN}} \rightarrow \sigma^{*}+\mathrm{d}_{x y}\right)+17 \%\left(\mathrm{~d}_{x z}+\pi \rightarrow \sigma^{*}+\mathrm{d}_{x y}\right)$ \\
& BP86 & 2.53 & $72 \%\left(\mathrm{~d}_{y z}+\pi \rightarrow \pi^{*}\right)$ \\
& LC-BLYP $(\mu=0)$ & 2.54 & $83 \%\left(\pi+\mathrm{d}_{y z} \rightarrow \pi^{*}\right)$ \\
$\mathrm{S}_{3}$ & B3LYP & 2.93 & $24 \%\left(\pi \rightarrow \sigma^{*}\left(\mathrm{~d}_{z^{2}}\right)\right)+20 \%\left(\mathrm{~d}_{x z}+\pi_{\mathrm{CN}} \rightarrow \sigma^{*}\left(\mathrm{~d}_{z^{2}}\right)\right)+10 \%\left(\pi_{\mathrm{Im}}+\pi_{\mathrm{CN}} \rightarrow \sigma^{*}\left(\mathrm{~d}_{z^{2}}\right)\right)$ \\
& BP86 & 2.62 & $56 \%\left(\mathrm{~d}_{x z}+\pi_{\mathrm{CN}}+\pi \rightarrow \pi^{*}\right)+18 \%\left(\pi \rightarrow \pi^{*}\right)+14 \%\left(\pi \rightarrow \mathrm{d}_{x y}+\mathrm{n}\right)$ \\
& LC-BLYP $(\mu=0)$ & 2.62 & $65 \%\left(\mathrm{~d}_{x z}+\pi \rightarrow \pi^{*}\right)+22 \%\left(\pi \rightarrow \pi^{*}\right)$ \\
$\mathrm{S}_{4}$ & B3LYP & 3.10 & $48 \%\left(\mathrm{~d}_{x y}+\sigma \rightarrow \sigma^{*}+\mathrm{d}_{x y}\right)+16 \%\left(\mathrm{~d}_{x y}+\sigma \rightarrow \sigma^{*}\left(\mathrm{~d}_{z^{2}}\right)\right)+10 \%\left(\pi+\mathrm{d}_{x z} \rightarrow \sigma^{*}+\mathrm{d}_{x y}\right)$ \\
& BP86 & 2.73 & $74 \%\left(\pi \rightarrow \mathrm{d}_{x y}+\mathrm{n}\right)$ \\
& LC-BLYP $(\mu=0)$ & 2.74 & $86 \%\left(\pi \rightarrow \sigma^{*}+\mathrm{d}_{x y}\right)$ \\
\hline \hline
\end{tabular}

interpreted as a vibrational progression of one intense $\pi \rightarrow \pi^{*}$ electronic transition, giving rise to the corrin $-\mathrm{C}=\mathrm{C}$ - stretching vibrations. However, according to previous findings based on CD and MCD spectra, it is not necessary to make such an assumption in order to understand the absorption spectrum of CNCbl. The lowest energy singlet states $S_{1}-S_{10}$ are transitions involving several of the highest occupied MOs and lowest unoccupied MOs (Figure 2.11). As described in the previous section, the first three of them are dominated by both $\mathrm{d} / \pi \rightarrow \pi^{*}$ and $\pi \rightarrow \pi^{*}$ transitions. In addition, the $S_{3}$ state at $474 \mathrm{~nm}$ also involves a $14 \%$ contribution from a HOMO to LUMO +1 transition that corresponds to a $\pi \rightarrow \mathrm{d}$ transition. It is interesting to note that the $\mathrm{S}_{4}$ transition at $455 \mathrm{~nm}$ is primarily comprised of a $\pi \rightarrow \mathrm{d}$ excitation and may also be included in the $\alpha / \beta$ band. In fact, experiments based on ultrafast transient absorption spectroscopy have indicated the significant role of CT in the photolysis of cobalamin species. In particular, the metastable $S_{1}$ state of CNCbl was found to have a ligand-to-metal charge transfer (LMCT) character. Although the BP86-computed results suggest such contributions to only the third or fourth state, their presence should be interpreted with caution, especially since our COSMO results (Table 2.5) show very high $40 \%$ and $53 \%$ contribution of $\pi \rightarrow \mathrm{d}$ transition to the $S_{2}$ and $S_{3}$ excitations, respectively. 
TABLE 2.4. TD-DFT/BP86/6-31G(d)-based electronic transitions ${ }^{a}$ for the singlet states of $\mathrm{Im}-\left[\mathrm{Co}{ }^{\mathrm{III}}\right.$ (corrin) $]-\mathrm{CN}^{+}$.

\begin{tabular}{|c|c|c|c|c|c|c|c|c|}
\hline & $\overline{\mathrm{E}(\mathrm{eV})}$ & $\lambda(\mathrm{nm})$ & $f$ & $\%$ & Character $^{b}$ & & & $\begin{array}{l}\text { Experiment }^{c} \\
\mathrm{E}(\mathrm{eV}) \lambda(\mathrm{nm})\end{array}$ \\
\hline $\mathrm{S}_{1}$ & 2.43 & 510.4 & 0.0207 & $\begin{array}{l}30 \\
60\end{array}$ & $\begin{array}{l}117 \rightarrow 120 \\
119 \rightarrow 120\end{array}$ & $\begin{array}{l}\mathrm{H}-2 \rightarrow \mathrm{L} \\
\mathrm{H} \rightarrow \mathrm{L}\end{array}$ & $\begin{array}{l}\mathrm{d}_{x z}+\pi_{\mathrm{CN}}+\pi \rightarrow \pi^{*} \\
\pi \rightarrow \pi^{*}\end{array}$ & $2.25(550)$ \\
\hline $\mathrm{S}_{2}$ & 2.53 & 490.8 & 0.0237 & 72 & $118 \rightarrow 120$ & $\mathrm{H}-1 \rightarrow \mathrm{L}$ & $\mathrm{d}_{y z}+\pi \rightarrow \pi^{*}$ & \\
\hline \multirow[t]{3}{*}{$\mathrm{S}_{3}$} & 2.62 & 474.1 & 0.0435 & 56 & $117 \rightarrow 120$ & $\mathrm{H}-2 \rightarrow \mathrm{L}$ & $\mathrm{d}_{x z}+\pi_{\mathrm{CN}}+\pi \rightarrow \pi^{*}$ & $2.40(517)$ \\
\hline & & & & 18 & $119 \rightarrow 120$ & $\mathrm{H} \rightarrow \mathrm{L}$ & $\pi \rightarrow \pi^{*}$ & \\
\hline & & & & 14 & $119 \rightarrow 121$ & $\mathrm{H} \rightarrow \mathrm{L}+1$ & $\pi \rightarrow \mathrm{d}_{x y}+\mathrm{n}$ & \\
\hline $\mathrm{S}_{4}$ & 2.73 & 454.7 & 0.0137 & 74 & $119 \rightarrow 121$ & $\mathrm{H} \rightarrow \mathrm{L}+1$ & $\pi \rightarrow \mathrm{d}_{x y}+\mathrm{n}$ & $2.56(485)$ \\
\hline $\mathrm{S}_{5}$ & 2.85 & 434.5 & 0.0034 & 72 & $118 \rightarrow 121$ & $\mathrm{H}-1 \rightarrow \mathrm{L}+1$ & $\mathrm{~d}_{y z}+\pi \rightarrow \mathrm{d}_{x y}+\mathrm{n}$ & \\
\hline $\mathrm{S}_{6}$ & 2.89 & 429.3 & 0.0039 & 84 & $116 \rightarrow 120$ & $\mathrm{H}-3 \rightarrow \mathrm{L}$ & $\mathrm{d}_{x^{2}-y^{2}}+\pi_{\mathrm{CN}} \rightarrow \pi^{*}$ & \\
\hline $\mathrm{S}_{7}$ & 3.00 & 413.7 & 0.0071 & 76 & $118 \rightarrow 122$ & $\mathrm{H}-1 \rightarrow \mathrm{L}+2$ & $\mathrm{~d}_{y z}+\pi \rightarrow \sigma^{*}\left(\mathrm{~d}_{z^{2}}\right)+\mathrm{n}$ & \\
\hline \multirow[t]{2}{*}{$\mathrm{S}_{8}$} & 3.00 & 413.0 & 0.0124 & 70 & $119 \rightarrow 122$ & $\mathrm{H} \rightarrow \mathrm{L}+2$ & $\pi \rightarrow \sigma^{*}\left(\mathrm{~d}_{z^{2}}\right)+\mathrm{n}$ & $2.71(457)$ \\
\hline & & & & 14 & $119 \rightarrow 123$ & $\mathrm{H} \rightarrow \mathrm{L}+3$ & $\pi \rightarrow \pi^{*}$ & \\
\hline \multirow[t]{2}{*}{$\mathrm{S}_{9}$} & 3.11 & 399.1 & 0.0013 & 68 & $115 \rightarrow 120$ & $\mathrm{H}-4 \rightarrow \mathrm{L}$ & $\mathrm{d}_{x^{2}-y^{2}}+\pi_{\mathrm{CN}}+\pi \rightarrow \pi^{*}$ & \\
\hline & & & & 18 & $119 \rightarrow 123$ & $\mathrm{H} \rightarrow \mathrm{L}+3$ & $\pi \rightarrow \pi^{*}$ & \\
\hline \multirow[t]{2}{*}{$S_{10}$} & 3.20 & 387.2 & 0.0091 & 10 & $113 \rightarrow 120$ & $\mathrm{H}-6 \rightarrow \mathrm{L}$ & $\pi_{\mathrm{CN}}+\pi \rightarrow \pi^{*}$ & $3.04(408)$ \\
\hline & & & & 66 & $118 \rightarrow 123$ & $\mathrm{H}-1 \rightarrow \mathrm{L}+3$ & $\mathrm{~d}_{y z}+\pi \rightarrow \pi^{*}$ & \\
\hline \multirow[t]{2}{*}{$S_{15}$} & 3.54 & 350.2 & 0.1052 & 16 & $115 \rightarrow 121$ & $\mathrm{H}-4 \rightarrow \mathrm{L}+1$ & $\mathrm{~d}_{x^{2}-y^{2}}+\pi_{\mathrm{CN}}+\pi \rightarrow \mathrm{d}_{y z}+\mathrm{n}$ & \\
\hline & & & & 30 & $119 \rightarrow 123$ & $\mathrm{H} \rightarrow \mathrm{L}+3$ & $\pi \rightarrow \pi^{*}$ & $3.44(380)$ \\
\hline \multirow[t]{2}{*}{$S_{16}$} & 3.56 & 348.3 & 0.0399 & 60 & $113 \rightarrow 120$ & $\mathrm{H}-6 \rightarrow \mathrm{L}$ & $\pi_{\mathrm{CN}}+\pi \rightarrow \pi^{*}$ & \\
\hline & & & & 10 & $119 \rightarrow 124$ & $\mathrm{H} \rightarrow \mathrm{L}+4$ & $\pi \rightarrow \pi^{*}$ & \\
\hline $\mathrm{S}_{23}$ & 3.91 & 316.8 & 0.0302 & 70 & $118 \rightarrow 124$ & $\mathrm{H}-1 \rightarrow \mathrm{L}+4$ & $\mathrm{~d}_{y z}+\pi \rightarrow \pi^{*}$ & \\
\hline \multirow[t]{3}{*}{$\mathrm{S}_{24}$} & 3.92 & 316.0 & 0.0157 & 18 & $113 \rightarrow 121$ & $\mathrm{H}-6 \rightarrow \mathrm{L}+1$ & $\pi_{\mathrm{CN}}+\pi \rightarrow \mathrm{d}_{x y}+\mathrm{n}$ & $3.85(322)$ \\
\hline & & & & 26 & $115 \rightarrow 123$ & $\mathrm{H}-4 \rightarrow \mathrm{L}+3$ & $\mathrm{~d}_{x^{2}-y^{2}}+\pi_{\mathrm{CN}}+\pi \rightarrow \pi^{*}$ & \\
\hline & & & & 28 & $119 \rightarrow 124$ & $\mathrm{H} \rightarrow \mathrm{L}+4$ & $\pi \rightarrow \pi^{*}$ & \\
\hline \multirow[t]{4}{*}{$\mathrm{S}_{29}$} & 4.10 & 302.7 & 0.0150 & 30 & $111 \rightarrow 120$ & $\mathrm{H}-8 \rightarrow \mathrm{L}$ & $\pi_{\mathrm{CN}}+\pi+\mathrm{d}_{y z} \rightarrow \pi^{*}$ & \\
\hline & & & & 12 & $113 \rightarrow 122$ & $\mathrm{H}-6 \rightarrow \mathrm{L}+2$ & $\pi_{\mathrm{CN}}+\pi \rightarrow \sigma^{*}\left(\mathrm{~d}_{z^{2}}\right)+\mathrm{n}$ & \\
\hline & & & & 12 & $117 \rightarrow 124$ & $\mathrm{H}-2 \rightarrow \mathrm{L}+4$ & $\mathrm{~d}_{x z}+\pi_{\mathrm{CN}}+\pi \rightarrow \pi^{*}$ & \\
\hline & & & & 12 & $119 \rightarrow 125$ & $\mathrm{H} \rightarrow \mathrm{L}+5$ & $\pi \rightarrow \pi_{\mathrm{Im}}^{*}$ & \\
\hline \multirow[t]{2}{*}{$\mathrm{S}_{30}$} & 4.11 & 301.8 & 0.0129 & 34 & $117 \rightarrow 124$ & $\mathrm{H}-2 \rightarrow \mathrm{L}+4$ & $\mathrm{~d}_{x z}+\pi_{\mathrm{CN}}+\pi \rightarrow \pi^{*}$ & \\
\hline & & & & 58 & $118 \rightarrow 125$ & $\mathrm{H}-1 \rightarrow \mathrm{L}+5$ & $\mathrm{~d}_{y z}+\pi \rightarrow \pi_{\mathrm{Im}}^{*}$ & \\
\hline \multirow[t]{2}{*}{$\mathrm{S}_{31}$} & 4.12 & 300.7 & 0.0261 & 46 & $117 \rightarrow 124$ & $\mathrm{H}-2 \rightarrow \mathrm{L}+4$ & $\mathrm{~d}_{x z}+\pi_{\mathrm{CN}}+\pi \rightarrow \pi^{*}$ & \\
\hline & & & & 38 & $118 \rightarrow 125$ & $\mathrm{H}-1 \rightarrow \mathrm{L}+5$ & $\mathrm{~d}_{y z}+\pi \rightarrow \pi_{\mathrm{Im}}^{*}$ & $4.05(306)$ \\
\hline \multirow{2}{*}{$\begin{array}{l}\cdots \\
S_{33}\end{array}$} & 4.25 & 291.6 & 0.0146 & 68 & $112 \rightarrow 121$ & $\mathrm{H}-7 \rightarrow \mathrm{L}+1$ & $\sigma\left(\mathrm{d}_{z^{2}}\right)+\mathrm{n} \rightarrow \mathrm{d}_{x y}+\mathrm{n}$ & \\
\hline & & & & 12 & $113 \rightarrow 122$ & $\mathrm{H}-6 \rightarrow \mathrm{L}+2$ & $\pi_{\mathrm{CN}}+\pi \rightarrow \sigma^{*}\left(\mathrm{~d}_{z^{2}}\right)+\mathrm{n}$ & \\
\hline \multirow[t]{3}{*}{$\mathrm{S}_{34}$} & 4.28 & 289.5 & 0.0505 & 14 & $111 \rightarrow 120$ & $\mathrm{H}-8 \rightarrow \mathrm{L}$ & $\pi_{\mathrm{CN}}+\pi+\mathrm{d}_{y z} \rightarrow \pi^{*}$ & \\
\hline & & & & 24 & $112 \rightarrow 121$ & $\mathrm{H}-7 \rightarrow \mathrm{L}+1$ & $\sigma\left(\mathrm{d}_{z^{2}}\right)+\mathrm{n} \rightarrow \mathrm{d}_{x y}+\mathrm{n}$ & \\
\hline & & & & 26 & $113 \rightarrow 122$ & $\mathrm{H}-6 \rightarrow \mathrm{L}+2$ & $\pi_{\mathrm{CN}}+\pi \rightarrow \sigma^{*}\left(\mathrm{~d}_{z^{2}}\right)+\mathrm{n}$ & \\
\hline \multirow[t]{2}{*}{$\mathrm{S}_{38}$} & 4.48 & 277.0 & 0.0330 & 16 & $113 \rightarrow 123$ & $\mathrm{H}-8 \rightarrow \mathrm{L}+3$ & $\pi_{\mathrm{CN}}+\pi \rightarrow \pi^{*}$ & \\
\hline & & & & 22 & $117 \rightarrow 125$ & $\mathrm{H}-2 \rightarrow \mathrm{L}+5$ & $\mathrm{~d}_{x z}+\pi_{\mathrm{CN}}+\pi \rightarrow \pi_{\mathrm{Im}}^{*}$ & \\
\hline $\mathrm{S}_{39}$ & 4.50 & 275.4 & 0.0343 & 32 & $113 \rightarrow 123$ & $\mathrm{H}-6 \rightarrow \mathrm{L}+3$ & $\pi_{\mathrm{CN}}+\pi \rightarrow \pi^{*}$ & \\
\hline \multirow[t]{2}{*}{$\mathrm{S}_{43}$} & 4.66 & 266.2 & 0.0307 & 42 & $108 \rightarrow 120$ & $\mathrm{H}-11 \rightarrow \mathrm{L}$ & $\pi_{\operatorname{Im}}+\pi+\mathrm{n} \rightarrow \pi^{*}$ & $4.70(264)$ \\
\hline & & & & 20 & $115 \rightarrow 124$ & $\mathrm{H}-4 \rightarrow \mathrm{L}+4$ & $\mathrm{~d}_{x^{2}-y^{2}}+\pi_{\mathrm{CN}}+\pi \rightarrow \pi^{*}$ & \\
\hline \multirow[t]{3}{*}{$\mathrm{S}_{44}$} & 4.68 & 264.8 & 0.0422 & 24 & $108 \rightarrow 120$ & $\mathrm{H}-11 \rightarrow \mathrm{L}$ & $\pi_{\mathrm{Im}}+\pi+\mathrm{n} \rightarrow \pi^{*}$ & \\
\hline & & & & 10 & $109 \rightarrow 120$ & $\mathrm{H}-10 \rightarrow \mathrm{L}$ & $\pi+\mathrm{n} \rightarrow \pi^{*}$ & \\
\hline & & & & 26 & $110 \rightarrow 121$ & $\mathrm{H}-9 \rightarrow \mathrm{L}+1$ & $\pi+\pi_{\mathrm{CN}}+\mathrm{d}_{x z} \rightarrow \mathrm{d}_{x y}+\mathrm{n}$ & \\
\hline
\end{tabular}

${ }^{a}$ The proposed assignment includes only transitions that can be correlated with experimental bands. Note that for higher energy regions of the spectrum, several states can be associated with excitations observed in experiment.

${ }^{b} \mathrm{n}$ denotes free electron pairs of corrin nitrogen atoms.

${ }^{c}$ Experimental values were taken from Ref. 68. 
The further part of the low-energy region of CNCbl spectrum reveals excitations with very small intensities. Among them, only two possess $\sim 0.01$ oscillator strengths. The first, $\mathrm{S}_{8}$ state, can be characterized as a $\pi \rightarrow \sigma^{*}$ excitation. The same transition in the simulated solution spectrum is shifted to a lower energy region and found to be the fifth excited state. The second intensity corresponds to the $S_{10}$ state, calculated in gas phase, and is composed mainly of $\mathrm{d} / \pi \rightarrow \mathrm{p}^{*}$ transition. Nevertheless, two additional states, $\mathrm{S}_{11}$ and $\mathrm{S}_{12}$ calculated in solution, possess almost identical energies to the $S_{10}$ state, with the $S_{12}$ having the highest intensity. Although $\mathrm{S}_{12}$ reveals a large, $41 \%$ contribution from a $\pi \rightarrow \pi^{*}$ transition, it is, similarly to remaining two excitations, also composed of mixed $\mathrm{d} / \pi \rightarrow \pi^{*}$ transitions. Taking into account only the intensity pattern, two gas phase excitations $\left(S_{8}\right.$ and $\left.S_{10}\right)$ could be attributed to the $\mathrm{D}$ and $\mathrm{E}$ bands in the CNCbl absorption spectrum. Previous $\mathrm{CD} / \mathrm{MCD}$ reports, however, suggested that such estimations have to be done very carefully as some of the excitations, especially those including $d \rightarrow d$ transitions, might not obtain sufficient intensities in the calculated absorbance profiles. In fact, in the analyzed region of the spectrum (first 12 excited states) there are several other electronic transitions that can be described as $\mathrm{d} / \pi \rightarrow \mathrm{d}, \mathrm{d} / \pi \rightarrow \pi^{*}, \mathrm{~d} / \pi \rightarrow \sigma^{*}$ and $\pi \rightarrow \pi^{*}$ and should not be excluded unambiguously from the assignment of the D/E band.

At higher energies, the $\gamma$ part of the spectrum reveals absorption features at 360, 322, and $306 \mathrm{~nm}$. The two calculated transitions with the highest intensities in this region are found at 350 and $348 \mathrm{~nm}$. The former has an oscillator strength more than twice as large as the latter and is mainly a $\pi \rightarrow \pi^{*}$ transition. At the same time, the excitation at $348 \mathrm{~nm}$ includes some contribution from $\pi$ orbitals localized on the axial cyano group, leading to a mixed $\pi_{\mathrm{CN}} / \pi \rightarrow \pi^{*}$ nature of the discussed transition. As mentioned in Section 2.3.1, the change of the environment leads to the presence of a single electronic transition corresponding to the $\gamma$ part of the spectrum. A detailed analysis of the BP86 transitions calculated in the presence of a solvent model (Table 2.5) indicates that the most intense peak corresponds to the $\mathrm{S}_{17}$ state possessing 32,29 , and $12 \%$ contributions from $\mathrm{d}_{y z} / \pi / \pi_{\mathrm{CN}} \rightarrow \pi^{*}$, 


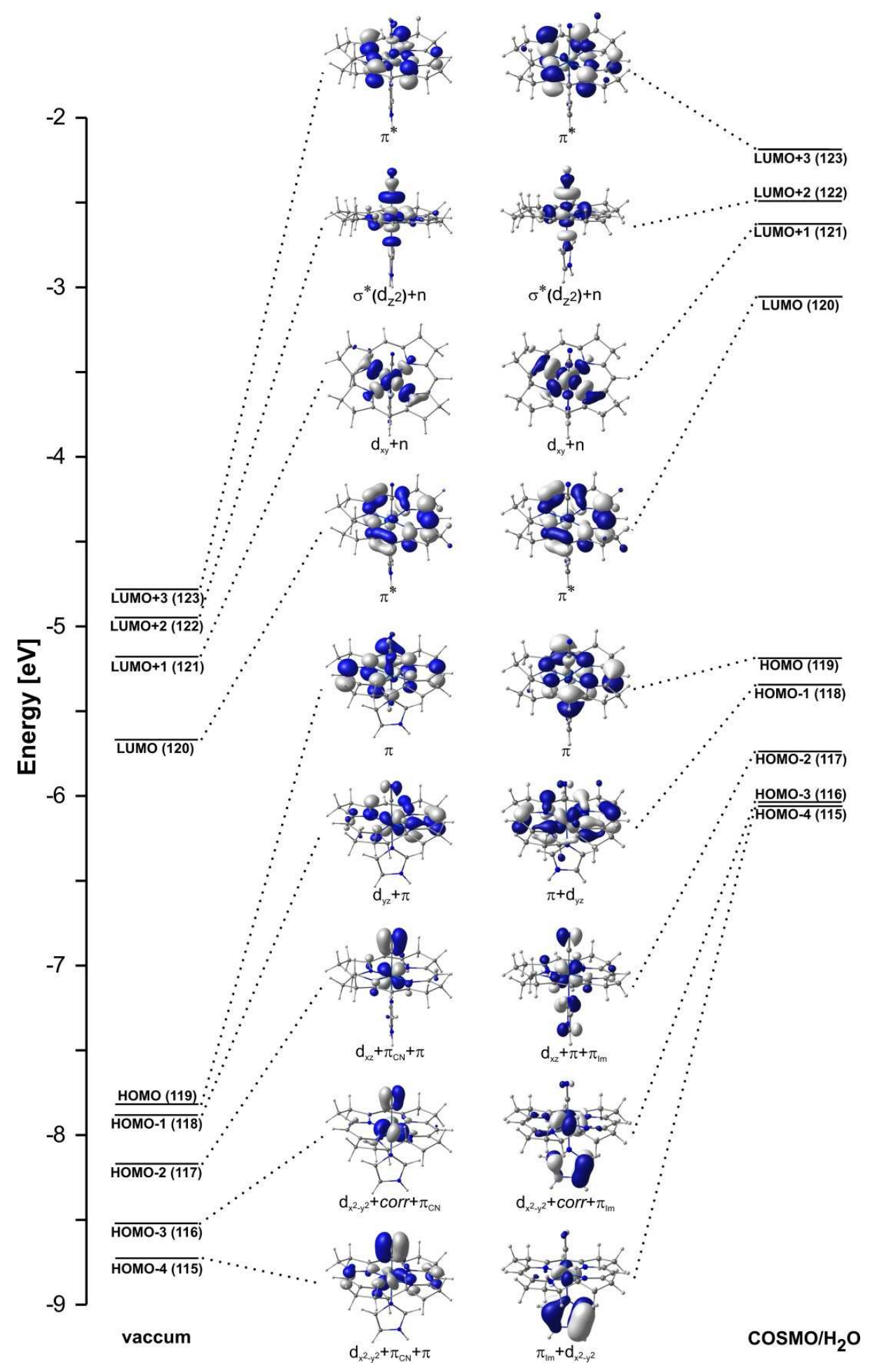

Figure 2.11. Isosurface plots of frontier MOs for the ground state of CNCbl model based on BP86/6-31G(d) calculations in vacuo and with the COSMO/ $\mathrm{H}_{2} \mathrm{O}$ model. 
$\pi \rightarrow \pi^{*}$ and $\mathrm{d}_{x z} / \pi / \pi_{\mathrm{Im}} \rightarrow \pi^{*}$, respectively. This observation leads to the conclusion that the $\gamma$ band of CNCbl is not necessarily a pure $\pi \rightarrow \pi^{*}$ electronic transition. When describing the nature of this excitation, one has to consider the involvement of orbitals localized on the corrin ring $(\pi)$, as well as axial bonds of the system (d, $\left.\pi_{\mathrm{CN}}\right)$ as indicated by both in vacuo and COSMO data.

Together with the increase in energy, two additional spectral features, at 322 and $306 \mathrm{~nm}$ have been extracted from the experimental absorption profiles of CNCbl. However, the unambiguous assignment of these bands is hampered by the congestion of calculated electronic transitions in the high-energy part of the spectra, which have very small oscillator strengths. Following the intensity pattern, the band at $322 \mathrm{~nm}$ can be attributed to $S_{23}$ obtained from calculations in gas phase and described as a $\mathrm{d} / \pi \rightarrow \pi^{*}$ transition. The same state in solution, however, is blue shifted by $\sim 7 \mathrm{~nm}$, and its oscillator strength decreases significantly. At the same time, the following two excitations $\left(\mathrm{S}_{24}\right.$ and $\left.\mathrm{S}_{25}\right)$ gain in solution almost similar intensities, making them plausible contributors to the experimentally observed band. However, their nature is mixed due to the involvement of several occupied and unoccupied MOs (Table 2.5). The transitions are composed not only of $d / \pi / \pi_{\mathrm{CN}} \rightarrow \mathrm{d}$ and $\mathrm{d} / \pi / \pi_{\mathrm{CN}} \rightarrow \pi^{*}$ excitations but also of $\mathrm{d} / \pi / \pi_{\mathrm{CN}} \rightarrow \sigma^{*}\left(\mathrm{~d}_{z^{2}}\right),\left(\mathrm{S}_{24}\right)$ and $\pi \rightarrow \pi^{*}$, $\left(\mathrm{S}_{25}\right)$, making the assignment of the experimental band (at $322 \mathrm{~nm}$ ) a very difficult task. Assignment of the experimental band at $306 \mathrm{~nm}$ is even less straightforward as there are a number of excitations having comparable oscillator strengths, in the calculated spectral range from $\sim 303$ to $\sim 290 \mathrm{~nm}$ in gas phase. In addition, most of these bands possess small contributions from several electronic transitions and, hence, none of them has a pure character. The most intense calculated transition ascribed to the $306 \mathrm{~nm}$ experimental band is the transition at $289 \mathrm{~nm}$. This transition is comprised of $\pi_{\mathrm{CN}} / \pi \rightarrow \sigma_{\mathrm{Co}-\mathrm{CN}}^{*}$ and $\sigma_{\mathrm{Co}-\mathrm{CN}} \rightarrow \mathrm{d}$ excitations.

The inclusion of solvent lowers the oscillator strengths of the particular excitations in this region. The most intense excitation corresponds to the $S_{32}$ state which refers to a $\pi / \pi_{\mathrm{CN}} \rightarrow \pi^{*}$ transition. Taking into account only the intensity, this transition might be attributed to the experimental band at $306 \mathrm{~nm}$. However, its 
significant ( $\sim 25 \mathrm{~nm}$ ) blue shift as compared to experiment, as well as the lack of a similar counterpart obtained from calculations in the gas phase, makes such an assignment speculative.

In the energy range of the $\delta$ band, there are several calculated transitions of relatively large oscillator strengths $277,275,266$, and $265 \mathrm{~nm}$. The former two transitions are $\mathrm{d} / \pi_{\mathrm{CN}} / \pi \rightarrow \pi_{\mathrm{Im}}^{*}$ and $\pi_{\mathrm{CN}} / \pi \rightarrow \pi^{*}$ excitations. The transition at $266 \mathrm{~nm}$ involves excitations of $\sigma_{\mathrm{Corr}} / \pi_{\mathrm{Im}} \rightarrow \pi^{*}$ and $\pi / \mathrm{d} / \pi_{\mathrm{CN}} \rightarrow \pi^{*}$ character, and the transition at $265 \mathrm{~nm}$ is composed of $\sigma_{\mathrm{Corr}} / \pi_{\mathrm{Im}} \rightarrow \pi^{*}$ and $\pi / \mathrm{d} / \pi_{\mathrm{CN}} \rightarrow \mathrm{d}$ excitations. Similarly to the lower-energy region containing bands at 322 and $306 \mathrm{~nm}$, the change of the environment decreased the oscillator strengths of the individual excitations contributing to the $\delta$ band as well. In addition, the calculated transitions change their character, making the final assignment of this region in the absorption spectrum of $\mathrm{CNCbl}$ very counterintuitive. The most intense band in this spectral part, resulting from calculations in solution (Table 2.5), corresponds to the $\mathrm{S}_{40}$ state which is composed of $\pi_{\mathrm{Im}} / \pi_{\mathrm{CN}} \rightarrow \mathrm{d}$ and $\pi_{\mathrm{CN}} / \pi \rightarrow \mathrm{d}$ transitions. Its presence, at 260 $\mathrm{nm}$, suggests a possible correspondence to the experimental band at $264 \mathrm{~nm}$ but in order to support or refute this proposal, further studies are required including both experimental and theoretical investigations. 
TABLE 2.5. TD-DFT/BP86/6-31G(d)-based electronic transitions ${ }^{a}$ for the singlet states of Im-[Co ${ }^{\text {III }}$ (corrin) $]-\mathrm{CN}^{+}$calculated with the use of the COSMO $\left(\mathrm{H}_{2} \mathrm{O}\right)$ solvation model.

\begin{tabular}{|c|c|c|c|c|c|c|c|c|}
\hline & $\overline{E(e V)}$ & $\overline{\lambda \lambda(\mathrm{nm})}$ & $\overline{f f}$ & $\%$ & Character $^{b}$ & & & $\begin{array}{l}\text { Experiment }{ }^{c} \\
\mathrm{E}(\mathrm{eV}) \lambda(\mathrm{nm})\end{array}$ \\
\hline $\mathrm{S}_{1}$ & 2.47 & 502.2 & 0.0409 & 88 & $119 \rightarrow 120$ & $\mathrm{H} \rightarrow \mathrm{L}$ & $\pi \rightarrow \pi^{*}$ & $2.25(550)$ \\
\hline \multirow[t]{2}{*}{$\mathrm{S}_{2}$} & 2.61 & 474.5 & 0.0155 & 50 & $118 \rightarrow 120$ & $\mathrm{H}-1 \rightarrow \mathrm{L}$ & $\pi+\mathrm{d}_{y z} \rightarrow \pi^{*}$ & \\
\hline & & & & 40 & $119 \rightarrow 121$ & $\mathrm{H} \rightarrow \mathrm{L}+1$ & $\pi \rightarrow \mathrm{d}_{x y}+\mathrm{n}$ & \\
\hline \multirow[t]{2}{*}{$\mathrm{S}_{3}$} & 2.65 & 467.5 & 0.0136 & 53 & $119 \rightarrow 121$ & $\mathrm{H} \rightarrow \mathrm{L}+1$ & $\pi \rightarrow \mathrm{d}_{x y}+\mathrm{n}$ & $2.40(517)$ \\
\hline & & & & 29 & $118 \rightarrow 120$ & $\mathrm{H}-1 \rightarrow \mathrm{L}$ & $\pi+\mathrm{d}_{y z} \rightarrow \pi^{*}$ & \\
\hline $\mathrm{S}_{4}$ & 2.79 & 444.7 & 0.0267 & 75 & $117 \rightarrow 120$ & $\mathrm{H}-2 \rightarrow \mathrm{L}$ & $\mathrm{d}_{x z}+\pi+\pi_{\operatorname{Im}} \rightarrow \pi^{*}$ & $2.56(485)$ \\
\hline $\mathrm{S}_{5}$ & 2.83 & 438.8 & 0.0121 & 83 & $119 \rightarrow 122$ & $\mathrm{H} \rightarrow \mathrm{L}+2$ & $\pi \rightarrow \sigma^{*}\left(\mathrm{~d}_{z^{2}}\right)+\mathrm{n}$ & \\
\hline $\mathrm{S}_{6}$ & 2.86 & 433.6 & 0.0039 & 78 & $118 \rightarrow 121$ & $\mathrm{H}-1 \rightarrow \mathrm{L}+1$ & $\pi+\mathrm{d}_{y z} \rightarrow \mathrm{d}_{x y}+\mathrm{n}$ & \\
\hline \multirow[t]{2}{*}{$\mathrm{S}_{7}$} & 2.94 & 421.3 & 0.0078 & 78 & $118 \rightarrow 122$ & $\mathrm{H}-1 \rightarrow \mathrm{L}+2$ & $\pi+\mathrm{d}_{y z} \rightarrow \sigma^{*}\left(\mathrm{~d}_{z^{2}}\right)+\mathrm{n}$ & \\
\hline & & & & 10 & $118 \rightarrow 121$ & $\mathrm{H}-1 \rightarrow \mathrm{L}+1$ & $\pi+\mathrm{d}_{y z} \rightarrow \mathrm{d}_{x y}+\mathrm{n}$ & $2.71(457)$ \\
\hline $\mathrm{S}_{8}$ & 3.01 & 411.8 & 0.0002 & 89 & $116 \rightarrow 120$ & $\mathrm{H}-3 \rightarrow \mathrm{L}$ & $\mathrm{d}_{x^{2}-y^{2}}+\pi_{I m} \rightarrow \pi^{*}$ & \\
\hline $\mathrm{S}_{9}$ & 3.05 & 406.8 & 0.0075 & 86 & $115 \rightarrow 120$ & $\mathrm{H}-4 \rightarrow \mathrm{L}$ & $\pi_{I m}+\mathrm{d}_{x^{2}-y^{2}} \rightarrow \pi^{*}$ & \\
\hline \multirow[t]{3}{*}{$S_{10}$} & 3.30 & 375.4 & 0.0095 & 30 & $118 \rightarrow 123$ & $\mathrm{H}-1 \rightarrow \mathrm{L}+3$ & $\pi+\mathrm{d}_{y z} \rightarrow \pi^{*}$ & \\
\hline & & & & 28 & $117 \rightarrow 121$ & $\mathrm{H}-2 \rightarrow \mathrm{L}+1$ & $\mathrm{~d}_{x z}+\pi+\pi_{\operatorname{Im}} \rightarrow \mathrm{d}_{x y}+\mathrm{n}$ & \\
\hline & & & & 26 & $117 \rightarrow 122$ & $\mathrm{H}-2 \rightarrow \mathrm{L}+2$ & $\mathrm{~d}_{x z}+\pi+\pi_{\operatorname{Im}} \rightarrow \sigma^{*}\left(\mathrm{~d}_{z^{2}}\right)+\mathrm{n}$ & \\
\hline \multirow[t]{3}{*}{$S_{11}$} & 3.32 & 373.3 & 0.0095 & 49 & $118 \rightarrow 123$ & $\mathrm{H}-1 \rightarrow \mathrm{L}+3$ & $\pi+\mathrm{d}_{y z} \rightarrow \pi^{*}$ & $3.04(408)$ \\
\hline & & & & 18 & $117 \rightarrow 122$ & $\mathrm{H}-2 \rightarrow \mathrm{L}+2$ & $\mathrm{~d}_{x z}+\pi+\pi_{\operatorname{Im}} \rightarrow \sigma^{*}\left(\mathrm{~d}_{z^{2}}\right)+\mathrm{n}$ & \\
\hline & & & & 13 & $117 \rightarrow 121$ & $\mathrm{H}-2 \rightarrow \mathrm{L}+1$ & $\mathrm{~d}_{x z}+\pi+\pi_{\operatorname{Im}} \rightarrow \mathrm{d}_{x y}+\mathrm{n}$ & \\
\hline \multirow[t]{2}{*}{$S_{12}$} & 3.33 & 372.5 & 0.0256 & 41 & $119 \rightarrow 123$ & $\mathrm{H} \rightarrow \mathrm{L}+3$ & $\pi \rightarrow \pi^{*}$ & \\
\hline & & & & 40 & $114 \rightarrow 120$ & $\mathrm{H}-5 \rightarrow \mathrm{L}$ & $\mathrm{d}_{y z}+\pi+\pi_{\mathrm{CN}} \rightarrow \pi^{*}$ & \\
\hline \multirow[t]{3}{*}{$S_{17}$} & 3.68 & 337.4 & 0.1367 & 32 & $114 \rightarrow 120$ & $\mathrm{H}-5 \rightarrow \mathrm{L}$ & $\mathrm{d}_{y z}+\pi+\pi_{\mathrm{CN}} \rightarrow \pi^{*}$ & $3.44(360)$ \\
\hline & & & & 29 & $119 \rightarrow 123$ & $\mathrm{H} \rightarrow \mathrm{L}+3$ & $\pi \rightarrow \pi^{*}$ & \\
\hline & & & & 12 & $117 \rightarrow 123$ & $\mathrm{H}-2 \rightarrow \mathrm{L}+3$ & $\mathrm{~d}_{x z}+\pi+\pi_{\mathrm{Im}} \rightarrow \pi^{*}$ & \\
\hline \multirow[t]{2}{*}{$\mathrm{S}_{22}$} & 3.99 & 310.8 & 0.0162 & 33 & $118 \rightarrow 124$ & $\mathrm{H}-1 \rightarrow \mathrm{L}+4$ & $\pi+\mathrm{d}_{y z} \rightarrow \pi^{*}$ & \\
\hline & & & & 26 & $114 \rightarrow 122$ & $\mathrm{H}-5 \rightarrow \mathrm{L}+2$ & $\mathrm{~d}_{y z}+\pi+\pi_{\mathrm{CN}} \rightarrow \sigma^{*}\left(\mathrm{~d}_{z^{2}}\right)+\mathrm{n}$ & \\
\hline \multirow[t]{2}{*}{$\mathrm{S}_{23}$} & 4.01 & 309.6 & 0.0048 & 46 & $118 \rightarrow 124$ & $\mathrm{H}-1 \rightarrow \mathrm{L}+4$ & $\pi+\mathrm{d}_{y z} \rightarrow \pi^{*}$ & \\
\hline & & & & 12 & $114 \rightarrow 121$ & $\mathrm{H}-5 \rightarrow \mathrm{L}+1$ & $\mathrm{~d}_{y z}+\pi+\pi_{\mathrm{CN}} \rightarrow \mathrm{d}_{x y}+\mathrm{n}$ & \\
\hline \multirow[t]{3}{*}{$\mathrm{S}_{24}$} & 4.08 & 304.2 & 0.0218 & 20 & $114 \rightarrow 121$ & $\mathrm{H}-5 \rightarrow \mathrm{L}+1$ & $\mathrm{~d}_{y z}+\pi+\pi_{\mathrm{CN}} \rightarrow \mathrm{d}_{x y}+\mathrm{n}$ & \\
\hline & & & & 18 & $114 \rightarrow 122$ & $\mathrm{H}-5 \rightarrow \mathrm{L}+2$ & $\mathrm{~d}_{y z}+\pi+\pi_{\mathrm{CN}} \rightarrow \sigma^{*}\left(\mathrm{~d}_{z^{2}}\right)+\mathrm{n}$ & $3.85(322)$ \\
\hline & & & & 15 & $114 \rightarrow 123$ & $\mathrm{H}-5 \rightarrow \mathrm{L}+3$ & $\mathrm{~d}_{y z}+\pi+\pi_{\mathrm{CN}} \rightarrow \pi^{*}$ & \\
\hline \multirow[t]{4}{*}{$\mathrm{S}_{25}$} & 4.15 & 298.7 & 0.0204 & 20 & $113 \rightarrow 121$ & $\mathrm{H}-6 \rightarrow \mathrm{L}+1$ & $\pi+\pi_{\mathrm{CN}}+\mathrm{d}_{x z} \rightarrow \mathrm{d}_{x y}+\mathrm{n}$ & \\
\hline & & & & 18 & $114 \rightarrow 123$ & $\mathrm{H}-5 \rightarrow \mathrm{L}+3$ & $\mathrm{~d}_{y z}+\pi+\pi_{\mathrm{CN}} \rightarrow \pi^{*}$ & \\
\hline & & & & 13 & $114 \rightarrow 121$ & $\mathrm{H}-5 \rightarrow \mathrm{L}+1$ & $\mathrm{~d}_{y z}+\pi+\pi_{\mathrm{CN}} \rightarrow \mathrm{d}_{x y}+\mathrm{n}$ & \\
\hline & & & & 12 & $119 \rightarrow 124$ & $\mathrm{H} \rightarrow \mathrm{L}+4$ & $\pi \rightarrow \pi^{*}$ & \\
\hline $\mathrm{S}_{26}$ & 4.20 & 295.5 & 0.0092 & 64 & $113 \rightarrow 121$ & $\mathrm{H}-6 \rightarrow \mathrm{L}+1$ & $\pi+\pi_{\mathrm{CN}}+\mathrm{d}_{x z} \rightarrow \mathrm{d}_{x y}+\mathrm{n}$ & \\
\hline $\begin{array}{l}\cdots \\
\mathrm{S}_{29}\end{array}$ & 4.30 & 288.6 & 0.0176 & 85 & $117 \rightarrow 124$ & $\mathrm{H}-2 \rightarrow \mathrm{L}+4$ & $\mathrm{~d}_{x z}+\pi+\pi_{\operatorname{Im}} \rightarrow \pi^{*}$ & \\
\hline $\mathrm{S}_{30}$ & 4.32 & 286.8 & 0.0043 & 83 & $113 \rightarrow 122$ & $\mathrm{H}-6 \rightarrow \mathrm{L}+2$ & $\pi+\pi_{\mathrm{CN}}+\mathrm{d}_{x z} \rightarrow \sigma^{*}\left(\mathrm{~d}_{z^{2}}\right)+\mathrm{n}$ & \\
\hline \multirow[t]{2}{*}{$S_{31}$} & 4.42 & 280.3 & 0.0079 & 78 & $119 \rightarrow 125$ & $\mathrm{H} \rightarrow \mathrm{L}+5$ & $\pi \rightarrow \pi_{I m}^{*}$ & \\
\hline & & & & 10 & $114 \rightarrow 123$ & $\mathrm{H}-5 \rightarrow \mathrm{L}+3$ & $\mathrm{~d}_{y z}+\pi+\pi_{\mathrm{CN}} \rightarrow \pi^{*}$ & \\
\hline$S_{32}$ & 4.44 & 279.2 & 0.0291 & 81 & $111 \rightarrow 120$ & $\mathrm{H}-8 \rightarrow \mathrm{L}$ & $\pi_{\mathrm{CN}}+\pi \rightarrow \pi^{*}$ & $4.05(306)$ \\
\hline \multirow[t]{3}{*}{$\mathrm{S}_{33}$} & 4.50 & 275.3 & 0.0193 & 49 & $110 \rightarrow 120$ & $\mathrm{H}-9 \rightarrow \mathrm{L}$ & $\pi_{I m}+\pi_{\mathrm{CN}}+\pi \rightarrow \pi^{*}$ & \\
\hline & & & & 14 & $119 \rightarrow 125$ & $\mathrm{H} \rightarrow \mathrm{L}+5$ & $\pi \rightarrow \pi_{I m}^{*}$ & \\
\hline & & & & 13 & $116 \rightarrow 124$ & $\mathrm{H}-3 \rightarrow \mathrm{L}+4$ & $\mathrm{~d}_{x^{2}-y^{2}}+\pi_{I m} \rightarrow \pi^{*}$ & \\
\hline \multirow[t]{2}{*}{$\begin{array}{l}\cdots \\
\mathrm{S}_{40}\end{array}$} & 4.76 & 260.3 & 0.0780 & 27 & $112 \rightarrow 121$ & $\mathrm{H}-7 \rightarrow \mathrm{L}+1$ & $\pi_{I m}+\pi_{\mathrm{CN}} \rightarrow \mathrm{d}_{x y}+\mathrm{n}$ & $4.70(264)$ \\
\hline & & & & 19 & $111 \rightarrow 121$ & $\mathrm{H}-8 \rightarrow \mathrm{L}+1$ & $\pi_{\mathrm{CN}}+\pi \rightarrow \mathrm{d}_{x y}+\mathrm{n}$ & \\
\hline
\end{tabular}

${ }^{a}$ The proposed assignment includes only transitions that can be correlated with experimental bands. Note that for higher energy regions of the spectrum, several states can be associated with excitations observed in experiment.

${ }^{b} \mathrm{n}$ denotes free electron pairs of corrin nitrogen atoms.

${ }^{c}$ Experimental values were taken from Ref. 68. 


\subsection{Summary and Conclusions}

Although electronically excited states of vitamin $\mathrm{B}_{12}$ derivatives have been probed using a variety of experimental techniques, their exact nature remains poorly understood from an electronic structure point of view. To rectify this situation, several groups have applied the TD-DFT framework to determine properties of the electronically excited states. At this stage, the TD-DFT method is probably the only practical tool that can be applied to study excited states of bioinorganic molecules such as vitamin $B_{12}$. Nevertheless, a critical step in TD-DFT calculations is the choice of an appropriate functional which was the focal point of the present study.

In the present contribution, the choice of the proper density functional for vitamin $\mathrm{B}_{12}$ was evaluated by comparing it with experimental data as well as correlated $a b$ initio wave function-based calculations. Three different methods received special attention: GGA (BP86), hybrid (B3LYP), and the range-separated (LC-BLYP) functionals, which was tested as a function of the damping parameter, $\mu$. A comprehensive analysis revealed that only results based on the BP86 and LC-BLYP, with $\mu$ being close to zero, are consistent with both experimental results and high-level $a b$ initio calculations. The finding that a GGA-type functional gives a better description than a hybrid one may at first appear surprising. However, it reflects the fact that the BP86 functional gives a reliable description of the energy gap between the occupied and virtual orbitals in organometallic complexes, thus making the virtual-occupied energy difference a good estimation for excitations energies. In order to assess contributions due to CT-type excitations, which are often found to be inaccurately predicted by TD-DFT calculations, we also validated the nature of the excitations by $\Lambda$ diagnostic test. The results of our findings dispelled suspicions about possible CT problems and confirmed the legitimacy of TD-DFT in studying electronic properties of CNCbl. Consequently, the use of BP86 is suggested as an expedient and accurate choice in calculating electronically excited states of CNCbl. 
The conclusion reached in the present study regarding the BP86 functional may have several important implications for computational studies involving the modeling of cobalt corrinoids. First, it questions earlier studies where assignment of electronic transitions for vitamin $\mathrm{B}_{12}$ has been proposed using the B3LYP functional. ${ }^{107,108}$ Although, traditionally, electronic transitions of $B_{12}$ cofactors have been assigned mostly as having $\pi \rightarrow \pi^{*}$ character, the BP86 results indicate that many excitations possess significant contributions from transitions involving cobalt d orbitals. Second, it further supports the conclusions reached in our recent study, where CD and MCD data were analyzed (see Chapter 4 or Ref. 140), showing the advantage of GGA over hybrid functionals in modeling excited states of $\mathrm{B}_{12}$ derivatives. This particularly applies to the lowest energy $\alpha / \beta$ band, where multiple electronic transitions are observed rather than the vibrational progression of a single electronic excitation. Third, from a theoretical perspective, targeting a particular electronically excited state becomes a moot case when there is a lack of experimental evidence supporting computed results. This particularly applies to photochemical and photophysical events where low-lying excited states play a significant role. It has been noted that in CNCbl these states are related to LMCT transitions. ${ }^{64,65}$ The examination of these excitations at different levels of theory presented in this study confirms the applicability of the BP86 functional in describing electronic properties of $\mathrm{CNCbl}$ due to their best correspondence to CASSCF/MC-XQDPT2 results. This conclusion may assist in prospective studies explaining the photochemical processes of CNCbl. 


\section{CHAPTER 3}

\section{ELECTRONIC STRUCTURE OF THE $S_{1}$ STATE IN METHYLCOBALAMIN ${ }^{1}$}

\subsection{Introduction}

Derivatives of vitamin $\mathrm{B}_{12}$ ( $\mathrm{CNCbl)}$, such as the MeCbl or AdoCbl cofactors (Figure 1.1), catalyze complex molecular transformations in which the cleavage of the cobalt-carbon bond constitutes one of the key steps. ${ }^{9}, 10,13-28,33$ Both cofactors possess a complex manifold of electronically excited states that have been probed by various experimental techniques. Whereas the analysis of the Abs, CD, and MCD spectra has brought considerable advancement in understanding the electronic structure of vitamin $\mathrm{B}_{12}$ derivatives, the experimental and computational results summarized in the Introdution must also be reconciled with other types of studies targeting the photochemical and photophysical properties of cobalamins, particularly those carried out by Sension and co-workers. ${ }^{56-60,62-65,162}$ As demonstrated by Sension et al., an useful information about the low-lying excited electronic states of $\mathrm{B}_{12}$ derivatives can be obtained with the aid of transient absorption spectroscopy (TAS). The TAS measurements by Sension et al. have indicated a diverse sensitivity of cobalamins to the excitation wavelength depending on the upper axial ligand. In the case of $\mathrm{MeCbl}$, considered in this work, an examination of the nature of metastable photoproduct has revealed a metal-to-ligand charge transfer (MLCT) character of the $\mathrm{S}_{1}$ state, i.e., a $\mathrm{d} \rightarrow \pi^{*}$ transition. $^{56,58,60,65}$

\footnotetext{
${ }^{1}$ The material presented in this Chapter was reprinted (adapted) with permission from K. Kornobis, N. Kumar, P. Lodowski, M. Jaworska, P. Piecuch, J. J. Lutz, B. M. Wong and P. M. Kozlowski, J. Comput. Chem., article first published online: 19 Jan 2013, DOI: 10.1002/jcc.23204. Copyright 2013 John Wiley and Sons.
} 
Such an assignment is in clear contrast with the earlier proposals implying the $\pi \rightarrow \pi^{*}$ character of the $\mathrm{S}_{0} \rightarrow \mathrm{S}_{1}$ transition.

Although TD-DFT calculations employing the hybrid B3LYP functional ${ }^{114,115}$ tend to support a $\pi \rightarrow \pi^{*}$ assignment of the lowest-energy spectral band, there has been growing skepticism regarding the applicability of hybrid functionals for studying the electronic properties of $\mathrm{B}_{12}$ cofactors. ${ }^{100,155,156,163}$ For this reason, the electronically excited states of $B_{12}$ cofactors ${ }^{105,106,118,119}$ have also been investigated using generalized-gradient-approximation-type (GGA-type) functionals, such as BP86. ${ }^{123,124}$ It has been deduced that if one is interested in a larger number of MeCbl excited states, the utilization of at least two functionals (i.e., B3LYP and BP86) was required. ${ }^{105}$ No single functional was capable of providing a uniformly accurate description of the entire electronic spectrum. However, the photochemistry of $\mathrm{B}_{12}$ cofactors, which is governed by the lowest-energy transitions only, was more appropriately described by BP86. ${ }^{118,119}$ The BP86 functional has been shown to perform better than B3LYP in modeling axial bond properties ${ }^{155,164}$ and has turned out to be more adequate in predicting the electronic excitations involving $\mathrm{d}$ orbitals of the cobalt metal. The studies reported in Ref. 105 have also identified the $\mathrm{S}_{0}$ $\rightarrow \mathrm{S}_{1}$ excitation in MeCbl as a predominantly $\pi \rightarrow \pi^{*}$ transition when the B3LYP functional was applied in TD-DFT calculations, although some $\mathrm{d} \rightarrow \pi^{*}$ contribution was also present due to the slight contribution of the $d_{z^{2}}$ orbital in the HOMO participating in this transition. In contrast, the TD-DFT/BP86 results ${ }^{118,119}$ have revealed a large amount of electron density originating from the Co d contributions to the HOMO and HOMO-1 that were associated with the first electronic transition in MeCbl, implying that the $\mathrm{S}_{1}$ state of MeCbl should be characterized as an MLCT $\left(\mathrm{d} / \pi \rightarrow \pi^{*}\right)$ transition. Thus, the TD-DFT/B3LYP calculations were in line with the traditional $\pi \rightarrow \pi^{*}$ interpretation of the $\mathrm{S}_{0} \rightarrow \mathrm{S}_{1}$ band, whereas the TD-DFT/BP86 implied an MLCT transition, consistently with the analysis of TAS data. ${ }^{56,58,60,65}$

The significant step toward understanding the nature of excited states in $\mathrm{B}_{12}$ derivatives has been achieved when, in addition to the Abs data, the $\mathrm{CD}$ and MCD spectra of CNCbl and MeCbl were computed and compared with experiment 
(see Chapter 4 or Ref. 140). Two different functionals, i.e., CAM-B3LYP and BP86, were applied to simulate the electronic spectra of $\mathrm{CNCbl}$ and $\mathrm{MeCbl}$, and it has been concluded that better agreement with experiment was provided by the BP86 functional. It has also been noticed, however, that the Abs data alone were not sufficient to justify the use of one functional over another. Moreover, it has been determined that multiple electronic transitions contribute to the $\alpha / \beta$ band observed for both cobalamins. ${ }^{140}$

The above discussion demonstrates that the nature of the $S_{1}$ state of $\mathrm{MeCbl}$ cofactor remains elusive, since the existing experimental and theoretical predictions do not yield a consistent picture regarding its electronic structure. Clearly, a proper description of the energetically lowest excited state of MeCbl requires additional independent analyses, particularly those, based on higher-level ab initio wave-function methodologies. The purpose of the studies presented in this chapter is to provide such new insights. Two different higher-level $a b$ initio methods are employed to determine the low-lying states of MeCbl, namely the modified version of the second-order multi-configurational quasi-degenerate perturbation theory (MC-QDPT2), ${ }^{154}$ abbreviated as MC-XQDPT2, using complete active space self-consistent field (CASSCF) ${ }^{165}$ reference wave functions, which has previously been applied in the study of $\mathrm{CNCbl}^{161}$ and the single-reference equation-of-motion coupled-cluster singles and doubles (EOM-CCSD) approach, ${ }^{166,167}$ which has never been used before to examine the cobalamin spectra, but is known to provide a reasonable description of excited states dominated by one-electron transitions, including charge-transfer (CT) excitations. Taking into account the strong dependence of the TD-DFT results on the applied functional, a number of TD-DFT calculations have been performed and benchmarked against the MC-XQDPT2 and EOM-CCSD $a b$ initio data. In addition to B3LYP ${ }^{114,115}$ and BP86, ${ }^{123,124}$ a few other functionals, including hybrid (MPW1PW91 136,168 and TPSSh ${ }^{169}$ ), GGA-type (BLYP ${ }^{115,123}$ and MPWPW91 ${ }^{168,170}$ ), meta-GGA (TPSS ${ }^{171}$ ), and range-separated (CAM-B3LYP ${ }^{135}$ and LC-BLYP ${ }^{172}$ ) approaches, have been employed in the present computations. 
The latter two functionals were used to address the potential problem caused by underestimation of the TD-DFT excitation energies having a significant CT character. ${ }^{144,145,173}$ In particular, the effect of varying the Hartree-Fock (HF) exchange and correlation contributions in the exchange-correlation functional was investigated by computing the excited-state properties of $\mathrm{MeCbl}$ as a function of the rangeseparation parameter $\mu$.

\subsection{Computational details}

\subsubsection{Structural models}

To reduce computational costs, the majority of the previous theoretical studies of the $B_{12}$ cofactors employed simplified models with respect to side chains. Such an approach demonstrated satisfactory results in predicting their structural and electronic properties. ${ }^{100,104-112,118,119,155,156,163,164}$ Consequently, a truncated structure of the MeCbl cofactor, i.e., the $\mathrm{Im}-\left[\mathrm{Co}{ }^{\mathrm{III}}\right.$ (corrin) $]-\mathrm{CH}_{3}^{+}$model having 58 atoms shown in Figure 3.1, was exploited in the present study. Using the X-ray crystallographic data for the full MeCbl structure ${ }^{147}$ as a starting point for designing the Im-[Co ${ }^{\text {III }}$ (corrin) $]-\mathrm{CH}_{3}^{+}$model, we replaced all amide side chains of the corrin ring with the hydrogen atoms while truncating the nucleotide loop at $\mathrm{C}_{17}$, leaving the resulting structure positively charged. Under enzymatic conditions, in $\mathrm{B}_{12}$-dependent

methyltransferases, ${ }^{16,19,26,27}$ the dimethylbenzimidazole (DBI) base is replaced by the histidine (His) residue from the protein side chain. Thus, the lower axial DBI fragment was substituted by imidazole (Im). The final Im- $\left[\mathrm{Co}^{\mathrm{III}}\right.$ (corrin) $]-\mathrm{CH}_{3}^{+}$structure was optimized at the BP86/6-31G(d) level (using spherical d functions). As shown in Ref. 163, the optimized structural parameters of the Im-[CoIII (corrin) $]-\mathrm{CH}_{3}^{+}$ system are almost insensitive to the functional employed, and the structure of $\mathrm{Im}-\left[\mathrm{Co}^{\mathrm{III}}\right.$ (corrin) $]-\mathrm{CH}_{3}^{+}$obtained in the BP86/6-31G(d) calculations is in good agreement with the X-ray data for $\operatorname{Co} \alpha$-( $1 H$-Imidazolyl)-Co $\beta$-methylcob(III)amide. ${ }^{174}$ 


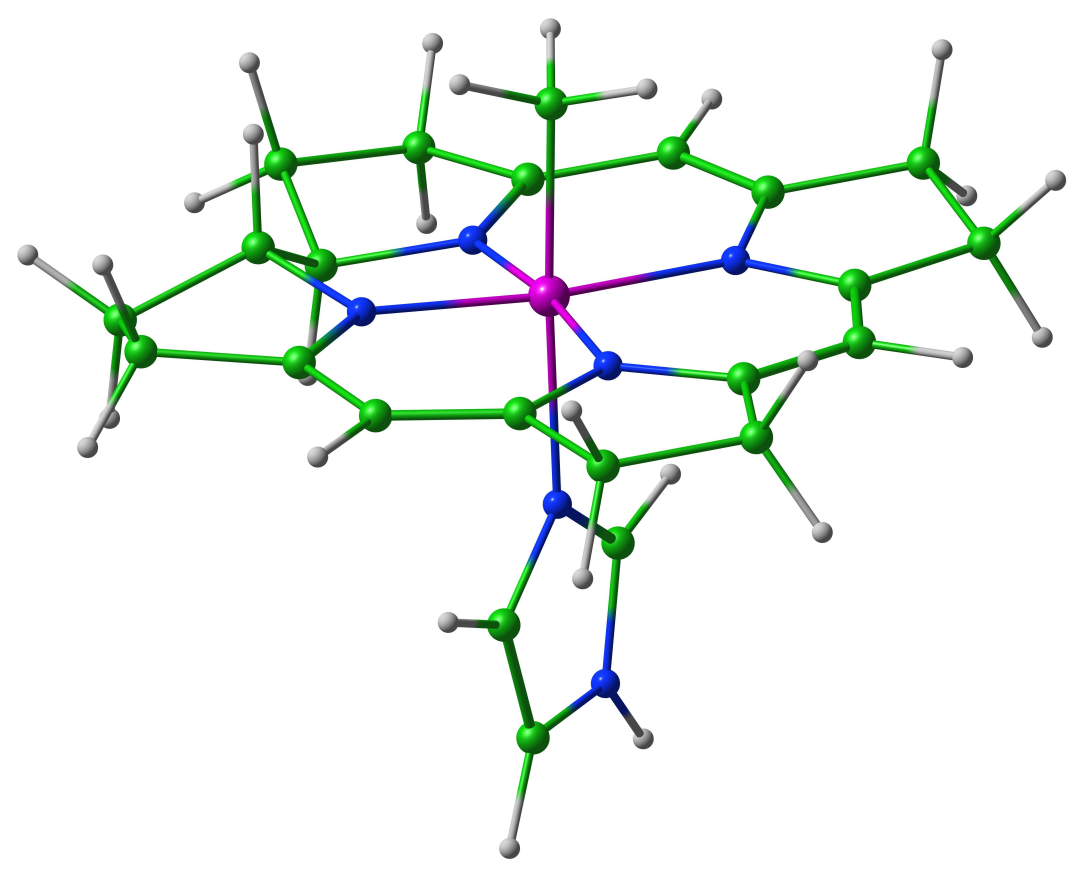

Figure 3.1. Structural model of MeCbl, abbreviated as Im-[CoII (corrin) $]-\mathrm{CH}_{3}^{+}$, used in the present study.

\subsubsection{CASSCF/MC-XQDPT2 calculations}

To examine the properties of the low-lying excited states of MeCbl, as modeled by the Im-[Co ${ }^{\mathrm{III}}$ (corrin) $]-\mathrm{CH}_{3}^{+}$system shown in Figure 3.1, both CASSCF and MC-XQDPT2-level calculations were carried out. The latter calculations, aimed at correcting the CASSCF excitation energies for the leading dynamical correlation effects, were performed using the modified version of the MC-QDPT2 approach of Ref. 154, abbreviated as MC-XQDPT2, as implemented in the PCGAMESS/Firefly QC package. ${ }^{153}$ We recall that the MC-XQDPT2 approach is a genuine multi-root (i.e., perturb-then-diagonalize) multi-reference perturbation theory technique using the CASSCF state-averaged (SA) electron density over the ground and excited states of interest, which allows a true mixing of the CASSCF zero-order states via the construction and the subsequent diagonalization of the effective Hamiltonian. Typically, in order to obtain accurate results, the excited-state, CASSCFbased, MC-XQDPT2 calculations require a determination of a manifold of electronic states for the proper analysis of the low-lying excited states of interest, particularly 
for transition-metal systems, such as cobalamins, ${ }^{161}$ where there may be several low-lying electronic states that interact one with another. As a result, 20 electronic states were considered in the SA-CASSCF calculations preceding the final MC-XQDPT2 work in order to investigate the nature of the four lowest-energy excited states at the MC-XQDPT2 level of theory. The CASSCF/MC-XQDPT2 calculations were performed using the 6-31G(d) basis with spherical d orbitals, assuming the BP86/6-31G(d) optimized geometry of the Im-[Co ${ }^{\mathrm{III}}$ (corrin) $]-\mathrm{CH}_{3}^{+}$system. The details of the active orbital space used in the CASSCF/MC-XQDPT2 calculations reported in this work are discussed in Section 3.3.2. The 38 lowest-energy core orbitals that correlate with the 1s shells of the carbon, and nitrogen atoms, and the 1 s, 2s, 2p, 3s, and 3p shells of Co were frozen in the MC-XQDPT2 calculations.

\subsubsection{EOM-CCSD calculations}

The EOM-CC-type calculations were carried out at the basic EOM-CCSD ${ }^{166,167}$ or the equivalent linear response CCSD level, ${ }^{175,176}$ which is considered to be appropriate if the excited states of interest are dominated by one-electron transitions, ${ }^{177-179}$ as is the case in this study, where the low-lying states of the Im-[CoII(corrin)] $-\mathrm{CH}_{3}^{+}$model of MeCbl are examined. The EOM-CCSD approximation provides reasonable results for such states, with errors in the excitation energies on the order of $0.1 \mathrm{eV}$ in calculations for smaller systems ${ }^{166,167,175-179}$ and typically in the 0.3-0.5 eV range for larger polyatomic molecules (see Refs. 180, 181, 182, 183 or 184 for examples).

Let us recall that in the EOM-CCSD method, one represents excited electronic

states $\left|\Psi_{\mu}\right\rangle$ by applying the linear excitation operator $R_{\mu}^{(\mathrm{CCSD})}=R_{\mu, 0}+R_{\mu, 1}+R_{\mu, 2}$ ( $R_{\mu, n}$ is the $n$-body or $n$-tuply excited component of $R_{\mu}^{(\mathrm{CCSD})}$ ) to the ground state $\left|\Psi_{0}\right\rangle=e^{T_{1}+T_{2}}|\Phi\rangle$ resulting from the standard CCSD ${ }^{185-188}$ calculations. Here, $T_{1}$ and $T_{2}$ are the singly and doubly excited components of the cluster operator $T^{(\mathrm{CCSD})}=$ $T_{1}+T_{2}$ obtained in the ground-state CCSD calculations and $|\Phi\rangle$ is the reference determinant. Note that we used the restricted HF (RHF) determinant as a reference in our calculations. The EOM-CCSD excitation operators $R_{\mu}^{(\mathrm{CCSD})}$ and the 
corresponding vertical excitation energies $\omega_{\mu}^{(\mathrm{CCSD})}=E_{\mu}^{(\mathrm{CCSD})}-E_{0}^{(\mathrm{CCSD})}$ are obtained by diagonalizing the similarity-transformed Hamiltonian $\bar{H}^{(\mathrm{CCSD})}=e^{-T^{(\mathrm{CCSD})}} H e^{T^{(\mathrm{CCSD})}}$ resulting from the CCSD calculations in a space of singly and doubly excited determinants relative to $|\Phi\rangle$.

The EOM-CCSD calculations reported in this chapter and the corresponding ground-state CCSD calculations were performed using the CC and EOM-CC computer programs described in Refs. 189 and 190 that are part of the GAMESS package. ${ }^{150,191}$ In analogy to the MC-XQDPT2 case, the 38 lowest-energy core orbitals that correlate with the 1s shells of the carbon and nitrogen atoms, and the $1 s, 2 s, 2 p, 3 s$, and $3 p$ shells of cobalt were frozen in the CCSD and subsequent EOM-CCSD iterations. As in the case of the CASSCF/MC-XQDPT2 computations and due to the enormous computer costs of the CCSD and EOM-CCSD calculations for molecular systems of the Im-[Co ${ }^{\mathrm{III}}$ (corrin) $]-\mathrm{CH}_{3}^{+}$size, where we had to correlate 158 electrons outside the frozen core, the 6-31G(d) basis set was used (using spherical components of the $d$ functions). Again, the ground-state nuclear geometry of $\mathrm{Im}-\left[\mathrm{Co}^{\mathrm{III}}\right.$ (corrin) $]-\mathrm{CH}_{3}^{+}$employed in the EOM-CCSD calculations of vertical excitation energies for this system was obtained from the aforementioned BP86/6-31G(d) optimization. We attempted to determine four excited states, but we could not converge the fourth one, so the EOM-CCSD results presented in this chapter are limited to the $\mathrm{S}_{0} \rightarrow \mathrm{S}_{n}$ excitations with $n=1,2$, and 3 .

\subsubsection{TD-DFT calculations with different functionals}

The low-lying excited states of MeCbl, as modeled by the Im-[Co ${ }^{\mathrm{III}}$ (corrin)]$\mathrm{CH}_{3}^{+}$structure, were also calculated using a variety of TD-DFT approaches. Nine different functionals were employed to examine the performance of TD-DFT against the high-level MC-XQDPT and EOM-CCSD data. In addition to the standard B3LYP and BP86 choices, seven other functionals, including BLYP, TPSSh, TPSS, MPW1PW91, MPWPW91, CAM-B3LYP, and LC-BLYP, were employed. Since the recent studies of $\mathrm{MeCbl}$, examining the performance of different functionals in predicting the Co- $\mathrm{C}_{\mathrm{Me}}$ bond dissociation energy, indicated that the GGA-type func- 
tionals were capable of producing the results that were quite close to the experimental and high-level $a b$ initio dissociation energies, ${ }^{163}$ three such functionals, namely, BLYP, MPWPW91, and TPSS, were tested and compared with their hybrid B3LYP, MPW1PW91, and TPSSh analogs, having different fractions of the exact HF exchange (20\%, 25\% and 10\%, respectively). The Coulomb-attenuating CAM-B3LYP ${ }^{135}$ method and range-separated LC-BLYP approach ${ }^{136,144,145,173}$ were employed to address the possible underestimation of the TD-DFT excitation energies for CT transitions. The LC-BLYP approach allows for an effective tuning of the relative contributions to the exchange-correlation potential originating from the exchange and correlation terms via the range separation parameter $\mu$. Thus, the LC-BLYP vertical excitation energies were calculated as a function of $\mu$ ranging from 0 to $0.90 \mathrm{Bohr}^{-1}$, with an increment of $0.10 \mathrm{Bohr}^{-1}$.

The TD-DFT calculations of the low-lying excited states of Im-[Co ${ }^{\mathrm{III}}$ (corrin)]$\mathrm{CH}_{3}^{+}$were carried out using the 6-31G(d) basis set employing the spherical $\mathrm{d}$ orbitals. The basis set dependence of the resulting excitation energies was examined by performing TD-DFT calculations with a larger 6-311++G(d,p) basis. As shown below, the use of a larger basis set has essentially a negligible effect on the key characteristics of the low-lying states, particularly on the $S_{1}$ state, which is the main subject of the work presented in this chapter.

To verify the influence of the ground-state geometry on the TD-DFT vertical excitations energies, two different approaches were explored, namely, (i) the TDDFT calculations using different functionals, but utilizing the same, BP86/6-31G(d) optimized ground state structure of the Im-[Co ${ }^{\mathrm{III}}$ (corrin) $]-\mathrm{CH}_{3}^{+}$model, and (ii) the TD-DFT calculations using the ground-state geometries of Im-[Co ${ }^{\mathrm{III}}$ (corrin) $]-\mathrm{CH}_{3}^{+}$ optimized with the same functional as applied in the excited-state calculations. For each functional explored in this study, the excitation energies obtained using the above two approaches turned out to be practically identical. For this reason, in our discussion below, we focus on the results obtained with the second approach. The exceptions are the LC-BLYP vertical excitation energies, which are based on the BP86/6-31G(d) optimized structure. The TD-DFT calculations employing the 
B3LYP, BP86, BLYP, TPSSh, TPSS, MPW1PW91, MPWPW91, and CAM-B3LYP functionals were performed using the Gaussian 09 suite of programs. ${ }^{148}$ The LC-BLYP calculations using different values of $\mu$ were carried out using a locally-modified version of the GAMESS software. ${ }^{150,191}$ Finally, the TD-DFT/BP86 geometry optimization of the $S_{1}$ state was performed with Turbomole. ${ }^{151}$

\subsection{Results and discussion}

\subsubsection{Experimental information about the low-lying excited states of MeCbl}

The analysis of the low-lying excited states of MeCbl cofactor has been the subject of several experimental investigations. The most recent results originate from the TAS studies by Sension et al. ${ }^{56,58,60,65}$ and the spectroscopic studies by Brunold and co-workers. ${ }^{108,113}$ Since the latter group analyzed the details of the Abs as well as the CD and MCD spectra, our computed excitations are compared with the results obtained by Brunold et al.

According to the analysis of Abs, CD, and MCD data reported by Brunold and co-workers, ${ }^{108}$ at least four bands contribute to the Abs envelope in the $\alpha / \beta$ spectral range. The excitation energies, which correspond to the maxima of the four Gaussian bands found by Brunold et al. using the iterative fitting of the Abs, CD, and MCD spectra, are $2.22 \mathrm{eV}$ (band 1), $2.35 \mathrm{eV}$ (band 2), $2.55 \mathrm{eV}$ (band 3), and $2.70 \mathrm{eV}$ (band 4) (see Table 3.1 and footnote $\mathrm{b}$ in it). The splitting between bands 2 and 3 , of about $0.20 \mathrm{eV}$, is larger than that between bands 1 and $2(\approx 0.13 \mathrm{eV})$, implying that the four bands identified by Brunold et al. do not represent vibrational progressions associated with a single electronic transition. Furthermore, a detailed analysis of the CD and MCD data shows that bands 1 and 2 have opposite signs in comparison with bands 3 and 4 . Based on all of this information, Brunold and coworkers arrived at the conclusion that one should associate two distinct electronic transitions with the $\alpha / \beta$ spectral range, which are characterized by the $0-0$ excitation energies of 2.22 and $2.55 \mathrm{eV}$ and that the lowest one should be interpreted as $\pi \rightarrow \pi^{*}$ excitation of corrin. ${ }^{108}$ 
TABLE 3.1. Vertical excitation energies (eV) of the lowest four excited states of Im$\left[\mathrm{Co}^{\mathrm{III}}(\right.$ corrin $\left.)\right]-\mathrm{CH}_{3}^{+}$calculated with different methods and the 6-31G(d) basis set.

\begin{tabular}{|c|c|c|c|c|}
\hline Method & $\overline{\mathrm{S}_{1}}$ & $\overline{\mathrm{S}_{2}}$ & 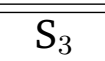 & $\overline{\mathrm{S}_{4}}$ \\
\hline \multicolumn{5}{|l|}{$A b$-initio } \\
\hline $\operatorname{CASSCF}(12,12)$ & 3.21 & 3.34 & 4.47 & 4.60 \\
\hline MC-XQDPT2 & 2.48 & 2.67 & 2.79 & 3.42 \\
\hline EOM-CCSD & 2.65 & 3.12 & 3.23 & $a$ \\
\hline \multicolumn{5}{|l|}{ TD-DFT } \\
\hline B3LYP (20\% HF) & 2.65 & 2.82 & 2.95 & 3.02 \\
\hline CAM-B3LYP & 2.87 & 2.92 & 3.00 & 3.06 \\
\hline BLYP & 2.19 & 2.33 & 2.48 & 2.58 \\
\hline LC-BLYP $(\mu=0.00)$ & 2.20 & 2.35 & 2.48 & 2.62 \\
\hline LC-BLYP $(\mu=0.10)$ & 2.27 & 2.44 & 2.53 & 2.67 \\
\hline MPW1PW91 (25\% HF) & 2.70 & 2.85 & 2.96 & 3.02 \\
\hline MPWPW91 & 2.19 & 2.35 & 2.48 & 2.58 \\
\hline TPSSh $(10 \% \mathrm{HF})$ & 2.52 & 2.79 & 2.85 & 3.14 \\
\hline TPSS & 2.29 & 2.44 & 2.55 & 2.75 \\
\hline BP86 & 2.18 & 2.34 & 2.48 & 2.59 \\
\hline \multicolumn{5}{|l|}{ Experiment $t^{b}$} \\
\hline Abs, CD and MCD & 2.22 & $2.35^{c}$ & 2.55 & $2.70^{d}$ \\
\hline
\end{tabular}

${ }^{a}$ The fourth EOM-CCSD root did not converge.

${ }^{b}$ The results obtained in Ref. 108 using the Gaussian deconvolution of the Abs spectrum in the $\alpha / \beta$ range, which gives four bands with the maxima at $17,870,18,950,20,550$, and $21,800 \mathrm{~cm}^{-1}$.

${ }^{c}$ Interpreted in Ref. 108 as a vibrational progression of the transition at $2.22 \mathrm{eV}$.

${ }^{d}$ Interpreted in Ref. 108 as a vibrational progression of the transition at $2.55 \mathrm{eV}$.

According to this interpretation, the remaining two bands that result from the Gaussian deconvolution of the $\alpha / \beta$ spectral region carried out in Ref. 108, with the maxima at 2.35 and $2.70 \mathrm{eV}$, belong to the overlapping vibrational progressions associated with the two electronic transitions that have the $0-0$ excitation energies of 2.22 and $2.55 \mathrm{eV}$, respectively. Although this certainly is a plausible explanation, there are potential issues with it, some acknowledged by the authors of Ref. 108, what consequently leaves room for alternative interpretations. First of all, one has to consider the above interpretation with caution, since the analysis of the Abs, $C D$, and MCD data reported in Ref. 108 is based on the Gaussian deconvolution technique. Gaussian deconvolution is an iterative fit procedure which assumes the smallest possible number of Gaussian bands to resolve the major electronic transitions con- 
tributing to the particular spectral window and does not guarantee that certain low-intensity electronic transitions are not omitted from the fitting procedure. In other words, one cannot exclude the scenario that the number of electronically excited states of $\mathrm{MeCbl}$ which contribute to the $\alpha / \beta$ spectral region is greater than that predicted by the Gaussian deconvolution exploited in Ref. 108. Another issue related to the interpretation of the $\alpha / \beta$ spectral region, as originating from $\pi \rightarrow \pi^{*}$ electronic transition accompanied by the corresponding vibrational progressions, is intensity ratio. Typically, one observes intensity lowering when the second member of the vibrational progression is compared with the $0-0$ transition. However, in the $\mathrm{MeCbl}$ case, the opposite pattern is observed, i.e., the second band centered at 2.35 $\mathrm{eV}$ has a larger intensity than the first one at $2.22 \mathrm{eV}$. The next potential problem with the interpretation of the $\alpha / \beta$ spectral region, as originating from only $\pi \rightarrow \pi^{*}$ electronic transition, is related to the strong enhancement of the $\mathrm{Co}-\mathrm{C}_{\mathrm{Me}}$ stretching mode, which resembles an isolated harmonic oscillator perpendicular to the corrin plane. If the $\mathrm{S}_{1}$ state was a $\pi \rightarrow \pi^{*}$ transition, an in-plane mode would be strongly enhanced in the RR spectrum when the laser excitation was tuned to this particular spectral region. This, however, contradicts the available RR data acquired for the resonance with the longer-wavelength absorption band at $530.9 \mathrm{~nm}$, where strong enhancement of the Co- $\mathrm{C}_{\mathrm{Me}}$ stretch at $506 \mathrm{~cm}^{-1}$ is observed. ${ }^{192}$ The RR experiments also suggest that the $\pi$ system of corrin should be electronically coupled to the Co$\mathrm{C}_{\mathrm{Me}}$ bond to weaken it with the electronic excitation. It is, in principle, possible that one of the higher excited states $S_{n}$ with $n>1$, with a noticeable transition dipole, provides such a coupling. This, however, seems rather unlikely, since, at least according to TD-DFT calculations with hybrid functional, ${ }^{108}$ the states of interest are energetically higher and may, in fact, lie outside of the $\alpha / \beta$ spectral range. It is true that such a coupling could also be provided by the $\mathrm{S}_{1}$ state, as implied by the findings in Ref. 108, but this would then contradict the interpretation of the $S_{0} \rightarrow S_{1}$ transition as a $\pi \rightarrow \pi^{*}$ excitation in corrin. 
All of this suggests that the $\pi \rightarrow \pi^{*}$ character of the low-lying excited states of MeCbl, particularly of the $S_{1}$, can be questioned and that the alternative, MLCT interpretation of the $S_{1}$ state, postulated by Sension et al. ${ }^{56,58,60,65}$ is possible as well.

\subsubsection{Electronic excitations resulting from the CASSCF/MC-XQDPT2 calcula- tions}

$A b$ initio methods based on the CASSCF methodology are frequently regarded as useful tools for studying electronically excited states. ${ }^{193-196}$ However, when performing CASSCF and other CASSCF-based multi-reference computations, such as those based on the MC-XQDPT2 approach, one has to select active orbitals in a judicious manner, particularly in the case of larger molecules containing transition metals and tetrapyrrolic ligands, such as corrin. The number of active orbitals that may have to be considered in these systems can easily become very large, making the CASSCF-based calculations prohibitively expensive or even technically impossible. At the same time, the results of CASSCF-based calculations may strongly depend on the choice of the active space. It is, therefore, important to find a proper balance between chemical intuition used to identify meaningful active orbitals, relevant to the problem of interest, and the size of the resulting active space that has to be computationally manageable.

In the case of the CASSCF and CASSCF-based MC-XQDPT2 calculations for the Im- $\left[\mathrm{Co}^{\mathrm{III}}\right.$ (corrin) $]-\mathrm{CH}_{3}^{+}$model of MeCbl, reported in this work, the process of selecting active orbitals was guided by the active spaces that were previously used for the various derivatives of cobalt corrinoids. ${ }^{159-161,197,198}$ Let us recall that although the MeCbl cofactor belongs to cobalamins, a class of $\mathrm{Co}^{\mathrm{III}}$ octahedral complexes, the local environment of cobalt in Im- $[\mathrm{Co}$ orbitals consistent with the square-planar structure, which has a $d^{6}$ configuration as the dominant electronic configuration. According to the crystal field theory, such an electronic configuration leaves the highest $d_{x^{2}-y^{2}}$ orbital empty, i.e., in designing meaningful active spaces for the CASSCF and CASSCF-based MC-XQDPT2 calcula- 
tions for the Im-[Co ${ }^{\mathrm{III}}$ (corrin) $]-\mathrm{CH}_{3}^{+}$system one may focus on the $d_{x y}, d_{x z}, d_{y z}$, and $d_{z^{2}}$ orbitals of Co, mixed up with the appropriate orbitals of the corrin and methyl fragments. Here and elsewhere in this description, in labeling the d orbitals of Co we are assuming that the $\mathrm{z}$ axis is perpendicular to the corrin macrocycle. Furthermore, the $\mathrm{x}$ and $\mathrm{y}$ axes that define the xy plane of the corrin backbone are oriented such that the $\mathrm{C}_{2}$ axis of symmetry coincides with the $\mathrm{x}$ axis. This choice of the coordinate system corresponds to a 45 degree rotation of the $\mathrm{x}$ and $\mathrm{y}$ axes compared to the previous study of cob(I)alamin by Jensen. ${ }^{159}$ Keeping in mind the above coordinate frame and adding the $4 \mathrm{~d}$ correlating orbitals to the doubly occupied $3 \mathrm{~d}$ orbitals to account for the double shell effect in transition metal complexes, ${ }^{196}$ we ended up with the following four subgroups of orbitals in our final active space design (see Figure 3.2): (i) the doubly occupied $3 d_{x z}$ and $3 d_{y z}$ orbitals centered on the Co atom and the corresponding correlating orbitals of the $4 \mathrm{~d}$ type, i.e., $4 d_{x z}$ and $4 d_{y z}$, (ii) the bonding Co- $\mathrm{C}_{\mathrm{Me}} \sigma\left(d_{z^{2}}\right)$ orbital and its antibonding $\sigma^{*}\left(d_{z^{2}}\right)$ counterpart, (iii) the occupied $3 d_{x y}+\mathrm{n}$ and unoccupied $3 d_{x y}-\mathrm{n}$ orbitals describing the respective bonding and antibonding combinations of the $3 d_{x y}$ orbital centered on the Co atom with the lone pairs (n) centered on the four equatorial nitrogens in the corrin plane, and (iv) the two occupied $\pi$ and two unoccupied $\pi^{*}$ orbitals of corrin. Whereas the inclusion of active orbitals that belong to the (ii) and (iii) subgroups was mandatory for a proper description of the equatorial and axial $\sigma$-interactions, leaving no room for maneuver, the selection of active orbitals that has ultimately resulted in subgroups (i) and (iv) turned out to be less straightforward. Therefore, we had to come up with an active space which was chemically meaningful and, at the same time, did not, have more than a dozen orbitals to make the resulting CASSCF and CASSCF MC-XQDPT2 calculations computationally feasible. Thus, in the initial stages of our work, several active orbital spaces were tested, mostly by selecting different subsets of orbitals that correlate with the $3 \mathrm{~d}$ and $4 \mathrm{~d}$ shells of Co and $\pi$ network of corrin, and additional analyses were carried out to eliminate those molecular orbitals (MOs) that did not significantly contribute to the CASSCF wave functions relevant to the electronic states of interest (as measured, for example, by the oc- 


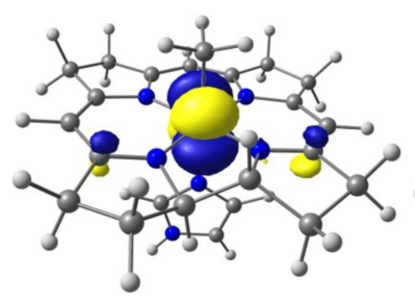

(112) $3 d_{x z}$

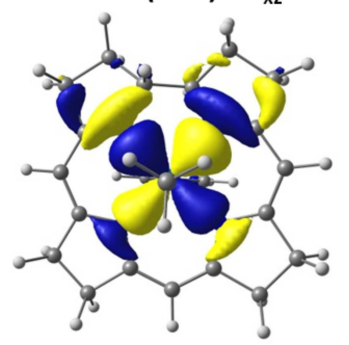

(116) $3 d_{x y}+n$

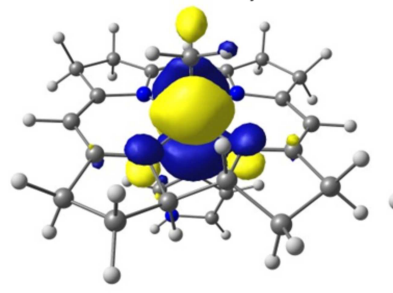

(120) $4 d_{x z}$

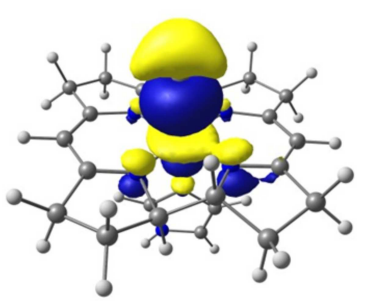

(113) $\sigma\left(d_{z} 2\right)$

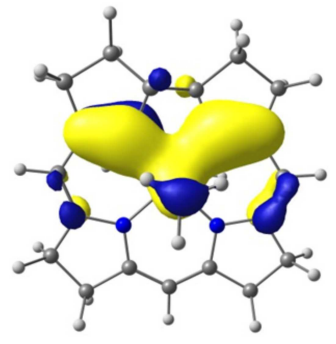

(117) $\pi$

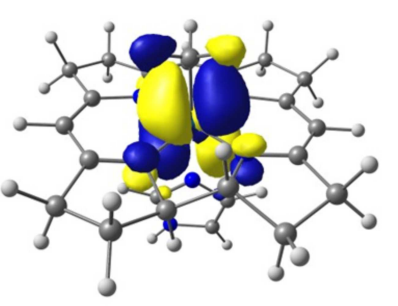

(121) $4 d_{y z}$

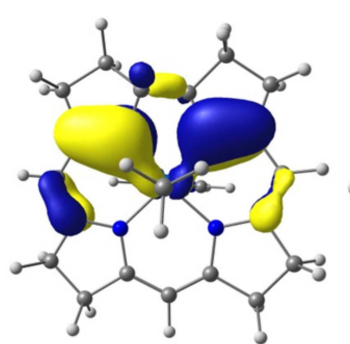

(114) $\pi$

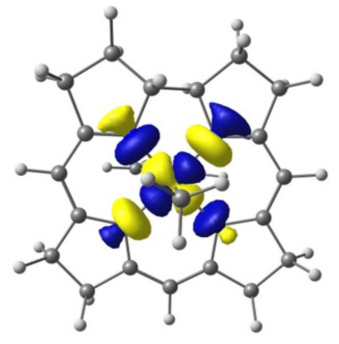

(118) $3 d_{x y}-n$

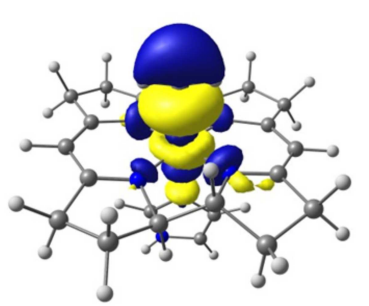

(122) $\sigma^{*}\left(d_{z} 2\right)$

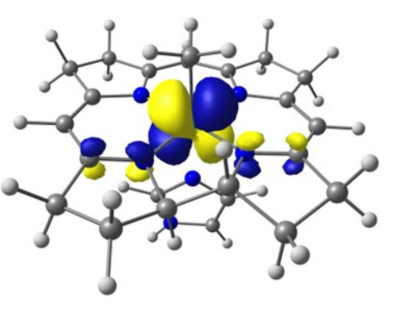

(115) $3 d_{y z}$

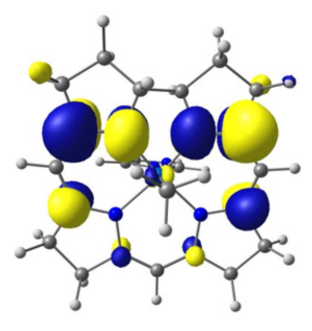

(119) $\pi^{*}$

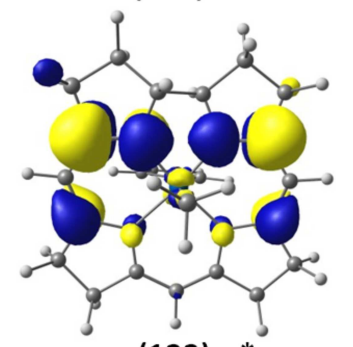

(123) $\pi^{*}$

Figure 3.2. Active orbitals of $\mathrm{Im}-\left[\mathrm{Co}{ }^{\mathrm{III}}\right.$ (corrin) $]-\mathrm{CH}_{3}^{+}$used in the $\mathrm{CASSCF}(12,12)$ and subsequent MC-XQDPT2 $(12,12)$ calculations.

cupation numbers of the natural orbitals of CASSCF). As a result, the final active space used in the CASSCF and MC-XQDPT2 calculations for Im-[Co ${ }^{\mathrm{III}}$ (corrin) $]-\mathrm{CH}_{3}^{+}$ reported in this work consisted of 12 active electrons distributed among 12 active orbitals, which corresponded to the above subgroups (i)-(iv). Four occupied d-type orbitals of Co and two occupied $\pi$-type orbitals localized on corrin were combined with the six corresponding virtual orbitals to obtain the optimal set of active MOs (Figure 3.2) for elucidating the nature of the $S_{1}$ state.

As shown in Table 3.1, the vertical excitation energies obtained in our CASSCF $(12,12)$ calculations are considerably higher than those observed experimentally. For example, the energy of the lowest excited $S_{1}$ state is almost $1 \mathrm{eV}$ higher than the corresponding experimental energy. The CASSCF results obtained here for the Im- $\left[\mathrm{Co}{ }^{\mathrm{III}}\right.$ (corrin) $]-\mathrm{CH}_{3}^{+}$model of $\mathrm{MeCbl}$ are similar to those charac- 
terizing the $\mathrm{CNCbl}$ derivative, where the analogous overestimation of the excitation energies was observed and subsequently improved by including the dynamical correlation effects via the MC-XQDPT2 theory. In addition, the inspection of the lowest-energy excited states of MeCbl predicted by CASSCF reveals that they are dominated by $\mathrm{d} \rightarrow \mathrm{d}$ transitions (see Table A.4 in the Appendix), which is a problematic finding. Such a poor description of the low-lying electronic states reflects a downside of using the CASSCF method alone, as previously argued in the case of CNCbl (see Chapter 2 or Ref. 161). One has to calculate many more excited states at the CASSCF level, enable their intrinsic mixing at the correlated theory level to improve the zero-order description, and include dynamical correlation effects on top of CASSCF to obtain a more quantitative description. This can be done through the use of the multi-reference, multi-root (i.e., perturb-then-diagonalize), perturbation theory, represented in this study by the MC-XQDPT2 approach.

As shown in Tables 3.1 and 3.2, the inclusion of dynamical correlation effects via the MC-XQDPT2 methodology dramatically improves the excitation energies characterizing the Im-[Co ${ }^{\mathrm{III}}$ (corrin) $]-\mathrm{CH}_{3}^{+}$system. Indeed, the MC-XQDPT2 approach reduces the CASSCF vertical excitation energies corresponding to the $\mathrm{S}_{0}$ $\rightarrow \mathrm{S}_{1}, \mathrm{~S}_{0} \rightarrow \mathrm{S}_{2}, \mathrm{~S}_{0} \rightarrow \mathrm{S}_{3}$, and $\mathrm{S}_{0} \rightarrow \mathrm{S}_{4}$ transitions, of 3.21, 3.34, 4.47, and 4.60 $\mathrm{eV}$, by $0.73,0.67,1.68$, and $1.18 \mathrm{eV}$, respectively, bringing at least three of them to a reasonably good agreement with the excitation energies observed experimentally. In particular, the MC-XQDPT2 result for the $S_{1}$ state, which is the main focus of this chapter, differs from the corresponding experimental excitation energy by only $0.26 \mathrm{eV}$, which is an excellent description. We note, however, that these considerable improvements would not be possible if we did not calculate many additional CASSCF states and if we did not allow their intrinsic mixing at the multireference perturbation theory level. As shown in Figure 3.3, the electronic states of the Im- $\left[\mathrm{Co}^{\mathrm{III}}\right.$ (corrin) $]-\mathrm{CH}_{3}^{+}$system undergo a significant reordering when the dynamical correlation effects are turned on via the MC-XQDPT2 theory. In particular, the CASSCF states that contribute to the four lowest-energy excited states of Im$\left[\mathrm{Co}^{\mathrm{III}}\right.$ (corrin) $]-\mathrm{CH}_{3}^{+}$in the MC-XQDPT2 description most significantly, can be iden- 
tified as roots $10-14$ (or $S_{9}-S_{13}$ ) in the CASSCF spectrum (see Figure 3.3 and Table 3.2) This has several interesting consequences. One of the most important consequences is the observation that the physical nature of the computed excited states alters when the reordering and mixing of the CASSCF states is allowed and when the dynamical correlation effects are turned on. As shown in Table 3.2, the four lowest-energy excited states of the $\mathrm{Im}$ - $\left[\mathrm{Co}{ }^{\mathrm{III}}\right.$ (corrin) $]-\mathrm{CH}_{3}^{+}$calculated at the MC-XQDPT2 level have substantial components from single $\mathrm{d} \rightarrow \pi^{*}$ and double $\mathrm{d} / \pi \rightarrow \pi^{*}$ transitions. In particular, the MC-XQDPT2 wave function of the $\mathrm{S}_{1}$ state, which interests us here the most, is primarily composed of the $10^{\text {th }}(16 \%)$ and $12^{\text {th }}(52 \%)$ states from the CASSCF spectrum. The main contribution to the latter CASSCF wave function, which dominates the MC-XQDPT2 $S_{1}$ state, is a $d \rightarrow \pi^{*}$ transition, whereas the former CASSCF state has a predominantly $d / \pi \rightarrow d+\sigma / \pi^{*}$ character (see Table 3.2). In other words, the lowest-energy excited state of the Im-[Co ${ }^{\mathrm{III}}$ (corrin) $]-\mathrm{CH}_{3}^{+}$system in the MC-XQDPT2 description can be characterized as a MLCT $d \rightarrow \pi^{*}$ transition that originates from large $d \rightarrow \pi^{*}$ CT excitations defining the $12^{\text {th }}$ root in the underlying CASSCF spectrum. As shown in Table 3.2, both the $S_{2}$ and $S_{3}$ states include substantial contributions from the $11^{\text {th }}$ CASSCF state, which has a $\mathrm{d}_{y z} / \mathrm{d}_{x z} \rightarrow \pi^{*}$ character, and the $\mathrm{S}_{2}$ state has also very large (in fact, dominant) contributions from the $14^{\text {th }}$ CASSCF state, which is mainly composed of $\mathrm{d}_{x z} \rightarrow \pi^{*}$ and $\mathrm{d}_{y z} \rightarrow \pi^{*}$ transitions. Thus, the three lowest-energy excited states of the Im-[Co ${ }^{\text {III }}$ (corrin) $]-\mathrm{CH}_{3}^{+}$system resulting from the MC-XQDPT2 computations, which fall within about $0.2-0.3 \mathrm{eV}$ from the bands observed experimentally in the $\alpha / \beta$ region, can be interpreted as the MLCT $\mathrm{d} \rightarrow \pi^{*}$ transitions. The same applies to the $\mathrm{S}_{4}$ state (see Table 3.2). According to our MC-XQDPT2 calculations, none of the lowest-energy excited states of Im- $\left[\mathrm{Co}^{\mathrm{III}}\right.$ (corrin) $]-\mathrm{CH}_{3}^{+}$can be described as $\pi \rightarrow \pi^{*}$ transitions localized on corrin; they all are of the $d \rightarrow \pi^{*}$ type, instead. 


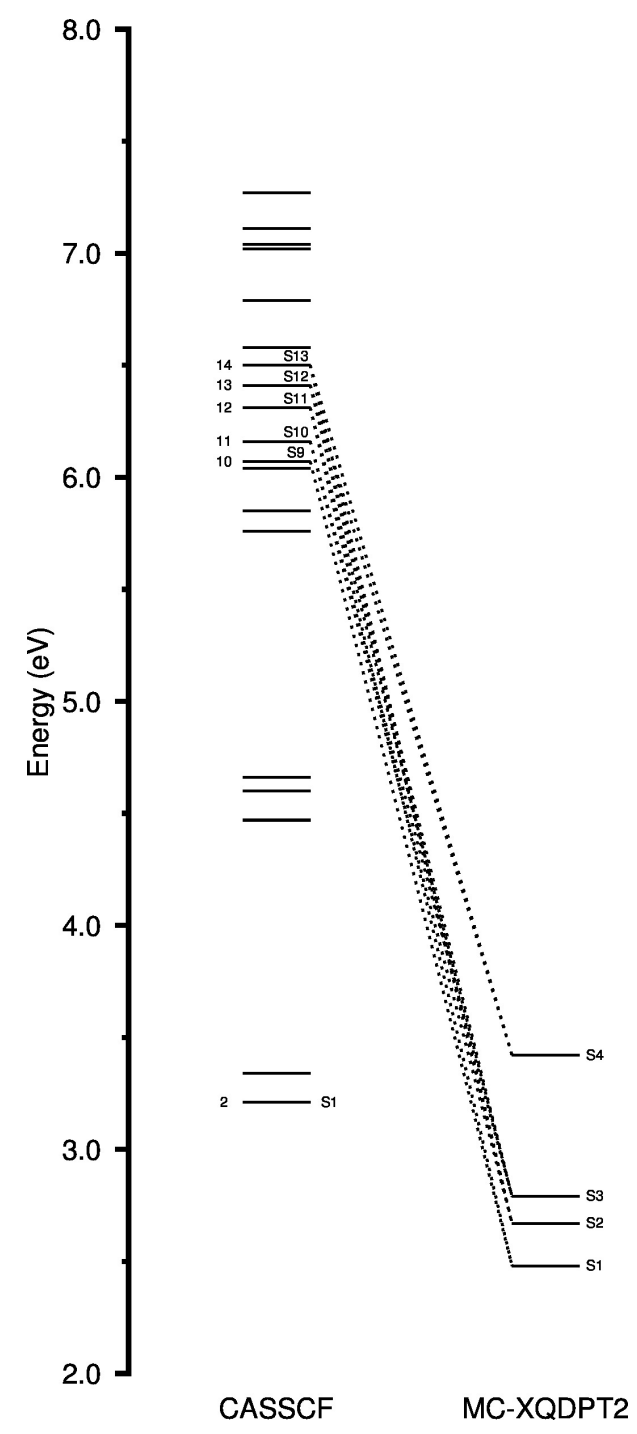

Figure 3.3. The lowest twenty excited states computed at the $\operatorname{CASSCF}(12,12)$ level and the four lowest-energy excited states resulting from the corresponding MCXQDPT2 calculations. The lines between the CASSCF and MC-XQDPT2 energy levels indicate mixing of states upon the incorporation of dynamical correlation effects via the second order perturbation theory (see Table 3.2 for further details). 
TABLE 3.2. The ground and four lowest excited states of $\mathrm{Im}-\left[\mathrm{Co}^{\mathrm{III}}\right.$ (corrin) $]-\mathrm{CH}_{3}^{+}$ obtained from the CASSCF-based MC-XQDPT2 calculations. CSF stands for a configuration state function.

\begin{tabular}{|c|c|c|c|c|c|c|}
\hline State & $\mathrm{E}(\mathrm{eV})$ & f & $\begin{array}{l}\text { weight (\%) of } \\
\text { CAS state in } \\
\text { MC-XQDPT2 }\end{array}$ & $\begin{array}{l}\text { CAS } \\
\text { state }\end{array}$ & $\begin{array}{l}\text { Coeff. of } \\
\text { CSF }(\%)\end{array}$ & Character \\
\hline $\mathrm{S}_{0}$ & 0 & & 88 & 1 & & \\
\hline \multirow[t]{2}{*}{$\mathrm{S}_{1}$} & 2.48 & 0.0022 & 52 & 12 & 22 & $\mathrm{~d}_{x z} \rightarrow \pi^{*}$ \\
\hline & & & 16 & 10 & $\begin{array}{l}24 \\
18\end{array}$ & $\begin{array}{l}\mathrm{d}_{y z} / \pi \rightarrow \mathrm{d}_{x y}+\sigma^{*} / \pi^{*} \\
\mathrm{~d}_{x z} / \pi \rightarrow \mathrm{d}_{x y}+\sigma^{*} / \pi^{*}\end{array}$ \\
\hline \multirow[t]{3}{*}{$\mathrm{S}_{2}$} & 2.67 & 0.0452 & 45 & 14 & $\begin{array}{l}12 \\
30\end{array}$ & $\begin{array}{l}\mathrm{d}_{y z} \rightarrow \pi^{*} \\
\mathrm{~d}_{x z} \rightarrow \pi^{*}\end{array}$ \\
\hline & & & 27 & 11 & $\begin{array}{r}36 \\
4 \\
11\end{array}$ & $\begin{array}{l}\mathrm{d}_{y z} \rightarrow \pi^{*} \\
\pi \rightarrow \pi^{*} \\
\mathrm{~d}_{x z} \rightarrow \pi^{*}\end{array}$ \\
\hline & & & 19 & 13 & $\begin{array}{r}26 \\
4 \\
9\end{array}$ & $\begin{array}{l}\mathrm{d}_{y z} \rightarrow \pi^{*} \\
\pi \rightarrow \pi^{*} \\
\mathrm{~d}_{x z} \rightarrow \pi^{*}\end{array}$ \\
\hline \multirow[t]{3}{*}{$\mathrm{S}_{3}$} & 2.79 & 0.2536 & 39 & 11 & $\begin{array}{r}36 \\
4 \\
11\end{array}$ & $\begin{array}{l}\mathrm{d}_{y z} \rightarrow \pi^{*} \\
\pi \rightarrow \pi^{*} \\
\mathrm{~d}_{x z} \rightarrow \pi^{*}\end{array}$ \\
\hline & & & 23 & 13 & $\begin{array}{r}26 \\
4 \\
9\end{array}$ & $\begin{array}{l}\mathrm{d}_{y z} \rightarrow \pi^{*} \\
\pi \rightarrow \pi^{*} \\
\mathrm{~d}_{x z} \rightarrow \pi^{*}\end{array}$ \\
\hline & & & 20 & 12 & 22 & $\mathrm{~d}_{x z} \rightarrow \pi^{*}$ \\
\hline \multirow[t]{2}{*}{$\mathrm{S}_{4}$} & 3.42 & 0.0421 & 32 & 14 & $\begin{array}{l}12 \\
30\end{array}$ & $\begin{array}{l}\mathrm{d}_{y z} \rightarrow \pi^{*} \\
\mathrm{~d}_{x z} \rightarrow \pi^{*}\end{array}$ \\
\hline & & & 30 & 13 & $\begin{array}{r}26 \\
4 \\
9\end{array}$ & $\begin{array}{l}\mathrm{d}_{y z} \rightarrow \pi^{*} \\
\pi \rightarrow \pi^{*} \\
\mathrm{~d}_{x z} \rightarrow \pi^{*}\end{array}$ \\
\hline
\end{tabular}




\subsubsection{Electronic excitations resulting from the EOM-CCSD calculations}

The low-lying excited states of the Im-[CoII (corrin) $]-\mathrm{CH}_{3}^{+}$model of MeCbl were also examined using EOM-CCSD. The ground-state CCSD and excited-state EOM-CCSD calculations were carried out using a ground-state RHF determinant as a reference. Four excited states were requested, but we could not converge the fourth EOM-CCSD root. Consequently, only three lowest excited states of Im-[Co ${ }^{\mathrm{III}}$ (corrin) $]-\mathrm{CH}_{3}^{+}$found in the EOM-CCSD calculations were taken into consideration for further analysis.

TABLE 3.3. The lowest three excited states of Im- $\left[\mathrm{Co}^{\mathrm{III}}\right.$ (corrin) $]-\mathrm{CH}_{3}^{+}$obtained from EOM-CCSD calculations.

\begin{tabular}{|c|c|c|c|c|c|}
\hline State & $\mathrm{E}(\mathrm{eV})$ & & plitude $^{a}$ & Transition & Character \\
\hline \multirow[t]{6}{*}{$\mathrm{S}_{1}$} & 2.65 & R1 & -0.0912 & $114 \rightarrow 118$ & $\sigma\left(\mathrm{d}_{z^{2}} / \mathrm{d}_{x^{2}-y^{2}}\right) / \pi \rightarrow \pi^{*}$ \\
\hline & & $\mathrm{R} 1$ & -0.6232 & $117 \rightarrow 118$ & $\pi / \sigma\left(\mathrm{d}_{z^{2}}\right) \rightarrow \pi^{*}$ \\
\hline & & R1 & -0.1506 & $116 \rightarrow 119$ & $\pi \rightarrow \pi^{*}$ \\
\hline & & R1 & 0.0595 & $113 \rightarrow 120$ & $\pi / \sigma\left(\mathrm{d}_{z^{2}} / \mathrm{d}_{x z}\right) \rightarrow \pi^{*}$ \\
\hline & & R2 & -0.0552 & $117,116 \rightarrow 118,118$ & $\pi / \sigma\left(\mathrm{d}_{z^{2}}\right), \pi \rightarrow \pi^{*}$ \\
\hline & & $\mathrm{R} 2$ & -0.0552 & $116,117 \rightarrow 118,118$ & $\pi, \pi / \sigma\left(\mathrm{d}_{z^{2}}\right) \rightarrow \pi^{*}$ \\
\hline \multirow[t]{7}{*}{$\mathrm{S}_{2}$} & 3.12 & $\mathrm{R} 1$ & 0.3076 & $80 \rightarrow 139$ & $\mathrm{~d}_{x^{2}-y^{2}} / \sigma_{\text {Corr }} / \sigma_{\operatorname{Im}} \rightarrow \mathrm{d}_{x y}+\mathrm{n}$ \\
\hline & & R1 & -0.1683 & $95 \rightarrow 139$ & $\sigma_{\text {Corr }} / \mathrm{d}_{x^{2}-y^{2}} \rightarrow \mathrm{d}_{x y}+\mathrm{n}$ \\
\hline & & R1 & -0.1494 & $79 \rightarrow 139$ & $\mathrm{~d}_{y z} / \sigma_{\mathrm{Im}} \rightarrow \mathrm{d}_{x y}+\mathrm{n}$ \\
\hline & & R1 & 0.1383 & $80 \rightarrow 131$ & $\mathrm{~d}_{x^{2}-y^{2}} / \sigma_{\text {Corr }} / \sigma_{\text {Im }} \rightarrow \sigma_{\text {Corr }}^{*}$ \\
\hline & & $\mathrm{R} 1$ & -0.1363 & $75 \rightarrow 139$ & $\sigma_{\text {Corr }} / \mathrm{d}_{x^{2}-y^{2}} \rightarrow \mathrm{d}_{x y}+\mathrm{n}$ \\
\hline & & R1 & -0.1320 & $85 \rightarrow 139$ & $\sigma_{\text {Corr }} / \mathrm{d}_{x^{2}-y^{2}} \rightarrow \mathrm{d}_{x y}+\mathrm{n}$ \\
\hline & & R1 & 0.1018 & $81 \rightarrow 139$ & $\sigma_{\mathrm{Im}} / \mathrm{d}_{y z} \rightarrow \mathrm{d}_{x y}+\mathrm{n}$ \\
\hline \multirow[t]{5}{*}{$\mathrm{S}_{3}$} & 3.23 & $\mathrm{R} 1$ & 0.1494 & $81 \rightarrow 139$ & $\sigma_{\mathrm{Im}} / \mathrm{d}_{y z} \rightarrow \mathrm{d}_{x y}+\mathrm{n}$ \\
\hline & & R1 & 0.1335 & $80 \rightarrow 139$ & $\mathrm{~d}_{x^{2}-y^{2}} / \sigma_{\text {Corr }} / \sigma_{\text {Im }} \rightarrow \mathrm{d}_{x y}+\mathrm{n}$ \\
\hline & & R1 & -0.1220 & $75 \rightarrow 139$ & $\sigma_{\text {Corr }} / \mathrm{d}_{x^{2}-y^{2}} \rightarrow \mathrm{d}_{x y}+\mathrm{n}$ \\
\hline & & R1 & 0.1103 & $81 \rightarrow 132$ & $\sigma_{\mathrm{Im}} / \mathrm{d}_{y z} \rightarrow \pi^{*} / \sigma\left(\mathrm{d}_{z^{2}}\right)$ \\
\hline & & R1 & 0.1090 & $78 \rightarrow 139$ & $\mathrm{~d}_{x z} / \sigma_{\mathrm{Im}} \rightarrow \mathrm{d}_{x y}+\mathrm{n}$ \\
\hline
\end{tabular}

${ }^{a}$ For the $\mathrm{S}_{2}$ and $\mathrm{S}_{3}$ states, only the transitions with excitation amplitudes R greater than 0.1000 are presented.

As mentioned earlier, the EOM-CCSD method is considered to be appropriate when the excited states of interest are dominated by one-electron (including CT) transitions. Careful inspection of the largest $R_{1}$ and $R_{2}$ amplitudes defining the EOM-CCSD wave functions of the $S_{1}, S_{2}$, and $S_{3}$ states reveals that dominant 
contributions to these states indeed, originate from one-electron transitions (see Table 3.3 and Figure 3.4). As pointed out in Section 3.2, for such states, particularly when large polyatomic molecules, such as Im- $\left[\mathrm{Co}^{\mathrm{III}}\right.$ (corrin) $]-\mathrm{CH}_{3}^{+}$, are considered, one can anticipate that errors in the excitation energies resulting from the EOM-CCSD calculations are in the 0.3-0.5 eV range. As shown in Table 3.1, the first electronic excitation in the EOM-CCSD spectrum is $0.43 \mathrm{eV}$ above the experimental $S_{0} \rightarrow S_{1}$ transition, i.e., it falls within the expected error range. However, larger differences, of $0.77 \mathrm{eV}$ in the case of $\mathrm{S}_{2}$ and $0.68 \mathrm{eV}$ in the case of $\mathrm{S}_{3}$, are observed when the EOM-CCSD results for these two states are compared with experiment. It is, therefore, unclear whether the $S_{2}$ and $S_{3}$ states found in our calculations significantly contribute to the observed $\alpha / \beta$ bands. The EOM-CCSD excitation energy characterizing the $S_{1}$ state is also in excellent agreement with that obtained in the MC-XQDPT2 calculations (a small, $0.17 \mathrm{eV}$, difference), but the energies of the $S_{2}$ and $S_{3}$ states are again rather different, with differences falling into the 0.4-0.5 eV range (see Table 3.1). One should re-emphasize though that such an overestimation of excitation energies in the EOM-CCSD calculations for larger molecular systems is not unusual and that the EOM-CCSD wave functions remain perfectly meaningful in spite of such energy differences as long as the electronically excited states of interest are dominated by one-electron transitions. ${ }^{180-184}$ For example, the similarly overestimated excitation energies have been observed in the highly successful calculations of the electronic spectra of metalloporphyrins using the symmetry-adapted-cluster configuration-interaction ${ }^{199-201}$ and linear-response coupled-cluster ${ }^{175,176}$ methods with singles and doubles, which are closely related to the EOM-CCSD approach used in this work (see Refs. 202, 203, and 204 for a few representative examples).

The nature of the lowest excited states of MeCbl resulting from the EOMCCSD calculations for the Im- $\left[\mathrm{Co}^{\mathrm{III}}\right.$ (corrin) $]-\mathrm{CH}_{3}^{+}$system can be elaborated on by examining the RHF orbitals (displayed in Figure 3.4) involved in the largest $\mathrm{R}_{1}$ and $\mathrm{R}_{2}$ amplitudes defining the EOM-CCSD wave functions. As shown in Table 3.3, the $S_{1}$ state resulting from the EOM-CCSD calculations has several large contributions 


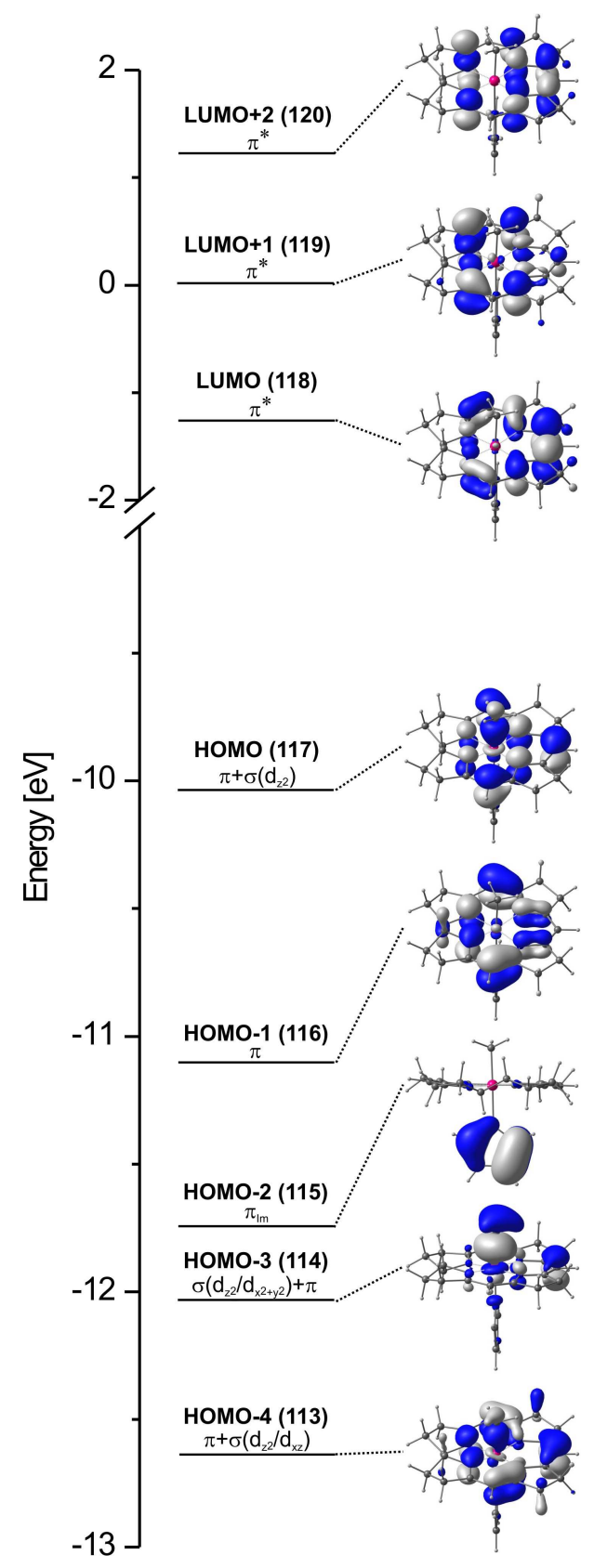

Figure 3.4. Canonical RHF orbitals used in the CCSD and EOM-CCSD calculations. 
from single excitations and some much smaller contributions from double excitations. Closer inspection of the largest excitation amplitudes in the EOM-CCSD description of the $S_{1}$ state shows that the main contribution to this state originates from a $\pi / \sigma\left(\mathrm{d}_{z^{2}}\right) \rightarrow \pi^{*}$ excitation. The second largest $\mathrm{R}_{1}$ value represents a $\pi \rightarrow \pi^{*}$ transition. The remaining singly as well as doubly excited amplitudes contributing to the $S_{1}$ state, although smaller than the leading two, have a mixed $\mathrm{d} / \pi \rightarrow \pi^{*}$ character. Taking into account all of these contributions together, we can conclude that the $S_{1}$ state of MeCbl resulting from the EOM-CCSD calculations has a predominantly $\mathrm{d} / \pi \rightarrow \pi^{*}$ (i.e., MLCT) character. This MLCT character of the $\mathrm{S}_{1}$ state is consistent with the assignment based on the independent MC-XQDPT2 calculations.

Unlike in the $S_{1}$ case, the EOM-CCSD results for the $S_{2}$ and $S_{3}$ states do not coincide with the results of the MC-XQDPT2 calculations. Indeed, the EOM-CCSD $\mathrm{S}_{2}$ state is dominated by the $\mathrm{d}_{x^{2}-y^{2}} / \sigma_{\text {Corr }} / \sigma_{\operatorname{Im}} \rightarrow \mathrm{d}_{x y}+\mathrm{n}$ contributions and it also has significant contributions that correspond to transfer of an electron from the Co $\mathrm{d}$ and corrin $\sigma$ orbitals to the unoccupied MOs of a similar character. Analogous remarks apply to the EOM-CCSD $S_{3}$ state, which also has significant excitations involving the $\pi^{*}$ orbital. We can conclude that the $S_{2}$ and $S_{3}$ states can be characterized as mainly single $\mathrm{d} \rightarrow \mathrm{d}+\mathrm{n}$ transitions in the EOM-CCSD description. Although this particular finding is not consistent with the results obtained with the MC-XQDPT2 approach, it is reassuring that the independent EOM-CCSD and MCXQDPT2 methodologies agree in the interpretation of the lowest excited $\mathrm{S}_{1}$ state of MeCbl of interest in this study, which they both classify as a predominantly MLCT $\mathrm{d} \rightarrow \pi^{*}$ or $\mathrm{d} / \pi \rightarrow \pi^{*}$ transition. The $\mathrm{S}_{2}$ and $\mathrm{S}_{3}$ states resulting from the EOM-CCSD calculations are considerably higher in energy and, as such, probably do not contribute to the $\alpha / \beta$ bands, although it would be useful to examine if the incorporation of triple excitations in the EOM-CC calculations could change this conclusion. Unfortunately, we are unable to perform the EOM-CC calculations corrected for triples at this time due to prohibitive computer costs. 


\subsubsection{Comparison of TD-DFT and $a b$ initio wave-function}

The TD-DFT formalism offers a relatively inexpensive approach to electronic excitations in complex molecular systems, but, as pointed out in the Introduction (Section 3.1), the results of TD-DFT calculations for cobalamins strongly depend on the choice of the functional. Therefore, selection of the proper functional represents a critical step prior to any extensive investigation directed toward the photochemistry or photophysics of $\mathrm{B}_{12}$ cofactors. The recent benchmark analysis of the parent vitamin $\mathrm{B}_{12}$ complex provided arguments supporting the superiority of BP86 over the B3LYP functional in terms of its electronically excited states (Chapter 2 or Ref. 161). The question arises whether a similar conclusion applies to MeCbl.

It has previously been noticed that while hybrid functionals overestimate excitation energies, their pure GGA counterparts tend to underestimate them. ${ }^{105-107,161}$ As shown in Table 3.1, a similar trend is observed when the low-lying excited states of MeCbl are examined, although one also has to point out that the excitation energies obtained with pure functionals, represented in this work by the BLYP, MPWPW91, TPSS, and BP86 approaches, are in much better agreement with the experimentally derived data of Ref. 108 than the excitations energies obtained with hybrid functionals. A comparison of the $S_{0} \rightarrow S_{1}$ excitation energies computed with the hybrid and the corresponding non-hybrid approaches, i.e., MPW1PW91 and MPWPW91, B3LYP and BLYP, and TPSSh and TPSS, shows differences of 0.51, 0.46, and $0.23 \mathrm{eV}$ for the respective pairs of functionals. These differences correlate with the amounts of the HF exchange in the hybrid functionals under consideration, which are 25\%,20\%, and 10\% for MPW1PW91, B3LYP, and TPSSh, respectively, implying that functionals with smaller fractions of the HF exchange provide a more accurate description of the $S_{1}$ state of MeCbl. A comparison of the $S_{0} \rightarrow S_{1}$ excitation energy obtained with the B3LYP functional and its long-range corrected CAM-B3LYP counterpart shows the difference of $0.22 \mathrm{eV}$, where the lower excitation energy value of $2.65 \mathrm{eV}$, which itself is considerably higher than the $S_{0} \rightarrow S_{1}$ excitation energies obtained with pure GGAs, is provided by the former functional. 
The analogous computations employing the long-range corrected LC-BLYP methods indicate a systematic increase in the excitation energies for the larger values of the range separation parameter $\mu$ (see Table 3.4). When $\mu$ is close to zero, the LC-BLYP functional approximates its pure

exchange-correlation counterpart, while increasing $\mu$ produces excitation energies similar to hybrid functionals. Based on the above analysis, we may conclude that by lowering excitation energies the pure DFT functionals, such as MPWPW91, TPSS, and BP86, work better than the corresponding hybrid approaches when the lowlying excited states of $\mathrm{MeCbl}$ are examined. However, the final conclusion regarding the suitability of various functionals for studying electronic properties of $\mathrm{MeCbl}$ cannot be based on examining the energetics only. The nature of electronic transitions has to be considered as well and we discuss this aspect of our calculations next.

TABLE 3.4. LC-BLYP/6-31G(d) electronic transitions of Im- $\left[\mathrm{Co}^{\mathrm{III}}\right.$ (corrin) $]-\mathrm{CH}_{3}^{+}$calculated as a function of the $\mu$ range-separation parameter together with their composition and character.

\begin{tabular}{llcl}
\hline \hline State & LC-BLYP & Energy [eV] & Character \\
\hline $\mathrm{S}_{1}$ & $\mu=0.00$ & 2.20 & $70 \%\left(\pi+\mathrm{d}_{y z} \rightarrow \pi^{*}+\mathrm{d}_{y z}\right)+28 \%\left(\mathrm{~d}_{x z} \rightarrow \pi^{*}+\mathrm{d}_{y z}\right)$ \\
& $\mu=0.10$ & 2.27 & $50 \%\left(\pi+\mathrm{d}_{z^{2}} \rightarrow \pi^{*}+\mathrm{d}_{y z}\right)+48 \%\left(\mathrm{~d}_{x z} \rightarrow \pi^{*}+\mathrm{d}_{y z}\right)$ \\
& $\mu=0.20$ & 2.57 & $65 \%\left(\pi+\mathrm{d}_{z^{2}} \rightarrow \pi^{*}\right)+30 \%\left(\mathrm{~d}_{x z} \rightarrow \pi^{*}\right)$ \\
& $\mu=0.30$ & 2.76 & $88 \%\left(\pi+\mathrm{d}_{z^{2}} \rightarrow \pi^{*}\right)$ \\
& $\mu=0.40$ & 2.86 & $78 \%\left(\pi+\mathrm{d}_{z^{2}} \rightarrow \pi^{*}\right)$ \\
$\mathrm{S}_{2}$ & $\mu=0.00$ & 2.35 & $86 \%\left(\mathrm{~d}_{y z} \rightarrow \pi^{*}+\mathrm{d}_{y z}\right)$ \\
& $\mu=0.10$ & 2.44 & $87 \%\left(\mathrm{~d}_{y z} \rightarrow \pi^{*}+\mathrm{d}_{y z}\right)$ \\
& $\mu=0.20$ & 2.72 & $65 \%\left(\mathrm{~d}_{x z} \rightarrow \pi^{*}\right)+26 \%\left(\pi+\mathrm{d}_{z^{2}} \rightarrow \pi^{*}\right)$ \\
& $\mu=0.30$ & 3.04 & $22 \%\left(\mathrm{~d}_{y z}+\pi \rightarrow \mathrm{d}_{x y}+\pi^{*}\right)+19 \%\left(\mathrm{~d}_{y z}+\pi \rightarrow \mathrm{d}_{x y}+\pi^{*}\right)+14 \%\left(\mathrm{~d}_{x z} \rightarrow \mathrm{d}_{x y}+\pi^{*}\right)$ \\
& $\mu=0.40$ & 2.94 & $26 \%\left(\mathrm{~d}_{x z} \rightarrow \mathrm{d}_{x y}+\mathrm{n}\right)+23 \%\left(\mathrm{~d}_{y z}+\pi \rightarrow \mathrm{d}_{x y}+\mathrm{n}\right)+15 \%\left(\mathrm{~d}_{x^{2}-y^{2}} \rightarrow \mathrm{d}_{x y}+\mathrm{n}\right)$ \\
$\mathrm{S}_{3}$ & $\mu=0.00$ & 2.48 & $62 \%\left(\mathrm{~d}_{x z} \rightarrow \pi^{*}+\mathrm{d}_{y z}\right)+26 \%\left(\pi+\mathrm{d}_{y z} \rightarrow \pi^{*}+\mathrm{d}_{y z}\right)$ \\
& $\mu=0.10$ & 2.53 & $44 \%\left(\mathrm{~d}_{x z} \rightarrow \pi^{*}+\mathrm{d}_{y z}\right)+43 \%\left(\pi+\mathrm{d}_{z 2} \rightarrow \pi^{*}+\mathrm{d}_{y z}\right)$ \\
& $\mu=0.20$ & 3.76 & $85 \%\left(\mathrm{~d}_{y z}+\pi \rightarrow \pi^{*}\right)$ \\
& $\mu=0.30$ & 3.16 & $27 \%\left(\mathrm{~d}_{x z} \rightarrow \mathrm{d}_{x y}+\pi^{*}\right)+24 \%\left(\mathrm{~d}_{y z}+\pi \rightarrow \mathrm{d}_{z^{2}}\right)+10 \%\left(\mathrm{~d}_{x z} \rightarrow \mathrm{d}_{z^{2}}\right)$ \\
& $\mu=0.40$ & 3.06 & $25 \%\left(\mathrm{~d}_{x z} \rightarrow \mathrm{d}_{x y}+\mathrm{n}\right)+22 \%\left(\mathrm{~d}_{y z}+\pi \rightarrow \mathrm{d}_{x y}+\mathrm{n}\right)+15 \%\left(\mathrm{~d}_{x^{2}-y^{2}} \rightarrow \mathrm{d}_{x y}+\mathrm{n}\right)$ \\
& & & \\
$\mathrm{S}_{4}$ & $\mu=0.00$ & 2.62 & $92 \%\left(\mathrm{~d}_{x^{2}-y^{2}} \rightarrow \pi^{*}+\mathrm{d}_{y z}\right)$ \\
& $\mu=0.10$ & 2.67 & $91 \%\left(\mathrm{~d}_{x^{2}}-y^{2} \rightarrow \pi^{*}+\mathrm{d}_{y z}\right)$ \\
& $\mu=0.20$ & 3.04 & $79 \%\left(\mathrm{~d}_{x^{2}-y^{2}} \rightarrow \pi^{*}\right)+13 \%\left(\mathrm{~d}_{x z} \rightarrow \mathrm{d}_{x y}+\mathrm{n}\right)$ \\
& $\mu=0.30$ & 3.29 & $69 \%\left(\mathrm{~d}_{x^{2}}-y^{2} \rightarrow \mathrm{d}_{x y}+\pi^{*}\right)$ \\
$\mu=0.40$ & 3.18 & $83 \%\left(\mathrm{~d}_{x^{2}-y^{2}} \rightarrow \mathrm{d}_{x y}+\mathrm{n}\right)$ \\
\hline \hline
\end{tabular}


TABLE 3.5. Orbital composition of the $\mathrm{S}_{1}$ excited state of Im-[Co ${ }^{\mathrm{III}}$ (corrin) $]-\mathrm{CH}_{3}^{+}$ resulting from the TD-DFT calculations with different density functionals and basis sets.

\begin{tabular}{|c|c|c|c|c|c|c|}
\hline \multirow{3}{*}{$\begin{array}{l}\text { Functional }^{a} \\
\text { B3LYP }(20 \% \mathrm{HF})\end{array}$} & \multicolumn{3}{|r|}{ 6-31G(d) } & \multicolumn{3}{|c|}{$6-311++G(d, p)$} \\
\hline & $\mathrm{E}(\mathrm{eV})$ & & Character & $\mathrm{E}(\mathrm{eV})$ & & Character \\
\hline & 2.65 & $94 \%$ & $\left(\pi+\mathrm{d}_{z^{2}} \rightarrow \pi^{*}\right)$ & 2.68 & $93 \%$ & $\left(\pi+\mathrm{d}_{z^{2}} \rightarrow \pi^{*}\right)$ \\
\hline CAM-B3LYP $^{b}$ & 2.87 & $\begin{array}{l}22 \% \\
17 \% \\
16 \% \\
15 \%\end{array}$ & $\begin{array}{l}\left(\mathrm{d}_{x z}+\pi \rightarrow \pi^{*}+\mathrm{d}_{x y} / \mathrm{d}_{z^{2}}\right) \\
\left(\pi+\mathrm{d}_{y z} \rightarrow \pi^{*}+\mathrm{d}_{x y} / \mathrm{d}_{z^{2}}\right) \\
\left(\mathrm{d}_{y z}+\pi \rightarrow \pi^{*}+\mathrm{d}_{x y} / \mathrm{d}_{z^{2}}\right) \\
\left(\pi+\mathrm{d}_{z^{2}} \rightarrow \pi^{*}\right)\end{array}$ & & & \\
\hline BLYP & 2.19 & $\begin{array}{l}71 \% \\
27 \%\end{array}$ & $\begin{array}{l}\left(\pi+\mathrm{d}_{x z} \rightarrow \pi^{*}\right) \\
\left(\mathrm{d}_{x z}+\pi \rightarrow \pi^{*}\right)\end{array}$ & 2.26 & $\begin{array}{r}61 \% \\
8 \%\end{array}$ & $\begin{array}{l}\left(\pi+\mathrm{d}_{x z} \rightarrow \pi^{*}\right) \\
\left(\mathrm{d}_{x z}+\pi \rightarrow \pi^{*}\right)\end{array}$ \\
\hline LC-BLYP $(\mu=0.00)$ & 2.20 & $\begin{array}{l}70 \% \\
28 \%\end{array}$ & $\begin{array}{l}\left(\pi+\mathrm{d}_{x z} \rightarrow \pi^{*}+\mathrm{d}_{y z}\right) \\
\left(\mathrm{d}_{x z} \rightarrow \pi^{*}+\mathrm{d}_{y z}\right)\end{array}$ & & & \\
\hline LC-BLYP $(\mu=0.10)$ & 2.27 & $\begin{array}{l}50 \% \\
48 \%\end{array}$ & $\begin{array}{l}\left(\pi+\mathrm{d}_{z^{2}} \rightarrow \pi^{*}+\mathrm{d}_{y z}\right) \\
\left(\mathrm{d}_{x z} \rightarrow \pi^{*}+\mathrm{d}_{y z}\right)\end{array}$ & & & \\
\hline MPW1PW91 (25\% HF) & 2.70 & $95 \%$ & $\left(\pi+\mathrm{d}_{z^{2}} \rightarrow \pi^{*}\right)$ & 2.73 & $94 \%$ & $\left(\pi+\mathrm{d}_{z^{2}} \rightarrow \pi^{*}\right)$ \\
\hline MPWPW91 & 2.19 & $\begin{array}{l}78 \% \\
20 \%\end{array}$ & $\begin{array}{l}\left(\pi+\mathrm{d}_{x z} \rightarrow \pi^{*}\right) \\
\left(\mathrm{d}_{x z}+\pi \rightarrow \pi^{*}\right)\end{array}$ & 2.25 & $\begin{array}{r}69 \% \\
9 \%\end{array}$ & $\begin{array}{l}\left(\pi+\mathrm{d}_{x z} \rightarrow \pi^{*}\right) \\
\left(\mathrm{d}_{x z}+\pi \rightarrow \pi^{*}\right)\end{array}$ \\
\hline TPSSh (10\% HF) & 2.52 & $\begin{array}{r}89 \% \\
9 \%\end{array}$ & $\begin{array}{l}\left(\pi+\mathrm{d}_{z^{2}} \rightarrow \pi^{*}\right) \\
\left(\mathrm{d}_{x z}+\pi \rightarrow \pi^{*}\right)\end{array}$ & 2.55 & $\begin{array}{r}93 \% \\
4 \%\end{array}$ & $\begin{array}{l}\left(\pi+\mathrm{d}_{z^{2}} \rightarrow \pi^{*}\right) \\
\left(\mathrm{d}_{x z}+\pi \rightarrow \pi^{*}\right)\end{array}$ \\
\hline TPSS & 2.29 & $\begin{array}{l}67 \% \\
31 \%\end{array}$ & $\begin{array}{l}\left(\pi+\mathrm{d}_{x z} / \mathrm{d}_{z^{2}} \rightarrow \pi^{*}\right) \\
\left(\mathrm{d}_{x z}+\pi \rightarrow \pi^{*}\right)\end{array}$ & 2.35 & $\begin{array}{l}69 \% \\
26 \%\end{array}$ & $\begin{array}{l}\left(\pi+\mathrm{d}_{x z} / \mathrm{d}_{z^{2}} \rightarrow \pi^{*}\right) \\
\left(\mathrm{d}_{x z}+\pi \rightarrow \pi^{*}\right)\end{array}$ \\
\hline BP86 & 2.18 & $\begin{array}{l}76 \% \\
22 \% \\
\end{array}$ & $\begin{array}{l}\left(\pi+\mathrm{d}_{x z} / \mathrm{d}_{z^{2}} \rightarrow \pi^{*}\right) \\
\left(\mathrm{d}_{x z}+\pi \rightarrow \pi^{*}\right)\end{array}$ & 2.25 & $\begin{array}{l}69 \% \\
29 \%\end{array}$ & $\begin{array}{l}\left(\pi+\mathrm{d}_{x z} / \mathrm{d}_{z^{2}} \rightarrow \pi^{*}\right) \\
\left(\mathrm{d}_{x z}+\pi \rightarrow \pi^{*}\right)\end{array}$ \\
\hline
\end{tabular}

${ }^{a}$ All TD-DFT calculations (apart from LC-BLYP, see below) were performed using the ground-state geometries of $\mathrm{Im}-\left[\mathrm{Co}{ }^{\mathrm{III}}\right.$ (corrin) $]-\mathrm{CH}_{3}^{+}$optimized with the same functional and basis set as applied in the excited-state calculations. LC-BLYP calculations were performed using the ground-state geometry of $\mathrm{Im}-\left[\mathrm{Co}^{\mathrm{III}}\right.$ (corrin) $]-\mathrm{CH}_{3}^{+}$optimized with BP86/6-31G(d).

${ }^{b}$ The $\alpha=0.19, \beta=0.46$, and $\mu=0.33$ parameters, recommended in Ref. 135 and implemented in Gaussian 09, were used in the CAM-B3LYP calculations.

When the physical nature of the $\mathrm{S}_{1}$ state, which the high-level MC-XQDPT2 and EOM-CCSD wave-function methods characterize as a MLCT excitation, is examined with TD-DFT, it becomes clear that the results strongly depend on the applied functional (see Table 3.5). Indeed, while hybrid functionals support the interpretation in which the $S_{1}$ state represents a $\pi \rightarrow \pi^{*}$ transition localized on corrin, pure GGA, meta-GGA, and range-separated LC-BLYP approaches produce the results which are consistent with a MLCT assignment suggested by MC-XQDPT2 and EOM-CCSD. Before discussing the nature of the $S_{1}$ state any further, it is important to emphasize that its character resulting from the TD-DFT calculations is, to a large extent, insensitive to the quality of the basis set. The results based on the smaller 6-31G(d) basis set are virtually identical to those obtained with the larger $6-311++G(d, p)$ basis (see Table 3.5), most likely reflecting on the fact that the $S_{1}$ state of MeCbl is localized within the corrin macrocycle. Two hybrid functionals un- 
der consideration, B3LYP and MPW1PW91, describe the $\mathrm{S}_{1}$ state as a single HOMO $\rightarrow$ LUMO excitation (see Tables 3.5 and 3.6). The orbital composition of this excitation indicates that contributions from the Co d orbitals are relatively small and that the $S_{0} \rightarrow S_{1}$ transition predicted by hybrid functionals can be characterized as belonging to the corrin $\pi \rightarrow \pi^{*}$ type (see the Appendix Tables A.6 and A.11 for MOs decomposition). Although similar remarks apply to the TPSSh functional, which describes the $S_{1}$ state as the predominantly $\pi / d_{z^{2}} \rightarrow \pi^{*}$ transition, one can also see additional contributions involving the excitations from HOMO-2, in which the electron density is largely localized on the Co d orbitals, with some admixture of the corrin's $\pi$ orbitals (see Table A.8 in the Appendix).

The results based on the pure GGA-type functionals (BLYP, MPWPW91, TPSS, and BP86) were found to be very similar in terms of both the energy and the nature of the electronic excitation (Table 3.5). All four pure functionals used in this study predict significant $\pi / \mathrm{d} \rightarrow \pi^{*}$ (MLCT-like) contributions to the $\mathrm{S}_{1}$ state of MeCbl. In contrast to hybrid functionals, the $\mathrm{S}_{0} \rightarrow \mathrm{S}_{1}$ transitions resulting from the GGA-type calculations have a more complex character and originate from HOMO- $1 \rightarrow$ LUMO and HOMO-2 $\rightarrow$ LUMO excitations. The composition of frontier MOs involved in these electronic excitations indicates larger participation of the $d$ orbitals of Co (see Tables A.5, A.7, A.9, and A.10 in the Appendix). This observation is consistent with the EOM-CCSD calculations, where the $\mathrm{S}_{1}$ state is dominated by a $\pi / \sigma\left(\mathrm{d}_{z^{2}}\right) \rightarrow \pi^{*}$ transition, with a small admixture of other excitations, particularly those originating from the transitions from the occupied Co $\mathrm{d}$ and corrin $\pi$ orbitals to the unoccupied $\pi^{*}$ orbital of the corrin macrocycle (cf. Table 3.3). In the MC-XQDPT2 calculations, the same state appears as a predominantly $\mathrm{d} \rightarrow \pi^{*}$ excitation, with the corrin $\pi$ occupied orbitals also participating in the transition (see Table 3.2). Based on the distribution of the electron density among the Co d orbitals, it can be concluded that the $\mathrm{d}_{z^{2}}$ orbital is not the only one that is required for a proper description of the $S_{1}$ state. 
TABLE 3.6. Electronic transitions of $\mathrm{Im}-\left[\mathrm{Co}{ }^{\mathrm{III}}(\mathrm{corrin})\right]-\mathrm{CH}_{3}^{+}$calculated with B3LYP/6-31G(d).

\begin{tabular}{|c|c|c|c|c|c|c|c|c|c|c|}
\hline \multirow{2}{*}{$\begin{array}{l}\text { State } \\
S_{1}\end{array}$} & \multirow{2}{*}{$\begin{array}{c}\mathrm{E}(\mathrm{eV}) \\
2.65\end{array}$} & \multirow{2}{*}{$\begin{array}{c}\lambda(\mathrm{nm}) \\
468.5\end{array}$} & \multirow{2}{*}{$\begin{array}{c}f \\
0.0643\end{array}$} & \multirow{2}{*}{$\begin{array}{r}\% \\
94\end{array}$} & \multicolumn{6}{|c|}{ Character } \\
\hline & & & & & 117 & $\rightarrow$ & 118 & $\mathrm{H}$ & $\rightarrow$ & $\mathrm{L}$ \\
\hline \multirow[t]{10}{*}{$\mathrm{S}_{2}$} & 2.82 & 439.1 & 0.0024 & 19 & 115 & $\rightarrow$ & 121 & $\mathrm{H}-2$ & $\rightarrow$ & $\mathrm{L}+3$ \\
\hline & & & & 17 & 116 & $\rightarrow$ & 120 & $\mathrm{H}-1$ & $\rightarrow$ & $\mathrm{L}+2$ \\
\hline & & & & 16 & 116 & $\rightarrow$ & 121 & $\mathrm{H}-1$ & $\rightarrow$ & $\mathrm{L}+3$ \\
\hline & & & & 14 & 115 & $\rightarrow$ & 120 & $\mathrm{H}-2$ & $\rightarrow$ & $\mathrm{L}+2$ \\
\hline & & & & 6 & 113 & $\rightarrow$ & 121 & $\mathrm{H}-4$ & $\rightarrow$ & $\mathrm{L}+3$ \\
\hline & & & & 5 & 113 & $\rightarrow$ & 120 & $\mathrm{H}-4$ & $\rightarrow$ & $\mathrm{L}+2$ \\
\hline & & & & 4 & 111 & $\rightarrow$ & 120 & $\mathrm{H}-6$ & $\rightarrow$ & $\mathrm{L}+2$ \\
\hline & & & & 3 & 111 & $\rightarrow$ & 121 & $\mathrm{H}-6$ & $\rightarrow$ & $\mathrm{L}+3$ \\
\hline & & & & 2 & 115 & $\rightarrow$ & 122 & $\mathrm{H}-2$ & $\rightarrow$ & $\mathrm{L}+4$ \\
\hline & & & & 2 & 116 & $\rightarrow$ & 119 & $\mathrm{H}-1$ & $\rightarrow$ & $\mathrm{L}+1$ \\
\hline \multirow[t]{9}{*}{$\mathrm{S}_{3}$} & 2.95 & 420.5 & 0.0009 & 23 & 116 & $\rightarrow$ & 122 & $\mathrm{H}-1$ & $\rightarrow$ & $\mathrm{L}+4$ \\
\hline & & & & 17 & 115 & $\rightarrow$ & 122 & $\mathrm{H}-2$ & $\rightarrow$ & $\mathrm{L}+4$ \\
\hline & & & & 12 & 111 & $\rightarrow$ & 120 & H-6 & $\rightarrow$ & $\mathrm{L}+2$ \\
\hline & & & & 12 & 111 & $\rightarrow$ & 122 & $\mathrm{H}-6$ & $\rightarrow$ & $\mathrm{L}+4$ \\
\hline & & & & 11 & 115 & $\rightarrow$ & 120 & $\mathrm{H}-2$ & $\rightarrow$ & $\mathrm{L}+2$ \\
\hline & & & & 7 & 116 & $\rightarrow$ & 120 & $\mathrm{H}-1$ & $\rightarrow$ & $\mathrm{L}+2$ \\
\hline & & & & 4 & 113 & $\rightarrow$ & 122 & $\mathrm{H}-4$ & $\rightarrow$ & $\mathrm{L}+4$ \\
\hline & & & & 3 & 113 & $\rightarrow$ & 121 & $\mathrm{H}-4$ & $\rightarrow$ & $\mathrm{L}+3$ \\
\hline & & & & 2 & 111 & $\rightarrow$ & 121 & H-6 & $\rightarrow$ & $L+3$ \\
\hline \multirow[t]{8}{*}{$\mathrm{S}_{4}$} & 3.02 & 411.2 & 0.0008 & 25 & 113 & $\rightarrow$ & 120 & $\mathrm{H}-4$ & $\rightarrow$ & $\mathrm{L}+2$ \\
\hline & & & & 20 & 111 & $\rightarrow$ & 120 & H-6 & $\rightarrow$ & $\mathrm{L}+2$ \\
\hline & & & & 15 & 113 & $\rightarrow$ & 122 & $\mathrm{H}-4$ & $\rightarrow$ & $L+4$ \\
\hline & & & & 10 & 111 & $\rightarrow$ & 121 & H-6 & $\rightarrow$ & $\mathrm{L}+3$ \\
\hline & & & & 7 & 115 & $\rightarrow$ & 122 & $\mathrm{H}-2$ & $\rightarrow$ & $\mathrm{L}+4$ \\
\hline & & & & 7 & 113 & $\rightarrow$ & 121 & $\mathrm{H}-4$ & $\rightarrow$ & $L+3$ \\
\hline & & & & 4 & 116 & $\rightarrow$ & 122 & $\mathrm{H}-1$ & $\rightarrow$ & $\mathrm{L}+4$ \\
\hline & & & & 3 & 111 & $\rightarrow$ & 122 & $\mathrm{H}-6$ & $\rightarrow$ & $\mathrm{L}+4$ \\
\hline \multirow{3}{*}{$\mathrm{S}_{5}$} & 3.15 & 394.2 & 0.0446 & 80 & 116 & $\rightarrow$ & 118 & $\mathrm{H}-1$ & $\rightarrow$ & $\mathrm{L}$ \\
\hline & & & & 12 & 117 & $\rightarrow$ & 119 & $\mathrm{H}$ & $\rightarrow$ & $\mathrm{L}+1$ \\
\hline & & & & 2 & 113 & $\rightarrow$ & 118 & $\mathrm{H}-4$ & $\rightarrow$ & $\mathrm{L}$ \\
\hline $\mathrm{S}_{6}$ & 3.30 & 376.1 & 0.0307 & 89 & 115 & $\rightarrow$ & 118 & $\mathrm{H}-2$ & $\rightarrow$ & $\mathrm{L}$ \\
\hline & & & & 3 & 116 & $\rightarrow$ & 119 & $\mathrm{H}-1$ & $\rightarrow$ & $\mathrm{L}+1$ \\
\hline $\mathrm{S}_{7}$ & 3.51 & 353.1 & 0.0067 & 18 & 115 & $\rightarrow$ & 120 & $\mathrm{H}-2$ & $\rightarrow$ & $\mathrm{L}+2$ \\
\hline & & & & 18 & 115 & $\rightarrow$ & 121 & $\mathrm{H}-2$ & $\rightarrow$ & $\mathrm{L}+3$ \\
\hline & & & & 12 & 116 & $\rightarrow$ & 121 & $\mathrm{H}-1$ & $\rightarrow$ & $L+3$ \\
\hline & & & & 6 & 116 & $\rightarrow$ & 122 & $\mathrm{H}-1$ & $\rightarrow$ & $\mathrm{L}+4$ \\
\hline & & & & 6 & 116 & $\rightarrow$ & 119 & $\mathrm{H}-1$ & $\rightarrow$ & $\mathrm{L}+1$ \\
\hline & & & & 6 & 116 & $\rightarrow$ & 120 & $\mathrm{H}-1$ & $\rightarrow$ & $\mathrm{L}+2$ \\
\hline & & & & 5 & 113 & $\rightarrow$ & 121 & $\mathrm{H}-4$ & $\rightarrow$ & $\mathrm{L}+3$ \\
\hline & & & & 3 & 113 & $\rightarrow$ & 120 & $\mathrm{H}-4$ & $\rightarrow$ & $\mathrm{L}+2$ \\
\hline & & & & 3 & 111 & $\rightarrow$ & 118 & H-6 & $\rightarrow$ & $\mathrm{L}$ \\
\hline & & & & 3 & 115 & $\rightarrow$ & 119 & $\mathrm{H}-2$ & $\rightarrow$ & $\mathrm{L}+1$ \\
\hline & & & & 2 & 113 & $\rightarrow$ & 118 & $\mathrm{H}-4$ & $\rightarrow$ & $\mathrm{L}$ \\
\hline $\mathrm{S}_{8}$ & 3.65 & 340.0 & 0.0042 & 41 & 115 & $\rightarrow$ & 122 & $\mathrm{H}-2$ & $\rightarrow$ & $L+4$ \\
\hline & & & & 16 & 116 & $\rightarrow$ & 122 & $\mathrm{H}-1$ & $\rightarrow$ & $\mathrm{L}+4$ \\
\hline & & & & 7 & 116 & $\rightarrow$ & 120 & $\mathrm{H}-1$ & $\rightarrow$ & $\mathrm{L}+2$ \\
\hline & & & & 7 & 113 & $\rightarrow$ & 118 & $\mathrm{H}-4$ & $\rightarrow$ & $\mathrm{L}$ \\
\hline & & & & 4 & 113 & $\rightarrow$ & 122 & $\mathrm{H}-4$ & $\rightarrow$ & $\mathrm{L}+4$ \\
\hline & & & & 4 & 115 & $\rightarrow$ & 121 & $\mathrm{H}-2$ & $\rightarrow$ & $\mathrm{L}+3$ \\
\hline & & & & 3 & 115 & $\rightarrow$ & 120 & $\mathrm{H}-2$ & $\rightarrow$ & $\mathrm{L}+2$ \\
\hline & & & & 3 & 110 & $\rightarrow$ & 122 & $\mathrm{H}-7$ & $\rightarrow$ & $\mathrm{L}+4$ \\
\hline & & & & 2 & 113 & $\rightarrow$ & 120 & $\mathrm{H}-4$ & $\rightarrow$ & $\mathrm{L}+2$ \\
\hline $\mathrm{S}_{9}$ & 3.72 & 333.4 & 0.0992 & 60 & 117 & $\rightarrow$ & 119 & $\mathrm{H}$ & $\rightarrow$ & $\mathrm{L}+1$ \\
\hline & & & & 11 & 117 & $\rightarrow$ & 120 & $\mathrm{H}$ & $\rightarrow$ & $\mathrm{L}+2$ \\
\hline & & & & 5 & 116 & $\rightarrow$ & 118 & $\mathrm{H}-1$ & $\rightarrow$ & $\mathrm{L}$ \\
\hline & & & & 4 & 117 & $\rightarrow$ & 122 & $\mathrm{H}$ & $\rightarrow$ & $\mathrm{L}+4$ \\
\hline & & & & 3 & 116 & $\rightarrow$ & 119 & $\mathrm{H}-1$ & $\rightarrow$ & $\mathrm{L}+1$ \\
\hline & & & & 3 & 111 & $\rightarrow$ & 118 & $\mathrm{H}-6$ & $\rightarrow$ & $\mathrm{L}$ \\
\hline & & & & 3 & 117 & $\rightarrow$ & 121 & $\mathrm{H}$ & $\rightarrow$ & $\mathrm{L}+3$ \\
\hline $\mathrm{S}_{10}$ & 3.84 & 323.2 & 0.0207 & 23 & 117 & $\rightarrow$ & 120 & $\mathrm{H}$ & $\rightarrow$ & $\mathrm{L}+2$ \\
\hline & & & & 23 & 116 & $\rightarrow$ & 119 & $\mathrm{H}-1$ & $\rightarrow$ & $\mathrm{L}+1$ \\
\hline & & & & 16 & 113 & $\rightarrow$ & 118 & $\mathrm{H}-4$ & $\rightarrow$ & L \\
\hline & & & & 13 & 117 & $\rightarrow$ & 119 & $\mathrm{H}$ & $\rightarrow$ & $\mathrm{L}+1$ \\
\hline & & & & 6 & 117 & $\rightarrow$ & 121 & $\mathrm{H}$ & $\rightarrow$ & $L+3$ \\
\hline & & & & 3 & 112 & $\rightarrow$ & 118 & $\mathrm{H}-5$ & $\rightarrow$ & L \\
\hline & & & & 2 & 116 & $\rightarrow$ & 121 & $\mathrm{H}-1$ & $\rightarrow$ & $\mathrm{L}+3$ \\
\hline
\end{tabular}


According to both types of the high-level $a b$ initio wave-function calculations performed in this study, the $S_{0} \rightarrow S_{1}$ electronic transition involves the Co d orbitals along the axis connecting the two axial ligands of MeCbl. Such a contribution (i.e., $\mathrm{d}_{x z}+\pi \rightarrow \pi^{*}$ ) is also observed when the results obtained with the pure GGA-type (BLYP, MPWPW91, TPSS, and BP86) functionals are examined. Similarly, when the $\mathrm{S}_{0} \rightarrow \mathrm{S}_{1}$ excitation is examined using the LC-BLYP approaches, the $\mathrm{d}_{z^{2}}$ and $\mathrm{d}_{x z}$ occupied orbitals contribute when $\mu=0.10$ and 0.20 , but when $\mu$ is set at zero, the $\mathrm{d}_{z^{2}}$ orbital does not participate in it. Interestingly, the CAM-B3LYP functional introduces significant contributions from the Co d orbitals not only to the occupied but also to the unoccupied MOs participating in the $S_{0} \rightarrow S_{1}$ excitation. This leads to a mixed $\mathrm{d} / \pi \rightarrow \pi^{*} / \mathrm{d}$ character of the $\mathrm{S}_{1}$ state. Since the EOM-CCSD calculations clearly indicate that the unoccupied orbitals participating in the lowest excited state of MeCbl are largely corrin-localized (i.e., of the $\pi^{*}$ type) and since according to the MC-XQDPT2 calculations the contributions from the $\pi^{*}$ orbitals localized on corrin to the unoccupied MOs involved in the $\mathrm{S}_{0} \rightarrow \mathrm{S}_{1}$ transition are larger than the contributions from the Co d orbitals, the results obtained with the CAM-B3LYP functional disagree with both EOM-CCSD and MC-XQDPT2. They are not only inconsistent with a MLCT assignment suggested by the EOM-CCSD and MC-XQDPT2 calculations, but also with a traditional $\pi \rightarrow \pi^{*}$ description of the $S_{0} \rightarrow S_{1}$ excitation.

TABLE 3.7. The first three excited states of $\mathrm{Im}-\left[\mathrm{Co}^{\mathrm{III}}\right.$ (corrin) $]-\mathrm{CH}_{3}^{+}$calculated with BP86/6-311++G(d,p).

\begin{tabular}{ccccrclc}
\hline \hline State & $\mathrm{E}(\mathrm{eV})$ & $\lambda(\mathrm{nm})$ & $f$ & Contribution & Transition \\
\hline $\mathrm{S}_{1}$ & 2.25 & 551.4 & 0.0035 & -0.380 & $115 \rightarrow 118$ & $29 \%\left(\mathrm{~d}_{x z}+\pi \rightarrow \pi^{*}\right)$ & Exp (eV) \\
& & & & 0.589 & $116 \rightarrow 118$ & $69 \%\left(\pi+\mathrm{d}_{x z} / \mathrm{d}_{z^{2}} \rightarrow \pi^{*}\right)$ & 2.22 \\
$\mathrm{~S}_{2}$ & 2.38 & \multirow{2}{*}{521.4} & 0.0271 & 0.150 & $116 \rightarrow 119$ & $5 \%\left(\pi+\mathrm{d}_{x z} / \mathrm{d}_{z^{2}} \rightarrow \mathrm{d}_{x y}+\mathrm{n}+\pi^{*}\right)$ & 2.35 \\
& & & & -0.153 & $116 \rightarrow 120$ & $5 \%\left(\pi+\mathrm{d}_{x z} / \mathrm{d}_{z^{2}} \rightarrow \pi^{*}+\mathrm{d}_{x y}\right)$ & \\
& & & 0.660 & $117 \rightarrow 118$ & $87 \%\left(\mathrm{~d}_{y z}+\pi \rightarrow \pi^{*}\right)$ & 2.55 \\
$\mathrm{~S}_{3}$ & \multirow{2}{*}{496.6} & \multirow{2}{*}{0.0565} & 0.254 & $114 \rightarrow 118$ & $13 \%\left(\mathrm{~d}_{x^{2}}-y^{2} \rightarrow \pi^{*}\right)$ & \\
& & & & 0.532 & $115 \rightarrow 118$ & $57 \%\left(\mathrm{~d}_{x z}+\pi \rightarrow \pi^{*}\right)$ & \\
& & & & 0.343 & $116 \rightarrow 118$ & $24 \%\left(\pi+\mathrm{d}_{x z} / \mathrm{d}_{z^{2}} \rightarrow \pi^{*}\right)$ & \\
& & 0.116 & $117 \rightarrow 120$ & $3 \%\left(\mathrm{~d}_{y z}+\pi \rightarrow \pi^{*}+\mathrm{d}_{x y}\right)$ & \\
\hline \hline
\end{tabular}

${ }^{a}$ The isosurface plots of the corresponding MOs are shown in Figure 3.5.

${ }^{b}$ The natural transition orbitals (NTOs) corresponding to the presented electronic transitions are shown in Figure 3.6.

${ }^{c}$ The experimental excitation-energy values reported in Ref. 108 are 17870,18950 , and $20550 \mathrm{~cm}^{-1}$, respectively. 


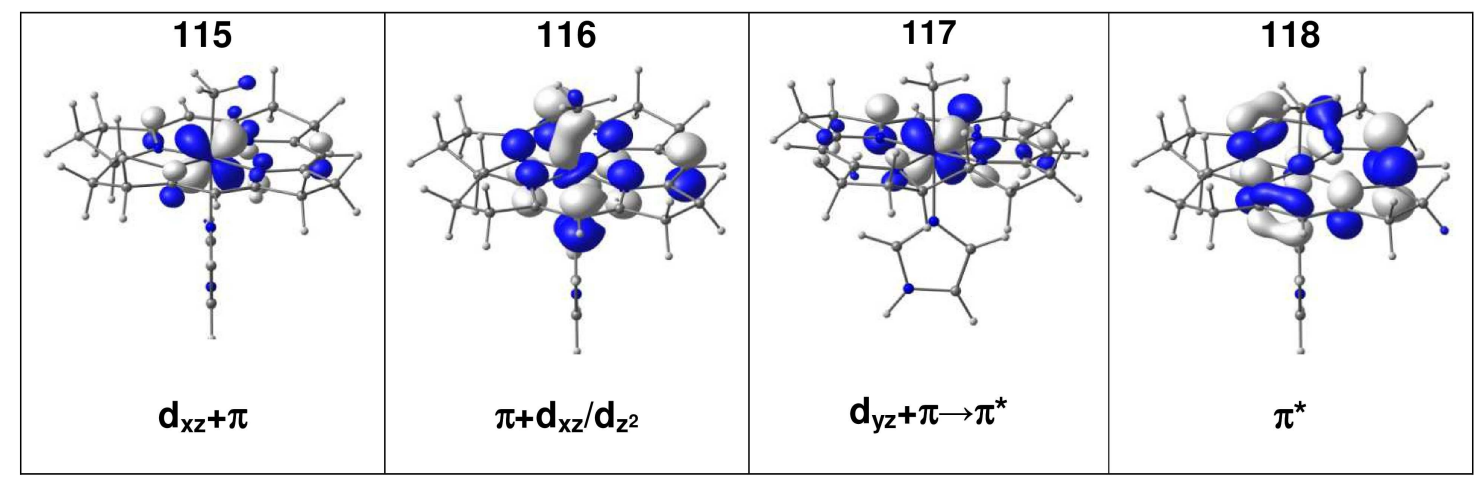

Figure 3.5. Molecular orbitals obtained with BP86/6-311++G(d,p).

The reliability of the MLCT assignment provided by the BP86 and other pure GGA functionals can be further confirmed by considering the nature of the three lowest-energy excited states of MeCbl, as shown in Table 3.7. According to the BP86 calculations and independently of the basis set employed in these calculations, $\mathrm{S}_{1}$, $\mathrm{S}_{2}$, and $\mathrm{S}_{3}$ are close lying states and the $\mathrm{S}_{0} \rightarrow \mathrm{S}_{1}$ and $\mathrm{S}_{0} \rightarrow \mathrm{S}_{3}$ transitions involve the same subsets of orbitals. Although the relative contributions from various orbital excitations vary from state to state, both $S_{0} \rightarrow S_{1}$ and $S_{0} \rightarrow S_{3}$ can be characterized as the $\mathrm{d} / \pi \rightarrow \pi^{*}$ transitions. A very similar description applies to the $\mathrm{S}_{2}$ state, which possesses an even larger contribution from the d orbitals of Co. All of this allows us to conclude that not only $S_{1}$ but also the next two electronic excitations in MeCbl, which have larger intensities than $S_{1}$, share the MLCT character. The MLCT nature of the $S_{1}, S_{2}$, and $S_{3}$ states resulting from the use of BP86 and other pure functionals is in a good agreement with the MC-XQDPT2 calculations and there also is a great degree of consistency in the characterization of the $S_{1}$ state as a MLCT transition by pure GGAs and EOM-CCSD. It is, therefore, quite certain that the lowest-energy excitations in MeCbl are the MLCT transitions.

Additional insights into the nature of the three lowest-energy excited states of MeCbl can be obtained through the use of the natural transition orbitals (NTOs) of Martin, ${ }^{205}$ which are shown for the Im-[Co ${ }^{\mathrm{III}}$ (corrin) $]-\mathrm{CH}_{3}^{+}$model of MeCbl in Figure 3.6. According to the NTO analysis, the $S_{1}$ state can be described as an out-of-phase $d_{z^{2}}-d_{x z}$ combination of the Co $d$ orbitals, whereas $S_{3}$ is an in-phase 


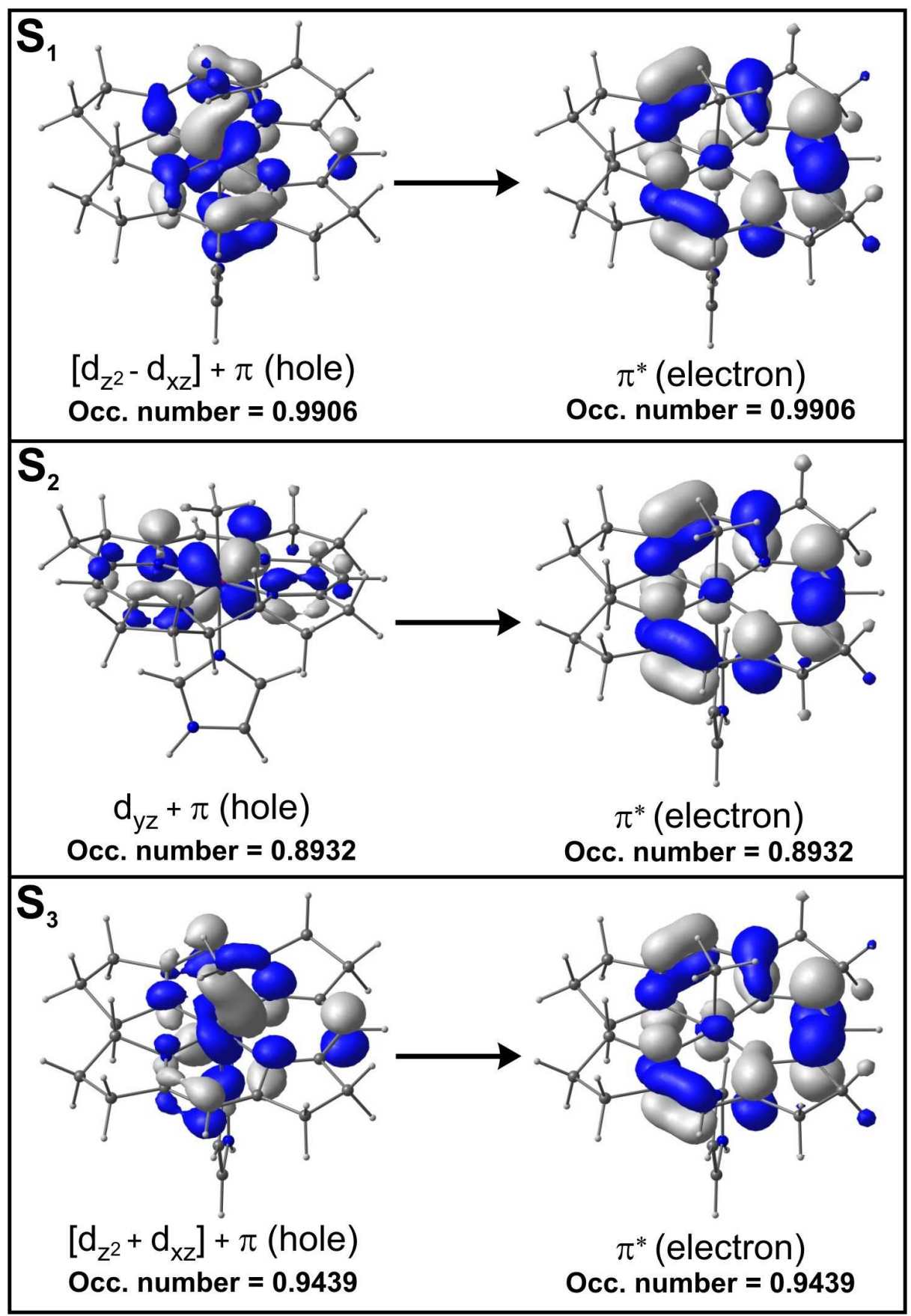

Figure 3.6. Natural transition orbitals (NTOs) describing the three lowest singlet excited states for the Im- $\left[\mathrm{Co}{ }^{\mathrm{III}}\right.$ (corrin) $]-\mathrm{CH}_{3}^{+}$model complex. Results obtained from the TD-DFT/BP86/6-311++G(d,p) calculations. 
$\mathrm{d}_{z^{2}}+\mathrm{d}_{x z}$ combination. This explains why the intensity of the $\mathrm{S}_{1}$ state is much lower in comparison to $\mathrm{S}_{3}$; the transition dipole moments cancel out in the former case and add up in the case of the latter excitation. The nature of NTOs shown in Figure 3.6 also reveals that none of the low-lying excitations in MeCbl can be classified as pure MLCT or pure $\pi \rightarrow \pi^{*}$ transitions. These transitions always appear together and, due to the low symmetry of the corrin ligand, they cannot be completely separated. Thus, to understand the relative magnitudes of the MLCT and $\pi \rightarrow \pi^{*}$ contributions to the low-lying excited states of MeCbl, we calculated the electron density differences between $S_{0}$ and $S_{1}, S_{0}$ and $S_{2}$, and $S_{0}$ and $S_{3}$ using the BP86/6-311++G(d,p) approach (see Figure 3.7). A direct inspection of these electron density differences corroborates our main conclusion that the $\mathrm{S}_{1}$ state of $\mathrm{MeCbl}$ has indeed a MLCT character. There is a visible outflow of the electron density from the Co atom and along the $\mathrm{N}_{\mathrm{Im}}-\mathrm{Co}-\mathrm{C}_{\mathrm{Me}}$ axis and there also is a noticeable electron density inflow toward the corrin ring. Similar decreases of the electron density on Co with concurrent density increases on corrin are also observed for the $S_{0} \rightarrow S_{2}$ and $\mathrm{S}_{0} \rightarrow \mathrm{S}_{3}$ transitions (see Figure 3.7), confirming the $\mathrm{d} / \pi \rightarrow \pi^{*}$ nature of the low-lying excited states of MeCbl postulated in this chapter.

\subsubsection{Adiabatic nature of the $S_{1}$ transition}

Up to this point, we have mainly focused on comparing the results obtained with the two high-level $a b$ initio wave-function methods with those obtained with TD-DFT in order to understand the nature of the low-lying excited states of the $\mathrm{Im}$ - $\left[\mathrm{Co}^{\mathrm{III}}\right.$ (corrin) $]-\mathrm{CH}_{3}^{+}$complex. Relying on the overall agreement between the MC-XQDPT2 and EOM-CCSD results and based on the reasonable agreement between the TD-DFT calculations using pure GGAs and the high-level $a b$ initio data, the lowest-energy $S_{1}$ state has been characterized as a MLCT-type transition. The MC-XQDPT2 and EOM-CCSD $S_{0} \rightarrow S_{1}$ excitation energies of 2.48 and $2.65 \mathrm{eV}$, respectively, are in good agreement with the experimental value of $2.22 \mathrm{eV}$ (see Table 3.1). The non-hybrid functionals, including BLYP, MPWPW91, TPSS, BP86, and LC-BLYP ( $\mu=0.00$ or 0.10 ), provide a description which is consistent with the $\mathrm{S}_{1}$ 


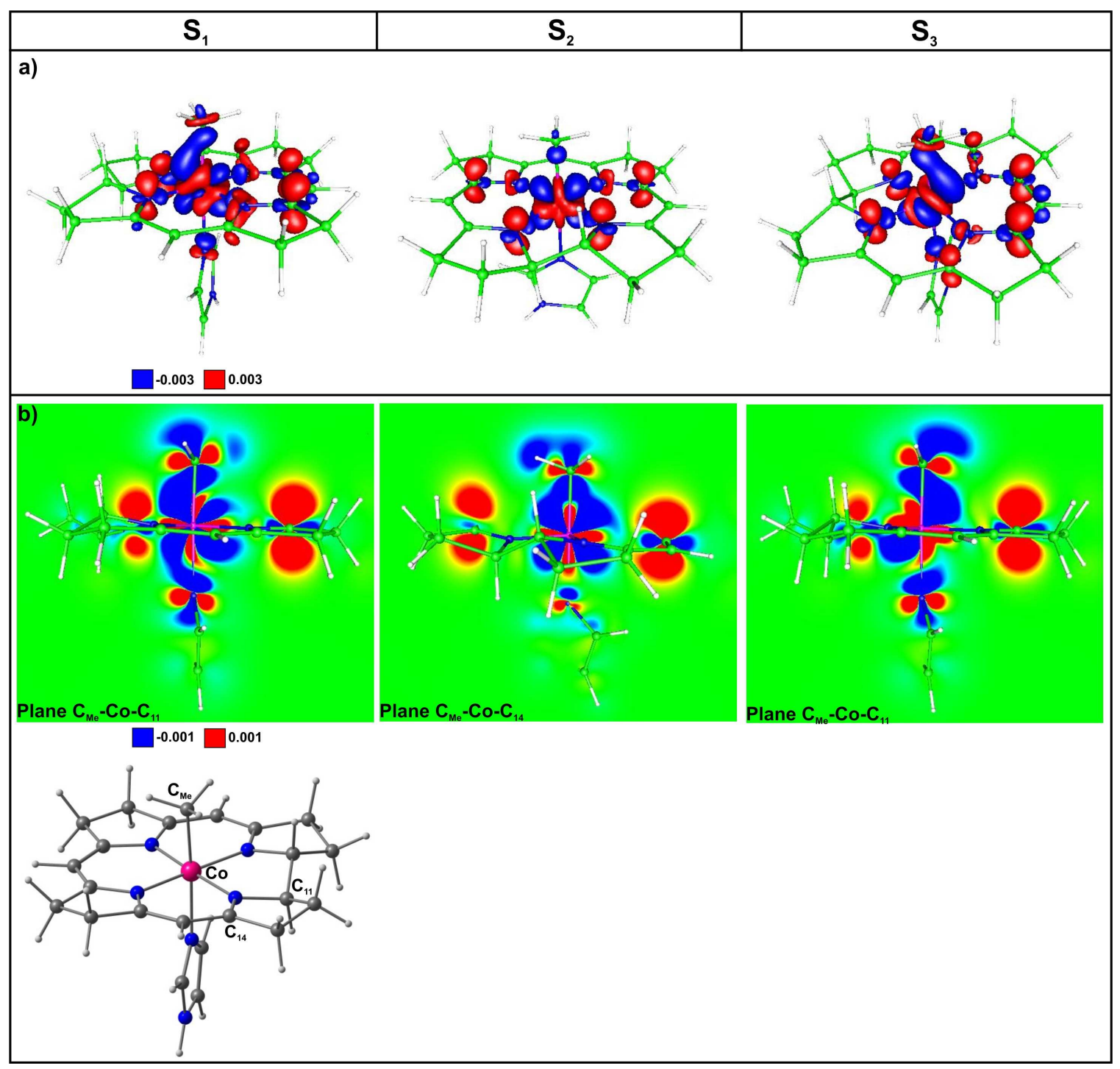

Figure 3.7. Electron density differences between the $S_{0}$ and $S_{1}, S_{0}$ and $S_{2}$, and $\mathrm{S}_{0}$ and $\mathrm{S}_{3}$ states for the Im-[CoIII (corrin)]- $\mathrm{CH}_{3}^{+}$model complex: (a) isosurface plot value of 0.003 and (b) cross-section contour plot along the axial bonding. Results obtained from the TD-DFT/BP86/6-311++G(d,p) calculations. 
state being a MLCT transition. The corresponding excitation energies of 2.19, 2.19, $2.29,2.18$, and 2.20 or $2.27 \mathrm{eV}$, respectively, obtained with the $6-31 \mathrm{G}(\mathrm{d})$ basis set are remarkably close to the experimental value of $2.22 \mathrm{eV}$ (see Table 3.1), and the use of larger basis sets, such as $6-311 \mathrm{G}(\mathrm{d}, \mathrm{p})$ and $6-311++\mathrm{G}(\mathrm{d}, \mathrm{p})$, has virtually no effect on these findings (cf. Tables 3.7, A.12, and A.13 in the Appendix). Based on the energetics and the physical nature of the electronic transitions in MeCbl obtained with different methods, as discussed above, the present study supports the MLCT interpretation of the lowest $\mathrm{S}_{1}$ state rather than the traditional interpretation of this state as a $\pi \rightarrow \pi^{*}$ transition localized on corrin.

To provide a more complete understanding of the $S_{0} \rightarrow S_{1}$ transition, we also examined the mechanism governing this transition. In the lowest-order description, the Abs, $\mathrm{CD}$, and MCD spectroscopies are guided by the Franck-Condon (FC) principle, which assumes that the nuclear configuration does not change upon electronic excitation. In contrast, TAS explores the photoproducts at different time delays. The absorbance at a particular wavelength (or range of wavelengths) is measured as a function of time after excitation by a flash of light. Thus, in examining the results of TAS experiments, it is more appropriate to discuss the adiabatic nature of the $\mathrm{S}_{0} \rightarrow \mathrm{S}_{1}$ transition, focusing on the electronic properties of MeCbl in terms of the photo-induced geometrical changes.

Since one can expect that the most significant changes in the electronic structure of the $\mathrm{S}_{1}$ state of $\mathrm{MeCbl}$ are associated with the photo-induced geometrical changes in the axial ligands, ${ }^{119}$ the additional calculations evaluating the energy changes in response to the variations of the $\mathrm{Co}-\mathrm{C}_{\mathrm{Me}}$ and $\mathrm{Co}-\mathrm{N}_{\mathrm{Im}}$ distances were performed. For each point on the two-dimensional potential energy surface (PES) cut defined by fixed $\mathrm{Co}-\mathrm{C}_{\mathrm{Me}}$ and $\mathrm{Co}-\mathrm{N}_{\mathrm{Im}}$ distances, the ground-state structure of $\mathrm{Im}-\left[\mathrm{Co}^{\mathrm{III}}\right.$ (corrin) $]-\mathrm{CH}_{3}^{+}$was re-optimized. The $\mathrm{Co}-\mathrm{C}_{\mathrm{Me}}$ and $\mathrm{Co}-\mathrm{N}_{\mathrm{Im}}$ distances were varied between 1.9 to $2.6 \AA$, with a step of $0.1 \AA$, in the former case and from 2.0 to $2.6 \AA$, with a step of $0.1 \AA$ as well, in the latter case, allowing for the full relaxation of remaining geometrical parameters. Taking into account the fact that the character of the $S_{1}$ state is not significantly affected by the basis set, only the smaller 

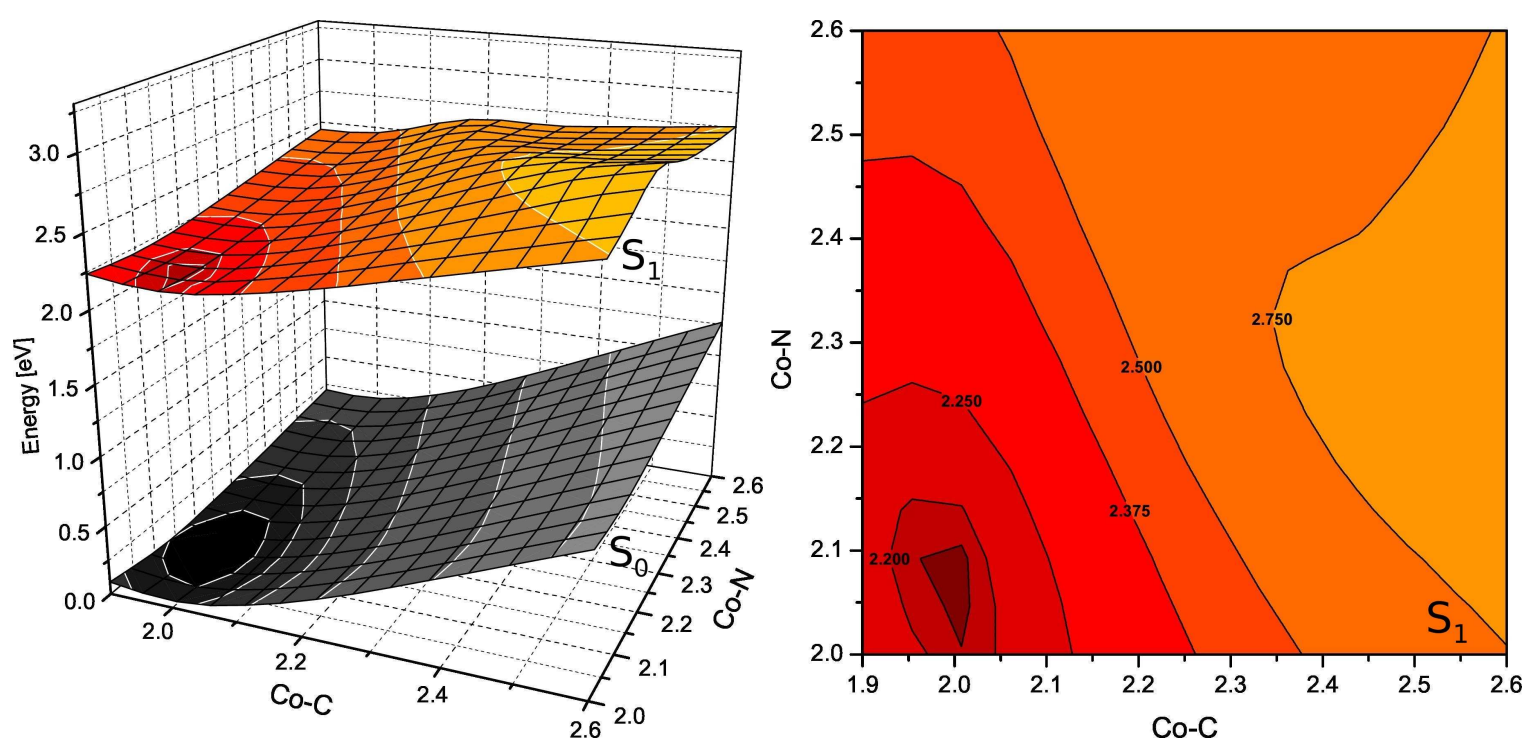

Figure 3.8. Left panel - potential energy surfaces of the $S_{0}$ and $S_{1}$ states resulting from the BP86 and TD-DFT/BP86/6-31G(d) calculations plotted as a function of axial bond lengths (in $\AA$ ). Right panel - vertical projection of the potential energy surface of the $S_{1}$ state.

6-31G(d) basis set was used in these calculations. The two-dimensional PES cuts of the $\mathrm{S}_{0}$ and $\mathrm{S}_{1}$ states along the $\mathrm{Co}-\mathrm{C}_{\mathrm{Me}}$ and $\mathrm{Co}-\mathrm{N}_{\mathrm{Im}}$ coordinates, resulting from the BP86/6-31G(d) and subsequent TD-DFT/BP86/6-31G(d) calculations, are shown in Figure 3.8. Considering the fact that upon the electronic excitation the geometry of MeCbl might relax to another stable structure (as in the case of $\mathrm{CNCbl}^{206}$ ), the presence of additional minima on the PES of the $S_{1}$ state might lead to questions about the characterization of the $S_{0} \rightarrow S_{1}$ transition as a MLCT excitation based on the vertical excitations only. However, careful inspection of Figure 3.8 shows that there is only one minimum on the PES of the $S_{1}$ state. Compared to the groundstate equilibrium geometry of the $\mathrm{Im}-\left[\mathrm{Co}{ }^{\mathrm{III}}\right.$ (corrin) $]-\mathrm{CH}_{3}^{+}$system, the minimum on the $\mathrm{S}_{1}$ PES is only slightly shifted toward shorter Co- $\mathrm{N}_{\mathrm{Im}}$ distances (from $2.15 \AA$ in the case of $\mathrm{S}_{0}$ state to about $2.05 \AA$ in the $\mathrm{S}_{1}$ case) and almost unchanged with respect to the Co- $\mathrm{C}_{\mathrm{Me}}$ bond-length (which is $1.98 \AA$ for the $\mathrm{S}_{0}$ state and $2.00 \AA$ for the $S_{1}$ state). 


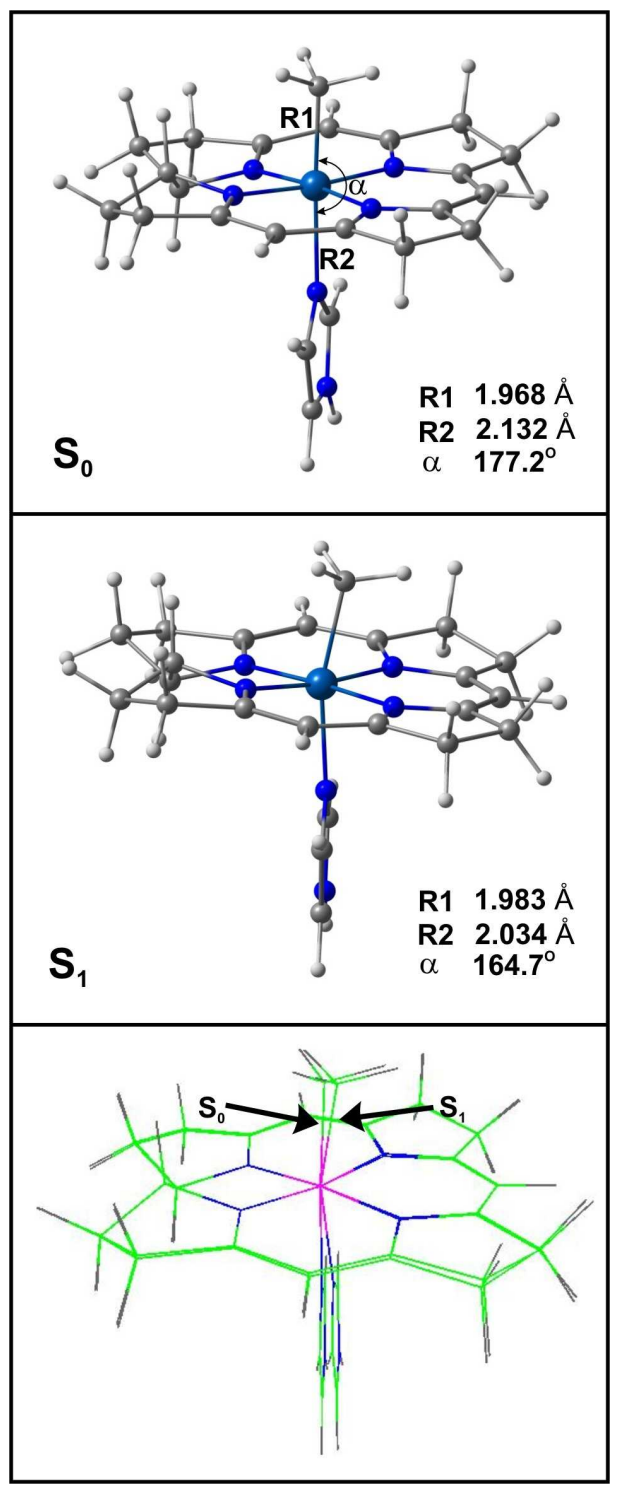

Figure 3.9. A comparison of the BP86/6-31G(d)-optimized geometries of the ground (top) and first excited (middle) state of $\mathrm{Im}-\left[\mathrm{Co}{ }^{\mathrm{III}}\right.$ (corrin) $]-\mathrm{CH}_{3}^{+}$, along with the alignment of their structures (bottom). 


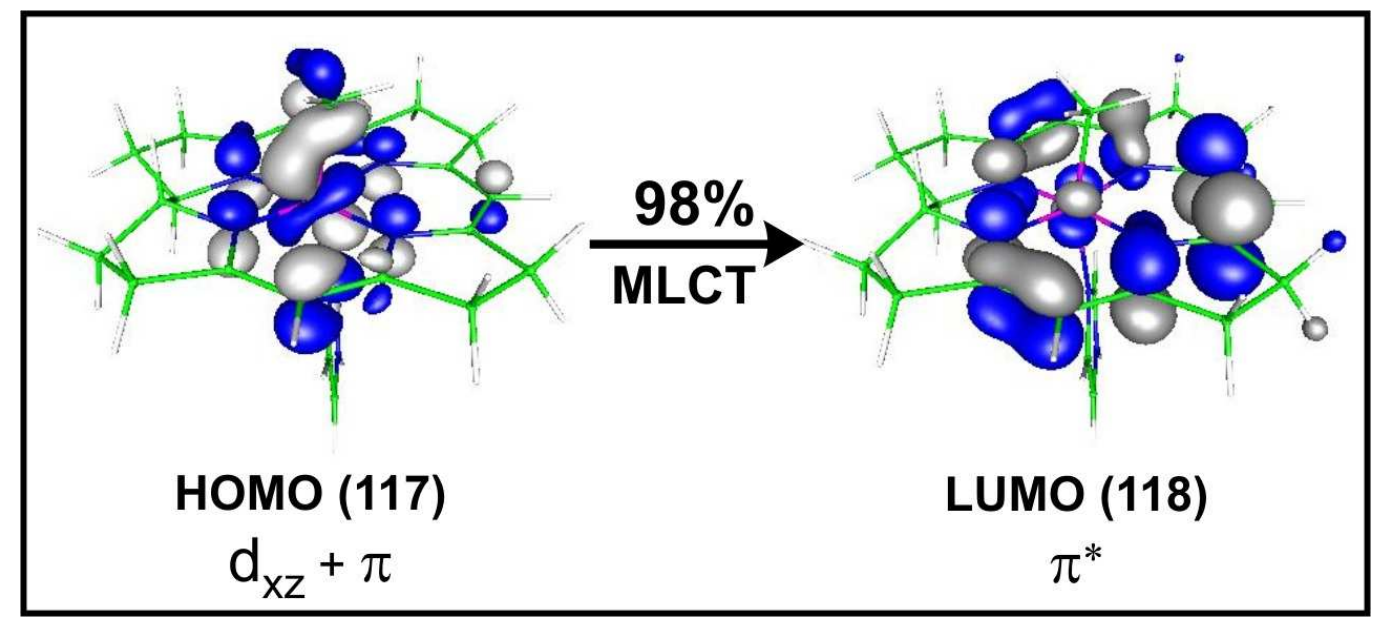

Figure 3.10. The isosurface plots of dominant MOs corresponding to the first electronic transition $\left(\mathrm{S}_{0} \rightarrow \mathrm{S}_{1}\right)$ of Im-[Co ${ }^{\mathrm{III}}$ (corrin) $]-\mathrm{CH}_{3}^{+}$at the TD-DFT/BP86/6$31 \mathrm{G}(\mathrm{d})$-optimized geometry of the $\mathrm{S}_{1}$ excited state.

These small geometrical changes upon the electronic excitation support the validity of the previously discussed MLCT interpretation of the $S_{1}$ state, which has been based on the vertical $S_{0} \rightarrow S_{1}$ excitation calculated using several pure DFT functionals, EOM-CCSD, and MC-XQDPT2.

To re-confirm the MLCT nature of the $S_{0} \rightarrow S_{1}$ transition in yet another way, the geometry of the Im- $\left[\mathrm{Co}{ }^{\mathrm{III}}\right.$ (corrin) $]-\mathrm{CH}_{3}^{+}$complex in its $\mathrm{S}_{1}$ state was also fully reoptimized at the TD-DFT/BP86/6-31G(d) level. Only small structural changes between the $S_{0}$ and $S_{1}$ states, mostly in the axial ligands, were observed (Figure 3.9). The $S_{0} \rightarrow S_{1}$ excitation resulted in the $0.015 \AA$ elongation of the Co- $\mathrm{C}_{\mathrm{Me}}$ bond, the $0.092 \AA$ shortening of the Co- $\mathrm{N}_{\text {Im }}$ bond, and the $12.5^{\circ}$ change in the $\mathrm{N}_{\mathrm{Im}}-\mathrm{Co}-\mathrm{C}_{\mathrm{Me}}$ angle value. The subsequent analysis of MOs participating in the lowest electronic excitation showed once again that the $S_{1}$ state is dominated by the $d / \pi \rightarrow \pi^{*}$ MLCT excitations (see Figure 3.10), in perfect agreement with the TAS experiments of Sension et al. ${ }^{56,58,60,65}$ 


\subsection{Summary and Conclusions}

The goal of the study presented in this chapter was to provide insights into the nature of the $S_{1}$ state of the MeCbl cofactor, which was previously characterized as either the $\pi \rightarrow \pi^{*}$ excitation localized on corrin $^{66,68,108,113}$ or the MLCT excitation. ${ }^{56,58,60,65}$ To resolve the existing controversy, we performed high-level $a b$ initio calculations, including the MC-XQDPT2 and EOM-CCSD wave-function-based methods, and several TD-DFT calculations using the hybrid (B3LYP, MPW1PW91, TPSSh), GGA-type (BP86, BLYP, MPWPW91), meta-GGA (TPSS), and range-

separated (CAM-B3LYP and LC-BLYP) functionals. We have demonstrated that the MC-XQDPT2 and EOM-CCSD approaches provide a consistent description of the $\mathrm{S}_{1}$ state as a MLCT transition, in agreement with the TAS experiments. ${ }^{56,58,60,65}$ In contrast to the high-level wave function approaches, the TD-DFT results vary significantly depending on the applied functional. The commonly used hybrid functionals, including B3LYP, MPW1PW91, and TPSS-h, suggest a predominantly $\pi \rightarrow \pi^{*}$ character of the $S_{0} \rightarrow S_{1}$ transition, as opposed to the MLCT interpretation resulting from the MC-XQDPT2 and EOM-CCSD calculations. On the other hand, the GGAtype (BP86, BLYP, and MPWPW91), meta-GGA (TPSS), and range-separated pure GGA (LC-BLYP) functionals characterize it as the predominantly MLCT, $d / \pi \rightarrow \pi^{*}$, excitation, in perfect agreement with the MC-XQDPT2 and EOM-CCSD calculations. Thus, the previous assignments of the low-lying excitations of MeCbl derived from the experimental Abs, $\mathrm{CD}$, and MCD spectra coupled with the theoretical analysis based on the functionals other than GGAs ${ }^{108,113}$ may require revision. Clearly, additional experimental and theoretical work is needed to fully understand the nature of the electronically excited states of the MeCbl cofactor. However, in view of the highlevel $a b$ initio calculations reported in this work and the recent TAS studies, ${ }^{56,58,60,65}$ it is reasonable to assume that the $\mathrm{S}_{1}$ state of MeCbl is a MLCT transition. 


\section{CHAPTER 4}

\section{ELECTRONICALLY EXCITED STATES OF VITAMIN B ${ }_{12}$ AND METHYLCOBALAMIN: THEORETICAL ANALYSIS OF ABSORPTION, CD AND MCD DATA ${ }^{1}$}

\subsection{Introduction}

Vitamin $\mathrm{B}_{12}$ derivatives have for a long time intrigued chemists as one of the most extraordinary groups of organometallic complexes. ${ }^{9,14,16,20,23,28}$ Cobalamins are deeply colored and exhibit a great variation in their colors. This can be attributed to a manifold of low-lying excited states whose energies are sensitive to the nature of the axial ligands. ${ }^{66,68}$ In strongly acidic conditions, the protonated DBI (lower axial ligand) is replaced by water, leading to a blue shift of the lowest absorption band. The ability to monitor these changes makes Abs spectroscopy a particularly useful spectroscopic technique in the context of the biologically important enzymes that use the derivatives of vitamin $\mathrm{B}_{12}$ as cofactors. In addition to Abs spectroscopy, circular dichroism (CD) and magnetic CD (MCD) techniques have also been applied to probe the nature of the excited states in cobalamins. ${ }^{67,108,110,112,113}$ The CD and MCD spectroscopic probes are complementary to Abs, particularly with respect to ligand field ( $\mathrm{d} \rightarrow \mathrm{d}$ ) transitions that show very little intensity in Abs spectra, but might in some cases gain significant intensity in the MCD spectrum. The nature of the low-lying electronically excited states and photochemistry of cobalamins have been also studied by ultrafast transient absorption spectroscopy. ${ }^{56-65,162,207}$ The dynamics of the photoinduced homolysis of $\mathrm{B}_{12}$ cofactors were investigated on

\footnotetext{
${ }^{1}$ The material presented in this Chapter was reprinted (adapted) with permission from H. Solheim, K. Kornobis, K. Ruud, and P. M. Kozlowski, J. Phys. Chem. B 115, 737 (2011). Copyright 2011 American Chemical Society.
} 
a time scale ranging from femtoseconds to nanoseconds, allowing for the detailed mechanism of $\mathrm{Co}-\mathrm{C}$ bond photolysis to be obtained. In the case of $\mathrm{CNCbl}$, it was found that the metastable $S_{1}$ state has a ligand-to-metal charge transfer (LMCT) character. ${ }^{64}$ The $S_{1}$ state in MeCbl was on the other hand characterized as a metalto-ligand charge transfer (MLCT). ${ }^{56,58,60}$ For coenzyme $\mathrm{B}_{12}$ it was demonstrated that depending on the environment, $\mathrm{S}_{1}$ may have either LMCT or MLCT character. ${ }^{65}$

By applying different experimental techniques, remarkable progress has been made in generating and characterizing electronically excited states of cobalamins. However, the detailed understanding of these excited states still remains insufficient from an electronic structure point of view. In order to rectify this situation, several groups have started to use time-dependent density functional theory (TDDFT ${ }^{80}$ to determine the properties of the electronically excited states. It should be noted that the TD-DFT framework is currently probably the only practical tool that can be used to routinely study excited states of these fairly large and complex molecules. ${ }^{81,84}$ The primary target of earlier investigations has been the Abs data. ${ }^{105-107,109,111}$ The reason for this is that TD-DFT calculations can provide reliable estimates of the electronic excitation energies and transition dipole moments that are required for simulating Abs spectra. Consequently, the simulated Abs spectra can be directly compared with experiment in order to assign electronic transitions. Using such a procedure, an identification of the main absorption features has been obtained. ${ }^{105-109,118,119}$ However, several issues still remain open and require further analysis. In particular, the simulated Abs spectra depend strongly on the type of functional used in the TD-DFT calculations. This strong dependence has been noticed not only in terms of the quantitative description of the states, but more importantly in terms of the qualitative description. For example, taking into consideration the lowest-energy band (the so-called $\alpha / \beta$ band), very different assignments regarding to the number of electronic transitions were predicted, depending on the type of functional applied, i.e. hybrid or gradient-corrected functionals. ${ }^{105,106}$ In Chapters 2 and 3 we discussed these issues in detail, pointing out the applicability of BP86 functional to explore the electronically excited states of CNCbl and MeCbl. 
We based this conclusion on the assignments of their lowest electronic transitions which were examined at different level of theories. The more comprehensive understanding of electronic structure of cobalamins can be reached by elucidation of the excitations having higher energies which contribute to the spectral bands following the lowest-energy $\alpha / \beta$ region.

Based on these earlier theoretical studies, it has become apparent that the analysis of electronically excited states based on Abs data and TD-DFT calculations alone is not sufficient. In order to obtain a more reliable description of the electronic excitations, more independent information is needed to validate the theoretical approach. In the present theoretical contribution, this independent insight is provided by taking into account not only Abs data, but also CD and MCD data. In particular, the properties of electronically excited states of $\mathrm{CNCbl}$ and $\mathrm{MeCbl}$ are analyzed in detail. For both cobalamins, accurate X-ray structures have been reported, ${ }^{147}$ and Abs, CD and MCD spectroscopic data are available in literature.

\subsection{Computational Details}

\subsubsection{Structural models}

In the majority of previously reported computational studies, truncated models with respect to the side-chains have been employed when describing the $\mathrm{B}_{12}$ cofactors. In the present work, such models were also used, based on the highresolution X-ray data of $\mathrm{CNCbl}$ and $\mathrm{MeCbl}$ cobalamins. ${ }^{147}$ To generate the initial structures of the DBI-[Co ${ }^{\mathrm{III}}($ corrin) $]-\mathrm{R}^{+}$models $(\mathrm{R}=\mathrm{CN}$ or $\mathrm{Me})$, the full $\mathrm{CNCbl}$ and $\mathrm{MeCbl}$ complexes were simplified by replacing the corrin side chains by hydrogen atoms (Figure 4.1). Full geometry optimization was carried out employing the BP86/6-31G(d) (5d) level of theory, which has been found to be appropriate for structural analysis of cobalt corrinoids. ${ }^{100,155,164}$ For each optimized geometry harmonic frequencies were calculated to verify that the optimized structure corresponds to a stable minimum. 

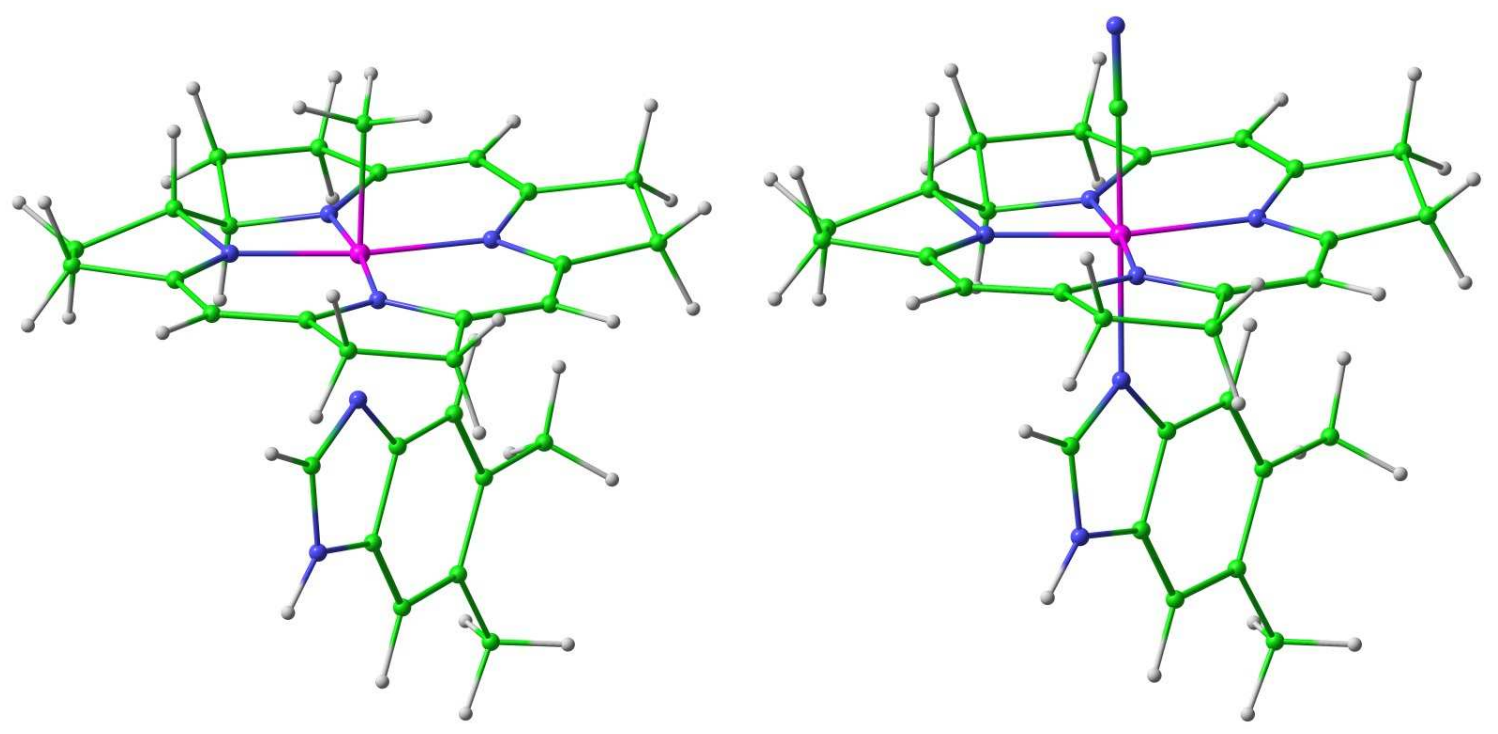

Figure 4.1. Simplified structural models of the MeCbl (left) and CNCbl (right) used in calculations and denoted as DBI-[Co ${ }^{\mathrm{III}}$ (corrin) $]-\mathrm{Me}^{+}$and DBI-[Co ${ }^{\mathrm{III}}$ (corrin)]$\mathrm{CN}^{+}$, respectively.

The geometry optimization and frequency calculations have been carried out using the Gaussian 03 suite of programs for the electronic structure calculations. ${ }^{148}$

\subsubsection{Abs and CD calculations}

To simulate Abs and CD spectra, several components, such as electronic transition energies, oscillator and rotational strengths need to be calculated. In the formalism of response theory, these properties are related to residues of linear response functions. ${ }^{208}$ Note that this approach does not describe the relaxation of the excited system back to the ground state, and may therefore be called the infinite lifetime approximation. This approximation leads to a stick spectrum that is typically broadened by a Lorentzian or Gaussian function to mimic the experimental spectrum. 


\subsubsection{MCD calculations}

The MCD spectrum is normally interpreted in terms of three contributions, labeled as the A, B, and C terms. For systems with no orbital degeneracies, such as $\mathrm{MeCbl}$ and $\mathrm{CNCbl}$ studied here, only the B term can be non-zero. Complex polarization propagator theory (CPP), also called damped response theory, which provides a unified framework for calculating the A and B terms, was employed to simulate MCD spectra. ${ }^{209-211}$ In this approach, an empirical damping parameter is included in the actual response calculations, making the response functions complex. For real perturbations, the real part of the response is related to scattering, i.e. the polarizability for linear response, and the imaginary part to the absorption at a given frequency. For MCD, one of the perturbing fields is imaginary, meaning that the relationship between the real and complex parts of the response function to the scattering and absorption processes is reversed. A Lorentzian broadening of the bands was inherited from the use of the CPP approach.

\subsubsection{Functionals and basis set}

Following previous TD-DFT studies of cobalt corrinoids, two different functionals, namely BP86 ${ }^{115,123}$ and CAM-B3LYP, ${ }^{135,212}$ have been employed to compute the components required to simulate Abs, CD and MCD spectra. The CAM-B3LYP functional was used instead of B3LYP, because it is more appropriate for charge transfer (CT) type excitations and gives a more uniform description of excited states of different character. Two different basis set, i.e. 6-31G(d) and aug-cc-pVDZ were used. $^{213,214}$ It should be noted that the smaller 6-31G(d) basis set ( $\sim 600$ contracted functions) was used mainly as a reference with respect to previous studies. In order to obtain reliable predictions of molecular properties (including magnetic), calculations were carried out using the large aug-cc-pVDZ basis set ( $\sim 1200$ contracted functions). All Abs, CD and MCD calculations were performed using a development

version of the Dalton program. ${ }^{152}$ 


\subsection{Results and Discussion}

\subsubsection{Absorption spectra of $\mathrm{CNCbl}$ and $\mathrm{MeCbl}$}

We start by focusing on the Abs data and related spectral properties of the $\mathrm{CNCbl}$ and MeCbl complexes. Visible spectra of both cobalamins exhibit many distinctive absorption features that can be attributed to a manifold of low-lying excited states. Traditionally, they have been interpreted as $\pi \rightarrow \pi^{*}$ transitions of the corrin ring with some participation of cobalt $\mathrm{d}$ orbitals. ${ }^{66,68,72}$ However, there is a noticeable difference between the Abs spectrum of CNCbl and MeCbl. The Abs spectrum of CNCbl has been referred to as "typical", being dominated by two spectral features, the so-called $\alpha / \beta$ band in the visible region and the $\gamma$ band in the UV. In contrast, the MeCbl cofactor exhibits a "unique" Abs spectrum. ${ }^{108}$ The main difference between them lies in the $\gamma$ band, which in the case of MeCbl consists of several much less intense and comparable (in magnitude) transitions in the UV spectral region.

Several TD-DFT calculations focusing on the electronic structure and Abs spectra of CNCbl and MeCbl have appeared in the literature. ${ }^{105,107,108}$ Although these calculations do not yet provide a complete picture of the electronic structure of these cobalamins, they provide significant insight into the electronic excitations of these complex biological molecules. The TD-DFT method, which takes into account only single-electron excitations, typically gives accurate results for valenceexcited states that lie below the ionization potential. For such states, the typical error of TD-DFT is within the range of $0.1-0.5 \mathrm{eV}$, which can be effectively compensated by a scaling. ${ }^{105-107}$ A critical step in TD-DFT is the choice of an appropriate functional. Recently reported excited-state calculations highlighted some of the difficulties one may experience in modeling the electronic spectra of $\mathrm{B}_{12}$ cofactors. ${ }^{105,106}$ It was concluded that the B3LYP functional was more appropriate for modeling the $\pi \rightarrow \pi^{*}$ transitions of the corrin ring, but it was somehow problematic for describing $\mathrm{d} / \pi \rightarrow \pi^{*} / \mathrm{d}$ transitions involving axial bonding. On the other hand, it was found that the performance of the BP86 functional was better, particularly for 
describing excitations related to the axial bonding. For certain types of molecules it has been recognized that CT excitations based on TD-DFT calculations tend to be significantly underestimated (see, for example Refs. 128, 131 or 144). More specifically, long-range excitations between orbitals with low overlap are significantly underestimated while for short-range excitations with high overlap errors tend to be small. Also, the problem is more pronounced for GGA-type functionals than for hybrid functionals. It has been suggested that a $\Lambda$ parameter (which measures the degree of spatial overlap between the occupied and virtual orbitals involved in an excitation) can be used as a diagnostic test. ${ }^{138}$ Consequently, for each excitation, the value of $\Lambda$ was computed. While a more detailed analysis will be presented in the next section, we note here that for the large basis set calculations, the values of the $\Lambda$ parameter were predicted to be greater than 0.4 , indicating that the computed excitations do not suffer from any CT failure. ${ }^{212}$ Note, however, that a large $\Lambda$ value does not necessarily guarantee that the calculated excitation energy is of high quality in comparison to experiment.

As a prerequisite for analyzing the $\mathrm{CD}$ and MCD spectra, we first computed TD-DFT excitations and simulated the Abs spectra for a direct comparison with experiment. Truncated complexes with respect to the side chains (Figure 4.1) served as models when performing these calculations. Two different functionals (i.e. BP86 and CAM-B3LYP) have been used, and the TD-DFT vertical excitation energies together with the corresponding oscillator strengths were calculated for the 35 lowest excited states. To simulate the Abs spectra, each electronic transition was associated with a Lorentzian function having an appropriate band width $(\Gamma)$. For both systems studied a band width of $\Gamma=0.075 \mathrm{eV}$ was used.. In order to improve the agreement between experiment and theory, a small scaling was applied to adjust computed electronic transitions. A more detailed description of the scaling procedure will be presented along with the analysis of the CD data in the next Section. 

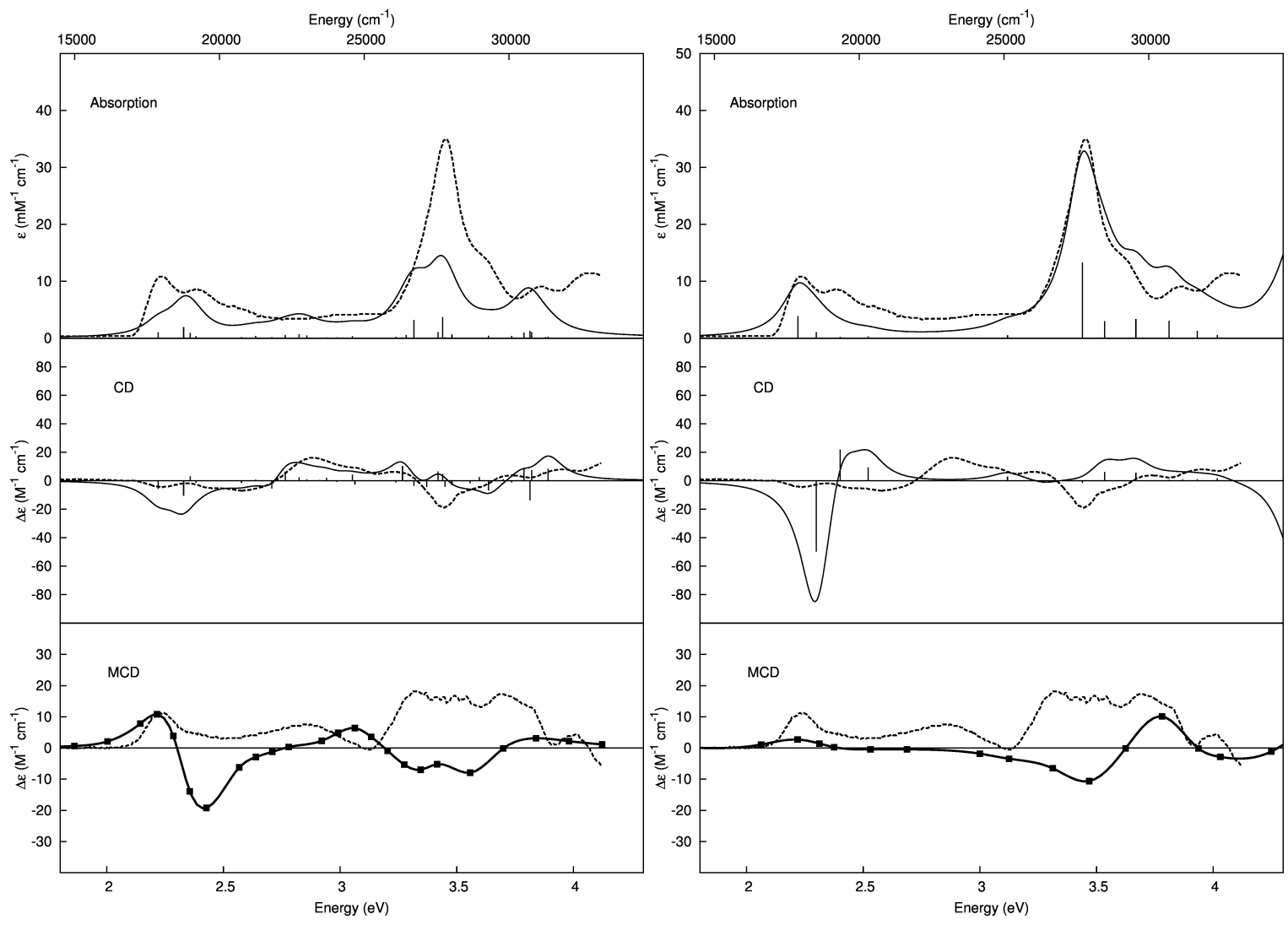

Figure 4.2. Abs (top), CD (middle) and MCD (bottom) computed spectra (solid line) and experimental (dotted line) of CNCbl. Simulated TD-DFT spectra correspond to gas phase calculations with BP86/aug-cc-pVDZ (left panel) and CAMB3LYP/aug-cc-pVDZ (right panel). Experimental spectra were reproduced from Ref. 108 with permission.

Comparison with experimental data shows that in the case of $\mathrm{CNCbl}$, the simulated spectra based on both the BP86 and CAM-B3LYP functionals reproduce the experimental spectrum quite well (Figure 4.2), although there are noticeable differences between the quantities computed with the two functionals. In particular, the BP86 functional predicts a much higher density of states in the D/E region than CAM-B3LYP does. Both functionals predict several transitions in the $\alpha / \beta$ region. In the simulated spectrum based on the CAM-B3LYP functional, the first transition gives the dominant contribution to this band, while BP86 is predicting 3-4 states with contributions of similar magnitude. Note that the relative intensities of the transitions contributing to the $\alpha / \beta$ band are strongly affected by solvent effects as will be discussed in Section 4.3.5. For the $\gamma$ band, the CAM-B3LYP functional 

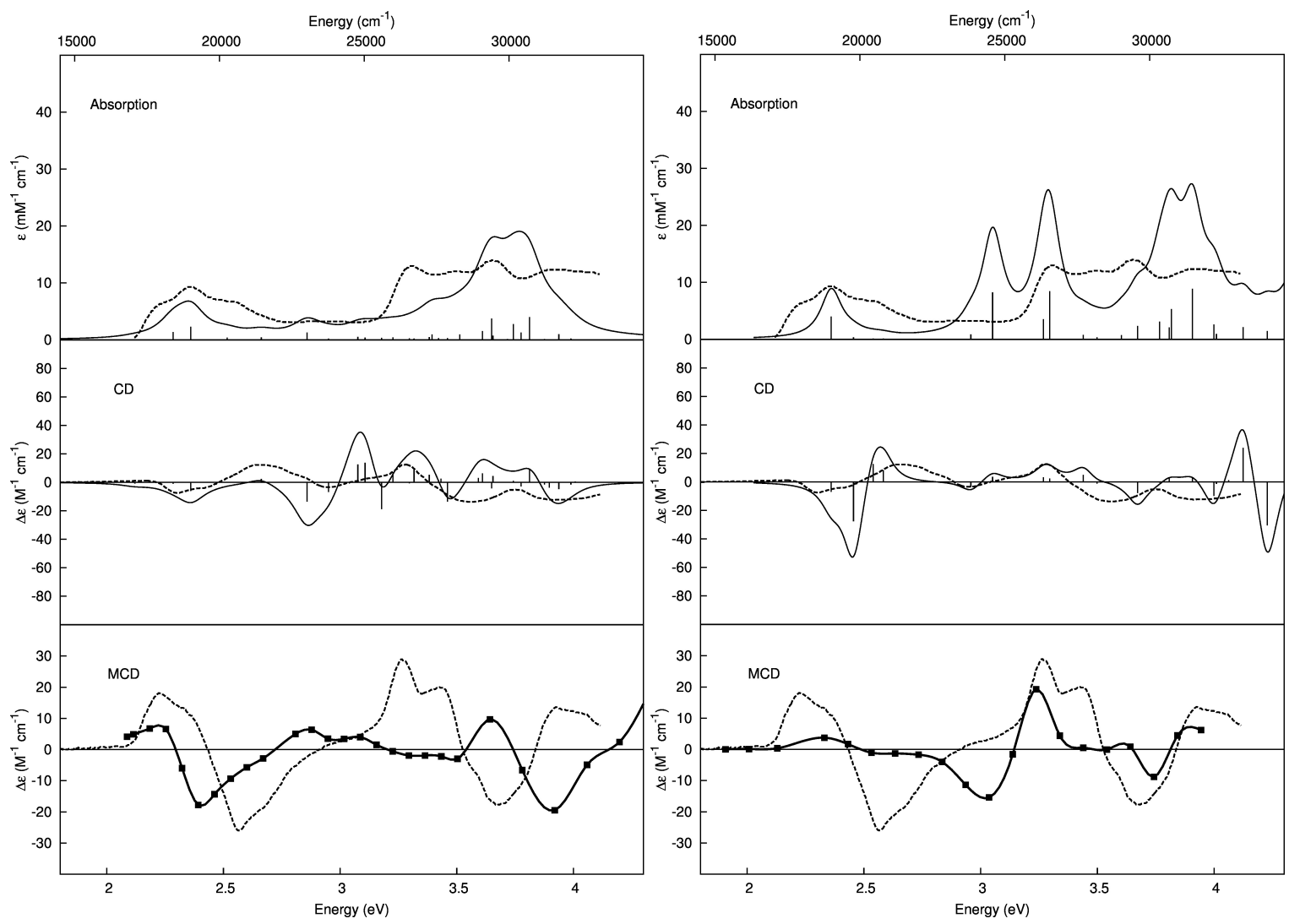

Figure 4.3. Abs (top), CD (middle) and MCD (bottom) computed spectra (solid line) and experimental (dotted line) of MeCbl. Simulated TD-DFT spectra correspond to gas phase calculations with BP86/aug-cc-pVDZ (left panel) and CAMB3LYP/aug-cc-pVDZ (right panel). Experimental spectra were reproduced from Ref. 108 with permission.

correctly predicts domination by one strong transition, while BP86 seems to have some difficulties, predicting two electronic transitions of comparable intensities.

Figure 4.3 shows the experimental and simulated spectra for MeCbl. The overall agreement with experiment here seems to be better for the BP86 functional than for CAM-B3LYP, although with the scaling we have chosen for the calculated energies, the $\gamma$ band starts at too high energy in the simulation based on the BP86 results (see the following section for a discussion of the scaling procedure). In addition, the CAM-B3LYP functional predicts a very intense transition in the D/E region of the Abs spectrum that is not observed in the experimental spectrum. 

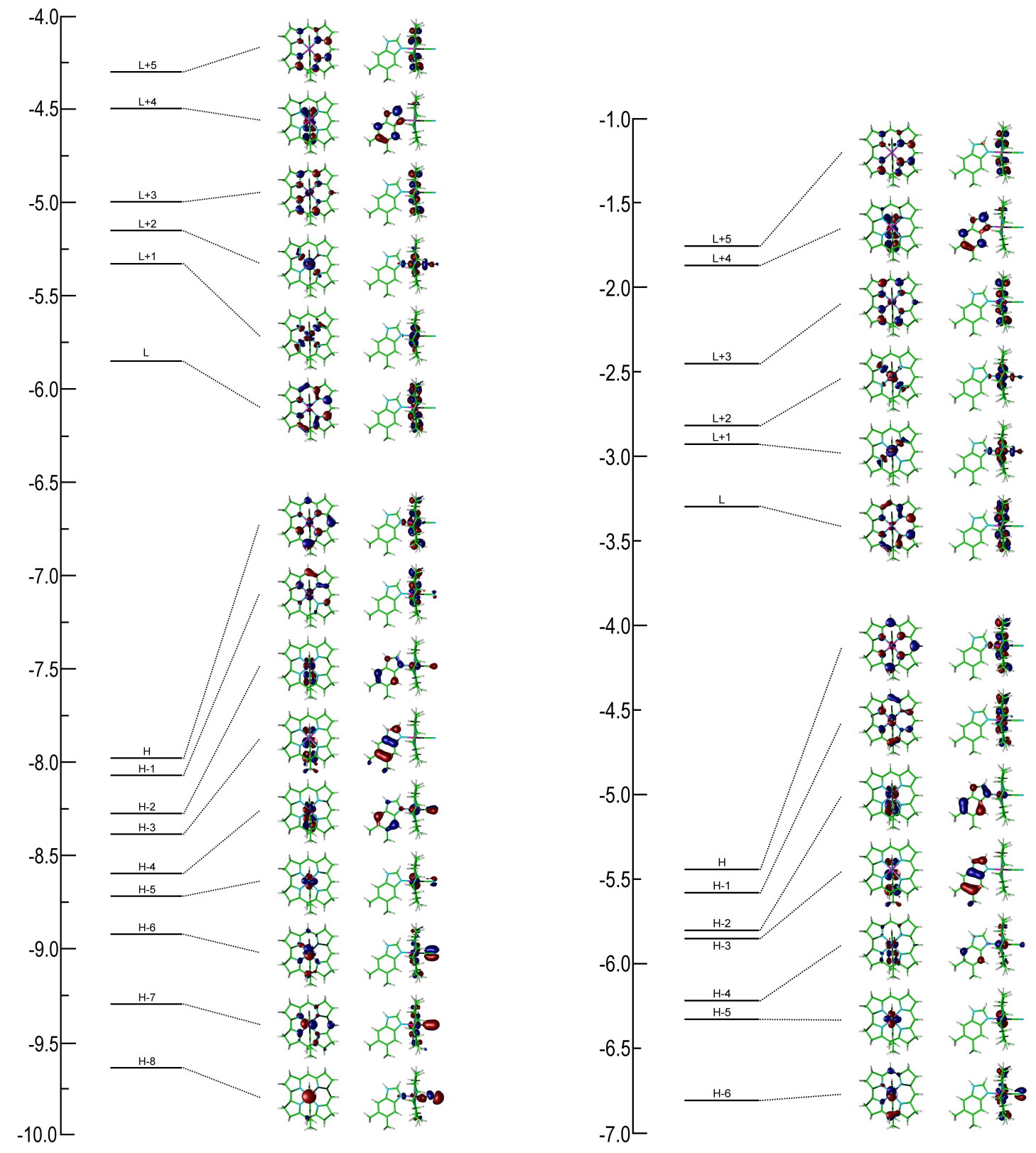

Figure 4.4. Energy (eV) diagram representing frontier MOs of DBI-[Co ${ }^{\mathrm{III}}$ (corrin)]$\mathrm{CN}^{+}$based on BP86/aug-cc-pVDZ calculations: left panel in gas phase and right panel in water solution, PCM. 


\subsubsection{CD spectra of $\mathrm{CNCbl}$ and $\mathrm{MeCbl}$}

The CD spectra for both cobalamins were simulated employing the TD-DFT electronic transitions and rotational strengths (Tables 4.1 and 4.2). Each transition was associated with the Lorentzian band having a $0.075 \mathrm{eV}$ band width. Although a comparable computational cost is required to obtain the components needed to simulate the Abs and CD spectra, the reproduction of CD data is more challenging than Abs data. This is because the rotational strengths have different signs making the overall shape of the simulated CD spectrum much more sensitive to the accuracy of the computed quantities.

To bring the computational Abs and CD results closer to the experimental data, we have as already pointed out, applied a simple scaling procedure. ${ }^{105,107}$ Each electronic transition computed via TDDFT was scaled as $E_{\text {scaled }}^{i}=\chi E_{\mathrm{TDDFT}}^{i}+$ $E_{\text {shift}}$, where $\chi$ and $E_{\text {shift }}$ are empirical parameters used to adjust the simulated spectrum. It should be noted that scaling does not alter the order of electronic tran-

sitions, but brings simulated and experimental spectra close one to another. Hence, the scaling parameters were selected to reproduce accurately the excitations associated with both the $\alpha / \beta$ and $\gamma$ bands, taking into account the Abs, CD, and MCD spectra. In addition, for the sake of consistency we have tried to avoid large differences in the scaling parameters used for $\mathrm{CNCbl}$ and $\mathrm{MeCbl}$ and a given functional.

Figures 4.2 and 4.3 show the comparison between the simulated and experimental CD spectra. Overall, taking into account the complexity of both systems, reliable agreement has been reached, though qualitatively less satisfactory than the one received for the Abs data. The $\mathrm{CD}$ data for $\mathrm{CNCbl}$ computed with the BP86 functional are much better reproduced than those calculated with CAM-B3LYP. Comparison of BP86 and CAM-B3LYP simulated spectra with the experimental CD spectrum for MeCbl indicates that BP86 better captures the shape of the experimental spectrum in low-energy part (up to $3 \mathrm{eV}$ ), while in the higher energy spectral region CAM-B3LYP outperforms BP86. However, the BP86 functional predicts a high density of transitions in the $\mathrm{D} / \mathrm{E}$ region of $\mathrm{MeCbl}$. 
TABLE 4.1. Electronic excitations of CNCbl based on BP86/aug-cc-pVDZ calculations in gas phase ${ }^{a}$.

\begin{tabular}{|c|c|c|c|c|c|}
\hline State & 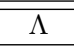 & $\overline{E(\mathrm{eV})}$ & Osc. str. & Rot. str. & Character $^{b}$ \\
\hline $\mathrm{S}_{1}$ & 0.57 & 2.39 & 0.018 & -20.881 & $31 \%(\mathrm{H} \rightarrow \mathrm{L})+18 \%(\mathrm{H}-2 \rightarrow \mathrm{L})$ \\
\hline $\mathrm{S}_{2}$ & 0.58 & 2.49 & 0.034 & -36.359 & $31 \%(\mathrm{H}-2 \rightarrow \mathrm{L})+13 \%(\mathrm{H} \rightarrow \mathrm{L})+9 \%(\mathrm{H}-1 \rightarrow \mathrm{L})$ \\
\hline $\mathrm{S}_{3}$ & 0.58 & 2.52 & 0.016 & 10.152 & $24 \%(\mathrm{H}-1 \rightarrow \mathrm{L})+11 \%(\mathrm{H}-3 \rightarrow \mathrm{L})+7 \%(\mathrm{H}-2 \rightarrow \mathrm{L})$ \\
\hline $\mathrm{S}_{4}$ & 0.56 & 2.55 & 0.006 & -4.912 & $38 \%(\mathrm{H}-3 \rightarrow \mathrm{L})+8 \%(\mathrm{H}-1 \rightarrow \mathrm{L})$ \\
\hline $\mathrm{S}_{5}$ & 0.50 & 2.73 & 0.003 & -5.064 & $35 \%(\mathrm{H} \rightarrow \mathrm{L}+1)+10 \%(\mathrm{H}-4 \rightarrow \mathrm{L})$ \\
\hline $\mathrm{S}_{6}$ & 0.56 & 2.79 & 0.007 & 2.382 & $35 \%(\mathrm{H}-4 \rightarrow \mathrm{L})+7 \%(\mathrm{H} \rightarrow \mathrm{L}+1)$ \\
\hline $\mathrm{S}_{7}$ & 0.48 & 2.86 & 0.003 & -16.292 & $36 \%(\mathrm{H}-1 \rightarrow \mathrm{L}+1)$ \\
\hline $\mathrm{S}_{8}$ & 0.56 & 2.91 & 0.010 & 18.753 & $40 \%(\mathrm{H}-5 \rightarrow \mathrm{L})$ \\
\hline $\mathrm{S}_{9}$ & 0.55 & 2.97 & 0.012 & 6.579 & $36 \%(\mathrm{H} \rightarrow \mathrm{L}+2)+5 \%(\mathrm{H} \rightarrow \mathrm{L}+3)$ \\
\hline$S_{10}$ & 0.55 & 3.00 & 0.008 & 2.461 & $36 \%(H-1 \rightarrow L+2)$ \\
\hline$S_{11}$ & 0.47 & 3.06 & 0.002 & 1.472 & $39 \%(\mathrm{H}-3 \rightarrow \mathrm{L}+1)+7 \%(\mathrm{H}-2 \rightarrow \mathrm{L}+1)$ \\
\hline $\mathrm{S}_{12}$ & 0.48 & 3.08 & 0.002 & 5.643 & $19 \%(\mathrm{H}-2 \rightarrow \mathrm{L}+1)+9 \%(\mathrm{H}-3 \rightarrow \mathrm{L}+1)+5 \%(\mathrm{H}-6 \rightarrow \mathrm{L})$ \\
\hline $\mathrm{S}_{13}$ & 0.54 & 3.13 & 0.003 & -1.881 & $28 \%(\mathrm{H}-6 \rightarrow \mathrm{L})+7 \%(\mathrm{H} \rightarrow \mathrm{L}+3)+5 \%(\mathrm{H}-2 \rightarrow \mathrm{L}+1)$ \\
\hline $\mathrm{S}_{14}$ & 0.58 & 3.19 & 0.006 & 11.500 & $26 \%(\mathrm{H}-1 \rightarrow \mathrm{L}+3)+12 \%(\mathrm{H}-2 \rightarrow \mathrm{L}+2)$ \\
\hline $\mathrm{S}_{15}$ & 0.55 & 3.20 & 0.002 & -7.489 & $26 \%(\mathrm{H}-2 \rightarrow \mathrm{L}+2)+12 \%(\mathrm{H}-1 \rightarrow \mathrm{L}+3)$ \\
\hline$S_{16}$ & 0.55 & 3.25 & 0.000 & 1.012 & $45 \%(\mathrm{H}-3 \rightarrow \mathrm{L}+2)$ \\
\hline $\mathrm{S}_{17}$ & 0.59 & 3.37 & 0.003 & -3.015 & $27 \%(\mathrm{H}-2 \rightarrow \mathrm{L}+3)+12 \%(\mathrm{H}-3 \rightarrow \mathrm{L}+3)$ \\
\hline $\mathrm{S}_{18}$ & 0.50 & 3.40 & 0.003 & 26.308 & $23 \%(\mathrm{H}-4 \rightarrow \mathrm{L}+1)+11 \%(\mathrm{H}-3 \rightarrow \mathrm{L}+3)+7 \%(\mathrm{H}-4 \rightarrow \mathrm{L}+2)$ \\
\hline $\mathrm{S}_{19}$ & 0.60 & 3.41 & 0.010 & -1.219 & $22 \%(\mathrm{H}-3 \rightarrow \mathrm{L}+3)+14 \%(\mathrm{H}-2 \rightarrow \mathrm{L}+3)$ \\
\hline $\mathrm{S}_{20}$ & 0.59 & 3.44 & 0.056 & -8.952 & $20 \%(\mathrm{H} \rightarrow \mathrm{L}+4)+11 \%(\mathrm{H} \rightarrow \mathrm{L}+3)+5 \%(\mathrm{H}-6 \rightarrow \mathrm{L})$ \\
\hline $\mathrm{S}_{21}$ & 0.47 & 3.50 & 0.000 & -11.026 & $35 \%(\mathrm{H}-5 \rightarrow \mathrm{L}+1)+5 \%(\mathrm{H}-6 \rightarrow \mathrm{L}+1)+5 \%(\mathrm{H}-5 \rightarrow \mathrm{L}+2)$ \\
\hline $\mathrm{S}_{22}$ & 0.55 & 3.54 & 0.019 & 15.516 & $32 \%(\mathrm{H}-7 \rightarrow \mathrm{L})+6 \%(\mathrm{H}-1 \rightarrow \mathrm{L}+4)+5 \%(\mathrm{H} \rightarrow \mathrm{L}+5)$ \\
\hline $\mathrm{S}_{23}$ & 0.59 & 3.56 & 0.064 & 11.355 & $24 \%(\mathrm{H} \rightarrow \mathrm{L}+4)+8 \%(\mathrm{H} \rightarrow \mathrm{L}+3)+5 \%(\mathrm{H}-1 \rightarrow \mathrm{L}+4)$ \\
\hline $\mathrm{S}_{24}$ & 0.52 & 3.57 & 0.002 & -10.296 & $30 \%(\mathrm{H}-4 \rightarrow \mathrm{L}+2)$ \\
\hline $\mathrm{S}_{25}$ & 0.59 & 3.60 & 0.011 & -7.284 & $38 \%(\mathrm{H}-1 \rightarrow \mathrm{L}+4)$ \\
\hline $\mathrm{S}_{26}$ & 0.53 & 3.68 & 0.001 & -4.446 & $28 \%(\mathrm{H}-6 \rightarrow \mathrm{L}+1)+12 \%(\mathrm{H}-5 \rightarrow \mathrm{L}+3)+5 \%(\mathrm{H}-4 \rightarrow \mathrm{L}+3)$ \\
\hline $\mathrm{S}_{27}$ & 0.58 & 3.72 & 0.001 & 5.771 & $27 \%(\mathrm{H}-4 \rightarrow \mathrm{L}+3)+7 \%(\mathrm{H}-5 \rightarrow \mathrm{L}+2)$ \\
\hline $\mathrm{S}_{28}$ & 0.55 & 3.75 & 0.008 & -15.620 & $11 \%(\mathrm{H}-5 \rightarrow \mathrm{L}+3)+8 \%(\mathrm{H}-6 \rightarrow \mathrm{L}+1)+7 \%(\mathrm{H} \rightarrow \mathrm{L}+5)$ \\
\hline $\mathrm{S}_{29}$ & 0.59 & 3.81 & 0.001 & 2.405 & $42 \%(\mathrm{H}-8 \rightarrow \mathrm{L})$ \\
\hline$S_{30}$ & 0.56 & 3.85 & 0.006 & -2.793 & $18 \%(\mathrm{H}-6 \rightarrow \mathrm{L}+2)+11 \%(\mathrm{H}-5 \rightarrow \mathrm{L}+3)+9 \%(\mathrm{H}-1 \rightarrow \mathrm{L}+5)$ \\
\hline$S_{31}$ & 0.58 & 3.90 & 0.017 & 19.560 & $28 \%(\mathrm{H}-1 \rightarrow \mathrm{L}+5)$ \\
\hline $\mathrm{S}_{32}$ & 0.59 & 3.92 & 0.022 & -30.158 & $21 \%(\mathrm{H}-2 \rightarrow \mathrm{L}+4)+8 \%(\mathrm{H}-6 \rightarrow \mathrm{L}+3)+6 \%(\mathrm{H}-1 \rightarrow \mathrm{L}+5)$ \\
\hline $\mathrm{S}_{33}$ & 0.56 & 3.93 & 0.019 & 16.096 & $11 \%(\mathrm{H} \rightarrow \mathrm{L}+5)+10 \%(\mathrm{H}-6 \rightarrow \mathrm{L}+3)+10 \%(\mathrm{H}-2 \rightarrow \mathrm{L}+4)$ \\
\hline $\mathrm{S}_{34}$ & 0.43 & 3.99 & 0.003 & 0.296 & $40 \%(\mathrm{H}-7 \rightarrow \mathrm{L}+1)$ \\
\hline $\mathrm{S}_{35}$ & 0.57 & 4.00 & 0.005 & 17.715 & $24 \%(\mathrm{H}-2 \rightarrow \mathrm{L}+5)+7 \%(\mathrm{H}-10 \rightarrow \mathrm{L})+7 \%(\mathrm{H}-9 \rightarrow \mathrm{L})+6 \%(\mathrm{H}-6 \rightarrow \mathrm{L}+3)$ \\
\hline
\end{tabular}

${ }^{a}$ Rotational strengths are given in $\left(10^{-40} \mathrm{esu}^{2} \mathrm{~cm}^{2}\right)$ and oscillator strengths in dimensionless units. Energies presented in table were not scaled.

${ }^{b} \mathrm{H}, \mathrm{L}, \mathrm{H}-1, \mathrm{~L}+1, \ldots$, etc. correspond to energy diagram shown in Figure 4.4.

The oscillator strengths for these transitions are not very large as compared to the main transitions in the $\alpha / \beta$ and $\gamma$ bands. Their BP86-predicted rotational strengths are, however, quite substantial with some transitions having large positive rotational strengths and some large negative rotational strengths. The shapes of the broadened bands strongly depend on the relative positions of the individual transitions. On the other hand, CAM-B3LYP gives a very different picture with few transitions in the $\mathrm{D} / \mathrm{E}$ region of the $\mathrm{MeCbl}$ spectra. In contrast to $\mathrm{BP} 86$, these transitions are predicted to have small rotational strengths. 


\subsubsection{MCD spectra of CNCbl and MeCbl}

Our initial attempt was to perform MCD calculations using the single residue of the conventional quadratic response function, ${ }^{215,216}$ which refers to the infinite lifetime approximation. However, for the calculations using conventional response theory, we encountered problems due to singularities in the response functions. When the excited states are close in energy, the MCD B term diverges since the denominator includes a difference between excited-state energies. We were therefore forced to use the CPP formalism, which is significantly more time consuming (see Section 4.2 for details). In this formalism, as already noted, we calculated both the real (absorption) and imaginary (scattering) parts of a quadratic response function for a given incoming frequency of the light. Since a Lorentzian broadening is inherent in the calculation in this approach, it does not identify directly the individual transitions. The spectrum is simulated by running calculations for a large number of incoming frequencies. The broadening is an input parameter in the response calculation and it is empirical in the same way as the lifetime broadening parameter $\Gamma$, used when broadening a stick spectrum. The value of $\Gamma$ is chosen such that it best reproduced the experimental shape of the spectrum. Several transitions may contribute to each band in the simulated spectrum, and the contribution from the individual transitions is not identified directly. This is in contrast to the more conventional residue approach used for the simulation of the Abs and CD spectra above, where the individual transitions is what is actually calculated, and where a broadening is applied afterwards in order to simulate the spectrum.

For the strong MCD transitions, we can rather easily determine the excitations involved in the calculated band structure from the CPP calculations, whereas for weaker and more closely spaced excitations, it gets difficult to reproduce experiment and one may not be able to isolate all excitations involved. In these cases, our possibilities for identifying which of the close-lying excitations actually dominates the observed MCD signal become somewhat limited. 
The computed MCD spectra (Figures 4.2 and 4.3) show reasonable with experiment, though a few difficulties can be noticed. The BP86-based MCD spectrum for MeCbl agrees better with the experiment than the one based on CAM-B3LYP. This is particularly pronounced for the low-energy part of the spectrum. It can be seen that the negative band observed around $2.55 \mathrm{eV}$ in the experiment, is by CAM-B3LYP shifted into the D/E region. Actually, for CAM-B3LYP this negative MCD band seems to be related to the same transition that gives a strong band in the $\mathrm{D} / \mathrm{E}$ region of the Abs spectrum. On the other hand, the reproduction of the MCD spectrum for CNCbl is less satisfactory for BP86. The experimental MCD spectrum for CNCbl looks very different from $\mathrm{MeCbl}$ and it appears that the experimental baseline is somehow shifted. In contrast, the simulated spectra for CNCbl and MeCbl based on the BP86 functional look rather similar since the same basic corrin model has been used, and since the excitations only to a very limited extent involve orbitals on the axial CN or Me ligands.

\subsubsection{Comparison between BP86 and CAM-B3LYP results}

A direct comparison of computed and experimental data reveals that the overall performance of BP86 is better than CAM-B3LYP, though the former also has difficulties in correctly reproducing some spectroscopic features. This observation corroborates previous studies that concluded that the BP86 functional does a good job in modeling electronic transitions in cobalamins.

Regarding the quality of CAM-B3LYP, this functional is specifically designed to handle CT excitations well, where, in general, it would give slightly too low (by about $0.2 \mathrm{eV}$ ) excitation energies. For more "conventional" excitations, CAMB3LYP may perform somewhat worse than other functionals. ${ }^{212}$ Consequently, a shift may be required to adjust computed excitations. However, in terms of data presented here, one can observe that the agreement was not improved even when scaling was applied. Thus, we turned to another alternative, namely to the change of the amount of exchange that was treated using the DFT XC functional and the amount treated by Hartree-Fock exchange, as compared to the regular CAM-B3LYP 
functional. As a result, the CAM-B3LYP* calculations were performed based on the recommended values for $\alpha$ and $\beta$ in the parameterization of the CAM-B3LYP functional given in Ref. 135. At the same time, the range separation parameter $\mu$ was changed from 0.33 to 0.20 . Only Abs and CD data have been simulated (Figure 4.5). The conclusion coming from these results is that the Abs data are somehow improved, but the overall picture remains unchanged.

\subsubsection{Solvent effects}

Previous studies have demonstrated that certain electronic excitations in cobalamins are very sensitive to the environment. Consequently, a series of calculations have been carried out in the presence of water as a solvent using the PCM model. ${ }^{217-219}$ Only the Abs and CD spectra have been analyzed, since the MCD computations are significantly much more expensive, even though such calculations are in principle possible. In the case of MeCbl, results involving the $\mathrm{PCM} / \mathrm{H}_{2} \mathrm{O}$ model (Figure 4.6 and Figures A.1 and A.5 in the Appendix) do not lead to any noticeable changes in spectral properties, implying that the solvent essentially has a negligible effect as described by a dielectric continuum model. On the other hand, the presence of the solvent has noticeable influence on the CNCbl spectral properties. In particular, the lowest energy band is affected by the presence of water. This can be attributed to the key difference between $\mathrm{CNCbl}$ and $\mathrm{MeCbl}$ that lies in their axial bond involving cobalt and carbon. The $\sigma$-bonding in $\mathrm{Co}-\mathrm{C}_{\mathrm{CN}}$ is much stronger than in $\mathrm{Co}-\mathrm{C}_{\mathrm{Me}}$ in addition to the fact that the former has a much more ionic character than the latter, thus making the CNCbl complex more sensitive with respect to the surrounding environment.

\subsubsection{Basis set effect}

To date, the majority of reported TD-DFT calculations have been carried out with a split-valence quality basis set, which results in approximately $\sim 600$ contracted functions for the truncated models of cobalamins (Figure 4.1). Taking into 

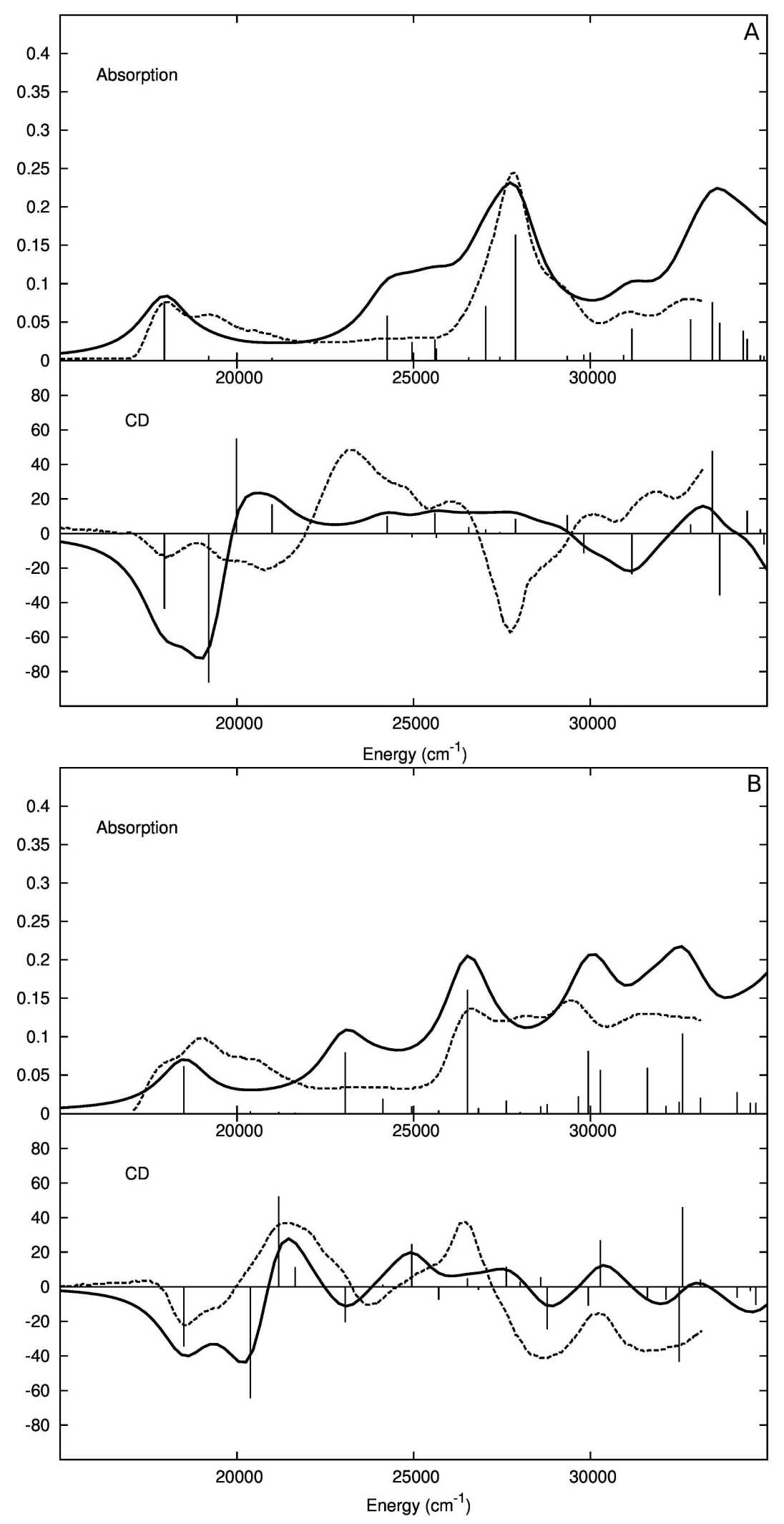

Figure 4.5. Absorption and CD spectra of CNCbl (panel A) and MeCbl (panel B) calculated with CAM-B3LYP*. Experimental spectra were reproduced from Ref. 108 with permission. 


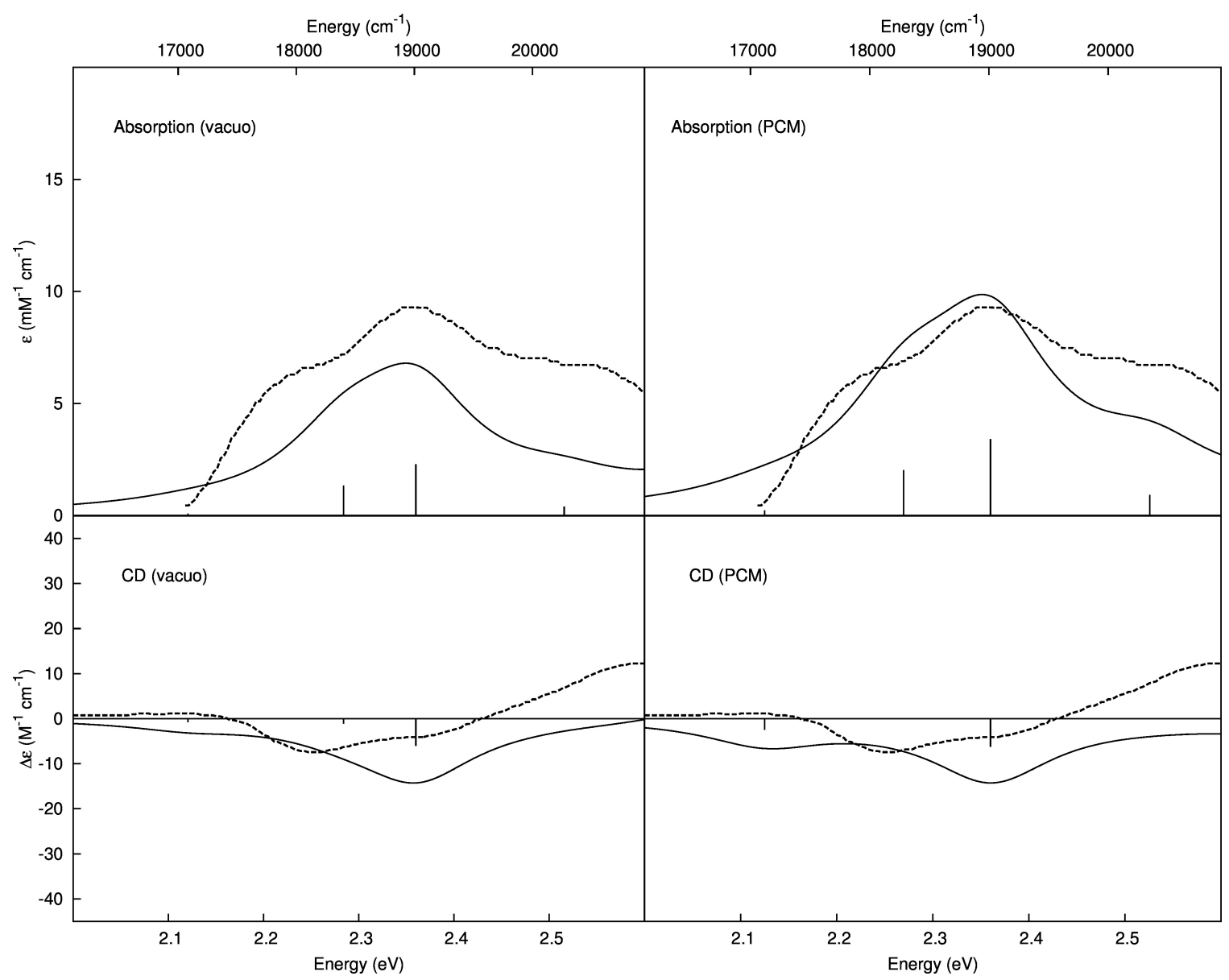

Figure 4.6. Comparison of low-energy regions of $\mathrm{MeCbl}$ absorption and $\mathrm{CD}$ spectra computed with BP86/aug-cc-pVDZ. Experimental data were taken from Ref. 108 with permission. 
account that molecular properties are more sensitive to the quality of the basis set, a question is to what extent our results are sensitive with respect to the quality of the basis set. Thus, in addition to the aug-cc-pVDZ ( $\sim 1200$ contracted functions), we also performed calculations with the 6-31G(d) basis set. Simulated Abs and CD spectra employing the small basis set can be found in Figures A.2, A.3, A.4, and A.5 (Appendix).

A visual inspection of the simulated spectroscopic data implies that calculations employing the small basis set do not significantly differ from those involving larger basis sets. However, a closer inspection of the $\Lambda$ values (Tables A.14 and A.15 in the Appendix) shows that certain electronic excitations are not adequately described by the small basis set.

\subsubsection{Assignment of electronic transitions}

Abs spectra of $\mathrm{CNCbl}$ and $\mathrm{MeCbl}$ exhibit striking similarities reflecting the fact that the corrin ligand is primarily responsible for nearly all observed spectral features. Their CD and MCD spectra differ more noticeably from each other pointing out that these properties are more sensitive to the nature of the axial ligands.

The main absorption bands of $\mathrm{CNCbl}$ as well as of MeCbl have been traditionally interpreted as being primarily of $\pi \rightarrow \pi^{*}$ type with some participation of cobalt d orbitals. ${ }^{68}$ The majority of electronic transitions were previously assigned based on TD-DFT calculations and compared with experimental data. The CD and MCD spectra were not analyzed to such an extent as it has been done for the Abs data and their spectra were not simulated for a direct comparison with experiment. In the present theoretical contribution, the 35 lowest singlet states have been computed for both cobalamins. This is sufficient to cover the experimental spectral range including the most intense $\gamma$ band. Also, according to the discussion presented above, only results based on the BP86 functional need to be taken into consideration. The solvent effect is important for CNCbl but not for MeCbl. The basis set quality (at least based on visual inspection) does not play a critical role and 6-31G(d)-based calculations can be used as a reference when assigning electronic transitions. Thus, 
the overall agreement between spectroscopic data and simulated spectra implies that a reliable assignment of electronic transitions (at least for certain spectral regions) can be achieved.

The assignment of electronic transitions is usually discussed in terms of occupied and virtual MOs involved in an excitation. Figures 4.4 and 4.7 display orbital energies along with the contours of relevant MOs extracted from the BP86/aug-ccpVDZ calculations for the $\mathrm{CNCbl}$ and MeCbl models, respectively. In the case of $\mathrm{CNCbl}$, the calculations in the presence of a solvent (as modeled via $\mathrm{H}_{2} \mathrm{O} / \mathrm{PCM}$ ) are also presented. Tables 4.1-4.3 show the summary of electronic transitions in terms of relevant MOs. Since assignment of electronic transitions has been presented previously in the literature, we do not discuss in details excitations that contribute to the Abs spectrum of $\mathrm{CNCbl}$ or MeCbl. The main motivation is to present complementary results (or reassignment), taking into account CD and MCD data. The analysis of the simulated Abs, CD and MCD spectra reveals relevant information and helps to solve bands that have remained enigmatic in their electronic origin, especially those related to the low-energetic regions of the spectra.

\section{Analysis of the $\alpha / \beta$ Band in CNCbl}

The lowest energy part of the CNCbl Abs spectrum ( $\alpha / \beta$ band, Figure 4.2) has been interpreted as a vibrational progression associated with a single corrincentered $\pi \rightarrow \pi^{*}$ electronic transition. ${ }^{66,68,108,113}$ Indeed, such an assignment seems to be consistent with the Abs spectrum alone when the B3LYP or CAM-B3LYP functionals were used to simulate the Abs spectra. However, this picture is somehow restricted in its applicability since the CD and MCD spectra of CNCbl are not well reproduced using the same level of theory. Better overall agreement was achieved when the BP86 functional was applied (Figure 4.2).

Figure 4.8 displays the close-up of the low-energy regions of CNCbl where the corresponding Abs and $\mathrm{CD}$ profiles were calculated in gas phase and in presence of a solvent described by $\mathrm{H}_{2} \mathrm{O} / \mathrm{PCM}$. Inclusion of the solvent changes the spectro- 


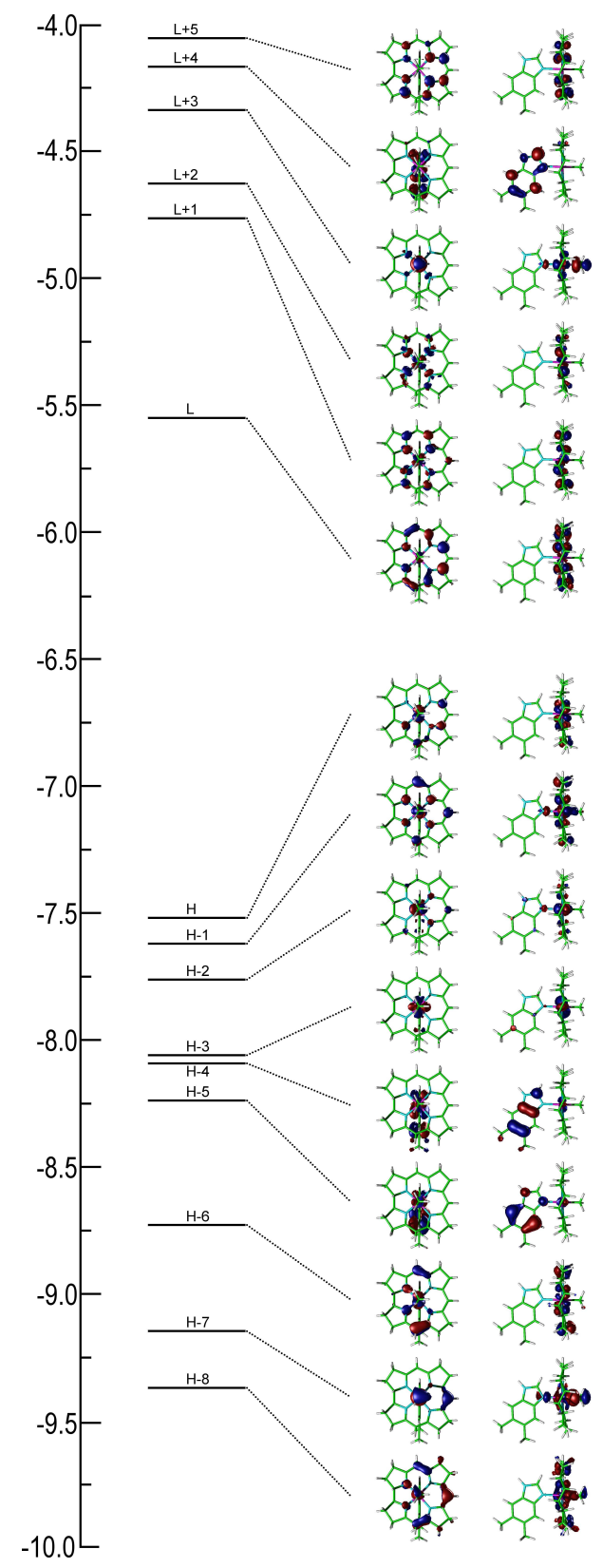

Figure 4.7. Energy (eV) diagram representing frontier MOs of DBI-[Co ${ }^{\mathrm{III}}$ (corrin)]$\mathrm{Me}^{+}$gas phase BP86/aug-cc-pVDZ calculations. 


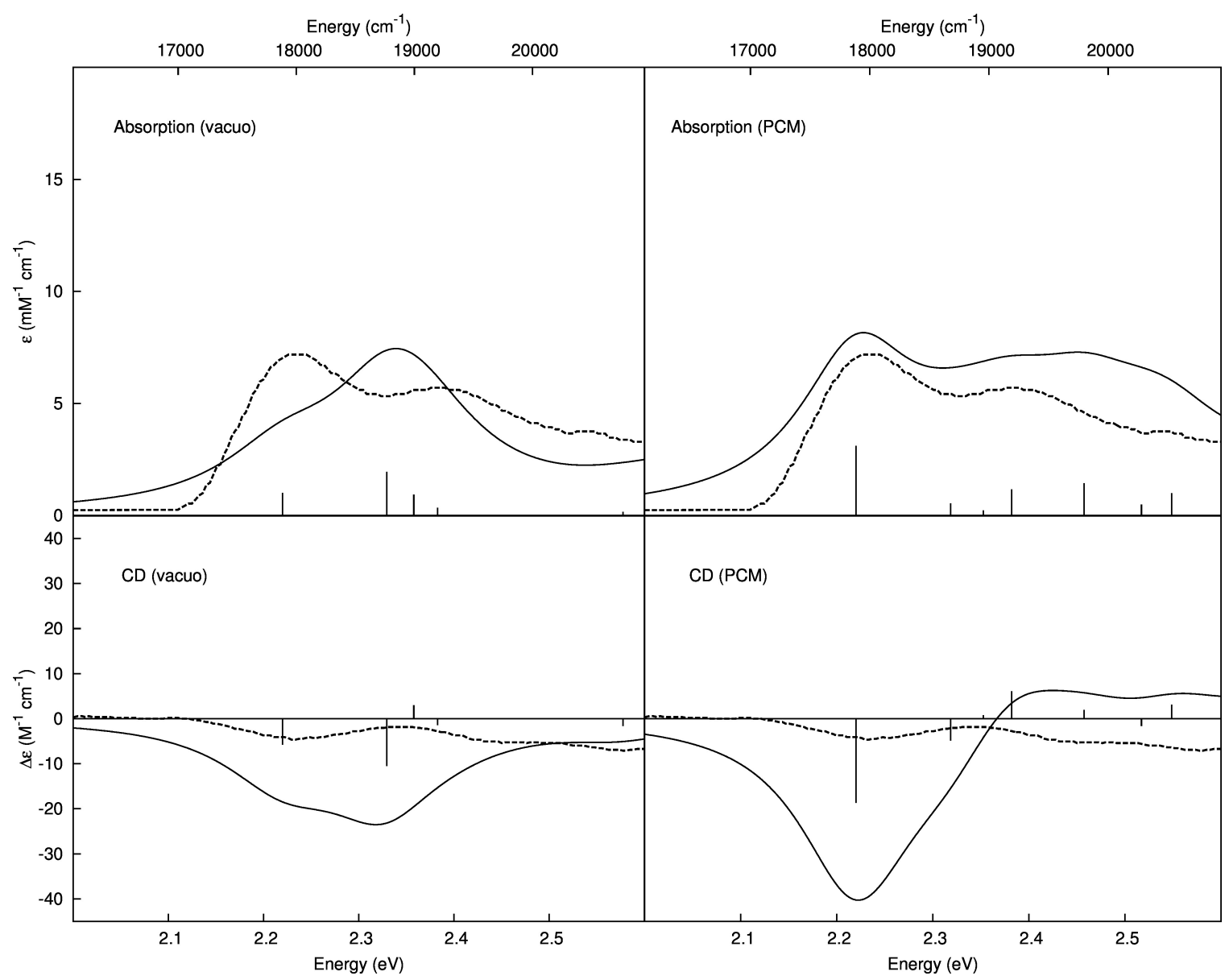

Figure 4.8. Comparison of low-energy regions of $\mathrm{CNCbl}$ absorption and $\mathrm{CD}$ spectra computed with BP86/aug-cc-pVDZ. Experimental data were taken from Ref. 108 with permission. 
TABLE 4.2. Electronic excitations of MeCbl based on BP86/aug-cc-pVDZ calculations in gas phase ${ }^{a}$.

\begin{tabular}{|c|c|c|c|c|c|}
\hline State & $\Lambda$ & $E(\mathrm{eV})$ & Osc. str. & Rot. str. & Character $^{b}$ \\
\hline $\mathrm{S}_{1}$ & 0.57 & 2.19 & 0.002 & -3.107 & $33 \%(\mathrm{H}-1 \rightarrow \mathrm{L})+12 \%(\mathrm{H}-2 \rightarrow \mathrm{L})$ \\
\hline $\mathrm{S}_{2}$ & 0.59 & 2.35 & 0.023 & -4.148 & $40 \%(\mathrm{H} \rightarrow \mathrm{L})$ \\
\hline$S_{3}$ & 0.57 & 2.42 & 0.040 & -21.387 & $31 \%(\mathrm{H}-2 \rightarrow \mathrm{L})+10 \%(\mathrm{H}-1 \rightarrow \mathrm{L})$ \\
\hline $\mathrm{S}_{4}$ & 0.57 & 2.54 & 0.000 & -0.236 & $33 \%(\mathrm{H}-4 \rightarrow \mathrm{L})+16 \%(\mathrm{H}-3 \rightarrow \mathrm{L})$ \\
\hline $\mathrm{S}_{5}$ & 0.55 & 2.57 & 0.007 & 0.317 & $29 \%(\mathrm{H}-3 \rightarrow \mathrm{L})+16 \%(\mathrm{H}-4 \rightarrow \mathrm{L})$ \\
\hline $\mathrm{S}_{6}$ & 0.50 & 2.72 & 0.008 & 7.751 & $29 \%(\mathrm{H}-5 \rightarrow \mathrm{L})+16 \%(\mathrm{H}-4 \rightarrow \mathrm{L})$ \\
\hline $\mathrm{S}_{7}$ & 0.47 & 2.91 & 0.022 & -40.109 & $38 \%(\mathrm{H} \rightarrow \mathrm{L}+1)$ \\
\hline $\mathrm{S}_{8}$ & 0.50 & 3.00 & 0.004 & -19.511 & $18 \%(\mathrm{H}-1 \rightarrow \mathrm{L}+1)+18 \%(\mathrm{H} \rightarrow \mathrm{L}+2)$ \\
\hline $\mathrm{S}_{9}$ & 0.51 & 3.12 & 0.009 & 34.588 & $16 \%(\mathrm{H} \rightarrow \mathrm{L}+2)+13 \%(\mathrm{H}-2 \rightarrow \mathrm{L}+1)$ \\
\hline$S_{10}$ & 0.54 & 3.15 & 0.007 & 37.304 & $25 \%(\mathrm{H}-1 \rightarrow \mathrm{L}+2)+7 \%(\mathrm{H}-6 \rightarrow \mathrm{L})+7 \%(\mathrm{H} \rightarrow \mathrm{L}+3)$ \\
\hline $\mathrm{S}_{11}$ & 0.52 & 3.22 & 0.006 & -50.533 & $20 \%(\mathrm{H} \rightarrow \mathrm{L}+3)+8 \%(\mathrm{H}-2 \rightarrow \mathrm{L}+2)+5 \%(\mathrm{H}-6 \rightarrow \mathrm{L})+5 \%(\mathrm{H}-1 \rightarrow \mathrm{L}+3)$ \\
\hline $\mathrm{S}_{12}$ & 0.50 & 3.27 & 0.007 & 18.756 & $16 \%(\mathrm{H}-2 \rightarrow \mathrm{L}+1)+6 \%(\mathrm{H} \rightarrow \mathrm{L}+3)+5 \%(\mathrm{H}-1 \rightarrow \mathrm{L}+1)$ \\
\hline$S_{13}$ & 0.53 & 3.34 & 0.004 & -1.874 & $35 \%(\mathrm{H}-4 \rightarrow \mathrm{L}+1)+8 \%(\mathrm{H}-3 \rightarrow \mathrm{L}+1)$ \\
\hline $\mathrm{S}_{14}$ & 0.55 & 3.36 & 0.003 & 22.631 & $15 \%(\mathrm{H}-3 \rightarrow \mathrm{L}+1)+12 \%(\mathrm{H} \rightarrow \mathrm{L}+4)+7 \%(\mathrm{H}-4 \rightarrow \mathrm{L}+1)+6 \%(\mathrm{H}-1 \rightarrow \mathrm{L}+3)$ \\
\hline$S_{15}$ & 0.50 & 3.37 & 0.000 & 0.263 & $35 \%(\mathrm{H} \rightarrow \mathrm{L}+4)+5 \%(\mathrm{H}-3 \rightarrow \mathrm{L}+1)$ \\
\hline$S_{16}$ & 0.52 & 3.42 & 0.008 & 13.198 & $19 \%(\mathrm{H}-3 \rightarrow \mathrm{L}+2)+8 \%(\mathrm{H}-2 \rightarrow \mathrm{L}+2)$ \\
\hline $\mathrm{S}_{17}$ & 0.55 & 3.43 & 0.016 & 0.080 & $15 \%(\mathrm{H}-1 \rightarrow \mathrm{L}+3)+8 \%(\mathrm{H}-2 \rightarrow \mathrm{L}+2)+6 \%(\mathrm{H}-5 \rightarrow \mathrm{L}+1)$ \\
\hline $\mathrm{S}_{18}$ & 0.47 & 3.46 & 0.005 & 1.048 & $30 \%(\mathrm{H}-1 \rightarrow \mathrm{L}+4)+8 \%(\mathrm{H}-4 \rightarrow \mathrm{L}+2)$ \\
\hline$S_{19}$ & 0.49 & 3.47 & 0.000 & 6.357 & $39 \%(\mathrm{H}-4 \rightarrow \mathrm{L}+2)+6 \%(\mathrm{H}-1 \rightarrow \mathrm{L}+4)$ \\
\hline $\mathrm{S}_{20}$ & 0.54 & 3.50 & 0.005 & -33.426 & $23 \%(\mathrm{H}-3 \rightarrow \mathrm{L}+2)+5 \%(\mathrm{H}-5 \rightarrow \mathrm{L}+2)$ \\
\hline$S_{21}$ & 0.56 & 3.55 & 0.016 & -0.984 & $21 \%(\mathrm{H}-5 \rightarrow \mathrm{L}+1)+7 \%(\mathrm{H}-1 \rightarrow \mathrm{L}+4)+5 \%(\mathrm{H}-6 \rightarrow \mathrm{L})+5 \%(\mathrm{H} \rightarrow \mathrm{L}+5)$ \\
\hline$S_{22}$ & 0.62 & 3.57 & 0.000 & -1.919 & $15 \%(\mathrm{H}-2 \rightarrow \mathrm{L}+3)+10 \%(\mathrm{H} \rightarrow \mathrm{L}+5)+5 \%(\mathrm{H}-3 \rightarrow \mathrm{L}+1)$ \\
\hline $\mathrm{S}_{23}$ & 0.59 & 3.63 & 0.000 & 7.131 & $23 \%(\mathrm{H}-7 \rightarrow \mathrm{L})+20 \%(\mathrm{H}-1 \rightarrow \mathrm{L}+5)$ \\
\hline $\mathrm{S}_{24}$ & 0.58 & 3.65 & 0.027 & 14.936 & $31 \%(\mathrm{H}-2 \rightarrow \mathrm{L}+4)+5 \%(\mathrm{H}-5 \rightarrow \mathrm{L}+2)$ \\
\hline $\mathrm{S}_{25}$ & 0.54 & 3.69 & 0.065 & -9.991 & $22 \%(\mathrm{H} \rightarrow \mathrm{L}+5)+10 \%(\mathrm{H}-2 \rightarrow \mathrm{L}+3)$ \\
\hline$S_{26}$ & 0.55 & 3.69 & 0.012 & 10.517 & $18 \%(\mathrm{H}-5 \rightarrow \mathrm{L}+2)+10 \%(\mathrm{H}-2 \rightarrow \mathrm{L}+4)$ \\
\hline $\mathrm{S}_{27}$ & 0.55 & 3.75 & 0.003 & -0.518 & $39 \%(\mathrm{H}-4 \rightarrow \mathrm{L}+3)+7 \%(\mathrm{H}-3 \rightarrow \mathrm{L}+3)$ \\
\hline $\mathrm{S}_{28}$ & 0.62 & 3.78 & 0.048 & 2.467 & $9 \%(H-5 \rightarrow L+2)+8 \%(H-6 \rightarrow L)$ \\
\hline $\mathrm{S}_{29}$ & 0.59 & 3.81 & 0.022 & -6.613 & $35 \%(\mathrm{H}-2 \rightarrow \mathrm{L}+5)$ \\
\hline$S_{30}$ & 0.56 & 3.84 & 0.070 & 20.608 & $15 \%(\mathrm{H}-7 \rightarrow \mathrm{L})+10 \%(\mathrm{H}-8 \rightarrow \mathrm{L})+9 \%(\mathrm{H}-1 \rightarrow \mathrm{L}+5)+5 \%(\mathrm{H}-2 \rightarrow \mathrm{L}+5)$ \\
\hline$S_{31}$ & 0.53 & 3.91 & 0.002 & -3.432 & $41 \%(\mathrm{H}-3 \rightarrow \mathrm{L}+4)+6 \%(\mathrm{H}-4 \rightarrow \mathrm{L}+4)$ \\
\hline $\mathrm{S}_{32}$ & 0.53 & 3.93 & 0.001 & -8.174 & $42 \%(\mathrm{H}-5 \rightarrow \mathrm{L}+3)$ \\
\hline$S_{33}$ & 0.59 & 3.97 & 0.017 & -10.440 & $22 \%(\mathrm{H}-6 \rightarrow \mathrm{L}+1)+16 \%(\mathrm{H}-8 \rightarrow \mathrm{L})$ \\
\hline $\mathrm{S}_{34}$ & 0.60 & 4.02 & 0.004 & -3.378 & $41 \%(H-3 \rightarrow L+5)$ \\
\hline $\mathrm{S}_{35}$ & & 4.04 & 0.001 & 0.909 & $42 \%(H-4 \rightarrow L+5)$ \\
\hline
\end{tabular}

${ }^{a}$ Rotational strengths are given in $\left(10^{-40} \mathrm{esu}^{2} \mathrm{~cm}^{2}\right)$ and oscillator strengths in dimensionless units. Energies presented in table were not scaled.

${ }^{b} \mathrm{H}, \mathrm{L}, \mathrm{H}-1, \mathrm{~L}+1, \ldots$, etc. correspond to energy diagram shown in Figure 4.7.

scopic data noticeably and a much better reproduction of the spectroscopic features is achieved in comparison to the spectra simulated based on gas-phase calculations. The most important conclusion that can be drawn from a comparison of experimental and simulated spectroscopic data is the number of electronic transitions associated with the $\alpha / \beta$ band. Its origin can not be attributed to a single specific electronic excitation but rather to multiple electronic transitions. According to the BP86 calculations, there are about seven electronic transitions that contribute to the low-energy spectral region below $2.6 \mathrm{eV}$. The most intense of these excitations is the first electronic transition, which can be ascribed as the $\alpha$ band, while the 
fourth excitation can be assigned to the $\beta$ band (Figure 4.8 and Table 4.3). The intensity distribution pattern is consistent with experiment and there is no need to assume a vibrational progression in order to understand the experimental spectrum. Further support comes from the comparison of simulated and experimental CD and MCD spectra (Figure 4.2). The number of transitions predicted in the $\alpha / \beta$ region by the BP86 functional affects the overall shape of the profile, giving better agreement with the experimental CD and MCD spectra.

TABLE 4.3. Electronic excitations of CNCbl based on BP86/aug-cc-pVDZ calculations in solution (water, PCM) ${ }^{a}$.

\begin{tabular}{|c|c|c|c|c|c|}
\hline State & $\bar{\Lambda}$ & $E(\mathrm{eV})$ & Osc. str. & Rot. str. & Character $^{b}$ \\
\hline $\mathrm{S}_{1}$ & 0.51 & 2.42 & 0.054 & -66.445 & $43 \%(\mathrm{H} \rightarrow \mathrm{L})$ \\
\hline $\mathrm{S}_{2}$ & 0.50 & 2.52 & 0.010 & -16.763 & $29 \%(\mathrm{H}-2 \rightarrow \mathrm{L})+11 \%(\mathrm{H}-1 \rightarrow \mathrm{L})+5 \%(\mathrm{H} \rightarrow \mathrm{L}+1)$ \\
\hline $\mathrm{S}_{3}$ & 0.48 & 2.55 & 0.004 & 2.608 & $37 \%(\mathrm{H}-3 \rightarrow \mathrm{L})+9 \%(\mathrm{H}-2 \rightarrow \mathrm{L})$ \\
\hline $\mathrm{S}_{4}$ & 0.53 & 2.58 & 0.020 & 20.328 & $15 \%(\mathrm{H}-1 \rightarrow \mathrm{L})+13 \%(\mathrm{H} \rightarrow \mathrm{L}+1)+9 \%(\mathrm{H}-3 \rightarrow \mathrm{L})+8 \%(\mathrm{H}-2 \rightarrow \mathrm{L})$ \\
\hline $\mathrm{S}_{5}$ & 0.57 & 2.66 & 0.025 & 6.306 & $25 \%(\mathrm{H} \rightarrow \mathrm{L}+1)+6 \%(\mathrm{H} \rightarrow \mathrm{L}+2)$ \\
\hline $\mathrm{S}_{6}$ & 0.59 & 2.72 & 0.009 & -5.213 & $37 \%(\mathrm{H} \rightarrow \mathrm{L}+2)+6 \%(\mathrm{H}-1 \rightarrow \mathrm{L}+1)$ \\
\hline $\mathrm{S}_{7}$ & 0.61 & 2.75 & 0.017 & 9.808 & $36 \%(\mathrm{H}-1 \rightarrow \mathrm{L}+1)+5 \%(\mathrm{H} \rightarrow \mathrm{L}+2)$ \\
\hline $\mathrm{S}_{8}$ & 0.60 & 2.88 & 0.005 & 8.296 & $36 \%(\mathrm{H}-1 \rightarrow \mathrm{L}+2)+9 \%(\mathrm{H}-2 \rightarrow \mathrm{L}+1)$ \\
\hline $\mathrm{S}_{9}$ & 0.59 & 2.93 & 0.000 & 0.316 & $48 \%(\mathrm{H}-3 \rightarrow \mathrm{L}+1)$ \\
\hline $\mathrm{S}_{10}$ & 0.59 & 2.96 & 0.001 & 2.098 & $36 \%(\mathrm{H}-2 \rightarrow \mathrm{L}+1)+6 \%(\mathrm{H}-1 \rightarrow \mathrm{L}+2)$ \\
\hline $\mathrm{S}_{11}$ & 0.51 & 2.99 & 0.022 & -14.039 & $39 \%(\mathrm{H}-4 \rightarrow \mathrm{L})+5 \%(\mathrm{H}-1 \rightarrow \mathrm{L}+3)$ \\
\hline $\mathrm{S}_{12}$ & 0.58 & 3.03 & 0.002 & -8.680 & $29 \%(\mathrm{H}-2 \rightarrow \mathrm{L}+2)+10 \%(\mathrm{H}-3 \rightarrow \mathrm{L}+2)+9 \%(\mathrm{H}-5 \rightarrow \mathrm{L})$ \\
\hline $\mathrm{S}_{13}$ & 0.59 & 3.05 & 0.001 & 0.562 & $37 \%(\mathrm{H}-3 \rightarrow \mathrm{L}+2)+8 \%(\mathrm{H}-5 \rightarrow \mathrm{L})$ \\
\hline $\mathrm{S}_{14}$ & 0.55 & 3.09 & 0.007 & 11.046 & $27 \%(\mathrm{H}-5 \rightarrow \mathrm{L})+13 \%(\mathrm{H}-2 \rightarrow \mathrm{L}+2)$ \\
\hline $\mathrm{S}_{15}$ & 0.61 & 3.30 & 0.031 & -20.045 & $37 \%(\mathrm{H}-1 \rightarrow \mathrm{L}+3)$ \\
\hline $\mathrm{S}_{16}$ & 0.60 & 3.32 & 0.073 & 9.205 & $26 \%(\mathrm{H} \rightarrow \mathrm{L}+3)+10 \%(\mathrm{H}-6 \rightarrow \mathrm{L})$ \\
\hline $\mathrm{S}_{17}$ & 0.60 & 3.38 & 0.006 & 32.537 & $39 \%(\mathrm{H}-4 \rightarrow \mathrm{L}+1)$ \\
\hline $\mathrm{S}_{18}$ & 0.60 & 3.40 & 0.004 & -6.417 & $28 \%(\mathrm{H}-3 \rightarrow \mathrm{L}+3)+20 \%(\mathrm{H}-2 \rightarrow \mathrm{L}+3)$ \\
\hline $\mathrm{S}_{19}$ & 0.60 & 3.43 & 0.015 & 0.804 & $23 \%(\mathrm{H}-2 \rightarrow \mathrm{L}+3)+20 \%(\mathrm{H}-3 \rightarrow \mathrm{L}+3)$ \\
\hline $\mathrm{S}_{20}$ & 0.61 & 3.53 & 0.001 & -6.646 & $25 \%(\mathrm{H}-5 \rightarrow \mathrm{L}+2)+17 \%(\mathrm{H}-5 \rightarrow \mathrm{L}+1)$ \\
\hline $\mathrm{S}_{21}$ & 0.65 & 3.58 & 0.014 & 4.946 & $34 \%(\mathrm{H} \rightarrow \mathrm{L}+4)+10 \%(\mathrm{H}-6 \rightarrow \mathrm{L})$ \\
\hline $\mathrm{S}_{22}$ & 0.60 & 3.60 & 0.001 & 11.180 & $30 \%(\mathrm{H}-4 \rightarrow \mathrm{L}+2)+6 \%(\mathrm{H}-5 \rightarrow \mathrm{L}+1)$ \\
\hline $\mathrm{S}_{23}$ & 0.60 & 3.66 & 0.126 & 20.425 & $18 \%(\mathrm{H}-6 \rightarrow \mathrm{L})+11 \%(\mathrm{H} \rightarrow \mathrm{L}+4)+7 \%(\mathrm{H} \rightarrow \mathrm{L}+3)$ \\
\hline $\mathrm{S}_{24}$ & 0.63 & 3.73 & 0.002 & 4.807 & $47 \%(\mathrm{H}-1 \rightarrow \mathrm{L}+4)$ \\
\hline $\mathrm{S}_{25}$ & 0.53 & 3.78 & 0.007 & -8.290 & $26 \%(\mathrm{H}-7 \rightarrow \mathrm{L})+22 \%(\mathrm{H} \rightarrow \mathrm{L}+5)$ \\
\hline $\mathrm{S}_{26}$ & 0.63 & 3.87 & 0.019 & -27.809 & $28 \%(\mathrm{H}-5 \rightarrow \mathrm{L}+3)+5 \%(\mathrm{H}-5 \rightarrow \mathrm{L}+2)+5 \%(\mathrm{H}-5 \rightarrow \mathrm{L}+1)$ \\
\hline $\mathrm{S}_{27}$ & 0.62 & 3.91 & 0.002 & -7.645 & $17 \%(\mathrm{H}-6 \rightarrow \mathrm{L}+1)+9 \%(\mathrm{H}-5 \rightarrow \mathrm{L}+3)+5 \%(\mathrm{H}-5 \rightarrow \mathrm{L}+1)+5 \%(\mathrm{H}-4 \rightarrow \mathrm{L}+3)$ \\
\hline $\mathrm{S}_{28}$ & 0.61 & 3.95 & 0.005 & -8.287 & $26 \%(\mathrm{H}-1 \rightarrow \mathrm{L}+5)+11 \%(\mathrm{H}-4 \rightarrow \mathrm{L}+3)$ \\
\hline $\mathrm{S}_{29}$ & 0.62 & 3.98 & 0.001 & 0.122 & $14 \%(\mathrm{H}-1 \rightarrow \mathrm{L}+5)+10 \%(\mathrm{H}-6 \rightarrow \mathrm{L}+1)+7 \%(\mathrm{H}-4 \rightarrow \mathrm{L}+3)+5 \%(\mathrm{H}-5 \rightarrow \mathrm{L}+3)$ \\
\hline $\mathrm{S}_{30}$ & 0.63 & 4.06 & 0.003 & -6.905 & $37 \%(\mathrm{H}-2 \rightarrow \mathrm{L}+5)+10 \%(\mathrm{H}-2 \rightarrow \mathrm{L}+4)$ \\
\hline $\mathrm{S}_{31}$ & 0.62 & 4.09 & 0.007 & -2.275 & $30 \%(\mathrm{H}-3 \rightarrow \mathrm{L}+5)+6 \%(\mathrm{H}-3 \rightarrow \mathrm{L}+4)$ \\
\hline $\mathrm{S}_{32}$ & 0.61 & 4.10 & 0.024 & -39.737 & $15 \%(\mathrm{H}-6 \rightarrow \mathrm{L}+2)+10 \%(\mathrm{H}-3 \rightarrow \mathrm{L}+5)$ \\
\hline $\mathrm{S}_{33}$ & 0.59 & 4.12 & 0.025 & 35.374 & $24 \%(\mathrm{H}-7 \rightarrow \mathrm{L}+1)+5 \%(\mathrm{H}-6 \rightarrow \mathrm{L}+3)$ \\
\hline $\mathrm{S}_{34}$ & 0.60 & 4.15 & 0.003 & 7.376 & $13 \%(\mathrm{H}-4 \rightarrow \mathrm{L}+3)+11 \%(\mathrm{H}-5 \rightarrow \mathrm{L}+1)+8 \%(\mathrm{H}-5 \rightarrow \mathrm{L}+2)$ \\
\hline $\mathrm{S}_{35}$ & 0.63 & 4.21 & 0.039 & 4.437 & $19 \%(\mathrm{H}-2 \rightarrow \mathrm{L}+4)+17 \%(\mathrm{H}-4 \rightarrow \mathrm{L}+4)+8 \%(\mathrm{H}-2 \rightarrow \mathrm{L}+5)$ \\
\hline
\end{tabular}

${ }^{a}$ Rotational strengths are given in $\left(10^{-40} \mathrm{esu}^{2} \mathrm{~cm}^{2}\right)$ and oscillator strengths in dimensionless units. Energies presented in table were not scaled.

${ }^{b} \mathrm{H}, \mathrm{L}, \mathrm{H}-1, \mathrm{~L}+1, \ldots$, etc. correspond to energy diagram shown in Figure 4.4. 


\section{Analysis of $\alpha / \beta$ Band in MeCbl}

The interpretation of the $\alpha / \beta$ band in MeCbl is less straightforward than in CNCbl. There is a noticeable difference in terms of intensity distribution between $\mathrm{MeCbl}$ and $\mathrm{CNCbl}$. The $\alpha$ band in MeCbl is less intense than the $\beta$ band, in contrast to CNCbl where the opposite pattern is observed (see Figures 4.8 and 4.6 for comparison). It has been suggested that the prominent shoulder of the $\beta$ band in the $\mathrm{MeCbl}$ Abs spectrum corresponds to the origin of another electronic transition. ${ }^{108}$ Consequently, two different electronic transitions have been proposed in order to explain the vibrational progression associated with the low-energy band.

It has been previously concluded that TD-DFT calculations only partially support the assignment that invokes a vibrational progression: ${ }^{107,108,113}$ the B3LYPbased calculation gives one intense transition in the $\alpha / \beta$ part, while there are three such transitions in the BP86-based analysis. The B3LYP functional for MeCbl describes the lowest energy transitions as mainly of $\mathrm{d} / \pi \rightarrow \mathrm{d}$ character (only the first one is a $\pi / \mathrm{d} \rightarrow \pi^{*}$ transition). On the other hand, according to the BP86-based calculations, the first three transitions are of $\mathrm{d} / \pi \rightarrow \pi^{*}$ character, and several of the subsequent ones are of $\mathrm{d} / \pi \rightarrow \pi^{*} / \mathrm{d}$ type.

According to the present analysis, it can be further concluded that the $\alpha / \beta$ band in MeCbl is composed of several electronic transitions (Figures 4.3 and 4.6). The first two correspond to the transitions involving mainly the HOMO-1, HOMO and LUMO orbitals (Table 4.2). Both occupied MOs are composed of cobalt $\mathrm{d}$ and corrin $\pi$ orbitals, although the ratio of that mixture is slightly different. Consequently, the $S_{1}$ and $S_{2}$ states can be described as $\pi / d \rightarrow \pi^{*}$ and $d / \pi \rightarrow \pi^{*}$ transitions, respectively. Such an assignment is consistent with transient absorption spectroscopy where the $S_{1}$ state was identified as MLCT. ${ }^{65}$ Moreover, taking into account the intensities of these two excitations in the simulated absorption spectra, the $\mathrm{S}_{2}$ state can be attributed to the $\alpha$ band. As noted before, the $\beta$ band in MeCbl is more intense than the $\alpha$ band. In our BP86 Abs and CD spectra, the b band appears at $\sim 2.37 \mathrm{eV}$ (Figure 4.6) and corresponds to the third electronic excitation 
$\left(\mathrm{S}_{3}\right)$. The analysis of its character indicates involvement of HOMO-2 and HOMO-1 MOs, both $\pi / d$ in nature. Hence, the character of the transition contributing to the $\beta$ part of the Abs spectrum is $\pi / d \rightarrow \pi^{*}$, though it should be noted that the orbital composition of the $S_{3}$ state looks very similar to $S_{1}$.

\section{Analysis of D/E and $\gamma$ band in CNCbl}

The Abs spectrum of CNCbl in the energetically higher region does not allow for a certain assignment of electronic transitions due to the very low intensities of such bands. However, a few features can be isolated from the CD spectra. As noted before better agreement with experiment was achieved with the BP86 functional where there are a few electronic transitions pronounced as positive signals in the region between 2.70-3.25 eV (Figure 4.2). Following the rotational strengths, the band at $\sim 2.75 \mathrm{eV}\left(\mathrm{S}_{8}\right)$ can be assigned to the $\mathrm{D}$ band. The corresponding electronic transition refers to HOMO-5 $\rightarrow$ LUMO (Table 4.1) which makes the excitation to be of $\mathrm{d} / \pi \rightarrow \pi^{*}$ character.

Further inspection of the $\mathrm{CD}$ spectrum revealed two additional transitions of noticeable rotational strengths in this region. The first one $\left(\mathrm{S}_{14}\right)$ is composed to $26 \%$ of a $\pi / \mathrm{d} \rightarrow \pi^{*}$ transition and its location at $\sim 3.05 \mathrm{eV}$ suggests the plausible assignment to the E band. At the same time, the second one is located at $\sim 3.25 \mathrm{eV}$ $\left(\mathrm{S}_{18}\right)$ and may, hence, also be considered as a transition contributing to the $\gamma$ band in "typical" Abs spectrum. However, the BP86-simulated Abs spectrum for CNCbl indicates that there are two other (i.e., $\mathbf{S}_{20}$ and $\mathbf{S}_{23}$ ) main excitations in $\gamma$ band at $\sim 3.30$ and $\sim 3.45 \mathrm{eV}$, respectively. Although they give signals with opposite signs in the $\mathrm{CD}$ spectra, their origin may be related to the same electronic transition (Table 4.1), where the main contribution comes from $(H \rightarrow L+4)$ and $(H \rightarrow L+3)$ transitions that are of $\pi \rightarrow \pi_{(\mathrm{DBI})}^{*}$ and $\pi \rightarrow \pi^{*}$ character, respectively. 


\section{Analysis of the $\mathrm{D} / \mathrm{E}$ and $\gamma$ bands in $\mathrm{MeCbl}$}

The Abs spectra of MeCbl are characterized as "unique" not only because of the interchange of the $\alpha$ and $\beta$ intensities, but also due to the number of transitions in the $\gamma$ region. Moreover, the description of the D/E part observed in "typical" Abs profiles is also more complex for this system.

Based on partially resolved CD and MCD spectra, it was suggested that at least two electronic transitions contribute to the D/E band. ${ }^{108}$ Our BP86-based CD spectra reveal the presence of at least five signals of significant rotational strengths between 2.75-3.25 eV. The first negative one, at $\sim 2.85 \mathrm{eV}\left(\mathrm{S}_{7}\right)$, corresponds to the excitation in which the electron is transferred from HOMO to LUMO+1. Both MOs possess contributions from cobalt $\mathrm{d}$ orbitals and $\mathrm{p}$ corrin, hence the excitation becomes $\mathrm{d} / \pi \rightarrow \pi^{*} / \mathrm{d}$ in character. Furthermore, its presence in the Abs spectrum suggests that it should be assigned to the D part of the spectrum. On the other hand, the E part shows three opposite features visible in the CD profiles. Whereas the main contributions to $S_{9}$ and $S_{10}$ are again transitions including both $\mathrm{d}$ and $\pi$ corrin orbitals, the negative band at $\sim 3.20 \mathrm{eV}$ refers to an excitation mainly between HOMO $(\mathrm{d} / \pi)$ and axial-based $\mathrm{d}_{z^{2}} / \sigma_{\text {(DBI-Co-Me) }}^{*}$. However, the strong signal of that band in the CD spectrum does not have a counterpart in the Abs spectrum, indicating the possible exclusion of that transition from the E region.

The $\gamma$ part of MeCbl consists of several electronic excitations that by analogy to "typical" Abs spectra (such as CNCbl) have been described as $\pi \rightarrow \pi^{*}$ transitions. ${ }^{108}$ Based on the present analysis, the manifold of transitions in the $g$ band of $\mathrm{MeCbl}$ has also contributions from excitations of different nature. Among the three most intense excitations, the energetically lowest $\left(\mathrm{S}_{25}\right)$ can be described as a $\mathrm{HOMO} \rightarrow \mathrm{LUMO}+5$ (22\%) and HOMO-2 $\rightarrow$ LUMO +3 (10\%) excitation. Whereas both occupied MOs are of mixed $d$ and $\pi$ corrin character, only $L+5$ possess significant contribution from the $\pi$ corrin orbital. The LUMO +3 , on the other hand, is the orbital localized along the axial bonds and hence described as $\mathrm{d}_{z^{2}} / \sigma_{\text {(DBI-Co-Me) }}^{*}$. The other two excitations corresponding to the $S_{28}$ and $S_{30}$ excited states are also 
mixed-type transitions involving several occupied and unoccupied MOs (Table 4.2). However, only HOMO-8 and LUMO can be denoted as clear $\pi$ corrin orbitals, pointing out that the electronic spectrum of $\mathrm{MeCbl}$ is far from being dominated primarily by $\pi \rightarrow \pi^{*}$ transitions.

\subsection{Summary and Conclusions}

The spectroscopic studies of cobalamins involving electronically excited states have been an active field of research during the past few years. Both experimental and theoretical investigations have brought significant new insight, but the question related to a complete assignment of the electronic bands and the nature of the transitions contributing to the individual excitations still remains only partially answered. In particular, information coming from the Abs spectra of $B_{12}$ derivatives is limited to transitions that have noticeable intensities. The excitations that dominate Abs spectra, i.e. have high oscillator strengths, correspond mainly to the corrin $\pi$ $\rightarrow \pi^{*}$ transitions. Transitions involving cobalt $\mathrm{d}$ orbitals are not prominent in the Abs profiles. In this respect, $\mathrm{CD}$ and MCD spectra are complementary to Abs profiles and hence, their theoretical analysis for $\mathrm{CNCbl}$ and $\mathrm{MeCbl}$ provides additional, independent insight into their electronic transitions.

In this chapter, a theoretical attempt was made to compute for the first time the $\mathrm{CD}$ and MCD spectra of the $\mathrm{CNCbl}$ and MeCbl cobalamins. In order to simulate the Abs, CD and MCD spectra of these molecules, 35 excited states were computed. The comparison with experimental data revealed that the performance of BP86 was noticeably better than CAM-B3LYP, which is specifically designed to handle CT-type excitations. This is a rather surprising outcome, because it is generally expected (at least for molecules having second-row atoms only) that the performance of the CAM-B3LYP functional is typically better than GGA-type functionals such as BP86. The hereby presented study corroborates the previously reached conclusion that BP86 remains the better functional for describing the electronically excited states of the cobalamins. 
It should be emphasized that both Abs and CD spectra of CNCbl simulated with the use of BP86 give satisfactory agreement with the experiment. Furthermore, the analysis of the transitions contributing to the $\alpha / \beta$ band shows multiple transitions rather than a single electronic excitation followed by a vibrational progression in that region. Similarly, the $\alpha / \beta$ part of MeCbl spectrum tends to be composed of several excitations as confirmed by the BP86 data.

There are several other factors that may affect the final assignment of electronic transitions. We managed to elucidate some of them in the present study. First, the calculations were performed with different basis sets. The use of the bigger aug-cc-pVDZ basis set did not affect the overall spectral shape but helped to reassign the electronic transitions contributing to the main bands in the "typical" and "unique" absorption profiles of $\mathrm{MeCbl}$ and $\mathrm{CNCbl}$, respectively. Secondly, the change of the environment implied a difference in the axial bonding, as well as the nature of the $\mathrm{Co}-\mathrm{C}$ bond in both systems. The $\mathrm{CNCbl}$, having a more ionic character of its axial bond, is expected to be more sensitive to the change of dielectric constant. Consequently, the simulation of the CNCbl spectra in water solution (PCM model) revealed some improvements, especially in the low-energy region. At the same time, Abs and $\mathrm{CD}$ spectra of MeCbl did not show significant environmental dependency. Having in mind the above observations, the question arises to what extent the truncated models (Figure 4.1) are valid for the analysis of the spectroscopic properties. The overall good agreement of simulated and experimental Abs data and some discrepancies visible in $\mathrm{CD}$ and MCD profiles may have their roots in the simplifications made to the structures. However, an analysis based on the full structures goes beyond the scope of the study presented in this chapter. Instead, this aspect is discussed in Chapter 5 where Abs, CD, and MCD spectra of cob(I)alamin were simulated with the use of both simplified and full structure of the system. 
In conclusion, the study described in this chapter presents significant improvements in the understanding of the nature of the electronic excited states in $\mathrm{CNCbl}$ and MeCbl. It is the first attempt to simulate and analyze CD and MCD spectra of these complex molecules. Although the problem still demands further analysis, our study provides additional reliable assignments of the pronounced electronic bands in "typical" and "unique" Abs spectra of cobalamins. 


\section{CHAPTER 5}

\section{COB(I)ALAMIN: INSIGHT INTO THE NATURE OF ELECTRONICALLY EXCITED STATES ${ }^{1}$}

\subsection{Introduction}

The super-reduced form of vitamin $B_{12}$ (i.e., cob(I)alamin or $B_{12 s}$, Figure 5.1) generated via two-electron reduction of Co(III)-based cobalamins (such as hydroxycobalamin) or by a one-electron reduction of $\operatorname{cob}(\mathrm{II})$ alamin $\left(\mathrm{B}_{12 \mathrm{r}}\right)$ appears as a universal intermediate in many areas of vitamin $B_{12}$ chemistry. ${ }^{159,197,220-233}$ For example, it is formed during methyl transfer reactions catalyzed by methyltransferases, ${ }^{224-230}$ which utilize the methylcobalamin (MeCbl) cofactor as a methyl carrier. The most representative of these is methionine synthase (MetH), ${ }^{11,224,234}$ which is responsible for the regeneration of methionine from homocysteine. Other examples involve methanol-dependent cobalamin or corrinoid/Fe-S protein. ${ }^{223,225}$ Another key function played by cob(I)alamin is the biosynthesis of adenosylcobalamin (AdoCbl), in a process guided by ATP:corrinoid adenosyltransferases

(ACAs). ${ }^{230-233}$ The pivotal role of cob(I)alamin is also revealed in reductive dehalogenation of halogenated compounds, a process involved in dehalorespiration in several anaerobic bacteria. ${ }^{235-241}$ Finally, the reactions with $\mathrm{N}_{2} \mathrm{O}, \mathrm{NO}, \mathrm{NO}_{2}^{-}, \mathrm{NO}_{3}^{-}$, sucralose, epoxides and $\mathrm{SO}_{2}^{-}$have also been explored, adding to the diversity of processes that rely on the super-reduced form of vitamin $\mathrm{B}_{12} \cdot{ }^{242-248}$

Although cob(I)alamin is doubtlessly one of the most important components of vitamin $B_{12}$ chemistry, it is, at the same time, a very challenging system to study. The $\mathrm{B}_{12 \mathrm{~s}}$ (where the central cobalt has formally a +1 oxidation state) possesses

\footnotetext{
${ }^{1}$ The material presented in this Chapter was reprinted (adapted) with permission from K. Kornobis, K. Ruud, and P. M. Kozlowski, J. Phys. Chem. A 117, 863 (2013). Copyright 2013 American Chemical Society.
} 


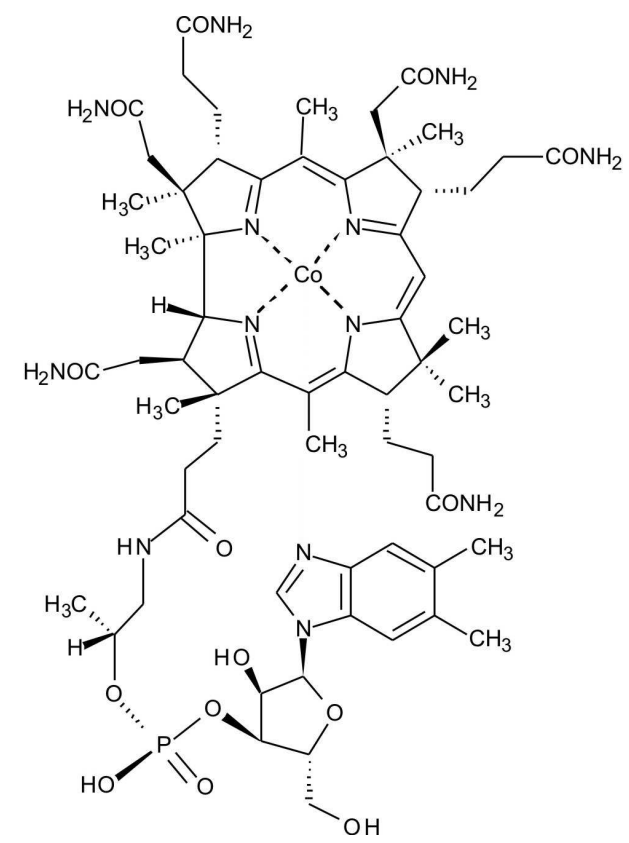

Figure 5.1. Molecular structure of super-reduced form of vitamin $B_{12}$ (cob(I)alamin or $\mathrm{B}_{12 \mathrm{~s}}$ ). Note that the lower axial position is unoccupied as opposed to another reduced derivative, i.e., $\operatorname{cob}(\mathrm{II})$ alamin $\left(\mathrm{B}_{12 \mathrm{r}}\right)$. The nucleotide loop is connected to $\mathrm{C}_{17}$ of the corrin ring.

unusually high reactivity, hindering structural and biophysical investigations. Studies comparing its nucleophilic rate constant toward methyl iodide ${ }^{249}$ and against the other methyl transfer reactions revealed extremely strong nucleophilic properties, being 14.4 according to the Pearson scale ${ }^{250}$ or 10.7 based on the SwainScott scale, ${ }^{251}$ and for this reason $\mathrm{B}_{12 \mathrm{~s}}$ is often called a supernucleophile. ${ }^{252}$ Since cob(I)alamin can be generated in transient form (which remains in equilibrium with molecular hydrogen and $\mathrm{B}_{12 \mathrm{r}}$ ), the experimental data available for cob(I)alamin on its structure in solution include X-ray edge as well as extended X-ray absorption fine structure (EXAFS) spectroscopy. ${ }^{253}$ According to these findings, the Co(I) form of $\mathrm{B}_{12 \mathrm{~s}}$ appears to be a distorted square-planar structure. Because structural data is not currently available for cob(I)alamin, only a few theoretical studies have addressed this issue using density functional theory (DFT). ${ }^{100,109,110,254}$ Calculations using the local spin-density approximation (LSDA) indicated a non-planar structure with $\mathrm{C}_{2}$ symmetry. B3LYP optimizations of a simplified model of cob(I)alamin have also shown deviations from planarity of the corrin macrocycle, ${ }^{109}$ while the PBE- 
optimized structure of the system was determined as "nearly planar". ${ }^{110}$ However, taking into account the tendency of Co(I) complexes to appear in square pyramidal coordination, as already observed in the case of cob(I)aloximes, ${ }^{255}$ most likely cob(I)alamin would adopt a similar geometry. Indeed, computational studies support the strong tendency of $\mathrm{B}_{12 s}$ to form an axial hydrogen bond, i.e. Co(I) $-\mathrm{H}$, thus adopting a square-pyramidal structure. ${ }^{197,256,257}$

At present, the main sources of knowledge about cob(I)alamin are spectroscopic techniques. The early attempts to characterize the system with absorption spectra date back the fifties, when different groups reported results for reduced forms of vitamin $\mathrm{B}_{12} \cdot{ }^{220,221,258,259}$ The following decades brought forth a number of studies focused more on explaining the conditions at which the reduced form of vitamin $B_{12}$ can be generated, ${ }^{222,260}$ its stability, ${ }^{261}$ nucleophilicity, ${ }^{249,252,262,263}$ role in catalytic cycles, ${ }^{11,234,264,265}$ the exploration of electron transfer between different $\mathrm{B}_{12}$ derivatives, ${ }^{266-268}$ reducing capabilities ${ }^{269,270}$ and more, rather than a detailed analysis of the electronic spectra of $\mathrm{B}_{12 \mathrm{~s}}$. Only recently have there been theoretical efforts to explore electronically excited states of cob(I)alamin at a molecular level employing electronic-structure calculations. ${ }^{100,109,110,159,197}$ Such studies face certain challenges due to the limitations of commonly used computational tools that can be applied to complex systems such as $\mathrm{B}_{12}$ derivatives. Whereas a detailed discussion of previous results will be provided in Section 5.3, it is important to note the work of Jensen, ${ }^{159}$ as well a recent study in our group, ${ }^{197}$ where the internal complexity of the cob(I)alamin wave function has been revealed using multi-reference CASSCF calculations. It was shown ${ }^{159}$ that the ground-state wave function consisted of only $67 \%$ closed-shell $\mathrm{Co}^{\mathrm{I}}\left(\mathrm{d}^{8}\right)$ configuration and of about $23 \%$ diradical i.e., $\mathrm{Co}^{\mathrm{II}}\left(\mathrm{d}^{7}\right)$-radical and $\operatorname{corrin}\left(\pi^{*}\right)^{1}$ character. Although the severely multi-configurational nature of the wave function makes an analysis of electronic excitations challenging, one should take into account that the partial diradical character of the ground state may be the key component responsible for the high reactivity of cob(I)alamin. 
As outlined above, our knowledge of cob(I)alamin is still very limited and the aim of the study presented in this chapter is therefore to provide a comprehensive analysis of the electronically excited states of cob(I)alamin at a molecular level by performing quantum-chemical calculations and comparing these with existing experimental data including absorption (Abs), circular dichroism (CD) and magnetic $\mathrm{CD}$ (MCD). ${ }^{110}$ When performing quantum-chemical computations, several different issues have to be addressed. Taking into account the size and complexity of the system, time-dependent DFT (TD-DFT) was chosen as the most practical tool for characterizing the electronic properties of $\operatorname{cob}(\mathrm{I})$ alamin. However, previous experiences have shown that the nature of computed excited states strongly depends on the applied exchange-correlation (XC) functional. ${ }^{105,106,140,161,271}$ Thus, Abs, CD and MCD spectra have been simulated with the use of BP86 and CAM-B3LYP, being the representatives of two different classes of functionals. In addition, the nature of the low-lying electronic transitions was inspected in terms of their correspondence to the results obtained with the CASSCF/MC-XQDPT2 method, used for benchmarking purposes. Finally, not only a simplified structural model of cob(I)alamin, as opposed to previous theoretical studies, but also a full structure, including side chains of the corrin ring, has been investigated.

\subsection{Computational details}

\subsubsection{Structural models}

Two structural models of cob(I)alamin were employed to calculate the electronically excited states required to simulate the Abs, CD and MCD spectra. Initially, the high-resolution crystal structure of the MeCbl cofactor was used to build both models. ${ }^{272}$ After the removal of both axial ligands and truncation of the nucleotide loop at the phosphodiester, the structure of the cob(I)alamin contained 140 atoms, including the corrin ring and its side chains. In the present study, this system (Figure 5.2, right panel) served as a full model of cob(I)alamin. (Note that such a derivative of cob(I)alamin lacking a lower axial base, i.e., base-off form, is classi- 


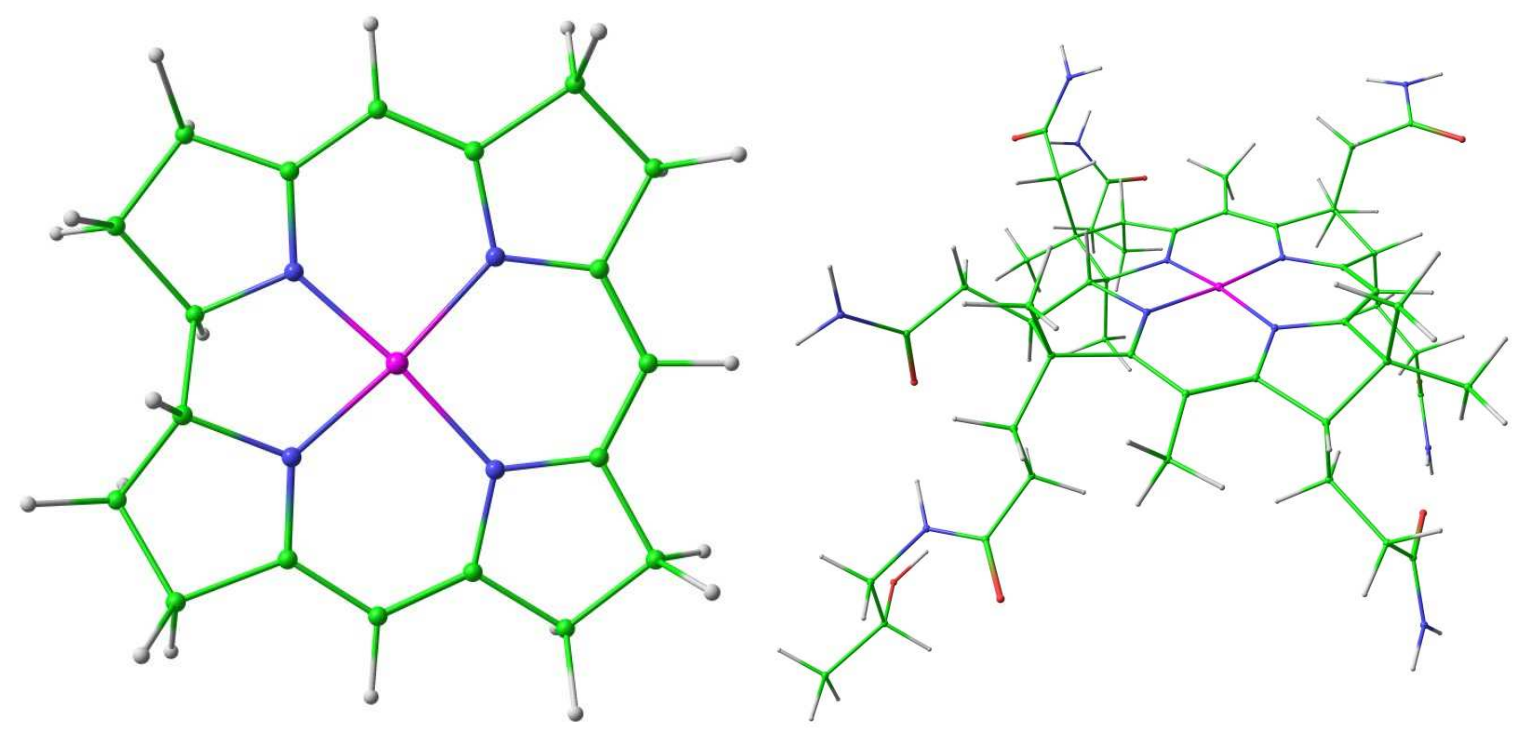

Figure 5.2. Structural models of cob(I)alamin employed in calculations: simplified (left) and full (right) without lower axial base (i.e., cob(I)inamide).

fied as cob(I)inamide but throughout the present study it will simply be referred to as cob(I)alamin.) The simplified model was obtained by further truncation of the corrin side chains and replacing them with hydrogen atoms, which reduced its size to 45 atoms. Both full and simplified structures of cob(I)alamin (Figure 5.2) have been optimized with the BP86 $6^{123,124}$ and B3LYP ${ }^{114,115}$ functionals, respectively, using the $6-31 G^{*}$ basis set. For both models, the singlet spin state was assumed, adjusting the number of electrons in order to be consistent with $a+1$ oxidation state of cobalt. The geometry optimizations have been performed in the gas phase using Gaussian 09. ${ }^{148}$

\subsubsection{Abs, CD and MCD spectra in response theory TD-DFT calculations}

The electronic spectra of $\operatorname{cob}(\mathrm{I})$ alamin were simulated with the use of response theory ${ }^{208,273}$ as implemented in the Dalton program. ${ }^{152}$ In this approach, the excited-state energies correspond to the poles of linear response functions. The transition dipole moments and the oscillator strengths were determined from the corresponding residues of the linear response functions. Scalar rotational strengths required for the simulation of the $\mathrm{CD}$ spectra were obtained in a similar man- 
ner, ${ }^{274,275}$ using London atomic orbitals ${ }^{276}$ to ensure gauge-origin independence of the calculated rotational strengths. The calculated poles and residues of the linear response functions lead to the Abs and $\mathrm{CD}$ spectra exposing the individual excitations contributing to experimental bands. In order to reproduce the shape of the Abs and CD spectra, a Lorenzian broadening was applied to each transition with a $0.1 \mathrm{eV}$ bandwidth.

The simulation of MCD spectra is more demanding as the transition matrix elements of the system are obtained from the residues of the quadratic response function. ${ }^{208,215,273,277}$ Moreover, in the regions where the calculated states are nearly degenerate in the excited-state spectrum, singularities in the response functions occur, leading to an unphysical behavior of the molecular properties near such accidental resonances. These divergences arise because of the implicit assumption of an infinite lifetime for the excited states in standard response theory. The finite lifetime of the excited states can be introduced by using the complex polarization propagator (CPP) approach ${ }^{209,278}$ in which an empirical damping parameter (chosen to be 0.004556 a.u. in our calculations) imposes a Lorenzian line-shape function, allowing for the direct calculation of MCD spectra with no divergences in the calculated spectra. ${ }^{210}$ Such an approach was here used to calculate the MCD spectra of cob(I)alamin. We recall that in this framework, the poles are moved into the complex plane, making the real-valued polarizability well defined. The MCD rotational strength is described by the imaginary part of the quadratic complex polarization propagator.

All spectra were simulated with TD-DFT/6-31G* on the previously optimized geometries employing the BP86 and B3LYP functionals. Subsequent excited-state calculations were performed with the BP86 and CAM-B3LYP ${ }^{135}$ functionals, respectively. Whereas the ground-state structural properties were essentially insensitive with respect to the functional used, in contrast, the excited states were very sensitive to the choice of XC functional. Recent theoretical studies ${ }^{140,161,163,271}$ on cobalamins support the use of gradient-corrected functionals, such as BP86, when studying excited states of these systems compared to, for instance, the use of hybrid functionals. 
On the other hand, the use of the Coulomb-attenuated CAM-B3LYP functional is often considered important due to the fact that TD-DFT calculations often suffer from an incorrect description of Rydberg and CT excitations. ${ }^{137,138}$ Both methods were used in the present chapter in calculations on the simplified and full structure of cob(I)alamin. In addition, the possibility of CT failure was analyzed by evaluation of the $\Lambda$ parameter. ${ }^{137}$ This simple test, measuring the degree of overlap of the occupied and virtual orbitals participating in an excitation in the system has been suggested as a diagnostic tool for whether computed TD-DFT excitation energies can be expected to be unreliable. It is, thus, only a negative test and does not guarantee that a computed result is reliable. It has been shown that its numerical value (ranging from 0 to 1 ) correlate well with the extent of the excitation energies errors, ${ }^{137,138}$ with smaller values signifying such a possibility. Consequently, the $\Lambda$ was computed for all excited states discussed in this study. The basis set used in all calculations was the 6-31G* set. Previous numerical experience has shown that results are not very sensitive to the size of the basis set in cases where the excitations are largely located in the core of the molecules, as the basis functions on the side chains then can act as the necessary polarization functions to the region in which the excitation takes place. ${ }^{279}$ Recent investigations targeting excitations of MeCbl and $\mathrm{CNCbl}$ indicated that even doubling the number of contracted functions (from $\sim 600$ in $6-31 G^{*}$ to $\sim 1200$ in aug-cc-pVDZ) did not significantly affect their spectra (see Chapter 4 or Ref. 140). The systematic analysis of low-lying excited states of MeCbl (see Chapter 3 or Ref 271), where results of different level of theories were compared, had also indicated a negligible effect of the size of the basis set on the transitions of the system. Taking this into account as well as the significant increase in the computational time when increasing the basis set, the use of $6-31 \mathrm{G}^{*}$ in the study presented in this is justified. 


\subsubsection{CASSCF/MC-XQDPT2 calculations}

Due to the multi-configurational character of the $\operatorname{cob}(\mathrm{I})$ alamin ground-state wave function, ${ }^{159,197}$ CASSCF calculations were performed on the simplified model of the system optimized at the BP86/6-31G* level of theory. The state-average (SA) CASSCF were carried out by computing 20 states using the same basis set. The resulting excitation energies together with their composition in terms of molecular orbitals (MOs) are listed in Table A.17 in the Appendix. Excitation energies obtained at the CASSCF level are not accurate and need to be corrected by second-order perturbation theory. To accomplish this, the quasi-degenerate perturbation theory of Nakano, ${ }^{154}$ (QDPT2) based on the multiconfigurational CASSCF reference function was applied. The MC-XQDPT2 version, which is based on the effective Hamiltonian approach, was used in our studies as implemented in the PC-GAMESS/Firefly quantum chemistry package. ${ }^{153}$ It should be noted that the CASSCF/MC-XQDPT2 formalism, which allows for true mixing of the zeroth-order CASSCF states, differs from commonly used CASSCF/CASPT2 approaches. ${ }^{165,194,280,281}$ This kind of mixing of states have to be considered particularly when the nature of the excited states is analyzed, and may be the origin of the differences in the character of the excitations obtained in the current work and the results of earlier studies based on CASSCF/CASPT2 $2^{159}$ approaches.

The selection of a proper active space becomes a challenging task when calculations of transition metal complexes are involved. Since the accuracy of CASSCF calculations for these molecules requires an inclusion of $4 \mathrm{~d}$ orbitals in the active space, in addition to $3 \mathrm{~d}$ (double-shell effect ${ }^{158}$ ), one can very quickly reach a size limit of the calculations for large molecules. The studies on corrin and corrolbased $^{159,160}$ complexes have guided a selection of active orbitals for cob(I)alamin in our previous investigations. ${ }^{197}$ The truncated structure of cob(I)alamin (Figure 5.2, left) has $\mathrm{C}_{2}$ symmetry with a two-fold axis passing through $\mathrm{C}_{10}$, cobalt and bisecting the short corrin bond. According to the DFT calculations, the single-reference wave function consists of 50 doubly occupied orbitals of symmetry $a$ and 45 of sym- 
metry $b$, and possesses dominant $\mathrm{d}^{8}$ ground-state electronic configuration. Taking into account an approximate square-planar structure of $\operatorname{cob}(\mathrm{I})$ alamin together with crystal field theory, such a $\mathrm{d}^{8}$ electronic configuration would have the $\mathrm{d}_{x^{2}-y^{2}}$ orbital empty. (Note, that this particular labeling of the empty d orbital assumes that the $\mathrm{z}$ axis is taken as the normal vector to the corrin macrocycle and the $\mathrm{C}_{2}$ two-fold symmetry is between the $\mathrm{x}$ and $\mathrm{y}$ axes. Such a labeling was applied in former studies of cob(I)alamin by Jensen. ${ }^{159}$ In order to obtain a consistent description of the molecular orbitals in the current work between the CASSCF results and previously reported TD-DFT calculations for cobalamins with axial ligands lacking symmetry, the cob(I)alamin frame was assumed to be oriented within the xy plane, while the $\mathrm{C}_{2}$ axis coincided with the $\mathrm{x}$ axis (Figure 5.3). This corresponds to a 45 deg rotation of the active orbitals described previously ${ }^{159,197}$ and exchanges an unoccupied orbital $\mathrm{d}_{x^{2}-y^{2}}$ for $\mathrm{d}_{x y}$. Despite the difference in the definition of the reference coordinate frame between the current work and previous CASSCF studies, the same set of 10 active electrons distributed among 11 active orbitals, as depicted on Figure 5.3, was used for calculations of 20 excited states of cob(I)alamin. More specifically, $6 \mathrm{a}+4 \mathrm{~b}$ orbitals with $\mathrm{d}$ character were included in the active space in order to ensure that the double shell effect is captured. In addition, the active space contains an extra corrin orbital obtained as the lowest unoccupied linear combination of the corrin $\pi$ orbitals. 


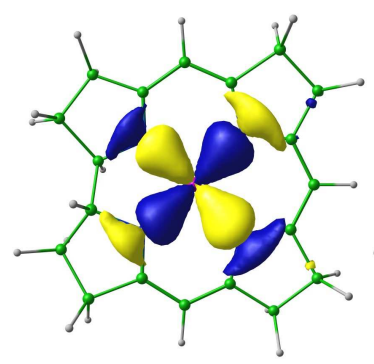

(91b) $3 \mathrm{~d}_{x y}+\mathrm{n}$

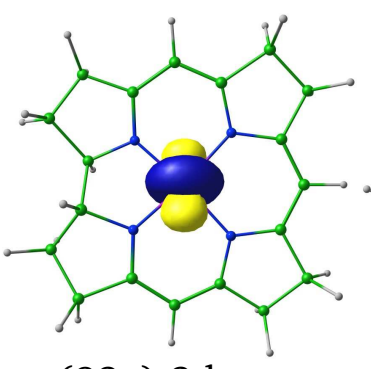

(92a) $3 \mathrm{~d}_{x^{2}-y^{2}}$ (distorted)

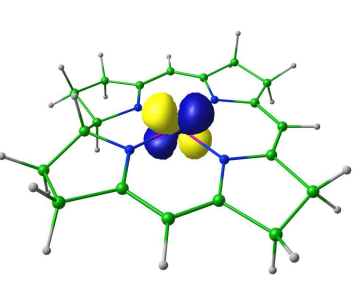

(93b) $3 d_{x z}$

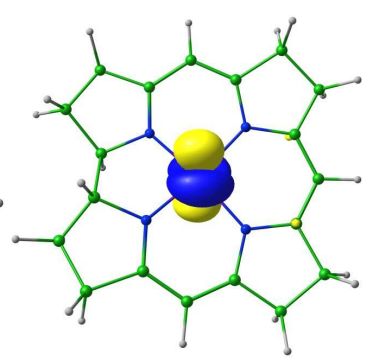

(94a) $3 d_{y z}$

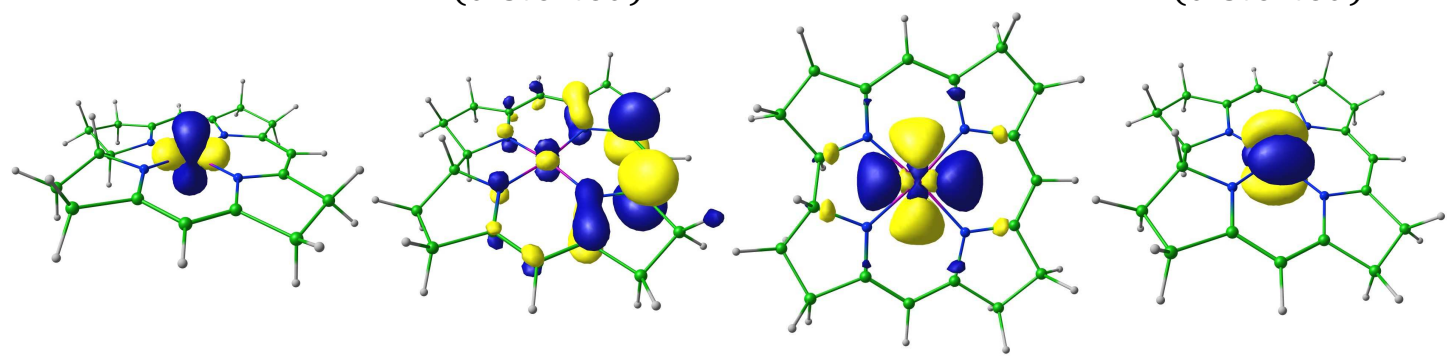

(95a) $3 d_{z^{2}}$

(distorted)

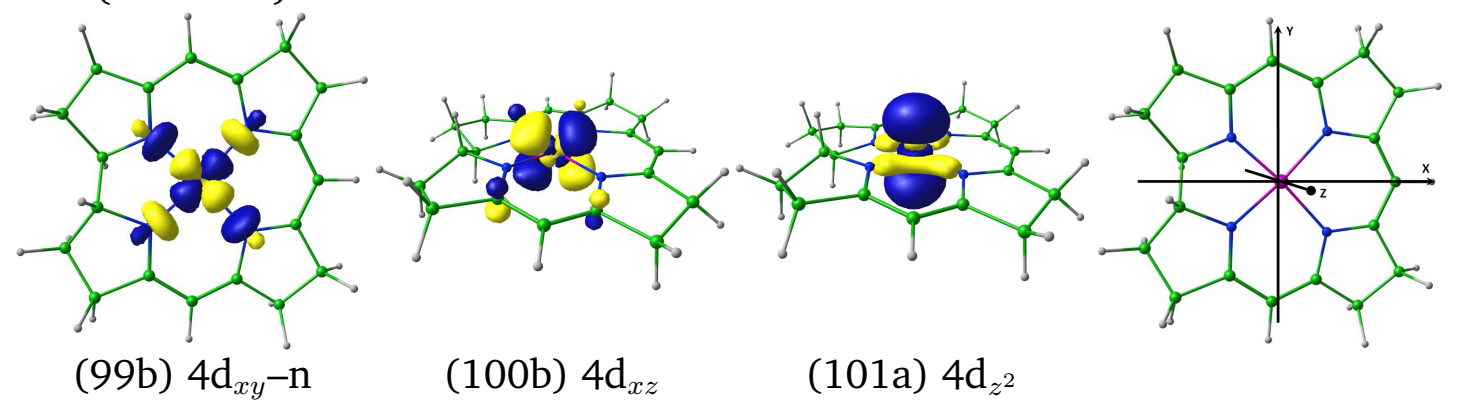

Figure 5.3. CASSCF Active Space Orbitals used in the calculations of the cob(I)alamin model system. The last inset shows the coordinate system used to label MOs in present study. Note that $\mathrm{C}_{2}$ symmetry axis coincides with $\mathrm{x}$ axis in this frame while $\mathrm{n}$ denotes lone pair on corrin nitrogens. See Section 5.2.3 for details. 

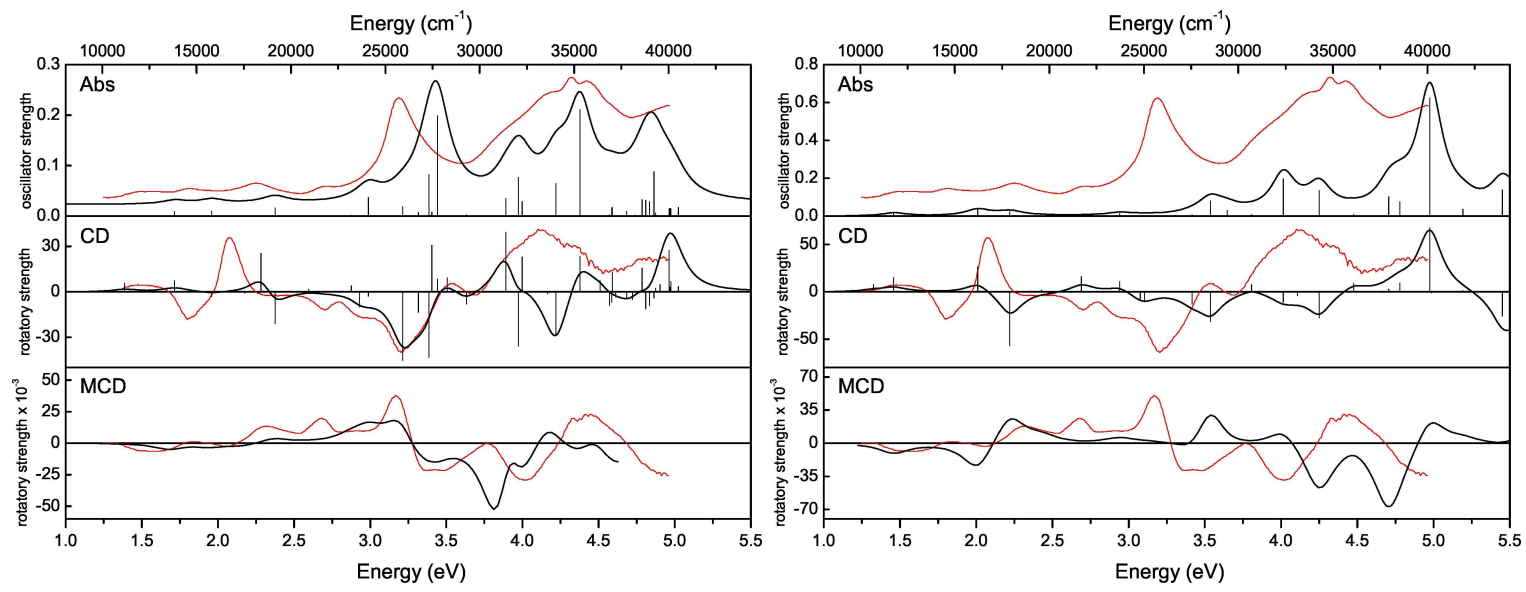

Figure 5.4. Abs, $\mathrm{CD}$ and MCD spectra of cob(I)alamin (simplified model) calculated with BP86/6-31G* (left) and CAM-B3LYP/6-31G* (right). Experimental data (plotted in red) were taken from Ref. 110.
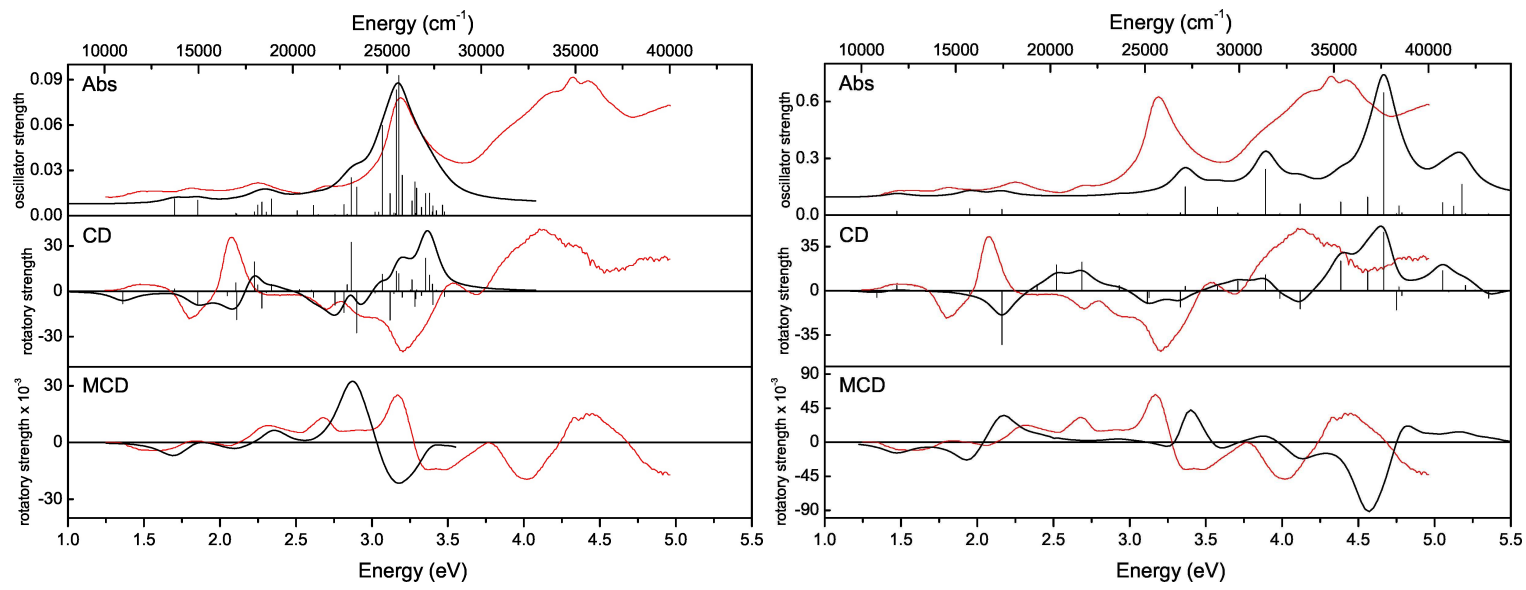

Figure 5.5. Abs, CD and MCD spectra of cob(I)alamin (full structure) calculated with BP86/6-31G* (left) and CAM-B3LYP/6-31G* (right). Experimental data (plotted in red) were taken from Ref. 110. 


\subsection{Results and discussion}

\subsubsection{Brief summary of earlier work}

Electronically excited states of cob(I)alamin have been the subject of several experimental and theoretical investigations. Although its Abs spectrum was reported long time ago, ${ }^{220,221,258,259}$ the assignment of individual bands in terms of electronic transitions remains elusive. Jaworska and co-workers ${ }^{109}$ performed the first systematic analysis where the origin of the major bands was explored in the TD-DFT framework using the B3LYP functional. The calculated electronic transitions were compared with experimental ones and the dependence on the basis set was also tested. It turned out that the quality of the basis set has a relatively small influence on the results, especially for the lower-energy regions. However, some problems were noticed in the case of the higher-energy Rydberg states, which may be attributed to the incorrect asymptotic behavior of the B3LYP functional. To rectify this problem, the CAM-B3LYP functional, which was designed to correct the long-range asymptotic behavior, ${ }^{137,138}$ was employed in the present study.

An important step in characterizing the electronic spectrum of cob(I)alamin was made by Jensen, who applied CASSCF/CASPT2 calculations to describe the six most intense peaks in the visible part of the Abs spectrum. ${ }^{159}$ The main absorption features were analyzed and the character of the lowest electronic transitions was discussed in terms of relevant molecular orbitals (MOs). Based on this analysis, the electronic transitions involve excitations of electrons between corrin $\pi$ and Co $d$ MOs what emphasized the importance of $d$ orbitals. Moreover, this study (as well as the recent in our group ${ }^{198}$ ) have brought attention to the complexity of the ground-state wave function of cob(I)alamin. As already noted in the Introduction (Section 5.1), the CASSCF wave function was found to consist of less than $70 \%$ closed-shell $\mathrm{Co}^{\mathrm{I}}\left(\mathrm{d}^{8}\right)$ configuration and of more than $20 \%$ diradical $\mathrm{Co}^{\mathrm{II}}\left(\mathrm{d}^{7}\right)$-radical corrin $\left(\pi^{*}\right)^{1}$ contribution. 
The appearance of the diradical contribution was attributed to the overlap of the low-lying metal d orbitals with ligand orbitals in the singlet ground state, allowing for the transfer of an electron from the Co to the corrin ligand.

The subsequent experimental and computational study of Brunold and coworkers enriched our knowledge about the electronically excited states of cob(I)alamin by providing supplementary results from CD and MCD spectroscopy. ${ }^{110}$ Due to different selection rules, some low-lying transitions gained measurable intensities in the $\mathrm{CD}$ and $\mathrm{MCD}$ spectra, allowing for an identification of additional excitations, particularly those contributing to the $\alpha / \beta$ and D/E parts. These authors also performed an analysis of the electronic transitions based on a Gaussian deconvolution of the experimental bands and TD-DFT calculations employing the PBE functional. The comparison with earlier B3LYP/TD-DFT ${ }^{109}$ and CASSCF/CASPT2 ${ }^{159}$ reports, where excitations of cob(I)alamin were addressed, reveals some differences. According to the B3LYP data by Jaworska and Lodowski, ${ }^{109}$ the nature of the low-lying excited states was mainly associated with metal-to-ligand charge transfer (MLCT). Similarly, the PBE results obtained by Brunold and co-workers implied participation of Co d orbitals in the main bands of cob(I)alamin Abs spectra as MLCT excitations. ${ }^{110}$ However, they refuted the previously implied ${ }^{159}$ partially open-shell character (i.e. antiferromagnetically coupled $\operatorname{Co}\left(\mathrm{d}^{7}\right)$-radical with corrin $\left(\pi^{*}\right)^{1}$ contribution to the ground state), arguing that PBE/TD-DFT calculations support a closedshell $\operatorname{Co}\left(\mathrm{d}^{8}\right)$ singlet description of $\operatorname{cob}(\mathrm{I})$ alamin. The same authors also pointed out an inconsistency related to the assignment of the low-energy peaks (footnote 85 in Ref. 110). Although two oppositely signed features were observed in this region of the MCD spectrum, they were assigned as parts of a vibrational progression on the basis of their very weak signals rather than two independent electronic transitions. Although possible, as sign changes due to Herzberg-Teller interactions have been observed in chiroptical spectra, ${ }^{282,283}$ this remains a rather unlikely explanation for the observed oppositely signed features.

Taking into account the above summary, it is clear that a consensus regarding the nature of the electronic excitations in $\operatorname{cob}(\mathrm{I})$ alamin has not yet been reached. 


\subsubsection{Abs spectra}

The Abs spectrum of cob(I)alamin possesses many distinct bands with the largest-intensity one located at $3.22 \mathrm{eV}$. In $\mathrm{B}_{12}$ cofactors, this peak is commonly referred to as the $\gamma$ band and is the main spectral feature which serves as a reference for distinguishing the "unique" Abs of alkylcobalamins from "typical" (alternatively, the terms "anomalous" and "normal" have been used ${ }^{67}$ ) ones characteristic for nonalkylcobalamins. The $\gamma$ band of cob(I)alamin resembles the corresponding region of the electronic spectrum in aquacobalamin $\left(\mathrm{H}_{2} \mathrm{OCbl}^{+}\right)$and $\mathrm{CNCbl}$ because of the sharp, highly intense peak that is recurrent for non-alkylcobalamins. The typical Abs spectrum of cobalamins is characterized by several bands that by analogy with porphyrins have been categorized as $\alpha, \beta, \gamma, \delta$ in accordance with their increasing energy order. ${ }^{66,68}$ The lower-energy region of the spectrum contains several bands with small intensities. They can be divided into two groups: the $\alpha / \beta$ part which covers the 2.07-2.95 eV (600-420 nm) range and the following additional features in the 2.95-3.18 eV (420-390 $\mathrm{nm}$ ) region commonly referenced as D/E bands. The higher energy part is associated with the $\gamma$ band located between 3.35-3.54 eV (420-350 $\mathrm{nm}$ ) followed by the $\delta$ band being present around 3.76-4.13 eV (330-300 $\mathrm{nm}) .{ }^{66,68}$ Depending on the nature of the axial ligands, these bands are shifted on the energy scale. In the particular case of cob(I)alamin the largest intensity peak is present at $3.22 \mathrm{eV}$ in the Abs spectrum reported by Brunold et al. The other five main absorption features indicated in the same study for cob(I)alamin were centered at 1.55, 1.86, 2.23 and $2.73 \mathrm{eV}$, respectively.

The $\alpha / \beta$ part of the cobalamin spectra has traditionally been interpreted as a single corrin-centered $\pi \rightarrow \pi^{*}$ electronic transition followed by its vibrational progression. ${ }^{66,68}$ However, recent theoretical studies question such an interpretation, and the inconsistency of such an interpretation has also been noted by Brunold and co-workers, ${ }^{110}$ who observed two weak but oppositely signed features in the low-energy region of the measured MCD spectrum of cob(I)alamin. A comprehensive analysis of the $S_{1}$ state of MeCbl revealed a metal-to-ligand charge transfer 
(MLCT) nature, ${ }^{271}$ as opposed to a $\pi \rightarrow \pi^{*}$ character. Moreover, calculations of the Abs, CD and MCD data and comparison with experiment showed that the $\alpha / \beta$ band for $\mathrm{CNCbl}$ and $\mathrm{MeCbl}$ did not consist of a single electronic transition, but rather multiple excited-state absorption bands. ${ }^{140}$ A similar conclusion can be expected for $\operatorname{cob}(\mathrm{I})$ alamin. Both the $\alpha / \beta$ and the neighboring $\mathrm{D} / \mathrm{E}$ part of the Abs spectrum exhibit features that are hardly visible due to their low intensities.

The comparison of TD-DFT/CAM-B3LYP- and TD-DFT/BP86-based simulated spectra with experimental Abs data highlights significant differences (Figure 5.4). Both XC functionals overestimate the excitation energies. However, the results based on BP86 calculations provide a better agreement with experiment (Figure 5.4, Table 5.1). The most intense excitation calculated in a range from 1.0 to $4.0 \mathrm{eV}$ has been found at $3.44 \mathrm{eV}$. This transition can be attributed to the $\gamma$ band as it is blueshifted by only $0.22 \mathrm{eV}$ as compared to experiment, which is within the typical range of TD-DFT excitation energy errors for valence transitions, being normally about 0.1-0.5 eV. ${ }^{137}$ However, another transition, with approximately half the intensity, is also present in this region. The $\mathrm{S}_{13}$ appears at $3.39 \mathrm{eV}$ and has a similar character as the most intense $S_{15}$ transition (mixed $\pi \rightarrow \pi^{*} / d$ and $d \rightarrow d$ ) with the main difference compared to the higher-energy excitation being the larger contribution of $d \rightarrow d$ instead of $\pi \rightarrow \pi^{*} / d$. The lower-energy part of the BP86-computed spectrum indicates the presence of six excitations with oscillator strengths larger than 0.005 . Judging by the energy values, the $S_{2}, S_{3}, S_{6}$ states could be attributed to the $\alpha / \beta$ part as they are slightly blue-shifted with respect to experiment. Similarly, the $S_{10}, S_{11}, S_{12}$ could contribute to the D/E band. However, this assumption cannot be based only on the analysis of the Abs spectrum. Some of the states with small transition dipole moments do not appear in the Abs spectrum, but gain larger rotational strengths and, therefore, become visible in the $\mathrm{CD}$ and MCD spectra (vide infra). 
TABLE 5.1. Excitation energies ${ }^{a}$ together with oscillator and rotatory strengths ${ }^{b}$ (CD) of 38 states of cob(I)alamin (simplified model) calculated with BP86/6-31G*.

\begin{tabular}{|c|c|c|c|c|c|}
\hline State & $\Lambda$ & Energy $(\mathrm{eV})$ & o.s. & r.s. & Character \\
\hline $\mathrm{S}_{1}$ & 0.45 & 1.387 & 0.000 & 5.72 & $93 \%\left(\mathrm{~d}_{z 2} \rightarrow \pi^{*}+\mathrm{d}_{y z}\right)$ \\
\hline $\mathrm{S}_{2}$ & 0.56 & 1.715 & 0.009 & 7.44 & $92 \%\left(\mathrm{~d}_{x z}^{z}+\pi \rightarrow \pi^{*}+\mathrm{d}_{y z}\right)$ \\
\hline $\mathrm{S}_{3}$ & 0.72 & 1.959 & 0.010 & -3.02 & $76 \%\left(\mathrm{~d}_{y z}+\pi \rightarrow \pi^{*}+\mathrm{d}_{y z}\right)+11 \%\left(\mathrm{~d}_{x z}+\pi \rightarrow \pi^{*}+\mathrm{d}_{x z}\right)$ \\
\hline $\mathrm{S}_{4}$ & 0.40 & 2.176 & 0.000 & -0.75 & $95 \%\left(\mathrm{~d}_{z^{2}} \rightarrow \pi^{*}+\mathrm{d}_{x z}\right)^{9}$ \\
\hline $\mathrm{S}_{5}$ & 0.44 & 2.283 & 0.001 & 25.37 & $94 \%\left(\mathrm{~d}_{x^{2}-y^{2}}^{z^{2}} \rightarrow \pi^{*}+\mathrm{d}_{y z}\right)$ \\
\hline $\mathrm{S}_{6}$ & 0.58 & 2.375 & 0.016 & -21.18 & $82 \%\left(\mathrm{~d}_{y z}+\pi \rightarrow \pi^{*}+\mathrm{d}_{x z}\right)$ \\
\hline $\mathrm{S}_{7}$ & 0.30 & 2.596 & 0.000 & 1.38 & $89 \%\left(\mathrm{~d}_{z^{2}} \rightarrow \pi^{*}\right)$ \\
\hline $\mathrm{S}_{8}$ & 0.52 & 2.876 & 0.002 & 3.83 & $59 \%\left(\mathrm{~d}_{x z}^{z^{2}}+\pi \rightarrow \pi^{*}\right)+37 \%\left(\pi \rightarrow \pi^{*}+\mathrm{d}_{y z}\right)$ \\
\hline $\mathrm{S}_{9}$ & 0.47 & 2.928 & 0.000 & -9.94 & $54 \%\left(\mathrm{~d}_{x^{2}-y^{2}} \rightarrow \pi^{*}+\mathrm{d}_{x z}\right)+37 \%\left(\mathrm{~d}_{y z}+\pi \rightarrow \mathrm{d}_{x y}+\mathrm{n}\right)$ \\
\hline $\mathrm{S}_{10}$ & 0.62 & 2.988 & 0.037 & -2.83 & $70 \%\left(\mathrm{~d}_{y z}+\pi \rightarrow \pi^{*}\right)+16 \%\left(\mathrm{~d}_{x z}+\pi \rightarrow \pi^{*}+\mathrm{d}_{x z}\right)$ \\
\hline$S_{11}$ & 0.63 & 3.214 & 0.018 & -45.39 & $40 \%\left(\mathrm{~d}_{x z}+\pi \rightarrow \pi^{*}+\mathrm{d}_{x z}\right)+17 \%\left(\mathrm{~d}_{x z}+\pi \rightarrow \mathrm{d}_{x y}+\mathrm{n}\right)$ \\
\hline $\mathrm{S}_{12}$ & 0.48 & 3.317 & 0.007 & -13.61 & $41 \%\left(\mathrm{~d}_{x^{2}-y^{2}} \rightarrow \pi^{*}+\mathrm{d}_{x z}\right)+36 \%\left(\mathrm{~d}_{y z}+\pi \rightarrow \mathrm{d}_{x y}+\mathrm{n}\right)+14 \%\left(\mathrm{~d}_{z^{2}} \rightarrow \mathrm{d}_{x y}+\mathrm{n}\right)$ \\
\hline $\mathrm{S}_{13}$ & 0.53 & 3.385 & 0.082 & -43.16 & $49 \%\left(\mathrm{~d}_{z^{2}} \rightarrow \mathrm{d}_{x y}+\mathrm{n}\right)+21 \%\left(\pi \rightarrow \pi^{*}+\mathrm{d}_{y z}\right)+15 \%\left(\mathrm{~d}_{x z}+\pi \rightarrow \pi^{*}\right)$ \\
\hline $\mathrm{S}_{14}$ & 0.39 & 3.405 & 0.008 & 30.65 & $61 \%\left(\mathrm{~d}_{x^{2}-y^{2}} \rightarrow \pi^{*}\right)+21 \%\left(\mathrm{~d}_{x z}+\pi \rightarrow \mathrm{d}_{x y}+\mathrm{n}\right)$ \\
\hline $\mathrm{S}_{15}$ & 0.56 & 3.442 & 0.199 & 8.40 & $28 \%\left(\pi \rightarrow \pi^{*}+\mathrm{d}_{y z}\right)+19 \%\left(\mathrm{~d}_{z} 2 \rightarrow \mathrm{d}_{x y}+\mathrm{n}\right)+14 \%\left(\mathrm{~d}_{x z}+\pi \rightarrow \pi^{*}\right)+13 \%\left(\mathrm{~d}_{y z}+\pi \rightarrow \mathrm{d}_{x y}+\mathrm{n}\right)$ \\
\hline$S_{16}$ & 0.46 & 3.506 & 0.001 & 9.16 & $54 \%\left(\mathrm{~d}_{x z}+\pi \rightarrow \mathrm{d}_{x y}+\mathrm{n}\right)+34 \%\left(\mathrm{~d}_{x^{2}-y^{2}} \rightarrow \pi^{*}\right)$ \\
\hline $\mathrm{S}_{17}$ & 0.58 & 3.633 & 0.003 & -8.01 & $98 \%\left(\mathrm{~d}_{x^{2}-y^{2}} \rightarrow \mathrm{d}_{x y}+\mathrm{n}\right)$ \\
\hline $\mathrm{S}_{18}$ & 0.71 & 3.690 & 0.000 & 0.74 & $50 \%\left(\pi \rightarrow \pi^{*}+\mathrm{d}_{y z}\right)+21 \%\left(\pi \rightarrow \pi^{*}+\mathrm{d}_{x z}\right)$ \\
\hline$S_{19}$ & 0.42 & 3.890 & 0.035 & 39.24 & $96 \%\left(\mathrm{~d}_{z_{2}} \rightarrow \pi^{*}+\mathrm{d}_{x y}\right)$ \\
\hline $\mathrm{S}_{20}$ & 0.52 & 3.972 & 0.076 & -35.82 & $88 \%\left(\mathrm{~d}_{y z}+\pi \rightarrow \pi^{*}+\mathrm{d}_{x y}\right)$ \\
\hline$s_{21}$ & 0.54 & 3.997 & 0.028 & 22.87 & $61 \%\left(\mathrm{~d}_{x z}+\pi \rightarrow \pi^{*}+\mathrm{d}_{x y}\right)+22 \%\left(\pi \rightarrow \pi^{*}+\mathrm{d}_{x z}\right)$ \\
\hline $\mathrm{S}_{22}$ & 0.73 & 4.165 & 0.000 & -1.27 & $67 \%\left(\pi \rightarrow \pi^{*}+\mathrm{d}_{y z}\right)+24 \%\left(\pi \rightarrow \pi^{*}+\mathrm{d}_{x z}\right)$ \\
\hline $\mathrm{S}_{23}$ & 0.69 & 4.218 & 0.015 & -27.69 & $48 \%\left(\pi \rightarrow \pi^{*}\right)+40 \%\left(\pi \rightarrow \pi^{*}+\mathrm{d}_{x z}\right)$ \\
\hline $\mathrm{S}_{24}$ & 0.43 & 4.220 & 0.064 & -13.05 & $66 \%\left(\pi \rightarrow \mathrm{d}_{x y}+\mathrm{n}\right)+17 \%\left(\mathrm{~d}_{x z}+\pi \rightarrow \pi^{*}+\mathrm{d}_{x y}\right)$ \\
\hline$S_{25}$ & 0.52 & 4.378 & 0.211 & 23.26 & $29 \%\left(\pi \rightarrow \mathrm{d}_{x y}+\mathrm{n}\right)+20 \%\left(\pi \rightarrow \pi^{*}+\mathrm{d}_{x z}\right)+13 \%\left(\mathrm{~d}_{x z}+\pi \rightarrow \pi^{*}+\mathrm{d}_{x y}\right)+10 \%\left(\pi \rightarrow \pi^{*}+\mathrm{d}_{y z}\right)$ \\
\hline $\mathrm{S}_{26}$ & 0.25 & 4.510 & 0.001 & 7.20 & $97 \%\left(\mathrm{~d}_{z^{2}} \rightarrow \pi^{*}\right)$ \\
\hline$S_{27}$ & 0.39 & 4.571 & 0.002 & -9.06 & $68 \%\left(\mathrm{~d}_{y z}+\pi \rightarrow \sigma^{*}+\mathrm{d}_{z^{2}}\right)+17 \%\left(\mathrm{~d}_{z^{2}} \rightarrow \sigma^{*}+\mathrm{d}_{z^{2}}\right)$ \\
\hline $\mathrm{S}_{28}$ & 0.57 & 4.586 & 0.016 & -6.90 & $57 \%\left(\mathrm{~d}_{y z}+\pi \rightarrow \pi^{*}\right)+13 \%\left(\pi \rightarrow \pi^{*}\right)+12 \%\left(\pi \stackrel{z^{2}}{\rightarrow} \pi^{*}+\mathrm{d}_{x z}\right)$ \\
\hline$S_{29}^{20}$ & 0.36 & 4.590 & 0.018 & 12.94 & $43 \%\left(\mathrm{~d}_{z^{2}} \rightarrow \pi^{*}\right)+31 \%\left(\mathrm{~d}_{z^{2}} \rightarrow \sigma^{*}+\mathrm{d}_{z^{2}}\right)$ \\
\hline $\mathrm{S}_{30}$ & 0.34 & 4.607 & 0.000 & -0.02 & $46 \%\left(\mathrm{~d}_{z^{2}}^{z^{2}} \rightarrow \pi^{*}\right)+25 \%\left(\mathrm{~d}_{y z}^{z^{2}}+\pi \rightarrow \sigma^{*^{2}}+\mathrm{d}_{z^{2}}\right)$ \\
\hline$s_{31}$ & 0.28 & 4.611 & 0.000 & -3.43 & $97 \%\left(\mathrm{~d}_{x^{2}-y^{2}}^{z^{2}} \rightarrow \pi^{*}+\mathrm{d}_{x y}\right)$ \\
\hline $\mathrm{S}_{32}$ & 0.22 & 4.685 & 0.009 & -4.23 & $94 \%\left(\mathrm{~d}_{z} 2 \stackrel{y}{\rightarrow} \sigma^{*}\right)$ \\
\hline$s_{33}$ & 0.35 & 4.722 & 0.001 & -4.93 & $96 \%\left(\mathrm{~d}_{x z}^{z^{2}}+\pi \rightarrow \sigma^{*}+\mathrm{d}_{z^{2}}\right)$ \\
\hline $\mathrm{S}_{34}$ & 0.42 & 4.739 & 0.001 & -0.35 & $52 \%\left(\mathrm{~d}_{x z}+\pi \rightarrow \pi^{*}\right)+42 \%\left(\mathrm{~d}_{y z}+\pi \rightarrow \pi^{*}\right)$ \\
\hline $\mathrm{S}_{35}$ & 0.23 & 4.760 & 0.001 & -1.02 & $88 \%\left(\mathrm{~d}_{z^{2}} \rightarrow \sigma^{*}\right)$ \\
\hline $\mathrm{s}_{36}$ & 0.35 & 4.785 & 0.032 & 15.68 & $48 \%\left(\mathrm{~d}_{y z}^{z^{2}}+\pi \rightarrow \sigma^{*}\right)+28 \%\left(\mathrm{~d}_{z^{2}} \rightarrow \pi^{*}+\sigma^{*}\right)$ \\
\hline$s_{37}$ & 0.27 & 4.809 & 0.031 & -11.19 & $61 \%\left(\mathrm{~d}_{z^{2}} \rightarrow \pi^{*}+\sigma^{*}\right)+34 \%\left(\mathrm{~d}_{y z}+\pi \rightarrow \sigma^{*}\right)$ \\
\hline $\mathrm{S}_{38}$ & 0.43 & 4.835 & 0.028 & -9.10 & $52 \%\left(\mathrm{~d}_{x z}^{z^{2}}+\pi \rightarrow \pi^{*}\right)+12 \%\left(\mathrm{~d}_{y z}+\pi \rightarrow \sigma^{*}\right)+11 \%\left(\mathrm{~d}_{y z}+\pi \rightarrow \pi^{*}+\sigma^{*}\right)$ \\
\hline
\end{tabular}

${ }^{a}$ The main experimental bands (in Ref. 110) were observed at: 1.55, 1.86, 2.23. 2.73, $3.22 \mathrm{eV}$.

${ }^{b}$ Here, o.s and r.s. correspond to oscillator (dimensionless) and rotatory strengths $\left(10^{-40} \mathrm{esu}^{2} \mathrm{~cm}^{2}\right)$, respectively.

The Abs spectrum obtained with CAM-B3LYP differs significantly from the one based on BP86 calculations (Figure 5.4, Table 5.2). The most intense transition among the computed states up to $4.5 \mathrm{eV}$, is observed at $4.01 \mathrm{eV}$. Although this excitation shows up as a single electronic transition, possibly indicating a better match with the experimentally observed $\gamma$ band, its location on the energy scale makes such an assignment questionable. The difference between the calculated and measured excitation energy indicates a quite large, i.e., $0.79 \mathrm{eV}$ blue-shift, of the computed result, which is much larger than the error normally observed for valence transitions with the CAM-B3LYP functional. Therefore, the more appropriate assignment of the $\gamma$ peak seems to be the $S_{12}$ state, higher in energy by 0.31 $\mathrm{eV}$ than the corresponding experimental value. Of the lower-energy excited states, the $S_{2}, S_{3}, S_{4}$ and $S_{8}$ states possess oscillator strengths above 0.010, making them 
suitable candidates for the $\alpha / \beta$ and $\mathrm{D} / \mathrm{E}$ bands. The $\mathrm{S}_{2}$ underestimates the experimental energy of the first band by $0.09 \mathrm{eV}$, whereas the $S_{3}$ overestimates it by 0.46 $\mathrm{eV}$ but is, at the same time, larger than the second measured peak by only 0.15 $\mathrm{eV}$. The remaining transitions, extracted from the experimental spectrum via Gaussian deconvolution, were observed at 2.23 and $2.72 \mathrm{eV} .{ }^{110}$ The CAM-B3LYP-based excitation energies for the $S_{4}$ and $S_{8}$ are 2.22 and $2.94 \mathrm{eV}$, respectively, indicating a possible correspondence to the measured results. However, these assignments must be used with caution due to the lack of systematic overestimation of excitation energies obtained with TD-DFT calculations as well as the possible omission of low-intensity absorption bands of $\mathrm{d} \rightarrow \mathrm{d}$ character. Based on the Abs spectrum alone, a better overall agreement between the simulated and experimental spectra is obtained by BP86 compared to CAM-B3LYP.

\subsubsection{CD and MCD spectra}

The comparison of the experimental and computed CD and MCD spectra also shows rather different spectra obtained with the two functionals (Figure 5.4 and 5.5). As for the Abs spectra, better agreement with experiment was obtained using the BP86 functional. A visual comparison of the measured and calculated spectra indicates that the features observed at higher energies, including the $\gamma$ band, show better agreement with experiment when BP86 was applied, both for the excitation energies and the sign of the rotational strengths.

The CD spectra calculated with both functionals uncovered at least two electronic transitions near the first absorption band visible in experiment. The energy of the $S_{1}$ state was lower than the measured value of $1.55 \mathrm{eV}$ by 0.16 and $0.22 \mathrm{eV}$, for BP86 (Table 5.1) and CAM-B3LYP (Table 5.2), respectively. The energy of the $\mathrm{S}_{2}$ state obtained with CAM-B3LYP is $1.46 \mathrm{eV}$ and is also underestimated compared to the experimental value of $1.55 \mathrm{eV}$, but is slightly overestimated, i.e., $1.72 \mathrm{eV}$, in the case of BP86. Despite the zero oscillator strengths for the $S_{1}$ and low intensity of the $S_{2}$ in the calculated Abs spectra, using both functionals, the larger rotational strengths support these states as contributing to the $\alpha$ band. A more detailed in- 
spection of the $\alpha / \beta$ part shows additional differences in the calculated spectra. In experiment, two opposite signals were observed at $\sim 1.85$ and $\sim 2.10 \mathrm{eV}$. The CAMB3LYP-based CD spectrum possesses a positive and negative feature appearing as the third and fourth excitations. However, their relative order (or sign) questions the reliability of the CAM-B3LYP results as the signs of the aforementioned experimental peaks occur in opposite sequence. Even if the $S_{3}$ state is attributed to the transition at $2.10 \mathrm{eV}$, due to similarity to the excitation energy at $2.01 \mathrm{eV}$, the lack of a matching transition for the excitation at $1.85 \mathrm{eV}$ should not be overlooked. In contrast, the BP86-computed spectra indicate the presence of two electronic transitions of negative rotational strengths in this region. Despite the relatively small intensities, their excitation energies $\left(1.96 \mathrm{eV}\right.$ and $2.18 \mathrm{eV}$ for $S_{3}$ and $S_{4}$, respectively) are much closer to the experimental value of $1.85 \mathrm{eV}$ than the following $\mathrm{S}_{6}$ state $(2.38 \mathrm{eV})$ with large negative value for the rotational strength. Consequently, it is more likely that the $S_{3}$ state may be associated with the transition at $1.85 \mathrm{eV}$, whereas the $2.10 \mathrm{eV}$ excitation could be attributed to the positively signed $\mathrm{S}_{5}$ feature with an excitation energy of $2.28 \mathrm{eV}$. The D/E part of the electronic spectrum of cob(I)alamin is also better reproduced with BP86. Although the $\mathrm{S}_{10}$ can only be proposed as a contributing state due to significant oscillator strength and negative sign of the rotational strength, the presence of neighboring excitations consistently signed with experimental features, observed in this part of the spectrum, indicate that BP86 performs quite well as compared to CAM-B3LYP. The two intense, positively signed excitations $\left(S_{7}\right.$ and $\left.S_{8}\right)$ predicted with the latter functional cannot be matched with the experimental spectrum, which is dominated by negative CD signals.

The MCD plots calculated with both methods show visible deviations from experiment. As for the Abs and CD spectra, the major electronic features correspond better with the BP86 results, especially for the low-energy part of the spectrum. However, with increasing excitation energy, the agreement with the measured MCD spectra decreases. This part of the CAM-B3LYP-computed MCD spectrum differs from experiment even more as the excitations have the opposite signs for the ro- 
tational strength. This supports our conclusion that a detailed analysis of the electronic transitions of cob(I)alamin should be provided based on the results obtained from BP86 calculations.

\subsubsection{Assignment of electronic transitions}

The comprehensive analysis of Abs, CD and MCD data for cob(I)alamin does not only demand accurate comparisons of computed and experimental excitation energies but also elucidation of the individual transitions contributing to the observed bands. Since the nature of these states has not been well established, an insight at the molecular level from theoretical calculations is required. This is not an easy task for several reasons. First, due to the complexity of the $\mathrm{B}_{12}$ cofactors, truncated structural models have been used in the majority of earlier calculations, and it is not clear to what extent these simplifications affect the calculation of electronic transitions. Second, the computed results may be sensitive to the quality of the basis set as well as environmental effects. In earlier studies, examining the Abs, CD and MCD spectra of MeCbl and CNCbl at the molecular level, both of these factors were considered. ${ }^{140}$ It was found that the increase from $\sim 600\left(6-31 \mathrm{G}^{*}\right)$ to $\sim 1200$ contracted basis functions (aug-cc-pVDZ) only led to minor changes in the calculated spectra. The inclusion of solvent effects improved the results for CNCbl but had only a minor influence in the case of MeCbl. Third, the level of theory, particularly the choice of density functional, has proven to be crucial for all $\mathrm{B}_{12}$ analogues studied to date. However, the lack of reliable benchmarks in the case of cob(I)alamin against which computed electronic excitations can be compared precludes final and unambiguous assignment of the isolated transitions. Although CASSCF/CASPT2 calculations have been previously reported and we used CASSCF/MC-XQDPT2 computations in this work, CASSCF-based electronic excitations corrected with second-order perturbation theory has limitations of their own. In particular, the energies of the higher excited states for complicated systems containing transition metals tend to be not very accurate. ${ }^{196}$ This may be due to restrictions with respect to the size of the active space, quality of basis set, inaccurate treat- 
ment of the correlation energy or even the approximations applied in the design of the particular form of the second-order perturbation theory approach. Fourth, for cob(I)alamin, the complexity of the ground-state wave function adds to the complexity of the problem. It has been shown that the electronic ground state consists of two dominating configurations, arising respectively from a closed-shell $\mathrm{Co}^{\mathrm{I}}\left(\mathrm{d}^{8}\right)$ and an open-shell, diradical, $\mathrm{Co}^{\text {II }}\left(\mathrm{d}^{7}\right)$-radical corrin $\left(\pi^{*}\right)^{1}$ configuration. ${ }^{159,197}$ Taking into account all the above considerations, the only reliable assignments of the numerous bands observed in the electronic spectra of cob(I)alamin can be obtained based on TD-DFT calculations with an appropriately chosen XC functional. Despite the aforementioned limitations of the CASSCF/MC-XQDPT2 approach in terms of energetically higher excited states, it can still be considered as a reliable approach for a limited number of low-lying excitations, and was therefore used to validate the results obtained with TD-DFT.

Previous TD-DFT/B3LYP calculations using different basis sets led to an assignment of the major electronic bands of cob(I)alamin by considering both energies and intensities. ${ }^{109}$ However, the validity of hybrid functionals with respect to electronic excitations of cobalamins have been questioned ${ }^{140,161,163,271}$ and numerical evidence suggests that GGA-type functionals perform better. From these investigations, the $S_{1}$ for MeCbl was assigned as a MLCT excitation, whereas LMCT described the lowest excitation of CNCbl, indicating the importance of Co d orbitals in the electronic transitions. Many studies have shown that such excitations are in most cases better described by GGA-type methods. PBE results for cob(I)alamin added support to the notion that transitions involving Co d orbitals might, indeed, contribute to the major absorption bands. ${ }^{110}$

The comparison of experimental and simulated Abs, CD and MCD spectra presented in the previous sections of this chapter confirms the superiority of BP86 as compared to CAM-B3LYP. However, both functionals display limitations in a direct comparison to experiment and additional calculations using CASSCF/MC-XQDPT2 were therefore performed. This wave function-based method served as a benchmark for the excitation energies computed with BP86 (Table 5.1) and CAM-B3LYP 
(Table 5.2). It is important to emphasize here that the assignments of electronic transitions based on CASSCF/MC-XQDPT2 depend on the set of MOs included in the active space, which implies that possible excitations of cob(I)alamin are limited to the active space presented on Figure 5.3. To make the present study consistent with previous work of Jensen as well as in accordance with preceding studies of cob(I)alamin performed in our group, the same set of active orbitals was used. As a result, the $S_{1}$ state obtained with CASSCF/MC-XQDPT2 (Table 5.3) was characterized mainly by $\mathrm{d}_{z^{2}} \rightarrow \pi^{*}$ transition and this was also the case for the $\mathrm{S}_{1}$ state predicted by TD-DFT/BP86. At the same time, TD-DFT/CAM-B3LYP finds such an excitation as the second electronic state, not as the lowest-energy state. Instead, the $S_{0} \rightarrow S_{1}$ transition involves the Co $d$ orbital, oriented in the $x z$ plane $\left(d_{x z}\right)$ in preference to the $\mathrm{z}$ axis $\left(\mathrm{d}_{z^{2}}\right)$, as predicted by the other two methods.

The next two excited states calculated with CASSCF/MC-XQDPT2 are close in energy but also in character. The data in Table 5.3 suggests a similar mix of $d \rightarrow \pi^{*} / d$ character for both the $S_{2}$ and $S_{3}$ states, although the percentage contribution from the participating CASSCF states varies (compare Tables A.16 and A.17 in the Appendix). The $S_{2}$ and $S_{3}$ states computed with CAM-B3LYP are more distinct from each other both in energy and nature, whereas the corresponding BP86-computed $\mathrm{S}_{2}$ and $\mathrm{S}_{3}$ states are described by the same sets of orbitals. According to the data in Table 5.1, both the $S_{2}$ and $S_{3}$ states are of mixed $d / \pi \rightarrow \pi^{*} / d$ type, suggesting better agreement between the CASSCF/MC-XQDPT2 and BP86 methods in describing the electronic excitations of cob(I)alamin. Taking into account the latter observation as well as the advantage of BP86 over CAM-B3LYP when calculated and experimental spectra were compared, it is reasonable to assume that a more reliable assignment of the electronic excitations is provided by TD-DFT/BP86. 
TABLE 5.2. Excitation energies ${ }^{a}$ together with oscillator and rotatory strengths ${ }^{b}$ (CD) of 38 states of cob(I)alamin (simplified model) calculated with CAM-B3LYP/6$31 G^{*}$.

\begin{tabular}{|c|c|c|c|c|c|}
\hline State & $\overline{\Lambda \Lambda}$ & Energy(eV) & o.s. & r.s. & Character \\
\hline $\mathrm{S}_{1}$ & 0.33 & 1.326 & 0.000 & 7.51 & $98 \%\left(\mathrm{~d}_{x z}+\pi \rightarrow \pi^{*}+\mathrm{d}_{y z}\right)$ \\
\hline $\mathrm{S}_{2}$ & 0.52 & 1.459 & 0.015 & 14.92 & $91 \%\left(\mathrm{~d}_{z^{2}} \rightarrow \pi^{*}+\mathrm{d}_{y z}\right)$ \\
\hline$S_{3}$ & 0.74 & 2.010 & 0.033 & 26.26 & $80 \%\left(\mathrm{~d}_{y z}+\pi \rightarrow \pi^{*}+\mathrm{d}_{y z}\right)+13 \%\left(\mathrm{~d}_{z^{2}} \rightarrow \pi^{*}\right)$ \\
\hline $\mathrm{S}_{4}$ & 0.55 & 2.220 & 0.022 & -56.77 & $68 \%\left(\mathrm{~d}_{y z}+\pi \rightarrow \pi^{*}\right)+18 \%\left(\mathrm{~d}_{x z}+\pi \rightarrow \sigma^{*}+\mathrm{d}_{y z}\right)$ \\
\hline $\mathrm{S}_{5}$ & 0.38 & 2.428 & 0.001 & 1.72 & $63 \%\left(\mathrm{~d}_{x z}+\pi \rightarrow \pi^{*}\right)+17 \%\left(\mathrm{~d}_{y z}+\pi \rightarrow \sigma^{*}+\mathrm{d}_{y z}\right)$ \\
\hline $\mathrm{S}_{6}$ & 0.50 & 2.473 & 0.000 & -1.99 & $45 \%\left(\mathrm{~d}_{z^{2}} \rightarrow \sigma^{*}+\mathrm{d}_{y z}\right)+32 \%\left(\mathrm{~d}_{x^{2}-y^{2}} \rightarrow \pi^{*}+\mathrm{d}_{y z}\right)$ \\
\hline$S_{7}$ & 0.45 & 2.690 & 0.000 & 15.92 & $42 \%\left(\mathrm{~d}_{y z}+\pi \rightarrow \sigma^{*}+\mathrm{d}_{y z}\right)+29 \%\left(\mathrm{~d}_{x z}+\pi \rightarrow \pi^{*}\right)$ \\
\hline $\mathrm{S}_{8}$ & 0.50 & 2.942 & 0.013 & 10.83 & $45 \%\left(\mathrm{~d}_{x z}+\pi \rightarrow \sigma^{*}+\mathrm{d}_{y z}\right)+22 \%\left(\mathrm{~d}_{y z}+\pi \rightarrow \pi^{*}\right)$ \\
\hline $\mathrm{S}_{9}$ & 0.51 & 3.076 & 0.000 & -7.99 & $65 \%\left(\mathrm{~d}_{x^{2}-y^{2}} \rightarrow \sigma^{*}+\mathrm{d}_{y z}\right)+12 \%\left(\mathrm{~d}_{x^{2}-y^{2}} \rightarrow \sigma^{*}\right)$ \\
\hline$S_{10}$ & 0.49 & 3.103 & 0.002 & -9.42 & $60 \%\left(\mathrm{~d}_{x^{2}-y^{2}} \rightarrow \pi^{*}+\mathrm{d}_{y z}\right)+23 \%\left(\mathrm{~d}_{z^{2}} \rightarrow \sigma^{*}+\mathrm{d}_{y z}\right)$ \\
\hline$S_{11}$ & 0.61 & 3.415 & 0.007 & -12.97 & $42 \%\left(\mathrm{~d}_{z^{2}} \rightarrow \pi^{*}\right)+35 \%\left(\mathrm{~d}_{y z}+\pi \rightarrow \pi^{*}\right)$ \\
\hline $\mathrm{S}_{12}$ & 0.59 & 3.536 & 0.080 & -31.40 & $70 \%\left(\pi \rightarrow \pi^{*}+\mathrm{d}_{y z}\right)+19 \%\left(\mathrm{~d}_{z^{2}} \rightarrow \pi^{*}\right)$ \\
\hline $\mathrm{S}_{13}$ & 0.52 & 3.647 & 0.030 & 0.42 & $52 \%\left(\mathrm{~d}_{y z}+\pi \rightarrow \pi^{*}\right)+19 \%\left(\mathrm{~d}_{z^{2}} \rightarrow \pi^{*}\right)+14 \%\left(\mathrm{~d}_{x z}+\pi \rightarrow \pi^{*}\right)$ \\
\hline $\mathrm{S}_{14}$ & 0.25 & 3.805 & 0.007 & 7.50 & $81 \%\left(\mathrm{~d}_{x z}+\pi \rightarrow \pi^{*}\right)$ \\
\hline $\mathrm{S}_{15}$ & 0.43 & 4.013 & 0.196 & -10.98 & $67 \%\left(\mathrm{~d}_{z^{2}} \rightarrow \pi^{*}\right)+14 \%\left(\mathrm{~d}_{y z}+\pi \rightarrow \pi^{*}+\mathrm{d}_{x y}\right)+10 \%\left(\pi \rightarrow \pi^{*}+\mathrm{d}_{y z}\right)$ \\
\hline$S_{16}$ & 0.43 & 4.107 & 0.001 & -3.82 & $77 \%\left(\mathrm{~d}_{x^{2}-y^{2}} \rightarrow \pi^{*}\right)+12 \%\left(\mathrm{~d}_{y z}+\pi \rightarrow \pi^{*}+\mathrm{d}_{x y}\right)$ \\
\hline $\mathrm{S}_{17}$ & 0.40 & 4.249 & 0.135 & -27.53 & $64 \%\left(\mathrm{~d}_{y z}+\pi \rightarrow \pi^{*}+\mathrm{d}_{x y}\right)+10 \%\left(\mathrm{~d}_{z^{2}} \rightarrow \pi^{*}\right)$ \\
\hline $\mathrm{S}_{18}$ & 0.36 & 4.475 & 0.009 & 9.00 & $83 \%\left(\mathrm{~d}_{z^{2}} \rightarrow \pi^{*}+\mathrm{d}_{x y}\right)$ \\
\hline$S_{19}$ & 0.35 & 4.663 & 0.000 & -0.89 & $88 \%\left(\mathrm{~d}_{y z}+\pi \rightarrow \mathrm{d}_{x y}+\mathrm{n}\right)$ \\
\hline $\mathrm{S}_{20}$ & 0.40 & 4.705 & 0.102 & 2.92 & $94 \%\left(\mathrm{~d}_{x z}+\pi \rightarrow \pi^{*}+\mathrm{d}_{x y}\right)$ \\
\hline$S_{21}$ & 0.72 & 4.779 & 0.075 & 9.12 & $67 \%\left(\pi+\mathrm{d}_{y z} \rightarrow \pi^{*}+\mathrm{d}_{y z}\right)+13 \%\left(\pi \rightarrow \pi^{*}\right)$ \\
\hline $\mathrm{S}_{22}$ & 0.61 & 4.974 & 0.623 & 67.50 & $62 \%\left(\pi \rightarrow \pi^{*}\right)$ \\
\hline $\mathrm{S}_{23}$ & 0.29 & 4.986 & 0.000 & -1.00 & $84 \%\left(\mathrm{~d}_{z^{2}} \rightarrow \mathrm{d}_{x y}+\mathrm{n}\right)$ \\
\hline $\mathrm{S}_{24}$ & 0.34 & 5.192 & 0.036 & 1.11 & $88 \%\left(\mathrm{~d}_{x^{2}-y^{2}} \rightarrow \pi^{*}\right)$ \\
\hline $\mathrm{S}_{25}$ & 0.63 & 5.451 & 0.138 & -25.45 & $68 \%\left(\pi \rightarrow \pi^{*}\right)+10 \%\left(\pi+\mathrm{d}_{y z} \rightarrow \pi^{*}\right)$ \\
\hline$S_{26}$ & 0.31 & 5.518 & 0.023 & -12.37 & $86 \%\left(\mathrm{~d}_{y z}+\pi \rightarrow \mathrm{d}_{z^{2}}+\sigma^{*}\right)$ \\
\hline$S_{27}$ & 0.68 & 5.665 & 0.004 & 1.42 & $41 \%\left(\pi+\mathrm{d}_{y z} \rightarrow \pi^{*}\right)+34 \%\left(\pi \rightarrow \pi^{*}+\mathrm{d}_{y z}\right)$ \\
\hline $\mathrm{S}_{28}$ & 0.28 & 5.698 & 0.010 & -8.79 & $87 \%\left(\mathrm{~d}_{y z}+\pi \rightarrow \sigma^{*}+\mathrm{d}_{x z}\right)$ \\
\hline $\mathrm{S}_{29}$ & 0.71 & 5.744 & 0.078 & -42.85 & $52 \%\left(\pi \rightarrow \pi^{*}+\mathrm{d}_{y z}\right)+26 \%\left(\pi+\mathrm{d}_{y z} \rightarrow \pi^{*}\right)+13 \%\left(\pi \rightarrow \pi^{*}\right)$ \\
\hline$S_{30}$ & 0.39 & 5.764 & 0.019 & -2.24 & $33 \%\left(\mathrm{~d}_{x z}+\pi \rightarrow \mathrm{d}_{x y}+\mathrm{n}\right)+31 \%\left(\mathrm{~d}_{z^{2}} \rightarrow \mathrm{d}_{z^{2}}+\sigma^{*}\right)$ \\
\hline$S_{31}$ & 0.27 & 5.902 & 0.000 & 1.06 & $89 \%\left(\mathrm{~d}_{x^{2}-y^{2}} \rightarrow \pi^{*}+\mathrm{d}_{x y}\right)$ \\
\hline $\mathrm{S}_{32}$ & 0.26 & 5.923 & 0.016 & 10.28 & $80 \%\left(\mathrm{~d}_{x z}+\pi \rightarrow \mathrm{d}_{z^{2}}+\sigma^{*}\right)$ \\
\hline$S_{33}$ & 0.33 & 5.944 & 0.009 & 2.47 & $61 \%\left(\mathrm{~d}_{z^{2}} \rightarrow \mathrm{d}_{z^{2}}+\sigma^{*}\right)+12 \%\left(\mathrm{~d}_{x z}+\pi \rightarrow \mathrm{d}_{x y}+\mathrm{n}\right)$ \\
\hline $\mathrm{S}_{34}$ & 0.43 & 6.023 & 0.090 & -18.28 & $75 \%\left(\mathrm{~d}_{y z}+\pi \rightarrow \pi^{*}\right)$ \\
\hline $\mathrm{S}_{35}$ & 0.43 & 6.093 & 0.006 & -0.35 & $78 \%\left(\pi \rightarrow \pi^{*}+\mathrm{d}_{x y}\right)$ \\
\hline$S_{36}$ & 0.21 & 6.102 & 0.001 & -3.53 & $91 \%\left(\mathrm{~d}_{z^{2}} \rightarrow \sigma^{*}+\mathrm{d}_{x z}\right)$ \\
\hline $\mathrm{S}_{37}$ & 0.49 & 6.118 & 0.001 & 3.47 & $57 \%\left(\sigma \rightarrow \pi^{*}+\mathrm{d}_{y z}\right)+15 \%\left((\pi / \sigma) \rightarrow \pi^{*}+\mathrm{d}_{y z}\right)$ \\
\hline $\mathrm{S}_{38}$ & 0.29 & 6.123 & 0.026 & -0.37 & $70 \%\left(\mathrm{~d}_{x z}+\pi \rightarrow \sigma^{*}+\mathrm{d}_{x z}\right)$ \\
\hline
\end{tabular}

${ }^{a}$ The main experimental bands (in Ref. 110) were observed at: $1.55,1.86,2.23 .2 .73,3.22 \mathrm{eV}$.

${ }^{b}$ Here, o.s and r.s. correspond to oscillator (dimensionless) and rotatory strengths $\left(10^{-40} \mathrm{esu}^{2} \mathrm{~cm}^{2}\right)$, respectively.

The data in Table 5.1 and the Abs, CD, and MCD spectra plotted on Figure 5.4 allow us to identify the electronic transitions that are likely to contribute to the major bands in the cob(I)alamin spectra. Based on the intensity and rotational strength pattern, the low-energy $\alpha / \beta$ band has contributions from multiple electronic transitions. Of the first four excited states, the $S_{2}$ and $S_{3}$ states have small oscillator strengths, while the $\mathrm{S}_{1}$ and $\mathrm{S}_{4}$ states are not visible in the Abs spectra. 
TABLE 5.3. First 10 excited states of cob(I)alamin calculated with MC-XQDPT2 presented in terms of $\%$ contributions. Note that only contributions larger than $5 \%$ were shown.

\begin{tabular}{|c|c|c|}
\hline State & $\mathrm{E}(\mathrm{eV})$ & Composition \\
\hline $\mathrm{S}_{1}$ & 0.94 & $56 \%\left(\mathrm{~d}_{z^{2}} \rightarrow \pi^{*}\right)+30 \%\left(\mathrm{~d}_{y z} / \mathrm{d}_{z^{2}} \rightarrow \pi^{*}\right)$ \\
\hline $\mathrm{S}_{2}$ & 1.91 & $22 \%\left(\mathrm{~d}_{y z} \rightarrow \pi^{*}\right)+15 \%\left(\mathrm{~d}_{x^{2}-y^{2}} \rightarrow \pi^{*}\right)+10 \%\left(\mathrm{~d}_{x z} / \mathrm{d}_{z^{2}} \rightarrow \pi^{*} / \mathrm{d}_{x y}-\mathrm{n}\right)+8 \%\left(\mathrm{~d}_{x^{2}-y^{2}} / \mathrm{d}_{y z} \rightarrow \pi^{*}\right)$ \\
\hline $\mathrm{S}_{3}$ & 2.17 & $28 \%\left(\mathrm{~d}_{x^{2}-y^{2}} \rightarrow \pi^{*}\right)+15 \%\left(\mathrm{~d}_{x^{2}-y^{2}} / \mathrm{d}_{y z} \rightarrow \pi^{*}\right)+13 \%\left(\mathrm{~d}_{y z} \rightarrow \pi^{*}\right)+8 \%\left(\mathrm{~d}_{x z} \rightarrow \mathrm{d}_{x y}-\mathrm{n}\right)$ \\
\hline $\mathrm{S}_{4}$ & 3.57 & $39 \%\left(\mathrm{~d}_{x z} / \mathrm{d}_{y z} \rightarrow \pi^{*} / \mathrm{d}_{x y}-\mathrm{n}\right)+31 \%\left(\mathrm{~d}_{x z} \rightarrow \mathrm{d}_{x y}-\mathrm{n}\right)$ \\
\hline $\mathrm{S}_{5}$ & 4.14 & $25 \%\left(\mathrm{~d}_{x z} \rightarrow \pi^{*}\right)+18 \%\left(\mathrm{~d}_{y z} / \mathrm{d}_{z^{2}} \rightarrow \pi^{*}\right)+14 \%\left(\mathrm{~d}_{z^{2}} \rightarrow \pi^{*}\right)+6 \%\left(\mathrm{~d}_{x^{2}-y^{2}} / \mathrm{d}_{z^{2}} \rightarrow \pi^{*}\right)$ \\
\hline $\mathrm{S}_{6}$ & 4.16 & $30 \%\left(\mathrm{~d}_{y z} / \mathrm{d}_{z^{2}} \rightarrow \pi^{*}\right)+19 \%\left(\mathrm{~d}_{z^{2}} \rightarrow \pi^{*}\right)+13 \%\left(\mathrm{~d}_{x z} \rightarrow \pi^{*}\right)$ \\
\hline $\mathrm{S}_{7}$ & 4.31 & $45 \%\left(\mathrm{~d}_{z^{2}} \rightarrow \pi^{*}\right)+10 \%\left(\mathrm{~d}_{x^{2}-y^{2}} / \mathrm{d}_{z^{2}} \rightarrow \pi^{*}\right)+9 \%\left(\mathrm{~d}_{x z} \rightarrow \pi^{*}\right)$ \\
\hline $\mathrm{S}_{8}$ & 4.39 & $33 \%\left(\mathrm{~d}_{x^{2}-y^{2}} / \mathrm{d}_{x z} \rightarrow \pi^{*} / \mathrm{d}_{x y}-\mathrm{n}\right)+21 \%\left(\mathrm{~d}_{x^{2}-y^{2}} / \mathrm{d}_{x z} / \mathrm{d}_{y z} \rightarrow \pi^{*} / \mathrm{d}_{x y}-\mathrm{n}\right)+17 \%\left(\mathrm{~d}_{x z} / \mathrm{d}_{y z} \rightarrow \pi^{*} / \mathrm{d}_{x y}-\mathrm{n}\right)$ \\
\hline $\mathrm{S}_{9}$ & 4.47 & $42 \%\left(\mathrm{~d}_{x z} / \mathrm{d}_{y z} \rightarrow \pi^{*} / \mathrm{d}_{x y}-\mathrm{n}\right)+13 \%\left(\mathrm{~d}_{x z} \rightarrow \mathrm{d}_{x y}-\mathrm{n}\right)$ \\
\hline $\mathrm{S}_{10}$ & 4.82 & $32 \%\left(\mathrm{~d}_{x z} / \mathrm{d}_{z^{2}} \rightarrow \pi^{*} / \mathrm{d}_{x y}-\mathrm{n}\right)+15 \%\left(\mathrm{~d}_{x^{2}-y^{2}} / \mathrm{d}_{x z} \rightarrow \pi^{*} / \mathrm{d}_{x y}-\mathrm{n}\right)+20 \%\left(\mathrm{~d}_{x z} / \mathrm{d}_{y z} / \mathrm{d}_{z^{2}} \rightarrow \pi^{*} / \mathrm{d}_{x y}-\mathrm{n}\right)$ \\
\hline
\end{tabular}

The mixed $\mathrm{d} / \pi \rightarrow \pi^{*} / \mathrm{d}$ character of the $\mathrm{S}_{2}$ and $\mathrm{S}_{3}$ states correlates nicely with the results of the CASSCF/MC-XQDPT2 calculations. On the other hand, the orbital from which the $S_{1}$ and $S_{4}$ excitations occur is localized along the $\mathrm{z}$ axis of the corrin ring, and both transitions are described as $\mathrm{d}_{z^{2}} \rightarrow \pi^{*}$ with small contributions of unoccupied d orbitals. The $\mathrm{S}_{4}$ is hardly visible in both the Abs and CD spectra, although its energy could suggest its resemblance to the experimental peak observed at $\sim 2.10 \mathrm{eV}$ in the $\mathrm{CD}$ spectrum. However, a positively signed peak in the experimental CD spectrum contradicts the negative rotational strength of $S_{4}$ predicted with BP86. At the same time, the positive sign carried by $S_{5}$ in the computed CD spectrum suggests that this $\mathrm{d} \rightarrow \pi^{*} / \mathrm{d}$ transition may correspond to the experimental absorption band. There are four excitations found in the region between 2.5 and $3.0 \mathrm{eV}$ in the computed spectra. The D/E band in the experimental CD spectrum is negative. Only two of the four computed transitions follow this pattern. The $S_{9}$ is described as a mixed $\mathrm{d} \rightarrow \pi^{*} / \mathrm{d}$ and $\mathrm{d} / \pi \rightarrow \mathrm{d}$ and is better visible in the CD spectrum due to the magnitude of its rotational strength, while the $S_{10}$ gains intensity in the Abs spectrum and can be characterized as $\mathrm{d} / \pi \rightarrow \pi^{*}$ and $\mathrm{d} / \pi \rightarrow \pi^{*} / \mathrm{d}$. The presence of the next two negatively signed $S_{11}$ and $S_{12}$ states brings the attention to possible contributions from other transitions to the $\mathrm{D} / \mathrm{E}$ band. The $\mathrm{S}_{11}$ stays in the range of the expected TD-DFT error as its excitation energy $(3.21 \mathrm{eV})$ overestimates the experimental value for the isolated peak $(\sim 2.72 \mathrm{eV})$ in this region by $0.49 \mathrm{eV}$. The $\mathrm{S}_{11}$ transition includes $40 \% \mathrm{~d} / \pi \rightarrow \pi^{*} / \mathrm{d}$ but a contribution from Co orbitals of $\mathrm{d}$ char- 
acter $(17 \% \mathrm{~d} / \pi \rightarrow \mathrm{d}+\mathrm{n})$ is also observed. This suggests that the transition metal in cob(I)alamin not only serves as a moiety from which electrons depart during excitations, as is the case in most of the lower states where MLCT predominate, but that it also can be a center to which electrons are excited, as in the case of LMCT or $\mathrm{d} \rightarrow \mathrm{d}$ transitions. This becomes particularly important when the high-energy $\gamma$ band is considered. This band gains the largest intensity in the Abs spectra for all $\mathrm{B}_{12}$ derivatives and was characterized mainly as a $\pi \rightarrow \pi^{*}$ transition. However, the data obtained in our calculations indicate that the corrin $\pi$ orbitals might not be the only orbitals participating. According to the intensity pattern in the Abs spectrum, the $S_{15}$ transition should be considered as the state contributing to the $\gamma$ band. Data from Table 5.1 clearly show that $S_{15}$ is significantly mixed by several orbitals as both corrin $\pi$ occupied and unoccupied as well as cobalt $\mathrm{d}$ orbitals are necessary to define this transition. At the same time, the shape of the experimental CD spectrum requires us to look for a negative feature in the $\gamma$ region of the spectrum. This suggests that the positively signed $S_{15}$ should be excluded from the analysis. There is indeed a strong negative signal observed in the computed CD spectrum coming from $S_{13}$. In fact, this state also possesses a quite large oscillator strength and its energy differs by only $0.06 \mathrm{eV}$ from the corresponding value of $S_{15}$. More importantly, however, the nature of $S_{13}$ is very similar to $S_{15}$. It is described by the same sets of orbitals and the main difference lays in a larger contribution coming from $\mathrm{d}_{z^{2}} \rightarrow \mathrm{d}_{x y}+\mathrm{n}$ rather than $\pi \rightarrow \pi^{*}+\mathrm{d}_{y z}$ as is the case for $\mathrm{S}_{15}$. These observation clearly demonstrates that the $\gamma$ band of $\operatorname{cob}(\mathrm{I})$ alamin should be interpreted with caution, as both $\pi \rightarrow \pi^{*}$ as well as $\mathrm{d} \rightarrow \mathrm{d}$ transitions may be crucial.

\subsubsection{Truncated versus full model}

Let us now examine the effect of the simplifications introduced to the structure of $\operatorname{cob}(\mathrm{I})$ alamin on the Abs, $\mathrm{CD}$ and MCD spectra by comparing the results for both the simplified model and the full structure of the system (Figure 5.2). 
The first major difference in the results obtained for the simplified and the full structure is a significant congestion of the transitions observed in the BP86 computed spectra for the full structure (Figure 5.5). This is particularly prominent for the high-energy $\gamma$ band, which shows a number of intense excitations in the case of the full structure. Two of these, $S_{28}$ and $S_{29}$, are dominated by the same $(\sigma / \pi)_{\mathrm{R} 3} \rightarrow \pi^{*}$ transition and have transition energies of 3.16 and $3.18 \mathrm{eV}$, respectively (see Table 5.4). Judging by both the excitation energies and the value of the oscillator strength, they could be identified as the main contributors to the $\gamma$ region. However, their nature suggests long-range charge-transfer (CT) excitation from the side chain including the $\mathrm{PO}_{4}^{-}$group to the corrin moiety. Such transitions, where the degree of overlap between occupied and virtual orbitals involved in the excitation is relatively small, are very often incorrectly predicted by TD-DFT and will normally have rather modest oscillator strengths. The excitation energies for CT transitions tend to be significantly underestimated with these methods and can lead to an incorrect prediction of electronic transitions. In order to validate the possibility of such errors, we use the so-called $\Lambda$ diagnostic. Previous numerical experience suggests that if $\Lambda<0.4$, the particular excitation is most likely a potential suspect of CT failure. Taking this into account, the assignment of the $\gamma$ band cannot be considered as conclusive based on the results of the full structure of cob(I)alamin. All excitations in a range between 3.0 and $3.5 \mathrm{eV}$ except the $\mathrm{S}_{25}$ possess $\Lambda<0.4$ (Table 5.4). The situation improved significantly when CAM-B3LYP data were analyzed (Table 5.5). The majority of the excited states have $\Lambda$ values larger than 0.4. This is not surprising, as Coulomb-attenuated functionals are designed to improve the description of charge-transfer excitations. However, one should remember that the $\Lambda$ test is only a diagnosis and therefore does not guarantee the accuracy of the results for the excitation energies. Of the computed CAM-B3LYP excitations, $S_{12}$ can be expected to contribute to the $\gamma$ band due to its non-negligible oscillator strength and transition energy $(3.36 \mathrm{eV})$ being close to the experimental value of $3.22 \mathrm{eV}$. It is described as a $\pi \rightarrow \pi^{*} / \mathrm{d}$ transition which was also one of the contributions observed for the states associated with this region based on the BP86 
results for the truncated model. However, the CAM-B3LYP $S_{12}$ for the full structure misses the significant involvement of $\mathrm{d} \rightarrow \mathrm{d}$ transitions which was obtained in the case of the BP86 small model calculations. Moreover, this state gives a positive signal in the calculated CAM-B3LYP CD spectrum, in contrast to the negative one observed in experiment, questioning the assignments based on the full structure of cob(I)alamin in the high-energy region of the spectra.

TABLE 5.4. Excitation energies ${ }^{a}$ together with oscillator and rotatory strengths ${ }^{b}$ (CD) of 43 states of cob(I)alamin (full structure) calculated with BP86/6-31G*.

\begin{tabular}{|c|c|c|c|c|c|}
\hline State & 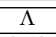 & Energy(eV) & o.s. & r.s. & Character $^{\mathrm{C}}$ \\
\hline $\mathrm{S}_{1}$ & 0.43 & 1.361 & 0.000 & -8.25 & $97 \%\left(\mathrm{~d}_{z^{2}} \rightarrow \pi^{*}\right)$ \\
\hline $\mathrm{S}_{2}$ & 0.56 & 1.702 & 0.011 & 1.48 & $89 \%\left(\mathrm{~d}_{x z}^{z}+\pi \rightarrow \pi^{*}\right)$ \\
\hline $\mathrm{s}_{3}$ & 0.69 & 1.854 & 0.010 & -8.19 & $80 \%\left(\mathrm{~d}_{x z}+\pi \rightarrow \pi^{*}\right)$ \\
\hline $\mathrm{S}_{4}$ & 0.27 & 2.049 & 0.000 & -2.88 & $81 \%\left(\mathrm{~d}_{z 2} \rightarrow \pi_{\mathrm{R} 1} *\right)+18 \%\left(\mathrm{~d}_{z} 2 \rightarrow \pi_{\mathrm{R} 1} * \pi^{*}\right)$ \\
\hline $\mathrm{S}_{5}$ & 0.38 & 2.105 & 0.002 & 5.41 & $59 \%\left(\mathrm{~d}_{x z}+\pi \rightarrow \pi_{\mathrm{R} 1} *\right)+18 \%\left(\mathrm{~d}_{z} 2 \rightarrow \pi_{\mathrm{R} 1} *+\pi^{*}\right)+17 \%\left(\mathrm{~d}_{x z}+\pi \rightarrow \pi_{\mathrm{R} 1} *+\pi^{*}\right)$ \\
\hline $\mathrm{S}_{6}$ & 0.34 & 2.110 & 0.001 & -18.67 & $61 \%\left(\mathrm{~d}_{z^{2}} \rightarrow \pi_{\mathrm{R} 1}{ }^{*}+\pi^{*}\right)+14 \%\left(\mathrm{~d}_{z^{2}} \rightarrow \pi_{\mathrm{R} 1}{ }^{*}\right)+12 \%\left(\mathrm{~d}_{x z}+\pi \rightarrow \pi_{\mathrm{R} 1}{ }^{*}+\pi^{*}\right)+10 \%\left(\mathrm{~d}_{x z}+\pi \rightarrow \pi_{\mathrm{R} 1}{ }^{*}\right)$ \\
\hline $\mathrm{S}_{7}$ & 0.46 & 2.226 & 0.003 & 19.37 & $78 \%\left(\mathrm{~d}_{x^{2}-y^{2}} \rightarrow \pi^{*}\right)+10 \%\left(\mathrm{~d}_{x z}+\pi \rightarrow \pi_{\mathrm{R} 1}^{*}+\pi^{*}\right)$ \\
\hline $\mathrm{S}_{8}$ & 0.23 & 2.250 & 0.007 & 4.12 & $64 \%\left(\mathrm{~d}_{z}^{2} \rightarrow \pi_{\mathrm{R} 1}\right)+15 \%\left(\mathrm{~d}_{x z}+\pi \rightarrow \pi_{\mathrm{R} 1} *+\pi^{*}\right)$ \\
\hline $\mathrm{S}_{9}$ & 0.29 & 2.276 & 0.009 & -11.13 & $33 \%\left(\mathrm{~d}_{z^{2}}^{z} \rightarrow \pi_{\mathrm{R} 1}^{*}\right)+22 \%\left(\mathrm{~d}_{x z}+\pi \rightarrow \pi_{\mathrm{R} 1}{ }^{*}+\pi^{*}\right)+19 \%\left(\mathrm{~d}_{x z}+\pi \rightarrow \pi_{\mathrm{R} 1} *\right)$ \\
\hline$s_{10}$ & 0.51 & 2.305 & 0.002 & -0.75 & $62 \%\left(\mathrm{~d}_{x z}+\pi \rightarrow \pi_{\mathrm{R} 1} *\right)+36 \%\left(\mathrm{~d}_{x z}+\pi \rightarrow \pi_{\mathrm{R} 1} * \pi^{*}\right)$ \\
\hline $\mathrm{S}_{11}$ & 0.28 & 2.338 & 0.011 & 4.50 & $73 \%\left(\mathrm{~d}_{x z}+\pi \rightarrow \pi_{\mathrm{R} 1} *\right)+10 \%\left(\mathrm{~d}_{x z}+\pi \rightarrow \pi_{\mathrm{R} 1} * \pi^{*}\right)$ \\
\hline $\mathrm{S}_{12}$ & 0.23 & 2.508 & 0.003 & -0.02 & $92 \%\left(\mathrm{~d}_{x z}+\pi \rightarrow \pi_{\mathrm{R} 1} *\right)$ \\
\hline $\mathrm{S}_{13}$ & 0.26 & 2.522 & 0.000 & 1.17 & $95 \%\left(\mathrm{~d}_{z^{2}} \rightarrow \pi^{*}+\pi_{\mathrm{R} 1}^{*}\right)$ \\
\hline $\mathrm{S}_{14}$ & 0.12 & 2.602 & 0.000 & 0.70 & $98 \%\left(\mathrm{~d}_{z^{2}}^{z} \rightarrow \pi_{\mathrm{R} 2}{ }^{*}\right)$ \\
\hline $\mathrm{S}_{15}$ & 0.48 & 2.617 & 0.007 & -3.87 & $51 \%\left(\pi+(\sigma / \pi)_{\mathrm{R} 2} \rightarrow \pi^{*}\right)+38 \%\left((\sigma / \pi)_{\mathrm{R} 2} \rightarrow \pi^{*}\right)$ \\
\hline $\mathrm{S}_{16}$ & 0.27 & 2.647 & 0.001 & -0.34 & $83 \%\left(\mathrm{~d}_{x z}+\pi \rightarrow \pi_{\mathrm{R} 2} *\right)+10 \%\left(\mathrm{~d}_{x z}+\pi \rightarrow \pi^{*}+\pi_{\mathrm{R} 1} *\right)$ \\
\hline $\mathrm{S}_{17}$ & 0.42 & 2.756 & 0.001 & -9.15 & $49 \%\left(\mathrm{~d}_{x z}+\pi \rightarrow \pi^{*}+\pi_{\mathrm{R} 1}{ }^{*}\right)+35 \%\left((\sigma / \pi)_{\mathrm{R} 2} \rightarrow \pi^{*}\right)$ \\
\hline $\mathrm{S}_{18}$ & 0.36 & 2.816 & 0.007 & -14.00 & $34 \%\left(\mathrm{~d}_{x z}+\pi \rightarrow \pi_{\mathrm{R} 2} *\right)+25 \%\left(\mathrm{~d}_{x z}+\pi \rightarrow \pi^{*}+\pi_{\mathrm{R} 1} *\right)+15 \%\left(\mathrm{~d}_{x^{2}}-y^{2} \rightarrow \pi_{\mathrm{R} 1} *\right)$ \\
\hline $\mathrm{S}_{19}$ & 0.29 & 2.838 & 0.001 & 4.37 & $63 \%\left(\mathrm{~d}_{x^{2}-y^{2}} \rightarrow \pi_{\mathrm{R} 1} *\right)+16 \%\left(\mathrm{~d}_{x z}+\pi \rightarrow \pi_{\mathrm{R} 2} *\right)+15 \%\left(\mathrm{~d}_{x^{2}-y^{2}} \rightarrow \pi_{\mathrm{R} 1} * \pi^{*}\right)$ \\
\hline $\mathrm{S}_{20}$ & 0.37 & 2.865 & 0.025 & 32.41 & $31 \%\left(\mathrm{~d}_{x^{2}-y^{2}} \rightarrow \pi_{\mathrm{R} 1} *+\pi^{*}\right)+29 \%\left(\mathrm{~d}_{x z}+\pi \rightarrow \pi_{\mathrm{R} 2} *\right)+21 \%\left(\mathrm{~d}_{x z}+\pi \rightarrow \pi^{*}+\pi_{\mathrm{R} 1} *\right)$ \\
\hline $\mathrm{S}_{21}$ & 0.42 & 2.900 & 0.019 & -27.45 & $34 \%\left(\mathrm{~d}_{x^{2}-y^{2}} \rightarrow \pi_{\mathrm{R} 1}{ }^{*}+\pi^{*}\right)+21 \%\left(\mathrm{~d}_{x z}+\pi \rightarrow \mathrm{d}_{x y}+\mathrm{n}\right)+17 \%\left(\mathrm{~d}_{x z}+\pi \rightarrow \pi^{*}+\pi_{\mathrm{R} 1} *\right)+10 \%\left(\mathrm{~d}_{x^{2}-y^{2}} \rightarrow \pi_{\mathrm{R} 1} *\right)$ \\
\hline $\mathrm{S}_{22}$ & 0.22 & 3.021 & 0.002 & -1.19 & $82 \%\left(\mathrm{~d}_{z^{2}} \stackrel{9}{\rightarrow} \pi_{\mathrm{R} 1} *\right)$ \\
\hline $\mathrm{S}_{23}$ & 0.16 & 3.045 & 0.002 & 0.36 & $95 \%\left(\mathrm{~d}_{x^{2}-y^{2}} \rightarrow \pi_{\mathrm{R} 1} *\right)$ \\
\hline $\mathrm{S}_{24}$ & 0.28 & 3.068 & 0.060 & 11.19 & $65 \%\left(\mathrm{~d}_{x z}+\pi \rightarrow \pi_{\mathrm{R} 1} *\right)$ \\
\hline $\mathrm{S}_{25}$ & 0.41 & 3.119 & 0.015 & -19.00 & $21 \%\left(\pi+\pi_{\mathrm{R} 1} \rightarrow \pi^{*}\right)+15 \%\left(\mathrm{~d}_{x z}+\pi \rightarrow \pi_{\mathrm{R} 1}{ }^{*}+\pi^{*}\right)+12 \%\left(\mathrm{~d}_{z} 2 \rightarrow \pi_{\mathrm{R} 3}{ }^{*}\right)$ \\
\hline $\mathrm{S}_{26}$ & 0.11 & 3.145 & 0.002 & -1.51 & $84 \%\left(\mathrm{~d}_{z^{2}} \rightarrow \pi_{\mathrm{R} 3}{ }^{*}\right)$ \\
\hline$S_{27}$ & 0.21 & 3.155 & 0.001 & 0.11 & $92 \%\left((\sigma / \pi)_{\mathrm{R} 2} \rightarrow \pi^{*}\right)$ \\
\hline $\mathrm{S}_{28}$ & 0.30 & 3.161 & 0.083 & 13.16 & $53 \%\left((\sigma / \pi)_{\mathrm{R} 3} \rightarrow \pi^{*}\right)$ \\
\hline $\mathrm{S}_{29}$ & 0.29 & 3.178 & 0.093 & 11.66 & $32 \%\left((\sigma / \pi)_{\mathrm{R} 3} \rightarrow \pi^{*}\right)+13 \%\left(\mathrm{~d}_{x z}+\pi \rightarrow \pi_{\mathrm{R} 3} *\right)$ \\
\hline $\mathrm{S}_{30}$ & 0.08 & 3.195 & 0.000 & -0.17 & $77 \%\left(\mathrm{~d}_{z^{2}} \rightarrow \pi_{\mathrm{R} 2} *+\pi_{\mathrm{R} 1} *\right)+20 \%\left(\mathrm{~d}_{x z}+\pi \rightarrow \pi_{\mathrm{R} 3}{ }^{*}\right)$ \\
\hline$s_{31}$ & 0.13 & 3.199 & 0.027 & -3.86 & $62 \%\left(\mathrm{~d}_{x z}+\pi \rightarrow \pi_{\mathrm{R} 3}{ }^{*}\right)+17 \%\left(\mathrm{~d}_{z 2} \rightarrow \pi_{\mathrm{R} 2}{ }^{*}+\pi_{\mathrm{R} 1}{ }^{*}\right)$ \\
\hline $\mathrm{S}_{32}$ & 0.22 & 3.253 & 0.000 & 0.77 & $71 \%\left(\mathrm{~d}_{x z}+\pi \rightarrow \pi_{\mathrm{R} 2} *+\pi_{\mathrm{R} 1} *\right)+12 \%\left(\mathrm{~d}_{x z}+\pi \rightarrow \pi_{\mathrm{R} 1} *\right)$ \\
\hline $\mathrm{s}_{33}$ & 0.39 & 3.264 & 0.010 & 7.63 & $18 \%\left(\pi+(\sigma / \pi)_{\mathrm{R} 2} \rightarrow \pi_{\mathrm{R} 1}^{*}\right)+13 \%\left(\pi+\pi_{\mathrm{R} 1} \rightarrow \pi^{*}\right)+12 \%\left(\mathrm{~d}_{x^{2}}-y^{2} \rightarrow \pi^{*}+\pi_{\mathrm{R} 1}{ }^{*}\right)+12 \%\left(\pi_{\mathrm{R} 1}+\pi \rightarrow \pi^{*}\right)$ \\
\hline $\mathrm{S}_{34}$ & 0.31 & 3.282 & 0.022 & -10.00 & $41 \%\left(\pi+(\sigma / \pi)_{\mathrm{R} 2} \rightarrow \pi_{\mathrm{R} 1} *\right)+27 \%\left(\pi_{\mathrm{R} 2}+\pi \rightarrow \pi^{*}\right)+12 \%\left(\pi+(\sigma / \pi)_{\mathrm{R} 2} \rightarrow \pi_{\mathrm{R} 1}^{*}+\pi^{*}\right)$ \\
\hline $\mathrm{S}_{35}$ & 0.35 & 3.290 & 0.002 & -4.84 & $25 \%\left(\mathrm{~d}_{x z}+\pi \rightarrow \mathrm{d}_{x y}+\mathrm{n}\right)+17 \%\left(\mathrm{~d}_{x z}+\pi \rightarrow \pi_{\mathrm{R} 1} *\right)+16 \%\left(\mathrm{~d}_{x^{2}}-y^{2} \rightarrow \pi^{*}+\pi_{\mathrm{R} 1} *\right)$ \\
\hline $\mathrm{S}_{36}$ & 0.22 & 3.293 & 0.018 & 0.86 & $63 \%\left(\pi_{\mathrm{R} 2}+\pi \rightarrow \pi^{*}\right)+7 \%\left(\pi_{\mathrm{R} 1}+\pi \rightarrow \pi^{*}\right)$ \\
\hline $\mathrm{S}_{37}$ & 0.38 & 3.327 & 0.005 & -2.75 & $32 \%\left(\pi_{\mathrm{R} 1}+\pi \rightarrow \pi^{*}\right)+29 \%\left(\mathrm{~d}_{x z}+\pi \rightarrow \pi_{\mathrm{R} 1} *\right)+12 \%\left(\mathrm{~d}_{x^{2}}-y^{2} \rightarrow \pi^{*}+\pi_{\mathrm{R} 1} *\right)$ \\
\hline $\mathrm{S}_{38}$ & 0.34 & 3.354 & 0.015 & 21.81 & $31 \%\left(\mathrm{~d}_{x^{2}-y^{2}} \rightarrow \pi^{*}+\pi_{\mathrm{R} 1} *\right)+22 \%\left(\mathrm{~d}_{x z}+\pi \rightarrow \mathrm{d}_{x y}+\mathrm{n}\right)$ \\
\hline $\mathrm{S}_{39}$ & 0.07 & 3.365 & 0.000 & 0.02 & $99 \%\left((\pi / \sigma)_{\mathrm{R} 3} \rightarrow \pi^{*}\right)$ \\
\hline $\mathrm{S}_{40}$ & 0.31 & 3.380 & 0.015 & 10.57 & $28 \%\left((\sigma / \pi)_{\mathrm{R} 2} \rightarrow \pi_{\mathrm{R} 1}{ }^{*}\right)+20 \%\left(\pi+(\sigma / \pi)_{\mathrm{R} 2} \rightarrow \pi_{\mathrm{R} 1}{ }^{*}+\pi^{*}\right)+15 \%\left((\sigma / \pi)_{\mathrm{R} 2} \rightarrow \pi_{\mathrm{R} 1}{ }^{*}+\pi^{*}\right)$ \\
\hline $\mathrm{S}_{41}$ & 0.17 & 3.398 & 0.003 & 4.65 & $76 \%\left(\mathrm{~d}_{x^{2}-y^{2}} \rightarrow \pi_{\mathrm{R} 2} *\right)+10 \%\left((\sigma / \pi)_{\mathrm{R} 2} \rightarrow \pi_{\mathrm{R} 1} *\right)$ \\
\hline $\mathrm{S}_{42}$ & 0.12 & 3.401 & 0.006 & -8.75 & $88 \%\left(\mathrm{~d}_{x z}+\pi \rightarrow \pi_{\mathrm{R} 3}{ }^{*}\right)$ \\
\hline $\mathrm{S}_{43}$ & 0.29 & 3.424 & 0.003 & 0.68 & $45 \%\left((\sigma / \pi)_{\mathrm{R} 2} \rightarrow \pi_{\mathrm{R} 1} *\right)+44 \%\left((\sigma / \pi)_{\mathrm{R} 2} \rightarrow \pi_{\mathrm{R} 1}^{*}+\pi^{*}\right)$ \\
\hline
\end{tabular}

${ }^{a}$ The main experimental bands (in Ref. 110) were observed at: 1.55, 1.86, 2.23. 2.73, $3.22 \mathrm{eV}$.

${ }^{b}$ Here, o.s and r.s. correspond to oscillator (dimensionless) and rotatory strengths $\left(10^{-40} \mathrm{esu}^{2} \mathrm{~cm}^{2}\right)$, respectively.

${ }^{c}$ The abbreviations: R1, R2, R3 refer to the same side chains of corrin as presented on Figure 5.6. 


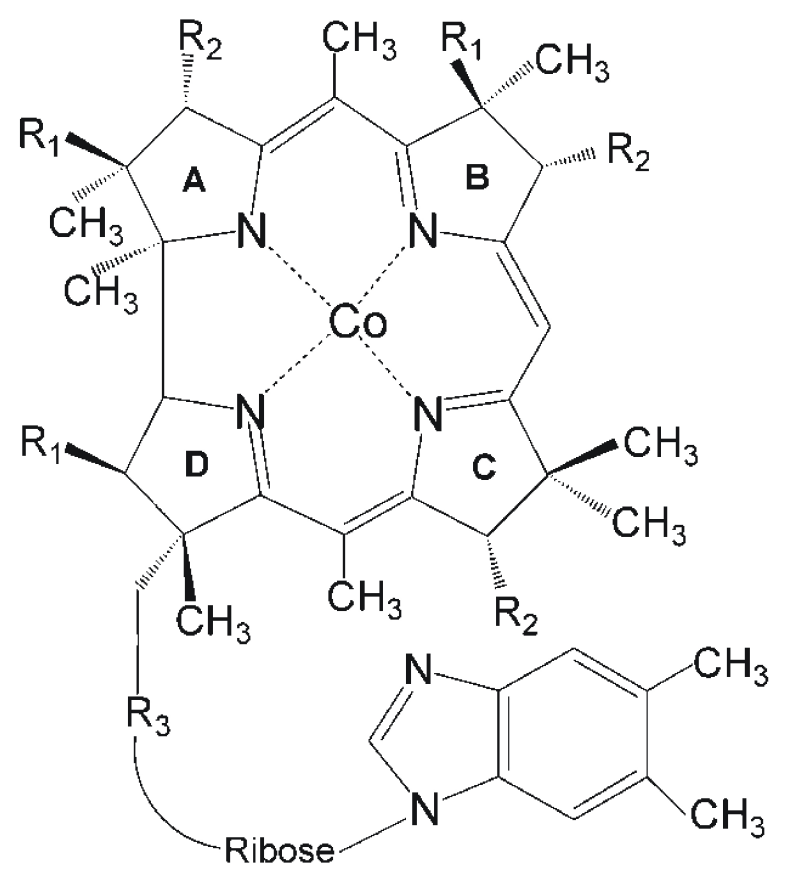

Figure 5.6. Molecular structure of vitamin $\mathrm{B}_{12 s}$ (cob(I)alamin). Note that both axial positions are unoccupied. The nucleotide loop is connected to $\mathrm{C}_{17}$ of the corrin ring. $\mathrm{R}_{1}=\mathrm{CH}_{2} \mathrm{CONH}_{2}, \mathrm{R}_{2}=\mathrm{CH}_{2} \mathrm{CH}_{2} \mathrm{CONH}_{2}, \mathrm{R}_{3}=\left(\mathrm{CH}_{2}\right)_{2} \mathrm{CONHCH}_{2} \mathrm{CH}\left(\mathrm{CH}_{3}\right) \mathrm{OPO}_{3}^{-}$.

TABLE 5.5. Excitation energies ${ }^{a}$ together with oscillator and rotatory strengths ${ }^{b}$ (CD) of 30 states of cob(I)alamin (full structure) calculated with CAM-B3LYP/6$31 G^{*}$.

\begin{tabular}{|c|c|c|c|c|c|}
\hline State & $\Lambda$ & Energy(eV) & o.s. & r.s. & Character $^{c}$ \\
\hline $\mathrm{S}_{1}$ & 0.35 & 1.341 & 0.000 & -5.26 & $94 \%\left(\mathrm{~d}_{x z}+\pi \rightarrow \pi^{*}+\mathrm{d}_{y z}\right)$ \\
\hline $\mathrm{S}_{2}$ & 0.53 & 1.473 & 0.021 & 4.22 & $87 \%\left(\mathrm{~d}_{z} \rightarrow \pi^{*}+\mathrm{d}_{y z}\right)$ \\
\hline $\mathrm{S}_{3}$ & 0.73 & 1.950 & 0.034 & -2.14 & $80 \%\left(\mathrm{~d}_{y z}+\pi \rightarrow \pi^{*}+\mathrm{d}_{y z}\right)+12 \%\left(\mathrm{~d}_{z^{2}} \rightarrow \pi^{*}+\mathrm{d}_{x z}\right)$ \\
\hline $\mathrm{S}_{4}$ & 0.54 & 2.162 & 0.030 & -42.55 & $75 \%\left(\mathrm{~d}_{y z}+\pi \rightarrow \pi^{*}+\mathrm{d}_{x z}\right)$ \\
\hline $\mathrm{S}_{5}$ & 0.35 & 2.394 & 0.000 & 4.84 & $81 \%\left(\mathrm{~d}_{x z}+\pi \rightarrow \pi^{*}+\mathrm{d}_{x z}\right)$ \\
\hline $\mathrm{S}_{6}$ & 0.48 & 2.520 & 0.001 & 20.59 & $35 \%\left(\mathrm{~d}_{x^{2}-y^{2}} \rightarrow \pi^{*}+\mathrm{d}_{y z}\right)+28 \%\left(\mathrm{~d}_{z^{2}} \rightarrow \sigma_{\mathrm{R} 1}{ }^{*}\right)+13 \%\left(\mathrm{~d}_{z} 2 \rightarrow \sigma_{\mathrm{R} 1} * \sigma_{\mathrm{R} 2}+\pi^{*}\right)$ \\
\hline $\mathrm{S}_{7}$ & 0.42 & 2.686 & 0.001 & 22.62 & $37 \%\left(\mathrm{~d}_{y z}+\pi \rightarrow \sigma_{\mathrm{R} 1}{ }^{*}\right)+18 \%\left(\mathrm{~d}_{y z}+\pi \rightarrow \sigma_{\mathrm{R} 1}{ }^{*}+\sigma_{\mathrm{R} 2}{ }^{*}+\pi^{*}\right)+13 \%\left(\mathrm{~d}_{x z}+\pi \rightarrow \pi^{*}+\mathrm{d}_{x z}\right)$ \\
\hline $\mathrm{S}_{8}$ & 0.44 & 2.933 & 0.008 & 4.11 & $37 \%\left(\mathrm{~d}_{x z}+\pi \rightarrow \sigma_{\mathrm{R} 1}^{*}\right)+18 \%\left(\mathrm{~d}_{x z}+\pi \rightarrow \sigma_{\mathrm{R} 1}{ }^{*}+\sigma_{\mathrm{R} 2}{ }^{*}+\pi^{*}\right)+16 \%\left(\mathrm{~d}_{y z}+\pi \rightarrow \pi^{*}+\mathrm{d}_{x z}\right)$ \\
\hline $\mathrm{S}_{9}$ & 0.48 & 3.116 & 0.005 & -10.16 & $38 \%\left(\mathrm{~d}_{x^{2}-y^{2}} \rightarrow \pi^{*}+\mathrm{d}_{y z}\right)+16 \%\left(\mathrm{~d}_{z} 2 \rightarrow \sigma_{\mathrm{R} 1} *\right)$ \\
\hline $\mathrm{S}_{10}$ & 0.44 & 3.128 & 0.002 & -5.33 & $34 \%\left(\mathrm{~d}_{x^{2}-y^{2}} \rightarrow \sigma_{\mathrm{R} 1}{ }^{*}\right)+16 \%\left(\mathrm{~d}_{x^{2}-y^{2}} \rightarrow \sigma_{\mathrm{R} 1} * \sigma_{\mathrm{R} 2} * \pi^{*}\right)$ \\
\hline$S_{11}$ & 0.53 & 3.333 & 0.011 & -12.80 & $34 \%\left(\mathrm{~d}_{y z}+\pi \rightarrow \pi_{\mathrm{R} 1}{ }^{*}+\pi^{*}\right)+31 \%\left(\mathrm{~d}_{z}{ }^{2} \rightarrow \pi^{*}+\mathrm{d}_{x z}\right)$ \\
\hline$S_{12}$ & 0.59 & 3.364 & 0.148 & 3.49 & $67 \%\left(\pi \rightarrow \pi^{*}+\mathrm{d}_{y z}\right)$ \\
\hline $\mathrm{S}_{13}$ & 0.47 & 3.577 & 0.041 & 3.55 & $30 \%\left(\mathrm{~d}_{y z}+\pi \rightarrow \pi_{\mathrm{R} 1}^{*}+\pi^{*}\right)+30 \%\left(\mathrm{~d}_{z} 2 \rightarrow \pi^{*}+\mathrm{d}_{x z}\right)$ \\
\hline $\mathrm{S}_{14}$ & 0.18 & 3.709 & 0.010 & 7.58 & $63 \%\left(\mathrm{~d}_{x z}+\pi \rightarrow \pi_{\mathrm{R} 1}{ }^{*}+\pi^{*}\right)+13 \%\left(\mathrm{~d}_{x z}+\pi \rightarrow \pi^{*}+\pi_{\mathrm{R} 1} *\right)$ \\
\hline $\mathrm{S}_{15}$ & 0.33 & 3.890 & 0.242 & 12.66 & $59 \%\left(\mathrm{~d}_{z^{2}} \rightarrow \pi_{\mathrm{R} 1} * \pi^{*}\right)+11 \%\left(\mathrm{~d}_{z^{2}} \rightarrow \pi^{*}+\pi_{\mathrm{R} 1}{ }^{*}\right)$ \\
\hline $\mathrm{S}_{16}$ & 0.46 & 3.986 & 0.006 & -5.91 & $77 \%\left(\mathrm{~d}_{x^{2}-y^{2}} \rightarrow \pi^{*}+\mathrm{d}_{x z}\right)$ \\
\hline $\mathrm{S}_{17}$ & 0.27 & 4.117 & 0.058 & -14.40 & $61 \%\left(\mathrm{~d}_{y z}+\pi \rightarrow \pi_{\mathrm{R} 1} *\right)$ \\
\hline $\mathrm{S}_{18}$ & 0.32 & 4.384 & 0.069 & 23.64 & $52 \%\left(\mathrm{~d}_{z 2} \rightarrow \pi_{\mathrm{R} 1}^{*}\right)+10 \%\left(\pi \rightarrow \pi^{*}+\mathrm{d}_{x z}\right)$ \\
\hline $\mathrm{S}_{19}$ & 0.58 & 4.560 & 0.094 & 15.58 & $47 \%\left(\pi+\mathrm{d}_{y z} \rightarrow \pi^{*}+\mathrm{d}_{y z}\right)+22 \%\left(\mathrm{~d}_{x z}+\pi \rightarrow \pi_{\mathrm{R} 1} *\right)$ \\
\hline $\mathrm{S}_{20}$ & 0.54 & 4.666 & 0.646 & 46.24 & $56 \%\left(\pi \rightarrow \pi^{*}+\mathrm{d}_{x z}\right)+14 \%\left(\mathrm{~d}_{z} \rightarrow \pi_{\mathrm{R} 1} *\right)$ \\
\hline $\mathrm{S}_{21}$ & 0.23 & 4.750 & 0.007 & -15.00 & $30 \%\left(\mathrm{~d}_{y z}+\pi \rightarrow \pi_{\mathrm{R} 1} *\right)+19 \%\left(\mathrm{~d}_{y z}+\pi \rightarrow \sigma_{\mathrm{R} 2} * \sigma_{\mathrm{R} 1} *\right)+11 \%\left(\mathrm{~d}_{y z}+\pi \rightarrow \sigma_{\mathrm{R} 1} *+\mathrm{d}_{x y}+\mathrm{n}\right)$ \\
\hline $\mathrm{S}_{22}$ & 0.37 & 4.766 & 0.048 & 2.81 & $36 \%\left(\mathrm{~d}_{x z}+\pi \rightarrow \pi_{\mathrm{R} 1} *\right)+15 \%\left(\pi+\mathrm{d}_{y z} \rightarrow \pi^{*}+\mathrm{d}_{y z}\right)+12 \%\left(\mathrm{~d}_{x z}+\pi \rightarrow \sigma_{\mathrm{R} 2} * \sigma_{\mathrm{R} 1}{ }^{*}\right)$ \\
\hline $\mathrm{S}_{23}$ & 0.24 & 4.786 & 0.012 & -3.72 & $49 \%\left(\mathrm{~d}_{y z}+\pi \rightarrow \pi_{\mathrm{R} 1}^{*}\right)$ \\
\hline $\mathrm{S}_{24}$ & 0.34 & 5.053 & 0.066 & 15.81 & $43 \%\left(\mathrm{~d}_{x^{2}-y^{2}} \rightarrow \pi_{\mathrm{R} 1}^{*}+\pi^{*}\right)+19 \%\left(\pi \rightarrow \pi_{\mathrm{R} 1} * \pi^{*}\right)$ \\
\hline $\mathrm{S}_{25}$ & 0.24 & 5.093 & 0.001 & -0.83 & $34 \%\left(\mathrm{~d}_{z 2} \rightarrow \pi_{\mathrm{R} 1} *\right)+29 \%\left(\mathrm{~d}_{y z}+\pi \rightarrow \pi^{*}+\pi_{\mathrm{R} 1} *\right)+10 \%\left(\mathrm{~d}_{y z}+\pi \rightarrow \pi_{\mathrm{R} 1} * \pi^{*}\right)$ \\
\hline $\mathrm{S}_{26}$ & 0.25 & 5.125 & 0.046 & 0.36 & $22 \%\left(\mathrm{~d}_{z^{2}} \rightarrow \sigma_{\mathrm{R} 2} * \sigma_{\mathrm{R} 1} *\right)+14 \%\left(\mathrm{~d}_{z^{2}} \rightarrow \sigma_{\mathrm{R} 1}^{*}+\mathrm{d}_{x y}+\mathrm{n}\right)+11 \%\left(\mathrm{~d}_{z^{2}} \rightarrow \sigma_{\mathrm{R} 2} *\right)$ \\
\hline$S_{27}$ & 0.42 & 5.179 & 0.162 & 0.09 & $38 \%\left(\pi \rightarrow \pi_{\mathrm{R} 1} *+\pi^{*}\right)+18 \%\left(\mathrm{~d}_{x^{2}-u^{2}} \rightarrow \pi_{\mathrm{R} 1} * \pi^{*}\right)$ \\
\hline $\mathrm{S}_{28}$ & 0.23 & 5.202 & 0.006 & 4.32 & $44 \%\left(\mathrm{~d}_{z} 2 \rightarrow \pi_{\mathrm{R} 1} *\right)+27 \%\left(\mathrm{~d}_{y z}+\pi \stackrel{y}{\rightarrow} \pi^{*}+\pi_{\mathrm{R} 1} *\right)$ \\
\hline $\mathrm{S}_{29}$ & 0.09 & 5.265 & 0.002 & 0.56 & $83 \%\left(\mathrm{~d}_{x z}^{z}+\pi \rightarrow \pi_{\mathrm{R} 1} *\right)$ \\
\hline $\mathrm{S}_{30}$ & 0.18 & 5.355 & 0.005 & -5.90 & $78 \%\left(\mathrm{~d}_{y z}+\pi \rightarrow \pi_{\mathrm{R} 2} *\right)$ \\
\hline
\end{tabular}

${ }^{a}$ The main experimental bands (in Ref. 110) were observed at: 1.55, 1.86, 2.23. 2.73, $3.22 \mathrm{eV}$.

${ }^{b}$ Here, o.s and r.s. correspond to oscillator (dimensionless) and rotatory strengths $\left(10^{-40} \mathrm{esu}^{2} \mathrm{~cm}^{2}\right)$, respectively.

${ }^{c}$ The abbreviations: R1, R2, R3 refer to the same side chains of corrin as presented on Figure 5.6. 


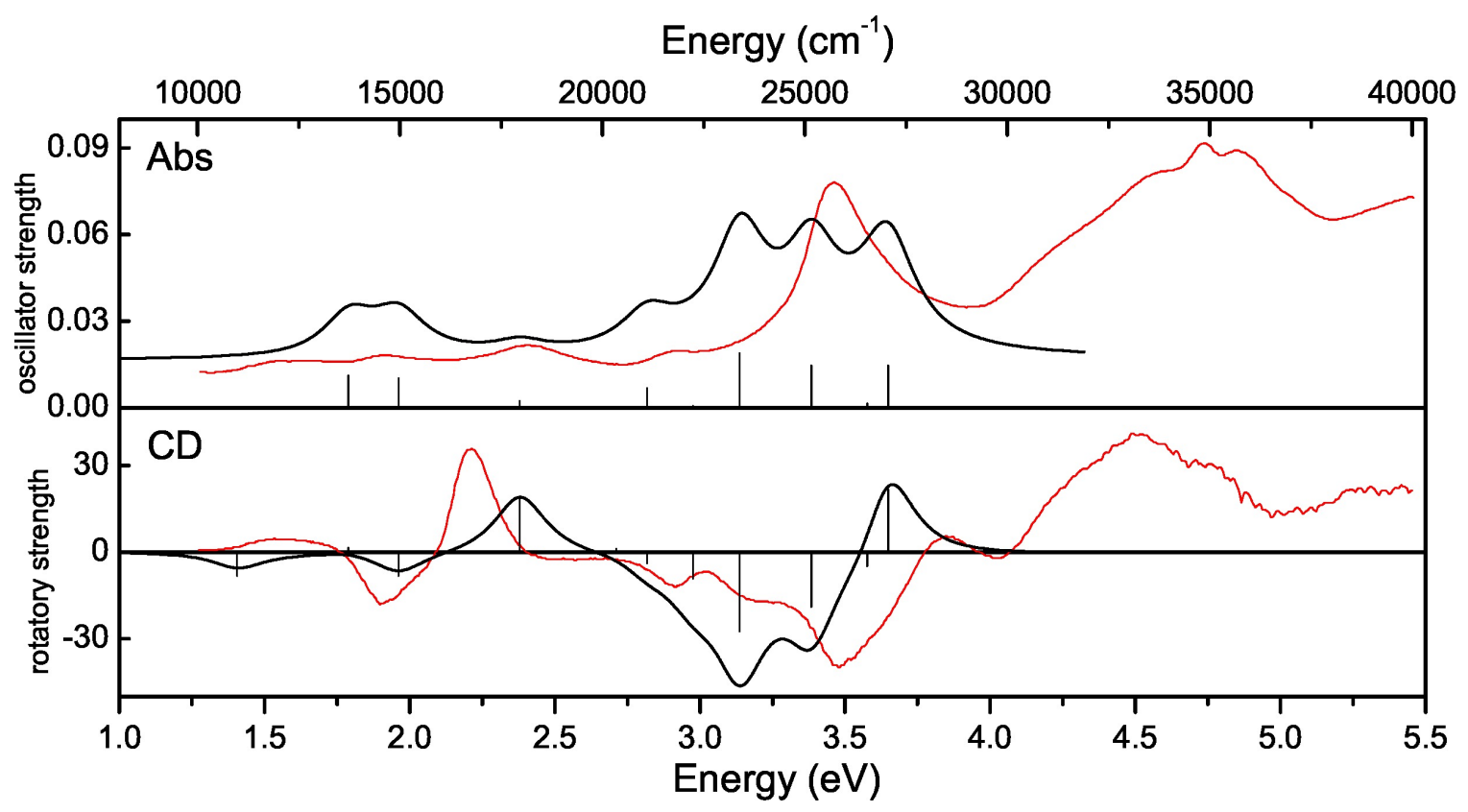

Figure 5.7. BP86/6-31G*-computed electronic spectra of cob(I)alamin (full structure) based on 11 isolated excitations localized mainly on corrin.

The visual inspection of the computed spectra for the full structure (Figure 5.5) further supports the BP86 functional in producing results correlating better to experiment than those generated using CAM-B3LYP. As was the case for the small model of cob(I)alamin, the D/E band has mainly positive features in the $\mathrm{CD}$ spectrum with the CAM-B3LYP functional. At the same time, this region is in much better agreement with experiment using BP86, as several negative signals are obtained. A closer look to the nature of the excitations indicates a significant participation of the side chains of the corrin ring in the majority of these transitions. However, the interpretation of the individual bands is limited due to the fact that only two states in the range from 2.25 to $2.75 \mathrm{eV}$ have $\Lambda$ values larger than 0.4 . Whereas the $\mathrm{S}_{10}$ is described as a mixture of $\mathrm{d} / \pi \rightarrow \pi_{\mathrm{R} 1}^{*} / \pi^{*}, \mathrm{~S}_{15}$ is dominated by $\pi /(\sigma / \pi)_{\mathrm{R} 2} \rightarrow \pi^{*}$. Although the $\mathrm{S}_{15}$ has an energy of $2.62 \mathrm{eV}$, which is close to the experimental peak found at $\sim 2.72 \mathrm{eV}$, the presence of a number of neighboring states with $\Lambda<0.4$ precludes an unambiguous assignment. 
The low-energy part of the spectrum seems to be quite well reproduced with calculations using the full structure, especially the MCD spectrum. Whereas the nature of the first three excitations remains almost intact in both structural models, the excitation energies for the full structure are slightly shifted towards lower values when BP86 is used. These three lowest excited states also have satisfactory $\Lambda$ values. The low-lying excited states based on CAM-B3LYP do not vary significantly between the two structures used in our calculations. Nevertheless, the better correlation of the small model of cob(I)alamin between the BP86 results and CASSCF/MC-XQDPT2 for the low-lying excited states and their agreement with the same states calculated for the full structure confirms the better applicability of BP86 than CAM-B3LYP to study excitations on this system and strengthens the reliability of the assignments made in the earlier sections.

In addition, an interesting finding appears when spectra are simulated excluding transitions involving side-chain contributions. Based on BP86 calculations for the full structure, we isolated 11 excitations that were either localized on the corrin or where the participation of the side-chain orbitals was relatively small. The spectra obtained are shown in Figure 5.7 and are still in satisfactory agreement with experiment. In addition to the first three electronic excitations, already discussed, the $S_{7}$ has $\Lambda>0.4$. It also has a similar energy (2.23 as compared to 2.28 $e V$ ), the $d \rightarrow \pi^{*}$ nature and the same sign of the rotational strength as the $S_{5}$ state $(2.28 \mathrm{eV})$ calculated for the simplified model of cob(I)alamin, which we proposed as a potential transition associated with the experimental band at $2.10 \mathrm{eV}$. The remaining 7 states possess small contributions from side chains and therefore, a conclusive assignment of the $\mathrm{D} / \mathrm{E}$ and $\gamma$ bands based on the calculations on the full structure cannot be unambiguously made. However, the excitation energies and negative sign of the rotational strengths of $\mathrm{S}_{15}, \mathrm{~S}_{17}$, and $\mathrm{S}_{21}$ suggest that these states might possibly contribute to the experimental D/E part. For similar reasons, the $S_{25}$ and $\mathrm{S}_{35}$ could be considered as excitations associated with the $\gamma$ band. The mixed $\mathrm{d} / \pi \rightarrow \pi^{*} / \mathrm{d}$ nature of $\mathrm{S}_{38}$ suggests that this state could contribute as well. However, both $S_{35}$ and $S_{38}$ possess $\Lambda<0.4$, leaving these assignments questionable. Clearly, 
the high-energy parts of the cob(I)alamin spectra cannot be resolved based on the calculations employing the full structure. However, the arguments brought up for the low-energy regions further confirm that BP86 is an appropriate functional for studying electronic properties of cobalamins.

\subsection{Conclusions}

The high reactivity of cob(I)alamin prevents a detailed structural characterization and spectroscopic techniques are therefore important tools for gaining insight into the electronic properties of the system. Despite the many studies dealing with the electronic spectra of the super-reduced form of vitamin $B_{12}$, the origins of the different bands have not been elucidated. The aim of the study presented in this chapter was to provide a comprehensive assignment of individual transitions at the molecular level. First, the agreement between calculated TD-DFT and experimental Abs, CD and MCD spectra was examined, where we noted the better overall agreement obtained with the BP86 XC functional. Second, the quality of the TD-DFT results was evaluated by comparing them against CASSCF/MC-XQDPT2 calculations, adding support to the superiority of BP86 over CAM-B3LYP. Third, two different molecular models were examined in order to assess the influence of structural simplifications on the computed excited states. To the best of our knowledge, such comparisons have not yet been reported for electronically excited states. It was shown that when the side chains of the corrin ring are considered, the number of longrange excitations, associated with these moieties, increases, but the charge-transfer nature of these excitations suggests that the corresponding excitation energies may be significantly underestimated and thus these contributions to the high-energy part of the spectrum may be artifacts of the BP86 XC functional. This conclusion applies in particular to high-energy states, whereas reliable comparisons could be made for the low-lying excitations. As a result, not only was the BP86 functional again found to be the most appropriate method for describing excitations in $\mathrm{B}_{12}$ cofactors, but transitions involving also partly Co d orbitals were shown to contribute to the 
major bands, despite normally being assigned as $\pi \rightarrow \pi^{*}$ transitions. In addition, the origin of the $\alpha / \beta$ band, commonly interpreted as a vibrational progression, was addressed. We find several electronic transitions, with both functionals as well as for both structural models, matching well the peaks observed in the Abs, CD and MCD spectra. It is worth to mention that multiple electronic excitations have also been associated with the $\alpha / \beta$ bands of $\mathrm{MeCbl}$ and $\mathrm{CNCbl},{ }^{140}$ undermining the suggestion of a vibrational progression as a common spectroscopic feature of the low-energy part of cobalamins photoabsorption spectra. 


\section{CHAPTER 6}

\section{ELECTRONIC AND STRUCTURAL PROPERTIES OF LOW-LYING EXCITED STATES OF VITAMIN $B_{12}^{1}$}

\subsection{Introduction}

Vitamin $\mathrm{B}_{12}$ (CNCbl, Figure 1.1) is an indispensable human nutrient and its properties have been probed both experimentally and theoretically. From photophysical point of view, the presence of the CN group makes the CNCbl distinguishable from other cobalamins in several ways. In contrast to MeCbl or AdoCbl, the $\mathrm{Co}-\mathrm{C}$ bond in $\mathrm{CNCbl}$ and related non-alkylcob(III)alamins such as azidocobalamin $\left(\mathrm{N}_{3} \mathrm{Cbl}\right)$ or aquocobalamin $\left(\mathrm{H}_{2} \mathrm{OCbl}^{+}\right)$, is photostable and does not undergo photodissociation under conditions of simple photon excitation. ${ }^{64}$ Instead, excitation results in internal emission from the initial excited state, which has dominant $\pi \rightarrow \pi^{*}$ character, to intermediate state $\left(\mathrm{S}_{1}\right)$ presumably, lacking an axial base. In contrast to MeCbl, where the $\mathrm{S}_{1}$ state has predominantly $\mathrm{d}_{\mathrm{Co}} \rightarrow \pi^{*}$ metal-to-ligand charge transfer (MLCT) character, ${ }^{58,62,65}$ it has been proposed that $S_{1}$ intermediate state in $\mathrm{CNCbl}$ is best described as $\pi \rightarrow \mathrm{d}_{z^{2} \text { Co }}$ ligand-to-metal charge transfer (LMCT). ${ }^{64,65}$

In the present chapter the electronic and structural properties of low-lying excited states of $\mathrm{CNCbl}$ were studied by means of time-dependent density functional theory (TD-DFT) ${ }^{80-84}$ Electronically excited states of vitamin $\mathrm{B}_{12}$ has been subject of several theoretical investigations. Initially, electronic absorption spectrum (Abs) has been assigned based on the B3LYP functional. ${ }^{107}$ However, when

\footnotetext{
${ }^{1}$ The material presented in this Chapter was reprinted (adapted) with permission from P. Lodowski, M. Jaworska, K. Kornobis, T. Andruniów, and P. M. Kozlowski, J. Phys. Chem. B 115, 13304 (2011). Copyright 2011 American Chemical Society.
} 


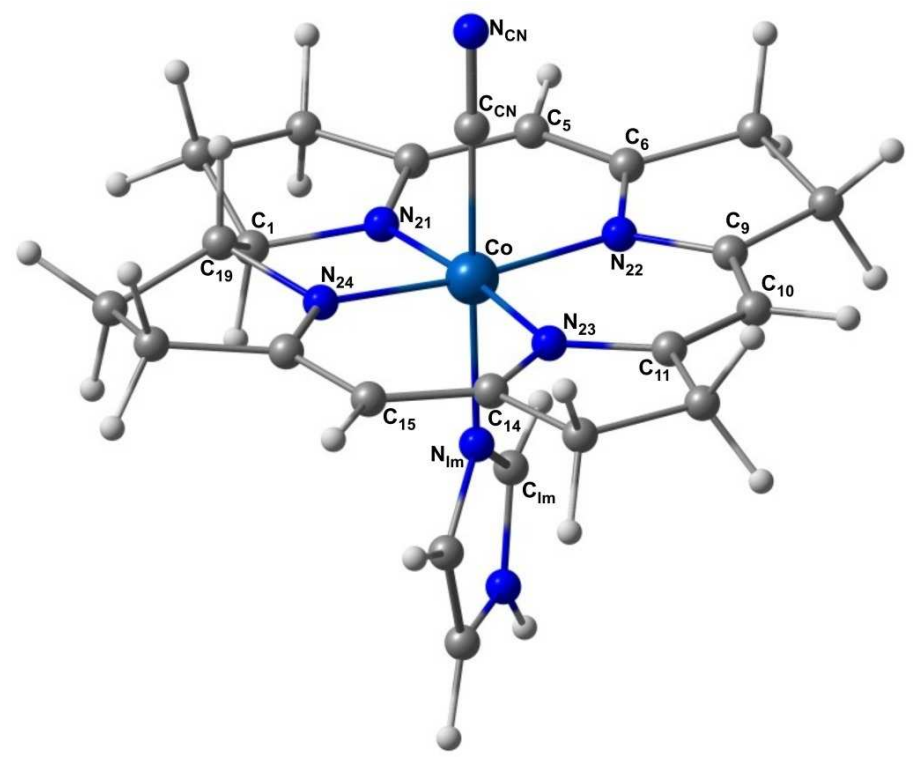

Figure 6.1. Structural model of vitamin $\mathrm{B}_{12}$ used in calculations and denoted as Im-[Co ${ }^{\mathrm{III}}$ (corrin) $]-\mathrm{CN}^{+}$.

linear and quadratic response TD-DFT was applied to investigate circular dichroism (CD) and magnetic CD (MCD) spectra along with Abs data, it turned out that the BP86 functional (rather than CAM-B3LYP) gave much better overall agreement with experiment (see Chapter 4 or Ref. 140). This particularly applies to the lowenergy part of spectra so-called $\alpha / \beta$ region. Furthermore, based on the BP86 it was concluded that the $\alpha / \beta$ part of Abs spectrum was associated with several electronic transitions rather than vibrational progression of single electronic excitation in accordance with earlier proposals. Recent benchmark analysis investigated the performance of different functional where three i.e., B3LYP, BP86 and LC-BLYP received special attention (see Chapter 2 or Ref. 161). In addition the effect of relative contributions of DFT and HF to the exchange correlation functional was investigated as a function of range separation parameter $(\mu)$. A comprehensive analysis revealed that only results based on the BP86 and LC-BLYP, with $\mu$ being close to zero, are consistent with both experimental results and high-level CASSCF/MC-XQDPT2 calculations. Consequently, the BP86 functional was applied in studies presented in this chapter where properties of low-lying singlet and triplet excited states of CNCbl were analyzed as a function of $\mathrm{Co}-\mathrm{C}$ bond distance. 
In all previous investigations the nature of the electronic transitions and spectral assignments has been discussed in terms of vertical excitations based on the ground state optimized geometry. ${ }^{107,140,161}$ This approach allowed for assignment of the majority of experimental bands observed in CNCbl Abs spectrum (referred to as "typical") in comparison to $\mathrm{MeCbl}$, that was considered as "unique". ${ }^{108}$ It should be noticed that structurally CNCbl differs noticeably from its alkylcobalamin analogs. The source lies in the nature of axial bonding. According to X-ray crystallographic studies, the Co-C bond in CNCbl (1.868 or $1.886 \AA$ ) is shorter than in MeCbl (1.979 ̊̊) or AdoCbl (2.033 $̊$ ), respectively. ${ }^{147,284}$ These differences have been previously explored by DFT methods, emphasizing the importance of inverse trans influence. ${ }^{88,164}$ In the study presented in this chapter geometrical changes associated with electronically excited states of CNCbl were also investigated in order to explain distinct photophysical properties of $\mathrm{CNCbl}$ when compared to MeCbl. The mechanism of the Co-C bond photodissociation in MeCbl has been examined previously by TD-DFT. ${ }^{118,119}$ It was found that its photolysis is mediated by the dissociative ${ }^{3} \sigma_{\mathrm{Co}-\mathrm{C}} \rightarrow \sigma_{\mathrm{Co}-\mathrm{C}}^{*}$ triplet state that drops in energy as the Co-C bond lengthens and the first excited state $\left(\mathrm{S}_{1}\right)$ was identified as the key intermediate, having dominant MLCT character. Consequently, the similar protocol was applied in present studies where properties of low-lying singlet and triplet excited states of $\mathrm{CNCbl}$ were analyzed as a function of $\mathrm{Co}-\mathrm{C}$ bond distance. Studies presented in this chapter shade new light on the photophysical properties of CNCbl and an effort was put to explain the differences, observed experimentally, between alkyl and non-alkylcobalamins.

\subsection{Computational Details}

All of the calculations were carried out using non-local DFT with the gradientcorrected Becke-Perdew (BP86) functional ${ }^{114,124}$ and the 6-31G(d) basis set (5d components) as implemented in Gaussian $03,{ }^{148}$ Turbomole, ${ }^{151}$ or GAMESS ${ }^{285}$ suite of programs. Recent benchmark analysis has demonstrated that the BP86/6-31G(d) 
level of theory was appropriate theoretical platform for describing electronic properties of vitamin $B_{12}$ (see Chapter 2 or Ref. 161). Simplified model of vitamin $B_{12}$, in which all side chains of corrin were substituted by hydrogen atoms, was used in all calculations (Figure 6.1). The structure was initially optimized in gas phase and subsequent vertical excitations were computed with TD-DFT both in vacuo and in water solution modeled via conductor-like screening model (COSMO). ${ }^{149}$

In order to simulate the dissociation process, the $\mathrm{Co}-\mathrm{C}_{\mathrm{CN}}$ bond was repeatedly stretched with the step size of $0.05 \AA$ and the ground state geometry was re-optimized at every point. Next, each geometry was used for calculating the manifold of low-lying singlet and triplet states using the TD-DFT/BP86/6-31G(d) level of theory. The potential energy curves were then generated as a function of Co-C bond distance. Because the aforementioned benchmark study (Chapter 2 or Ref. 161) evaluated only the quality of the local excited states of vitamin $B_{12}$ and did not address dissociation of a CN ligand, additional evidence refuting the likelihood of charge transfer (CT) problem was provided. Namely, the $\Lambda$ parameter was computed as function of the Co-CN distance. It has been previously suggested that simple diagnostic test based on orbital overlap (i.e., $\Lambda$ parameter ${ }^{137}$ ) may be used to judge the reliability of excitation energies in TD-DFT. The $\Lambda$ parameter measures the degree of overlap between the occupied and virtual orbitals involved in an excitation. Its numerical value can range between 0 and 1 . A value near zero signifies a long-range excitation while a value near unity signifies a short-range excitation and energies are significantly underestimated when $\Lambda$ is very small. It has been further proposed $^{138}$ that an excitation with $\Lambda<0.4$ from a GGA or $\Lambda<0.3$ from a hybrid functional is likely to be significantly underestimated.

In addition, the electronic and structural properties of low-lying excited states were further investigated by analyzing the corresponding potential energy surfaces (PES) computed as a function of both $\mathrm{Co}-\mathrm{C}$ and $\mathrm{Co}-\mathrm{N}_{\mathrm{Im}}$ axial bonds. Finally, the geometry of low-lying excited states has also been optimized in vacuo as well as in water solution, employing the $\mathrm{COSMO} / \mathrm{H}_{2} \mathrm{O}$ model. 


\subsection{Results and Discussion}

\subsubsection{Structural Model of CNCbl}

The optimized geometrical parameters (Table 6.1) of simplified CNCbl model (Figure 6.1) can be directly compared with the available data corresponding to high-resolution crystal structure of CNCbl (Tables 6.2 and 6.3 provide a detailed list of structural parameters). The optimized $\mathrm{Co}-\mathrm{C}_{\mathrm{CN}}$ bond length of $1.844 \AA$ matched well the distances of 1.868(8) $\AA$ or 1.886(4) reported for the crystal structures of $\mathrm{CNCbl}(\mathrm{KCl})$ or $\mathrm{CNCbl}(\mathrm{LiCl}),{ }^{147}$ respectively. At the same time, the computed Co- $\mathrm{N}_{\mathrm{Im}}$ bond distance of $2.048 \AA$ correlated nicely with the experimental values of 2.029(6) $\AA$ or 2.041(3) $\AA$, respectively. Furthermore, the $\mathrm{C}_{\mathrm{CN}}-\mathrm{N}_{\mathrm{CN}}$ bond distance of $1.181 \AA$ compared well with the experimental value of $1.161 \AA$. Inclusion of water solvent via COSMO model left the Co- $\mathrm{C}_{\mathrm{CN}}$ and $\mathrm{C}_{\mathrm{CN}}-\mathrm{N}_{\mathrm{CN}}$ bond distances practically intact. The most noticeable change was found for the Co- $\mathrm{N}_{\mathrm{Im}}$ bond, that was decreased by $0.027 \AA$, giving a value of $2.021 \AA$ and improving its agreement with $\mathrm{CNCbl}(\mathrm{KCl})$ X-ray bond distance of 2.029(6) $\AA{ }^{147}$ In summary, the ground state geometry of CNCbl was reasonably well reproduced by the DFT/BP86-6-31G(d) calculations.

\subsubsection{NBO Charges Analysis of CNCbl}

The structural model of CNCbl has the total charge equal to +1 because the nucleotide side chain containing negative $\mathrm{PO}_{4}^{-}$group has been truncated (compare Figures 1.1 and 6.1). It is, therefore, of interest to understand how this charge is distributed and how this distribution changes upon inclusion of solvent. The NBO charges for the ground state $\left(\mathrm{S}_{0}\right)$ of CNCbl model, extracted from DFT calculations, have been listed in Table 6.4. For the $S_{0}$ state the formal charge on cobalt is +3 , however, the computed NBO charge was lower, i.e. 1.330 in gas phase and 1.335 in water solution (COSMO). At the same time, the corrin and Im moieties (Im = imidazole) also gained positive charges, i.e. 0.031 and 0.136 , or 0.077 and 0.177 , without or with solvent, respectively. 
TABLE 6.1. Selected geometrical parameters ${ }^{a}$ of ground state and low-lying excited states corresponding to axial bonding in $\mathrm{Im}-\left[\mathrm{Co}^{\mathrm{III}}(\right.$ corrin) $]-\mathrm{CN}^{+}$.

\begin{tabular}{|c|c|c|c|c|c|}
\hline & $\overline{S_{0}^{b}}$ & $\mathrm{~S}_{1}(\mathrm{~A})$ & $\mathrm{S}_{1}(\mathrm{C})$ & $\mathrm{T}_{1}(\mathrm{~A})$ & $\mathrm{T}_{1}(\mathrm{C})$ \\
\hline & \multicolumn{5}{|c|}{ gas phase } \\
\hline & \multicolumn{5}{|c|}{ Bond distances $[\AA]$} \\
\hline $\mathrm{Co}-\mathrm{C}_{\mathrm{CN}}$ & 1.844 & 1.830 & 2.104 & 1.850 & 1.978 \\
\hline $\mathrm{Co}-\mathrm{N}_{\mathrm{Im}}$ & 2.048 & 2.006 & 2.290 & 2.058 & 2.533 \\
\hline \multirow[t]{2}{*}{$\mathrm{C}_{\mathrm{CN}}-\mathrm{N}_{\mathrm{CN}}$} & 181 & 1.184 & 1.186 & 1.181 & 1.185 \\
\hline & \multicolumn{5}{|c|}{ Bond angles [deg] } \\
\hline $\mathrm{C}_{\mathrm{CN}}-\mathrm{Co}-\mathrm{N}_{\mathrm{Im}}$ & 178.8 & 169.8 & 175.9 & 179.0 & 175.9 \\
\hline \multirow[t]{3}{*}{$\mathrm{Co}-\mathrm{C}_{\mathrm{CN}}-\mathrm{N}_{\mathrm{CN}}$} & 179.2 & 177.6 & 150.6 & 179.0 & 176.5 \\
\hline & \multicolumn{5}{|c|}{$\mathrm{COSMO} / \mathrm{H}_{2} \mathrm{O}$} \\
\hline & \multicolumn{5}{|c|}{ Bond distances $[\AA]$} \\
\hline $\mathrm{Co}-\mathrm{C}$ & 1.845 & 1.875 & 2.130 & 1.852 & 2.068 \\
\hline $\mathrm{Co}-\mathrm{N}$ & 2.021 & 2.023 & 2.236 & 1.995 & 2.417 \\
\hline $\mathrm{C}_{\mathrm{CN}}-\mathrm{N}_{\mathrm{CN}}$ & 1.182 & 1.184 & 1.187 & 1.182 & 1.184 \\
\hline & \multicolumn{5}{|c|}{ Bond angles [deg] } \\
\hline $\mathrm{C}_{\mathrm{CN}}-\mathrm{Co}-$ & 178.5 & 177.5 & 177.1 & 176.2 & 176.4 \\
\hline $\mathrm{Co}-\mathrm{C}_{\mathrm{CN}}-\mathrm{N}_{\mathrm{CN}}$ & 179.4 & 178.1 & 160.6 & 179.6 & 179.4 \\
\hline
\end{tabular}

${ }^{a}$ Tables 6.3 and 6.5 provide a detailed description of structural parameters (A and C refer to different minima on the $\mathrm{S}_{1}$ and $\mathrm{T}_{1}$ energy surfaces, see Sections 6.3.5 and 6.3.6).

${ }^{b}$ The experimental values based on high-resolution X-ray crystal structures (Ref. 147) are: Co-C $=1.868 \AA$, Co- $\mathrm{N}_{\mathrm{DBI}}=$ $2.029 \AA$ for $\mathrm{CNCbl}(\mathrm{KCl})$; $\mathrm{Co}-\mathrm{C}=1.886 \AA, \mathrm{Co}-\mathrm{N}_{\mathrm{DBI}}=2.041 \AA$ for $\mathrm{CNCbl}(\mathrm{LiCl})$, respectively.

On the other hand, the cyanide group obtained the negative charge in both environments with the value lower by 0.086 in solution. The $\mathrm{Co}-\mathrm{C}$ bond is of particular interest and, as it can be seen from calculated data, the presence of solvent resulted in enhanced polarization of axial bonding. It appeared that the charge was shifted from the corrin and Im to the cobalt and CN ligand. In addition, the inclusion of solvent magnified the charge separation within Co-C by increasing the positive charge on Co and the negative charge on $\mathrm{CN}^{-}$. 
TABLE 6.2. Selected geometrical parameters for $\mathrm{Im}-\left[\mathrm{Co}^{\mathrm{III}}\right.$ (corrin) $]-\mathrm{CN}^{+}$.

\begin{tabular}{|c|c|c|c|c|c|}
\hline & $\mathrm{S}_{0}$ & $\overline{S S_{1}(\mathrm{~A})}$ & $2 \mathrm{~S}_{1}(\mathrm{C})$ & $\overline{\mathrm{T}_{1}(\mathrm{~A})}$ & $\overline{\mathrm{T}_{1}(\mathrm{C})}$ \\
\hline & \multicolumn{5}{|c|}{$\mathrm{r}[\AA]$} \\
\hline $\mathrm{Co}-\mathrm{C}_{\mathrm{CN}}$ & 1.844 & 1.830 & 2.104 & 1.850 & 1.978 \\
\hline $\mathrm{Co}-\mathrm{N}_{\mathrm{Im}}$ & 8 & 006 & 2.290 & 058 & 2.533 \\
\hline Co-- & 886 & 1.867 & 1.877 & 891 & 1.897 \\
\hline $\mathrm{Co}-\mathrm{N}_{24}$ & 879 & 1.883 & 1.844 & 1.871 & 1.862 \\
\hline $\mathrm{Co}-\mathrm{N}_{22}$ & 939 & 1.968 & 1.949 & 1.939 & 1.917 \\
\hline $\mathrm{Co}-\mathrm{N}_{23}$ & 940 & 1.989 & 1.936 & 1.958 & 2.002 \\
\hline \multirow[t]{2}{*}{$\mathrm{C}_{\mathrm{CN}}-\mathrm{N}_{\mathrm{CN}}$} & 1 & .184 & 1.186 & 1.181 & 1.185 \\
\hline & \multicolumn{5}{|c|}{ Bond angles } \\
\hline $\mathrm{C}_{\mathrm{CN}}-\mathrm{Co}$ & .8 & 169.8 & 175.9 & 179.0 & 175.9 \\
\hline $\mathrm{N}_{21}-\mathrm{Co}$ & .3 & 90.8 & 91.1 & 90.8 & 91.7 \\
\hline $\mathrm{N}_{22}-\mathrm{Co}-$ & 5.1 & 95.6 & 94.9 & 95.1 & 94.7 \\
\hline $\mathrm{N}_{23}-\mathrm{Co}$ & 91.3 & 90.7 & 90.8 & 90.9 & 90.7 \\
\hline $\mathrm{N}_{24}-\mathrm{Co}$ & .5 & 3.0 & 83.5 & 83.3 & 82.7 \\
\hline $\mathrm{Cc}$ & .8 & 6.8 & 116.5 & 116.5 & 117.3 \\
\hline $\mathrm{Cc}$ & 4.5 & 123.5 & 124.9 & 124.4 & 124.7 \\
\hline $\mathrm{Co}-$ & 4.4 & 122.8 & 124.8 & 123.7 & 123.6 \\
\hline $\mathrm{Co}-\mathrm{N}_{24}-$ & 1 & 114.7 & 116.1 & 115.1 & 115.8 \\
\hline \multirow[t]{2}{*}{$\mathrm{Co}-\mathrm{C}_{\mathrm{CN}}-\mathrm{N}_{\mathrm{CN}}$} & 9.2 & 177.6 & 150.6 & 179.0 & 176.5 \\
\hline & \multicolumn{5}{|c|}{ Torsion angles } \\
\hline $\mathrm{N}_{21}-\mathrm{N}_{22}$ & -1.2 & -0.8 & -2.6 & -1.3 & -11.1 \\
\hline $\mathrm{N}_{21}-\mathrm{N}_{22}-\mathrm{N}_{23}$ & -4.1 & -3.3 & -5.5 & -4.0 & -10.0 \\
\hline $\mathrm{Co}-\mathrm{N}_{22}-\mathrm{C}_{9}-\mathrm{C}$ & 1.7 & -2.5 & 3.4 & -3.6 & 5.4 \\
\hline $\mathrm{Co}-\mathrm{N}_{22}-\mathrm{C}_{6}-\mathrm{C}_{5}$ & -8.1 & -7.7 & -7.5 & -9.2 & -9.9 \\
\hline$-\mathrm{N}_{23}-\mathrm{C}_{14}-\mathrm{C}_{1}$ & -0.4 & 1.9 & -5.2 & 1.0 & 1.5 \\
\hline 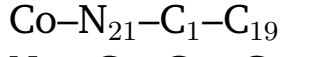 & 31.8 & 30.3 & 29.4 & 27.1 & 25.8 \\
\hline $\mathrm{N}_{\mathrm{Im}}-\mathrm{Co}-$ & 179.3 & -176.9 & 175.9 & -179.5 & -177.9 \\
\hline $\mathrm{C}_{10}-\mathrm{Co}-\mathrm{N}_{\mathrm{Im}}-\mathrm{C}_{\mathrm{Im}}$ & -88.2 & -85.3 & -89.0 & -84.7 & -82.0 \\
\hline
\end{tabular}


TABLE 6.3. Selected geometrical parameters for $\mathrm{Im}-\left[\mathrm{Co}^{\mathrm{III}}\right.$ (corrin) $]-\mathrm{CN}^{+}$(the values obtained from calculations with COSMO solvation model and water as solvent).

\begin{tabular}{|c|c|c|c|c|c|}
\hline & $\overline{S_{0}}$ & $\mathrm{~S}_{1}(\mathrm{~A})$ & 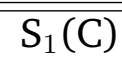 & $\mathrm{T}_{1}(\mathrm{~A})$ & $\bar{T}_{1}(\mathrm{C})$ \\
\hline & \multicolumn{5}{|c|}{$\mathrm{r}[\AA]$} \\
\hline $\mathrm{Co}-\mathrm{C}_{\mathrm{CN}}$ & 1.845 & 1.875 & 2.130 & 1.852 & 2.068 \\
\hline $\mathrm{Co}-\mathrm{N}_{\mathrm{Im}}$ & 2.021 & 2.023 & 2.236 & 1.995 & 2.417 \\
\hline $\mathrm{Co}-\mathrm{N}_{21}$ & 1.884 & 1.995 & 1.874 & 1.883 & 1.870 \\
\hline $\mathrm{Co}-\mathrm{N}_{24}$ & 1.883 & 1.856 & 1.855 & 1.891 & 1.867 \\
\hline $\mathrm{Co}-\mathrm{N}_{22}$ & 1.939 & 1.920 & 1.949 & 1.935 & 1.927 \\
\hline $\mathrm{Co}-\mathrm{N}_{23}$ & 1.940 & 2.036 & 1.939 & 1.978 & 1.976 \\
\hline \multirow[t]{2}{*}{$\mathrm{C}_{\mathrm{CN}}-\mathrm{N}_{\mathrm{CN}}$} & 1.182 & 1.184 & 1.187 & 1.182 & 1.184 \\
\hline & \multicolumn{5}{|c|}{ Bond angles } \\
\hline $\mathrm{C}_{\mathrm{CN}}-\mathrm{Co}-\mathrm{N}_{\mathrm{Im}}$ & 178.5 & 177.5 & 177.1 & 176.2 & 176.4 \\
\hline $\mathrm{N}_{21}-\mathrm{Co}-\mathrm{N}_{22}$ & 91.2 & 91.7 & 90.9 & 91.1 & 91.2 \\
\hline $\mathrm{N}_{22}-\mathrm{Co}-\mathrm{N}_{23}$ & 95.2 & 94.5 & 94.9 & 95.2 & 94.9 \\
\hline $\mathrm{N}_{23}-\mathrm{Co}-\mathrm{N}_{24}$ & 91.4 & 91.2 & 91.1 & 90.8 & 90.8 \\
\hline $\mathrm{N}_{24}-\mathrm{Co}-\mathrm{N}_{21}$ & 82.4 & 83.0 & 83.3 & 83.0 & 82.9 \\
\hline Co- $-\mathrm{N}_{21}-\mathrm{C}_{1}$ & 116.5 & 115.5 & 116.3 & 116.8 & 117.2 \\
\hline $\mathrm{Co}-\mathrm{N}_{22}-\mathrm{C}_{9}$ & 124.5 & 125.2 & 125.0 & 124.3 & 124.6 \\
\hline $\mathrm{Co}-\mathrm{N}_{23}-\mathrm{C}_{11}$ & 124.3 & 123.6 & 124.8 & 123.1 & 124.1 \\
\hline $\mathrm{Co}-\mathrm{N}_{24}-\mathrm{C}_{19}$ & 116.5 & 116.4 & 116.6 & 115.1 & 116.1 \\
\hline \multirow[t]{2}{*}{$\mathrm{Co}-\mathrm{C}_{\mathrm{CN}}-\mathrm{N}_{\mathrm{CN}}$} & 179.4 & 178.1 & 160.6 & 179.6 & 179.4 \\
\hline & \multicolumn{5}{|c|}{ Torsion angles } \\
\hline $\mathrm{N}_{21}-\mathrm{N}_{22}-\mathrm{N}_{23}-\mathrm{Co}$ & -2.0 & -3.6 & -3.3 & -1.2 & -7.7 \\
\hline $\mathrm{N}_{21}-\mathrm{N}_{22}-\mathrm{N}_{23}-\mathrm{N}_{24}$ & -4.1 & -6.2 & -5.1 & -3.2 & -6.7 \\
\hline $\mathrm{Co}-\mathrm{N}_{22}-\mathrm{C}_{9}-\mathrm{C}_{10}$ & 0.5 & -0.3 & 2.1 & -5.9 & 3.1 \\
\hline $\mathrm{Co}-\mathrm{N}_{22}-\mathrm{C}_{6}-\mathrm{C}_{5}$ & -8.1 & -6.9 & -7.2 & -7.7 & -7.6 \\
\hline $\mathrm{Co}-\mathrm{N}_{23}-\mathrm{C}_{14}-\mathrm{C}_{15}$ & 0.5 & -7.4 & -4.8 & -0.2 & -1.2 \\
\hline $\mathrm{Co}-\mathrm{N}_{21}-\mathrm{C}_{1}-\mathrm{C}_{19}$ & 34.0 & 20.5 & 32.9 & 30.1 & 30.0 \\
\hline $\mathrm{N}_{\mathrm{Im}}-\mathrm{Co}-\mathrm{C}_{10}-\mathrm{C}_{\mathrm{CN}}$ & 179.5 & 177.8 & 177.7 & -177.0 & -178.2 \\
\hline $\mathrm{C}_{10}-\mathrm{Co}-\mathrm{N}_{\mathrm{Im}}-\mathrm{C}_{\mathrm{Im}}$ & -87.0 & -92.1 & -87.7 & -84.9 & -82.1 \\
\hline
\end{tabular}


The distribution of charge based on the Mulliken analysis was found to be very similar (Table 6.5), although the separation of charges between the central atom and axial ligands was smaller and the total charge on the corin ring was more positive. According to Mulliken analysis, much of the positive charge is distributed throughout the corrin ring, while the NBO analysis indicates the localization of the positive charge essentially on the cobalt atom. It can, therefore, be noted that in the first case, the positive charge on the cobalt atom, which is the result of polarization of Co-CN bonds, is strongly compensated by the displacement of the charge from the corrin ring to the central atom. This displacement of the charge is smaller when taking into account the results of NBO analysis.

TABLE 6.4. NBO charges for Im- $\left[\mathrm{Co}{ }^{\mathrm{III}}\right.$ (corrin) $]-\mathrm{CN}^{+}$calculated in gas phase and in water solution based on COSMO/ $\mathrm{H}_{2} \mathrm{O}$ model.

\begin{tabular}{|c|c|c|c|c|c|c|c|c|c|}
\hline & $\mathrm{S}_{0}$ & & & & & & & & \\
\hline & & & & & gas phas & & & & \\
\hline & $q_{S_{0}}$ & $q_{S_{0}}$ & $q_{S_{1}(A)}$ & $q_{S_{0}}$ & $q_{S_{1}(C)}$ & $q_{S_{0}}$ & $q_{T_{1}(A)}$ & $q_{S_{0}}$ & $q_{T_{1}(C)}$ \\
\hline Co & 1.330 & 1.332 & 1.391 & 1.388 & 1.356 & 1.336 & 1.393 & 1.365 & 1.486 \\
\hline $\mathrm{C}_{\mathrm{CN}}$ & -0.091 & -0.100 & -0.055 & -0.077 & -0.202 & -0.094 & -0.086 & -0.087 & -0.215 \\
\hline $\mathrm{CN}$ & -0.497 & -0.509 & -0.402 & -0.481 & -0.678 & -0.501 & -0.473 & -0.479 & -0.594 \\
\hline Im & 0.136 & 0.138 & 0.205 & 0.137 & 0.073 & 0.138 & 0.149 & 0.093 & 0.061 \\
\hline Corr & 0.031 & 0.038 & -0.194 & -0.043 & 0.248 & 0.027 & -0.069 & 0.020 & 0.047 \\
\hline & & & & & $\Delta q^{a}$ & & & & \\
\hline Co & & & 0.059 & & -0.032 & & 0.057 & & 0.121 \\
\hline $\mathrm{C}_{\mathrm{CN}}$ & & & 0.046 & & -0.125 & & 0.008 & & -0.128 \\
\hline $\mathrm{CN}$ & & & 0.107 & & -0.197 & & 0.028 & & -0.115 \\
\hline $\mathrm{Im}$ & & & 0.066 & & -0.063 & & 0.011 & & -0.032 \\
\hline Corr & & & -0.232 & & 0.291 & & -0.096 & & 0.027 \\
\hline & & & & & $\mathrm{OSMO} / \mathrm{H}$ & & & & \\
\hline & $q_{S_{0}}$ & $q_{S_{0}}$ & $q_{S_{1}(A)}$ & $q_{S_{0}}$ & $q_{S_{1}(C)}$ & $q_{S_{0}}$ & $q_{T_{1}(A)}$ & $q_{S_{0}}$ & $q_{T_{1}(C)}$ \\
\hline Co & 1.335 & 1.362 & 1.312 & 1.404 & 1.353 & 1.341 & 1.360 & 1.394 & 1.492 \\
\hline $\mathrm{C}_{\mathrm{CN}}$ & -0.049 & -0.070 & -0.114 & -0.061 & -0.183 & -0.057 & -0.056 & -0.051 & -0.176 \\
\hline $\mathrm{CN}$ & -0.590 & -0.606 & -0.677 & -0.584 & -0.772 & -0.598 & -0.582 & -0.575 & -0.736 \\
\hline $\operatorname{Im}$ & 0.177 & 0.181 & 0.162 & 0.183 & 0.094 & 0.182 & 0.204 & 0.143 & 0.081 \\
\hline Corr & 0.077 & 0.063 & 0.203 & -0.003 & 0.326 & 0.075 & 0.018 & 0.038 & 0.163 \\
\hline & & & & & $\Delta q^{a}$ & & & & \\
\hline Co & & & -0.050 & & -0.051 & & 0.019 & & 0.098 \\
\hline $\mathrm{C}_{\mathrm{CN}}$ & & & -0.044 & & -0.122 & & 0.001 & & -0.125 \\
\hline $\mathrm{CN}$ & & & -0.070 & & -0.189 & & 0.016 & & -0.160 \\
\hline $\mathrm{Im}$ & & & -0.020 & & -0.089 & & 0.023 & & -0.062 \\
\hline Corr & & & 0.140 & & 0.329 & & -0.057 & & 0.125 \\
\hline
\end{tabular}

${ }^{a} \Delta q=$ difference between charge in excited state and ground state in excited state geometry. 
TABLE 6.5. Mulliken charges for Im-[Co ${ }^{\mathrm{III}}$ (corrin) $]-\mathrm{CN}^{+}$calculated in gas phase and in water solution based on COSMO $/ \mathrm{H}_{2} \mathrm{O}$ model.

\begin{tabular}{|c|c|c|c|c|c|c|c|c|c|}
\hline & 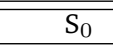 & \multicolumn{2}{|c|}{$\overline{S_{1}(\mathrm{~A})}$} & \multicolumn{2}{|c|}{$\overline{S_{1}(\mathrm{C})}$} & \multicolumn{2}{|c|}{$\overline{\mathrm{T}_{1}(\mathrm{~A})}$} & \multicolumn{2}{|c|}{$\overline{\mathrm{T}_{1}(\mathrm{C})}$} \\
\hline & \multicolumn{9}{|c|}{ gas phase } \\
\hline & $q_{S_{0}}$ & $q_{S_{0}}$ & $q_{S_{1}(A)}$ & $q_{S_{0}}$ & $q_{S_{1}(C)}$ & $q_{S_{0}}$ & $q_{T_{1}(A)}$ & $q_{S_{0}}$ & $q_{T_{1}(C)}$ \\
\hline Co & 0.380 & 0.385 & 0.414 & 0.505 & 0.463 & 0.384 & 0.398 & 0.537 & 0.531 \\
\hline $\mathrm{C}_{\mathrm{CN}}$ & 0.243 & 0.237 & 0.290 & 0.121 & 0.036 & 0.240 & 0.256 & 0.162 & 0.092 \\
\hline $\mathrm{CN}$ & -0.188 & -0.196 & -0.097 & -0.211 & -0.348 & -0.190 & -0.161 & -0.211 & -0.291 \\
\hline $\operatorname{Im}$ & 0.258 & 0.263 & 0.331 & 0.245 & 0.182 & 0.264 & 0.290 & 0.191 & 0.151 \\
\hline \multirow[t]{2}{*}{ Corr } & 0.551 & 0.548 & 0.352 & 0.461 & 0.703 & 0.542 & 0.473 & 0.482 & 0.609 \\
\hline & \multicolumn{9}{|c|}{$\Delta q^{a}$} \\
\hline Co & & & 0.028 & & -0.041 & & 0.015 & & 0.004 \\
\hline $\mathrm{C}_{\mathrm{CN}}$ & & & 0.053 & & -0.085 & & 0.013 & & -0.044 \\
\hline $\mathrm{CN}$ & & & 0.099 & & -0.137 & & 0.028 & & -0.035 \\
\hline $\operatorname{Im}$ & & & 0.068 & & -0.063 & & 0.014 & & -0.033 \\
\hline \multirow[t]{3}{*}{ Corr } & & & -0.196 & & 0.242 & & -0.058 & & 0.064 \\
\hline & \multicolumn{9}{|c|}{$\mathrm{COSMO} / \mathrm{H}_{2} \mathrm{O}$} \\
\hline & $q_{S_{0}}$ & $q_{S_{0}}$ & $q_{S_{1}(A)}$ & $q_{S_{0}}$ & $q_{S_{1}(C)}$ & $q_{S_{0}}$ & $q_{T_{1}(A)}$ & $q_{S_{0}}$ & $q_{T_{1}(C)}$ \\
\hline Co & 0.348 & 0.385 & 0.378 & 0.484 & 0.442 & 0.351 & 0.356 & 0.489 & 0.481 \\
\hline $\mathrm{C}_{\mathrm{CN}}$ & 0.278 & 0.248 & 0.206 & 0.154 & 0.061 & 0.272 & 0.275 & 0.203 & 0.138 \\
\hline $\mathrm{CN}$ & -0.274 & -0.294 & -0.354 & -0.312 & -0.448 & -0.279 & -0.265 & -0.299 & -0.386 \\
\hline $\operatorname{Im}$ & 0.311 & 0.314 & 0.286 & 0.301 & 0.214 & 0.319 & 0.339 & 0.256 & 0.195 \\
\hline \multirow[t]{2}{*}{ Corr } & 0.615 & 0.595 & 0.690 & 0.527 & 0.792 & 0.609 & 0.569 & 0.554 & 0.710 \\
\hline & & & & & $\Delta q^{a}$ & & & & \\
\hline Co & & & -0.007 & & -0.043 & & 0.006 & & -0.008 \\
\hline $\mathrm{C}_{\mathrm{CN}}$ & & & -0.043 & & -0.093 & & 0.002 & & -0.065 \\
\hline $\mathrm{CN}$ & & & -0.060 & & -0.136 & & 0.014 & & -0.087 \\
\hline $\operatorname{Im}$ & & & -0.028 & & -0.087 & & 0.020 & & -0.062 \\
\hline Corr & & & 0.094 & & 0.266 & & -0.040 & & 0.156 \\
\hline
\end{tabular}

${ }^{a} \Delta q=$ difference between charge in excited state and ground state in excited state geometry.

In order to exclude the possible misguidance in charge distribution that could have been induced by the structural truncations introduced to the CNCbl model, the full structure of the molecule was also analyzed (Table 6.6). The NBO analysis shows a significant separation of charge between the framework and side chains of the corrin ring. The total charge of the corrin is negative in contrast to the slightly positive charge on the ring for a simplified model. Nevertheless, the sum of charges of corrin ring and the side chains remains as to the value, close to the charge on the corrin ring in a truncated model. The charge distribution for the axial ligands and cobalt atom is very similar to that observed in the truncated model. In addition, according to the Mulliken analysis, the distribution of charge between the full structure and truncated model is similar. 
TABLE 6.6. NBO and Mulliken charges for the Im-[Co ${ }^{\mathrm{III}}$ (corrin) $]-\mathrm{CN}^{+}$model with side chains of the corrin ring calculated in gas phase and in water solution based on the COSMO $/ \mathrm{H}_{2} \mathrm{O}$ model (overall charge on the side chains is denoted as $\mathrm{R}$ ).

\begin{tabular}{|c|c|c|c|c|}
\hline & \multicolumn{2}{|c|}{$\mathrm{NBO}$} & \multicolumn{2}{|c|}{ Mulliken } \\
\hline & Gas & COSMO & Gas & COSMO \\
\hline & phase & $\mathrm{H}_{2} \mathrm{O}$ & phase & $\mathrm{H}_{2} \mathrm{O}$ \\
\hline Co & 1.329 & 1.335 & 0.373 & 0.360 \\
\hline $\mathrm{C}_{\mathrm{CN}}$ & -0.087 & -0.056 & 0.257 & 0.280 \\
\hline CN & -0.523 & -0.583 & -0.201 & -0.260 \\
\hline Im & 0.141 & 0.166 & 0.279 & 0.311 \\
\hline Corr & -0.647 & -0.646 & 0.449 & 0.433 \\
\hline $\mathrm{R}$ & 0.701 & 0.727 & 0.100 & 0.156 \\
\hline Corrin $+\mathrm{R}$ & 0.053 & 0.082 & 0.549 & 0.588 \\
\hline
\end{tabular}

\subsubsection{Potential Energy Curves along the Co-C Bond Stretch}

In order to understand the lack of photolytic cleavage of $\mathrm{Co}-\mathrm{C}$ in $\mathrm{CNCbl}$, which was revealed by transient absorption measurements, ${ }^{64}$ the dissociation process was simulated by repeated elongation of $\mathrm{Co}-\mathrm{C}_{\mathrm{CN}}$ bond. The calculated energies of the relevant 10 singlet and 15 triplet excited states along the $\mathrm{Co}-\mathrm{C}_{\mathrm{CN}}$ coordinate were plotted in Figure 6.2. Such strategy has been previously applied to understand photodissociation of $\mathrm{CO}$ or $\mathrm{O}_{2}$ ligands from heme. ${ }^{286-289}$ The underlying idea of calculating the plethora of low-lying excited states along a specific coordinate was to trace repulsive electronic state that can be directly connected with dissociation pathway. To ensure that computed TD-DFT excitations have not been affected by CT failure, the $\Lambda$ diagnostic test was carried out for low-lying singlet states computed as function of the Co-CN stretched coordinate. In fact, such a TD-DFT-imposed problem could be expected for excitations involving CT transfer between corrin ring and axial ligands. However, the main electronic transitions in $\mathrm{CNCbl}$ are rather localized within the corrin macrocycle. As can be inferred from Figure 6.3, none of the low-lying excitations should be considered as problematic ones as the calculated $\Lambda$ parameters exceed the recently suggested threshold value of $0.4 .{ }^{138}$ 

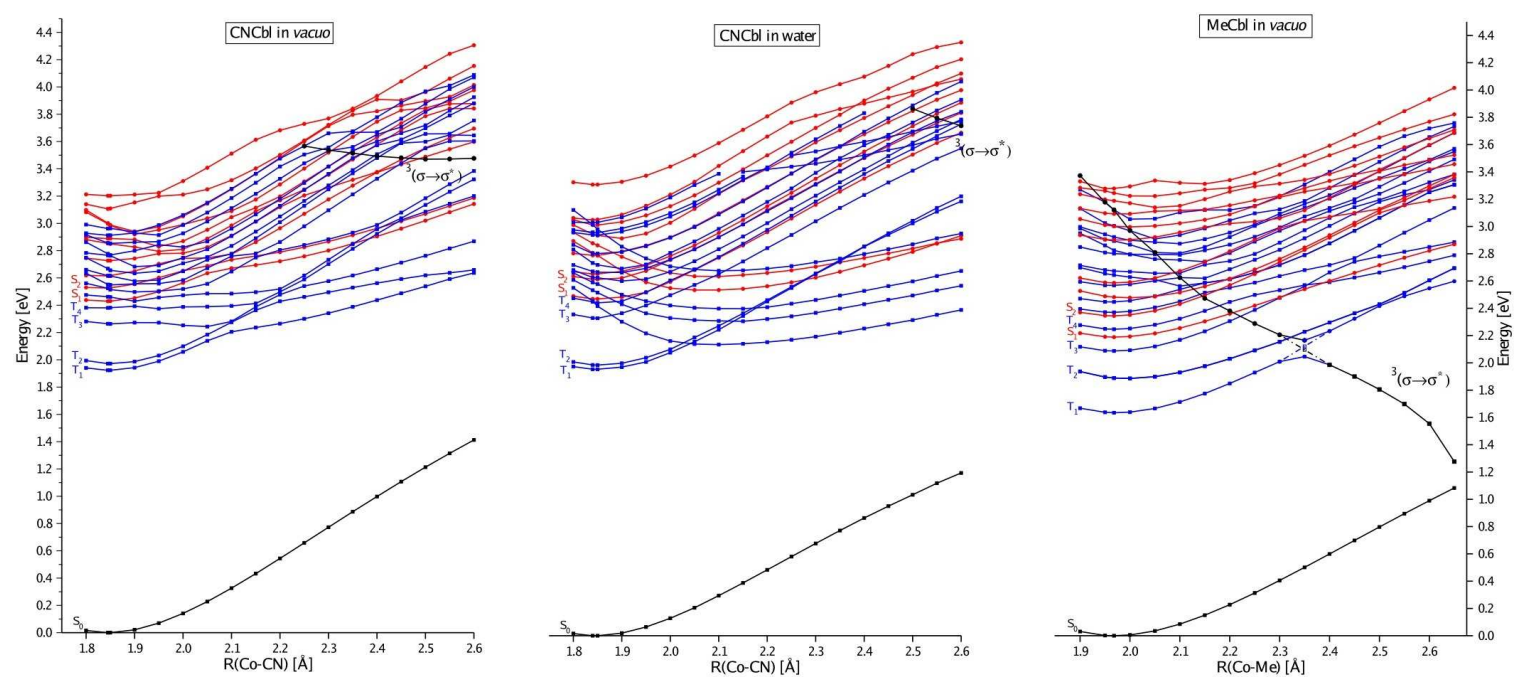

Figure 6.2. Potential energy curves of low-lying singlet (red) and triplet (blue) states of CNCbl (vacuo and water) and MeCbl (vacuo) generated as vertical excitations along the optimized $\mathrm{Co}-\mathrm{C}$ distance of $\mathrm{S}_{0}$ state. See Figure 6.3 for the corresponding $\Lambda$ diagnostic test analysis.

The potential energy curves of CNCbl were calculated and compared with the corresponding data for MeCbl. Both sets of curves were generated assuming structural models simplified with respect to corrin side chains (Figure 6.1). These models have been denoted as Im-[CoII (corrin) $]-\mathrm{R}^{+}$, where $\mathrm{R}=\mathrm{CN}, \mathrm{CH}_{3}$ for $\mathrm{CNCbl}$ and $\mathrm{MeCbl}$, respectively. Based on the TD-DFT calculations, it was concluded that in the case of $\mathrm{MeCbl}$, the photodissociation of the $\mathrm{Co}-\mathrm{C}$ bond is mediated by the repulsive ${ }^{3} \sigma_{\mathrm{Co}-\mathrm{C}} \rightarrow \sigma_{\mathrm{Co}-\mathrm{C}}^{*}$ triplet state. For the Im-[Co ${ }^{\mathrm{III}}($ corrin) $]-\mathrm{CN}^{+}$, such triplet state was also located by connecting the transitions with significant contributions of excitations arising from occupied orbitals of $\sigma_{\mathrm{Co}-\mathrm{CN}}$ to unoccupied orbitals of $\sigma_{\mathrm{Co}-\mathrm{CN}}^{*}$, which was marked with a black line and labeled as ${ }^{3}\left(\sigma \rightarrow \sigma^{*}\right)$ (Figure 6.2). Although this triplet state has a repulsive character and drops in energy as the $\mathrm{Co}-\mathrm{C}_{\mathrm{CN}}$ bond length increases, yet it does not become the lowest electronic state with dissociative character as it does happen in the case of $\mathrm{MeCbl}$ for the $\mathrm{Co}-\mathrm{C}_{\mathrm{CH}_{3}}$ distance greater than $2.34 \AA$. Clearly, the manifold of low-lying electronic states obtained for Im$\left[\mathrm{Co}^{\mathrm{III}}\right.$ (corrin) $]-\mathrm{R}^{+}\left(\mathrm{R}=\mathrm{CN}\right.$ or $\left.\mathrm{CH}_{3}\right)$ provides a rationale why the $\mathrm{Co}-\mathrm{C}_{\mathrm{CN}}$ bond in $\mathrm{CNCbl}$ is photostable and does not undergo photodissociation under conditions of simple photon excitation at 520 or $400 \mathrm{~nm}$ and why the $\mathrm{Co}-\mathrm{C}_{\mathrm{CH}_{3}}$ bond in $\mathrm{MeCbl}$ 


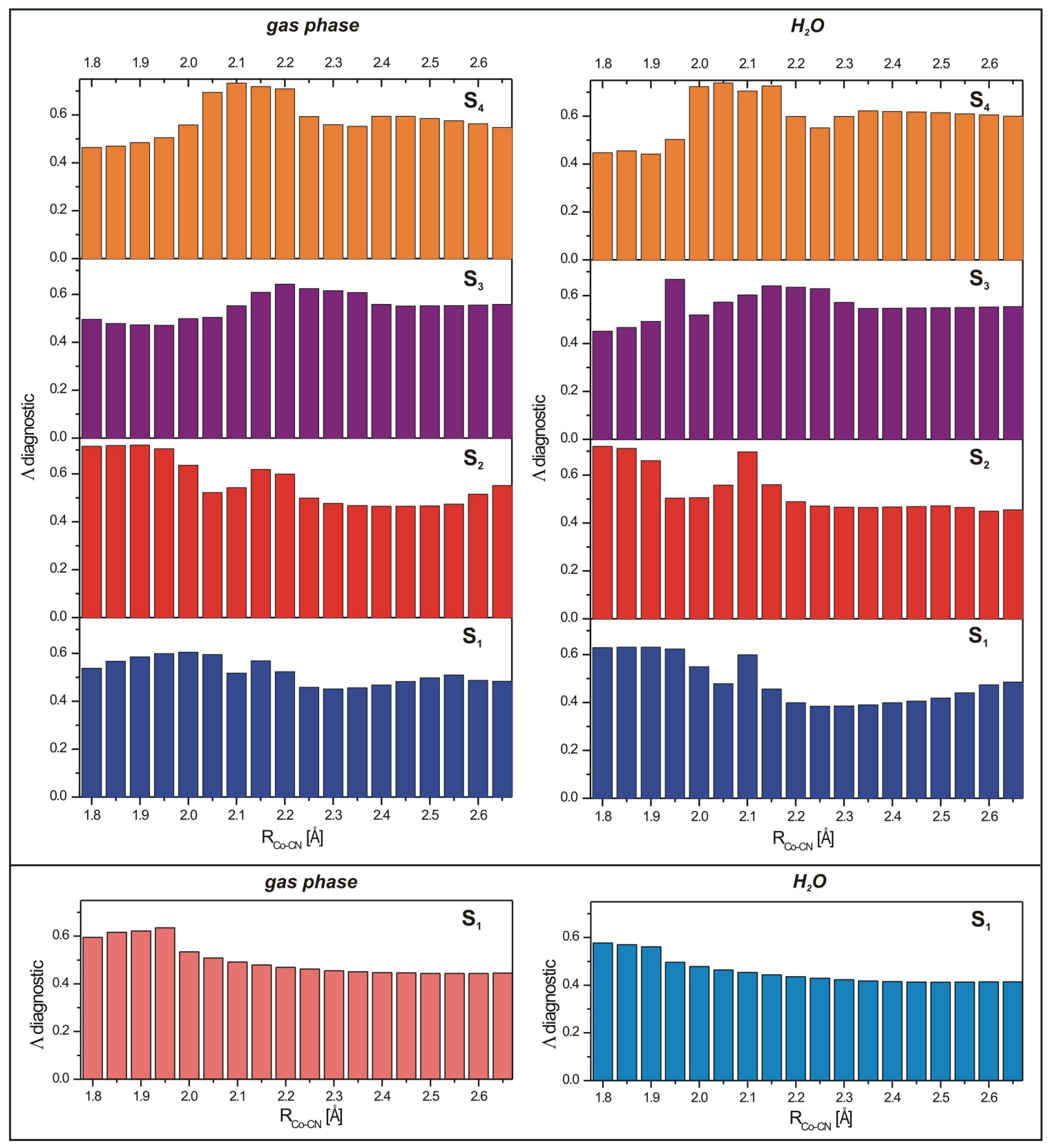

Figure 6.3. The $\Lambda$ diagnostic along the $\mathrm{Co}-\mathrm{C}_{\mathrm{CN}}$ bond stretch computed at BP86/6$31 \mathrm{G}(\mathrm{d})$. The values are presented for the four lowest vertical excitations (upper panel) and for the $S_{1}$ state in the optimized geometry (lower panel) calculated in gas phase and in water solution. 
undergoes photolytic scission. In contrast to MeCbl, very small decrease in energy of repulsive triplet state of $\mathrm{CNCbl}$ is observed when the $\mathrm{Co}-\mathrm{C}_{\mathrm{CN}}$ bond becomes elongated. It should be also added that the same applies for CNCbl excitations calculated in solution. In fact, there are amount of states that cross along the $\mathrm{Co}-\mathrm{C}_{\mathrm{CN}}$ coordinate but none of them becomes dissociative. Moreover, the repulsive state lies even higher in energy than its counterpart obtained in gas phase calculation, supporting the above conclusions.

Further inspection of energy curves computed as function of Co-C bond distance reveals that low-lying excited states for $\mathrm{CNCbl}$ and $\mathrm{MeCbl}$ are quite different (Figure 6.2). The lowest singlet states for $\mathrm{CNCbl}$ are closer in energy as comparing to these for $\mathrm{MeCbl}$ and the same observation can be extended to lowest triplet states. Another important difference is the shape of $\mathrm{CNCbl}$ and $\mathrm{MeCbl}$ energy curves of the lowest excited states. While $\mathrm{S}_{1}$ and $\mathrm{T}_{1}$ for MeCbl appear as rather smooth lines, the corresponding curvature for the $\mathrm{CNCbl}$ indicates possibly additional minima.

\subsubsection{Comparative Analysis of Axial Bonding in CNCbl and MeCbl}

The key difference between $\mathrm{CNCbl}$ and MeCbl lies in their axial bond involving cobalt and carbon. The $\sigma$-bonding is much stronger in $\mathrm{Co}-\mathrm{C}_{\mathrm{CN}}$ than in $\mathrm{Co}-\mathrm{C}_{\mathrm{Me}}$ as can be inferred from the corresponding equilibrium bond distances as well as dissociation energies. The optimized $\mathrm{Co}-\mathrm{C}_{\mathrm{CN}}$ bond length of $1.844 \AA$ is shorter $\sim 0.12 \AA$ than the optimized $\mathrm{Co}-\mathrm{CH}_{3}$ which is $1.968 \AA$. The computed $\mathrm{Co}-\mathrm{C}_{\mathrm{CN}}$ bond dissociation energy (BDE) of $81.13 \mathrm{kcal} / \mathrm{mol}(78.05 \mathrm{kcal} / \mathrm{mol}$ upon zero-point correction) is about two times higher than $37.0 \mathrm{kcal} / \mathrm{mol}$ predicted for the $\mathrm{MeCbl}$. The homolytic splitting of the $\mathrm{Co}-\mathrm{C}_{\mathrm{CN}}$ bond was computed as energy difference between the $\mathrm{Im}-\left[\mathrm{Co}{ }^{\mathrm{III}}\right.$ (corrin) $]-\mathrm{CN}^{+}$and fragments, i.e., $\mathrm{Im}-\left[\mathrm{Co}^{\mathrm{II}} \text { (corrin) }\right]^{+}$and $\mathrm{CN}$ radical, respectively. The difference in dissociation energy can be attributed to the fact that $\mathrm{Co}-\mathrm{C}_{\mathrm{CN}}$ bond has more ionic character as compared to $\mathrm{Co}-\mathrm{C}_{\mathrm{Me}}$. To obtain more quantitative description of $\mathrm{Co}-\mathrm{C}$ bonds in both cobalamins, the NBO calculations were performed. 
The NBO analysis of cobalt-axial ligands bonds was summarized in Table 6.7. It can be seen that the Co-C $\sigma$ bond in $\mathrm{CNCbl}$ is more ionic than in MeCbl. The participation of hybridized carbon orbital in this bond is $10 \%$ larger in the former case. At the same time, the participation of cobalt in this bond is lower in case of CNCbl. The data coming from calculations incorporating solvent effect indicates that the polarity of the bond is augmented in the polar environment. The increased polarization between the cobalt atom and the $\mathrm{CN}$ group is also visible based on the NBO charges analysis (Table 6.4) in solvent. The enhancement of $\mathrm{Co}-\mathrm{C}$ bond polarity under the influence of solvent is smaller in the case of $\mathrm{MeCbl}$, and slightly higher for the CNCbl. The participation of hybridized carbon orbital in $\sigma$ bond increases by about $1.6 \%$ for $\mathrm{MeCbl}$ and about $2.7 \%$ in $\mathrm{CNCbl}$, as compared to the calculations in the gas phase. The participation of cobalt orbitals for both $\mathrm{Co}-\mathrm{C}_{\mathrm{Me}}$ and $\mathrm{Co}-\mathrm{C}_{\mathrm{CN}}$ bonds is obviously reduced adequately. The NBO analysis based on calculations in both environments indicates a higher polarity of the Co-C bond in the case of cyanocobalamin than methylcobalamin. In addition, there is also a back donation from $\mathrm{d}_{x z}$ and $\mathrm{d}_{y z}$ orbitals to $\pi^{*}$ orbitals of cyanide, although it is rather small, yet it leads to further decrease of electron density on cobalt.

The difference in the Co-C bond polarity for the axial ligand $\mathrm{CN}$ and $\mathrm{CH}_{3}$ should also be noticeable by the donations effect from the imidazole nitrogen lone pair to $\sigma^{*}$ orbitals. The increase in Co-C $\sigma$ bond polarity leads to the strong localization of $\sigma^{*}$ orbital on the cobalt atom, and this, in turn, increases the efficiency of orbital overlap between the free electron pair of nitrogen and the $\sigma^{*}$ orbital. The perturbative donor-acceptor interaction, involving a filled orbital of free electron pair of nitrogen and unfilled orbital $\sigma^{*} \mathrm{Co}-\mathrm{C}$ is larger in CNCbl than in MeCbl. The perturbative stabilization energy ${ }^{290} \Delta E_{i j}$ for this donation is about $42 \mathrm{kcal} / \mathrm{mol}$ and $30 \mathrm{kcal} / \mathrm{mol}$ for $\mathrm{CNCbl}$ and $\mathrm{MeCbl}$, respectively. Taking into account the solvent effect, the perturbative stabilization energy increases due to the larger polarity of the $\sigma$ bond. The stabilization energy is higher by about $5 \mathrm{kcal} / \mathrm{mol}$ for CNCbl and $2.5 \mathrm{kcal} / \mathrm{mol}$ for $\mathrm{MeCbl}$, with respect to the same calculations in gas phase. 
TABLE 6.7. NBO analysis for cobalt axial bonds in gas phase and in water solution.

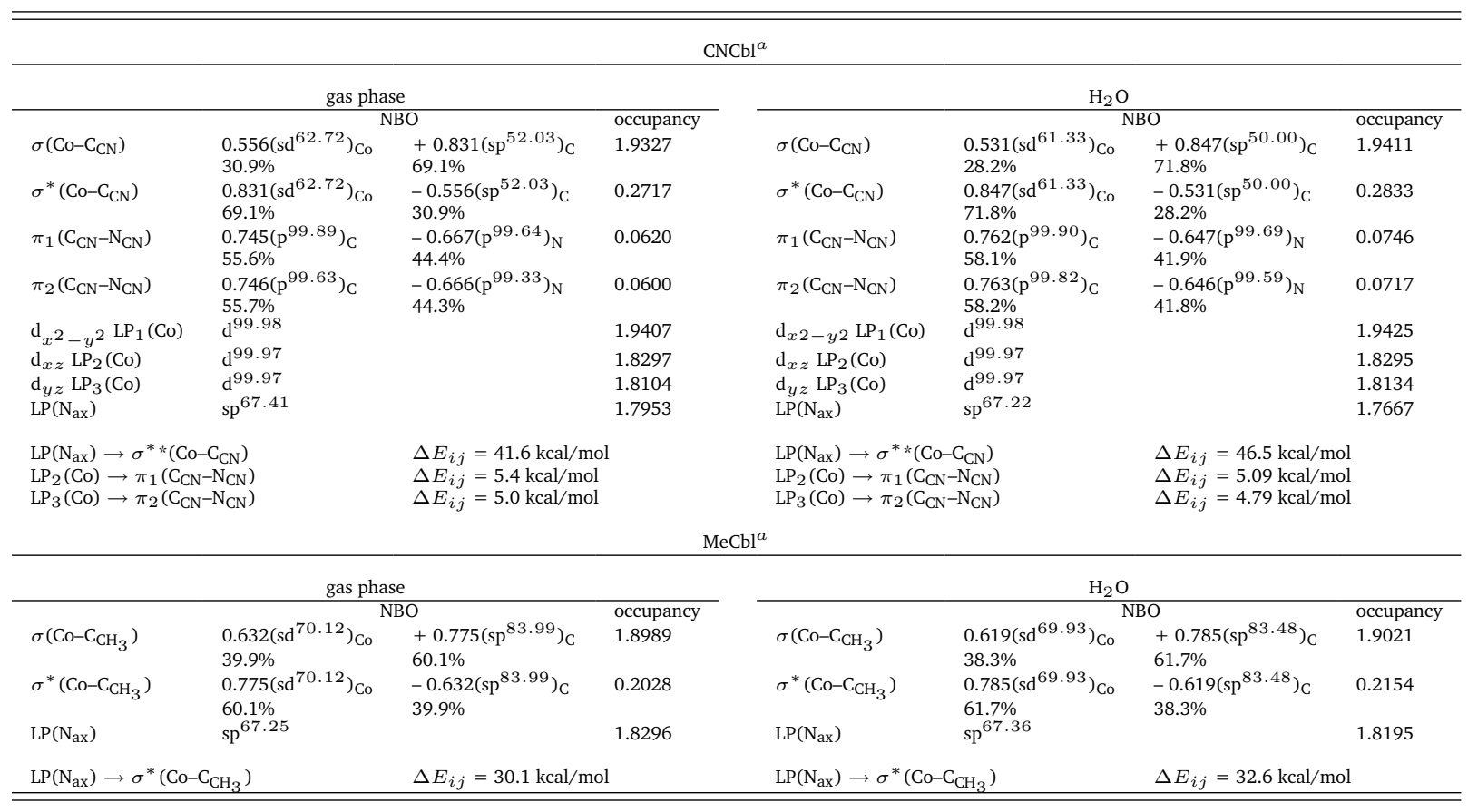

${ }^{a}$ The hybridization of the atoms is indicated by the percent contribution of the metal-centered d or (and) p orbitals as a superscript.

A stronger delocalization of nitrogen electron pairs towards the central atom should result in higher bond strength of Co- $\mathrm{N}_{\mathrm{Im}}$ bond in the case of cyanocobalamin as compared to the methylcobalamin. The energy of Co- $\mathrm{N}_{\mathrm{Im}}$ bond computed at BP86/6-31G(d) with solvent equals to $19.0 \mathrm{kcal} / \mathrm{mol}$ and $11.4 \mathrm{kcal} / \mathrm{mol}$ for $\mathrm{CNCbl}$ and $\mathrm{MeCbl}$, respectively. Stronger interaction between imidazole and cobalt for $\mathrm{CNCbl}$, as compared to $\mathrm{MeCbl}$ is also visible in terms of the Co- $\mathrm{N}_{\text {Im }}$ bond length. It is slightly shorter (by about $0.1 \AA$ ) in the CNCbl as compared to the MeCbl (Table 6.1, for MeCbl the Co- $\mathrm{N}_{\mathrm{Im}}$ bond lengths are $2.134 \AA$ and $2.110 \AA$ in calculation without and with solvent, respectively). 
It is worth noting that the charge distribution and the polarity of the Co-C axial bonds may significantly determine the nature and stability of the excited state. Deficiency of electron density on the cobalt, which increases with growth in the polarity of Co-C $\sigma$ bonds, will be energetically preferable rather than the stabilization of the excited states with charge transfer coming from equatorial ligand to the cobalt.

\subsubsection{Excited States of CNCbl Computed as a Function of Axial Bonds}

To further explore the nature of low-lying excited states, we computed their properties as a function of both axial bond lengths. Such approach was undertaken because the biggest structural change upon electronic excitation occurs mainly in the $\mathrm{N}_{\mathrm{Im}}-\mathrm{Co}-\mathrm{C}_{\mathrm{CN}}$ moiety, as it was found in the case of MeCbl. ${ }^{119}$ Consequently, potential energy surfaces (PESs) were generated as function of $\mathrm{Co}-\mathrm{C}_{\mathrm{CN}}$ and $\mathrm{Co}-$ $\mathrm{N}_{\text {Im }}$ by systematic stretching of these axial bonds with an increment of $0.10 \AA$. . The optimized equilibrium structures of the lowest singlet $\left(\mathrm{S}_{0}\right)$ and triplet $\left(\mathrm{T}_{1}\right)$ states were used to generate corresponding PESs. The geometries corresponding to the points on the grid were optimized and vertical excitation energies were computed for each point employing the TD-DFT/BP86/6-31G(d) level of theory (Figures 6.4 and 6.5).

The PES representing the $S_{1}$ state in CNCbl exhibits two minima (Figure 6.4). The first one was abbreviated as $\mathrm{A}$ and referred to the minimum located at the shorter value of $\mathrm{Co}-\mathrm{C}_{\mathrm{CN}}$ bond. Together with the increase of this distance the $\mathrm{S}_{1}$ state crossed with another, initially higher in energy, excited state and the crossing point was marked as $\mathrm{B}$. The following minimum that appeared at larger value of Co- $\mathrm{C}_{\mathrm{CN}}$ was designated as C. Consequently, the $\mathrm{S}_{1}(\mathrm{~A})$ and $\mathrm{S}_{1}(\mathrm{C})$ stood for minima for the $S_{1} / C N C b l$ singlet state. The $S_{1}(A)$ minimum appears to lie right above the $S_{0}$ minimum, within the subspace of geometrical parameters relevant for the considered photo-dissociation process. Based on the energy projection, it can be estimated that for the $\mathrm{S}_{1}(\mathrm{~A})$ minimum: $\mathrm{Co}-\mathrm{C}_{\mathrm{CN}} \approx 1.85 \AA$, while Co- $\mathrm{N}_{\mathrm{Im}} \approx 2.00 \AA$, in comparison to the minimum at the ground state potential energy surface, where 

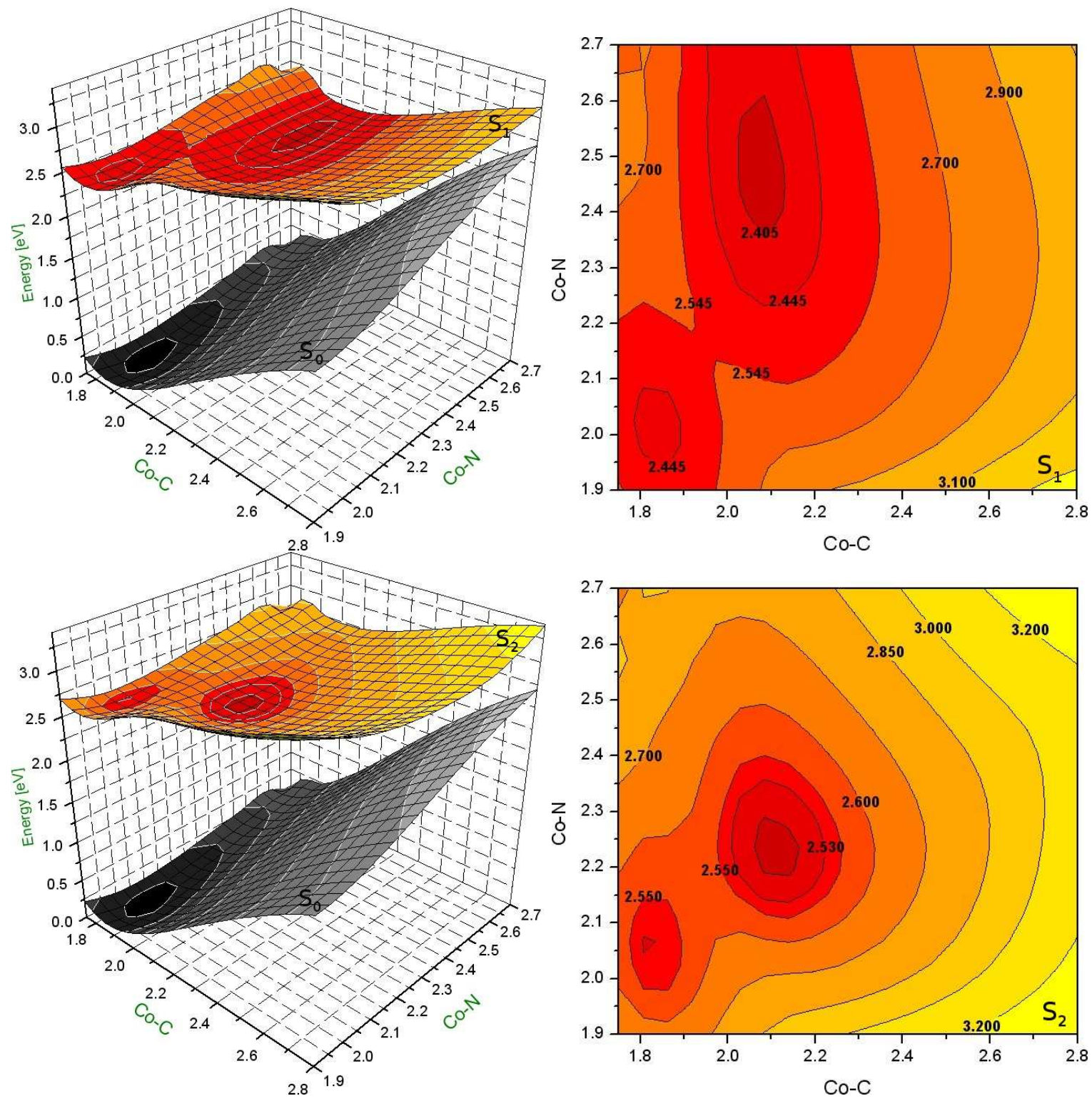

Figure 6.4. (Left) Potential energy surfaces for the two lowest singlet states generated as vertical excitations and plotted as a function of axial bond lengths (expressed in $\AA$ ). (Right) Vertical projections of the $S_{1}$ and $S_{2}$ potential energy surfaces. 
Co- $\mathrm{C}_{\mathrm{CN}}=1.84 \AA$ and $\mathrm{Co}-\mathrm{N}_{\mathrm{Im}}=2.05 \AA$ (note that bond lengths corresponding to the $\mathrm{S}_{0}$ minimum have been optimized). The $\mathrm{S}_{1}(\mathrm{C})$ minimum differs noticeably from $\mathrm{S}_{1}(\mathrm{~A})$ and the estimate gives $\mathrm{Co}-\mathrm{C}_{\mathrm{CN}} \approx 2.05 \AA$ and Co- $\mathrm{N}_{\mathrm{Im}} \approx 2.50 \AA$ lengths, respectively (Figure 6.4). When the similar analysis was carried out for MeCbl cofactor, only one minimum was found. The minimum on $\mathrm{S}_{1} / \mathrm{MeCbl}$ corresponds to $\mathrm{Co}-\mathrm{C}_{\mathrm{CN}}$ $=1.98 \AA$ and $\mathrm{Co}-\mathrm{N}=2.04 \AA$, which can be compared to $1.97 \AA$ and $2.13 \AA$, respectively, when the $\mathrm{S}_{0} / \mathrm{MeCbl}$ surface was considered. Based on the axial bonding changes in $\mathrm{MeCbl}$, as compared to $\mathrm{CNCbl}$, it can be concluded that the $\mathrm{S}_{1}$ minimum in MeCbl more closely resembles the $S_{1}(A)$ minimum rather than $S_{1}(C)$ in CNCbl.

The PES representing the $S_{2}$ state in CNCbl looks nearly parallel to the $S_{1}$. The $S_{2}$ state exhibits also two minima with respect to axial bond lengths. The estimates based on the $S_{2}$ projection (Figure 6.4) indicate that the first minimum, assigned as $\mathrm{S}_{2}(\mathrm{~A})$, lies right above the minimum for the ground state and for the $\mathrm{S}_{1}(\mathrm{~A})$. In fact, the $\mathrm{Co}-\mathrm{C}_{\mathrm{CN}}$ and $\mathrm{Co}-\mathrm{N}_{\mathrm{Im}}$ values corresponding to $\mathrm{S}_{2}(\mathrm{~A}): \approx 1.82 \AA$ and $\approx 2.06 \AA$, respectively, differ by no more than $0.06 \AA$ from the bond lengths describing the $S_{0}$ and $S_{1}(A)$ points. Similarly to the $S_{1} / C N C b l$ case, the second minimum is located at longer $\mathrm{Co}-\mathrm{C}_{\mathrm{CN}}$ and $\mathrm{Co}-\mathrm{N}_{\mathrm{Im}}$ distances. Whereas the estimated Co- $\mathrm{C}_{\mathrm{CN}}$ bond for $\mathrm{S}_{2}(\mathrm{C})(\approx 2.12 \AA)$ is longer than the one for $\mathrm{S}_{1}(\mathrm{C})$, the Co- $\mathrm{N}_{\mathrm{Im}}$ bond $(\approx 2.24 \AA)$ is shortened by $\sim 0.26 \AA$.

Similar calculations with respect to axial bond distances have been also carried out for low-lying triplets (Figure 6.5). The surface representing the lowest $\mathrm{T}_{1}$ state (as the one having different multiplicity with respect to the ground state) was computed in a similar way as it took place for the $S_{0}$ state and both of them were plotted on the same energy scale (Figure 6.5). The first important observation regarding $\mathrm{T}_{1}$ is its very shallow minimum with respect to axial bonds. There is a $\sim 0.35 \AA$ long Co- $\mathrm{N}_{\mathrm{Im}}$ range at concurrent $\mathrm{Co}-\mathrm{C}_{\mathrm{CN}}=1.90-2.00 \AA$, in which the energy achieves similar value. In order to decrease the energy of the system in $T_{1}$ state, both Co- $\mathrm{C}_{\mathrm{CN}}$ and Co- $\mathrm{N}_{\mathrm{Im}}$ distances have to be extended as compared to those of $S_{0}$. Hence, the minimum of the $T_{1}$ does not lay right over the $S_{0}$ equilibrium, that resembles the $S_{1}(A)$, but is rather shifted to the region corresponding to the 

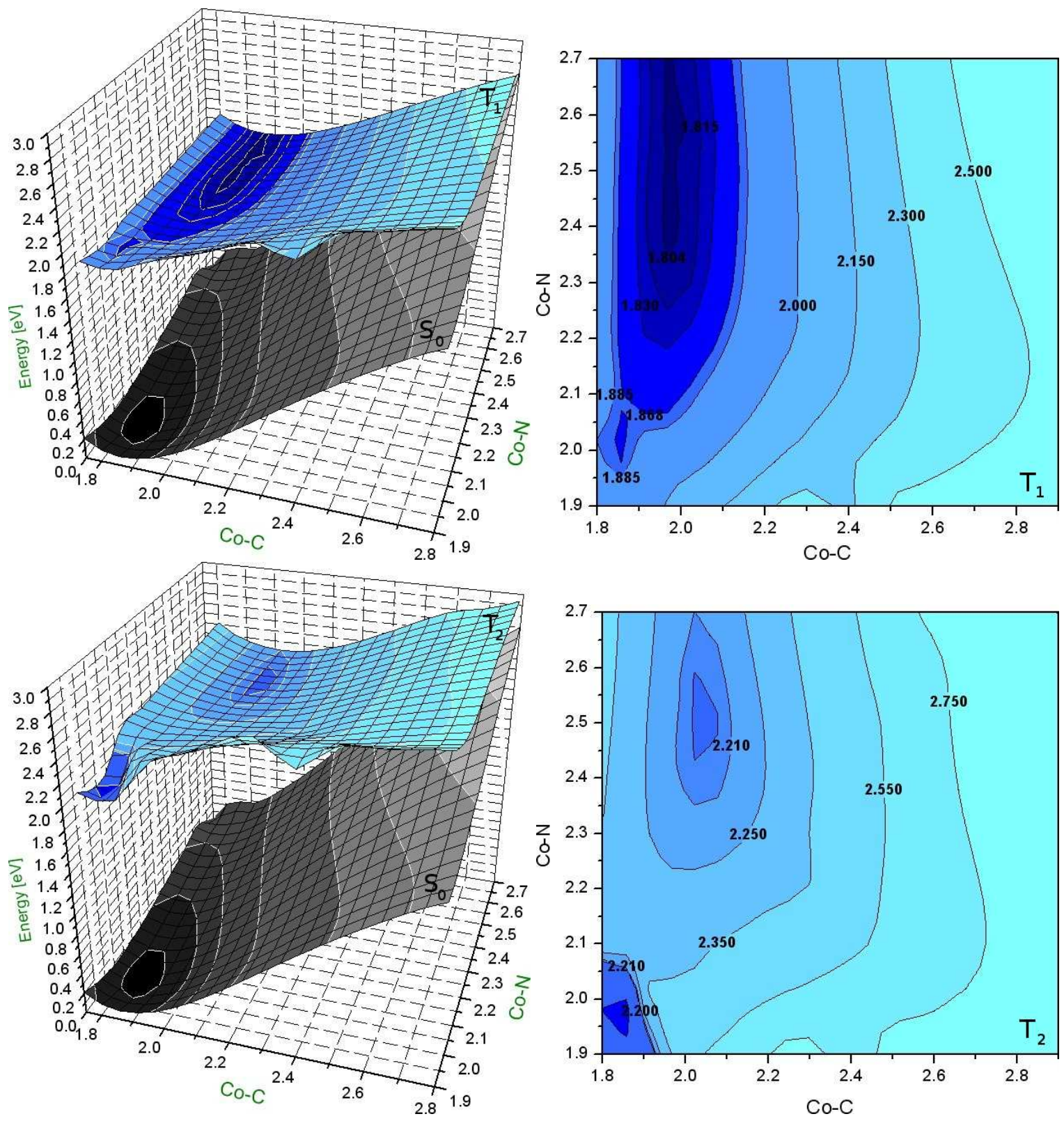

Figure 6.5. (Left) Potential energy surfaces for two lowest triplet states plotted as a function of axial bond lengths (expressed in $\AA$ ). (Right) Vertical projections of the $\mathrm{T}_{1}$ and $\mathrm{T}_{2}$ potential energy surfaces. Both singlet $\left(\mathrm{S}_{0}\right)$ and first triplet $\left(\mathrm{T}_{1}\right)$ geometries were optimized. The higher triplet states were obtained as vertical excitations from $\mathrm{T}_{1}$. 
$S_{1}(C)$. The axial bond lengths are slightly shorter than the estimated distances for the $\mathrm{S}_{1}(\mathrm{C})$ and are equal to: $1.97 \AA$ and $2.43 \AA$ for $\mathrm{Co}-\mathrm{C}_{\mathrm{CN}}$, and $\mathrm{Co}-\mathrm{N}_{\mathrm{Im}}$, respectively. Taking into account that the $T_{1}$ energy minimum is quite shifted with respect to the minimum representing the $S_{0}$, and it is more in line with the $S_{1}(C)$ rather than $\mathrm{S}_{1}(\mathrm{~A})$ minimum, it is reasonable to label it as $\mathrm{T}_{1}(\mathrm{C})$.

An attempt to locate minimum which looks like $S_{1}(A)$, i.e., $T_{1}(A)$, turned out to be difficult. This region of PES is rather shallow and the changes with respect to axial bonds do not cost much energy. In other words, at the short $\mathrm{Co}-\mathrm{C}_{\mathrm{CN}}$ distances (less than $2.10 \AA$ ) the energy of the system changes smoothly while varying Co- $\mathrm{N}_{\mathrm{Im}}$ bond length. Such topology of PES complicates the location of minima. However, appropriate congestion of the grid points representing vertical projection of the $T_{1}$ state onto the axial bonds plane, reveals the regions in which the energy is decreased as comparing to the neighboring areas. As a result, the expected minimum, $\mathrm{T}_{1}(\mathrm{~A})$, could be assigned to the point of PES at $\approx 1.85 \AA$ and $\approx 2.025 \AA$ for $\mathrm{Co}-\mathrm{C}_{\mathrm{CN}}$ and $\mathrm{Co}-\mathrm{N}_{\mathrm{Im}}$, respectively. Both axial bond lengths support such labeling due to the fact that the minimum is more in line with the $S_{0}$ and $S_{1}(A)$ rather than $S_{1}(C)$.

The contour of the $T_{2}$ state plotted together with $S_{0}$ state (Figure 6.5) looks somehow opposite to the $\mathrm{T}_{1}$ state, considering the energy changes as a consequence of axial bonds stretching. In contrast to the $\mathrm{T}_{1}$ state, the energetically lowest point on $\mathrm{T}_{2}$ PES corresponds to shorter axial bond distances: $\mathrm{Co}-\mathrm{C}_{\mathrm{CN}} \approx 1.85 \AA$ and $\mathrm{Co}-\mathrm{N} \approx 1.96 \AA$. Taking into account that the mentioned minimum is shifted by 0.01 and $0.09 \AA$ ( $\mathrm{Co}-\mathrm{C}_{\mathrm{CN}}$ and $\mathrm{Co}-\mathrm{N}_{\mathrm{Im}}$, respectively), as compared to $\mathrm{S}_{0}$, it was labeled as $\mathrm{T}_{2}(\mathrm{~A})$. The second minimum present on the $\mathrm{T}_{2}$ PES was easier to estimate as compared to $T_{1}(C)$ due to the fact that it was not as shallow. As opposed to the $T_{1}$ state, the second energetically lowest point at $\mathrm{T}_{2}$ PES was found at longer $\mathrm{Co}-\mathrm{C}_{\mathrm{CN}}$ and Co- $\mathrm{N}_{\text {Im }}$ distances, namely $\approx 2.05 \AA$ and $\approx 2.50 \AA$ and was, hence, denoted as $\mathrm{T}_{2}(\mathrm{C})$. 


\subsubsection{Energy Minima on $\mathrm{S}_{1} / \mathrm{CNCbl}$ and $\mathrm{T}_{1} / \mathrm{CNCbl}$ Surfaces}

Since the $S_{1}$ PES (Figure 6.4) was generated as vertical excitations based on the ground state geometry, the axial bonding changes corresponding to the $S_{1}(A)$ and $\mathrm{S}_{1}(\mathrm{C})$ could only be approximately described. Hence, to determine more accurate structures representing $S_{1}(A)$ and $S_{1}(C)$ points on the $S_{1} / C N C b l$ surface, their geometries were fully optimized employing structural model of CNCbl. The results of geometry optimization are summarized in Table 6.1. We focused our analysis mainly on the axial parameters since the biggest changes took place in the $\mathrm{N}_{\mathrm{Im}}-\mathrm{Co}-\mathrm{C}_{\mathrm{CN}}$ moiety. The full geometry optimization was also carried out for the $\mathrm{T}_{1}(\mathrm{~A})$ and $\mathrm{T}_{1}(\mathrm{C})$ points on $\mathrm{T}_{1} / \mathrm{CNCbl}$ surface. In addition, geometry optimization was performed in presence of water modeled via COSMO/ $\mathrm{H}_{2} \mathrm{O}$.

The $S_{0}$ and $S_{1}(A)$ minima were found to lay above each other when PES of the CNCbl was investigated as a function of axial bonds. The optimized axial bond distances (Table 6.1) confirm previous observation as compared bond lengths do not differ by more than $0.04 \AA$, regardless of the environment. In contrast, the bending angle $\mathrm{N}_{\mathrm{Im}}-\mathrm{Co}-\mathrm{C}_{\mathrm{CN}}$ between two axial bonds undergoes $\sim 9^{\circ}$ decrease in gas phase, whereas in water, the alignment does not change by more than $1^{\circ}$. The similar comparison was performed for the $S_{1}(C)$ and $T_{1}(C)$ minima and the computed data implied similar characteristics in axial bonds region. However, the closer inspection indicates rather remarkable change in the $\mathrm{Co}-\mathrm{C}_{\mathrm{CN}}-\mathrm{N}_{\mathrm{CN}}$ angle. The value of $176.5^{\circ}$ in gas phase and $179.4^{\circ}$ in water solution obtained for the $\mathrm{T}_{1}(\mathrm{C})$ state decreases by $\sim 26^{\circ}$ and $\sim 19^{\circ}$, respectively, in the case of $S_{1}(C)$. At the same time, the observed Co- $\mathrm{N}_{\mathrm{Im}}$ axial bond for $\mathrm{S}_{1}(\mathrm{C})$ shortens by $\sim 0.20 \AA$ and less than $0.10 \AA$ in gas phase or water solution, respectively. The geometry of $T_{1}(A)$ is very close to the one of $\mathrm{S}_{1}(\mathrm{~A})$. The compared bond lengths do not differ by more than $0.05 \AA$ although the $\mathrm{C}_{\mathrm{CN}}-\mathrm{Co}-\mathrm{N}_{\mathrm{Im}}$ angle changes significantly when the calculations are performed in gas phase. The corresponding $\mathrm{S}_{1}(\mathrm{~A})$ value of $169.8^{\circ}$ is lower than the one for $\mathrm{T}_{1}(\mathrm{~A})$ by $\sim 9^{\circ}$. Nevertheless, it should be noted that $\mathrm{S}_{1}(\mathrm{~A})$ is expected to have similar geometry as $\mathrm{S}_{0}$, where the $\mathrm{C}_{\mathrm{CN}}-\mathrm{Co}-\mathrm{N}_{\mathrm{Im}}$ angle was found to be $178.8^{\circ}$. The 
comparison of the results obtained in two different environments points out that the solvent may have influence on some structural parameters. The compared axial distances change by less than $0.12 \AA$, while the angle differences do not exceed $3^{\circ}$. The two exceptions are: $\mathrm{C}_{\mathrm{CN}}-\mathrm{Co}-\mathrm{N}_{\mathrm{Im}}$ for the $\mathrm{S}_{1}(\mathrm{~A})$ and $\mathrm{Co}-\mathrm{C}_{\mathrm{CN}}-\mathrm{N}_{\mathrm{CN}}$ for the $\mathrm{S}_{1}(\mathrm{C})$, where the change of the environment from water solution to gas phase leads to the bending by $7.7^{\circ}$ and by the $10^{\circ}$ respectively.

\subsubsection{Electronic Properties of $S_{1}$ and $T_{1}$ States in CNCbl}

To better understand the behavior of potential energy curves as a function of the $\mathrm{Co}-\mathrm{C}_{\mathrm{CN}}$ bond, the corresponding geometries of the lowest singlet and triplet states have been optimized (both in gas phase and in water solution) at sequentially elongated $\mathrm{Co}-\mathrm{C}_{\mathrm{CN}}$ distance (Figure 6.6). Note that previously computed curves (Figure 6.2) have been generated only as vertical excitations from the ground state equilibrium geometry. In order to confirm the reliability of the results obtained for optimized $\mathrm{S}_{1}$ state for different $\mathrm{Co}-\mathrm{C}_{\mathrm{CN}}$ bond distances and exclude the possibility of CT failure, the $\Lambda$ diagnostic test was performed (Figure 6.3). Although the monotonic decrease of its numerical value was observed together with the increased $\mathrm{Co}-\mathrm{C}_{\mathrm{CN}}$ distance, it did not drop below the threshold (as suggested in Ref. 138) value of 0.4. The newly obtained curves were characterized by the A, B and C points. The first minimum for the singlet and triplet states was found at $\mathrm{Co}-\mathrm{C}_{\mathrm{CN}}$ distance close to the equilibrium bond length of the ground state, while the second one appeared at extended $\mathrm{Co}-\mathrm{C}_{\mathrm{CN}}$. The curve representing $\mathrm{S}_{1}$ state computed without solvent was similar in shape to the curve of the low-lying singlet state generated as vertical excitation (Figure 6.2). However, in contrast to $S_{1}$ vertical excitation (Figure 6.6), the energy of the second minimum, $S_{1}(C)$, was higher than the energy of the first minimum, $S_{1}(A)$, when $S_{1}$ state was optimized. Nevertheless, inclusion of solvent caused the minimum $S_{1}(C)$ to become the energetically lowest point on $S_{1}$ potential energy curve. In contrast, when triplet was examined, the minimum at shorter Co- $\mathrm{C}_{\mathrm{CN}}$ distance, i.e. $\mathrm{T}_{1}(\mathrm{~A})$, was found to be energetically higher as compared to $\mathrm{T}_{1}(\mathrm{C})$, regardless of the environment. Furthermore, in comparison to gas phase 


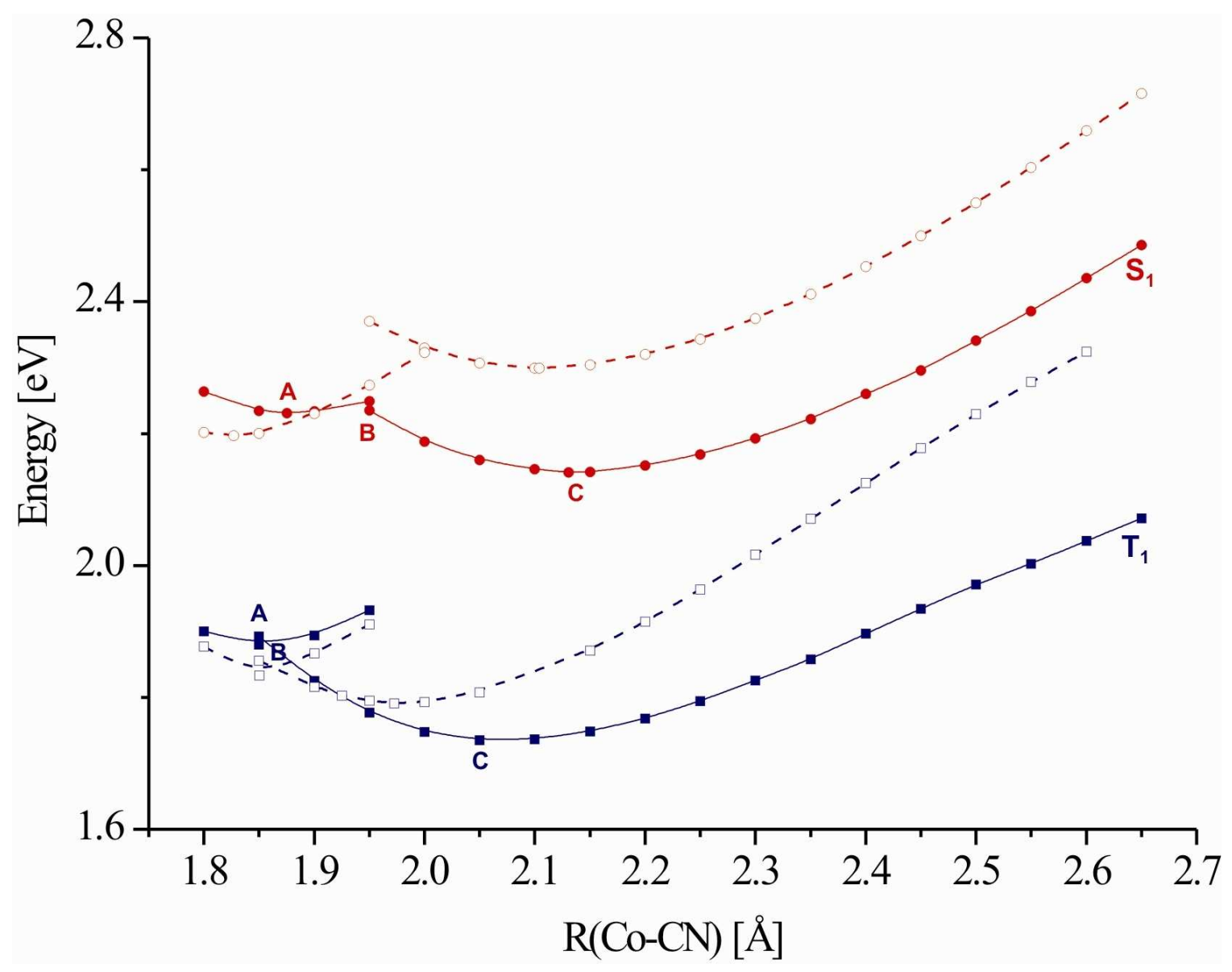

Figure 6.6. Potential energy curves of the lowest-excited singlet $\left(\mathrm{S}_{1}\right.$, red) and triplet $\left(\mathrm{T}_{1}\right.$, blue) states of the CNCbl model along the $\mathrm{Co}-\mathrm{C}_{\mathrm{CN}}$ bond stretch optimized at BP86/6-31G(d) without solvent (dashed lines) and with solvent (solid lines) using the COSMO/ $\mathrm{H}_{2} \mathrm{O}$ model. See Figure 6.3 for the corresponding $\Lambda$ diagnostic test analysis.

data, the second minimum for $\mathrm{T}_{1}$ was noticed to occur at largely extended $\mathrm{Co}-\mathrm{C}_{\mathrm{CN}}$ bond when solvent was included.

For the optimized geometry of singlet excited states with a minimum in $\mathrm{S}_{1}(\mathrm{~A})$ and $\mathrm{S}_{1}(\mathrm{C})$, the crossing took place at a $\mathrm{Co}-\mathrm{C}_{\mathrm{CN}}$ distance of about $1.94 \AA$ with solvent, and about $2.00 \AA$ in the case of gas phase calculations. The curves for the $\mathrm{T}_{1}(\mathrm{~A})$ and $\mathrm{T}_{1}(\mathrm{C})$ intersected at the point corresponding almost exactly to the $\mathrm{T}_{1}(\mathrm{~A})$ geometry. The energy barrier between the $S_{1}(A)$ and the point of intersection (B) with $S_{1}(C)$ curve was very small and corresponded to energy less than $1 \mathrm{kcal} / \mathrm{mol}$ in the calculation with the solvent. In the case of triplet states, this barrier was 
practically absent. Therefore, the internal conversion from higher to lower energy minimum located at points $S_{1}(C)$ and $T_{1}(C)$ is very probable for singlet and triplet, respectively.

To further explore the properties of the excited states the frontier orbitals have been extracted from TD-DFT calculations (Table A.18 in the Appendix). Figure 6.7 shows the MO diagram corresponding to the ground state geometry $\left(\mathrm{S}_{0}\right)$ and relaxed geometries at $S_{1}(A)$ and $S_{1}(C)$ minima in gas phase. Figure 6.8 displays similar diagram based on $\mathrm{COSMO} / \mathrm{H}_{2} \mathrm{O}$ calculations. As indicated by data in Table 6.8, the main contributions to the first few low-lying excited states of $\mathrm{CNCbl}$, calculated in both environments, are coming from the range of HOMO-2 up to LUMO+1 MOs. The first electronic excitation is dominated by HOMO $\rightarrow$ LUMO transition that vertically can be characterized as $\pi \rightarrow \pi^{*}$, regardless of the environment. It has to be noted, however, that $S_{1}$ state in gas phase possesses also $30 \%$ contribution of HOMO-2 $\rightarrow$ LUMO transition. The HOMO-2 orbital is localized mainly on Co atom with small contributions spread on corrin ring as well as cyano group (Table A.18 in the Appendix). Therefore, the $S_{1}$ vertical excitation in gas phase is of mixed, $\pi / \mathrm{d} \rightarrow \pi^{*}$, character. This observation is particularly important, when considering the nature of the transition from the ground state $\left(\mathrm{S}_{0}\right)$ to the first excited state in its $S_{1}(A)$ geometry. HOMO orbital corresponding to the relaxed $\mathrm{S}_{1}(\mathrm{~A})$ geometry is localized both on corrin ring and Co atom. Despite the participation of only one occupied orbital in this excitation, the same i.e., mixed $\pi / \mathrm{d} \rightarrow \pi^{*}$, nature is revealed that has been already assigned for vertical $S_{1}$ state. The situation changes dramatically upon inclusion of solvent. Although vertical $S_{1}$ state remains $\pi \rightarrow \pi^{*}$ type, the transition to $\mathrm{S}_{1}(\mathrm{~A})$ is partially described by LMCT. The difference between gas phase and COSMO/ $\mathrm{H}_{2} \mathrm{O}$ data lies in the changes in nature of orbitals HOMO and LUMO corresponding to $\mathrm{S}_{1}(\mathrm{~A})$ geometry. Whereas in gas phase Co $\mathrm{d}$ orbitals contribute to HOMO, solvent introduces them into LUMO. Consequently, transfer of electron from $\mathrm{S}_{0}$ to $\mathrm{S}_{1}(\mathrm{~A})$ is described by mixed $\pi \pi^{*} / \mathrm{LMCT}$ or $\pi \pi^{*} / \mathrm{MLCT}$ transitions with and without solvent, respectively. 

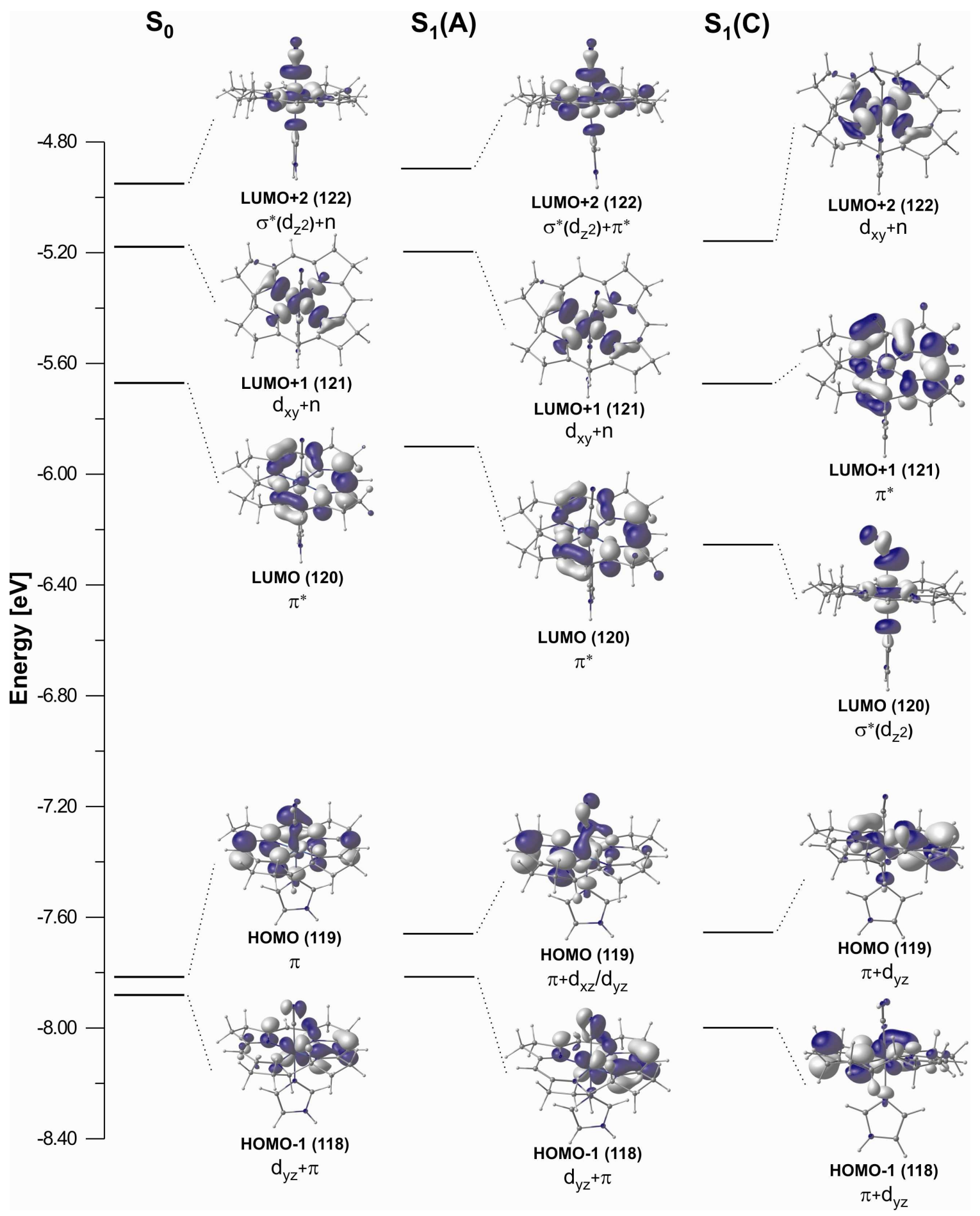

Figure 6.7. Molecular orbital diagram for $\mathrm{Im}-\left[\mathrm{Co}^{\mathrm{III}}\right.$ (corrin) $]-\mathrm{CN}^{+}$in the $\mathrm{S}_{0}, \mathrm{~S}_{1}(\mathrm{~A})$ and $\mathrm{S}_{1}(\mathrm{C})$ optimized geometry. 


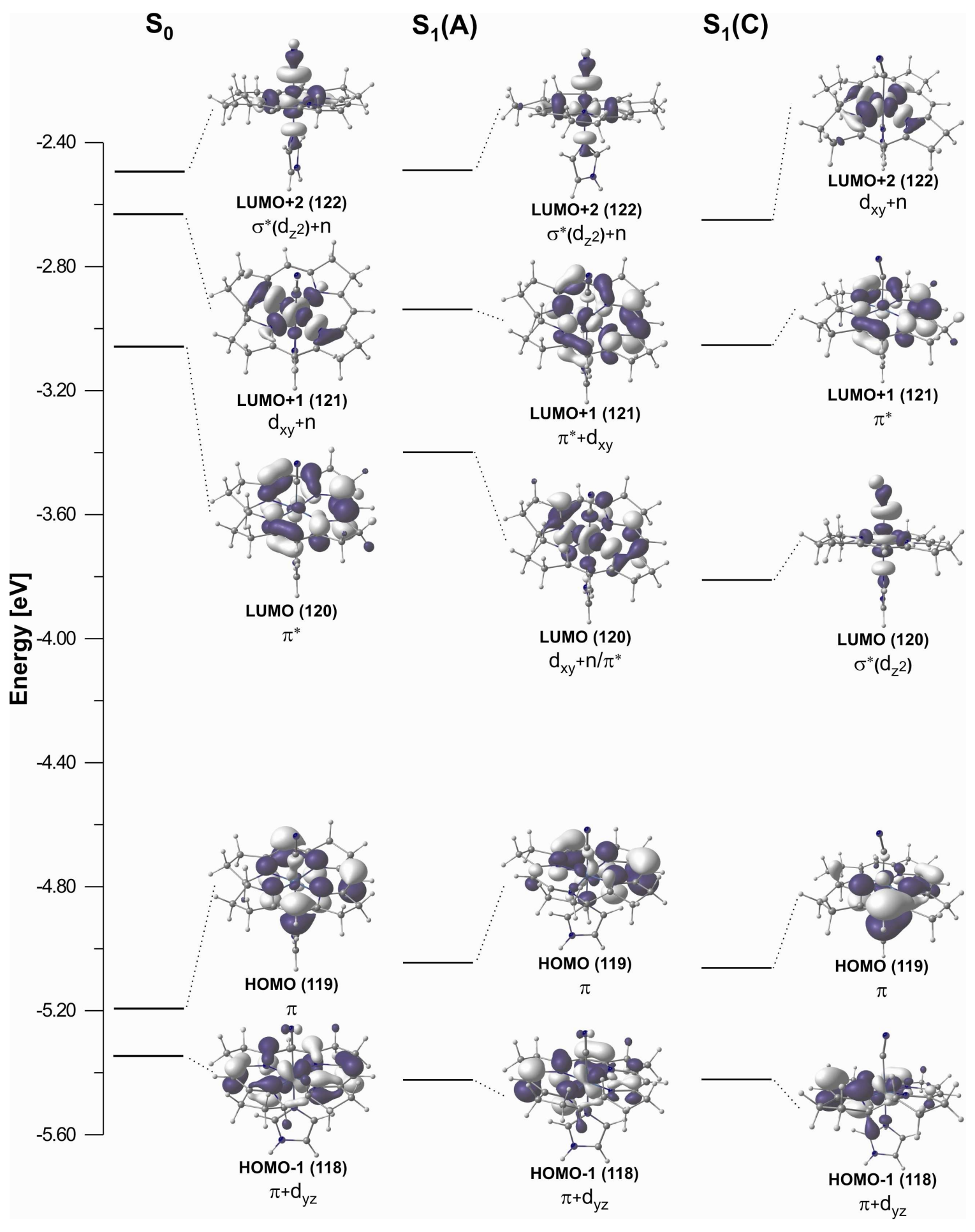

Figure 6.8. Molecular orbital diagram for $\mathrm{Im}-\left[\mathrm{Co}{ }^{\mathrm{III}}\right.$ (corrin) $]-\mathrm{CN}^{+}$in the $\mathrm{S}_{0}, \mathrm{~S}_{1}(\mathrm{~A})$ and $S_{1}(C)$ optimized geometry based on the BP86/6-31G(d) calculations with the use of COSMO/ $\mathrm{H}_{2} \mathrm{O}$ model. 
TABLE 6.8. Singlet electronic excitations calculated with BP86/6-31G(d).

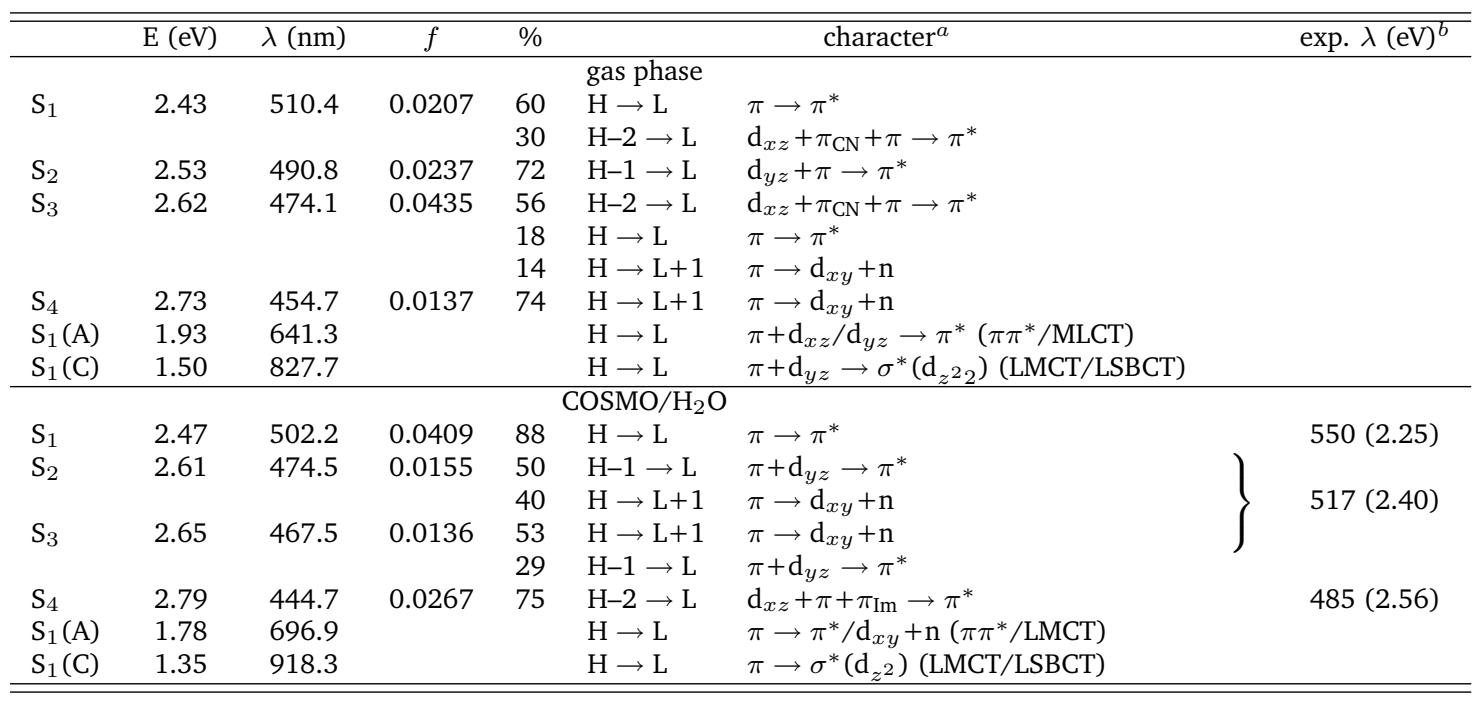

${ }^{a}$ Bold indicates transitions with dominant LMCT contributions.

${ }^{b}$ Experimental values taken from Ref. 68.

The optimization of the $S_{1}$ state revealed the presence of an additional minimum at longer $\mathrm{Co}-\mathrm{C}_{\mathrm{CN}}$ axial bond distance. The important outcome is that together with the relaxation of $S_{1}$ geometry, the nature of the first electronic transition may also change. Indeed, although the $S_{1}(C)$ refers to HOMO $\rightarrow$ LUMO transition the nature of LUMO does not agree neither with its vertical counterpart nor with the one that corresponds to $S_{1}(A)$ state. In fact, the orbital LUMO at $S_{1}(C)$ geometry is localized along the axial bonding and described as $\sigma^{*}\left(\mathrm{~d}_{z^{2}}\right)$. On the other hand, the orbital HOMO is mainly pure $\pi$ corrin, when solvent is taken into consideration, but possesses also some contribution from Co d orbitals when calculations are performed in gas phase. This observation confirms previous findings regarding the importance of solvent in modeling electronic properties of non-alkylcobalamins. In addition, the presence of the second minimum on the PES of $S_{1}$ state is in accordance with experimental results. The transient absorption spectroscopy measurements have indicated that the intermediate state could be characterized as LMCT. ${ }^{64}$ Our TD-DFT data imply such transition for electron transfer coming from the $\mathrm{S}_{0}$ to $\mathrm{S}_{1}(\mathrm{C})$. 


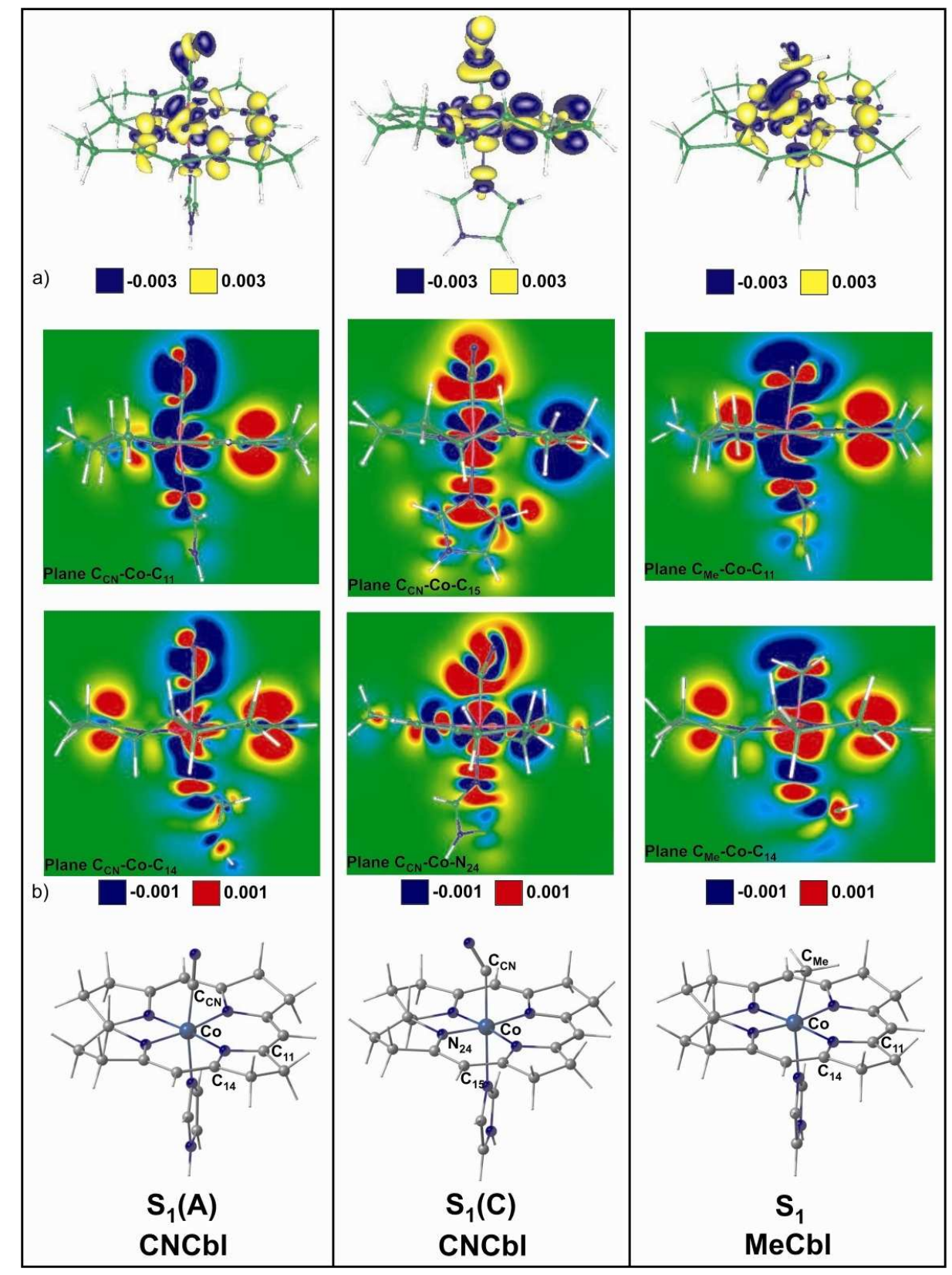

Figure 6.9. Electron density difference between the $S_{0}$ and $S_{1}$ states for $\mathrm{CNCbl}$ and MeCbl: (a) isosurface plot value of 0.003 and (b) cross-section contour plot along the axial bonding (in two different section planes). The yellow and red colors denote electron space, blue color - hole space. 
TABLE 6.9. Triplet electronic excitations calculated with BP86/6-31G(d).

\begin{tabular}{|c|c|c|c|c|c|}
\hline & $\mathrm{E}(\mathrm{eV})$ & $\lambda(\mathrm{nm})$ & $\%$ & & Character \\
\hline \multicolumn{6}{|c|}{ gas phase } \\
\hline \multirow[t]{2}{*}{$\mathrm{T}_{1}$} & 1.92 & 644.6 & 39 & $\mathrm{H}-1 \rightarrow \mathrm{L}$ & $\mathrm{d}_{y z}+\pi \rightarrow \pi^{*}$ \\
\hline & & & 16 & $\mathrm{H} \rightarrow \mathrm{L}$ & $\pi \rightarrow \pi^{*}$ \\
\hline \multirow[t]{2}{*}{$\mathrm{T}_{2}$} & 1.97 & 628.6 & 37 & $\mathrm{H} \rightarrow \mathrm{L}$ & $\pi \rightarrow \pi^{*}$ \\
\hline & & & 17 & $\mathrm{H}-1 \rightarrow \mathrm{L}$ & $\mathrm{d}_{y z}+\pi \rightarrow \pi^{*}$ \\
\hline \multirow[t]{2}{*}{$\mathrm{T}_{3}$} & 2.26 & 547.6 & 47 & $\mathrm{H}-1 \rightarrow \mathrm{L}+1$ & $\mathrm{~d}_{y z}+\pi \rightarrow \mathrm{d}_{x y}+\mathrm{n}$ \\
\hline & & & 6 & $\mathrm{H} \rightarrow \mathrm{L}+1$ & $\pi \rightarrow \mathrm{d}_{x y}+\mathrm{n}$ \\
\hline \multirow[t]{2}{*}{$\mathrm{T}_{4}$} & 2.38 & 520.8 & 34 & $\mathrm{H}-2 \rightarrow \mathrm{L}$ & $\mathrm{d}_{x z}+\pi_{\mathrm{CN}}+\pi \rightarrow \pi^{*}$ \\
\hline & & & 13 & $\mathrm{H}-2 \rightarrow \mathrm{L}+1$ & $\mathrm{~d}_{x z}+\pi_{\mathrm{CN}}+\pi \rightarrow \mathrm{d}_{x y}+\mathrm{n}$ \\
\hline $\mathrm{T}_{1}(\mathrm{~A})$ & 1.63 & 759.4 & & $\mathrm{H} \rightarrow \mathrm{L}$ & $\pi+\mathrm{d}_{y z} \rightarrow \pi^{*}$ \\
\hline $\mathrm{T}_{1}(\mathrm{C})$ & 1.11 & 1113.9 & & $\mathrm{H} \rightarrow \mathrm{L}$ & $\mathrm{d}_{y z}+\pi \rightarrow \sigma^{*}\left(\mathrm{~d}_{z^{2}}\right)$ \\
\hline \multicolumn{6}{|c|}{ COSMO $/ \mathrm{H}_{2} \mathrm{O}$} \\
\hline $\mathrm{T}_{1}(\mathrm{~A})$ & 1.67 & 743.1 & & $\mathrm{H} \rightarrow \mathrm{L}$ & $\pi+\mathrm{d}_{y z} \rightarrow \pi^{*}$ \\
\hline $\mathrm{T}_{1}(\mathrm{C})$ & 1.01 & 1229.4 & & $\mathrm{H} \rightarrow \mathrm{L}$ & $\pi+\mathrm{d}_{y z} \rightarrow \sigma^{*}\left(\mathrm{~d}_{z^{2}}\right)$ \\
\hline
\end{tabular}

The change in electronic structure is also visible in the electron density difference between the $S_{0}$ and $S_{1}$ states (Figures 6.9 and 6.10). As shown in Figure 6.9, electron density increases on the corrin ring and decreases on cobalt for $S_{1}(A)$, but rather reverse trend is observed for $S_{1}(C)$. Thus, in accordance with the orbital analysis, the $\mathrm{S}_{1}(\mathrm{~A})$ can be characterized as having mixed $\pi \pi^{*}$ /MLCT character while the $S_{1}(C)$ as LMCT-type excitation, respectively. However, neither of them can be viewed as pure MLCT or LMCT transitions because of the noticeable change of electron density along the axial bonds. For $\mathrm{S}_{1}(\mathrm{~A})$ the decrease while for $S_{1}(C)$ the increase could be noticed. Taking into consideration these differences, the $\mathrm{S}_{1}(\mathrm{~A})$ should be more accurately described as predominantly $\pi / \mathrm{d}_{\mathrm{Co}} \rightarrow \pi^{*}$ ( $\pi \pi^{*} /$ MLCT-type) with contribution from $\sigma$ bond to corrin CT ( $\pi \pi^{*} /$ SBLCT). On the other hand, the $S_{1}(C)$ should be characterized as predominantly $\pi / d \rightarrow \sigma^{*}\left(\mathrm{~d}_{z^{2}}\right)$ LMCT-type with contribution coming from corrin to $\sigma$ axial bonding CT (LSBCT). When the COSMO/ $\mathrm{H}_{2} \mathrm{O}$ was used to incorporate solvent effects (Figure 6.10) the nature of transition associated with $\mathrm{S}_{1}(\mathrm{~A})$ changed drastically. Inclusion of solvent reversed the character of $S_{1}(A)$ which became similar to $S_{1}(C)$. Therefore, $S_{1}(A)$ could be described as $\pi \pi^{*} / \mathrm{LMCT}$ and $\mathrm{S}_{1}(\mathrm{C})$ as LMCT/LSBCT. 


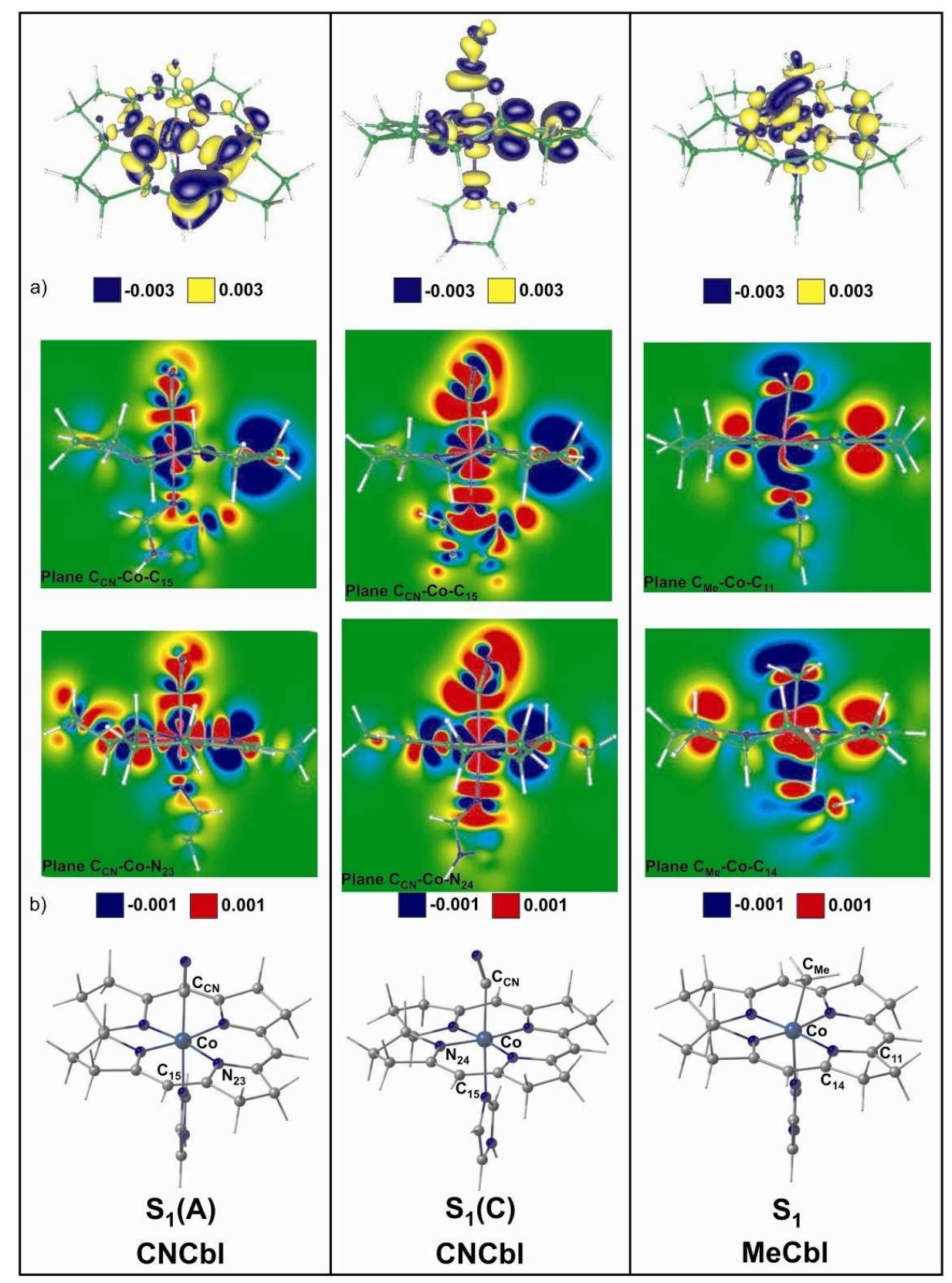

Figure 6.10. Electron density difference between the $\mathrm{S}_{0}$ and $\mathrm{S}_{1}$ states for CNCbl and MeCbl based on the BP86/6-31G(d) calculations with the use of COSMO solvatation model: (a) isosurface plot value of 0.003 and (b) cross-section contour plot along the axial bonding (in two different section planes). The yellow and red colors denote electron space, blue color - hole space. 
The electron density differences between the $S_{0}$ and $T_{1}$ states at $T_{1}(A)$ and $\mathrm{T}_{1}(\mathrm{C})$ geometries, based on the COSMO calculations are shown in Figure 6.11. For $\mathrm{T}_{1}$ (A) geometry the electron density increases in some parts of corrin ring and decreases in the remaining fragments of corrin macrocycle as well as around the cobalt atom. Taking into account the shape of electron density isosurface, the electronic excitation to $\mathrm{T}_{1}(\mathrm{~A})$ can be characterized as $\pi / \mathrm{d} \rightarrow \pi^{*}$ type. At $\mathrm{T}_{1}(\mathrm{C})$, the electron charge is shifted from the $d$ orbital of cobalt and $\pi$ orbital of corrin to $\sigma^{*}$ orbital, leading to $\pi / \mathrm{d} \rightarrow \sigma^{*}$ transition.

\subsection{Comparison with Transient Absorption Spectroscopy}

Sension and co-workers ${ }^{64}$ applied femtosecond transient IR and visible absorption spectroscopies to investigate the excited-state photophysics of vitamin $\mathrm{B}_{12}$ derivatives. In particular, it was demonstrated that the cobalt-upper ligand in nonalkyl cobalamins (including $\mathrm{CNCbl)}$ does not dissociate under conditions of single photon excitation neither at 400 nor $520 \mathrm{~nm}$. Regardless of the wavelength a sin-

gle transient excited state, formed on a subpicosecond time scale, was observed for each cobalamin. This state decayed to the ground state in 3-20 ps, depending on axial ligand and solvent. The transient intermediate was interpreted as short-lived LMCT state that was attributed to a corrin ring to $\operatorname{Co}\left(\mathrm{d}_{z^{2}}\right)$ transition. In this state the axial bonds were weakened and lengthened with respect to the corresponding structure of the ground state. The dynamics of this intermediate was also probed and it was found that it converted rapidly to the ground state without photolysis induced by electronic excitation.

In order to make a connection between the results of our TD-DFT calculations and transient absorption measurements ${ }^{64}$ the energy curves corresponding to low-lying excited states were plotted along the coordinate $q$ (Figure 6.12) both in a gas phase (left panel) and in water solution (right panel). Each plot contains two sets of energy curves where one is associated with vertical excitations based on the ground state geometry and the second depicts the energies based on the re- 


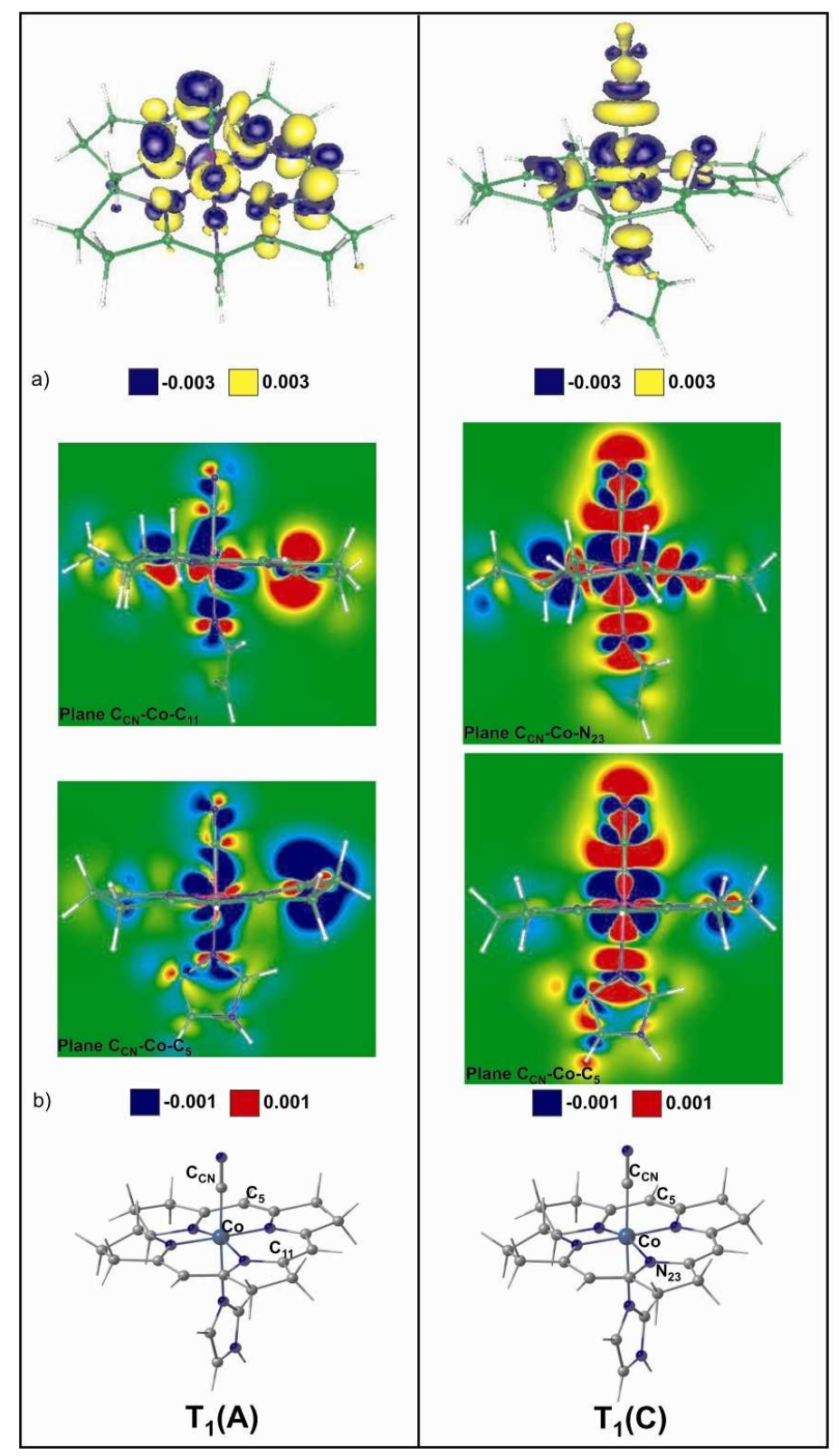

Figure 6.11. Electron density difference between the $S_{0}$ and $T_{1}$ states for CNCbl based on the BP86/6-31G(d) calculations with the use of COSMO solvatation model: (a) isosurface plot value of 0.003 and (b) cross-section contour plot along the axial bonding (in two different section planes). The yellow and red colors denote electron space, blue color - hole space. 

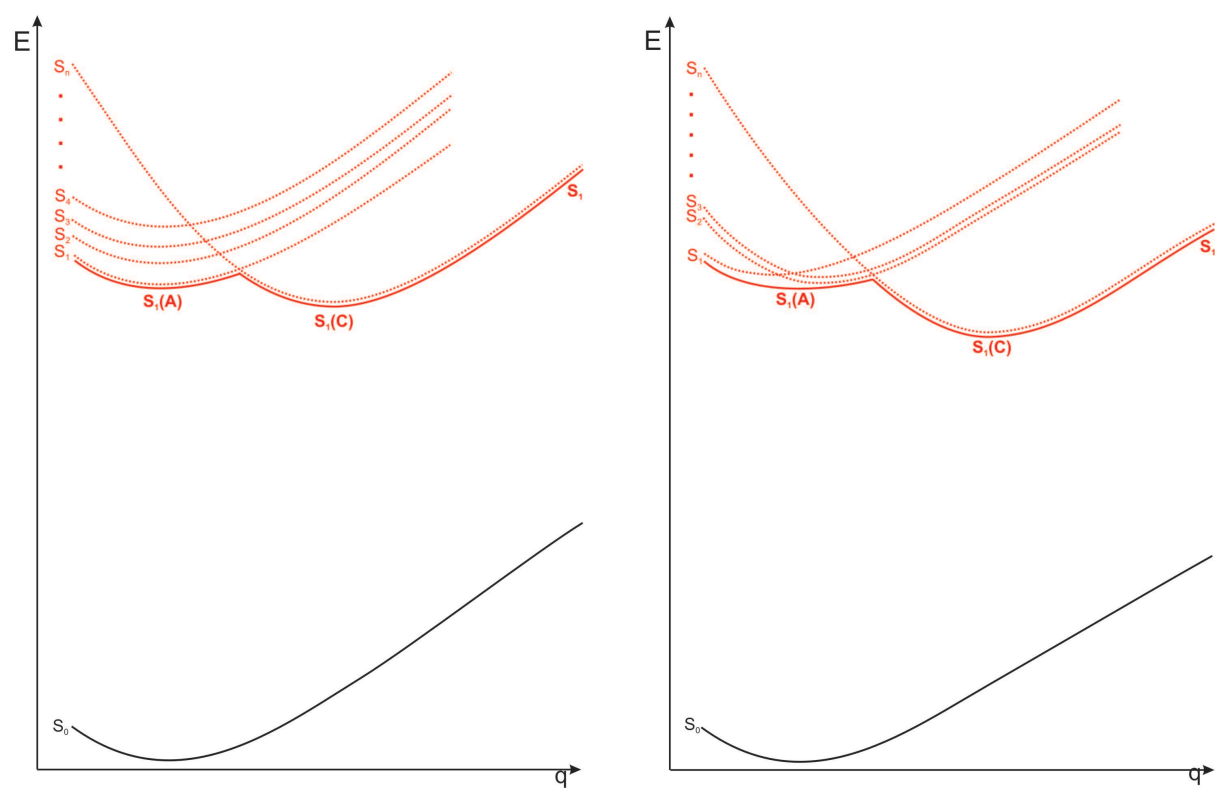

Figure 6.12. Potential energy curves of the lowest vertical excited singlet (red dot) and the optimized first excited state (red solid line) of the CNCbl model along the structural parameter q computed at BP86/6-31G(d) without solvent (left) and with solvent (right) using the COSMO/ $\mathrm{H}_{2} \mathrm{O}$ model.

laxed structures. The $q$ coordinate is not specified because it represents an internal geometric change along which the relaxation of geometry takes place. The energy curves can be partially connected with the energy surfaces characterizing the energy changes along the $\mathrm{Co}-\mathrm{C}_{\mathrm{CN}}$ and $\mathrm{Co}-\mathrm{N}_{\mathrm{Im}}$ distances, presented earlier in Figures 6.2 and 6.4, respectively. As revealed by calculations in which these two bond lengths were systematically stretched, the first minima for the four lowest excited states in gas phase lay almost one above another. The corresponding excitations can be found in Table 6.8.

Upon light absorption the $\mathrm{CNCbl}$ is excited from its ground state $\left(\mathrm{S}_{0}\right)$ to one of the vertical states, presumably to the $S_{1}$. After some short period of time, the early $S_{1}$ state, i.e., $S_{1}(A)$, overcomes some energetic barrier that is determined by the intersection with vertically higher excited state $S_{n}$. Afterwards, its geometry relaxes to the following stable structure, namely the $S_{1}(C)$. The $S_{1}(C)$ minimum has $\pi \rightarrow \sigma^{*}\left(\mathrm{~d}_{z^{2}}\right)$ character and therefore is probably a consequence of the crossing with energetically higher vertical state $\left(S_{n}\right)$. However, in contrast to gas phase, where 
the $\mathrm{S}_{1}(\mathrm{~A})$ is of mixed $\pi \pi^{*} / \mathrm{MLCT}$ type, the solvent data reveals $\pi \pi^{*} / \mathrm{LMCT}$ transition. Due to the $\pi \rightarrow \mathrm{d}_{x y}+\mathrm{n}$ contribution to this excitation it could be also correlated with LMCT observed in experiment. On the other hand, the participation of $\pi \rightarrow \pi^{*}$ transition associated with $S_{1}(A)$ can not be ignored. Although the outcomes, based on calculated vertical excitations, show $\pi \rightarrow \mathrm{d}_{x y}+\mathrm{n}$ transition to even second and third excited state, the $S_{1}$ is still described as $\pi \rightarrow \pi^{*}$ (Table 6.8). These findings allow to assume that $S_{1}, S_{2}$ and $S_{3}$ states lay close one to another and the possible crossing may occur at smaller values of $q$, explaining the mixed nature of $\mathrm{S}_{1}(\mathrm{~A})$. It should be also noted that $S_{2}$ and $S_{3}$ are composed of the exactly same transitions and have very close energies, indicating possible degeneracy.

The electronic properties of the lowest excited states of CNCbl can be also analyzed by taking into account the NBO charges of the excited and ground state (Table 6.4). For the $S_{1}(A)$, in calculations with solvent, there is a substantial increase of positive charge on corrin and the negative charge on axial ligands. This agrees with density difference and molecular orbital analysis, confirming the character of excited state associated with the $\mathrm{S}_{1}(\mathrm{~A})$ geometry, described as $\pi \pi^{*} / \mathrm{LMCT}$ type. Electronically excited state related to the $\mathrm{S}_{1}(\mathrm{C})$ geometry is similar and also exhibits the increase of positive charge on corrin ring and, at the same time, the increase of negative charge on cobalt and axial ligands, confirming the LMCT/LSBCT character. The largest charge difference was found for corrin, where the increase of positive charge is 0.329 in the calculations with solvent model. The increase of negative charge is the largest on the $\mathrm{CN}^{-}(-0.189)$, somewhat smaller on $\operatorname{Im}(-0.089)$ and quite small on cobalt (-0.051), indicating that the charge is shifted from corrin ring to the axial ligands and cobalt. Very similar effect can be noticed for $T_{1}(C)$, although the changes in atomic charges are smaller. In calculations with solvent, they are respectively: 0.125 for corrin, -0.160 for $\mathrm{CN}^{-}$and 0.098 for cobalt. Hence, the character of $T_{1}(C)$ is similar to $S_{1}(C)$, however in this case the negative charge from corrin is almost completely shifted towards the axial ligand. 


\subsection{Comparison of $S_{1}$ States in $\mathrm{CNCbl}$ and $\mathrm{MeCbl}$}

The electron density difference map between the $S_{0}$ and $S_{1}$ states in MeCbl has been plotted for comparison with CNCbl (Figures 6.10 and 6.11). It has been demonstrated that the $S_{1}$ state in MeCbl does not change its character upon inclusion of solvent. ${ }^{119}$ Therefore, only calculations without solvent were taken into account. Several interesting observations can be drawn from the density difference analysis. Without solvent the excited state corresponding to the $S_{1}(A)$ geometry shows a decrease of the electron density on corrin and, at the same time, an increase along the $\mathrm{N}_{\mathrm{Im}}-\mathrm{Co}-\mathrm{C}_{\mathrm{CN}}$ axis. Such density difference for the $\mathrm{S}_{1}(\mathrm{~A})$ closely resembles the difference observed for $S_{1} / \mathrm{MeCbl}$ state (Figure 6.9), which has been described as predominantly $\mathrm{d}_{\mathrm{Co}} \rightarrow \pi^{*}$ MLCT with contribution from $\sigma$ bond to corrin CT (SBLCT). Therefore, by analogy with $S_{1} / \mathrm{MeCbl}$ state the $S_{1}(A)$ state on $\mathrm{S}_{1} / \mathrm{CNCbl}$ PES should be described as having mixed $\pi \pi^{*} / \mathrm{MLCT} / \mathrm{SBLCT}$ character. On the other hand, the $S_{1}$ state in MeCbl is distinctively different from $S_{1}(C)$ (Figure 6.9). Inclusion of solvent via $\mathrm{COSMO} / \mathrm{H}_{2} \mathrm{O}$ changes the character of $\mathrm{S}_{1}(\mathrm{~A})$ to a significant degree, so that the resulting electron density difference does not resemble $S_{1} / \mathrm{MeCbl}$ anymore (Figure 6.10). In fact, the density difference looks more like the $S_{1}(C)$, which has been characterized as predominantly $\pi / d \rightarrow \sigma^{*}\left(d_{z^{2}}\right)$ LMCT-type, having contribution from corrin ligand to $\sigma$ axial bonding CT transition (LSBCT). Therefore, presence of solvent reverses the character of the $S_{1}(A)$ state, which consequently changes this excitation to be more in line with the $S_{1}(C)$, having $\pi \pi^{*} / \mathrm{LMCT} / \mathrm{LSBCT}$ character.

\subsection{Summary and Conclusions}

In the theoretical account presented in this chapter the structural and electronic properties of the CNCbl have been investigated by means of TD-DFT. Several interesting observations can be drawn from this computational analysis that may shed new light on some critical issues related to photophysical properties of CNCbl. In particular, the $\mathrm{Co}-\mathrm{C}$ bond in $\mathrm{CNCbl}$ and related non-alkyl cob(III)alamins is pho- 
tostable and does not undergo photodissociation under condition of simple photon excitation in contrast to $\mathrm{MeCbl}$ or AdoCbl. When electronically excited states were computed as a function of the $\mathrm{Co}-\mathrm{C}$ bond, it was found that in the case of $\mathrm{MeCbl}$ the photolysis is mediated by the dissociative ${ }^{3} \sigma_{\mathrm{Co}-\mathrm{C}} \rightarrow \sigma_{\mathrm{Co}-\mathrm{C}}^{*}$ triplet state. When similar calculations were carried out for $\mathrm{CNCbl}$, the repulsive triplet state, having ${ }^{3}\left(\sigma_{\mathrm{Co}-\mathrm{C}} \rightarrow \sigma_{\mathrm{Co}-\mathrm{C}}^{*}\right)$ character, was also located, but in contrast to MeCbl, this state did not become dissociative. A rationale was provided explaining why the repulsive triplet state lies energetically much higher in $\mathrm{CNCbl}$ as compared to $\mathrm{MeCbl}$ and why it does not become the lowest excited state as the Co-C lengthens. Although the $\mathrm{Co}-\mathrm{C}$ coordinate is a driving force for photolysis process, other coordinates, for example $\mathrm{N}_{\text {axial }}-\mathrm{Co}-\mathrm{C}$ bending, may also play a critical role. This issue, however, requires further computational analysis.

The structural and electronic analysis was carried out for the $S_{1}$ intermediate state and two energy minima were located on $\mathrm{S}_{1} / \mathrm{CNCbl}$ surface. In the absence of solvent, the transition to the $\mathrm{S}_{1}(\mathrm{~A})$ was described as excitation having $\pi \pi^{*} /$ MLCT/SBLCT character, while to the $\mathrm{S}_{1}(\mathrm{C})$ was characterized as LMCT/LSBCT excitation. It was also noticed that $S_{1}(A)$ on the $S_{1} / C N C b l$ PES closely resembles the $S_{1}$ state in MeCbl. However, inclusion of solvent altered the character of the $\mathrm{S}_{1}$ (A) state which became $\pi \pi^{*} / \mathrm{LMCT} / \mathrm{LSBCT}$-type transition. There are several reasons why presence of solvent changes character of the $S_{1}(A) / C N C b l$ but leaves $\mathrm{S}_{1}(\mathrm{C}) / \mathrm{CNCbl}$ and $\mathrm{S}_{1} / \mathrm{MeCbl}$ essentially intact. The difference in the electronic structure of the $\mathrm{S}_{1}$ state between $\mathrm{CNCbl}$ and $\mathrm{MeCbl}$ is visible if we take into account the order and the character of the frontier orbitals. Based on the NBO analysis, the $\mathrm{Co}-\mathrm{C} \mathrm{s}$ bond in $\mathrm{CNCbl}$ is more ionic than in MeCbl. The different character of $\mathrm{S}_{1}$ exited states of $\mathrm{CNCbl}$ and $\mathrm{MeCbl}$ is also related to different extent to polarization of $\mathrm{Co}-\mathrm{C}_{\mathrm{CN}}$ and $\mathrm{Co}-\mathrm{C}_{\mathrm{CH}_{3}}$ bond. It should also be mentioned that although two minima were located on $\mathrm{S}_{1} / \mathrm{CNCbl} \mathrm{PES}$, yet only one i.e., $\mathrm{S}_{1}(\mathrm{C}) / \mathrm{CNCbl}$ can be probed by transient Abs spectroscopy. This confirms the nature of the first excited state, as predominantly LMCT-type for CNCbl. 


\section{CHAPTER 7}

\section{CONCLUSIONS}

Although many years have passed since Whipple, Minot and Murphy were awarded the Nobel Prize for Medicine and Physiology in 1934 for the discovery of "antipernicious anaemia factor", ${ }^{291}$ vitamin $\mathrm{B}_{12}$ and its derivatives remain an attractive subject of research. Their enormous catalytic activity, an unique Co-C bond as well as variety of functions and the amount of diverse reactions that are catalyzed by enzyme-bound $B_{12}$ cofactors have placed these molecules as central players in studies directed towards understanding of biological organometallic processes. Although extensive scientific investigations have brought remarkable advances in the field, the mechanism of enzymatic catalysis continues to be unresolved and relies on proposals. This situation is to a large degree related to an elusive description of the Co-C bond activation as well as insufficient characterization of intermediates formed during the catalytic cycles. In response, the studies undertaken in this dissertation address structural, electronic and photochemical properties of $\mathrm{B}_{12}$ cofactors focusing on comprehensive analyses of spectroscopic data with the aim of a theoretical approach.

Spectroscopy as a research tool can be treated as a magnifying glass for electronic structure of the molecules. It allows to probe the quantum structure of molecular systems revealing their energetic states. The interpretation of recorded spectra might be however challenging, especially for systems as complex as transition metal compounds, hereby represented by vitamin $\mathrm{B}_{12}$ derivatives. Consequently, a theoretical approach becomes remarkably handy to explore the properties of these systems at molecular level. By analyzing the calculated spectroscopic parameters one can get insight into the properties of the molecules as these features are inti- 
mately tied to these parameters. However, in order to provide reliable conclusions based on calculated results, certain conditions have to be fulfilled starting with the application of a proper theoretical framework, including the utilization of reliable molecular models and adequate modeling of environmental effects and completing the thesis by clever correlation between the structural information and function of the systems and explanation of both experimental and theoretical data. Such a perspective was presented in this dissertation. Taking into account that the TD-DFT offers an attractive compromise between the accuracy and the cost of calculation, it was employed as a major theoretical tool to calculate electronic properties of diverse $\mathrm{B}_{12}$ cofactors. In the first two chapters following the Introduction the elucidation of the most appropriate density functional for these purposes was extensively discussed. Both CNCbl (Chapter 2) and MeCbl (Chapter 3) were investigated using various density functionals and higher level $a b$ initio calculations. The GGA functionals were proved to be the most adequate in describing the electronically excited states of both systems, revealing at the same time, the inadequacy of hybrid and range-separated ones. In particular, the nature of low-lying excitations predicted with BP86 matched the results obtained with CASSCF/MC-XQDPT2 (for CNCbl and $\mathrm{MeCbl}$. When the studies for MeCbl were extended to the EOM-CCSD calculations, the same functional was shown to be the most accurate one. These findings are of a remarkable meaning with respect to computational studies on cobalt corrinoids. It is due to the fact that the number of preceding investigations carried out for vitamin $\mathrm{B}_{12}$ and its derivatives relied on computations employing hybrid functionals. In the light of the studies presented it this dissertation, these previous assignments became questionable.

Consequently with the elucidation of BP86 as an appropriate density functional for cobalamin investigations, the TD-DFT/BP86 was utilized as the most practical tool to investigate their spectroscopic properties and to provide new assignments of their electronic spectra. The novel approach demonstrated in this dissertation was to apply linear and quadratic response TD-DFT to simulate CD and MCD spectra (in addition to Abs) of CNCbl, MeCbl and cob(I)alamin (Chapters 4 
and 5). Although electronically excited states of these systems have been probed before, the recent theoretical studies have left many unresolved issues related to the nature of individual transitions. Hereby the Abs but also CD and MCD spectra of aforementioned cobalamins were computed for the first time and compared with experiment. In the case of $\mathrm{MeCbl}$ and $\mathrm{CNCbl}$ (Chapter 4), the results were evaluated with respect to the choice of the exchange-correlation functional, basis set, and the environment (gas phase or solvent) used in the calculation. Taking into account the complexity of the $\mathrm{CNCbl}$ and $\mathrm{MeCbl}$ systems, reliable agreement between theory and experiment was achieved. Consequently the re-assignments of $\alpha, \beta, \mathrm{D} / \mathrm{E}$, and $\gamma$ bands based on calculations employing the BP86 functional were provided. In the case of $\operatorname{cob}(\mathrm{I})$ alamin several issues have been taken into considerations while performing the TD-DFT calculations, such as strong dependence on the applied exchange-correlation functional or structural simplification imposed on the cob(I)alamin. In addition, the low-lying transitions were also validated by performing CASSCF/MC-XQDPT2 calculations. By comparing computational results with existing experimental data a new level of understanding of electronic excitations has been established at the molecular level. This study extended and confirmed conclusions reached for other cobalamins. In particular, the better performance of the BP86 functional, rather than hybrid-type, was observed in terms of the excitations associated with both Co $\mathrm{d}$ and corrin $\pi$ localized transitions. The transitions including Co d orbitals were proved to possess significant contributions to the bands observed in calculated spectra. This observation is of big importance considering many years view suggesting the complete dominance of $\pi \rightarrow \pi^{*}$ transitions in electronic spectra of $\mathrm{B}_{12}$ cofactors. Finally, the use of the full cob(I)alamin structure, instead of simplified molecular models, shed new light on the spectral analyses of cobalamin systems and revealed new challenges of this approach related to longrange charge transfer excitations involving side chains.

A great amount of work presented in this dissertation was dedicated to a thorough analysis of the energetically lowest $\alpha / \beta$ band observed in Abs spectra due to the existing controversies with respect to its origin. Firstly, in Chapter 4, it was 
shown that the view about vibrational progression associated with a single electronic transition is unlikely as the number of electronic excitations was observed in simulated Abs and $\mathrm{CD}$ spectra of $\mathrm{CNCbl}$ and $\mathrm{MeCbl}$ in this region. This observation was strongly supported by joined TD-DFT and $a b$ initio studies described in Chapters 2 and 3 . In addition, the traditional $\pi \rightarrow \pi^{*}$ assignment of the lowest in energy $\alpha / \beta$ band was seriously undermined. Both TD-DFT/BP86 and CASSCF/MCXQDPT2 methods indicated that the excited states of CNCbl possessed contributions from mixed $\mathrm{d} / \pi \rightarrow \pi^{*}$ transitions. This allows to conclude that in contrast to the traditional interpretation as well as data obtained with hybrid functionals in earlier studies, which pointed only at corrin-localized excitations, the low-lying excited states of $\mathrm{CNCbl}$ include CTs. In particular, the lowest $\mathrm{S}_{1}$ state can be described not as a $\pi \rightarrow \pi^{*}$ but as a LMCT. Similar studies carried out for MeCbl (Chapter 3) with two independent $a b$ initio wave-function methods (CASSCF/MC-XQDPT2 and EOM-CCSD) provided a consistent MLCT description of its $S_{1}$ state. This again indicated that CT-type transitions describe the lowest $\alpha / \beta$ region of cobalamin spectra supporting the findings provided by TAS experiments.

This observation has significant implications for photophysical studies of vitamin $\mathrm{B}_{12}$ and its derivatives. The understanding of processes inducted by photon excitation requires a correct characterization of the nature of participating electronically excited states. Owing to the elucidation of BP86 as an appropriate density functional for these purposes (Chapters 2 and 3) and by confirming its applicability in analyses of the electronic spectra of MeCbl, CNCbl (Chapter 4) and cob(I)alamin (Chapter 5), the consequent investigation of the mechanism of events following photoexcitation in vitamin $B_{12}$ was presented in Chapter 6 .

To explain why the Co-C bond in CNCbl does not undergo photodissociation under conditions of simple photon excitation, electronically excited states have been computed along the $\mathrm{Co}-\mathrm{C}_{\mathrm{CN}}$ stretched coordinate. It was found that the repulsive ${ }^{3}\left(\sigma_{\mathrm{Co}-\mathrm{C}} \rightarrow \sigma_{\mathrm{Co}-\mathrm{C}}^{*}\right)$ triplet state drops in energy as the $\mathrm{Co}-\mathrm{C}_{\mathrm{CN}}$ bond lengthens, but it does not become dissociative. Low-lying excited states were also computed as function of two axial bond lengths. Two energy minima have been located on 
the $\mathrm{S}_{1} / \mathrm{CNCbl}$, as well as $\mathrm{T}_{1} / \mathrm{CNCbl}$, surfaces. Their presence indicated the possibility of alternative to photolysis channels of utilization of absorbed energy. It was proposed that an excitation to the $S_{1}$ state in $\mathrm{CNCbl}$ is followed by a relaxation of its geometry to another stable structure. This process gains credibility when one takes into account the possibility of an intersystem crossing between the $S_{1}$ and one of the energetically higher excited state, which is indicated by the occurrence of an additional minimum on the PES of CNCbl. In order to support this thesis the comparison with the data coming from TAS measurements were provided as the full geometry optimization was carried out for each minimum and electronic properties associated with each optimized structure were analyzed in detail. One minimum was described as excitation having mixed $\pi \pi^{*} /$ MLCT character, while the second as LMCT transition. Neither of them, however, can be viewed as pure MLCT or LMCT transitions since additional excitation to or from $\sigma$-bonds (SB) of $\mathrm{N}-\mathrm{Co}-\mathrm{C}$ unit have also noticeable contributions. Inclusion of solvent altered the character of one of the excitations from $\pi \pi^{*} / \mathrm{MLCT} / \mathrm{SBLCT}$ to $\pi \pi^{*} / \mathrm{LMCT} / \mathrm{LSBCT}$-type, and therefore, both of them gained significant contribution from LMCT/LSBCT transition. This observation agrees precisely with the TAS experiments which indicated the character of the intermediate created after photon excitation as a LMCT, strengthening our conclusions with respect to the photoinduced events in CNCbl. Finally, in the same chapter, the nature of $\mathrm{S}_{1}$ electronic state has been comparatively analyzed in $\mathrm{CNCbl}$ and MeCbl cobalamins, explaining the differences between the observed photolysis in the later system and the lack of photodissociation in the former one. 


\section{REFERENCES}

[1] G. H. Whipple and F. S. Robscheit-Robbins, Am. J. Physiol 72, 408 (1926).

[2] G. R. Minot and W. P. Murphy, JAMA 87, 470 (1926).

[3] E. L. Rickes, N. G. Brink, F. R. Konivszy, T. R. Wood, and K. Folkers, Science 107, 396 (1948).

[4] D. C. Hodgkin, J. Kamper, M. Mackay, J. W. Pickworth, K. N. Trueblood, and J. G. White, Nature 178, 64 (1956).

[5] R. B. Woodward, Synthetic vitamin $\mathrm{B}_{12}$, in Vitamin $B_{12}$, Proceedings of the Third European Symposium on Vitamin $B_{12}$ and Intrinsic Factors, edited by B. Zagalak and W. Friedrich, pages 37-88, Walter de Gruyter, Berlin, Germany, 1979.

[6] D. M. Matthews, in Vitamin $B_{12}$ : proceedings of the third European Symposium on Vitamin $B_{12}$ and Intrinsic Factor, University of Zürich, March 5-8, 1979, Zürich, Switzerland, edited by B. Zagalak and W. Friedrich, page 681, W. de Gruyter, 1979.

[7] L. Ellenbogen and B. A. Cooper, in Handbook of Vitamins, edited by L. J. Machlin, page 491, Marcel Dekker, New York \& Basel, 1991.

[8] M. I. Shevell and D. S. Rosenblatt, Can. J. Neurol. Sci. 19, 472 (1992).

[9] D. Dolphin (Ed.), $B_{12}$, Wiley-Interscience, New York, 1982.

[10] R. Banerjee, Chem. Biol. 66, 269 (1997).

[11] R. Banerjee, R. G. Matthews, and J. Faseb, FASEB J. 4, 1450 (1990).

[12] E. N. G. Marsh, BioEssays 17, 431 (1995).

[13] M. L. Ludwig and R. G. Matthews, Annu. Rev. Biochem. 66, 269 (1997).

[14] B. Kräutler, D. Arigoni, and B. T. G. (Eds.), Vitamin $B_{12}$ and $B_{12}$ Proteins, Wiley-VCH, New York, 1998.

[15] L. G. Marzilli, in Bioinorganic Catalysis, edited by J. Reedijk and E. Bouwman, pages 423-468, Marcel Dekker, New York, 1999. 
[16] R. Banerjee, Chemistry and Biochemistry of $B_{12}$, John Wiley \& Sons, New York, 1999.

[17] T. Toraya, Cell. Mol. Life Sci. 57, 106 (2000).

[18] R. Banerjee, Biochemistry 40, 6191 (2001).

[19] R. G. Matthews, Acc. Chem. Res. 34, 681 (2001).

[20] R. Banerjee and S. W. Ragsdale, Annu. Rev. Biochem. 72, 209 (2003).

[21] R. Banerjee, Chem. Rev. 103, 2083 (2003).

[22] T. Toraya, Chem. Rev. 103, 2095 (2003).

[23] K. L. Brown, Chem. Rev. 105, 2075 (2005).

[24] L. Randaccio, S. Geremia, G. Nardin, and J. Wuerges, Coord. Chem. Rev. 250, 1332 (2006).

[25] L. Randaccio, S. Geremia, and J. Wuerges, J. Organomet. Chem. 692, 1198 (2007).

[26] R. G. Matthews, M. Koutmos, and S. Datta, Curr. Opin. Struct. Biol. 18, 658 (2008).

[27] R. G. Matthews, Cobalamin-and corrinoid-dependent enzymes, in Metal Ions in Life Sciences, Volume 6, edited by A. Sigel, H. Sigel, and R. K. O. Sigel, pages 53-114, Royal Society of Chemistry, Cambridge, UK, 2009.

[28] L. Randaccio, S. Geremia, N. Demitri, and J. Wuerges, Molecules 15, 3228 (2010).

[29] P. A. Frey, M. K. Essenberg, and R. H. Abeles, J. Biol. Chem. 242, 5369 (1967).

[30] J. Rétey and D. Arigoni, Experientia 22, 783 (1966).

[31] H. P. C. Hogenkamp, in Chemistry and Biochemistry of $B_{12}$, edited by R. Banerjee, pages 3-8, John Wiley \& Sons, New York, NY, USA, 1999.

[32] B. Kräutler, Organometallic chemistry of $\mathrm{B}_{12}$ coenzymes., in Metal Ions in Life Sciences, edited by A. Sigel, H. Sigel, and R. K. O. Sigel, volume 6, pages 1-51, Royal Society of Chemistry, Cambridge, UK, 2009.

[33] L. Randaccio, S. Geremia, N. Demitri, and J. Wuerges, Trends Inorg. Chem. 11, 1 (2009). 
[34] J. F. Kolhouse and R. H. Allen, P. Natl. Acad. Sci. 74, 921 (1977).

[35] I. S. Mellman, P. Youngdahl-Turner, H. F. Willard, and L. E. Rosenberg, P. Natl. Acad. Sci. 74, 916 (1977).

[36] F. Mancia et al., Structure 4, 339 (1996).

[37] Y. Zhao, A. Abend, M. K. M, P. Such, and J. Rétey, Eur. J. Biochem. 225, 891 (1994).

[38] R. Padmakumar and R. Banerjee, J. Biol. Chem. 270, 9295 (1995).

[39] G. Russell-Jones, K. McTavish, J. McEwan, J. Rice, and D. Novotnik, J. Inorg. Biochem. 98, 1625 (2004).

[40] R. Waibel et al., Cancer Res. 68, 2904 (2008).

[41] A. K. Petrus, T. J. Fairchild, and R. P. Doyle, Angew. Chem. Int. Edit. 48, 1022 (2009).

[42] K. L. Brown and S. Peck-Siler, Inorg. Chem. 27, 3548 (1988).

[43] P. G. Lenhert and D. C. Hodgkin, Nature 192, 937 (1961).

[44] M. Rossi, J. P. Glusker, L. Randaccio, M. F. Summers, P. J. Toscano, and L. G. Marzilli, J. Am. Chem. Soc. 107, 1729 (1985).

[45] L. Randaccio, Comment. Inorg. Chem. 21, 327 (1999).

[46] L. Randaccio, N. B. Pahor, E. Zangrando, and L. Marzilli, Chem. Soc. Rev. 18, 225 (1989).

[47] L. G. Marzilli, M. F. Summers, N. Bresciani-Pahor, E. Zangrando, J. P. Charland, and L. Randaccio, J. Am. Chem. Soc. 107, 6880 (1985).

[48] C. B. Perry and H. M. Marques, S. Afr. J. Sci. 100, 368 (2004).

[49] G. Garau, S. N. Fedosov, T. E. Petersen, S. Geremia, and L. Randaccio, Acta Crystallogr. D 57, 1890 (2001).

[50] J. Masuda, N. Shibata, Y. Morimoto, T. Toraya, and N. Yasuoka, Structure 8, 775 (2000).

[51] J. Halpern, S. H. Kim, and T. Leung, J. Am. Chem. Soc. 106, 8317 (1984).

[52] C. D. Garr, J. M. Sirovatka, and R. G. Finke, J. Am. Chem. Soc. 118, 11142 (1996). 
[53] B. P. Hay and R. G. Finke, J. Am. Chem. Soc. 108, 4820 (1986).

[54] B. P. Hay and R. G. Finke, J. Am. Chem. Soc. 109, 8012 (1987).

[55] R. G. Finke, Coenzyme $\mathrm{B}_{12}$-based chemical precedent for Co-C bond homolysis and other key elementary steps., in Vitamin $B_{12}$ and $B_{12}$ Proteins, edited by B. Kräutler, D. Arigoni, and B. T. G. (Eds.), pages 383-403, Wiley-VCH, New York, 1998.

[56] L. A. Walker, II, J. T. Jarrett, N. A. Anderson, S. H. Pullen, R. G. Matthews, and R. J. Sension, J. Am. Chem. Soc. 120, 3597 (1998).

[57] L. A. Walker, II, J. J. Shiang, N. A. Anderson, S. H. Pullen, and R. J. Sension, J. Am. Chem. Soc. 120, 7286 (1998).

[58] J. J. Shiang, L. A. Walker, II, N. A. Anderson, A. G. Cole, and R. J. Sension, J. Phys. Chem. B 103, 10532 (1999).

[59] L. M. Yoder, A. G. Cole, L. A. Walker, II, and R. J. Sension, J. Phys. Chem. B 105, 12180 (2001).

[60] A. G. Cole, L. M. Yoder, J. J. Shiang, N. A. Anderson, L. A. Walker, II, M. M. B. Holl, and R. J. Sension, J. Am. Chem. Soc. 124, 434 (2002).

[61] R. J. Sension, A. G. Cole, D. A. Harris, C. C. Fox, N. W. Woodbury, S. Lin, and E. N. G. Marsh, J. Am. Chem. Soc. 126, 1598 (2004).

[62] R. J. Sension, D. A. Harris, A. Stickrath, A. G. Cole, C. C. Fox, and E. N. G. Marsh, J. Phys. Chem. B 109, 18146 (2005).

[63] R. J. Sension, D. A. Harris, and A. G. Cole, J. Phys. Chem. B 109, 21954 (2005).

[64] J. J. Shiang, A. G. Cole, R. J. Sension, K. Hang, Y. Weng, J. S. Trommel, L. G. Marzilli, T. Lian, and N. A. Anderson, J. Am. Chem. Soc. 128, 801 (2006).

[65] D. A. Harris, A. B. Stickrath, E. C. Carroll, and R. J. Sension, J. Am. Chem. Soc. 129, 7578 (2007).

[66] C. Giannotti, Chapter 11, in $B_{12}$, edited by D. Dolphin, pages 393-430, Wiley-Interscience, New York, 1982.

[67] R. A. Firth, H. A. O. Hill, J. M. Pratt, R. J. P. Williams, and W. R. Jacson, Biochemistry 6, 2178 (1967).

[68] J. M. Pratt, Chapter 5, in Chemistry and Biochemistry of B12, edited by R. Banerjee, pages 113-164, John Wiley \& Sons, New York, 1999. 
[69] P. Day, Theor. Chim. Acta 7, 328 (1967).

[70] P. Day, Coord. Chem. Rev. 2, 109 (1967).

[71] P. O. Offenhartz, B. H. Offenhartz, and M. M. Fung, J. Am. Chem. Soc. 92, 2966 (1970).

[72] J. I. Toohey, Proc. Natl. Acad. Sci. USA 54, 934 (1965).

[73] H. Kuhn, K. H. Drexhage, and H. Martin, Proc. R. Soc. London Ser. A 288, 348 (1965).

[74] B. H. Offenhartz, Proc. R. Soc. London Ser. A 288, 350 (1965).

[75] A. Veillard and B. Pullman, J. Theor. Biol. 8, 307 (1965).

[76] L. L. Ingraham and J. P. Fox, Ann. N.Y. Acad. Sci. 153, 738 (1969).

[77] G. N. Schrauzer, Naturwiss. 53, 459 (1969).

[78] G. N. Schrauzer, L. P. Lee, and J. W. Sibert, J. Am. Chem. Soc. 92, 2997 (1970).

[79] L. Salem, O. Eisenstein, N. T. Anh, H. B. Burgi, A. Devaquet, G. Segal, and A. Veillard, Nouv. J. Chim. 1, 335 (1977).

[80] E. Runge and E. K. U. Gross, Phys. Rev. Lett. 52, 997 (1984).

[81] E. J. Baerends, G. Ricciardi, A. Rosa, and S. J. A. van Gisbergen, Coord. Chem. Rev. 230, 5 (2002).

[82] A. Rosa, G. Ricciardi, O. Gritsenko, and E. J. Baerends, Struct. Bond. 112, 49 (2004).

[83] A. Dreuw and M. Head-Gordon, Chem. Rev. 105, 4009 (2005).

[84] A. Dreuw, ChemPhysChem 7, 2259 (2006).

[85] T. Andruniów, M. Z. Zgierski, and P. M. Kozlowski, Chem. Phys. Lett. 331, 509 (2000).

[86] T. Andruniów, M. Z. Zgierski, and P. M. Kozlowski, J. Phys. Chem. B 104, 10921 (2000).

[87] T. Andruniów, M. Z. Zgierski, and P. M. Kozlowski, J. Am. Chem. Soc. 123, 2679 (2001). 
[88] T. Andruniów, J. Kuta, M. Z. Zgierski, and P. M. Kozlowski, Chem. Phys. Lett. 410, 410 (2005).

[89] M. Freindorf and P. M. Kozlowski, J. Am. Chem. Soc. 126, 1930 (2004).

[90] P. M. Kozlowski, Curr. Opin. Chem. Biol. 5, 736 (2001).

[91] P. M. Kozlowski and M. Z. Zgierski, J. Phys. Chem. B 108, 14163 (2004).

[92] P. M. Kozlowski, T. Kamachi, T. Toraya, , and K. Yoshizawa, Angew. Chem. Int. Ed. 46, 980 (2007).

[93] N. Dölker, F. Maseras, and A. Lledós, J. Phys. Chem. B 105, 7564 (2001).

[94] N. Dölker, F. Maseras, and A. Lledós, J. Phys. Chem. B 107, 306 (2003).

[95] N. Dölker, F. Maseras, and P. E. M. Siegbahn, Chem. Phys. Lett. 386, 174 (2004).

[96] N. Dölker, A. Morreale, and F. Maseras, J. Biol. Inorg. Chem. 10, 509 (2005).

[97] L. Randaccio, S. Geremia, M. Stener, D. Toffoli, and E. Zangrando, Eur. J. Inorg. Chem. 93 (2002).

[98] K. P. Jensen, S. P. A. Sauer, T. Liljefors, and P.-O. Norrby, Organometallics 20, 550 (2001).

[99] K. P. Jensen and U. Ryde, J. Mol. Struct. (THEOCHEM) 585, 239 (2002).

[100] K. P. Jensen and U. Ryde, J. Phys. Chem. A 107, 7539 (2003).

[101] K. P. Jensen and U. Ryde, ChemBioChem 4, 413 (2003).

[102] K. P. Jensen and U. Ryde, J. Am. Chem. Soc. 127, 9117 (2005).

[103] C. Rovira, X. Biames, and K. Kunc, Inorg. Chem. 43, 6628 (2004).

[104] C. Rovira and P. M. Kozlowski, J. Phys. Chem. B 111, 3251 (2007).

[105] T. Andruniów, M. Jaworska, P. Lodowski, M. Z. Zgierski, R. Dreos, L. Randaccio, and P. M. Kozlowski, J. Chem. Phys. 129, 85101 (2008).

[106] T. Andruniów, M. Jaworska, P. Lodowski, M. Z. Zgierski, R. Dreos, L. Randaccio, and P. M. Kozlowski, J. Chem. Phys. 131, 105105 (2009).

[107] T. Andruniów, P. M. Kozlowski, and M. Z. Zgierski, J. Chem. Phys. 115, 7522 (2001). 
[108] T. R. Stich, A. J. Brooks, N. R. Buan, and T. C. Brunold, J. Am. Chem. Soc. 125, 5897 (2003).

[109] M. Jaworska and P. Lodowski, J. Mol. Struct. (THEOCHEM) 631, 209 (2003).

[110] M. D. Liptak and T. C. Brunold, J. Am. Chem. Soc. 128, 9144 (2006).

[111] M. Jaworska, G. Kozibut, and P. Lodowski, J. Phys. Chem. A 107, 1339 (2005).

[112] T. R. Stich, N. R. Buan, and T. C. Brunold, J. Am. Chem. Soc. 126, 9735 (2004).

[113] T. C. Brunold, K. S. Conrad, M. D. Liptak, and K. Park, Coord. Chem. Rev. 253, 779 (2009).

[114] A. D. Becke, J. Chem. Phys. 98, 5648 (1993).

[115] C. Lee, W. Yang, and R. G. Parr, Phys. Rev. B 37, 785 (1988).

[116] P. E. M. Siegbahn and T. Borowski, Acc. Chem. Res. 39, 729 (2006).

[117] J. Halpern, Science 227, 869 (1985).

[118] M. Jaworska, P. Lodowski, T. Andruniów, and P. M. Kozlowski, J. Phys. Chem. B (Letter) 111, 2419 (2007).

[119] P. Lodowski, M. Jaworska, T. Andruniów, M. Kumar, and P. M. Kozlowski, J. Phys. Chem. B 113, 6898 (2009).

[120] A. R. Jones, J. R. Woodward, and N. S. Scrutton, J. Am. Chem. Soc. 131, 17246 (2009).

[121] M. E. Casida, in Recent Advances in Density Functional Methods, Part I, edited by D. P. Chong, page 155, World Scientific, Singapore, 1995.

[122] M. A. L. Marques and E. K. U. Gross, Chapter 4, in A Primer in Density Functional Theory, edited by C. Fiolhais, F. Nogueira, and M. A. L. Marques, pages 144-184, Springer Verlag, Berlin, Heidelberg, 2003.

[123] A. D. Becke, Phys. Rev. A 38, 3098 (1988).

[124] J. P. Perdew, Phys. Rev. B 33, 8822 (1986).

[125] M. E. Casida, F. Gutierrez, J. Guan, F. X. Gadea, D. Salahub, and J. P. Daudey, J. Chem. Phys. 113, 7062 (2000). 
[126] A. Dreuw, J. L. Weisman, and M. Head-Gordon, J. Chem. Phys. 119, 2943 (2003).

[127] D. J. Tozer, J. Chem. Phys. 119, 12697 (2003).

[128] A. Dreuw and M. Head-Gordon, J. Am. Chem. Soc. 126, 4007 (2004).

[129] O. Gritsenko and E. J. Baerends, J. Chem. Phys. 121, 655 (2004).

[130] Y. Tawada, T. Tsuneda, and S. Yanagisawa, J. Chem. Phys. 120, 8425 (2004).

[131] J. Neugebauer, O. Gritsenko, and E. J. Baerends, J. Chem. Phys. 124, 214102 (2006).

[132] M. A. Rohrdanz, K. M. Martins, and J. M. Herbert, J. Chem. Phys. 130, 54112 (2009).

[133] T. Zielger, M. Seth, M. Krykunov, and J. Autschbach, J. Chem. Phys. 129, 184114 (2008).

[134] T. Zielger, M. Seth, M. Krykunov, J. Autschbach, and F. Wang, J. Mol. Struct. (THEOCHEM) 914, 106 (2009).

[135] T. Yanai, D. P. Tew, and N. C. Handy, Chem. Phys. Lett. 393, 51 (2004).

[136] H. Iikura, T. Tsuneda, T. Yanai, and K. Hirao, J. Chem. Phys. 115, 3540 (2001).

[137] M. J. G. Peach, P. Benfield, T. Helgaker, and D. Tozer, J. Chem. Phys. 128, 44118 (2008).

[138] M. J. G. Peach, C. R. L. Sueur, K. Ruud, M. Guillaume, and D. J. Tozer, Phys. Chem. Chem. Phys. 11, 4465 (2009).

[139] J. Plötner, D. J. Tozer, and A. Dreuw, J. Chem. Theory Comput. 6, 2315 (2010).

[140] H. Solheim, K. Kornobis, K. Ruud, and P. M. Kozlowski, J. Phys. Chem. B 115, 737 (2011).

[141] K. P. Jensen, B. O. Roos, and U. Ryde, J. Chem. Phys. 126, 14103 (2007).

[142] C. J. Cramer and D. G. Truhlar, Phys. Chem. Chem. Phys. 11, 10757 (2009).

[143] M. A. Rohrdanz and J. M. Herbert, J. Chem. Phys. 129, 34107 (2008).

[144] B. M. Wong and J. G. Cordaro, J. Chem. Phys. 129, 214703 (2008). 
[145] B. M. Wong, M. Piacenza, and F. D. Sala, Phys. Chem. Chem. Phys. 11, 4498 (2009).

[146] C. Clavaguéra, F. Piuzzi, and J. P. Dognon, J. Phys. Chem. B 113, 16443 (2009).

[147] L. Randaccio, M. Furlan, S. Geremia, M. Slouf, I. Srnova, and D. Toffoli, Inorg. Chem. 39, 3403 (2000).

[148] Gaussian 09, Revision A.1, M. J. Frisch, G. W. Trucks, H. B. Schlegel, G. E. Scuseria, M. A. Robb, J. R. Cheeseman, G. Scalmani, V. Barone, B. Mennucci, G. A. Petersson, H. Nakatsuji, M. Caricato, X. Li, H. P. Hratchian, A. F. Izmaylov, J. Bloino, G. Zheng, J. L. Sonnenberg, M. Hada, M. Ehara, K. Toyota, R. Fukuda, J. Hasegawa, M. Ishida, T. Nakajima, Y. Honda, O. Kitao, H. Nakai, T. Vreven, J. A. Montgomery, Jr., J. E. Peralta, F. Ogliaro, M. Bearpark, J. J. Heyd, E. Brothers, K. N. Kudin, V. N. Staroverov, R. Kobayashi, J. Normand, K. Raghavachari, A. Rendell, J. C. Burant, S. S. Iyengar, J. Tomasi, M. Cossi, N. Rega, N. J. Millam, M. Klene, J. E. Knox, J. B. Cross, V. Bakken, C. Adamo, J. Jaramillo, R. Gomperts, R. E. Stratmann, O. Yazyev, A. J. Austin, R. Cammi, C. Pomelli, J. W. Ochterski, R. L. Martin, K. Morokuma, V. G. Zakrzewski, G. A. Voth, P. Salvador, J. J. Dannenberg, S. Dapprich, A. D. Dan iels, O. Farkas, J. B. Foresman, J. V. Ortiz, J. Cioslowski, D. J. Fox, Gaussian, Inc., Wallingford, CT, 2009.

[149] A. Klamt and G. Schürmann, J. Chem. Soc. Perkin Trans. 2, 799 (1993).

[150] M. W. Schmidt, K. K. Baldridge, J. A. Boatz, S. T. Elbert, M. S. Gordon, J. H. Jensen, S. Koseki, N. Matsunaga, K. A. Nguyen, S. J. Su, T. L. Windus, M. Dupuis, and J. A. Montgomery, J. Comput. Chem. 14, 1347 (1993).

[151] (a) R. Ahlrichs, M. Bär, M. Häser, H. Horn, C. Kölmel, Chem. Phys. Lett. 1989, 162, 165; (b) O. Treutler, R. Ahlrichs, J. Chem. Phys. 1995, 102, 346; (c) F. Furche, R. Ahlrichs, J. Chem. Phys. 2002, 117, 7433; (d) F. Furche, D. Rappoport, in Theoretical and Computational Chemistry, Vol. 16 (M. Olivucci, Ed.), Elsevier, Amsterdam, 2005; (e) TURBOMOLE has been designed by the Quantum Chemistry Group, University of Karlsruhe, Germany, since 1988. The following members of the group have made contributions: Reinhart Ahlrichs, Michael Bär, Hans-Peter Baron, Rudiger Bauernschmitt, Stephan Bäcker, Nathan Crawford, Peter Deglmann, Michael Ehrig, Karin Eichkorn, Simon Elliott, Filipp Furche, Frank Haase, Marco Häser, Christof Hättig, Arnim Hellweg, Hans Horn, Christian Huber, Uwe Huniar, Marco Kattannek, Andreas Köhn, Christoph Kölmel, Markus Kollwitz, Klaus May, Paola Nava, Christian Ochsenfeld, Holger Ohm, Holger Patzelt, Dmitrij Rappoport, 
Oliver Rubner, Ansgar Schäfer, Uwe Schneider, Marek Sierka, Oliver Treutler, Barbara Unterreiner, Malte von Arnim, Florian Weigend, Patrick Weis, and Horst Weiss, available at: http://www.turbomole.com.

[152] DALTON, a molecular electronic structure program, Release 2.0 (2005); see http://www.daltonprogram.org.

[153] A. A. Granovsky, Firefly version 7.1.G, available at http://classic.chem.msu.su/gran/firefly/index.html.

[154] H. Nakano, J. Chem. Phys. 99, 7983 (1993).

[155] J. Kuta, S. Patchkovskii, M. Z. Zgierski, and P. M. Kozlowski, J. Comput. Chem. 27, 1429 (2006).

[156] K. Jensen and U. Ryde, Coord. Chem. Rev. 253, 769 (2009).

[157] C. L. Janssen and I. M. B. Nielsen, Chem. Phys. Lett. 290, 423 (1998).

[158] K. Pierloot, Nondynamic correlation eects in transition metal coordination compounds, in Computational Organometallic Chemistry, edited by T. R. Cundari, pages 123-158, Marcel Dekker, Inc., New York, 2001.

[159] K. P. Jensen, J. Phys. Chem. B 109, 10505 (2005).

[160] B. O. Roos, V. Veryazov, J. Conradie, P. R. Taylor, and A. Ghosh, J. Phys. Chem. B 112, 14099 (2008).

[161] K. Kornobis, N. Kumar, B. Wong, P. Lodowski, M. Jaworska, T. Andruniów, K. Ruud, and P. M. Kozlowski, J. Phys. Chem. A 115, 1280 (2011).

[162] J. Peng, K. C. Tang, K. McLoughlin, Y. Yang, D. Forgach, and R. J. Sension, J. Phys. Chem. B 114, 12398 (2010).

[163] P. M. Kozlowski, M. Kumar, P. Piecuch, W. Li, N. P. Bauman, J. A. Hansen, P. Lodowski, and M. Jaworska, J. Chem. Theory Comput. 8, 1870 (2012).

[164] J. Kuta, J. Wuerges, L. Randaccio, and P. M. Kozlowski, J. Phys. Chem. A 113, 11604 (2009).

[165] K. Andersson, P.-Å. Malmqvist, B. O. Roos, A. J. Sadlej, and K. Wolinski, J. Phys. Chem. 94, 5483 (1990).

[166] J. Geertsen, M. Rittby, and R. J. Bartlett, Chem. Phys. Lett. 164, 57 (1989).

[167] J. F. Stanton and R. J. Bartlett, J. Chem. Phys. 98, 7029 (1993). 
[168] C. Adamo and V. Barone, J. Chem. Phys. 108, 664 (1998).

[169] V. N. Staroverov, G. E. Scuseria, J. Tao, and J. P. Perdew, J. Chem. Phys. 119, 12129 (2003).

[170] J. P. Perdew, K. Burke, and Y. Wang, Phys. Rev. B 54, 16533 (1996).

[171] J. Tao, J. P. Perdew, V. N. Staroverov, and G. E. Scuseria, Phys. Rev. Lett. 91, 146401 (2003).

[172] J. P. Perdew, J. A. Chevary, S. H. Vosko, K. A. Jackson, M. R. Pederson, D. J. Singh, and C. Fiolhais, Phys. Rev. B 46, 6671 (1992).

[173] B. M. Wong and T. H. Hsieh, J. Chem. Theory Comput. 6, 3704 (2010).

[174] M. Fasching, W. Schmidt, B. Kräutler, E. Stupperich, A. Schmidt, and C. Kratky, Helv. Chim. Acta 83, 2295 (2000).

[175] H. Koch and P. Jörgensen, J. Chem. Phys. 93, 3333 (1990).

[176] H. Koch, H. J. A. Jensen, P. Jörgensen, and T. Helgaker, J. Chem. Phys. 93, 3345 (1990).

[177] J. Gauss, in Encyclopedia of Computational Chemistry, Vol. 1, edited by P. v. R. Schleyer, N. L. Allinger, T. Clark, J. Gasteiger, P. A. Kollman, I. H. F. Schaefer, and P. R. Schreiner, pages 615-636, Wiley, Chichester, UK, 1998.

[178] R. J. Bartlett and M. Musiał, Rev. Mod. Phys. 79, 291 (2007).

[179] P. Piecuch, M. Włoch, M. Lodriguito, and J. R. Gour, in Progress in Theoretical Chemistry and Physics, Vol. 15; Recent Advances in the Theory of Chemical and Physical Systems, edited by J.-P. Julien, J. Maruani, D. Mayou, S. Wilson, and G. Delgado-Barrio, pages 45-106, Springer, Dordrecht, 2006.

[180] M. Nooijen and R. J. Bartlett, J. Chem. Phys. 106, 6449 (1998).

[181] S. R. Gwaltey and R. J. Bartlett, J. Chem. Phys. 108, 6790 (1998).

[182] S. Coussan, Y. Ferro, A. Trivella, P. Roubin, R. Wieczorek, C. Manca, P. Piecuch, K. Kowalski, M. Włoch, S. A. Kucharski, and M. Musiał, J. Phys. Chem. A 110, 3920 (2006).

[183] K. Kowalski, S. Krishnamoorthy, O. Villa, J. R. Hammond, and N. Govind, J. Chem. Phys. 132, 154103 (2010). 
[184] G. Fradelos, J. J. Lutz, T. A. Wesołowski, P. Piecuch, and M. Włoch, J. Chem. Theor. Comput. 7, 1647 (2011).

[185] G. D. P. III and R. J. Bartlett, J. Chem. Phys. 76, 1910 (1982).

[186] J. M. Cullen and M. C. Zerner, J. Chem. Phys. 77, 4088 (1982).

[187] G. E. Scuseria, A. C. Scheiner, T. J. Lee, J. E. Rice, and H. F. S. III, J. Chem. Phys. 86, 2881 (1987).

[188] P. Piecuch and J. Paldus, Int. J. Quantum Chem. 36, 429 (1989).

[189] K. Kowalski and P. Piecuch, J. Chem. Phys. 120, 1715 (2004).

[190] P. Piecuch, S. A. Kucharski, K. Kowalski, and M. Musiał, Comput. Phys. Commun. 149, 71 (2002).

[191] M. S. Gordon and M. W. Schmidt, in Theory and Applications of Computational Chemistry: The First Forty Years, edited by C. E. Dykstra, G. Frenking, K. S. Kim, and G. E. Scuseria, pages 1167-1190, Elsevier, Amsterdam, 2005.

[192] S. Dong, R. Padmakumar, R. Banerjee, and T. G. Spiro, Inorg. Chem. Acta 270, 392 (1998).

[193] B. O. Roos, Chapter 69, in Ab Initio Methods in Quantum Chem.-II, edited by K. P. Lawley, page 399, Wiley, New York, 1987.

[194] B. O. Roos, K. Andersson, M. P. Fulscher, P.-Å. Malmqvist, L. S. Andrés, K. Pierloot, and M. Merchán, in Advances in Chemical Physics: New Methods in Computational Quantum Mechanics, Vol. 93, edited by I. Prigogine and S. A. Rice, pages 219-332, Wiley, New York, 1996.

[195] M. W. Schmidt and M. S. Gordon, Annu. Rev. Phys. Chem. 49, 233 (1998).

[196] K. Pierloot, Calculation of electronic spectra of transition metal complexes, in Computational Photochemistry, Vol. 16, edited by M. Olivucci, pages 279315, Elsevier, Amsterdam, 2005.

[197] N. Kumar, M. Jaworska, P. Lodowski, M. Kumar, and P. M. Kozlowski, J. Phys. Chem. B 115, 6722 (2011).

[198] N. Kumar, M. Alfonso-Prieto, C. Rovira, M. Jaworska, P. Lodowski, and P. M. Kozlowski, J. Chem. Theory Comput. 7, 1541 (2011).

[199] H. Nakatsuji, Chem. Phys. Lett. 59, 362 (1978).

[200] H. Nakatsuji, Chem. Phys. Lett. 67, 329 (1979). 
[201] H. Nakatsuji, Chem. Phys. Lett. 67, 334 (1979).

[202] J. Hasegawa, M. Hada, M. Nonoguchi, and H. Nakatsuji, Chem. Phys. Lett. 250, 159 (1996).

[203] T. Miyahara, H. Nakatsuji, J. Hasegawa, A. Osuka, N. Aratani, and A. Tsuda, J. Chem. Phys. 117, 11196 (2002).

[204] P. D. Fan, M. Valiev, and K. Kowalski, Chem. Phys. Lett. 458, 205 (2008).

[205] R. L. Martin, J. Chem. Phys. 118, 4775 (2003).

[206] P. Lodowski, M. Jaworska, K. Kornobis, T. Andruniów, and P. M. Kozlowski, J. Phys. Chem. B 115, 13304 (2011).

[207] A. B. Stickrath, E. C. Carroll, Z. Dai, D. A. Harris, A. Rury, B. Smith, K.-C. Tang, J. Wert, and R. J. Sension, J. Phys. Chem. A 113, 8513 (2009).

[208] J. Olsen and P. Jørgensen, J. Chem. Phys. 82, 3235 (1985).

[209] P. Norman, D. M. Bishop, H. J. Aa. Jensen, and J. Oddershede, J. Chem. Phys. 123, 194103 (2005).

[210] H. Solheim, K. Ruud, S. Coriani, and P. Norman, J. Chem. Phys. 128, 94103 (2008).

[211] H. Solheim, K. Ruud, S. Coriani, and P. Norman, J. Phys. Chem. A 112, 9615 (2008).

[212] M. J. G. Peach, A. J. Cohen, and D. J. Tozer, Phys. Chem. Chem. Phys. 8, 4543 (2006).

[213] D. E. Woon and J. T H. Dunning, J. Chem. Phys. 103, 4572 (1995).

[214] N. B. Balabanov and L. A. Peterson, J. Chem. Phys. 123, 64107 (2005).

[215] S. Coriani, P. Jørgensen, A. Rizzo, K. Ruud, and J. Olsen, Chem. Phys. Lett. 300, 61 (1999).

[216] H. Solheim, L. Frediani, K. Ruud, and S. Coriani, Theor. Chem. Acc. 119, 231 (2007).

[217] R. Cammi, L. Frediani, B. Mennucci, and K. Ruud, J. Chem. Phys. 119, 5818 (2003).

[218] L. Frediani, Z. Rinkevicius, and H. Ågren, J. Chem. Phys. 122, 244104 (2005). 
[219] L. Frediani, H. Ågren, L. Ferrighi, and K. Ruud, J. Chem. Phys. 123, 144117 (2005b).

[220] R. N. Boos, J. E. Carr, and J. B. Conn, Science 117, 603 (1953).

[221] J. H. Beaven and E. A. Johnson, Nature 176, 1264 (1955).

[222] E. L. Smith, L. Mervyn, A. W. Johnson, and N. Shaw, Nature 194, 1175 (1962).

[223] S. W. Ragsdale and H. G. Wood, J. Biol. Chem. 260, 3970 (1985).

[224] C. W. Goulding, D. Postigo, and R. G. Matthews, Biochemistry 36, 8082 (1997).

[225] K. Sauer and R. K. Thauer, in Chemistry and Biochemistry of $B_{12}$, edited by R. Banerjee, page 655, John Wiley \& Sons, New York, NY, USA, 1999.

[226] S. A. Burke, S. L. Lo, and J. A. Krzycki, J. Bacteriol. 180, 3432 (1998).

[227] D. J. F. Jr., N. Gorlatova, D. A. Grahame, and J. A. Krzycki, J. Biol. Chem. 275, 29053 (2000).

[228] L. Paul, D. J. Ferguson, and J. A. Krzycki, J. Bacteriol. 182, 2520 (2000).

[229] T. C. Tallant, L. Paul, and J. A. Krzyck, J. Biol. Chem. 276, 4485 (2001).

[230] J. C. Escalante-Semerena, S. J. Suh, and J. R. Roth, J. Bactriol. 172, 273 (1990).

[231] S. Suh and J. C. Escalante-Semerena, J. Bactriol. 177, 921 (1995).

[232] C. L. Johnson, M. L. Buszako, and T. A. Bobik, J. Bactriol. 186, 7781 (2004).

[233] N. R. Buan and J. C. Escalante-Semerena, J. Biol. Chem. 281, 16971 (2006).

[234] R. G. Matthews, A. E. Smith, Z. S. Zhou, R. E. Taurog, V. Bandarian, J. C. Evans, and M. Ludwig, Helv. Chim. Acta 86, 3939 (2003).

[235] A. Neumann, H. Wohlfahrt, and G. Diekert, Arch. Microbiol. 163, 276 (1995).

[236] W. Schumacher and C. Holliger, J. Bacteriol. 178, 2328 (1996).

[237] W. Schumacher, C. Holliger, A. J. Zehnder, and W. R. Hagen, FEBS. Lett. 409, 421 (1997). 
[238] D. R. Burris, C. A. Delcomyn, M. H. Smith, and A. L. Roberts, Environ. Sci. Technol. 30, 3047 (1996).

[239] D. R. Burris, C. A. Delcomyn, B. L. Deng, L. E. Buck, and K. Hatfield, Environ. Toxicol. Chem.. 17, 1681 (1998).

[240] J. Shey and W. A. van der Donk, J. Am. Chem. Soc. 122, 12403 (2000).

[241] R. P. H. Schmitz, J. Wolf, A. Habel, A. Neumann, K. Ploss, A. Svatos, W. Boland, and G. Diekert, Environ. Sci. Technol. 41, 7370 (2007).

[242] M. Wolak, A. Zahl, T. Schneppensieper, G. Stochel, and R. van Eldik, J. Am. Chem. Soc. 123, 9780 (2001).

[243] N. T. Plymale, R. S. Dassanayake, H. A. Hassanin, and N. E. Brasch, Eur. J. Inorg. Chem. 2012, 913 (2012).

[244] H. V. Motwani, S. R. Qiu, B. T. Golding, H. Kylin, and M. Tornqvist, Food Chem. Toxicol. 49, 750 (2011).

[245] W. P. Watson, T. Munter, and B. T. Golding, Chem. Res. Toxicol. 17, 1562 (2004).

[246] H. V. Motwani, C. Fred, J. Haglund, B. T. Golding, and M. Tornqvist, Chem. Res. Toxicol. 22, 1509 (2009).

[247] H. V. Motwani and M. Tornqvist, J. Chromatogr. A , 4389 (2011).

[248] D. S. Salnikov, R. Silaghi-Dumitrescu, S. V. Makarov, R. van Eldik, and G. R. Boss, Dalton Trans. 40, 9831 (2011).

[249] G. N. Schrauzer, E. Deutsch, and R. J. Windgassen, J. Am. Chem. Soc. 90, 2441 (1968).

[250] R. G. Pearson, H. Sobel, and J. Songstad, J. Am. Chem. Soc. 90, 319 (1968).

[251] C. G. Swain and C. B. Scott, J. Am. Chem. Soc. 75, 141 (1953).

[252] G. N. Schrauzer and E. Deutsch, J. Am. Chem. Soc. 91, 3341 (1969).

[253] M. D. Wirt, I. Sagi, and M. R. Chance, Biophys. J. 63, 412 (1992).

[254] C. Rovira, K. Kunc, J. Hutter, and M. Parrinello, Inorg. Chem. 40, 11 (2001).

[255] R. Dreos, S. Geremia, L. Randaccio, and P. Seiga, Chapter 15, in The Chemistry of hydroxylamines, oximes andhydroxamic acids, Vol. 2, page 903, John Wiley \& Sons Ltd., 2011. 
[256] M. Kumar, N. Kumar, H. Hirao, and P. M. Kozlowski, Inorg. Biochem. 51, 5533 (2012).

[257] M. Kumar, H. Hirao, and P. M. Kozlowski, J. Biol. Inorg. Chem. 17, 1107 (2012).

[258] H. Diehl, R. R. Sealock, and J. Morrison, Iowa State College J. Sci. 24, 433 (1950).

[259] H. Diehl, Record Chem. Progr. (Kresge-Hooker Sci. Lib.) 13, 9 (1952).

[260] G. N. Schrauzer and R. J. Holland, J. Am. Chem. Soc. 93, 4060 (1971).

[261] S. L. Tackett, J. W. Collat, and J. C. Abbott, Biochemistry 2, 919 (1963).

[262] J. M. Pratt, Chapter 13, in Inorganic Chemistry of Vitamin B12, Academic Press, New York, 1972.

[263] G. N. Schrauzer, Angew. Chem. Int. Ed. Engl. 15, 417 (1976).

[264] R. G. Eagar, B. G. Baltimore, M. M. Herbst, H. A. Barker, and J. H. Richards, Biochem. 11, 253 (1972).

[265] D. Lexa and J. M. Saveant, J. Am. Chem. Soc. 98, 2652 (1976).

[266] E. J. Kaufmann and J. H. Espenson, J. Am. Chem. Soc. 99, 7051 (1977).

[267] R. Blackburn, M. Kyaw, and A. J. Swalow, J. Chem. Soc. Faraday Trans. 73, 250 (1997).

[268] D. A. Ryan, J. H. Espenson, D. Meyerstein, and W. A. Mulac, Inorg. Chem. 17, 3725 (1978).

[269] S. K. Ghosh, P. N. Balasubramanian, G. C. Pillai, and E. S. Gould, Inorg. Chem. 30, 487 (1991).

[270] S. K. Ghosh, P. N. Balasubramanian, G. C. Pillai, and E. S. Gould, Inorg. Chem. 30, 1043 (1991).

[271] K. Kornobis, N. Kumar, P. Lodowski, M. Jaworska, P. Piecuch, J. J. Lutz, B. M. Wong and P. M. Kozlowski, J. Comput. Chem., article first published online: 19 Jan 2013, DOI: 10.1002/jcc.23204.

[272] C. L. Drennan, S. Huang, J. T. Drummond, R. G. Matthews, and M. L. Ludwig, Science 266, 1669 (1994).

[273] P. Sałek, O. Vahtras, T. Helgaker, and H. Ågren, J. Chem. Phys. 117, 9630 (2002). 
[274] K. L. Bak, Aa. E. Hansen, K. Ruud, T. Helgeker, J. Olsen, and P. Jørgensen, Theor. Chim. Acta 90, 441 (1995).

[275] M. Pecul, K. Ruud, and T. Helgaker, Chem. Phys. Lett. 388, 110 (2012).

[276] F. London, J. Phys. Radium 8, 397 (2006).

[277] T. Kjærgaard, S. Coriani, and K. Ruud, Wires Comput. Mol. Sci. 2, 443 (2012).

[278] P. Norman, D. M. Bishop, H. J. Aa. Jensen, and J. Oddershede, J. Chem. Phys. 115, 10323 (2001).

[279] D. Jonsson, P. Norman, K. Ruud, H. Ågren, and T. Helgaker, J. Chem. Phys. 109, 572 (1998).

[280] B. O. Roos, P. R. Taylor, and P. E. M. Siegbahn, Chem. Phys. 48, 157 (1980).

[281] K. Andersson, P.-Å. Malmqvist, and B. O. Roos, J. Chem. Phys. 96, 1218 (1992).

[282] N. Lin, Y. Luo, F. Santoro, X. Zhao, and A. Rizzo, Chem. Phys. Lett. 464, 144 (2008).

[283] M. Dierksen and S. Grimme, J. Chem. Phys. 124, 174301 (2006).

[284] L. Ouyang, P. Rulis, G. Ching, L. Nardin, and L. Randaccio, Inorg. Chem. 43, 1235 (2004).

[285] (a) M. W. Schmidt, K. K. Baldridge, J. A. Boatz, S. T. Elbert, M. S. Gordon, J. H. Jensen, S. Koseki, N. Matsunaga, K. A. Nguyen, S. J. Su, T. L. Windus, M. Dupuis, J. A. Montgomery, J. Comput. Chem. 1993, 14, 1347-1363. (b) M. S. Gordon, M. W. Schmidt, In Theory and Applications of Computational Chemistry, The First Forty Years; C. E. Dykstra, G. Frenking, K. S. Kim, G. E. Scuseria, Eds.; Elsevier, Amsterdam, 2005; Chapter 41, pp 1167-1189. (c) GAMESS, version 1 Oct 2010 (R1).

[286] A. Dreuw, B. D. Dunietz, and M. Head-Gordon, J. Am. Chem. Soc. 124, 12070 (2003).

[287] B. D. Dunietz, A. Dreuw, and M. Head-Gordon, J. Phys. Chem. B 107, 5623 (2003).

[288] F. D. Angelis, R. Carr, and T. G. Spiro, J. Am. Chem. Soc. 125, 15710 (2003).

[289] T. Ohta, B. Pal, and T. Kitagawa, J. Phys. Chem. B 109, 21110 (2005). 
[290] E. A. Reed, L. A. Curtiss, and F. Weinhold, Chem. Rev. 88, 899 (1988).

[291] K. Folkers, History of vitamin b12: Pernicious anemia to crystalline cyanocobalamin, in Vitamin $B_{12}$, edited by D. Dolphin, volume I, pages 1-15, John Wiley \& Sons, New York, 1982. 


\section{APPENDIX}

TABLE A.1. Scaling parameters used to simulate absorption spectra of Im$\left[\mathrm{Co}^{\mathrm{III}}\right.$ (corrin) $]-\mathrm{CN}^{+}$calculated in vacuo and water solution (PCM) with the 6$31 \mathrm{G}(\mathrm{d})$ basis set.

\begin{tabular}{llcc}
\hline \hline Phase & Functional & $\xi$ & $\mathrm{E}_{\text {shift }}(\mathrm{eV})$ \\
\hline vacuum & BP86 & 1.0785 & -0.3664 \\
& LC-BLYP & 1.0872 & -0.3908 \\
& B3LYP & 0.9909 & -0.4265 \\
\multirow{4}{*}{ water } & & & \\
& BP86 & 1.0167 & -0.2552 \\
& LC-BLYP & 1.0082 & -0.2241 \\
& B3LYP & 1.0467 & -0.5635 \\
\hline \hline
\end{tabular}

TABLE A.2. Scaling parameters used to simulate absorption spectra of Im$\left[\mathrm{Co}^{\mathrm{III}}\right.$ (corrin) $]-\mathrm{CN}^{+}$calculated in vacuo with the TZVP basis set.

\begin{tabular}{lcc}
\hline \hline Functional & $\xi$ & $\mathrm{E}_{\text {shift }}(\mathrm{eV})$ \\
\hline BP86 & 1.1194 & -0.4812 \\
LC-BLYP & 1.1174 & -0.4686 \\
B3LYP & 1.0652 & -0.6016 \\
\hline \hline
\end{tabular}


TABLE A.3. Twenty low-lying excited states of Im-[Co ${ }^{\text {III }}$ (corrin) $]-\mathrm{CN}^{+}$computed with the CASSCF method.

\begin{tabular}{|c|c|c|c|c|c|c|c|c|c|c|c|c|c|c|c|c|}
\hline 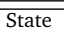 & $\overline{\mathrm{E}(\mathrm{eV})}$ & $\overline{\text { CSF coef. }}$ & $\overline{\text { Wgt.(\%) }}$ & $\overline{\mathrm{d}_{x y}}$ & $\overline{\mathrm{d}_{y z}}$ & $\overline{\mathrm{d}_{x^{2}-y^{2}}}$ & $\overline{\sigma \sigma\left(\mathrm{d}_{z^{2}}\right)}$ & $\overline{\bar{\pi}}$ & $\overline{\mathrm{d}_{x z}}$ & $\pi^{*}$ & $\overline{\mathrm{d}_{x y}+\mathrm{n}}$ & $\overline{\sigma^{*}\left(\mathrm{~d}_{z^{2}}\right)}$ & $\overline{4 \mathrm{~d}_{y}}$ & $\overline{z 4 \mathrm{~d}_{x}}$ & $\bar{z} 4 \mathrm{~d}_{x^{2}}$ & $2-y^{2}$ Character \\
\hline $\mathrm{S}_{0}$ & 0.00 & 0.9498 & 90 & 2 & 2 & 2 & 2 & 2 & 2 & 0 & 0 & 0 & 0 & 0 & 0 & \\
\hline \multirow[t]{3}{*}{$\mathrm{S}_{1}$} & 3.44 & 0.6996 & 49 & 2 & 2 & 1 & 2 & 2 & 2 & 0 & 1 & 0 & 0 & 0 & 0 & $\mathrm{~d}_{x^{2}-y^{2}} \rightarrow \mathrm{d}_{x y}+\mathrm{n}$ \\
\hline & & -0.5204 & 27 & 2 & 2 & 2 & 2 & 2 & 1 & 0 & 1 & 0 & 0 & 0 & 0 & $\mathrm{~d}_{x z} \rightarrow \mathrm{d}_{x y}+\mathrm{n}$ \\
\hline & & 0.2987 & 9 & 2 & 2 & 1 & 2 & 2 & 2 & 0 & 0 & 1 & 0 & 0 & 0 & $\mathrm{~d}_{x^{2}-y^{2}} \longrightarrow \sigma^{*}\left(\mathrm{~d}_{z^{2}}\right)$ \\
\hline \multirow[t]{5}{*}{$\mathrm{S}_{2}$} & 3.48 & 0.5113 & 26 & 2 & 2 & 1 & 2 & 2 & 2 & 0 & 1 & 0 & 0 & 0 & 0 & $\mathrm{~d}_{x^{2}-y^{2}} \rightarrow \mathrm{d}_{x y}+\mathrm{n}$ \\
\hline & & 0.5104 & 26 & 2 & 2 & 2 & 2 & 2 & 1 & 0 & 1 & 0 & 0 & 0 & 0 & $\mathrm{~d}_{x z} \rightarrow \mathrm{d}_{x y}+\mathrm{n}$ \\
\hline & & -0.3876 & 15 & 2 & 2 & 2 & 2 & 2 & 1 & 0 & 0 & 1 & 0 & 0 & 0 & $\mathrm{~d}_{x z} \rightarrow \sigma^{*}\left(\mathrm{~d}_{z^{2}}\right)$ \\
\hline & & 0.3190 & 10 & 2 & 1 & 2 & 2 & 2 & 2 & 0 & 1 & 0 & 0 & 0 & 0 & $\mathrm{~d}_{y z} \rightarrow \mathrm{d}_{x y}+\mathrm{n}$ \\
\hline & & -0.2856 & 8 & 2 & 1 & 2 & 2 & 2 & 2 & 0 & 0 & 1 & 0 & 0 & 0 & $\mathrm{~d}_{y z} \rightarrow \sigma^{*}\left(\mathrm{~d}_{2}\right)$ \\
\hline \multirow{3}{*}{$\mathrm{S}_{3}$} & & -0.4759 & 23 & 1 & 2 & 2 & 2 & 2 & 2 & 0 & 0 & 1 & 0 & 0 & 0 & $\mathrm{~d}_{x y} \rightarrow \sigma^{*}\left(\mathrm{~d}_{z^{2}}\right)$ \\
\hline & & -0.3887 & 15 & 2 & 2 & 2 & 2 & 2 & 1 & 0 & 0 & 1 & 0 & 0 & 0 & $\mathrm{~d}_{x z} \rightarrow \sigma^{*}\left(\mathrm{~d}_{z^{2}}^{z^{2}}\right)$ \\
\hline & & -0.3105 & 10 & 2 & 2 & 2 & 2 & 1 & 2 & 0 & 0 & 1 & 0 & 0 & 0 & $\pi \rightarrow \sigma^{*}\left(\mathrm{~d}_{\gamma_{2}}\right)^{2}$ \\
\hline \multirow[t]{4}{*}{$\mathrm{S}_{4}$} & 4.75 & 0.4808 & 23 & 1 & 2 & 2 & 2 & 2 & 2 & 0 & 1 & 0 & 0 & 0 & 0 & $\mathrm{~d}_{x y} \rightarrow \mathrm{d}_{x y}+\mathrm{n}$ \\
\hline & & -0.3909 & 15 & 2 & 1 & 2 & 2 & 2 & 2 & 0 & 1 & 0 & 0 & 0 & 0 & $\mathrm{~d}_{y z} \rightarrow \mathrm{d}_{x y}+\mathrm{n}$ \\
\hline & & 0.3896 & 15 & 2 & 2 & 2 & 2 & 1 & 2 & 0 & 1 & 0 & 0 & 0 & 0 & $\pi \rightarrow \mathrm{d}_{x y}+\mathrm{n}$ \\
\hline & & -0.2865 & 8 & 2 & 2 & 2 & 2 & 2 & 1 & 0 & 0 & 1 & 0 & 0 & 0 & $\mathrm{~d}_{x z} \rightarrow \sigma^{*}\left(\mathrm{~d}_{z^{2}}\right)$ \\
\hline \multirow[t]{2}{*}{$\mathrm{S}_{5}$} & 4.88 & 0.5509 & 30 & 2 & 2 & 2 & 2 & 2 & 1 & 0 & 0 & 1 & 0 & 0 & 0 & $\mathrm{~d}_{x z} \rightarrow \sigma^{*}\left(\mathrm{~d}_{z^{2}}^{z^{2}}\right)$ \\
\hline & & 0.4634 & 21 & 2 & 2 & 2 & 2 & 2 & 1 & 0 & 1 & 0 & 0 & 0 & 0 & $\mathrm{~d}_{x z} \rightarrow \mathrm{d}_{x y}+\mathrm{n}$ \\
\hline $\mathrm{S}_{6}$ & 5.17 & 0.3190 & 10 & 2 & 1 & 2 & 2 & 2 & 2 & 0 & 1 & 0 & 0 & 0 & 0 & $\mathrm{~d}_{y z} \rightarrow \mathrm{d}_{x y}+\mathrm{n}$ \\
\hline \multirow[t]{3}{*}{$\mathrm{s}_{7}$} & 5.35 & 0.7186 & 52 & 2 & 2 & 1 & 2 & 2 & 2 & 0 & 0 & 1 & 0 & 0 & 0 & $\mathrm{~d}_{x^{2}-y^{2}} \rightarrow \sigma^{*}\left(\mathrm{~d}_{z^{2}}\right)$ \\
\hline & & 0.2716 & 7 & 2 & 2 & 2 & 2 & 2 & 1 & 0 & 0 & 1 & 0 & 0 & 0 & $\mathrm{~d}_{x z} \mathrm{x}^{2}-\sigma^{*}\left(\mathrm{~d}_{2}\right)$ \\
\hline & & -0.1547 & 2 & 2 & 2 & 2 & 2 & 2 & 1 & 1 & 0 & 0 & 0 & 0 & 0 & $\mathrm{~d}_{x z} \rightarrow \pi^{*} z^{2}$ \\
\hline \multirow[t]{3}{*}{$\mathrm{S}_{8}$} & 5.45 & 0.2587 & 7 & 2 & 1 & 2 & 2 & 2 & 2 & 0 & 1 & 0 & 0 & 0 & 0 & $\mathrm{~d}_{y z} \rightarrow \mathrm{d}_{x y}+\mathrm{n}$ \\
\hline & & 0.2575 & 7 & 2 & 2 & 1 & 2 & 1 & 2 & 1 & 1 & 0 & 0 & 0 & 0 & $\mathrm{~d}_{x^{2}-y^{2}} / \pi \rightarrow \pi^{*} / \mathrm{d}_{x y}+\mathrm{n}$ \\
\hline & & 0.2292 & 5 & 2 & 2 & 2 & 2 & 1 & 1 & 1 & 1 & 0 & 0 & 0 & 0 & $\mathrm{~d}_{x z} / \pi \rightarrow \pi^{*} / \mathrm{d}_{x y}+\mathrm{n}$ \\
\hline \multirow[t]{4}{*}{$\mathrm{S}_{9}$} & 5.49 & 0.3073 & 9 & 2 & 2 & 2 & 2 & 1 & 1 & 1 & 1 & 0 & 0 & 0 & 0 & $\mathrm{~d}_{x z} / \pi \rightarrow \pi^{*} / \mathrm{d}_{x y}+\mathrm{n}$ \\
\hline & & -0.2927 & 9 & 2 & 2 & 1 & 2 & 1 & 2 & 1 & 1 & 0 & 0 & 0 & 0 & $\mathrm{~d}_{x^{2}-y^{2}} / \pi \rightarrow \mathrm{d}_{x y}+\mathrm{n} / \pi^{*}$ \\
\hline & & -0.2037 & 4 & 2 & 2 & 1 & 2 & 1 & 2 & 1 & 1 & 0 & 0 & 0 & 0 & $\mathrm{~d}_{y z} / \pi \rightarrow \mathrm{d}_{x y}+\mathrm{n} / \pi^{*}$ \\
\hline & & -0.2083 & 4 & 2 & 2 & 2 & 1 & 2 & 1 & 1 & 1 & 0 & 0 & 0 & 0 & $\sigma\left(\mathrm{d}_{z 2}\right) / \mathrm{d}_{x z} \rightarrow \pi^{*} / \mathrm{d}_{x y}+\mathrm{n}$ \\
\hline \multirow{3}{*}{$\mathrm{S}_{10}$} & 5.54 & 0.4281 & 18 & 2 & 2 & 2 & 2 & 1 & 2 & 1 & 0 & 0 & 0 & 0 & 0 & $\pi \rightarrow \pi^{*}$ \\
\hline & & -0.3241 & 11 & 2 & 2 & 2 & 1 & 2 & 2 & 1 & 0 & 0 & 0 & 0 & 0 & $\sigma\left(\mathrm{d}_{2}\right) \rightarrow \pi^{*}$ \\
\hline & & 0.3267 & 11 & 1 & 2 & 2 & 2 & 2 & 2 & 1 & 0 & 0 & 0 & 0 & 0 & $\mathrm{~d}_{x y} \stackrel{z}{\rightarrow} \pi^{*}$ \\
\hline $\mathrm{S}_{11}$ & 5.77 & 0.2749 & 8 & 2 & 2 & 2 & 2 & 1 & 2 & 1 & 0 & 0 & 0 & 0 & 0 & $\pi \rightarrow \pi^{*}$ \\
\hline & & -0.2270 & 5 & 2 & 2 & 2 & 1 & 2 & 2 & 1 & 0 & 0 & 0 & 0 & 0 & $\sigma\left(\mathrm{d}_{\sim 2}\right) \rightarrow \pi^{*}$ \\
\hline & & 0.2455 & 6 & 2 & 0 & 2 & 2 & 2 & 2 & 1 & 1 & 0 & 0 & 0 & 0 & $\mathrm{~d}_{y z} \rightarrow \pi^{*} / \mathrm{d}_{x y}+\mathrm{n}$ \\
\hline $\mathrm{S}_{12}$ & 6.09 & 0.2766 & 8 & 2 & 1 & 2 & 2 & 2 & 2 & 1 & 0 & 0 & 0 & 0 & 0 & $\mathrm{~d}_{y z} \rightarrow \pi^{*}$ \\
\hline & & 0.2628 & 7 & 2 & 2 & 2 & 2 & 2 & 1 & 1 & 0 & 0 & 0 & 0 & 0 & $\mathrm{~d}_{x z} \rightarrow \pi^{*}$ \\
\hline & & -0.2292 & 5 & 2 & 2 & 2 & 2 & 1 & 1 & 1 & 0 & 1 & 0 & 0 & 0 & $\mathrm{~d}_{x z} / \pi \rightarrow \sigma^{*}\left(\mathrm{~d}_{z^{2}}\right) / \pi^{*}$ \\
\hline $\mathrm{S}_{13}$ & 6.29 & 0.5581 & 31 & 2 & 2 & 1 & 2 & 2 & 2 & 1 & 0 & 0 & 0 & 0 & 0 & $\mathrm{~d}_{x^{2}-y^{2}} \rightarrow \pi^{*} z^{2}$ \\
\hline & & -0.4051 & 16 & 2 & 1 & 2 & 2 & 2 & 2 & 1 & 0 & 0 & 0 & 0 & 0 & $\mathrm{~d}_{y z} \rightarrow \pi^{*}$ \\
\hline & & 0.2548 & 6 & 2 & 2 & 1 & 2 & 2 & 2 & 1 & 0 & 0 & 0 & 0 & 0 & $\mathrm{~d}_{x^{2}-y^{2}} \rightarrow \pi^{*}$ \\
\hline & & 0.2360 & 6 & 1 & 2 & 2 & 2 & 2 & 2 & 1 & 0 & 0 & 0 & 0 & 0 & $\mathrm{~d}_{x y} \stackrel{-y^{2}}{\rightarrow} \pi^{*}$ \\
\hline $\mathrm{S}_{15}$ & 6.86 & 0.4894 & 24 & 2 & 1 & 2 & 2 & 2 & 2 & 1 & 0 & 0 & 0 & 0 & 0 & $\mathrm{~d}_{y z} \rightarrow \pi^{*}$ \\
\hline & & 0.3190 & 10 & 2 & 2 & 2 & 2 & 2 & 1 & 1 & 0 & 0 & 0 & 0 & 0 & $\mathrm{~d}_{x z} \rightarrow \pi^{*}$ \\
\hline & & 0.1912 & 4 & 2 & 2 & 2 & 2 & 1 & 1 & 1 & 0 & 1 & 0 & 0 & 0 & $\mathrm{~d}_{x z} / \pi \rightarrow \sigma^{*}\left(\mathrm{~d}_{2} 2\right) / \pi^{*}$ \\
\hline $\mathrm{S}_{16}$ & 7.08 & 0.4925 & 24 & 2 & 2 & 0 & 2 & 2 & 2 & 0 & 2 & 0 & 0 & 0 & 0 & $\mathrm{~d}_{x^{2}-y^{2}} \rightarrow \mathrm{d}_{x y}+\mathrm{n}$ \\
\hline & & -0.4597 & 21 & 2 & 2 & 1 & 2 & 2 & 1 & 0 & 2 & 0 & 0 & 0 & 0 & $\mathrm{~d}_{x^{2}-y^{2}} / \mathrm{d}_{x z} \rightarrow \mathrm{d}_{x y}+\mathrm{n}$ \\
\hline & & 0.2711 & 7 & 2 & 1 & 2 & 2 & 2 & 1 & 0 & 1 & 1 & 0 & 0 & 0 & $\mathrm{~d}_{y z} / \mathrm{d}_{x z} \rightarrow \mathrm{d}_{x y}+\sigma^{*} / \sigma^{*}\left(\mathrm{~d}_{z 2}\right)$ \\
\hline $\mathrm{S}_{17}$ & 7.13 & 0.4477 & 20 & 2 & 2 & 2 & 1 & 2 & 2 & 0 & 1 & 0 & 0 & 0 & 0 & $\sigma\left(\mathrm{d}_{z 2}\right) \rightarrow \mathrm{d}_{x y}+\mathrm{n}$ \\
\hline & & -0.2998 & 9 & 2 & 1 & 2 & 2 & 2 & 2 & 0 & 1 & 0 & 0 & 0 & 0 & $\mathrm{~d}_{y z} \rightarrow \mathrm{d}_{x y}+\mathrm{n}$ \\
\hline $\mathrm{S}_{18}$ & 7.16 & 0.3949 & 16 & 2 & 2 & 1 & 2 & 2 & 2 & 1 & 0 & 0 & 0 & 0 & 0 & $\mathrm{~d}_{x^{2}-y^{2}} \rightarrow \pi^{*}$ \\
\hline & & 0.3901 & 15 & 2 & 2 & 1 & 2 & 1 & 2 & 1 & 0 & 1 & 0 & 0 & 0 & $\mathrm{~d}_{x^{2}-y^{2}} / \pi \rightarrow \pi^{*} / \mathrm{d}_{x y}+\mathrm{n}$ \\
\hline & & 0.2633 & 7 & 1 & 2 & 1 & 2 & 2 & 2 & 1 & 0 & 1 & 0 & 0 & 0 & $\mathrm{~d}_{x y} / \mathrm{d}_{x^{2}-y^{2}} \rightarrow \pi^{*} / \sigma^{*}\left(\mathrm{~d}_{z^{2}}\right)$ \\
\hline $\mathrm{S}_{19}$ & 7.23 & 0.4651 & 22 & 2 & 2 & 1 & 2 & 2 & 1 & 0 & 2 & 0 & 0 & 0 & 0 & $\mathrm{~d}_{x^{2}-y^{2}} / \mathrm{d}_{x z} \rightarrow \mathrm{d}_{x y}+\mathrm{n}$ \\
\hline & & -0.4361 & 19 & 2 & 2 & 1 & 2 & 2 & 1 & 0 & 1 & 1 & 0 & 0 & 0 & $\mathrm{~d}_{x^{2}-y^{2}} / \mathrm{d}_{x z} \rightarrow \mathrm{d}_{x y}+\sigma^{*} / \sigma^{*}\left(\mathrm{~d}_{z^{2}}\right)$ \\
\hline & & -0.3067 & 9 & 2 & 2 & 2 & 2 & 2 & 0 & 0 & 2 & 0 & 0 & 0 & 0 & $\mathrm{~d}_{x z} \rightarrow \mathrm{d}_{x y}+\mathrm{n}$ \\
\hline
\end{tabular}


TABLE A.4. Twenty low-lying excited states of Im-[Co ${ }^{\mathrm{III}}$ (corrin) $]-\mathrm{CH}_{3}^{+}$computed with the CASSCF method.

\begin{tabular}{|c|c|c|c|c|c|c|c|c|c|c|c|c|c|c|c|c|}
\hline & $\mathrm{E}(\mathrm{eV})$ & & & $\mathrm{d}_{x z}$ & $\sigma\left(\mathrm{d}_{z^{2}}\right)$ & $\pi$ & $\mathrm{d}_{y z}$ & $\mathrm{~d}_{x y}$ & $\pi$ & $\mathrm{d}_{x y}+\sigma^{*}$ & $\pi^{*}$ & & $\overline{4 d}$ & $z \sigma^{*}$ & $\pi^{*}$ & Character \\
\hline $\mathrm{S}_{0}$ & 0.00 & 0.9029 & 0.815 & 2 & 2 & 2 & 2 & 2 & 2 & 0 & 0 & 0 & 0 & 0 & 0 & \\
\hline \multirow[t]{4}{*}{$\mathrm{S}_{1}$} & 3.21 & -0.3441 & 0.118 & 2 & 2 & 2 & 1 & 2 & 2 & 0 & 0 & 0 & 0 & 1 & 0 & $\mathrm{~d}_{y z} \rightarrow \sigma^{*}\left(\mathrm{~d}_{\gamma_{2}}\right)$ \\
\hline & & 0.3574 & 0.128 & 1 & 2 & 2 & 2 & 2 & 2 & 0 & 0 & 0 & 0 & 1 & 0 & $\mathrm{~d}_{x z} \rightarrow \sigma^{*}\left(\mathrm{~d}_{z^{2}}\right)$ \\
\hline & & 0.5976 & 0.357 & 2 & 2 & 2 & 1 & 2 & 2 & 1 & 0 & 0 & 0 & 0 & 0 & $\mathrm{~d}_{y z} \rightarrow \mathrm{d}_{x y}+\sigma^{*}$ \\
\hline & & -0.3749 & 0.141 & 1 & 2 & 2 & 2 & 2 & 2 & 1 & 0 & 0 & 0 & 0 & 0 & $\mathrm{~d}_{x z} \rightarrow \mathrm{d}_{x y}+\sigma^{*}$ \\
\hline \multirow[t]{4}{*}{$\mathrm{S}_{2}$} & 3.34 & 0.4044 & 0.164 & 2 & 2 & 2 & 1 & 2 & 2 & 0 & 0 & 0 & 0 & 1 & 0 & $\mathrm{~d}_{y z} \rightarrow \sigma^{*}\left(\mathrm{~d}_{z^{2}}\right)$ \\
\hline & & 0.3277 & 0.107 & 1 & 2 & 2 & 2 & 2 & 2 & 0 & 0 & 0 & 0 & 1 & 0 & $\mathrm{~d}_{x z} \rightarrow \sigma^{*}\left(\mathrm{~d}_{z^{2}}\right)$ \\
\hline & & 0.3897 & 0.152 & 2 & 2 & 2 & 1 & 2 & 2 & 1 & 0 & 0 & 0 & 0 & 0 & $\mathrm{~d}_{y z} \rightarrow \mathrm{d}_{x y}+\sigma^{*}$ \\
\hline & & 0.5619 & 0.316 & 1 & 2 & 2 & 2 & 2 & 2 & 1 & 0 & 0 & 0 & 0 & 0 & $\mathrm{~d}_{x z} \rightarrow \mathrm{d}_{x y}+\sigma^{*}$ \\
\hline \multirow[t]{4}{*}{$\mathrm{S}_{3}$} & 4.47 & 0.4678 & 0.219 & 2 & 2 & 2 & 1 & 2 & 2 & 0 & 0 & 0 & 0 & 1 & 0 & $\mathrm{~d}_{y z} \rightarrow \sigma^{*}\left(\mathrm{~d}_{z^{2}}\right)$ \\
\hline & & 0.4289 & 0.184 & 1 & 2 & 2 & 2 & 2 & 2 & 0 & 0 & 0 & 0 & 1 & 0 & $\mathrm{~d}_{x z} \rightarrow \sigma^{*}\left(\mathrm{~d}_{z^{2}}\right)$ \\
\hline & & -0.2453 & 0.060 & 2 & 2 & 2 & 1 & 2 & 2 & 1 & 0 & 0 & 0 & 0 & 0 & $\mathrm{~d}_{y z} \rightarrow \mathrm{d}_{x y}+\sigma^{*}$ \\
\hline & & -0.4265 & 0.182 & 1 & 2 & 2 & 2 & 2 & 2 & 1 & 0 & 0 & 0 & 0 & 0 & $\mathrm{~d}_{x z} \rightarrow \mathrm{d}_{x y}+\sigma^{*}$ \\
\hline \multirow[t]{2}{*}{$\mathrm{S}_{4}$} & 4.60 & 0.7762 & 0.603 & 2 & 1 & 2 & 2 & 2 & 2 & 1 & 0 & 0 & 0 & 0 & 0 & $\sigma\left(\mathrm{d}_{z 2}\right) \rightarrow \mathrm{d}_{x y}+\sigma^{*}$ \\
\hline & & -0.3296 & 0.109 & 2 & 0 & 2 & 2 & 2 & 2 & 1 & 0 & 0 & 0 & 1 & 0 & $\sigma\left(\mathrm{d}_{z^{2}}\right) \rightarrow \mathrm{d}_{x y}+\sigma^{*} / \sigma^{*}\left(\mathrm{~d}_{z^{2}}\right)$ \\
\hline \multirow[t]{5}{*}{$\mathrm{S}_{5}$} & 4.66 & -0.4184 & 0.175 & 2 & 2 & 2 & 1 & 2 & 2 & 0 & 0 & 0 & 0 & 1 & 0 & $\mathrm{~d}_{y z} \rightarrow \sigma^{*}\left(\mathrm{~d}_{z^{2}}\right)$ \\
\hline & & 0.4550 & 0.207 & 1 & 2 & 2 & 2 & 2 & 2 & 0 & 0 & 0 & 0 & 1 & 0 & $\mathrm{~d}_{x z} \rightarrow \sigma^{*}\left(\mathrm{~d}_{z^{2}}^{z}\right)$ \\
\hline & & -0.3448 & 0.119 & 2 & 2 & 2 & 1 & 2 & 2 & 1 & 0 & 0 & 0 & 0 & 0 & $\mathrm{~d}_{y z} \rightarrow \mathrm{d}_{x y}+\sigma^{*}$ \\
\hline & & -0.2980 & 0.089 & 2 & 1 & 2 & 2 & 2 & 2 & 1 & 0 & 0 & 0 & 0 & 0 & $\sigma\left(\mathrm{d}_{z^{2}}\right) \rightarrow \mathrm{d}_{x y}+\sigma^{*}$ \\
\hline & & 0.2975 & 0.089 & 1 & 2 & 2 & 2 & 2 & 2 & 1 & 0 & 0 & 0 & 0 & 0 & $\mathrm{~d}_{x z} \rightarrow \mathrm{d}_{x y}+\sigma^{*}$ \\
\hline \multirow{2}{*}{$\mathrm{S}_{6}$} & 5.76 & -0.2963 & 0.088 & 1 & 2 & 2 & 2 & 2 & 1 & 1 & 0 & 0 & 0 & 0 & 1 & $\mathrm{~d}_{x z} / \pi \rightarrow \mathrm{d}_{x y}+\sigma^{*} / \pi^{*}$ \\
\hline & & 0.3740 & 0.140 & 1 & 2 & 1 & 2 & 2 & 2 & 1 & 0 & 0 & 0 & 0 & 1 & $\mathrm{~d}_{x z} / \pi \rightarrow \mathrm{d}_{x y}+\sigma^{*} / \pi^{*}$ \\
\hline \multirow[t]{4}{*}{$\mathrm{S}_{7}$} & 5.85 & -0.3101 & 0.096 & 2 & 2 & 2 & 1 & 2 & 1 & 1 & 1 & 0 & 0 & 0 & 0 & $\mathrm{~d}_{y z} / \pi \rightarrow \mathrm{d}_{x y}+\sigma^{*} / \pi^{*}$ \\
\hline & & 0.2943 & 0.087 & 1 & 2 & 2 & 2 & 2 & 1 & 1 & 1 & 0 & 0 & 0 & 0 & $\mathrm{~d}_{x z} / \pi \rightarrow \mathrm{d}_{x y}+\sigma^{*} / \pi^{*}$ \\
\hline & & -0.2921 & 0.085 & 2 & 2 & 1 & 1 & 2 & 2 & 1 & 1 & 0 & 0 & 0 & 0 & $\mathrm{~d}_{y z} / \pi \rightarrow \mathrm{d}_{x y}+\sigma^{*} / \pi^{*}$ \\
\hline & & 0.3414 & 0.117 & 1 & 2 & 1 & 2 & 2 & 2 & 1 & 1 & 0 & 0 & 0 & 0 & $\mathrm{~d}_{x z} / \pi \rightarrow \mathrm{d}_{x y}+\sigma^{*} / \pi^{*}$ \\
\hline \multirow[t]{3}{*}{$\mathrm{S}_{8}$} & 6.04 & -0.3887 & 0.151 & 2 & 2 & 2 & 1 & 2 & 1 & 1 & 0 & 0 & 0 & 0 & 1 & $\mathrm{~d}_{y z} / \pi \rightarrow \mathrm{d}_{x y}+\sigma^{*} / \pi^{*}$ \\
\hline & & 0.4348 & 0.189 & 2 & 2 & 1 & 1 & 2 & 2 & 1 & 0 & 0 & 0 & 0 & 1 & $\mathrm{~d}_{y z} / \pi \rightarrow \mathrm{d}_{x y}+\sigma^{*} / \pi^{*}$ \\
\hline & & -0.3004 & 0.090 & 1 & 2 & 1 & 2 & 2 & 2 & 1 & 0 & 0 & 0 & 0 & 1 & $\mathrm{~d}_{x z} / \pi \rightarrow \mathrm{d}_{x y}+\sigma^{*} / \pi^{*}$ \\
\hline \multirow[t]{4}{*}{$\mathrm{S}_{9}$} & 6.07 & 0.3431 & 0.118 & 2 & 2 & 2 & 1 & 2 & 1 & 1 & 1 & 0 & 0 & 0 & 0 & $\mathrm{~d}_{y z} / \pi \rightarrow \mathrm{d}_{x y}+\sigma^{*} / \pi^{*}$ \\
\hline & & 0.2894 & 0.084 & 1 & 2 & 2 & 2 & 2 & 1 & 1 & 1 & 0 & 0 & 0 & 0 & $\mathrm{~d}_{x z} / \pi \rightarrow \mathrm{d}_{x y}+\sigma^{*} / \pi^{*}$ \\
\hline & & 0.3516 & 0.124 & 2 & 2 & 1 & 1 & 2 & 2 & 1 & 1 & 0 & 0 & 0 & 0 & $\mathrm{~d}_{y z} / \pi \rightarrow \mathrm{d}_{x y}+\sigma^{*} / \pi^{*}$ \\
\hline & & 0.3151 & 0.099 & 1 & 2 & 1 & 2 & 2 & 2 & 1 & 1 & 0 & 0 & 0 & 0 & $\mathrm{~d}_{x z} / \pi \rightarrow \mathrm{d}_{x y}+\sigma^{*} / \pi^{*}$ \\
\hline \multirow[t]{3}{*}{$\mathrm{S}_{10}$} & 6.16 & 0.5967 & 0.356 & 2 & 2 & 2 & 1 & 2 & 2 & 0 & 1 & 0 & 0 & 0 & 0 & $\mathrm{~d}_{y z} \rightarrow \pi^{*}$ \\
\hline & & -0.2034 & 0.041 & 2 & 2 & 1 & 2 & 2 & 2 & 0 & 1 & 0 & 0 & 0 & 0 & $\pi \rightarrow \pi \rightarrow *$ \\
\hline & & -0.3303 & 0.109 & 1 & 2 & 2 & 2 & 2 & 2 & 0 & 1 & 0 & 0 & 0 & 0 & $\mathrm{~d}_{x z} \rightarrow \pi^{*}$ \\
\hline$S_{11}$ & 6.31 & 0.4653 & 0.216 & 1 & 2 & 2 & 2 & 2 & 2 & 0 & 1 & 0 & 0 & 0 & 0 & $\mathrm{~d}_{x z} \rightarrow \pi^{*}$ \\
\hline \multirow[t]{3}{*}{$\mathrm{S}_{12}$} & 6.41 & 0.5138 & 0.264 & 2 & 2 & 2 & 1 & 2 & 2 & 0 & 0 & 0 & 0 & 0 & 1 & $\mathrm{~d}_{y z} \rightarrow \pi^{*}$ \\
\hline & & -0.2027 & 0.041 & 2 & 2 & 1 & 2 & 2 & 2 & 0 & 0 & 0 & 0 & 0 & 1 & $\pi \rightarrow \pi^{*}$ \\
\hline & & 0.3033 & 0.092 & 1 & 2 & 2 & 2 & 2 & 2 & 0 & 1 & 0 & 0 & 0 & 0 & $\mathrm{~d}_{x z} \rightarrow \pi^{*}$ \\
\hline \multirow[t]{2}{*}{$\mathrm{S}_{13}$} & 6.50 & -0.3384 & 0.115 & 2 & 2 & 2 & 1 & 2 & 2 & 0 & 0 & 0 & 0 & 0 & 1 & $\mathrm{~d}_{y z} \rightarrow \pi^{*}$ \\
\hline & & 0.5492 & 0.302 & 1 & 2 & 2 & 2 & 2 & 2 & 0 & 0 & 0 & 0 & 0 & 1 & $\mathrm{~d}_{x z} \rightarrow \pi^{*}$ \\
\hline $\mathrm{S}_{14}$ & 6.58 & 0.7066 & 0.499 & 1 & 2 & 2 & 1 & 2 & 2 & 1 & 0 & 0 & 0 & 1 & 0 & $\mathrm{~d}_{x z} / \mathrm{d}_{y z} \rightarrow \mathrm{d}_{x y}+\sigma^{*} / \sigma^{*}\left(\mathrm{~d}_{z^{2}}\right)$ \\
\hline $\mathrm{S}_{15}$ & 6.79 & 0.7399 & 0.547 & 1 & 2 & 2 & 1 & 2 & 2 & 1 & 0 & 0 & 0 & 1 & 0 & $\mathrm{~d}_{x z} / \mathrm{d}_{y z} \rightarrow \mathrm{d}_{x y}+\sigma^{*} / \sigma^{*}\left(\mathrm{~d}_{z^{2}}\right)$ \\
\hline \multirow[t]{2}{*}{$\mathrm{S}_{16}$} & 7.02 & 0.4152 & 0.172 & 2 & 1 & 2 & 1 & 2 & 2 & 1 & 0 & 0 & 0 & 1 & 0 & $\sigma\left(\mathrm{d}_{z^{2}}\right) / \mathrm{d}_{y z} \rightarrow \mathrm{d}_{x y} / \sigma^{*}\left(\mathrm{~d}_{z^{2}}\right)$ \\
\hline & & 0.7117 & 0.507 & 2 & 1 & 2 & 1 & 2 & 2 & 1 & 0 & 0 & 0 & 1 & 0 & $\sigma\left(\mathrm{d}_{z^{2}}\right) / \mathrm{d}_{y z} \rightarrow \mathrm{d}_{x y}+\sigma^{*} / \sigma^{*}\left(\mathrm{~d}_{z^{2}}\right)$ \\
\hline \multirow[t]{3}{*}{$\mathrm{S}_{17}$} & 7.04 & 0.5709 & 0.326 & 2 & 2 & 2 & 0 & 2 & 2 & 1 & 0 & 0 & 0 & 1 & 0 & $\mathrm{~d}_{y z} \rightarrow \mathrm{d}_{x y}+\sigma^{*} / \sigma^{*}\left(\mathrm{~d}_{z^{2}}\right)$ \\
\hline & & -0.3976 & 0.158 & 0 & 2 & 2 & 2 & 2 & 2 & 1 & 0 & 0 & 0 & 1 & 0 & $\mathrm{~d}_{x z} \rightarrow \mathrm{d}_{x y}+\sigma^{*} / \sigma^{*}\left(\mathrm{~d}_{z 2}\right)$ \\
\hline & & -0.2952 & 0.087 & 2 & 1 & 2 & 0 & 2 & 2 & 1 & 0 & 0 & 0 & 2 & 0 & $\sigma\left(\mathrm{d}_{z^{2}}\right) / \mathrm{d}_{y z} \rightarrow \mathrm{d}_{x y}+\sigma^{* *} / \sigma^{*}\left(\mathrm{~d}_{z^{2}}\right)$ \\
\hline $\mathrm{S}_{18}$ & 7.11 & -0.4118 & 0.170 & 1 & 1 & 2 & 2 & 2 & 2 & 1 & 0 & 0 & 0 & 1 & 0 & $\sigma\left(\mathrm{d}_{z^{2}}\right) / \mathrm{d}_{x z} \rightarrow \mathrm{d}_{x y}+\sigma^{*} / \sigma^{*}\left(\mathrm{~d}_{z^{2}}\right)$ \\
\hline & & 0.6949 & 0.483 & 1 & 1 & 2 & 2 & 2 & 2 & 1 & 0 & 0 & 0 & 1 & 0 & $\sigma\left(\mathrm{d}_{z^{2}}^{z^{2}}\right) / \mathrm{d}_{x z} \rightarrow \mathrm{d}_{x y}+\sigma^{*} / \sigma^{*}\left(\mathrm{~d}_{z^{2}}^{z^{2}}\right)$ \\
\hline $\mathrm{S}_{19}$ & 7.27 & 0.4602 & 0.212 & 0 & 2 & 2 & 2 & 2 & 2 & 1 & 0 & 0 & 0 & 1 & 0 & $\mathrm{~d}_{x z} \rightarrow \mathrm{d}_{x y}+\sigma^{*} / \sigma^{*}\left(\mathrm{~d}_{z^{2}}\right)$ \\
\hline & & 0.6329 & 0.401 & 1 & 2 & 2 & 1 & 2 & 2 & 2 & 0 & 0 & 0 & 0 & 0 & $\mathrm{~d}_{x z} / \mathrm{d}_{y z} \rightarrow \mathrm{d}_{x y}+\sigma^{*}$ \\
\hline
\end{tabular}


TABLE A.5. Orbital energies and composition of the Im- $\left[\mathrm{Co}{ }^{\text {III }}\right.$ (corrin) $]-\mathrm{CH}_{3}^{+}$molecular orbitals calculated with BLYP/6-31G(d).

\begin{tabular}{llcrrrrrr}
\hline \hline \multirow{2}{*}{ Orbital } & Character & $\mathrm{E}(\mathrm{eV})$ & \multicolumn{5}{c}{$\%$} \\
\cline { 4 - 8 } & & & & $\mathrm{Co}$ & $\mathrm{C}_{\mathrm{CH}_{3}}$ & $\mathrm{CH}_{3}$ & $\mathrm{Im}$ & Corrin \\
\hline 112 & HOMO-5 & $\pi_{\mathrm{Im}}$ & -8.29 & 5 & 0 & 1 & 80 & 15 \\
113 & HOMO-4 & $\pi+\mathrm{d}_{y z}+\pi_{\mathrm{Im}}$ & -8.24 & 22 & 1 & 3 & 18 & 58 \\
114 & HOMO-3 & $\mathrm{d}_{x^{2}-y^{2}}$ & -7.60 & 64 & 0 & 0 & 1 & 36 \\
115 & HOMO-2 & $\mathrm{d}_{x z}+\pi$ & -7.26 & 56 & 2 & 4 & 4 & 36 \\
116 & HOMO-1 & $\pi+\mathrm{d}_{x z}$ & -7.14 & 22 & 4 & 5 & 9 & 63 \\
117 & HOMO & $\mathrm{d}_{y z}+\pi$ & -7.01 & 52 & 1 & 3 & 8 & 37 \\
118 & LUMO & $\pi^{*}$ & -5.07 & 6 & 0 & 0 & 1 & 93 \\
119 & LUMO+1 & $\mathrm{d}_{x y}+\mathrm{n}$ & -4.32 & 32 & 1 & 1 & 2 & 66 \\
120 & LUMO+2 & $\pi^{*}+\mathrm{d}_{x y}+\mathrm{n}$ & -4.17 & 24 & 0 & 0 & 1 & 75 \\
121 & LUMO+3 & $\sigma^{*}\left(\mathrm{~d}_{z^{2}}\right)$ & -3.91 & 36 & 23 & 23 & 12 & 29 \\
\hline \hline
\end{tabular}



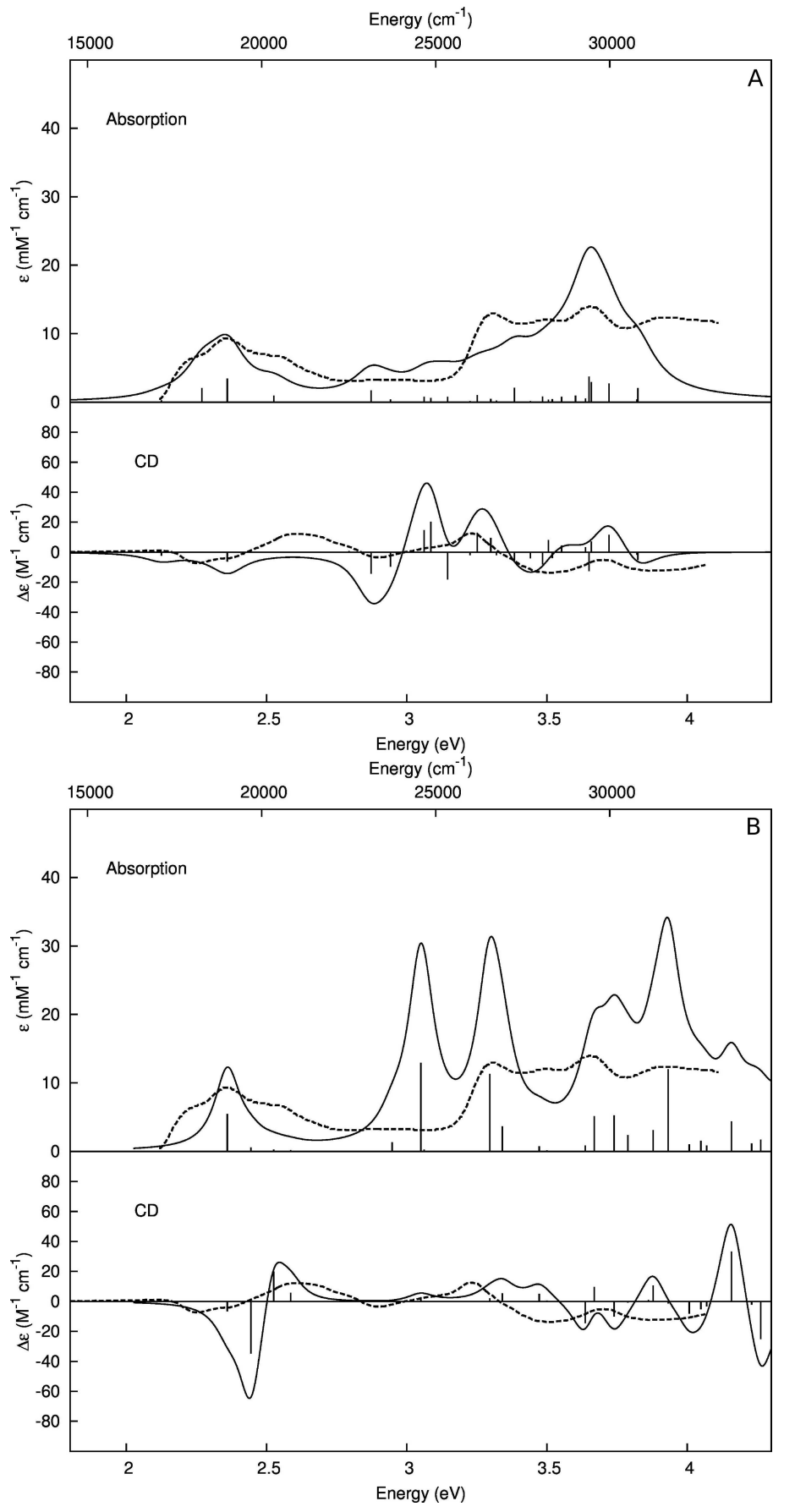

Figure A.1. Absorption and $\mathrm{CD}$ spectra of $\mathrm{MeCbl}$ calculated in solution (PCM) using: BP86/aug-cc-pVDZ (A) and CAM-B3LYP/aug-cc-pVDZ (B) levels of theory. Experimental spectra were reproduced from Ref. 108 with permission. 

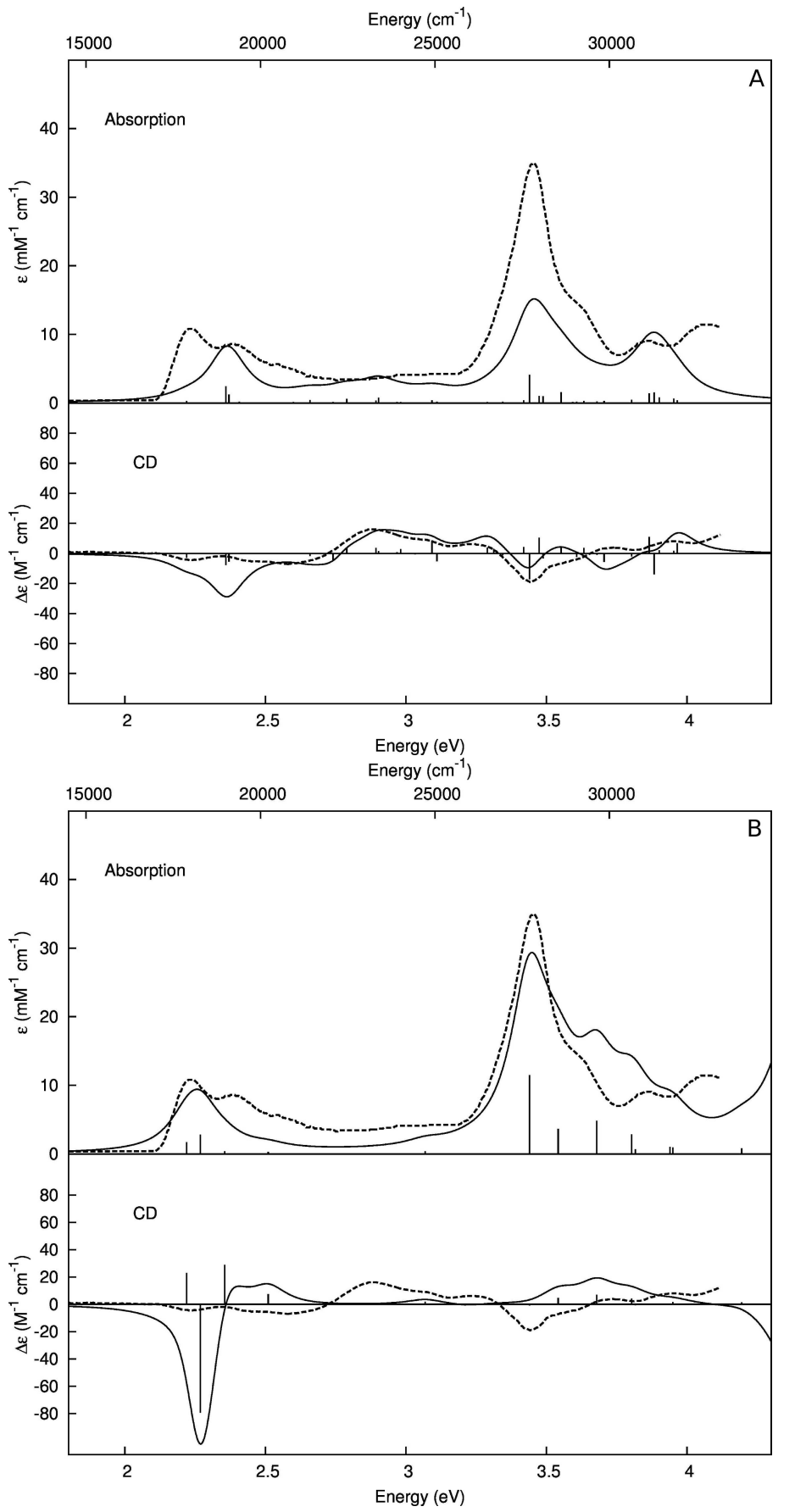

Figure A.2. Absorption and $\mathrm{CD}$ spectra of $\mathrm{CNCbl}$ calculated in gas phase using: BP86/6-31G(d) (A) and CAM-B3LYP/6-31G(d) (B) levels of theory. Experimental spectra were reproduced from Ref. 108 with permission. 

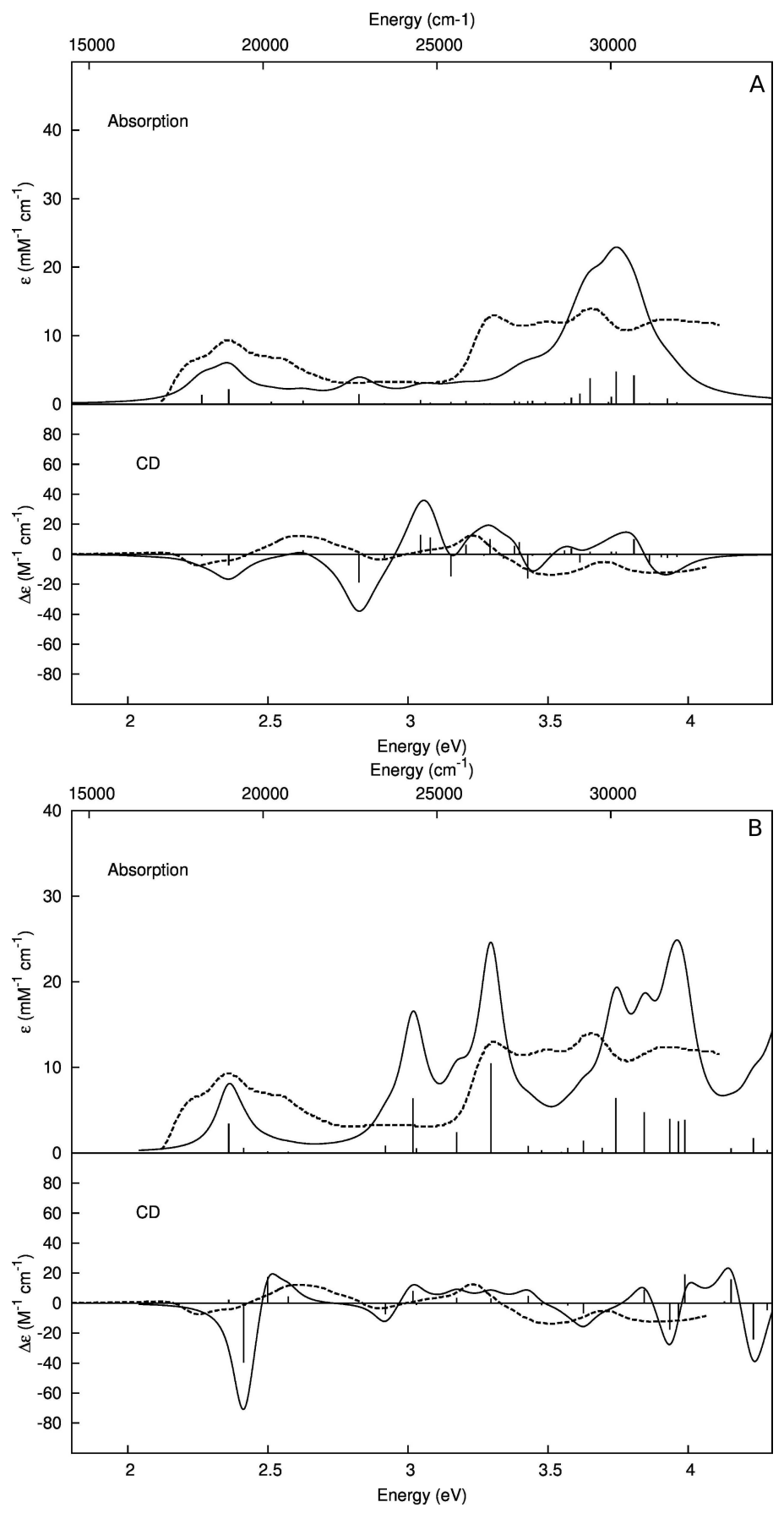

Figure A.3. Absorption and $\mathrm{CD}$ spectra of $\mathrm{MeCbl}$ calculated in gas phase using: BP86/6-31G(d) (A) and CAM-B3LYP/6-31G(d) (B) levels of theory. Experimental spectra were reproduced from Ref. 108 with permission. 

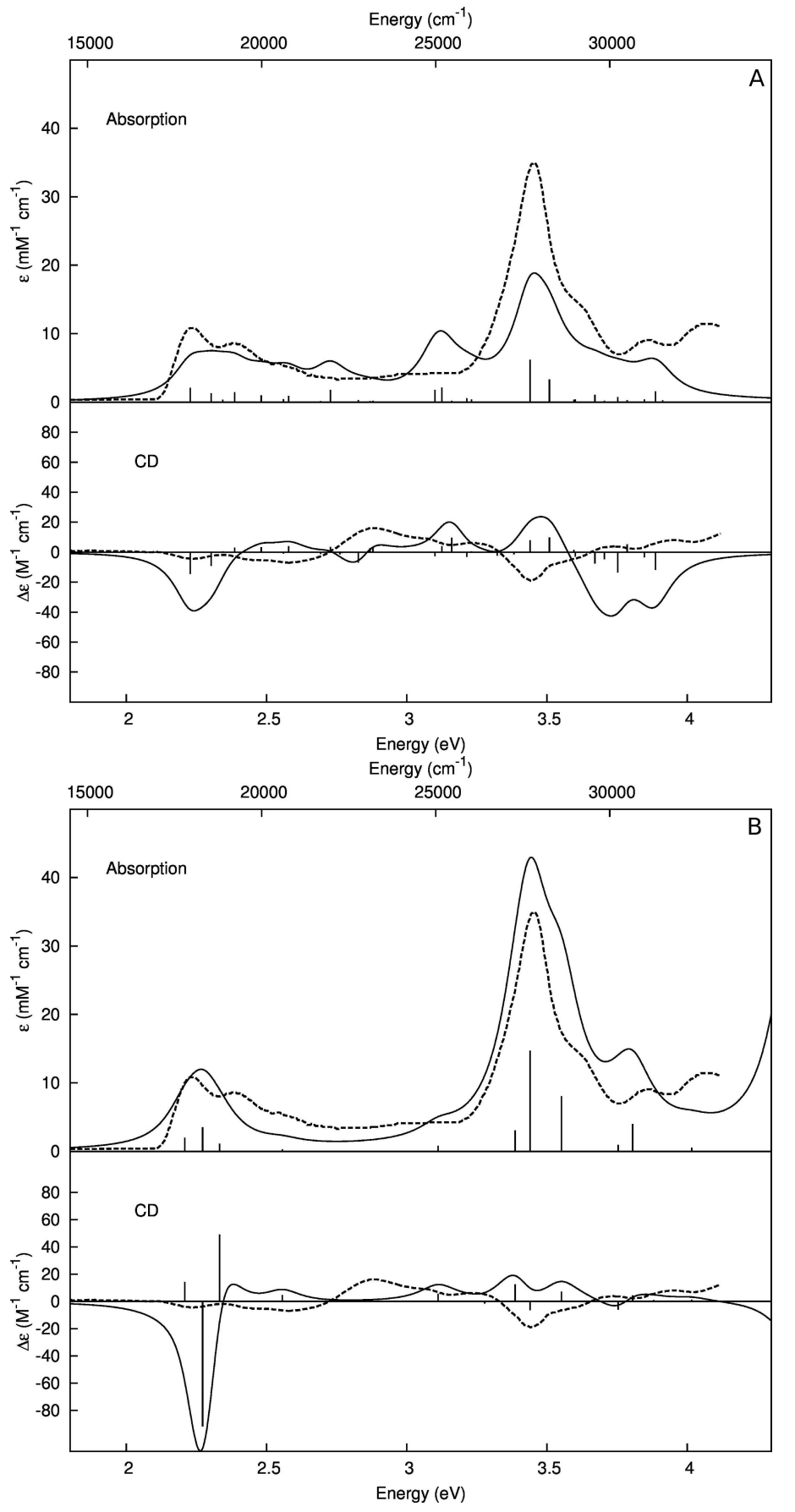

Figure A.4. Absorption and $\mathrm{CD}$ spectra of $\mathrm{CNCbl}$ calculated in solution (PCM) using: BP86/6-31G(d) (A) and CAM-B3LYP/6-31G(d) (B) levels of theory. Experimental spectra were reproduced from Ref. 108 with permission. 

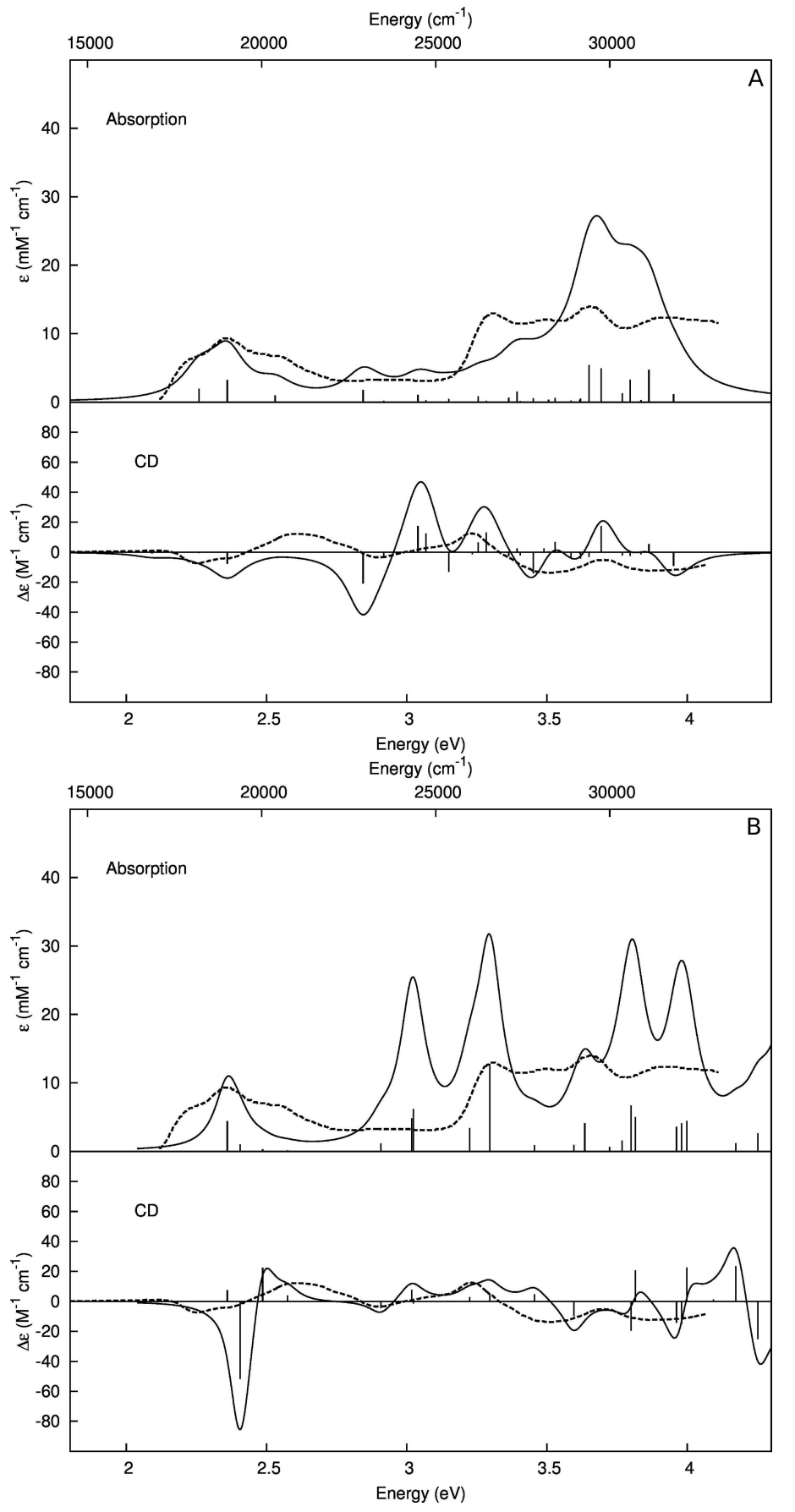

Figure A.5. Absorption and $\mathrm{CD}$ spectra of $\mathrm{MeCbl}$ calculated in solution (PCM) using: BP86/6-31G(d) (A) and CAM-B3LYP/6-31G(d) (B) levels of theory. Experimental spectra were reproduced from Ref. 108 with permission. 
TABLE A.6. Orbital energies and composition of the Im- $\left[\mathrm{Co}^{\mathrm{III}}\right.$ (corrin) $]-\mathrm{CH}_{3}^{+}$molecular orbitals calculated with MPW1PW91/6-31G(d).

\begin{tabular}{llcrrrrrr}
\hline \hline \multirow{2}{*}{ Orbital } & Character & $\mathrm{E}(\mathrm{eV})$ & \multicolumn{5}{c}{$\%$} \\
\cline { 4 - 8 } & & & & $\mathrm{Co}$ & $\mathrm{C}_{\mathrm{CH}_{3}}$ & $\mathrm{CH}_{3}$ & $\mathrm{Im}$ & Corrin \\
\hline 110 & HOMO-7 & $\pi+\mathrm{d}_{x z}$ & -10.46 & 19 & 2 & 5 & 7 & 69 \\
111 & HOMO-6 & $\mathrm{d}_{x^{2}-y^{2}}$ & -10.25 & 63 & 1 & 1 & 1 & 34 \\
112 & HOMO-5 & $\mathrm{d}_{y z} / \mathrm{d}_{x^{2}-y^{2}}+\pi$ & -10.06 & 61 & 1 & 5 & 7 & 27 \\
113 & HOMO-4 & $\sigma\left(\mathrm{d}_{z^{2}}\right)$ & -9.98 & 35 & 36 & 40 & 10 & 15 \\
114 & HOMO-3 & $\pi_{\mathrm{Im}}$ & -9.67 & 2 & 1 & 1 & 91 & 6 \\
115 & HOMO-2 & $\mathrm{d}_{x z}+\pi$ & -9.34 & 59 & 2 & 4 & 6 & 31 \\
116 & HOMO-1 & $\pi+\mathrm{d}_{y z}$ & -8.74 & 25 & 0 & 1 & 5 & 69 \\
117 & HOMO & $\pi+\mathrm{d}_{z^{2}}$ & -8.21 & 5 & 6 & 7 & 9 & 79 \\
118 & LUMO & $\pi^{*}$ & -4.61 & 3 & 0 & 0 & 1 & 96 \\
119 & LUMO+1 & $\pi^{*}$ & -3.58 & 4 & 0 & 0 & 1 & 95 \\
120 & LUMO+2 & $\pi^{*}$ & -2.72 & 5 & 0 & 0 & 3 & 92 \\
121 & LUMO+3 & $\mathrm{d}_{x y}+\mathrm{n}$ & -2.47 & 39 & 3 & 3 & 3 & 55 \\
122 & LUMO+4 & $\sigma\left(\mathrm{d}_{z^{2}}\right) / \mathrm{d}_{x y}+\mathrm{n}$ & -2.34 & 36 & 17 & 17 & 13 & 34 \\
\hline \hline
\end{tabular}

TABLE A.7. Orbital energies and composition of the Im- $\left[\mathrm{Co}^{\mathrm{III}}\right.$ (corrin) $]-\mathrm{CH}_{3}^{+}$molecular orbitals calculated with MPWPW91/6-31G(d).

\begin{tabular}{llcrrrrrr}
\hline \hline \multirow{2}{*}{ Orbital } & Character & $\mathrm{E}(\mathrm{eV})$ & \multicolumn{5}{c}{$\%$} \\
\cline { 4 - 8 } & & & & $\mathrm{Co}$ & $\mathrm{C}_{\mathrm{CH}_{3}}$ & $\mathrm{CH}_{3}$ & $\mathrm{Im}$ & Corrin \\
\hline 113 & HOMO-4 & $\pi+\mathrm{d}_{y z}+\pi_{\mathrm{Im}}$ & -8.47 & 23 & 1 & 3 & 13 & 61 \\
114 & HOMO-3 & $\mathrm{d}_{x^{2}-y^{2}}$ & -7.79 & 61 & 0 & 0 & 1 & 37 \\
115 & HOMO-2 & $\mathrm{d}_{x z}+\pi$ & -7.47 & 52 & 2 & 4 & 5 & 39 \\
116 & HOMO-1 & $\pi+\mathrm{d}_{x z}$ & -7.34 & 25 & 4 & 6 & 9 & 60 \\
117 & HOMO & $\mathrm{d}_{y z}+\pi$ & -7.21 & 50 & 1 & 3 & 10 & 37 \\
118 & LUMO & $\pi^{*}$ & -5.27 & 7 & 0 & 0 & 1 & 92 \\
119 & LUMO+1 & $\pi^{*}+\mathrm{d}_{x y}+\mathrm{n}$ & -4.47 & 27 & 1 & 1 & 1 & 71 \\
120 & LUMO+2 & $\pi^{*}+\mathrm{d}_{x y}+\mathrm{n}$ & -4.34 & 27 & 0 & 0 & 0 & 73 \\
121 & LUMO+3 & $\sigma^{*}\left(\mathrm{~d}_{z^{2}}\right)$ & -3.97 & 36 & 22 & 23 & 13 & 28 \\
\hline \hline
\end{tabular}


TABLE A.8. Orbital energies and composition of the Im- $\left[\mathrm{Co}^{\mathrm{III}}\right.$ (corrin) $]-\mathrm{CH}_{3}^{+}$molecular orbitals calculated with TPSSh/6-31G(d).

\begin{tabular}{llcrrrrrr}
\hline \hline \multirow{2}{*}{ Orbital } & Character & $\mathrm{E}(\mathrm{eV})$ & \multicolumn{5}{c}{$\%$} \\
\cline { 4 - 8 } & & & & $\mathrm{Co}$ & $\mathrm{C}_{\mathrm{CH}_{3}}$ & $\mathrm{CH}_{3}$ & $\mathrm{Im}$ & Corrin \\
\hline 112 & HOMO-5 & $\pi+\mathrm{d}_{y z} / \mathrm{d}_{x^{2}-y^{2}}$ & -9.12 & 39 & 1 & 4 & 8 & 49 \\
113 & HOMO-4 & $\pi_{\mathrm{Im}}$ & -9.00 & 4 & 0 & 0 & 89 & 7 \\
114 & HOMO-3 & $\mathrm{d}_{x^{2}-y^{2}}$ & -8.80 & 64 & 0 & 1 & 2 & 34 \\
115 & HOMO-2 & $\mathrm{d}_{x z}+\pi$ & -8.21 & 68 & 1 & 4 & 4 & 24 \\
116 & HOMO-1 & $\pi+\mathrm{d}_{y z}$ & -7.87 & 42 & 1 & 2 & 8 & 48 \\
117 & HOMO & $\pi+\mathrm{d}_{z^{2}}$ & -7.66 & 6 & 7 & 7 & 10 & 76 \\
118 & LUMO & $\pi^{*}$ & -4.93 & 4 & 0 & 0 & 1 & 95 \\
119 & LUMO+1 & $\pi^{*}$ & -3.98 & 6 & 0 & 0 & 1 & 92 \\
120 & LUMO+2 & $\mathrm{d}_{x y}+\mathrm{n}$ & -3.47 & 42 & 0 & 0 & 1 & 56 \\
121 & LUMO+3 & $\pi^{*}$ & -3.21 & 6 & 2 & 2 & 3 & 89 \\
122 & LUMO+4 & $\sigma^{*}\left(\mathrm{~d}_{z^{2}}\right)$ & -3.11 & 35 & 20 & 20 & 13 & 33 \\
\hline \hline
\end{tabular}

TABLE A.9. Orbital energies and composition of the Im- $\left[\mathrm{Co}^{\mathrm{III}}\right.$ (corrin) $]-\mathrm{CH}_{3}^{+}$molecular orbitals calculated with TPSS/6-31G(d).

\begin{tabular}{llcrrrrrr}
\hline \hline \multirow{2}{*}{ Orbital } & Character & $\mathrm{E}(\mathrm{eV})$ & \multicolumn{6}{c}{$\%$} \\
\cline { 4 - 9 } & & & & $\mathrm{Co}$ & $\mathrm{C}_{\mathrm{CH}_{3}}$ & $\mathrm{CH}_{3}$ & $\mathrm{Im}$ & Corrin \\
\hline 114 & HOMO-3 & $\mathrm{d}_{x^{2}-y^{2}}$ & -7.90 & 63 & 0 & 0 & 1 & 36 \\
115 & HOMO-2 & $\mathrm{d}_{x z}+\pi$ & -7.50 & 64 & 1 & 4 & 4 & 28 \\
116 & HOMO-1 & $\pi+\mathrm{d}_{x z} / \mathrm{d}_{z^{2}}$ & -7.32 & 13 & 6 & 7 & 10 & 70 \\
117 & HOMO & $\mathrm{d}_{y z}+\pi$ & -7.25 & 50 & 1 & 3 & 10 & 38 \\
118 & LUMO & $\pi^{*}$ & -5.18 & 6 & 0 & 0 & 1 & 93 \\
119 & LUMO+1 & $\pi^{*}+\mathrm{d}_{x y}+\mathrm{n}$ & -4.33 & 17 & 0 & 0 & 1 & 81 \\
120 & LUMO+2 & $\mathrm{d}_{x y}+\mathrm{n}$ & -4.17 & 37 & 0 & 0 & 0 & 63 \\
\hline \hline
\end{tabular}


TABLE A.10. Orbital energies and composition of the Im- $\left[\mathrm{Co}{ }^{\mathrm{III}}\right.$ (corrin) $]-\mathrm{CH}_{3}^{+}$molecular orbitals calculated with BP86/6-31G(d).

\begin{tabular}{llcrrrrrr}
\hline \hline \multirow{2}{*}{ Orbital } & Character & $\mathrm{E}(\mathrm{eV})$ & \multicolumn{5}{c}{$\%$} \\
\cline { 4 - 8 } & & & & $\mathrm{Co}$ & $\mathrm{C}_{\mathrm{CH}_{3}}$ & $\mathrm{CH}_{3}$ & $\mathrm{Im}$ & Corrin \\
\hline 111 & HOMO-6 & $\sigma\left(\mathrm{d}_{z^{2}}\right)+\pi$ & -8.98 & 24 & 28 & 31 & 12 & 32 \\
112 & HOMO-5 & $\pi_{\mathrm{Im}}$ & -8.63 & 4 & 0 & 1 & 82 & 13 \\
113 & HOMO-4 & $\pi+\mathrm{d}_{y z}+\pi_{\mathrm{Im}}$ & -8.54 & 23 & 1 & 3 & 15 & 59 \\
114 & HOMO-3 & $\mathrm{d}_{x^{2}-y^{2}}$ & -7.87 & 61 & 0 & 0 & 1 & 37 \\
115 & HOMO-2 & $\mathrm{d}_{x z}+\pi$ & -7.55 & 54 & 2 & 4 & 5 & 37 \\
116 & HOMO-1 & $\pi+\mathrm{d}_{x z} / \mathrm{d}_{z^{2}}$ & -7.41 & 23 & 5 & 6 & 10 & 61 \\
117 & HOMO & $\mathrm{d}_{y z}+\pi$ & -7.29 & 50 & 1 & 3 & 10 & 37 \\
118 & LUMO & $\pi^{*}$ & -5.35 & 6 & 0 & 0 & 1 & 93 \\
119 & LUMO+1 & $\mathrm{d}_{x y}+\mathrm{n}+\pi^{*}$ & -4.56 & 29 & 1 & 1 & 1 & 69 \\
120 & LUMO+2 & $\pi^{*}+\mathrm{d}_{x y}$ & -4.43 & 25 & 0 & 0 & 0 & 75 \\
121 & LUMO+3 & $\sigma^{*}\left(\mathrm{~d}_{z^{2}}\right)$ & -4.05 & 36 & 22 & 23 & 13 & 29 \\
122 & LUMO+4 & $\pi^{*}$ & -3.85 & 1 & 0 & 0 & 2 & 96 \\
\hline \hline
\end{tabular}

TABLE A.11. Orbital energies and composition of the Im-[CoII (corrin) $]-\mathrm{CH}_{3}^{+}$molecular orbitals calculated with B3LYP/6-31G(d).

\begin{tabular}{llcrrrrrr}
\hline \hline \multirow{2}{*}{ Orbital } & Character & $\mathrm{E}(\mathrm{eV})$ & \multicolumn{5}{c}{$\%$} \\
\cline { 4 - 8 } & & & & $\mathrm{Co}$ & $\mathrm{C}_{\mathrm{CH}_{3}}$ & $\mathrm{CH}_{3}$ & $\mathrm{Im}$ & Corrin \\
\hline 111 & HOMO-6 & $\mathrm{d}_{x^{2}-y^{2}}$ & -9.79 & 61 & 1 & 2 & 2 & 35 \\
112 & HOMO-5 & $\sigma\left(\mathrm{d}_{z^{2}}\right)$ & -9.68 & 34 & 37 & 41 & 10 & 15 \\
113 & HOMO-4 & $\mathrm{d}_{x^{2}}-y^{2} / \mathrm{d}_{y z}+\pi$ & -9.61 & 62 & 1 & 3 & 5 & 30 \\
114 & HOMO-3 & $\pi_{\mathrm{Im}}$ & -9.36 & 1 & 0 & 0 & 94 & 5 \\
115 & HOMO-2 & $\mathrm{d}_{x z}+\pi$ & -8.95 & 64 & 1 & 4 & 4 & 28 \\
116 & HOMO-1 & $\pi+\mathrm{d}_{y z}$ & -8.44 & 30 & 0 & 1 & 5 & 63 \\
117 & HOMO & $\pi+\mathrm{d}_{z^{2}}$ & -8.00 & 5 & 5 & 6 & 9 & 80 \\
118 & LUMO & $\pi^{*}$ & -4.71 & 3 & 0 & 0 & 1 & 96 \\
119 & LUMO+1 & $\pi^{*}$ & -3.72 & 5 & 0 & 0 & 1 & 94 \\
120 & LUMO+2 & $\mathrm{d}_{x y}+\mathrm{n}+\pi^{*}$ & -2.95 & 26 & 0 & 0 & 2 & 72 \\
121 & LUMO+3 & $\mathrm{d}_{x y} / \mathrm{d}_{z^{2}}+\pi^{*}$ & -2.82 & 23 & 6 & 6 & 6 & 65 \\
122 & LUMO+4 & $\sigma^{*}\left(\mathrm{~d}_{z^{2}} / \mathrm{d}_{x y}\right)+\pi^{*}$ & -2.74 & 34 & 15 & 15 & 10 & 41 \\
\hline \hline
\end{tabular}


TABLE A.12. The first five excited states calculated with BP86/6-311G(d,p).

\begin{tabular}{lcccrcl}
\hline \hline & $\mathrm{E}(\mathrm{eV})$ & $\lambda(\mathrm{nm})$ & $\mathrm{f}$ & Contribution & Transition & \\
\hline $\mathrm{S}_{1}$ & 2.21 & 559.9 & 0.0011 & 0.346 & $115 \rightarrow 118$ & $24 \%\left(\mathrm{~d}_{x z}+\pi \rightarrow \pi^{*}\right)$ \\
& & & & 0.609 & $116 \rightarrow 118$ & $74 \%\left(\pi+\mathrm{d}_{x z} / \mathrm{d}_{z^{2}} \rightarrow \pi^{*}\right)$ \\
$\mathrm{S}_{2}$ & 2.36 & 524.7 & 0.0277 & -0.163 & $116 \rightarrow 119$ & $5 \%\left(\pi+\mathrm{d}_{x z} / \mathrm{d}_{z^{2}} \rightarrow \mathrm{d}_{x y}+\mathrm{n}+\pi^{*}\right)$ \\
& & & & 0.144 & $116 \rightarrow 120$ & $4 \%\left(\pi+\mathrm{d}_{x z} / \mathrm{d}_{z^{2}} \rightarrow \pi^{*}+\mathrm{d}_{x y}\right)$ \\
& & & & 0.660 & $117 \rightarrow 118$ & $87 \%\left(\mathrm{~d}_{y z}+\pi \rightarrow \pi^{*}\right)$ \\
$\mathrm{S}_{3}$ & 2.49 & \multirow{4}{*}{497.8} & 0.0523 & 0.282 & $114 \rightarrow 118$ & $16 \%\left(\mathrm{~d}_{x^{2}-y^{2}} \rightarrow \pi^{*}\right)$ \\
& & & 0.539 & $115 \rightarrow 118$ & $58 \%\left(\mathrm{~d}_{x z}+\pi \rightarrow \pi^{*}\right)$ \\
& & & -0.310 & $116 \rightarrow 118$ & $19 \%\left(\pi+\mathrm{d}_{x z} / \mathrm{d}_{z^{2}} \rightarrow \pi^{*}\right)$ \\
& & & -0.102 & $117 \rightarrow 119$ & $2 \%\left(\mathrm{~d}_{y z}+\pi \rightarrow \mathrm{d}_{x y}+\mathrm{n}+\pi^{*}\right)$ \\
& & & 0.105 & $117 \rightarrow 120$ & $2 \%\left(\mathrm{~d}_{y z}+\pi \rightarrow \pi^{*}+\mathrm{d}_{x y}\right)$ \\
\hline \hline
\end{tabular}

TABLE A.13. The first five excited states calculated with BP86/6-311++G(d,p).

\begin{tabular}{lcccrcl}
\hline \hline & $\mathrm{E}(\mathrm{eV})$ & $\lambda(\mathrm{nm})$ & $\mathrm{f}$ & Contribution & Transition & \\
\hline $\mathrm{S}_{1}$ & 2.25 & 551.4 & 0.0035 & -0.380 & $115 \rightarrow 118$ & $29 \%\left(\mathrm{~d}_{x z}+\pi \rightarrow \pi^{*}\right)$ \\
& & & & 0.589 & $116 \rightarrow 118$ & $69 \%\left(\pi+\mathrm{d}_{x z} / \mathrm{d}_{z^{2}} \rightarrow \pi^{*}\right)$ \\
$\mathrm{S}_{2}$ & 2.38 & \multirow{2}{*}{521.4} & 0.0271 & 0.150 & $116 \rightarrow 119$ & $5 \%\left(\pi+\mathrm{d}_{x z} / \mathrm{d}_{z^{2}} \rightarrow \mathrm{d}_{x y}+\mathrm{n}+\pi^{*}\right)$ \\
& & & & -0.153 & $116 \rightarrow 120$ & $5 \%\left(\pi+\mathrm{d}_{x z} / \mathrm{d}_{z^{2}} \rightarrow \pi^{*}+\mathrm{d}_{x y}\right)$ \\
& & & & 0.660 & $117 \rightarrow 118$ & $87 \%\left(\mathrm{~d}_{y z}+\pi \rightarrow \pi^{*}\right)$ \\
$\mathrm{S}_{3}$ & 2.50 & \multirow{2}{*}{496.6} & \multirow{2}{*}{0.0565} & 0.254 & $114 \rightarrow 118$ & $13 \%\left(\mathrm{~d}_{x^{2}-y^{2}} \rightarrow \pi^{*}\right)$ \\
& & & & 0.532 & $115 \rightarrow 118$ & $57 \%\left(\mathrm{~d}_{x z}+\pi \rightarrow \pi^{*}\right)$ \\
& & & 0.343 & $116 \rightarrow 118$ & $24 \%\left(\pi+\mathrm{d}_{x z} / \mathrm{d}_{z^{2}} \rightarrow \pi^{*}\right)$ \\
& & & 0.116 & $117 \rightarrow 120$ & $3 \%\left(\mathrm{~d}_{y z}+\pi \rightarrow \pi^{*}+\mathrm{d}_{x y}\right)$ \\
\hline \hline
\end{tabular}


TABLE A.14. Lambda parameter for DBI-[Co ${ }^{\mathrm{III}}$ (corrin) $]-\mathrm{CN}^{+}$calculated with different methods. The values $\leq 0.30$ are indicated with red color, $\leq 0.40$ with green.

\begin{tabular}{|c|c|c|c|c|c|c|c|}
\hline & & & & & & B3LYP & \\
\hline & aug-cc-p & VDZ & $6-31 \mathrm{G}$ & & aug-cc-pVDZ & 6-31G( & \\
\hline & gas phase & PCM & gas phase & PCM & gas phase & gas phase & PCM \\
\hline $\mathrm{S}_{1}$ & 0.57 & 0.51 & 0.47 & 0.56 & 0.59 & 0.58 & 0.56 \\
\hline $\mathrm{S}_{2}$ & 0.58 & 0.50 & 0.51 & 0.39 & 0.57 & 0.58 & 0.56 \\
\hline $\mathrm{S}_{3}$ & 0.58 & 0.48 & 0.65 & 0.23 & 0.59 & 0.50 & 0.50 \\
\hline $\mathrm{S}_{4}$ & 0.56 & 0.53 & 0.15 & 0.54 & 0.59 & 0.54 & 0.54 \\
\hline $\mathrm{S}_{5}$ & 0.50 & 0.57 & 0.35 & 0.52 & 0.56 & 0.51 & 0.49 \\
\hline $\mathrm{S}_{6}$ & 0.56 & 0.59 & 0.49 & 0.49 & 0.59 & 0.53 & 0.49 \\
\hline$S_{7}$ & 0.48 & 0.61 & 0.56 & 0.52 & 0.61 & 0.62 & 0.49 \\
\hline $\mathrm{S}_{8}$ & 0.56 & 0.60 & 0.42 & 0.51 & 0.56 & 0.50 & 0.55 \\
\hline $\mathrm{S}_{9}$ & 0.55 & 0.59 & 0.56 & 0.47 & 0.57 & 0.49 & 0.50 \\
\hline$S_{10}$ & 0.55 & 0.59 & 0.55 & 0.28 & 0.56 & 0.43 & 0.50 \\
\hline$S_{11}$ & 0.47 & 0.51 & 0.24 & 0.28 & 0.61 & 0.40 & 0.59 \\
\hline$S_{12}$ & 0.48 & 0.58 & 0.40 & 0.39 & 0.57 & 0.42 & 0.25 \\
\hline $\mathrm{S}_{13}$ & 0.54 & 0.59 & 0.57 & 0.19 & 0.62 & 0.33 & 0.31 \\
\hline $\mathrm{S}_{14}$ & 0.58 & 0.55 & 0.56 & 0.33 & 0.59 & 0.48 & 0.61 \\
\hline $\mathrm{S}_{15}$ & 0.55 & 0.61 & 0.56 & 0.63 & 0.59 & 0.60 & 0.68 \\
\hline $\mathrm{S}_{16}$ & 0.55 & 0.60 & 0.15 & 0.60 & 0.59 & 0.26 & 0.63 \\
\hline $\mathrm{S}_{17}$ & 0.59 & 0.60 & 0.47 & 0.52 & 0.60 & 0.54 & 0.45 \\
\hline $\mathrm{S}_{18}$ & 0.50 & 0.60 & 0.45 & 0.34 & 0.60 & 0.54 & 0.54 \\
\hline $\mathrm{S}_{19}$ & 0.60 & 0.60 & 0.23 & 0.27 & 0.62 & 0.51 & 0.41 \\
\hline $\mathrm{S}_{20}$ & 0.59 & 0.61 & 0.57 & 0.54 & 0.59 & 0.48 & 0.42 \\
\hline$S_{21}$ & 0.47 & 0.65 & 0.50 & 0.51 & 0.58 & 0.47 & 0.44 \\
\hline $\mathrm{S}_{22}$ & 0.55 & 0.60 & 0.56 & 0.58 & 0.58 & 0.47 & 0.34 \\
\hline $\mathrm{S}_{23}$ & 0.59 & 0.60 & 0.49 & 0.52 & 0.59 & 0.50 & 0.38 \\
\hline $\mathrm{S}_{24}$ & 0.52 & 0.63 & 0.37 & 0.55 & 0.59 & 0.45 & 0.31 \\
\hline $\mathrm{S}_{25}$ & 0.59 & 0.53 & 0.32 & 0.59 & 0.60 & 0.41 & 0.42 \\
\hline $\mathrm{S}_{26}$ & 0.53 & 0.63 & 0.50 & 0.43 & 0.58 & 0.48 & 0.44 \\
\hline $\mathrm{S}_{27}$ & 0.58 & 0.62 & 0.44 & 0.54 & 0.55 & 0.54 & 0.41 \\
\hline $\mathrm{S}_{28}$ & 0.55 & 0.61 & 0.32 & 0.56 & 0.58 & 0.44 & 0.31 \\
\hline $\mathrm{S}_{29}$ & 0.59 & 0.62 & 0.52 & 0.55 & 0.60 & 0.41 & 0.34 \\
\hline $\mathrm{S}_{30}$ & 0.56 & 0.63 & 0.54 & 0.63 & 0.58 & 0.44 & 0.56 \\
\hline $\mathrm{S}_{31}$ & 0.58 & 0.62 & 0.57 & 0.55 & 0.58 & 0.42 & 0.43 \\
\hline $\mathrm{S}_{32}$ & 0.59 & 0.61 & 0.58 & 0.52 & 0.60 & 0.49 & 0.34 \\
\hline $\mathrm{S}_{33}$ & 0.56 & 0.59 & 0.57 & & 0.60 & 0.42 & \\
\hline $\mathrm{S}_{34}$ & 0.43 & 0.60 & 0.46 & & 0.58 & 0.49 & \\
\hline $\mathrm{S}_{35}$ & 0.57 & 0.63 & 0.48 & & 0.58 & 0.51 & \\
\hline
\end{tabular}


TABLE A.15. Lambda parameter for DBI-[Co ${ }^{\mathrm{III}}$ (corrin) $]-\mathrm{CH}_{3}^{+}$calculated with different methods. The values $\leq 0.30$ are indicated with red color, $\leq 0.40$ with green.

\begin{tabular}{|c|c|c|c|c|c|c|c|c|}
\hline & & & & & & CAN & SLYP & \\
\hline & aug-cc-p & DZ & $6-31 \mathrm{G}$ & & aug-cc-p & $\mathrm{DZ}$ & $6-31 G$ & \\
\hline & gas phase & PCM & gas phase & PCM & gas phase & PCM & gas phase & PCM \\
\hline $\mathrm{S}_{1}$ & 0.57 & 0.63 & 0.60 & 0.61 & 0.63 & 0.62 & 0.60 & 0.61 \\
\hline $\mathrm{S}_{2}$ & 0.59 & 0.62 & 0.71 & 0.73 & 0.60 & 0.57 & 0.55 & 0.55 \\
\hline $\mathrm{S}_{3}$ & 0.57 & 0.63 & 0.56 & 0.56 & 0.60 & 0.57 & 0.52 & 0.54 \\
\hline $\mathrm{S}_{4}$ & 0.57 & 0.66 & 0.21 & 0.39 & 0.60 & 0.57 & 0.57 & 0.57 \\
\hline $\mathrm{S}_{5}$ & 0.55 & 0.56 & 0.30 & 0.09 & 0.60 & 0.57 & 0.54 & 0.55 \\
\hline $\mathrm{S}_{6}$ & 0.50 & 0.61 & 0.28 & 0.33 & 0.61 & 0.63 & 0.61 & 0.61 \\
\hline $\mathrm{S}_{7}$ & 0.47 & 0.61 & 0.59 & 0.51 & 0.60 & 0.57 & 0.55 & 0.67 \\
\hline $\mathrm{S}_{8}$ & 0.50 & 0.63 & 0.64 & 0.65 & 0.60 & 0.56 & 0.46 & 0.46 \\
\hline $\mathrm{S}_{9}$ & 0.51 & 0.63 & 0.64 & 0.63 & 0.63 & 0.57 & 0.61 & 0.62 \\
\hline $\mathrm{S}_{10}$ & 0.54 & 0.62 & 0.62 & 0.59 & 0.61 & 0.58 & 0.49 & 0.47 \\
\hline $\mathrm{S}_{11}$ & 0.52 & 0.63 & 0.64 & 0.66 & 0.61 & 0.57 & 0.46 & 0.55 \\
\hline $\mathrm{S}_{12}$ & 0.50 & 0.54 & 0.58 & 0.23 & 0.60 & 0.58 & 0.50 & 0.46 \\
\hline $\mathrm{S}_{13}$ & 0.53 & 0.62 & 0.35 & 0.61 & 0.61 & 0.59 & 0.48 & 0.30 \\
\hline $\mathrm{S}_{14}$ & 0.55 & 0.62 & 0.50 & 0.58 & 0.59 & 0.57 & 0.30 & 0.32 \\
\hline $\mathrm{S}_{15}$ & 0.50 & 0.58 & 0.41 & 0.42 & 0.62 & 0.56 & 0.40 & 0.32 \\
\hline $\mathrm{S}_{16}$ & 0.52 & 0.62 & 0.51 & 0.53 & 0.60 & 0.57 & 0.47 & 0.47 \\
\hline $\mathrm{S}_{17}$ & 0.55 & 0.63 & 0.49 & 0.57 & 0.61 & 0.58 & 0.55 & 0.48 \\
\hline $\mathrm{S}_{18}$ & 0.47 & 0.62 & 0.33 & 0.55 & 0.60 & 0.57 & 0.46 & 0.46 \\
\hline $\mathrm{S}_{19}$ & 0.49 & 0.61 & 0.51 & 0.17 & 0.60 & 0.58 & 0.59 & 0.61 \\
\hline $\mathrm{S}_{20}$ & 0.54 & 0.58 & 0.50 & 0.49 & 0.58 & 0.57 & 0.59 & 0.60 \\
\hline $\mathrm{S}_{21}$ & 0.56 & 0.60 & 0.48 & 0.47 & 0.60 & 0.58 & 0.32 & 0.31 \\
\hline $\mathrm{S}_{22}$ & 0.62 & 0.62 & 0.53 & 0.46 & 0.58 & 0.53 & 0.48 & 0.50 \\
\hline $\mathrm{S}_{23}$ & 0.59 & 0.62 & 0.52 & 0.22 & 0.60 & 0.59 & 0.64 & 0.70 \\
\hline $\mathrm{S}_{24}$ & 0.58 & 0.60 & 0.46 & 0.48 & 0.61 & 0.56 & 0.57 & 0.63 \\
\hline $\mathrm{S}_{25}$ & 0.54 & 0.61 & 0.53 & 0.53 & 0.57 & 0.56 & 0.52 & 0.64 \\
\hline $\mathrm{S}_{26}$ & 0.55 & 0.62 & 0.54 & 0.47 & 0.55 & 0.60 & 0.64 & 0.43 \\
\hline $\mathrm{S}_{27}$ & 0.55 & 0.59 & 0.34 & 0.57 & 0.60 & 0.59 & 0.44 & 0.43 \\
\hline $\mathrm{S}_{28}$ & 0.62 & 0.62 & 0.59 & 0.43 & 0.60 & 0.56 & 0.50 & 0.38 \\
\hline $\mathrm{S}_{29}$ & 0.59 & & 0.57 & 0.22 & 0.60 & 0.59 & 0.43 & 0.26 \\
\hline $\mathrm{S}_{30}$ & 0.56 & & 0.57 & 0.59 & 0.60 & 0.59 & 0.45 & 0.43 \\
\hline $\mathrm{S}_{31}$ & 0.53 & & 0.36 & 0.17 & 0.59 & 0.57 & 0.53 & 0.44 \\
\hline $\mathrm{S}_{32}$ & 0.53 & & 0.52 & 0.63 & 0.60 & 0.56 & 0.45 & 0.43 \\
\hline $\mathrm{S}_{33}$ & 0.59 & & 0.61 & & 0.61 & & 0.47 & \\
\hline $\mathrm{S}_{34}$ & 0.60 & & 0.46 & & 0.52 & & 0.51 & \\
\hline $\mathrm{S}_{35}$ & & & 0.44 & & 0.61 & & 0.46 & \\
\hline
\end{tabular}


TABLE A.16. First 19 excited states of cob(I)alamin calculated with MC-XQDPT2 presented in terms of \% contributions of CAS states (see Table A.17 for the nature of individual states).

\begin{tabular}{lrl}
\hline \hline State & $\mathrm{E}(\mathrm{eV})$ & Composition \\
\hline $\mathrm{S}_{1}$ & 0.94 & $98.4 \% \mathrm{X}_{1}$ \\
$\mathrm{~S}_{2}$ & 1.91 & $38.6 \% \mathrm{X}_{8}+35.2 \% \mathrm{X}_{2}+15.2 \% \mathrm{X}_{9}$ \\
$\mathrm{~S}_{3}$ & 2.17 & $63.3 \% \mathrm{X}_{2}+24 \% \mathrm{X}_{8}+8.5 \% \mathrm{X}_{9}$ \\
$\mathrm{~S}_{4}$ & 3.57 & $65.9 \% \mathrm{X}_{3}+20 \% \mathrm{X}_{19}+11.1 \% \mathrm{X}_{5}$ \\
$\mathrm{~S}_{5}$ & 4.14 & $45.4 \% \mathrm{X}_{16}+36.6 \% \mathrm{X}_{14}+8.3 \% \mathrm{X}_{13}$ \\
$\mathrm{~S}_{6}$ & 4.16 & $59 \% \mathrm{X}_{14}+24.4 \% \mathrm{X}_{16}+6.8 \% \mathrm{X}_{13}$ \\
$\mathrm{~S}_{7}$ & 4.31 & $79 \% \mathrm{X}_{13}+17.4 \% \mathrm{X}_{16}$ \\
$\mathrm{~S}_{8}$ & 4.39 & $81.7 \% \mathrm{X}_{4}+14.6 \% \mathrm{X}_{5}$ \\
$\mathrm{~S}_{9}$ & 4.47 & $64.4 \% \mathrm{X}_{5}+15 \% \mathrm{X}_{3}+13 \% \mathrm{X}_{4}$ \\
$\mathrm{~S}_{10}$ & 4.82 & $94.3 \% \mathrm{X}_{6}$ \\
$\mathrm{~S}_{11}$ & 5.34 & $59.2 \% \mathrm{X}_{7}+30.4 \% \mathrm{X}_{19}+7.6 \% \mathrm{X}_{3}$ \\
$\mathrm{~S}_{12}$ & 5.39 & $45.4 \% \mathrm{X}_{19}+40.2 \% \mathrm{X}_{7}+9.2 \% \mathrm{X}_{3}$ \\
$\mathrm{~S}_{13}$ & 5.82 & $72.9 \% \mathrm{X}_{9}+25.7 \% \mathrm{X}_{8}$ \\
$\mathrm{~S}_{14}$ & 7.26 & $98 \% \mathrm{X}_{10}$ \\
$\mathrm{~S}_{15}$ & 7.54 & $89 \% \mathrm{X}_{11}$ \\
$\mathrm{~S}_{16}$ & 7.66 & $70.3 \% \mathrm{X}_{12}+16.9 \% \mathrm{X}_{18}+6.2 \% \mathrm{X}_{11}$ \\
$\mathrm{~S}_{17}$ & 8.37 & $36.7 \% \mathrm{X}_{18}+32.6 \% \mathrm{X}_{15}+14.2 \% \mathrm{X}_{17}+12.3 \% \mathrm{X}_{12}$ \\
$\mathrm{~S}_{18}$ & 8.45 & $63.2 \% \mathrm{X}_{15}+20.9 \% \mathrm{X}_{18}+6.4 \% \mathrm{X}_{17}$ \\
$\mathrm{~S}_{19}$ & 8.55 & $77.2 \% \mathrm{X}_{17}+20 \% \mathrm{X}_{18}$ \\
\hline \hline
\end{tabular}


TABLE A.17. First 19 excitations calculated with CASSCF.

\begin{tabular}{|c|c|c|}
\hline State & $\overline{E \mathrm{E}(\mathrm{eV})}$ & Character \\
\hline $\mathrm{X}_{1}$ & 0.36 & $\begin{array}{l}57 \%\left(\mathrm{~d}_{z^{2}} \rightarrow \pi^{*}\right) \\
30 \%\left(\mathrm{~d}_{y z} / \mathrm{d}_{z^{2}} \rightarrow \pi^{*}\right)\end{array}$ \\
\hline $\mathrm{X}_{2}$ & 1.80 & $\begin{array}{l}44 \%\left(\mathrm{~d}_{x^{2}-y^{2}} \rightarrow \pi^{*}\right) \\
24 \%\left(\mathrm{~d}_{x^{2}-y^{2}} / \mathrm{d}_{y z} \rightarrow \pi^{*}\right) \\
12 \%\left(\mathrm{~d}_{x z} \rightarrow \mathrm{d}_{x y}-\mathrm{n}\right)\end{array}$ \\
\hline $\mathrm{X}_{3}$ & 3.52 & $\begin{array}{l}46 \%\left(\mathrm{~d}_{x z} \rightarrow \mathrm{d}_{x y}-\mathrm{n}\right) \\
28 \%\left(\mathrm{~d}_{x z} / \mathrm{d}_{y z} \rightarrow \pi^{*} / \mathrm{d}_{x y}-\mathrm{n}\right)\end{array}$ \\
\hline $\mathrm{X}_{4}$ & 3.66 & $\begin{array}{l}41 \%\left(\mathrm{~d}_{x^{2}-y^{2}} / \mathrm{d}_{x z} \rightarrow \pi^{*} / \mathrm{d}_{x y}-\mathrm{n}\right) \\
26 \%\left(\mathrm{~d}_{x^{2}-y^{2}} / \mathrm{d}_{x z} / \mathrm{d}_{y z} \rightarrow \pi^{*} / \mathrm{d}_{x y}-\mathrm{n}\right) \\
11 \%\left(\mathrm{~d}_{x z} / \mathrm{d}_{y z} \rightarrow \pi^{*} / \mathrm{d}_{x y}-\mathrm{n}\right)\end{array}$ \\
\hline $\mathrm{X}_{5}$ & 3.91 & $\begin{array}{l}57 \%\left(\mathrm{~d}_{x z} / \mathrm{d}_{y z} \rightarrow \pi^{*} / \mathrm{d}_{x y}-\mathrm{n}\right) \\
10 \%\left(\mathrm{~d}_{x z} \rightarrow \mathrm{d}_{x y}-\mathrm{n}\right)\end{array}$ \\
\hline $\mathrm{X}_{6}$ & 3.99 & $\begin{array}{l}34 \%\left(\mathrm{~d}_{x z} / \mathrm{d}_{z^{2}} \rightarrow \pi^{*} / \mathrm{d}_{x y}-\mathrm{n}\right) \\
16 \%\left(\mathrm{~d}_{x^{2}-y^{2}} / \mathrm{d}_{x z} \rightarrow \pi^{*} / \mathrm{d}_{x y}-\mathrm{n}\right) \\
21 \%\left(\mathrm{~d}_{x z} / \mathrm{d}_{y z} / \mathrm{d}_{z^{2}} \rightarrow \pi^{*} / \mathrm{d}_{x y}-\mathrm{n}\right)\end{array}$ \\
\hline $\mathrm{X}_{7}$ & 4.64 & $\begin{array}{l}31 \%\left(\mathrm{~d}_{x^{2}-y^{2}} / \mathrm{d}_{x z} \rightarrow \pi^{*} / \mathrm{d}_{x y}-\mathrm{n}\right) \\
22 \%\left(\mathrm{~d}_{x z} / \mathrm{d}_{z^{2}} \rightarrow \pi^{*} / \mathrm{d}_{x y}-\mathrm{n}\right) \\
15 \%\left(\mathrm{~d}_{x z} / \mathrm{d}_{y z} / \mathrm{d}_{z^{2}} \rightarrow \pi^{*} / \mathrm{d}_{x y}-\mathrm{n}\right) \\
11 \%\left(\mathrm{~d}_{x^{2}-y^{2}} / \mathrm{d}_{x z} / \mathrm{d}_{y z} \rightarrow \pi^{*} / \mathrm{d}_{x y}-\mathrm{n}\right)\end{array}$ \\
\hline $\mathrm{X}_{8}$ & 4.96 & $\begin{array}{l}47 \%\left(\mathrm{~d}_{y z} \rightarrow \pi^{*}\right) \\
14 \%\left(\mathrm{~d}_{x z} / \mathrm{d}_{z^{2}} \rightarrow \pi^{*} / \mathrm{d}_{x y}-\mathrm{n}\right)\end{array}$ \\
\hline $\mathrm{X}_{9}$ & 5.36 & $\begin{array}{l}31 \%\left(\mathrm{~d}_{x z} / \mathrm{d}_{z^{2}} \rightarrow \pi^{*} / \mathrm{d}_{x y}-\mathrm{n}\right) \\
27 \%\left(\mathrm{~d}_{y z} \rightarrow \pi^{*}\right) \\
14 \%\left(\mathrm{~d}_{x z} / \mathrm{d}_{y z} / \mathrm{d}_{z^{2}} \rightarrow \pi^{*} / \mathrm{d}_{x y}-\mathrm{n}\right)\end{array}$ \\
\hline $\mathrm{X}_{10}$ & 6.74 & $\begin{array}{l}29 \%\left(\mathrm{~d}_{x^{2}-y^{2}} / \mathrm{d}_{z^{2}} \rightarrow \mathrm{d}_{x y}-\mathrm{n}\right) \\
29 \%\left(\mathrm{~d}_{x^{2}-y^{2}} \rightarrow \mathrm{d}_{x y}-\mathrm{n}\right) \\
11 \%\left(\mathrm{~d}_{x^{2}-y^{2}} / \mathrm{d}_{y z} / \mathrm{d}_{z^{2}} \rightarrow \pi^{*} / \mathrm{d}_{x y}-\mathrm{n}\right) \\
11 \%\left(\mathrm{~d}_{x^{2}-y^{2}} / \mathrm{d}_{y z} \rightarrow \pi^{*} / \mathrm{d}_{x y}-\mathrm{n}\right)\end{array}$ \\
\hline $\mathrm{X}_{11}$ & 6.93 & $\begin{array}{l}30 \%\left(\mathrm{~d}_{x^{2}-y^{2}} / \mathrm{d}_{y z} \rightarrow \mathrm{d}_{x y}-\mathrm{n}\right) \\
16 \%\left(\mathrm{~d}_{x^{2}-y^{2}} / \mathrm{d}_{y z} \rightarrow \pi^{*} / \mathrm{d}_{x y}-\mathrm{n}\right) \\
15 \%\left(\mathrm{~d}_{x^{2}-y^{2}} / \mathrm{d}_{x z} \rightarrow \pi^{*} / \mathrm{d}_{x y}-\mathrm{n}\right) \\
13 \%\left(\mathrm{~d}_{y z} / \mathrm{d}_{z^{2}} \rightarrow \mathrm{d}_{x y}-\mathrm{n}\right)\end{array}$ \\
\hline $\mathrm{X}_{12}$ & 7.17 & $\begin{array}{l}25 \%\left(\mathrm{~d}_{x^{2}-y^{2}} / \mathrm{d}_{y z} / \mathrm{d}_{z^{2}} \rightarrow \pi^{*} / \mathrm{d}_{x y}-\mathrm{n}\right) \\
16 \%\left(\mathrm{~d}_{x^{2}-y^{2}} / \mathrm{d}_{z^{2}} \rightarrow \mathrm{d}_{x y}-\mathrm{n}\right) \\
15 \%\left(\mathrm{~d}_{z^{2}} \rightarrow \mathrm{d}_{x y}-\mathrm{n}\right)\end{array}$ \\
\hline $\mathrm{X}_{13}$ & 7.31 & $\begin{array}{l}57 \%\left(\mathrm{~d}_{z^{2}} \rightarrow \pi^{*}\right) \\
10 \%\left(\mathrm{~d}_{x^{2}-y^{2}} / \mathrm{d}_{z^{2}} \rightarrow \pi^{*}\right)\end{array}$ \\
\hline $\mathrm{X}_{14}$ & 7.34 & $\begin{array}{l}50 \%\left(\mathrm{~d}_{y z} / \mathrm{d}_{z^{2}} \rightarrow \pi^{*}\right) \\
25 \%\left(\mathrm{~d}_{z^{2}} \rightarrow \pi^{*}\right)\end{array}$ \\
\hline $\mathrm{X}_{15}$ & 7.36 & $\begin{array}{l}46 \%\left(\mathrm{~d}_{x^{2}-y^{2}} / \mathrm{d}_{z^{2}} \rightarrow \pi^{*} / \mathrm{d}_{x y}-\mathrm{n}\right) \\
28 \%\left(\mathrm{~d}_{x^{2}-y^{2}} / \mathrm{d}_{y z} / \mathrm{d}_{z^{2}} \rightarrow \pi^{*} / \mathrm{d}_{x y}-\mathrm{n}\right)\end{array}$ \\
\hline $\mathrm{X}_{16}$ & 7.42 & $\begin{array}{l}54 \%\left(\mathrm{~d}_{x z} \rightarrow \pi^{*}\right) \\
12 \%\left(\mathrm{~d}_{x^{2}-y^{2}} / \mathrm{d}_{z^{2}} \rightarrow \pi^{*}\right)\end{array}$ \\
\hline $\mathrm{X}_{17}$ & 7.59 & $\begin{array}{l}34 \%\left(\mathrm{~d}_{x^{2}-y^{2}} / \mathrm{d}_{z^{2}} \rightarrow \pi^{*} / \mathrm{d}_{x y}-\mathrm{n}\right) \\
20 \%\left(\mathrm{~d}_{x^{2}-y^{2}} / \mathrm{d}_{x z} \rightarrow \pi^{*} / \mathrm{d}_{x y}-\mathrm{n}\right) \\
20 \%\left(\mathrm{~d}_{x^{2}-y^{2}} / \mathrm{d}_{y z} / \mathrm{d}_{z^{2}} \rightarrow \pi^{*} / \mathrm{d}_{x y}-\mathrm{n}\right) \\
12 \%\left(\mathrm{~d}_{x^{2}-y^{2}} / \mathrm{d}_{x z} / \mathrm{d}_{y z} \rightarrow \pi^{*} / \mathrm{d}_{x y}-\mathrm{n}\right)\end{array}$ \\
\hline $\mathrm{X}_{18}$ & 7.91 & $\begin{array}{l}22 \%\left(\mathrm{~d}_{x z} \rightarrow \mathrm{d}_{x y}-\mathrm{n}\right) \\
21 \%\left(\mathrm{~d}_{x^{2}-y^{2}} / \mathrm{d}_{y z} / \mathrm{d}_{z^{2}} \rightarrow \pi^{*} / \mathrm{d}_{x y}-\mathrm{n}\right) \\
11 \%\left(\mathrm{~d}_{x^{2}-y^{2}} / \mathrm{d}_{z^{2}} \rightarrow \mathrm{d}_{x y}-\mathrm{n}\right)\end{array}$ \\
\hline $\mathrm{X}_{19}$ & 8.15 & $74 \%\left(\mathrm{~d}_{x z} / \mathrm{d}_{y z} \rightarrow \pi^{*} / c\right.$ \\
\hline
\end{tabular}


TABLE A.18. MO energy and fragments contributions for singlet states of Im$\left[\mathrm{Co}^{\mathrm{III}}\right.$ (corrin) $]-\mathrm{CN}^{+}$in gas phase and $\mathrm{COSMO} / \mathrm{H}_{2} \mathrm{O}$. The results coming from calculations with solvent model are shown in parenthesis.

\begin{tabular}{|c|c|c|c|c|c|c|c|c|c|c|c|c|c|c|c|}
\hline & & \multicolumn{2}{|c|}{ Character } & \multirow{2}{*}{\multicolumn{2}{|c|}{$\mathrm{E}[\mathrm{eV}]$}} & \multicolumn{10}{|c|}{ Contributions of fragments in $\mathrm{MO}[\%]$} \\
\hline & & gas phase & COSMO & & & \multicolumn{2}{|c|}{ Co } & \multicolumn{2}{|c|}{$\mathrm{C}_{\mathrm{CN}}$} & \multicolumn{2}{|c|}{$\mathrm{CN}$} & \multicolumn{2}{|c|}{$\operatorname{Im}$} & \multicolumn{2}{|c|}{ Corr } \\
\hline \multicolumn{16}{|c|}{$\mathrm{s}_{0}$} \\
\hline HOMO-4 & $(115)$ & $\pi+\mathrm{d}_{x^{2}-y^{2}}+\pi_{\mathrm{CN}}$ & $\pi_{\operatorname{Im}}+\mathrm{d}_{x^{2}-y^{2}}$ & -8.72 & $(-6.07)$ & 31 & (14) & 7 & $(0)$ & 24 & (1) & 3 & (74) & 43 & $(11)$ \\
\hline HOMO-3 & $(116)$ & $\mathrm{d}_{x^{2}-y^{2}+\pi+\pi_{\mathrm{CN}}}$ & $\mathrm{d}_{x 2--y 2}+\pi+\pi_{\text {Im }}$ & -8.52 & $(-6.04)$ & 57 & (59) & 2 & (0) & 6 & (1) & 2 & (7) & 35 & (33) \\
\hline HOMO-2 & (117) & $\mathrm{d}_{x z}+\pi+\pi_{\mathrm{CN}}$ & $\mathrm{d}_{x z}+\pi+\pi_{\operatorname{Im}}$ & -8.17 & $(-5.73)$ & 62 & (61) & 3 & (1) & 15 & (7) & 4 & (10) & 19 & (22) \\
\hline HOMO-1 & (118) & $\pi+\mathrm{d}_{y z}$ & $\pi+\mathrm{d}_{y z}$ & -7.88 & $(-5.34)$ & 39 & (34) & 1 & (0) & 4 & (2) & 9 & (7) & 48 & (58) \\
\hline HOMO & (119) & $\pi$ & $\pi$ & -7.82 & $(-5.19)$ & 5 & $(2)$ & 2 & $(2)$ & 3 & (2) & 8 & (8) & 84 & (87) \\
\hline LUMO & (120) & $\pi^{*}$ & $\pi^{*}$ & -5.67 & $(-3.05)$ & 5 & (5) & 0 & (0) & 0 & (0) & 1 & (1) & 94 & (94) \\
\hline LUMO+1 & (121) & $\mathrm{d}_{x y}+\mathrm{n}$ & $\mathrm{d}_{x y}+\mathrm{n}$ & -5.18 & $(-2.62)$ & 43 & (43) & 1 & (1) & 1 & (1) & 2 & (2) & 55 & (53) \\
\hline $\mathrm{LUMO}+2$ & (122) & $\sigma^{*}\left(\mathrm{~d}_{2}\right)+\mathrm{n}$ & $\sigma^{*}\left(\mathrm{~d}_{2}\right)+\mathrm{n}$ & -4.95 & $(-2.48)$ & 40 & (43) & 11 & (12) & 14 & (16) & 12 & (11) & 35 & (30) \\
\hline $\mathrm{LUMO}+3$ & (123) & $\pi^{*}$ & $\pi^{*}$ & -4.78 & $(-2.18)$ & 11 & (6) & 2 & $(0)$ & 3 & $(1)$ & 2 & (0) & 85 & (93) \\
\hline \multicolumn{16}{|c|}{$\mathrm{S}_{1}(\mathrm{~A})$} \\
\hline HOMO-4 & (115) & $\pi+\mathrm{d}_{x^{2}-y^{2}}+\pi_{\mathrm{CN}}$ & $\pi_{\operatorname{Im}}+\mathrm{d}_{x^{2}-y^{2}}+\pi$ & -8.64 & $(-6.07)$ & 32 & (35) & 7 & (0) & 24 & (1) & 3 & (45) & 42 & $\overline{(19)}$ \\
\hline HOMO-3 & (116) & $\mathrm{d}_{x^{2}-y^{2}+\pi}^{x^{2}-y^{2}}$ & $\mathrm{~d}_{x^{2}-y^{2}+\pi+\pi_{\mathrm{Im}}}$ & -8.48 & $(-6.02)$ & 54 & (47) & 2 & (0) & 8 & (1) & 3 & (25) & 35 & (27) \\
\hline HOMO-2 & (117) & $\mathrm{d}_{x z}+\pi+\pi_{\mathrm{CN}}$ & $\mathrm{d}_{x z}+\pi+\pi_{\operatorname{Im}}$ & -8.20 & $(-5.75)$ & 42 & (62) & 3 & (1) & 13 & (7) & 3 & (11) & 41 & (20) \\
\hline HOMO-1 & (118) & $\pi+\mathrm{d}_{y z}$ & $\pi+\mathrm{d}_{y z}$ & -7.82 & $(-5.42)$ & 38 & (28) & 1 & (0) & 4 & (2) & 9 & (6) & 49 & (64) \\
\hline HOMO & (119) & $\pi+\mathrm{d}_{x z} / \mathrm{d}_{y z}$ & $\pi$ & -7.66 & $(-5.05)$ & 23 & $(8)$ & 2 & (1) & 7 & (2) & 9 & (7) & 61 & (83) \\
\hline LUMO & (120) & $\pi^{*}$ & $\pi^{*} / \mathrm{d}_{x y}+\mathrm{n}$ & -5.90 & $(-3.39)$ & 7 & (33) & 0 & (2) & 0 & (3) & 1 & (3) & 92 & (61) \\
\hline LUMO+1 & (121) & $\mathrm{d}_{x y}+\mathrm{n}$ & $\pi^{*}+\mathrm{d}_{x y}$ & -5.20 & $(-2.93)$ & 42 & (20) & 0 & (2) & 0 & (2) & 1 & (3) & 56 & (76) \\
\hline $\mathrm{LUMO}+2$ & (122) & $\sigma^{*}\left(\mathrm{~d}_{z 2}\right)+\pi^{*}$ & $\sigma^{*}\left(\mathrm{~d}_{z^{2}}\right)+\mathrm{n}$ & -4.90 & $(-2.47)$ & 29 & (43) & 8 & (10) & 10 & (13) & 10 & (9) & 50 & (35) \\
\hline $\mathrm{LUMO}+3$ & (123) & $\pi^{*}+\sigma^{*}\left(\mathrm{~d}_{z^{2}}\right)$ & $\pi^{*}$ & -4.80 & $(-2.16)$ & 20 & (8) & 5 & (0) & 7 & (0) & 4 & (0) & 69 & (92) \\
\hline \multicolumn{16}{|c|}{$S_{1}(C)$} \\
\hline HOMO-4 & (115) & $\pi_{\operatorname{Im}}$ & $\mathrm{d}_{x^{2}-y^{2}+\pi}$ & -8.92 & $(-6.12)$ & 18 & (64) & 3 & (0) & 9 & (1) & 53 & (1) & 19 & $\overline{(33)}$ \\
\hline HOMO-3 & (116) & $\mathrm{d}_{x^{2}-y^{2}}+\pi$ & $\begin{array}{c}x^{2}-y^{2} \\
\pi_{\operatorname{Im}}\end{array}$ & -8.60 & $(-6.04)$ & 64 & (4) & 0 & (0) & 1 & (0) & 1 & (90) & 34 & (6) \\
\hline HOMO-2 & (117) & $\mathrm{d}_{x z}+\pi+\pi_{\mathrm{CN}}$ & $\mathrm{d}_{x z}+\pi$ & -8.28 & $(-5.81)$ & 60 & (60) & 5 & (2) & 13 & (5) & 2 & (5) & 26 & (30) \\
\hline HOMO-1 & (118) & $\pi+\mathrm{d}_{y z}$ & $\pi+\mathrm{d}_{y z}$ & -8.00 & $(-5.42)$ & 22 & (22) & 1 & (1) & 2 & (2) & 6 & (5) & 70 & (71) \\
\hline HOMO & (119) & $\pi+\mathrm{d}_{y z}$ & $\pi$ & -7.66 & $(-5.06)$ & 20 & (13) & 1 & (1) & 1 & (2) & 7 & (7) & 72 & (78) \\
\hline LUMO & (120) & $\sigma^{*}\left(\mathrm{~d}_{z 2}\right)$ & $\sigma^{*}\left(\mathrm{~d}_{z_{2}}\right)$ & -6.25 & $(-3.80)$ & 50 & (50) & 15 & (16) & 20 & (21) & 12 & (13) & 17 & (16) \\
\hline LUMO+1 & (121) & $\pi^{*^{z^{2}}}$ & $\pi^{*^{z^{2}}}$ & -5.67 & $(-3.04)$ & 6 & (5) & 0 & (0) & 0 & (0) & 1 & (1) & 94 & (95) \\
\hline LUMO + 2 & (122) & $\mathrm{d}_{x y}+\mathrm{n}$ & $\mathrm{d}_{x y}+\mathrm{n}$ & -5.16 & $(-2.64)$ & 41 & (43) & 0 & (0) & 0 & (0) & 0 & (0) & 58 & (57) \\
\hline $\mathrm{LUMO}+3$ & (123) & $\pi^{*}$ & $\pi^{*}$ & -4.74 & $(-2.14)$ & 9 & (7) & 0 & (0) & 0 & (1) & 1 & (1) & 91 & (92) \\
\hline
\end{tabular}




\section{CURRICULUM VITAE}

NAME: $\quad$ Karina Kornobis

ADDRESS: $\quad 1800 \mathrm{~S}$ 2nd Street A 41

Louisville, KY 40208

EDUCATION Ph.D., Chemistry

\& TRAINING: University of Louisville

$2008-2013$

M.Eng., Biotechnology

Wroclaw University of Technology

$2008-2013$

Summer Research Internship

The Centre for Theoretical and Computational Chemistry (CTCC)

Tromsø, Norway

2011 (12 weeks)

$3^{\text {rd }}$ International Workshop and School on

Time-Dependent Density Functional Theory

Benasque Center for Science, Spain

2008 (2 weeks)

Summer Internship

P.F. Jelfa S.A., Pharmaceutical Company, Jelenia Góra, Poland 2005 (4 weeks)

TEACHING: Graduate Teaching Assistant

Courses taught:

Introduction to Chemical Analysis I (Chem-207),

Introduction to Chemical Analysis II (Chem-208),

Organic Chemistry Laboratory II (Chem-344),

Computational Chemistry (Chem-450),

Physical Chemistry I (Chem-465) 


\section{PUBLICATIONS}

\section{REFEREED JOURNALS}

H. Solheim, K. Kornobis, K. Ruud, and P. M. Kozlowski, "Electronically Excited States of Vitamin $\mathrm{B}_{12}$ and Methylcobalamin: Theoretical Analysis of Absorption, CD, and MCD Data," J. Phys. Chem. B, vol. 115, pp. 737-748, 2011.

P. Lodowski, M. Jaworska, K. Kornobis, T. Andruniów and P. M. Kozlowski, "Electronic and Structural Properties of Low-lying Excited States of Vitamin $\mathrm{B}_{12}$," J. Phys. Chem. B, vol. 115, pp. 13304-13319, 2011.

K. Kornobis, N. Kumar, B. M. Wong, P. Lodowski, M. Jaworska, T. Andruniów, K. Ruud and P. M. Kozlowski, "Electronically Excited States of Vitamin $\mathrm{B}_{12}$ : Benchmark Calculations Including Time-Dependent Density Functional Theory and Correlated ab Initio Methods," J. Phys. Chem. A, vol. 115, pp. 1280-1292, 2012.

K. Kornobis, N. Kumar, P. Lodowski, M. Jaworska, P. Piecuch, J. J. Lutz, B. M. Wong and P. M. Kozlowski, "Electronic structure of the $S_{1}$ state in methylcobalamin: Insight from CASSCF/MC-XQDPT2, EOM-CCSD, and TD-DFT calculations," J. Comput. Chem., article first published online: 19 Jan 2013, DOI: 10.1002/jcc.23204.

K. Kornobis, K. Ruud and P. M. Kozlowski, "Cob(I)alamin: Insight Into the Nature of Electronically Excited States Elucidated via Quantum Chemical Computations and Analysis of Absorption, CD and MCD Data," J. Phys. Chem. A, vol. 117, pp. 863-876, 2013.

\section{WORKSHOPS AND CONFERENCES}

Q-Chem Workshop, Pittsburgh, PA, USA, 2009.

K. Kornobis and T. Andruniów, "Poster", $3^{\text {rd }}$ International Workshop and School on Time-Dependent Density Functional Theory, Benasque, Spain, 2008.

K. Kornobis and T. Andruniów, "Poster," in Modeling Interactions in Biomolecules III, Conference, Prague, Czech Republic, 2007. 